\title{
The Physics of Ultraperipheral Collisions at the LHC
}

\author{
Editors and Conveners: K. Hencken ${ }^{7,8}$, M. Strikman ${ }^{18}$, \\ R. Vogt ${ }^{11,19,20}$, P. Yepes ${ }^{22}$ \\ Contributors: A. J. Baltz ${ }^{1}$, G. Baur ${ }^{2}$, D. d'Enterria ${ }^{3}$, \\ L. Frankfurt ${ }^{4}$, F. Gelis ${ }^{5}$, V. Guzey ${ }^{6}$, K. Hencken ${ }^{7,8}$, \\ Yu. Kharlov ${ }^{9}$, M. Klasen ${ }^{10}$, S. R. Klein ${ }^{11}$, V. Nikulin ${ }^{12}$, \\ J. Nystrand ${ }^{13}$, I. A. Pshenichnov ${ }^{14,15}$, S. Sadovsky ${ }^{9}$, \\ E. Scapparone ${ }^{16}$, J. Seger ${ }^{17}$, M. Strikman ${ }^{18}$, M. Tverskoy ${ }^{12}$, \\ R. Vogt ${ }^{11,19,20}$, S. N. White ${ }^{1}$, U. A. Wiedemann ${ }^{21}$, P. Yepes ${ }^{22}$, \\ M. Zhalov ${ }^{12}$ \\ ${ }^{1}$ Physics Department, Brookhaven National Laboratory, Upton, NY, USA \\ ${ }^{2}$ Institut fuer Kernphysik, Forschungszentrum Juelich, Juelich, Germany \\ ${ }^{3}$ Experimental Physics Division, CERN, Geneva, Switzerland \\ ${ }^{4}$ Nuclear Physics Department, Tel Aviv University, Tel Aviv, Israel \\ ${ }^{5} \mathrm{CEA} / \mathrm{DSM} / \mathrm{SPhT}$, Saclay, France \\ ${ }^{6}$ Institut für Theoretische Physik II, Ruhr-Universität Bochum, Bochum, Germany \\ ${ }^{7}$ University of Basel, Basel, Switzerland \\ ${ }^{8}$ ABB Corporate Research, Baden-Daettwil, Switzerland \\ ${ }^{9}$ Institute for High Energy Physics, Protvino, Russia \\ ${ }^{10}$ Laboratoire de Physique Subatomique et de Cosmologie, Université Joseph \\ Fourier/CNRS-IN2P3, Grenoble, France \\ ${ }^{11}$ Nuclear Science Division, Lawrence Berkeley National Laboratory, Berkeley, USA \\ ${ }^{12}$ Petersburg Nuclear Physics Institute, Gatchina, Russia \\ ${ }^{13}$ Department of Physics and Technology, University of Bergen, Bergen, Norway \\ ${ }^{14}$ Frankfurt Institute for Advanced Studies, Frankfurt am Main, Germany \\ ${ }^{15}$ Institute for Nuclear Research, Russian Academy of Sciences, Moscow, Russia \\ ${ }^{16}$ INFN, Sezione di Bologna, Bologna, Italy \\ ${ }^{17}$ Physics Department, Creighton University, Omaha, NE, USA \\ ${ }^{18}$ Physics Department, Pennsylvania State University, State College, PA, USA \\ ${ }^{19}$ Physics Department, University of California at Davis, Davis, CA, USA \\ ${ }^{20}$ Lawrence Livermore National Laboratory, Livermore, CA, USA \\ ${ }^{21}$ Theory Division, CERN, Geneva, Switzerland \\ ${ }^{22}$ Physics and Astronomy Department, Rice University, Houston, TX, USA
}

\begin{abstract}
We discuss the physics of large impact parameter interactions at the LHC: ultraperipheral collisions (UPCs). The dominant processes in UPCs are photonnucleon (nucleus) interactions. The current LHC detector configurations can explore small $x$ hard phenomena with nuclei and nucleons at photon-nucleon center-of-mass energies above $1 \mathrm{TeV}$, extending the $x$ range of HERA by a factor of ten. In particular, it will be possible to probe diffractive and inclusive parton densities in nuclei using several processes. The interaction of small dipoles with protons and nuclei can be
\end{abstract}


investigated in elastic and quasi-elastic $J / \psi$ and $\Upsilon$ production as well as in high $t \rho^{0}$ production accompanied by a rapidity gap. Several of these phenomena provide clean signatures of the onset of the new high gluon density QCD regime. The LHC is in the kinematic range where nonlinear effects are several times larger than at HERA. Two-photon processes in UPCs are also studied. In addition, while UPCs play a role in limiting the maximum beam luminosity, they can also be used a luminosity monitor by measuring mutual electromagnetic dissociation of the beam nuclei. We also review similar studies at HERA and RHIC as well as describe the potential use of the LHC detectors for UPC measurements. 


\section{Introduction}

Contributed by: K. Hencken, M. Strikman, R. Vogt and P. Yepes

In 1924 Enrico Fermi, 23 at the time, proposed the equivalent photon method [1] which treated the moving electromagnetic fields of a charged particle as a flux of virtual photons. A decade later, Weizsäcker and Williams applied the method [2] to relativistic ions. Ultraperipheral collisions, UPCs, are those reactions in which two ions interact via their cloud of virtual photons. The intensity of the electromagnetic field, and therefore the number of photons in the cloud surrounding the nucleus, is proportional to $Z^{2}$. Thus these types of interactions are highly favored when heavy ions collide. Figure 1 shows a schematic view of an ultraperipheral heavy-ion collision. The pancake shape of the nuclei is due to Lorentz contraction.

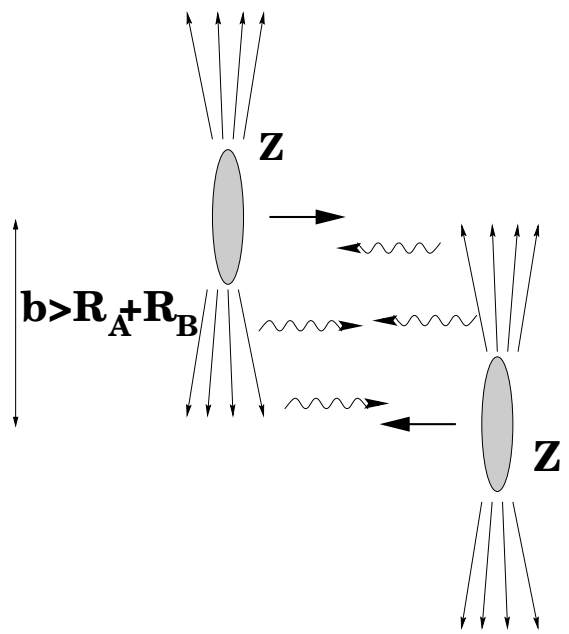

Figure 1. Schematic diagram of an ultraperipheral collision of two ions. The impact parameter, $b$, is larger than the sum of the two radii, $R_{A}+R_{B}$. Reprinted from Ref. [3] with permission from Elsevier.

Ultraperipheral photon-photon collisions are interactions where the radiated photons interact with each other. In addition, photonuclear collisions, where one radiated photon interacts with a constituent of the other nucleus, are also possible. The two processes are illustrated in Fig. 2(a) and (b). In these diagrams the nucleus that emits the photon remains intact after the collision. However, it is possible to have an ultraperipheral interaction in which one or both nuclei break up. The breakup may occur through the exchange of an additional photon, as illustrated in Fig. 2(c).

In calculations of ultraperipheral $A B$ collisions, the impact parameter is usually required to be larger than the sum of the two nuclear radii, $b>R_{A}+R_{B}$. Strictly speaking, an ultraperipheral electromagnetic interaction could occur simultaneously with a hadronic collision. However, since it is not possible to separate the hadronic and electromagnetic components in such collisions, the hadronic components are excluded by the impact parameter cut. 


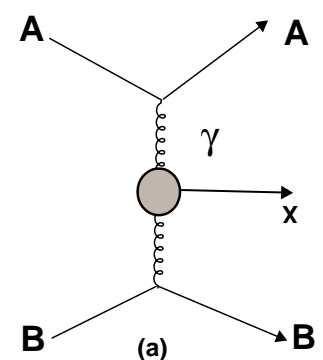

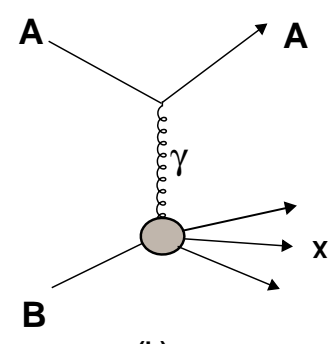

(b)

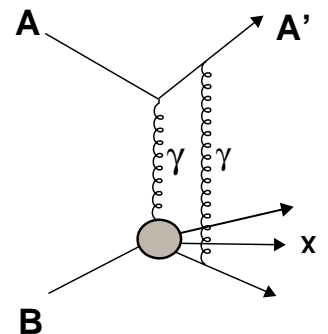

(c)

Figure 2. A schematic view of (a) an electromagnetic interaction where photons emitted by the ions interact with each other, (b) a photon-nuclear reaction in which a photon emitted by an ion interacts with the other nucleus, (c) photonuclear reaction with nuclear breakup due to photon exchange.

Photons emitted by ions are coherently radiated by the whole nucleus, imposing a limit on the minimum photon wavelength of greater than the nuclear radius. In the transverse plane, where there is no Lorentz contraction, the uncertainty principle sets an upper limit on the transverse momentum of the photon emitted by ion $A$ of $p_{T} \lesssim \hbar c / R_{A} \approx 28(330) \mathrm{MeV} / c$ for $\mathrm{Pb}(p)$ beams. In the longitudinal direction, the maximum possible momentum is multiplied by a Lorentz factor, $\gamma_{L}$, due to the Lorentz contraction of the ions in that direction: $k \lesssim \hbar c \gamma_{L} / R_{A}$. Therefore the maximum $\gamma \gamma$ collision energy in a symmetric $A A$ collision is $2 \hbar c \gamma_{L} / R_{A}$, about $6 \mathrm{GeV}$ at the Relativistic Heavy Ion Collider (RHIC) and $200 \mathrm{GeV}$ at the Large Hadron Collider (LHC).

The cross section for two-photon processes is [4]

$$
\sigma_{X}=\int d k_{1} d k_{2} \frac{d L_{\gamma \gamma}}{d k_{1} d k_{2}} \sigma_{X}^{\gamma \gamma}\left(k_{1}, k_{2}\right),
$$

where $\sigma_{X}^{\gamma \gamma}\left(k_{1}, k_{2}\right)$ is the two-photon production cross section of final state $X$ and $d L_{\gamma \gamma} / d k_{1} d k_{2}$ is the two-photon luminosity,

$$
\frac{d L_{\gamma \gamma}}{d k_{1} d k_{2}}=\int_{b>R_{A}} \int_{r>R_{A}} d^{2} b d^{2} r \frac{d^{3} N_{\gamma}}{d k_{1} d^{2} b} \frac{d^{3} N_{\gamma}}{d k_{2} d^{2} r},
$$

where $d^{3} N_{\gamma} / d k d^{2} r$ is the photon flux from a charge $Z$ nucleus at a distance $r$. The two-photon cross section can also be written in terms of the two-photon center-of-mass energy, $W_{\gamma \gamma}=\sqrt{s_{\gamma \gamma}}=\sqrt{4 k_{1} k_{2}}$ by introducing the delta function $\delta\left(s_{\gamma \gamma}-4 k_{1} k_{2}\right)$ to integrate over $k_{1}$ and changing the integration variable from $k_{2}$ to $W_{\gamma \gamma}$ so that

$$
\sigma_{X}=\int \frac{d L_{\gamma \gamma}}{d W_{\gamma \gamma}} W_{\gamma \gamma} \sigma_{X}^{\gamma \gamma}\left(W_{\gamma \gamma}\right) .
$$

(Note that we use $W$ and $\sqrt{s}$ for the center-of-mass energy interchangeably throughout the text.

The two-photon luminosity in Eq. (2) can be multiplied by the ion-ion luminosity, $L_{A A}$, yielding an effective two-photon luminosity, $d L_{\gamma \gamma}^{\text {eff }} / d W_{\gamma \gamma}$, which can be directly compared to two-photon luminosities at other facilities such as $e^{+} e^{-}$or $p p$ colliders [5]. 
Figure 3 shows the two-photon effective luminosities for various ion species and protons as a function of $W_{\gamma \gamma}$ for the LHC (left) and for RHIC (right) [3]. Note the difference in energy scales between the LHC and RHIC. The ion collider luminosities are also compared to the $\gamma \gamma$ luminosity at LEP II. The LHC will have significant energy and luminosity reach beyond LEP II and could be a bridge to $\gamma \gamma$ collisions at a future linear $e^{+} e^{-}$collider. Indeed, the LHC two-photon luminosities for light ion beams are higher than available elsewhere for energies up to $W_{\gamma \gamma} \approx 500 \mathrm{GeV} / c^{2}$.
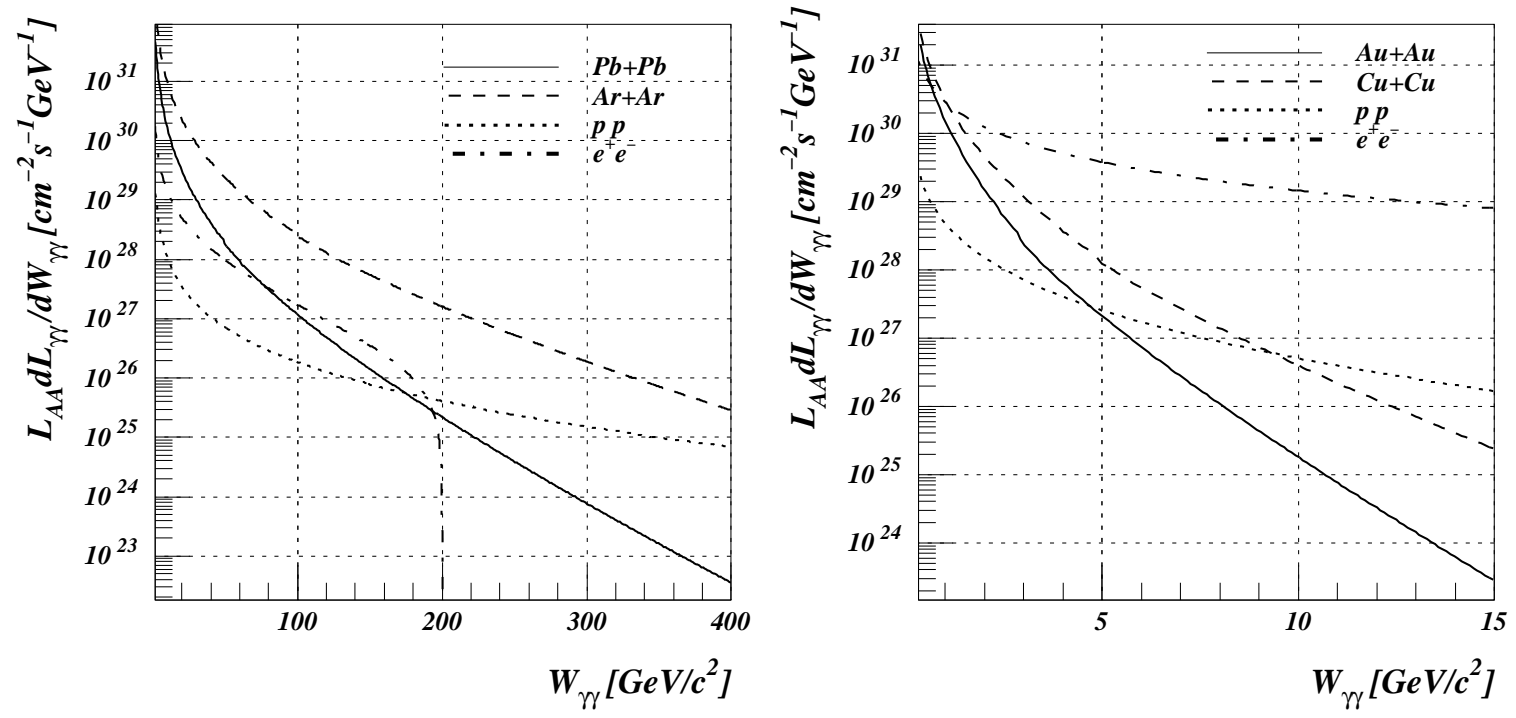

Figure 3. Effective $\gamma \gamma$ luminosity at LHC (left) and RHIC (right) for different ion species and protons as well as at LEP II. In $p p$ and $e^{+} e^{-}$collisions, $L_{A A}$ corresponds to the $p p$ or $e^{+} e^{-}$luminosity. Reprinted from Ref. [3] with permission from Elsevier.

The photoproduction cross section can also be factorized into the product of the photonuclear cross section and the photon flux, $d N_{\gamma} / d k$,

$$
\sigma_{X}=\int d k \frac{d N_{\gamma}}{d k} \sigma_{X}^{\gamma}(k)
$$

where $\sigma_{X}^{\gamma}(k)$ is the photonuclear cross section.

The photon flux used to calculate the two-photon luminosity in Eq. (2) and the photoproduction cross section in Eq. (4) is given by the Weizsäcker-Williams method [8]. The flux is evaluated in impact parameter space, as is appropriate for heavy-ion interactions $[9,10]$. The flux at distance $r$ away from a charge $Z$ nucleus is

$$
\frac{d^{3} N_{\gamma}}{d k d^{2} r}=\frac{Z^{2} \alpha w^{2}}{\pi^{2} k r^{2}}\left[K_{1}^{2}(w)+\frac{1}{\gamma_{L}^{2}} K_{0}^{2}(w)\right]
$$

where $w=k r / \gamma_{L}$ and $K_{0}(w)$ and $K_{1}(w)$ are modified Bessel functions. The photon flux decreases exponentially above a cutoff energy determined by the size of the nucleus. In the laboratory frame, the cutoff is $k_{\max } \approx \gamma_{L} \hbar c / R_{A}$. In the rest frame of the target nucleus, the cutoff is boosted to $E_{\max }=\left(2 \gamma_{L}^{2}-1\right) \hbar c / R_{A}$, about $500 \mathrm{GeV}$ at RHIC and $1 \mathrm{PeV}(1000 \mathrm{TeV})$ at the LHC. The photon flux for heavy ions at RHIC and the LHC 


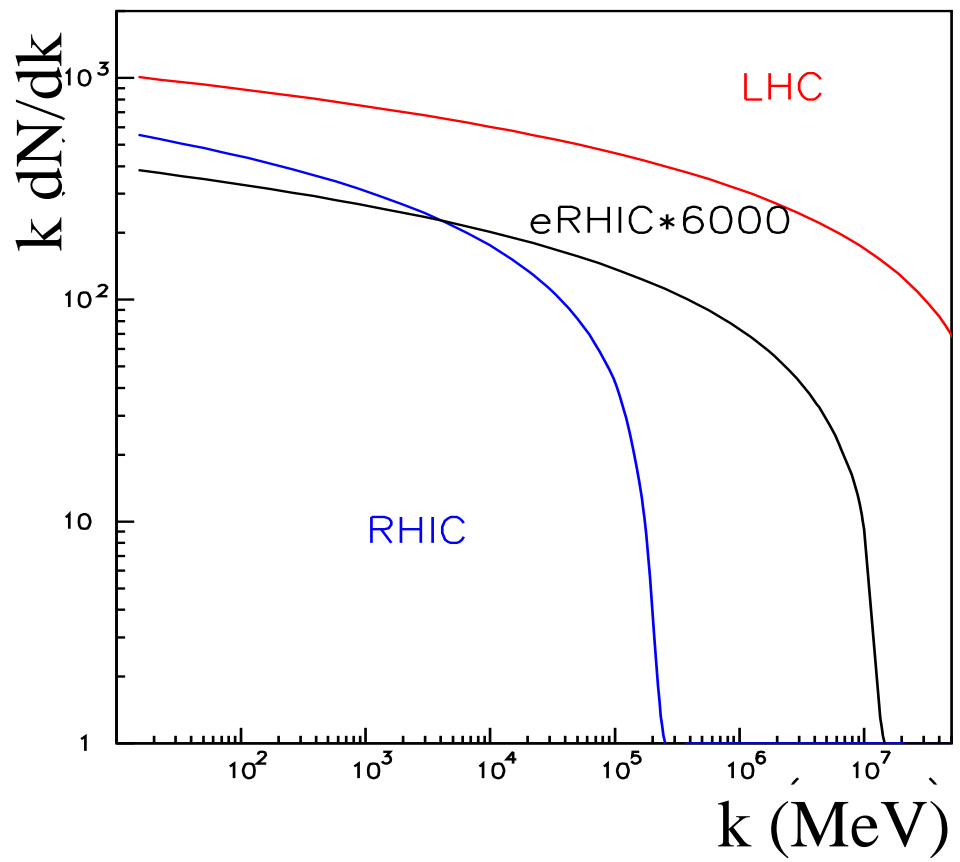

Figure 4. The photon flux from $\sqrt{s_{N N}}=200 \mathrm{GeV} \mathrm{Au}+\mathrm{Au}$ collisions at RHIC and $\sqrt{s_{N N}}=5.5 \mathrm{TeV} \mathrm{Pb}+\mathrm{Pb}$ collisions at the LHC, compared with that expected for 10 $\mathrm{GeV}+100 \mathrm{GeV}$ eAu collisions at the proposed eRHIC [6,7]. The eRHIC curve has been multiplied by 6000 to account for improved gold beam parameters at eRHIC. $k$ is given in the rest frame of the target nucleus in all three cases. Modified from Ref. [20] with permission from World Scientific.

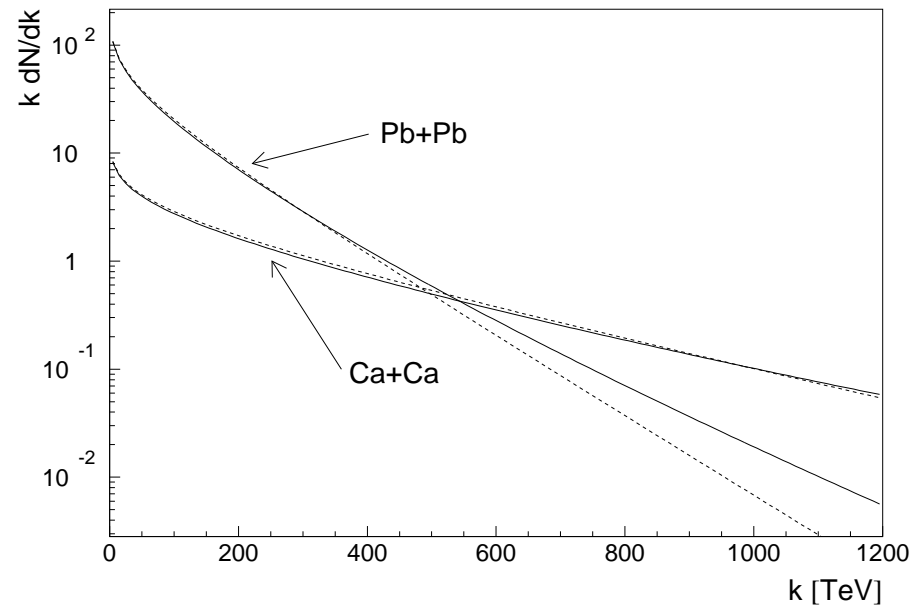

Figure 5. The equivalent photon spectrum in $\mathrm{Pb}+\mathrm{Pb}$ and $\mathrm{Ca}+\mathrm{Ca}$ interactions at the LHC, evaluated in the rest frame of the target nucleus. The solid curves correspond to the numerical result of Eq. (25) while the dashed curves are the analytical result, Eq. (6). 
is depicted in Fig. 4. Also shown, for comparison, is the flux for the proposed electronion collider at RHIC, eRHIC $\ddagger$. The $e A$ flux has been multiplied by 6000 to include the expected luminosity increase for eRHIC relative to RHIC. Although both RHIC and eRHIC are high luminosity $\gamma A$ colliders, the LHC has an energy reach far beyond other existing or planned machines.

In these collisions, the accelerated ion is surrounded by a cloud of almost real photons of virtuality $\left|q^{2}\right|<\left(\hbar c / R_{A}\right)^{2}$ where $R_{A}$ is the nuclear radius. The virtuality, less than $(60 \mathrm{MeV})^{2}$ for nuclei with $A>16$, can be neglected. Since the photon interaction is long range, photons can interact with partons in the opposite nucleus even when the nuclei themselves do not interpenetrate. Because the photon energies are less than those of the nucleons, these photonuclear interactions have a smaller average center-ofmass energy than hadronic parton-parton collisions. However, even though the energy is smaller, coherent photon beams have a flux proportional to the square of the nuclear charge, $Z$, enhancing the rates relative to those of photoproduction in $p p$ collisions. Although the photons are nearly real, their high energy allows interactions at high virtualities, $Q^{2}$, in the photon-parton center of mass. Thus, massive vector mesons, heavy quarks and jets can be produced with high rates in UPCs.

Table 1 shows the nucleon-nucleon center-of-mass energies, $\sqrt{s_{N N}}$, the beam energies in the center-of-mass frame, $E_{\text {beam }}$, Lorentz factors, $\gamma_{L}, k_{\max }$, and $E_{\max }$, as well as the corresponding maximum $\gamma A$ center-of-mass energy per nucleon, $\sqrt{s_{\gamma N}}=$ $W_{\gamma N}=\left[2 k_{\max } \sqrt{s_{N N}}\right]^{1 / 2}=\sqrt{2 E_{\max } m_{p}}$. We give the appropriate default kinematics for $A A, p A$ and $p p$ collisions at the LHC. The resulting values are compared to the fixedtarget kinematics of the SPS as well as the proton and gold beams at the RHIC collider. In fixed-target kinematics, $E_{\max }$ is obtained from $\gamma_{L} \hbar c / R_{A}$ with the Lorentz boost of the beam while $k_{\max }$ is calculated with $\gamma_{L}=\sqrt{s_{N N}} / 2 m_{p}$. In $p A$ collisions, the photon field of the nucleus is stronger so that the interacting photon almost always comes from the nucleus. Note also that the LHC $p A$ results are calculated in the center-of-mass kinematics although the different $Z / A$ ratios in asymmetric collisions mean that the beams have different velocities. In $p p$ collisions, we use $r_{p}=0.6 \mathrm{fm}$ to calculate $E_{\max }$ and $k_{\max }$. Note that, at high energy, the maximum photon energy is $25 \%$ of the proton energy for this choice of $r_{p}$, significantly increasing the probability of proton breakup. More work is required to understand the usable $p p$ luminosity in this case.

We have also included the best available estimates [11-13] of the beam-beam luminosities for $A A$ and $p p$ collisions in Table 1 to aid rate calculations. No beambeam luminosity is given for the fixed-target kinematics of the SPS. Only an estimate of the initial LHC $p A$ luminosities are given [12]. The maximum machine luminosities are applicable to CMS and ATLAS. Unfortunately the interaction rate in ALICE is limited to $200 \mathrm{kHz}$. Therefore its maximum $p p$ luminosities are significantly lower. The luminosities for collision modes other than $p p$ and $\mathrm{Pb}+\mathrm{Pb}$ are unofficial and, as such, are subject to revision.

$\ddagger$ We give estimates for the $10 \mathrm{GeV}+100 \mathrm{GeV}$ version of the proposed electron-ion collider eRHIC. 
Table 1. Pertinent parameters and kinematic limits for some projectile-target combinations at several accelerators. We first give the luminosities and the $N N$ collision kinematics, the nucleon-nucleon center-of-mass energies, $\sqrt{s_{N N}}$, the corresponding beam energies, $E_{\text {beam, }}$ and the Lorentz factors, $\gamma_{L}$. We then present the photon cutoff energies in the center-of-mass frame, $k_{\max }$, and in the nuclear rest frame, $E_{\max }$, as well as the equivalent maximum photon-nucleon and photon-photon center-of-mass energies, $\sqrt{s_{\gamma N}^{\max }}$ and $\sqrt{s_{\gamma \gamma}^{\max }}$ respectively.

\begin{tabular}{|c|c|c|c|c|c|c|c|c|}
\hline$A B$ & $\begin{array}{c}L_{A B} \\
\left(\mathrm{mb}^{-1} \mathrm{~s}^{-1}\right)\end{array}$ & $\begin{array}{c}\sqrt{s_{N N}} \\
(\mathrm{TeV})\end{array}$ & $\begin{array}{c}E_{\text {beam }} \\
(\mathrm{TeV})\end{array}$ & $\gamma_{L}$ & $\begin{array}{c}k_{\max } \\
(\mathrm{GeV})\end{array}$ & $\begin{array}{c}E_{\max } \\
(\mathrm{TeV})\end{array}$ & $\begin{array}{c}\sqrt{s_{\gamma N}^{\max }} \\
(\mathrm{GeV})\end{array}$ & $\begin{array}{c}\sqrt{s_{\gamma \gamma}^{\max }} \\
(\mathrm{GeV})\end{array}$ \\
\hline \multicolumn{8}{|c|}{ SPS } \\
\hline $\mathrm{In}+\mathrm{In}$ & - & 0.017 & 0.16 & 168 & 0.30 & $5.71 \times 10^{-3}$ & 3.4 & 0.7 \\
$\mathrm{~Pb}+\mathrm{Pb}$ & - & 0.017 & 0.16 & 168 & 0.25 & $4.66 \times 10^{-3}$ & 2.96 & 0.5 \\
\hline \multicolumn{8}{|c|}{ RHIC } \\
\hline $\mathrm{Au}+\mathrm{Au}$ & 0.4 & 0.2 & 0.1 & 106 & 3.0 & 0.64 & 34.7 & 6.0 \\
$p p$ & 6000 & 0.5 & 0.25 & 266 & 87 & 46.6 & 296 & 196 \\
\hline \multicolumn{8}{|c|}{ LHC } \\
\hline $\mathrm{O}+\mathrm{O}$ & 160 & 7 & 3.5 & 3730 & 243 & 1820 & 1850 & 486 \\
$\mathrm{Ar}+\mathrm{Ar}$ & 43 & 6.3 & 3.15 & 3360 & 161 & 1080 & 1430 & 322 \\
$\mathrm{~Pb}+\mathrm{Pb}$ & 0.42 & 5.5 & 2.75 & 2930 & 81 & 480 & 950 & 162 \\
\hline$p \mathrm{O}$ & 10000 & 9.9 & 4.95 & 5270 & 343 & 3620 & 2610 & 686 \\
$p \mathrm{Ar}$ & 5800 & 9.39 & 4.7 & 5000 & 240 & 2400 & 2130 & 480 \\
$p \mathrm{~Pb}$ & 420 & 8.8 & 4.4 & 4690 & 130 & 1220 & 1500 & 260 \\
\hline$p p$ & $10^{7}$ & 14 & 7 & 7455 & 2452 & 36500 & 8390 & 4504 \\
\hline
\end{tabular}

The total photon flux striking the target nucleus is the integral of Eq. (5) over the transverse area of the target for all impact parameters subject to the constraint that the two nuclei do not interact hadronically. A reasonable analytic approximation for $A B$ collisions is given by the photon flux integrated over radii larger than $R_{A}+R_{B}$. The analytic photon flux is

$$
\frac{d N_{\gamma}}{d k}=\frac{2 Z^{2} \alpha}{\pi k}\left[w_{R}^{i A} K_{0}\left(w_{R}^{i A}\right) K_{1}\left(w_{R}^{i A}\right)-\frac{\left(w_{R}^{i A}\right)^{2}}{2}\left(K_{1}^{2}\left(w_{R}^{i A}\right)-K_{0}^{2}\left(w_{R}^{i A}\right)\right)\right]
$$

where $w_{R}^{A A}=2 k R_{A} / \gamma_{L}$ and $w_{R}^{p A}=k\left(r_{p}+R_{A}\right) / \gamma_{L}$. This analytic flux is compared to the full numerical result, Eq. (25), in Fig. 5 for $\mathrm{Pb}+\mathrm{Pb}$ and $\mathrm{Ca}+\mathrm{Ca}$ collisions at the LHC. The numerical result gives a harder photon spectrum for $\mathrm{Pb}+\mathrm{Pb}$ collisions at the same $k$. On the other hand, there is little difference between the two results for $\mathrm{Ca}+\mathrm{Ca}$ collisions. (Note that there is some discussion of $\mathrm{Ca}+\mathrm{Ca}$ interactions in the text since some initial UPC studies were done before argon was chosen over calcium beams. While $A=40$ for both, the $Z$ is different, changing both the flux and the energy range for Ar relative to Ca.)

Since photonuclear rates increase more slowly than $A^{2}$, there may be advantages 
in $p A$ relative to $A A$ collisions. As presented above, event rates in ultraperipheral $A A$ collisions depend both on the photon flux, $d N_{\gamma} / d k$, which scales as $Z^{2}$ in photoproduction and $Z^{4}$ in two-photon processes, and on the beam-beam luminosity, $L_{A B}$. Lighter ions are favored for many UPCs since the higher luminosities [13] compensate for the larger $Z$ in lower luminosity $\mathrm{Pb}+\mathrm{Pb}$ collisions. In the case of $p \mathrm{~Pb}$ collisions, $L_{p \mathrm{~Pb}}$ is two orders of magnitude higher than $L_{\mathrm{PbPb}}$. While it is more probable for the photon to be emitted by the ion and interact with the proton $(\gamma p)$, it could also be emitted by the proton and interact with the ion $(\gamma \mathrm{Pb})$. The relevant figure of merit is the effective photon-nucleus luminosity, $L_{A B}\left(k d N_{\gamma} / d k\right)$. The left-hand side of Fig. 6 compares $L_{A B}\left(k d N_{\gamma} / d k\right)$ for $\gamma p$ (solid) and $\gamma \mathrm{Pb}$ (dashed) collisions in $p \mathrm{~Pb}$ interactions to the case where the photon is emitted from the ion in lower energy and lower luminosity $\mathrm{Pb}+\mathrm{Pb}$ collisions. The effective $\gamma p$ luminosities are enhanced by the larger $p \mathrm{~Pb}$ luminosity. Thus photonuclear processes on protons can be studied at energies beyond the HERA range so that e.g. the energy dependence of $\Upsilon$ production can be measured.

As shown on the right-hand side of Fig. 6, the two-photon luminosities for $p \mathrm{~Pb}$ collisions at the LHC are only slightly lower than those for $\mathrm{Pb}+\mathrm{Pb}$ collisions at low $W_{\gamma \gamma}$, and even become higher for $W_{\gamma \gamma}>250 \mathrm{GeV}$ due to the larger $p \mathrm{~Pb}$ energy. While these luminosities are lower than the $p p$ luminosities, heavy ions suppress the diffractive background. The potential for the discovery of new physics in $p A$ is rather limited at low $W_{\gamma \gamma}$ but there are again some advantages at higher $W_{\gamma \gamma}$. Thus two-photon studies are still possible, as are electroweak studies. When the photon is emitted from the proton, the luminosity could be further enhanced by allowing for inelastic processes such as proton breakup $[14,15]$.
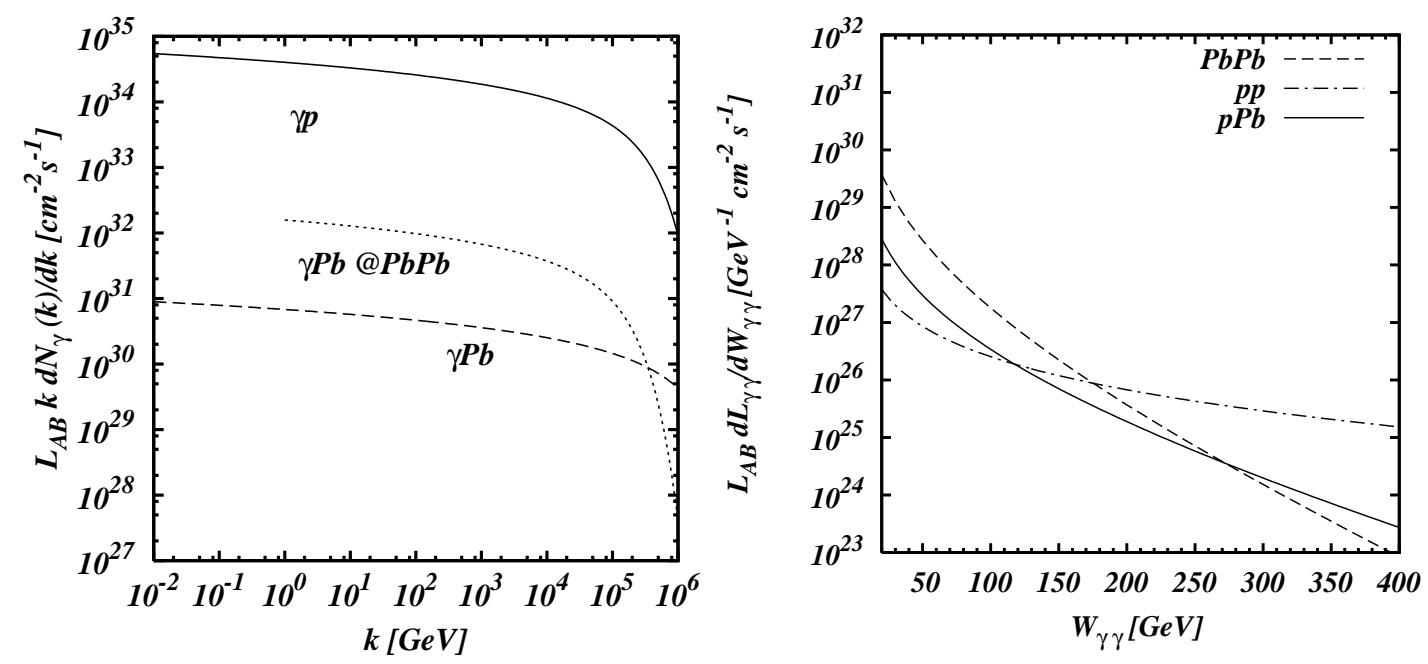

Figure 6. (left) The effective $\gamma A$ luminosity, $L_{A B}\left(k d N_{\gamma} / d k\right)$, is shown for the cases where the photon is emitted from the proton $(\gamma \mathrm{Pb})$ and the ion $(\gamma p)$ as well as when the photon is emitted from the ion in a $\mathrm{Pb}+\mathrm{Pb}$ collision $(\gamma \mathrm{Pb} @ \mathrm{~Pb}+\mathrm{Pb})$. (right) The photon-photon luminosities, $L_{A B}\left(d L_{\gamma \gamma} / d W_{\gamma \gamma}\right)$, are compared for $p p, p \mathrm{~Pb}$ and $\mathrm{Pb}+\mathrm{Pb}$ collisions at the LHC. 
The physics of UPCs has been reviewed by a number of groups. The first comprehensive study was by Baur and Bertulani in 1988 [4]. More recent reviews are by Krauss, Greiner and Soff [16], Baur and collaborators [3], and by Bertulani, Klein and Nystrand [17]. The LHC FELIX proposal also did much to advance UPCs [18], as did a UPC workshop in Erice, Italy [19,20]. Useful related material is discussed in a recent photoproduction review by Butterworth and Wing [21].

The remainder of this introduction will address some of the physics issues that can be studied with UPCs. A few of these will be described in more detail in the body of the report.

\subsection{Physics of photonuclear reactions}

Data from HERA show that the gluon and sea quark distributions rise quickly as their momentum fraction $x$ drops. At small enough $x$, the growth of the proton parton densities may decrease proportionally with $\ln (1 / x)$. The increase of the parton densities is regulated by phenomena such as shadowing, recombination reactions, e.g. $g g \rightarrow g$, as well as possible tunneling between different QCD vacua that are suppressed at large $x$. These phenomena are most significant in the central core of a nucleon. Scattering off the periphery of the nucleon will dominate at small $x$, causing the cross section to increase asymptotically as fast as $\propto \ln ^{3}(1 / x)$ [22]. The large diffractive gluon densities observed at HERA demonstrate nonlinear effects for squared momentum transfer of the virtual photon of up to $Q^{2} \sim 4 \mathrm{GeV}^{2}$ at the smallest $x$ values studied, $x \sim 10^{-4}$. At the LHC, these QCD phenomena should be visible at larger $x$ in central collisions of both protons and heavy ions.

Studies of small $x$ deep inelastic scattering (DIS) at HERA substantially improved our understanding of strong interactions at high energies. There are several key findings of HERA in this field. Rapid growth of the small $x$ parton densities was observed over a wide range of $Q^{2}$. A significant probability for hard diffraction was seen, consistent with approximate scaling and a logarithmic $Q^{2}$ dependence ("leading-twist" dominance). HERA also found a new class of hard exclusive processes - light vector meson production at large $Q^{2}$ and heavy $Q \bar{Q}$ vector mesons at all $Q^{2}$. These processes are described by the QCD factorization theorem $[23,24]$ and related to the generalized parton distributions in the target. In the small $x$ limit, they can be calculated for zero squared momentum transfer, $t$, using standard parton distributions. This new class of interactions probes small $q \bar{q}$ dipole interactions with hadrons. The $t$-dependence provides direct information on the gluon distribution of hadrons in the transverse plane as a function of $x$.

Combined analyses of inclusive DIS and hard vector meson production suggest that the strength of the interactions, especially in channels where a hard probe directly couples to low $x$ gluons, approaches the maximum possible strength - the black disk regime $(\mathrm{BDR})$ - for $Q^{2} \leq 4 \mathrm{GeV}^{2}$. This conclusion is confirmed by studies of hard inclusive diffraction $[22]$.

However, the $Q^{2}$ range over which the black disk regime holds is relatively small, 
with even smaller values for processes where a hard probe couples to a $q \bar{q}$ dipole with $Q^{2} \sim 1 \mathrm{GeV}^{2}$, making it difficult to separate perturbative from nonperturbative effects and draw unambiguous conclusions.

The interaction regime where hard probes of small target $x$ occur with high probability should be a generic feature of strong interactions at high energies. This feature is related to high gluon densities, reached for any target at sufficiently small $x$. Extended targets are expected to reach this high density regime at substantially higher $x$. At very high gluon density, even the notion of inclusive parton densities is ill-defined.

The onset of the BDR corresponds to a drastic departure from the linear regime of QCD. Observing the onset of nonlinear QCD dynamics at small $x$ would be of great importance. The problems which emerge in the BDR kinematics can be visualized by considering DIS interactions and exclusive diffractive processes in the language of small dipoles interacting with the target. In the leading-log approximation, the inelastic quark-antiquark (gluon-gluon) dipole-hadron cross section for a dipole of size $d$ has the form $[25-27]$

$$
\sigma_{\operatorname{dip} h}\left(s_{\operatorname{dip} h}, d^{2}\right)=\frac{\pi^{2}}{4} C_{F}^{2} d^{2} \alpha_{s}\left(Q_{\text {eff }}^{2}\right) x g\left(x, Q_{\text {eff }}^{2}\right)
$$

where $x=Q_{\text {eff }}^{2} / s_{\operatorname{dip} h}$ and $s_{\operatorname{dip} h}$ is the square of the dipole-hadron center-of-mass energy. Here $C_{F}^{2}$ is the Casimir operator, equal to $4 / 3$ for $q \bar{q}$ and 3 for $g g, \alpha_{s}\left(Q_{\text {eff }}^{2}\right)$ is the leading order (LO) strong coupling constant and $g\left(x, Q_{\text {eff }}^{2}\right)$ is the LO gluon density in the target. The coupling constant and the gluon density are evaluated at $Q_{\text {eff }}^{2} \propto d^{-2}$. Since the gluon densities increase at small $x$, the cross section in Eq. (7) ultimately becomes larger than allowed by the unitarity constraint, $\pi r_{h}^{2}$, where $r_{h}$ is the transverse radius of the gluon distribution in the hadron at the corresponding $x$. Since the unitarity bound corresponds to complete absorption at impact parameters $b \leq r_{h}$, the resulting diffractive cross section reflects absorption at small $b$. If the regime of complete absorption at $b \leq r_{h}$ is reached, the diffractive absorption cross section becomes nearly equal to the inelastic scattering cross section. At sufficiently high energies, the small $x$ gluon fields resolved by the small color dipole become so strong that the dipole cannot propagate through extended nuclear media without absorption, signaling the breakdown of the linear scaling regime of Eq. (7) and the onset of the BDR.

In the dipole picture, a high energy photon can be considered to be a superposition of large and small size dipoles. Smaller and smaller dipoles begin to interact in the BDR with increasing energy. Photons contain more small dipoles than hadrons such as pions, leading to faster growth of $\sigma_{\text {tot }}(\gamma p)$ than given by the Froissart bound for hadrons. Thus real photon interactions are sensitive to these small dipoles. As a result, a number of theoretical issues concerning the onset of the BDR can be studied using UPCs. The energy scale at which the dipole-target cross section in Eq. (7) is tamed by the unitarity constraint near the BDR and no longer undergoes rapid growth is unknown, as is the energy dependence of the cross section. The energy at which the dipole cross section makes the transition from color transparency (no screening) to color opacity (strong screening) and, ultimately, the BDR also needs to be determined. Answers may be 
found by selecting processes where gluons interact directly. High gluon densities may be achieved at lower energies using nuclei, as we now discuss.

To reach the regime where Eq. (7) breaks down, measurements need to be extended to higher energies, smaller $x$, and to higher gluon densities, at the same energy and $x$, using nuclei. Nuclear beams were discussed for HERA [28] but will not be implemented. Studies of small $x$ physics at the LHC using hadronic $p p$ or $p A$ collisions will be rather difficult because, at central rapidities, the backgrounds due to multiple hard collisions will likely prevent measurements at virtualities less than $Q_{\text {eff }}^{2} \sim 100-200 \mathrm{GeV}^{2}$. Although the fragmentation region at forward rapidity, with smaller backgrounds, is likely beyond the acceptance of the currently planned detectors, some small $x$ studies using the CMS forward hadron calorimeter, HF, or CASTOR have been performed $[29,30]$. Thus, instead of using $e A$ collisions to reach the small $x$ regime, many of the approaches used at HERA could be implemented at the LHC using UPCs in both $A A$ and $p A$ collisions.

A primary focus of UPC studies in $A B$ and $p A$ collisions is on hard interactions in the kinematics which probe high gluon densities in nucleons and nuclei. Hard scatterings on nuclear targets will extend the low $x$ range of previous studies by nearly three orders of magnitude. In $p A$ collisions, the HERA $x$ range could be extended to an order of magnitude smaller $x$. Thus all three HERA highlights: gluon density measurements, gluon-induced hard diffraction, and exclusive $J / \psi$ and $\Upsilon$ production can be studied in ultraperipheral $p A$ and $A B$ collisions. Figure 7 shows the $x$ and $Q^{2}$ ranges covered by UPCs at the LHC. For comparison, the kinematic range of both $Z^{0}$ production in $p p$ collisions at the LHC and the nuclear structure function at eRHIC are also shown. The $x$ range of $e p$ collisions at eRHIC is a factor of $\sim 30$ lower than at HERA for the same $p_{T}$. In the remainder of the introduction, we summarize some of the possible UPC measurements that could further our understanding of small $x$ dynamics.

\section{Measurements of parton distributions in nuclei/nucleons}

The studies in Section 4 will demonstrate that hard ultraperipheral collisions investigate hard photon-nucleus (proton) collisions at significantly higher energies than at HERA. The dominant process is photon-gluon fusion to two jets with leading light or heavy quarks, $\gamma g \rightarrow$ jet $_{1}+$ jet $_{2}$, fixing the gluon densities in protons/nuclei. The LHC rates will be high enough to measure dijets and $c$ and $b$ quarks, probing the gluon distribution at $x \sim 5 \times 10^{-5}$ for $p_{T} \geq 6 \mathrm{GeV} / c[31]$.

The virtualities that can be probed in UPCs will be much higher than those reached in lepton-nucleon/nucleus interactions. The larger $x$ range and direct gluon couplings will make these measurements competitive with those at HERA and the planned eRHIC as a way to probe nonlinear effects. Indeed if it is possible to go down to $p_{T} \sim 5 \mathrm{GeV} / c$, the nonlinear effects in UPCs would be a factor of six higher than at HERA and a factor of two larger than at eRHIC [31]. An example of the $b$ quark rate in the ATLAS detector [31] is presented in Fig. 8. 


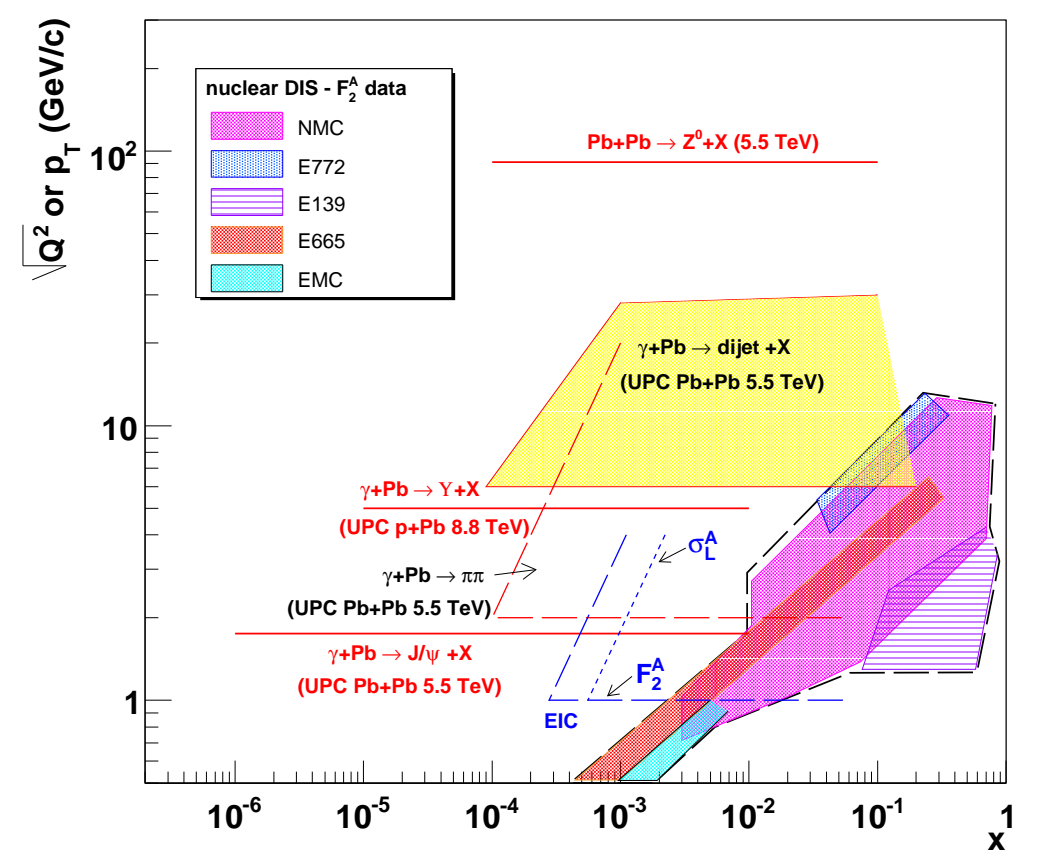

Figure 7. The kinematic range in which UPCs at the LHC can probe gluons in protons and nuclei in quarkonium production, dijet and dihadron production. The $Q$ value for typical gluon virtuality in exclusive quarkonium photoproduction is shown for $J / \psi$ and $\Upsilon$. The transverse momentum of the jet or leading pion sets the scale for dijet and $\pi \pi$ production respectively. For comparison, the kinematic ranges for $J / \psi$ at RHIC, $F_{2}^{A}$ and $\sigma_{L}^{A}$ at eRHIC and $Z^{0}$ hadroproduction at the LHC are also shown.

\section{Hard diffraction}

One of the cleanest signals of the proximity of the BDR is the ratio of the diffractive to total cross sections. In the cases we discuss, rapidity gap measurements will be straightforward in both ATLAS and CMS. If the diffractive rates are $\sim 20 \%$ of the total rate, as expected in current models, the statistics will be sufficient for inclusive measurements over most of the $x$ range. (Note that a $20 \%$ diffractive probability at $p_{T} \geq 5 \mathrm{GeV} / c$ suggests a $\sim 40 \%$ diffractive probability at $p_{T} \sim 2 \mathrm{GeV} / c$.) Production of two pions with $p_{T} \geq 2 \mathrm{GeV} / c$ will probe still further into the low $x$ regime, albeit at slightly higher $x$, see Fig. 7 .

\section{Exclusive quarkonium production}

Although calculations of the absolute cross section do involve significant highertwist corrections, the strong increase in the $J / \psi$ photoproduction cross section at HERA clearly indicates that heavy quarkonia are produced via coupling to small $x$ gluon fields. Thus $J / \psi$ and $\Upsilon$ photoproduction provide one of the cleanest tests of small $q \bar{q}$ dipole interactions with gluon fields. In the case of nuclear targets, several channels will be accessible: coherent processes, $\gamma A \rightarrow V A$; quasi-elastic processes, $\gamma A \rightarrow V A^{\prime}$; and rapidity gap processes such as large- $t$ light vector meson production, $\gamma A \rightarrow V X$.

A highly nontrivial prediction of QCD is that, at sufficiently high energies, even small dipoles should be strongly absorbed by extended targets both due to leading-twist 


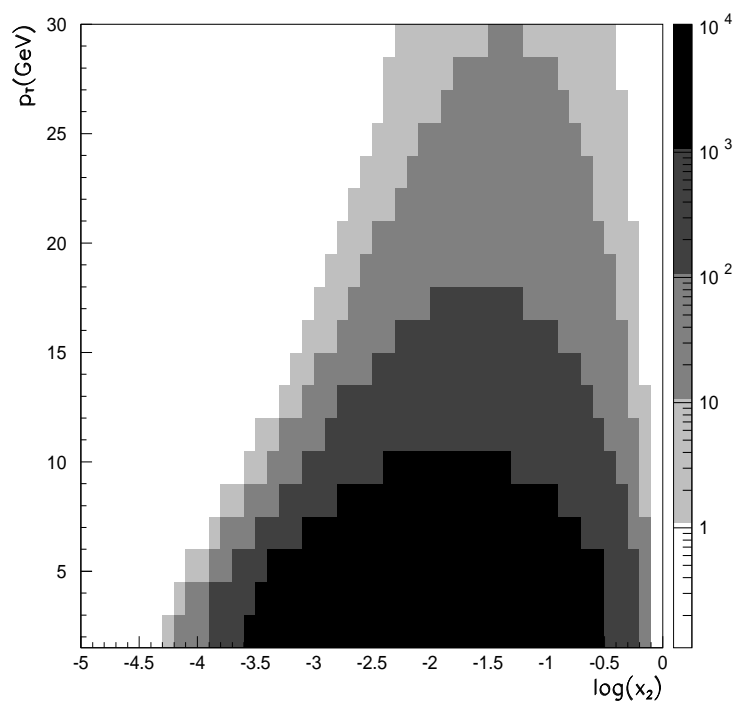

Figure 8. The rate for inclusive $b \bar{b}$ photoproduction for a one month $\mathrm{LHC} \mathrm{Pb}+\mathrm{Pb}$ run at $0.42 \times 10^{27} \mathrm{~cm}^{-2} \mathrm{~s}^{-1}$. Rates are in counts per bin of $\pm 0.25 x_{2}$ and \pm 0.75 $\mathrm{GeV}$ in $p_{T}$. From Ref. [31]. Copyright 2006 by the American Physical Society (http://link.aps.org/abstract/PRL/v96/e082001).

gluon shadowing and higher-twist multiple dipole rescattering. The $A$ dependence of the coherent and quasi-elastic reactions, both change by $A^{-2 / 3}$ when going from weak absorption to the regime of strong absorption, as we now illustrate. The coherent dipole scattering cross section is $\propto A^{4 / 3}$ in the weak absorption impulse approximation (a combination of $A^{2}$ from coherence at $t=0$ and $A^{-2 / 3}$ from the integral over $t$ ) and $\propto A^{2 / 3}$ for strong absorption over the surface area of the target. Likewise, the quasielastic $A$ dependence varies between $A$ (weak absorption: volume emission) and $A^{1 / 3}$ (strong absorption: edge emission).

Dipole absorption is expected to reveal itself through strong suppression of coherent quarkonium production at $x_{\text {eff }} \equiv m_{V}^{2} / s_{\gamma N} \leq 10^{-3}$ and at midrapidity for $x_{\text {eff }} \leq 5 \times 10^{-3}$. The $A A$ measurements probe $x_{\text {eff }}=m_{V} / 2 E_{N}$ since $s_{\gamma N}=2 E_{N} m_{V}$ when $E_{N} \gg m_{V}, m_{N}$, corresponding to $x_{\text {eff }} \equiv 2.5 \times 10^{-3}$ for $\Upsilon$ and $7.5 \times 10^{-4}$ for $J / \psi$. Measurements at lower $x_{\text {eff }}$ (higher effective energy) would require identifying which nucleus emitted the photon. An advantage of studying quasi-elastic reactions is the dissociation of the nucleus that absorbed the photon. As a result, the quasi-elastic $x_{\text {eff }}$ range is a factor of 10 higher than coherent processes because the measurement is not restricted to midrapidity. Measurements of low $p_{T} J / \psi$ production away from $y=0$ appear to be easier for several of the detectors. At forward rapidity, the difference between the minimum $x$ reached in breakup processes and coherent production is even larger.

Processes with rapidity gaps are most interesting for sufficiently large vector meson $p_{T}$ since they probe whether the elementary reaction $\gamma j \rightarrow V+$ jet where $j$ is a parton, leading to $\gamma A(N) \rightarrow V+$ rapidity gap $+X$, is dominated by elastic scattering of small $q \bar{q}$ 
dipole components of the photon wavefunction with partons in the nucleon. Light vector mesons, including the $\rho^{0}$, are then also effective probes. Such reactions are an effective way of studying the properties of perturbative colorless interactions in the vacuum (the "perturbative Pomeron") at finite $t$. The LHC kinematics and detector acceptances would greatly increase the energy range covered by HERA. Nuclear scattering would provide a complementary method of studying the dynamics of small dipole propagation through the nuclear medium. UPCs at the LHC are expected to reach both the large $t$ and moderate $W$ regime where the onset of the perturbative color transparency limit, $\sigma \propto A$, is expected as well as the onset of the BDR at large $W$ where $\sigma \propto A^{1 / 3}$.

$U P C s$ in $p A$ interactions

Proton-nucleus collisions are also an important part of the LHC program. Ultraperipheral $p A$ studies will further extend the HERA range for several important processes. The small $x$ gluon densities can be studied through heavy quark production by photon-gluon fusion when the gluon comes from the nucleus and, in the diffractive case, when the gluon comes from the Pomeron.

Exclusive $J / \psi$ production should be able to determine whether the growth of the $J / \psi$ cross section with $W$ decreases as the BDR is approached. If the proposed forward proton counters at $420 \mathrm{~m}$ downstream are approved [32], accurate measurements of the $t$-dependences of these reactions could determine the transverse gluon distribution over a wide $x$ range. In contrast, HERA could not directly detect protons and had to rely on vetoing. Measurements of the $\Upsilon$ photoproduction cross section could verify the prediction that the cross section should increase as $W_{\gamma p}^{1.7}[33,34]$.

The ATLAS and CMS detectors can study vector meson production both as functions of the vector meson rapidity and the rapidity gap, $\Delta y$, between the vector meson and other produced particles. While $\Delta y_{\max } \sim 2$ at HERA, at the LHC $\Delta y_{\max } \sim 8$, making studies of Pomeron dynamics much more effective.

In summary, UPC studies in $p A$ interactions will probe the small $x$ dynamics for $x \geq 10^{-4}$ in a number of complementary ways. They will address the high density regime, a primary motivation for the proposals to extend HERA running beyond 2007 [35], with the added advantage of much higher densities than accessible in ep collisions. Since these measurements will cover the $x$ range probed in $A A$ collisions at the LHC, these studies are also important for understanding the $A A$ collision dynamics.

\subsection{Overview of interesting $\gamma \gamma$ processes}

Two-photon collisions are fundamental processes that have previously been studied at every lepton collider, particularly in $e^{+} e^{-}$at the CERN LEP and also in ep at HERA. There are three areas of two-photon physics that may be studied using UPCs at the LHC: QED processes in strong electromagnetic fields; QCD processes; and new physics searches.

At low photon energies, QED processes in strong electromagnetic fields can be studied. The photon-ion coupling constant is $Z \alpha \approx 0.6$. Therefore Coulomb 
corrections, processes beyond leading order, can become important. In the case of $e^{+} e^{-}$ pair production, higher-order processes can be studied either as unitarity corrections, resulting in multiple pair production in single collisions, or as Coulomb corrections, giving a reduction relative to the Born cross section. Together with the possibility of tagging additional nuclear excitations, these processes can be studied at small impact parameter where the effects may be enhanced.

An important beam-physics effect is "bound-free pair production" or "electron capture from pair production", a pair production process where the electron is produced in a bound state with one of the ions. As the $Z / A$ ratio changes, the ion is no longer kept in the beam. These ions then hit the wall of the beam pipe, leading to large heating and potentially quenching the superconducting magnets. This is the dominant process restricting the maximum $\mathrm{Pb}+\mathrm{Pb}$ luminosity at the LHC. They also cause approximately half of the beam losses and therefore shorten the heavy-ion beam lifetime. Bound-free pair production was observed during the $2005 \mathrm{RHIC} \mathrm{Cu}+\mathrm{Cu}$ run [36].

At higher photon energies, QCD two-photon processes may be of interest. The large photon flux allows more detailed studies of processes that are separable from diffractive $\gamma A \rightarrow X A$ processes. In double vector meson production, not only light mesons like $\rho^{0} \rho^{0}$ but also $J / \psi J / \psi$ or pairs of two different vector mesons could be studied. Vector meson pair production can be distinguished from production of two independent vector mesons in coherent $\gamma A$ scattering since the transverse momenta of two vector mesons produced in $\gamma \gamma$ processes are much larger and back-to-back.

The high photon energies and the correspondingly large available two-photon invariant mass, together with the large photon flux, motivated previous new physics searches such as Higgs and supersymmetric particle production in two-photon interactions. However, experimental limits on the masses of many new particles have increased in recent years, making their discovery in $\gamma \gamma$ processes at the LHC unlikely. The parameter space for production beyond the Standard Model may still be explored. In $p p$ collisions, it is possible to tag the photons if they have lost more than $10 \%$ of their energy, making electroweak studies of $\gamma \gamma$ or $\gamma W$ processes possible. Although the cross section are not large, the higher energies, longer runs and high beam luminosities in $p p$ collisions offer some advantages.

\section{Exclusive photonuclear processes}

\subsection{Introduction}

Contributed by: L. Frankfurt, V. Guzey, M. Strikman, R. Vogt, and M. Zhalov

During the last decade, studies of small $x$ phenomena at HERA have revealed that, at the highest energies available in ep collisions, the interaction strength becomes comparable to the maximum allowed by unitarity over a wide range of $Q^{2}$. An increase in interaction energies and/or the extension to ion beams is needed to reach higher 
interaction strengths.

The most practical way to carry out such a program in the next decade appears to be investigation of photon-nucleus interactions at the LHC $[3,18,37]$. Though it is not possible to vary the virtuality of the photon in photonuclear interactions, as in lepton-nucleus scattering, the isolated nature of direct photon events provides an effective means of determining the virtuality of the probe. An important advantage of ultraperipheral heavy-ion collisions relative to the HERA program is the ability to simultaneously study $\gamma N$ and $\gamma A$ scattering, making it possible to investigate the onset of a variety of hard QCD phenomena leading to a new strong interaction regime including: color transparency and color opacity; leading-twist nuclear shadowing and the breakdown of linear QCD evolution, These phenomena will be clearer in hard scattering with nuclear beams since the onset should occur at larger $x$ than in nucleons. In general, nuclear targets are ideal probes of the space-time evolution of small dipoles of size $d$ which can be selected in high energy $\gamma N$ scattering either by considering small $x$ processes with $Q^{2} \propto 1 / d^{2}$, or by studying special diffractive processes such as quarkonium or dijet production. Understanding the space-time evolution has consequences for other branches of physics, including the early universe since the emergence of color-singlet clusters may play a role in the quark-hadron transition. This program makes it possible to study coherent (and some incoherent) photonuclear interactions at energies which exceed those at HERA by at least an order of magnitude. Thus coherent UPC studies at the LHC will answer a number of fundamental questions in QCD. They will identify and investigate a new regime of strong interactions by probing the dependence on the projectile, the final state, and the nuclear size and thickness.

Several QCD regimes may be accessible, depending on the incident energy, the $Q^{2}$ of the process and the nuclear thickness. High-energy interactions of hadrons with nuclei rapidly approach the black-disk regime (BDR) where the total interaction cross section is $\approx 2 \pi R_{A}^{2}$ where $R_{A} \simeq 1.2 A^{1 / 3}$. At another extreme, the photon interacts like a small color singlet dipole. In this case, the system remains small over a wide energy range while traversing the nucleus, known as color transparency. In this regime, small dipole interactions with nuclei are rather weak and proportional to $A$. Color transparency predicts that the forward scattering cross section in $\gamma A$ collisions should be proportional to $A^{2}$ since the amplitude is proportional to $A$. Color transparency has recently been observed in exclusive dijet production by coherent diffraction in $\pi A$ interactions [38]. A similar $A$ dependence has also been observed in coherent $J / \psi$ production in fixed-target $\gamma A$ interactions at FNAL [39]. At higher energies, the interactions of small color dipoles may be described in the perturbative color opacity regime. Here, the dipole still couples to the gluon field of the nucleus through the nuclear gluon density, $g_{A}\left(x, Q^{2}\right)$, as in the color transparency regime. However, the scattering amplitude is not $\propto A$ due to leading-twist (LT) shadowing, resulting in $g_{A}\left(x, Q^{2}\right) / A g_{N}\left(x, Q^{2}\right)<1$. The onset of LT gluon shadowing partially tames the increase of $g_{A}\left(x, Q^{2}\right)$ for $10^{-4}<x<10^{-2}$, slowing the increase of the dipole-nucleus cross section with energy. However, the reduction of $g_{A}\left(x, Q^{2}\right)$ at small $x$ is insufficient to prevent the LT approximation of the total inelastic 
cross section from reaching and exceeding its maximum value, violating unitarity, an unambiguous signal of the breakdown of the LT approximation at small $x$. We will discuss how to unambiguously distinguish between leading-twist nuclear shadowing and the blackening of hard interactions.

It is important to determine whether dipole-nuclear interactions are strongly modified by LT shadowing at small $x$ [40]. Some models neglect this effect [41] and focus on higher-twist effects, often modeled using the impact-parameter space eikonal approach $[42,43]$. If LT shadowing was small and only higher-twist effects reduced the increase of the dipole-nucleus cross section, the DGLAP approximation of parton evolution would break down at rather large $x$. On the other hand, the DGLAP breakdown may be due to the onset of the BDR, taming the dipole-nucleus cross section at smaller $x$. We argue that the relative importance of leading and higher-twist contributions could be experimentally resolved using coherent quarkonium photoproduction.

If LT gluon shadowing effects are small, $q \bar{q}$ dipoles with $d \geq 0.3-0.4 \mathrm{fm}$ could be in the BDR in central $A A$ collisions at $x \geq 10^{-3}$, the kinematic regime where $\ln x$ effects on the parton evolution are also small. In any case, the limiting behavior of the dipole-nuclear interaction is of great theoretical interest since it represents a new regime of strong interactions where the LT QCD approximation, and therefore the notion of parton distributions, becomes inapplicable at small $x$ even though $\alpha_{s}$ is small. We emphasize that, besides higher parton densities in nuclei, the dependence of the scattering amplitude on impact parameter is rather weak over a wide range of $b$. Thus the dependence of the amplitudes on the nuclear thickness can be studied by employing both heavy and light nuclear targets. On the other hand, nucleon scattering at large $b$ is important at small $x$, making the change of interaction regime at small $b$ and leading to different energy dependencies of the deep-inelastic scattering cross sections for nucleons $\left(\propto \ln ^{3} s\right)$ and nuclei $(\propto \ln s)$. In hard diffraction, the forward cross sections and the $t$ dependence of the slope parameter $B$ also increase rapidly with energy: $\sigma \propto \ln ^{4} s$ and $B \approx B_{0}+B_{1} \ln ^{2} s$ respectively.

Theoretical studies of the limiting behavior of the dipole-nucleus cross sections have so far not produced any definitive results. QCD dynamics may slow the increase of the dipole-nucleus cross section at central impact parameters $(b \sim 0)$ at significantly larger $x$ than allowed by the BDR. In the following discussion, we assume that the BDR is reached at small $b$ to emphasize the distinguishing features of the new regime where the elastic and inelastic cross sections are equal.

In many processes, the projectile wavefunction may be described as a superposition of different size configurations $(q \bar{q}, q \bar{q} g$, etc.) leading to fluctuations in the interaction strength. Interactions of real and virtual photons with heavy nuclei can therefore provide unique information since the photon wavefunction contains both "hadron-like" configurations (vector meson dominance) and "photon-like" configurations (light $q \bar{q}$ components and heavy $Q \bar{Q}$ components). In high-energy photonuclear interactions, the BDR is manifested by inelastic diffraction of the photon into a multitude of hadronic 
final states while elastic diffraction, $\gamma \rightarrow \gamma$, is negligible. On the other hand, only elastic hadron diffraction survives in the BDR, hiding the detailed dynamics. Moreover, it is possible to post-select a small or large configuration of the photon wavefunction by choosing a particular final state. Such post-selection is more difficult for hadrons since the configuration size distribution is wider for photons.

Spectacular manifestations of the BDR in (virtual) photon diffraction include strong enhancement of the high mass tail of the diffractive spectrum relative to the triple Pomeron limit and large dijet production cross sections at high $p_{T}$ [44]. We emphasize that the study of diffractive channels can distinguish between the two scenarios of strong cross section suppression: leading-twist shadowing and the black-disk regime. Studies of coherent diffraction in the BDR will uniquely measure components of the light-cone photon wavefunction, providing more detailed information than similar measurements where leading-twist dominates.

\subsection{Color transparency, nuclear shadowing and quarkonium production}

Contributed by: L. Frankfurt, V. Guzey, M. Strikman, R. Vogt, and M. Zhalov

The interaction of small color singlets with hadrons is one of the most actively studied issues in high-energy QCD. In exclusive electroproduction of mesons at high $Q^{2}$ as well as $J / \psi$ and $\Upsilon$ photoproduction, the QCD factorization theorem separates the vector meson wave function at zero transverse separation into the hard scattering amplitude and the generalized parton densities, making evaluation of the vector meson production amplitude possible $[23,24] \S$. The leading-twist approximation differs strongly from predictions based on the Glauber model and two-gluon exchange models. The LT approximation accounts for the dominance of the space-time evolution of small quark-gluon wave packets in electroproduction, leading to the formation of a softer gluon field which effectively increases the dipole size with energy.

In perturbative QCD, similar to QED, the total cross section for the interaction of small systems with hadrons is proportional to the area occupied by the color charge in the projectile hadron [47], predicting color transparency hard interactions with nuclei. Incoherent cross sections are expected to be proportional to the nuclear mass number, $A$, while the coherent amplitude is proportional to $A$ times the nuclear form factor, $F$. The approximation of a quarkonium projectile as a colorless $Q \bar{Q}$ dipole can be formally derived from QCD within the limit $m_{Q} \rightarrow \infty$ and a fixed, finite momentum fraction,

$x=4 m_{Q}^{2} / s$ [48]. In these kinematics, the quarkonium radius is sufficiently small to justify the applicability of pQCD.

It is important to determine the $Q^{2}$ in vector meson production where squeezing becomes effective and the dipole size decreases as $1 / Q$. Perhaps the most sensitive

$\S$ The proportionality of hard diffractive amplitudes to the nucleon gluon density was discussed for hard $p p$ diffraction [45], $J / \psi$ production [46] in the BFKL approximation, and pion diffraction into two jets [26] in the leading $\log Q^{2}$ approximation [26]. 
indicator of small dipole size is the $t$-dependence of vector meson production. The current HERA data are consistent with the prediction $[23,48]$ that the slopes of the $\rho^{0}$ and $J / \psi$ production amplitudes should converge to the same value. Thus configurations much smaller than average, $d \sim 0.6 \mathrm{fm}$ in light mesons, dominate small $x \rho^{0}$ production at $Q^{2} \geq 5 \mathrm{GeV}^{2}$. However, at all $Q^{2}, J / \psi$ production is dominated by small size configurations. Therefore, color transparency is expected for $x \geq 0.03$ where gluon shadowing is either very small or absent.

Color transparency (CT) was observed at Fermilab [38] with coherent dissociation in $\pi A \rightarrow$ jet $_{1}+$ jet $_{2}+A$ interactions at $500 \mathrm{GeV}$. Diffractive masses of up to $5 \mathrm{GeV}$ were observed, consistent with two jets. The results confirmed the $A$ dependence and the $p_{T}$ and longitudinal jet momentum distributions predicted in Ref. [26]. Color transparency was also previously observed in coherent $J / \psi$ photoproduction at $\left\langle E_{\gamma}\right\rangle=120 \mathrm{GeV}$ [39].

It is not clear whether CT will hold at arbitrarily high energies since two phenomena are expected to counter it at high energies: leading-twist gluon shadowing and the increase of the dipole-nucleon cross section with energy.

Leading-twist gluon shadowing predicts that the gluon distribution in a nucleus will be depleted at low $x$ relative to the nucleon, $g_{A}\left(x, Q^{2}\right) / A g_{N}\left(x, Q^{2}\right)<1$. Such expectations are tentatively supported by the current analyzes of nuclear DIS although the data does not extend deep enough into the shadowing region for confirmation. Shadowing should lead to a gradual but calculable disappearance of color transparency $[23,26]$ and the onset of a new regime, the color opacity regime. It is possible to consider color opacity to be generalized color transparency since the small $q \bar{q}$ dipole still couples to the gluon field of the target by two gluons with an amplitude proportional to the generalized nuclear gluon density.

The small dipole-nucleon cross section is expected to increase with energy as $x g_{N}\left(x, Q^{2}\right)$ where $x \propto 1 / s_{(q \bar{q}) N}$. For sufficiently large energies the cross section becomes comparable to the meson-nucleon cross sections which may result in significant suppression of hard exclusive diffraction relative to the leading-twist approximation.

While this suppression may be beyond the kinematics achievable for $\mathrm{J} / \psi$ photoproduction in UPCs at RHIC [63], $x \approx 0.015$ and $Q_{\text {eff }}^{2} \approx 4 \mathrm{GeV}^{2}$, it could be important in UPCs at the LHC. Thus systematic studies of coherent quarkonium production in ultraperipheral $A A$ interactions at collider energies should be very interesting. We emphasize that the eikonal (higher-twist) contributions die out quickly with decreasing quarkonium size for fixed $x$. In particular, for the $\Upsilon$, nuclear gluon fields at transverse scale $\sim 0.1 \mathrm{fm}\left(Q_{\text {eff }}^{2} \sim 40 \mathrm{GeV}^{2}\right)$ are probed. The $J / \psi$ is closer to the border between the perturbative and nonperturbative domains. As a result, the nonperturbative region appears to give a significant contribution to the production amplitude [49].

We now discuss the quarkonium photoproduction amplitude, $\gamma A \rightarrow J / \psi(\Upsilon) A$, in greater detail. The $W_{\gamma p}$ range probed at the LHC corresponds to rather small $x$. The key theoretical issue is how to properly incorporate nuclear shadowing. A number of coherent mechanisms have been suggested. Here leading-twist shadowing, shown 


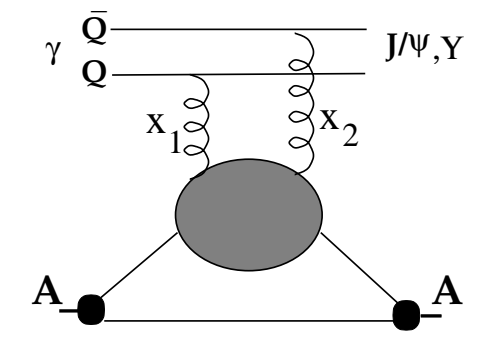

(a)

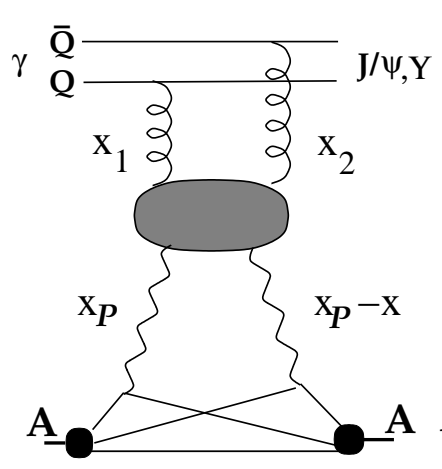

(b)

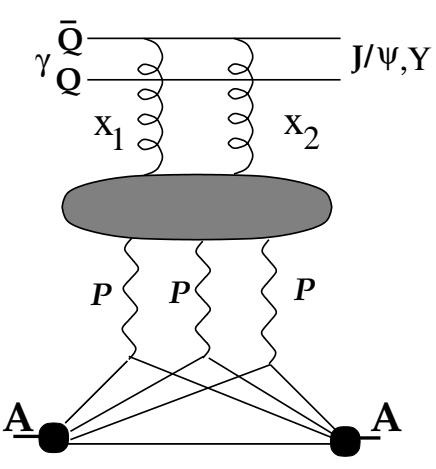

(c)

Figure 9. Leading-twist diagrams for quarkonium production from nuclear targets.

in the diagrams of Fig. 9, is employed. There is a qualitative difference between the interaction of a small dipole with several nucleons and a similar interaction with a single hadron. For example, we consider an interaction with two nucleons. The leading-twist contribution is described by diagrams where two gluons attach to the dipole. To ensure that the nucleus remains intact, color singlet lines should be attached to both nucleons. These diagrams, especially Fig. 9(b), are closely related to those describing diffractive gluon densities measured at HERA and thus also to similar diagrams for nuclear gluon shadowing [40].

The amplitude for coherent quarkonium photoproduction is proportional to the generalized gluon density of the target, $G_{A}\left(x_{1}, x_{2}, t, Q_{\text {eff }}^{2}\right)$, which depends on the lightcone fractions $x_{1}$ and $x_{2}$ of the two gluons attached to the quark loop, as shown in the top parts of the diagrams in Fig. 9. The momentum fractions satisfy the relation

$$
x_{1}-x_{2}=\frac{m_{V}^{2}}{s_{(q \bar{q}) N}} \equiv x .
$$

If Fermi motion and binding effects are negligible, $x_{2} \ll x_{1}$. The resolution scale, $Q_{\text {eff }}$, is large, $Q_{\text {eff }}^{2} \geq m_{Q}^{2}$ where $m_{Q}$ is the heavy quark mass. Numerical estimates of $J / \psi$ photoproduction give $Q_{\text {eff }}^{2} \sim 3-4 \mathrm{GeV}^{2}[48,49]$, reflecting the relatively small charm quark mass and indicating that this process bridges the nonperturbative and perturbative regimes. On the other hand, the bottom quark mass is very large on the scale of soft QCD. In this case, hard physics dominates and the effect of attaching more 
than two gluons to the $b \bar{b}$ is negligible. The QCD factorization theorem then provides a reliable description of $\Upsilon$ production. Higher-twist effects due to the overlap of the $b \bar{b}$ component of the photon and the $\Upsilon$ cancel in the ratio of $\Upsilon$ production on different targets. As a result, in the leading-twist shadowing approximation, the $\gamma A \rightarrow \Upsilon A$ cross section is proportional to the square of the generalized nuclear gluon density so that

$$
\begin{aligned}
\sigma_{\gamma A \rightarrow V A}\left(s_{\gamma N}\right)= & \left.\frac{d \sigma_{\gamma N \rightarrow V N}\left(s_{\gamma N}\right)}{d t}\right|_{t=t_{\min }}\left[\frac{G_{A}\left(x_{1}, x_{2}, t=0, Q_{\mathrm{eff}}^{2}\right)}{A G_{N}\left(x_{1}, x_{2}, t=0, Q_{\mathrm{eff}}^{2}\right)}\right]^{2} \\
& \times \int_{-\infty}^{t_{\min }} d t\left|\int d^{2} b d z e^{i \vec{q}_{T} \cdot \vec{b}} e^{-i q_{l} z} \rho_{A}(\vec{b}, z)\right|^{2} .
\end{aligned}
$$

Numerical estimates using realistic potential model wave functions indicate that for $J / \psi, x_{2} / x_{1} \sim 0.33$ [49] while for the $\Upsilon, x_{2} / x_{1} \sim 0.1$ [33]. Models of generalized parton distributions (GPDs) at moderate $Q^{2}$ suggest that, for any hadron or nucleus, $G\left(x_{1}, x_{2}, t=0, Q^{2}\right)$ can be approximated by the inclusive gluon density, $g\left(x, Q^{2}\right)$, at $x=\left(x_{1}+x_{2}\right) / 2[23,50]$. At large $Q^{2}$ and small $x$, the GPDs are dominated by evolution from $x_{i}^{\text {init }} \gg x_{i}$. Since evolution on the gluon ladder conserves $x_{1}-x_{2}$, the effect of skewedness $\left(x_{2} / x_{1}<1\right)$ is determined primarily by evolution from nearly diagonal $\left(x_{1} \sim x_{2}\right)$ distributions [51].

Skewedness increases the $\Upsilon$ cross section by a factor of $\sim 2[33,34]$, potentially obscuring the connection between the suppression of the cross section discussed above and nuclear gluon shadowing. However, Ref. [49] showed that the ratio $G_{A}\left(x_{1}, x_{2}, t, Q_{\text {eff }}^{2}\right) / A G_{N}\left(x_{1}, x_{2}, t, Q_{\text {eff }}^{2}\right)$ is a weak function of $x_{2}$ at $t=0$, slowly dropping from the diagonal value, $x_{2}=x_{1}$, for decreasing $x_{2}$. This observation suggests that it is more appropriate to compare the diagonal and non-diagonal (skewed) ratios at $x=\left(x_{1}+x_{2}\right) / 2$.

In the following, the ratio of generalized nuclear to nucleon gluon densities is approximated by the ratio of gluon densities at $x=m_{V}^{2} / s_{(q \bar{q}) N}$,

$$
\frac{G_{A}\left(x_{1}, x_{2}, t=0, Q_{e f f}^{2}\right)}{A G_{N}\left(x_{1}, x_{2}, t=0, Q_{e f f}^{2}\right)} \approx \frac{g_{A}\left(x, Q_{e f f}^{2}\right)}{A g_{N}\left(x, Q_{e f f}^{2}\right)} .
$$

For the $\Upsilon, x / 2$ may be more appropriate, leading to slightly larger shadowing effects than with $x$ alone.

Reference [40] showed that nuclear shadowing may be expressed in a modelindependent way, through the corresponding diffractive parton densities, using the Gribov theory of inelastic shadowing $[52,53]$ and the QCD factorization theorem for the hard diffraction [54]. HERA demonstrated that hard diffraction is dominated by the leading-twist contribution with gluons playing an important role in diffraction, referred to as "gluon dominance of the Pomeron". Analysis of diffractive HERA data indicates that the probability of diffraction in gluon-induced processes is significantly larger than in quark-induced processes [40]. The recent $\mathrm{H} 1$ data on diffractive dijet production [55] provide an additional confirmation of this observation. The large probability of diffraction in gluon-induced hard scattering can be understood in the $s$-channel language 
as the formation of large color-octet dipoles which can diffractively scatter with a correspondingly large cross section. The interaction strength can be quantified using the optical theorem, introducing the effective cross section,

$$
\begin{aligned}
\sigma_{\text {eff }}^{g}\left(x, Q_{0}^{2}\right) & =\frac{16 \pi}{\sigma_{\text {tot }}\left(x, Q_{0}^{2}\right)} \frac{d \sigma_{\text {diff }}\left(x, Q_{0}^{2}, t_{\text {min }}\right)}{d t} \\
& =\frac{16 \pi}{x g_{N}\left(x, Q_{0}^{2}\right)} \int_{x}^{x_{\mathbb{P}}^{0}} d x_{\mathbb{P}} \beta g_{N}^{D}\left(\frac{x}{x_{\mathbb{P}}}, x_{\mathbb{P}}, Q_{0}^{2}, t_{\text {min }}\right)
\end{aligned}
$$

for hard scattering of a virtual photon off the gluon field of the nucleon. Here $Q_{0}^{2}=4$ $\mathrm{GeV}^{2}$ is the resolution scale for the gluons; $x_{\mathbb{P}}$ is the longitudinal momentum fraction of the Pomeron; $x_{\mathbb{P}}^{0}=0.1$; and $g_{N}^{D}$ is the diffractive gluon density of the nucleon, known from the $\mathrm{H} 1$ Fit $\mathrm{B}$ diffractive analysis $[56,57]$. While this coherent mechanism may effectively be absent for $x \geq 0.02-0.03$, it may quickly become important at smaller $x$.

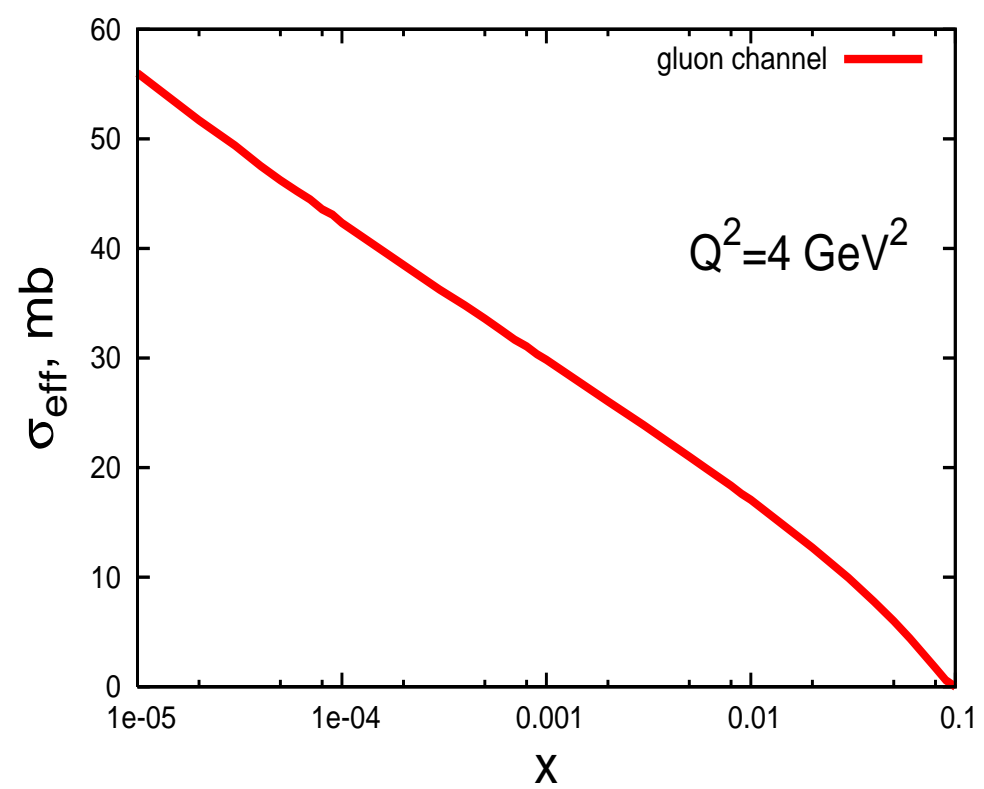

Figure 10. The effective gluon shadowing cross section, $\sigma_{\text {eff }}^{g}(x)$, at $Q^{2}=4 \mathrm{GeV}^{2}$ as a function of $x$ for the H1 parameterizations of the diffractive gluon density.

The ratio of the inclusive gluon densities in Eq. (10) is calculated using leadingtwist shadowing [40], see Ref. [58] for details. First, the nuclear gluon density, including leading-twist shadowing is calculated at the minimum scale, $Q_{0}^{2}=4 \mathrm{GeV}^{2}$

$$
\begin{aligned}
g_{A}\left(x, Q_{0}^{2}\right)= & A g_{N}\left(x, Q_{0}^{2}\right)-8 \pi \operatorname{Re}\left[\frac{(1-i \eta)^{2}}{1+\eta^{2}}\right. \\
& \times \int d^{2} b \int_{-\infty}^{\infty} d z_{1} \int_{z_{1}}^{\infty} d z_{2} \int_{x}^{x_{\mathbb{P}}^{0}} d x_{\mathbb{P}} \beta g_{N}^{D}\left(\frac{x}{x_{\mathbb{P}}}, x_{\mathbb{P}}, Q_{0}^{2}, t_{\min }\right) \\
& \left.\times \rho_{A}\left(b, z_{1}\right) \rho_{A}\left(b, z_{2}\right) e^{i x_{\mathbb{P}} m_{N}\left(z_{1}-z_{2}\right)} e^{-\frac{1-i \eta}{2} \sigma_{\mathrm{eff}}^{g}\left(x, Q_{0}^{2}\right) \int_{z_{1}}^{z_{2}} d z^{\prime} \rho_{A}\left(b, z^{\prime}\right)}\right],
\end{aligned}
$$

where $\eta$ is the ratio of the real to imaginary parts of the elementary diffractive amplitude. The H1 parametrization of $g_{N}^{D}\left(x / x_{\mathbb{P}}, x_{\mathbb{P}}, Q_{0}^{2}, t_{\mathrm{min}}\right)$ is used as input. The effective cross 
section, $\sigma_{\text {eff }}^{g}\left(x, Q_{0}^{2}\right)$, determined by Eq. (11), accounts for elastic rescattering of the produced diffractive state with a nucleon. Numerically, $\sigma_{\text {eff }}^{g}$ is very large at $Q_{0}^{2}$, see Fig. 10, and corresponds to a probability for gluon-induced diffraction of close to $\sim 50 \%$ at $x \sim 10^{-5}$ (see Fig. 44). Consequently at $Q_{0}^{2}$, gluon interactions with nucleons approach the BDR at $x \sim 10^{-4}-10^{-5}$ while, for nuclei, a similar regime should hold for $x \leq 10^{-3}$ over a large range of impact parameters.

The double scattering term in Eq. (12), proportional to $\sigma_{g \text { eff }}$, for the nuclear parton densities satisfies QCD evolution, while higher-order terms (higher powers of $\sigma_{g \text { eff }}$ ) do not. Thus if a different $Q_{0}^{2}$ is used, a different $g\left(x, Q^{2}\right)$ would be obtained since the higher-order terms, $\propto\left(\sigma_{\text {eff }}^{g}\right)^{n}, n \geq 2$ are sensitive to the $Q^{2}$-dependent fluctuations in the diffractive cross sections. The $Q^{2}$ dependence of the fluctuations are included in the QCD evolution, violating the Glauber-like structure of shadowing for $Q^{2}>Q_{0}^{2}$. The approximation for $n \geq 3$ in Eq. (12) corresponds to the assumption that the fluctuations are small at $Q_{0}^{2}$ since this scale is close to the soft interaction scale [40]. Thus we use NLO QCD evolution to calculate shadowing at larger $Q^{2}$ using the $Q_{0}^{2}$ result as a boundary condition. We also include gluon enhancement at $x \sim 0.1$ which influences shadowing at larger $Q^{2}$. The proximity to the BDR, reflected in large $\sigma_{g \text { eff }}$, may result in corrections to the LT evolution.
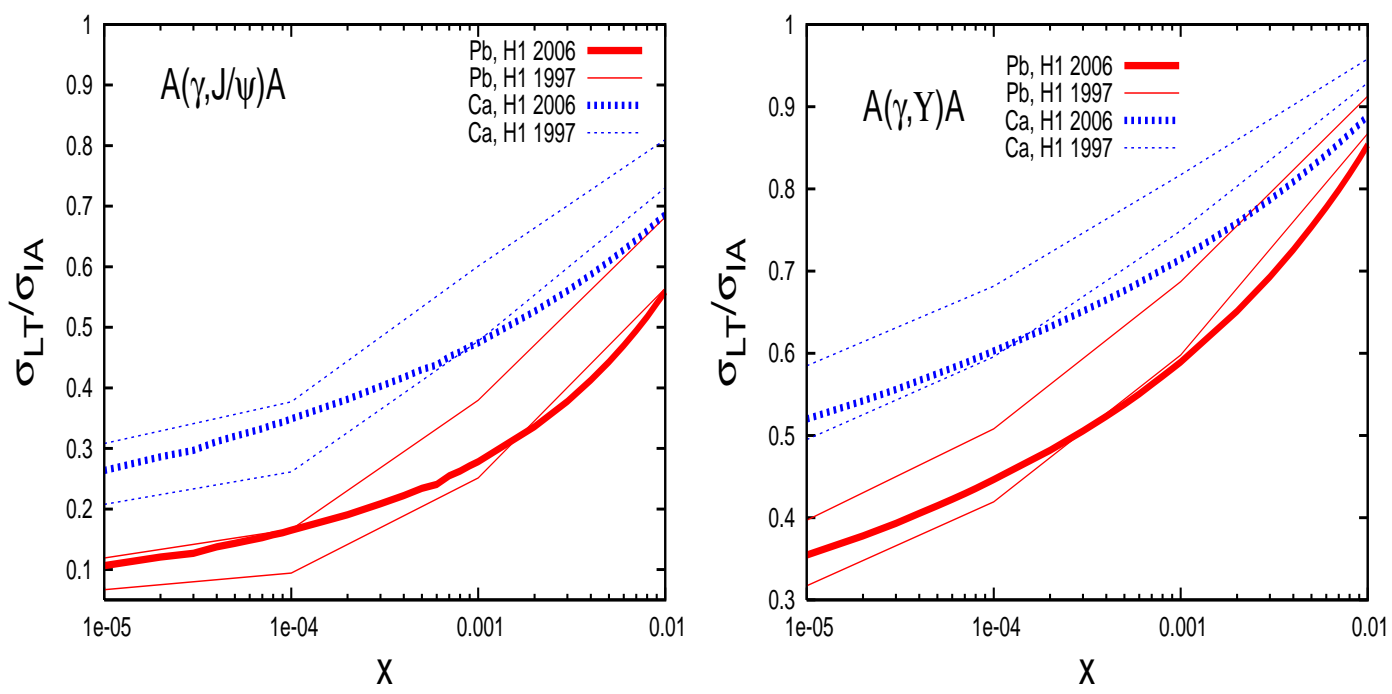

Figure 11. The $x$ dependence of the ratio of $J / \psi$ and $\Upsilon$ production in $\mathrm{Ca}+\mathrm{Ca}$ and $\mathrm{Pb}+\mathrm{Pb}$ interactions in the leading-twist Glauber model (LT) to that in the Impulse Approximation (IA), without shadowing. Calculations employing the new H1 parametrization $[56,57]$ of the diffractive PDFs are compared to those of Ref. [58].

We first present the coherent $J / \psi$ and $\Upsilon$ photoproduction cross section ratios in ultraperipheral $A A$ collisions, shown in Fig. 11. The thick curves show the leading-twist shadowing results using the recent $\mathrm{H} 1$ Fit $\mathrm{B}$ to the hard diffraction DIS data [56,57]. The thin curves are calculations [58] using the older H1 fits from 1995 [59]. The spread between the two thin curves corresponds to the difference between the "low" and "high" 
gluon shadowing scenarios introduced in Ref. [58].

We point out that, as shown in Fig. 11, the leading-twist shadowing predictions using the $2006 \mathrm{H} 1$ fits are consistent with the theoretical band determined from the 1997 H1 diffractive fits. Therefore, predictions made using the results of Ref. [58] elsewhere in this report should not change much when the more recent fits are used.

Finally, we comment on the difference between the present and earlier leading-twist shadowing predictions using the $\mathrm{H} 1$ fits to hard diffraction in DIS with protons. First, in the analysis of Ref. [58], the $1997 \mathrm{H} 1$ diffractive gluon PDF was multiplied by 0.75 to phenomenologically account for the difference between the 1994 and 1997 H1 hard diffractive gluon data. Second, in earlier analyses [60], a somewhat larger slope for the $t$-dependence of the gluon diffractive PDF was used. Thus, predictions of leadingtwist nuclear gluon shadowing made with the unmodified $1997 \mathrm{H} 1$ fits and the larger slope result in larger nuclear shadowing relative to Ref. [58]. Thus the FGS calculations presented in Section 4, based on the earlier predictions [60], somewhat overestimate the shadowing effect compared to predictions based on the most recent fits $[56,57]$, see Fig. 11.

The ratios in Fig. 11 are independent of uncertainties in the elementary cross sections, providing a sensitive test of LT shadowing effects. In the case of $J / \psi$ photoproduction, $Q^{2} \sim 4 \mathrm{GeV}^{2}$. A significant fraction of the amplitude comes from smaller virtualities [48, 49] which may result in a larger shadowing effect. We use $Q^{2}=40$ $\mathrm{GeV}^{2}$ to calculate $\Upsilon$ photoproduction. However, the result is not very sensitive to the precise $Q^{2}$, since the scale dependence of gluon shadowing at higher $Q^{2}$ is rather small. Despite the small $\Upsilon$ size, which precludes higher-twist shadowing at very small $x$, the perturbative color opacity effect is important. The effective rescattering cross section in the Glauber model is determined by the dipole-nucleon cross section, $\sigma_{\text {in }}^{(Q \bar{Q}) N}$, with $d \sim 0.25-0.3 \mathrm{fm}$ for $J / \psi$ and $\sim 0.1 \mathrm{fm}$ for the $\Upsilon$. These distances correspond to cross sections of $\sim 10-15 \mathrm{mb}$ for $J / \psi$ and $\sim 3 \mathrm{mb}$ for $\Upsilon$ at $x \sim 10^{-4}$, reducing the cross section by a factor of $\sim 1.5-2$ for $x \sim 10^{-3}$, see Fig. 13 in Ref. [49]. The cross sections are not reduced as much as those calculated using LT gluon shadowing.

The absolute quarkonium photoproduction cross sections were estimated over a wide range of photon energies. The energy dependence of the momentum-integrated cross sections, $\sigma\left(W_{\gamma N}\right)$ where $W_{\gamma N}=\sqrt{s_{\gamma N}}$, is presented in Fig. 12. At low $W_{\gamma N}$, there is a dip in the $\gamma \mathrm{Pb} \rightarrow J / \psi$ cross section due to the fast onset of shadowing at $x \sim 10^{-3}$ in the leading-twist parameterization employed in the calculation. The $J / \psi$ calculations are straightforward since accurate HERA data are available. However, the $\gamma N \rightarrow \Upsilon N$ data are very limited, with only ZEUS and H1 total cross section data for $\sqrt{s_{\gamma N}} \approx 100$ $\mathrm{GeV}$, complicating the $\Upsilon$ predictions. A simple parametrization is used to calculate the photoproduction cross section,

$$
\frac{d \sigma_{\gamma N \rightarrow V N}\left(s_{\gamma N}, t\right)}{d t}=10^{-4} B_{\Upsilon}\left(\frac{s_{\gamma N}}{s_{0}}\right)^{0.85} \exp \left(B_{\Upsilon} t\right) \mu \mathrm{b} / \mathrm{GeV}^{2}
$$

where $s_{0}=6400 \mathrm{GeV}^{2}$ and $B_{\Upsilon}=3.5 \mathrm{GeV}^{-2}$ were fixed from the analysis of the two-gluon form factor in Ref. [61]. The energy dependence follows from the $\Upsilon$ photoproduction 

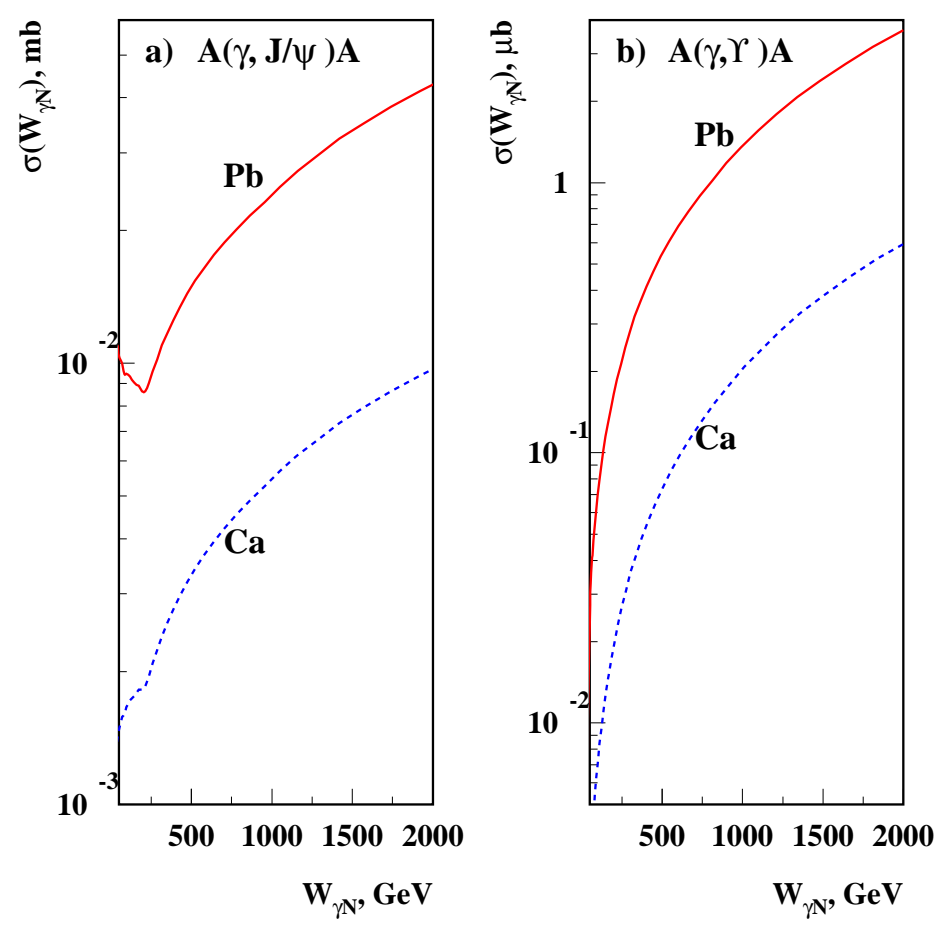

Figure 12. The energy dependence of coherent $J / \psi$ and $\Upsilon$ photoproduction in ultraperipheral $\mathrm{Ca}+\mathrm{Ca}$ and $\mathrm{Pb}+\mathrm{Pb}$ collisions in the $\mathrm{LT}$ approximation. Reprinted from Ref. [70] with permission from Acta Physica Polonica.

calculations of Ref. [33] in the leading $\log Q^{2}$ approximation, including the skewedness of the PDFs.

\subsection{Vector meson production}

Contributed by: L. Frankfurt, V. Guzey, S. R. Klein, J. Nystrand, M. Strikman, R. Vogt, and M. Zhalov

Exclusive photonuclear vector meson production in relativistic heavy-ion interactions are interactions of the type

$$
A+A \rightarrow A+A+V
$$

where the nuclei normally remain intact. These interactions typically occur for impact parameters much larger than the sum of the nuclear radii, $b \gg 2 R_{A}$, and proceed through an interaction between the electromagnetic field of one of the nuclei with the nuclear field of the other. The experimental feasibility of studying these interactions at heavy-ion colliders has been demonstrated by STAR [62] and PHENIX [63] at RHIC.

2.3.1. Vector Meson Dominance Model Coherent production of light vector mesons, $V$, off nucleons and nuclei, $\gamma A \rightarrow V A$, at high energies can be described within the 
framework of the Vector Meson Dominance Model (VDM) or the Generalized Vector Meson Dominance Model (GVDM) [64,65] reviewed in Refs. [66-68].

At low and moderate energies, the hadronic interaction of a low-virtuality photon is dominated by quantum mechanical fluctuations into a strongly interacting state, preferentially a vector meson, with the quantum numbers of the photon, $J^{P C}=1^{--}$.

The photon wavefunction can be written as a sum of Fock states,

$$
|\gamma\rangle=C_{\text {pure }}\left|\gamma_{\text {pure }}\right\rangle+C_{\rho^{0}}\left|\rho^{0}\right\rangle+C_{\omega}|\omega\rangle+C_{\phi}|\phi\rangle+C_{J / \psi}|J / \psi\rangle+\cdots+C_{q \bar{q}}|q \bar{q}\rangle,
$$

where $\left|\gamma_{\text {pure }}\right\rangle$ corresponds to a bare photon which may interact with a parton in the target, $C_{\text {pure }} \approx 1$. The amplitude, $C_{V}$, for the photon to fluctuate into vector meson $V$ is proportional to the inverse of the photon-vector meson coupling, $f_{V}$. This coupling can be related to the measured dilepton decay width, $\Gamma_{V \rightarrow e^{+} e^{-}}$,

$$
\left|C_{V}\right|^{2}=\frac{4 \pi \alpha}{f_{V}^{2}}=\frac{3 \Gamma_{V \rightarrow e^{+} e^{-}}}{\alpha^{2} M_{V}},
$$

where $\alpha$ is the electromagnetic coupling constant and $M_{V}$ the vector meson mass.

The VDM neglects contributions from non-diagonal transitions, i.e. $\left\langle\rho^{0} \mid \omega\right\rangle=0$. The GVDM includes these non-diagonal transitions. In such transitions, the photon fluctuates into a different hadronic state from the observed final-state vector meson. The observed final state is produced by hadronic rescattering, $V^{\prime} A \rightarrow V A$ where $V^{\prime}$ is the initially-produced vector meson and $V$ the final-state meson.

Squaring Eq. (15) and assuming the diagonal approximation of the VDM, the differential photoproduction cross section, $d \sigma_{\gamma A \rightarrow V A} / d t$, calculated using the Glauber scattering model, is

$$
\begin{aligned}
\frac{d \sigma_{\gamma A \rightarrow V A}}{d t}= & \left.\frac{d \sigma_{\gamma N \rightarrow V N}}{d t}\right|_{t=0} \\
& \times\left|\int d^{2} b d z e^{i \vec{q}_{T} \cdot \vec{b}} e^{i q_{L} z} \rho_{A}(b, z) e^{-\frac{1}{2} \sigma_{\mathrm{tot}}^{V N}(1-i \epsilon) \int_{z}^{\infty} d z^{\prime} \rho_{A}\left(b, z^{\prime}\right)}\right|^{2} .
\end{aligned}
$$

The square of the transverse momentum transfer in the $\gamma \rightarrow V$ transition, $\left|\vec{q}_{T}\right|^{2}=$ $\left|t_{T}\right|=\left|t_{\min }-t\right|$, depends on the photon energy, $\omega$, through $t_{\min }$ since $-t_{\min }=M_{V}^{4} / 4 \omega^{2}$. The ratio of the real to imaginary parts of the vector meson scattering amplitude is denoted $\epsilon$ in Eq. (17).

The longitudinal momentum transfer, $q_{L}$, reflects the large longitudinal distances over which the transition $\gamma \rightarrow V$ occurs. The hadronic fluctuation extends over distance $l_{c}$, the coherence length, restricted by the uncertainty principle so that

$$
l_{c}=1 / q_{L}=\Delta t c=\frac{\hbar c}{\Delta E}=\frac{\hbar c}{E_{V}-E_{\gamma}}=\frac{2 E_{\gamma}}{M_{V}^{2}+Q^{2}} \hbar c
$$

where $E_{V}$ is the vector meson energy while $E_{\gamma}$ and $Q$ are the energy and virtuality of the photon, respectively. In the limit where the coherence length is much larger than the nuclear radius, $l_{c} \gg R_{A}$, Eq. (17) is reduced to the usual Glauber expression for elastic hadron-nucleus scattering by making the substitutions $\left.\left(d \sigma_{\gamma N \rightarrow V N} / d t\right)\right|_{t=0} \rightarrow$ $\left.\left(d \sigma_{V N \rightarrow V N} / d t\right)\right|_{t=0}$ and $\left(d \sigma_{\gamma A \rightarrow V A} / d t\right) \rightarrow\left(d \sigma_{V A \rightarrow V A} / d t\right)$. 
In the nuclear rest frame, for light vector meson production at midrapidity the limit $l_{c} \gg R_{A}$ holds at RHIC and LHC so that

$$
\frac{d \sigma_{\gamma A \rightarrow V A}}{d t}=\left|C_{V}\right|^{2} \frac{d \sigma_{V A \rightarrow V A}}{d t} .
$$

The exclusive photo-nuclear scattering amplitude is thus proportional to the amplitude for elastic vector meson scattering. If two vector meson states, $V$ and $V^{\prime}$, contribute then non-diagonal transitions, $V^{\prime} A \rightarrow V A$, have to be considered in GVDM [69]. The more general expression for the scattering amplitude,

$$
\mathcal{M}_{\gamma A \rightarrow V A}=C_{V} \mathcal{M}_{V A \rightarrow V A}+C_{V^{\prime}} \mathcal{M}_{V^{\prime} A \rightarrow V A},
$$

is then needed.

The $t$-dependence of the differential cross section for coherent elastic scattering off a heavy nucleus is primarily determined by the nuclear form factor, $F(t)$,

$$
\frac{d \sigma_{\gamma A \rightarrow V A}}{d t}=\left.|F(t)|^{2} \frac{d \sigma_{\gamma A \rightarrow V A}}{d t}\right|_{t=0},
$$

where $F(t)$ is the Fourier transform of the nuclear density distribution The elastic cross section at $t=0$ is related to the total cross section, $\sigma_{\text {tot }}$, by the optical theorem,

$$
\left.\frac{d \sigma_{V A \rightarrow V A}}{d t}\right|_{t=0}=\frac{\sigma_{\text {tot }}^{2}}{16 \pi}\left(1+\epsilon^{2}\right) \text {. }
$$

The GVDM describes all available data at intermediate energies, see e.g. Fig. 13 from Ref. [70]. Hence vector meson production is very useful for checking the basic approximations of UPC theory.

2.3.2. Cross sections in heavy-ion colliders The first calculations of exclusive vector meson production at heavy-ion colliders were made in Ref. [72]. The model is briefly described here. The total photo-nuclear cross section is the convolution of the photon flux with the differential photo-nuclear cross section, integrated over the photon energy,

$$
\sigma_{A A \rightarrow A A V}=\left.\int_{0}^{\infty} d k \frac{d N_{\gamma}(k)}{d k} \frac{d \sigma_{\gamma A \rightarrow V A}}{d t}\right|_{t=0} \int_{-t_{\min }}^{\infty} d t|F(t)|^{2}
$$

Here $-t_{\min }=\left(M_{V}^{2} / 2 k\right)^{2}$ is the minimum momentum transfer squared needed to produce a vector meson of mass $M_{V}$. The nuclear form factor, $F(t)$, is significant only for $|t|<\left(\hbar c / R_{A}\right)^{2}$. Thus only photons with $k>M_{V}^{2} R_{A} / 2 \hbar c$ can contribute to coherent production.

The expression for $d N_{\gamma} / d k$ in Eq. (6) corresponds to the photon flux at the center of the target nucleus, $r=b$. The flux on the target surface will be higher near the photon-emitting projectile, $b-R_{A}<r<b$ and lower further away, $b<r<b+R_{A}$. In coherent interactions, where the fields couple to the entire nucleus or at least to the entire nuclear surface, a better estimate of the total flux is obtained by taking the average over the target surface

$$
\frac{d N_{\gamma}(k)}{d k}=2 \pi \int_{2 R_{A}}^{\infty} d b b \int_{0}^{R} \frac{d r r}{\pi R_{A}^{2}} \int_{0}^{2 \pi} d \phi \frac{d^{3} N_{\gamma}(k, b+r \cos \phi)}{d k d^{2} r} .
$$




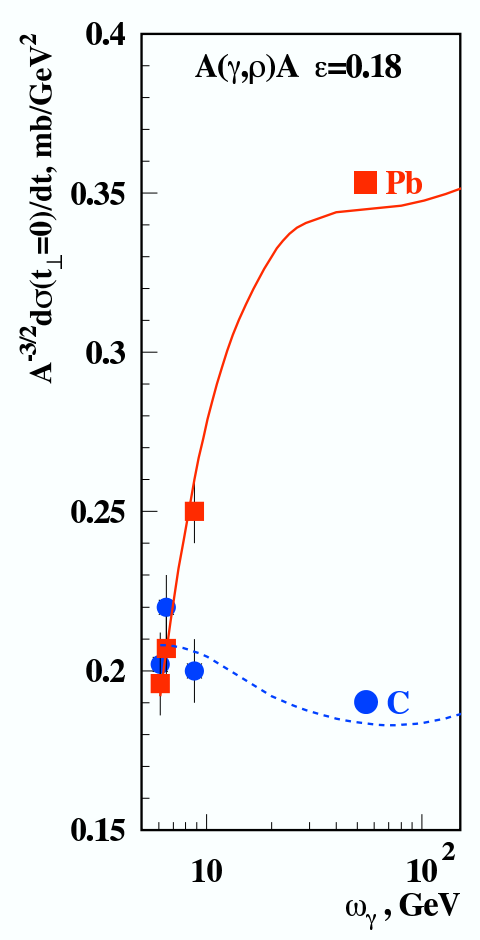

Figure 13. The energy dependence of the $\rho^{0}$ photoproduction cross section calculated in the GVDM with Glauber scattering. The data are from Ref. [71]. Reprinted from Ref. [76] with permission from Elsevier.

The $r$ and $\phi$ integrals, over the surface of the target nucleus for a given $b$, are evaluated numerically. A sharp cutoff at $b=2 R_{A}$ in the lower limit of the integral over $b$ treats the nuclei as hard spheres. In a more realistic model, accounting for the diffuseness of the nuclear surface, all impact parameters are included and the integrand is weighted by the probability for no hadronic interaction, $1-P_{H}(b)$,

$$
\frac{d N_{\gamma}(k)}{d k}=2 \pi \int_{0}^{\infty} d b b\left[1-P_{H}(b)\right] \int_{0}^{R_{A}} \frac{d r r}{\pi R_{A}^{2}} \int_{0}^{2 \pi} d \phi \frac{d^{3} N_{\gamma}(k, b+r \cos \phi)}{d k d^{2} r} .
$$

Here the probability of a hadronic interaction, $P_{H}(b)$, is often taken to be a step function, $P_{H}(b)=1$ for $b>2 R_{A}$ and 0 otherwise. Other, more sophisticated approaches, make a $(10-15) \%$ difference in the flux. This expression, used for the photon flux in the following calculations, is compared to the analytical approximation, Eq. (6), in Fig. 5 for $\mathrm{Pb}+\mathrm{Pb}$ and $\mathrm{Ca}+\mathrm{Ca}$ interactions at the $\mathrm{LHC}$.

As discussed previously, the optical theorem relates the forward scattering amplitude to the total interaction cross section, leading to the scaling

$$
\frac{d \sigma_{\gamma A \rightarrow V A} /\left.d t\right|_{t=0}}{d \sigma_{\gamma N \rightarrow V N} /\left.d t\right|_{t=0}}=\left(\frac{\sigma_{\text {tot }}^{V A}}{\sigma_{\text {tot }}^{V N}}\right)^{2}=A^{\beta}
$$

for $\gamma A$ relative to $\gamma N(\gamma p)$. The total interaction cross section in nuclei is a function of the total cross section on a nucleon and the absorption in nuclear medium. Two limits for the $A$ scaling can be obtained. First, if $\rho_{A} R_{A} \sigma_{\text {tot }}^{V N} \ll 1$, one expects scaling with 
target volume, $A$, and $\beta=2$. When $\rho_{A} R_{A} \sigma_{\text {tot }}^{V N} \gg 1$, the amplitude is proportional to the surface area of the target, $A^{2 / 3}$, and $\beta=4 / 3$.

A more accurate estimate of the effect of absorption on $\sigma_{\text {tot }}^{V A}$ is obtained by a Glauber calculation. In Refs. [72,73], the total cross section was calculated from the classical Glauber formula

$$
\sigma_{\text {tot }}^{V A}=\int d^{2} b\left[1-\exp \left(-\sigma_{\text {tot }}^{V N} T_{A}(b)\right)\right] .
$$

where $T_{A}(b)$ is the nuclear profile function, normalized so that $\int d^{2} b T_{A}(b)=A$. Equation (27) gives $\sigma_{\text {tot }}^{V A} \approx \pi R_{A}^{2}$ for $\rho^{0}$ and $\omega$ production. The model input is based on parameterizations of exclusive vector meson production data from HERA and lower energy, fixed-target experiments. We take $\sigma_{\text {tot }}^{J / \psi N}\left(W_{\gamma p}\right)=1.5 W_{\gamma p}^{0.8} \mathrm{nb}$ from HERA data. We use Eq. (13) for the $\Upsilon$, in agreement with the limited $\Upsilon$ HERA data $[74,75]$. The total production cross sections in different systems at RHIC and the LHC are given in Table 2.3.4.

References [76,77] compare the classical, Eq. (27), and quantum mechanical,

$$
\sigma_{\text {tot }}^{V A}=2 \int d^{2} b\left[1-\exp \left(-\sigma_{\text {tot }}^{V N} T_{A}(b) / 2\right)\right],
$$

Glauber formulas. They also include contributions from the cross term $\rho^{0 \prime} N \rightarrow \rho^{0} N$ and the finite coherence length, both of which are neglected above.

2.3.3. Comparison to RHIC data The STAR collaboration has measured the coherent $\rho^{0}$ production cross section in ultraperipheral $\mathrm{Au}+\mathrm{Au}$ collisions at $W_{N N}=\sqrt{s_{N N}}=130$ $\mathrm{GeV}$ [62], the first opportunity to check the basic model features. The primary assumptions include the Weizsäcker-Williams approximation of the equivalent photon spectrum and the vector meson production model in $\gamma A$ interactions. The basic process is better understood for $\rho^{0}$ production than other vector mesons. Hence, the $\rho^{0}$ study can prove that UPCs provide new information about photonuclear interactions. Inelastic shadowing effects remain a few percent correction at energies less than $100 \mathrm{GeV}$, relevant for the STAR kinematics. In the LHC energy range, the blackening of nuclear interactions should be taken into account. In this limit, inelastic diffraction in hadronnucleus collisions should approach zero. Therefore the $\rho^{0 \prime}$ contribution to diffractive $\rho^{0}$ photoproduction is negligible [76]. The $t$ distributions at $y=0$ and the $t$-integrated rapidity distribution for ultraperipheral $\mathrm{Au}+\mathrm{Au}$ collisions at $\sqrt{s_{N N}}=130 \mathrm{GeV}$ are presented in Fig. 14 [77]. The photon $p_{T}$ spread, which would smear the minimum in the $t$ distribution, and interference are neglected.

The total coherent $\rho^{0}$ production cross section at RHIC, calculated in the GVDM, is shown in Fig. 15 [77]. The cross section is $\sigma_{\mathrm{coh}}=540 \mathrm{mb}$ at $\sqrt{s_{N N}}=130 \mathrm{GeV}$. STAR measured $\sigma_{\mathrm{coh}}=370 \pm 170 \pm 80 \mathrm{mb}$ for $t_{\perp} \leq 0.02 \mathrm{GeV}^{2}$. This $t_{\perp}$ cut, reducing the cross section by $\sim 10 \%$, shown in the dashed curve in Fig. 15, should be included before comparing to the data. The $t_{\perp}$-dependence of the elementary amplitudes was not taken into account since it is relatively independent of energy in the RHIC regime compared 

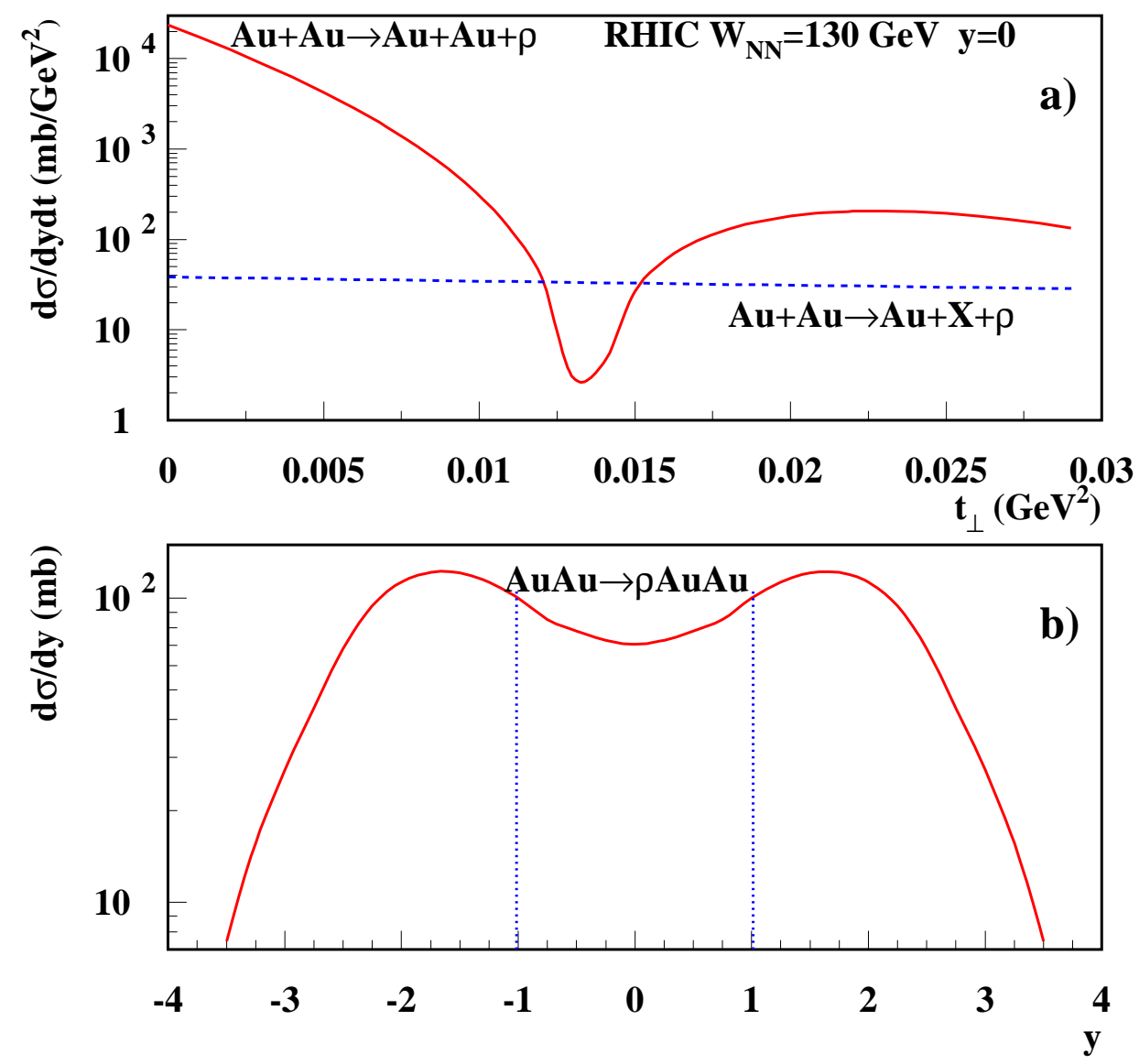

Figure 14. The $t$ dependence of coherent (solid) and incoherent (dashed) (a) and the coherent rapidity distribution (b) of $\rho^{0}$ production in $\mathrm{Au}+\mathrm{Au}$ UPCs at $\sqrt{s_{N N}}=130$ $\mathrm{GeV}$, calculated in the GVDM [77]. The photon $p_{T}$ is neglected. Copyright 2003 by the American Physical Society (http://link.aps.org/abstract/PRC/v67/e034901).

to the nuclear form factor. If included, it would further reduce the cross section slightly. Smearing due to the photon $p_{T}$ and interference of the production amplitudes of the two nuclei are also neglected [78].

Interference produces the narrow dip in the coherent $t_{\perp}$-distribution at $t_{\perp} \leq 5 \times 10^{-4}$ $\mathrm{GeV}^{2}$, in addition to the Glauber diffractive minimum at $\perp \sim 0.013$. While these effects do not strongly influence the $t_{\perp}$-integrated cross section, they can easily be taken into account, giving $\sigma_{\mathrm{coh}}=490 \mathrm{mb}$, closer to the STAR value. Since the calculation does not have any free parameters, the cross section is in reasonable agreement with the STAR data.

2.3.4. LHC Estimates References $[40,48]$ suggested using $J / \psi$ (electro)photoproduction to search for color opacity. However, this requires energies much larger than those available at fixed-target facilities, such as electron-nucleus colliders. FELIX rate estimates [18] demonstrated that the effective photon luminosities generated in peripheral heavy- 

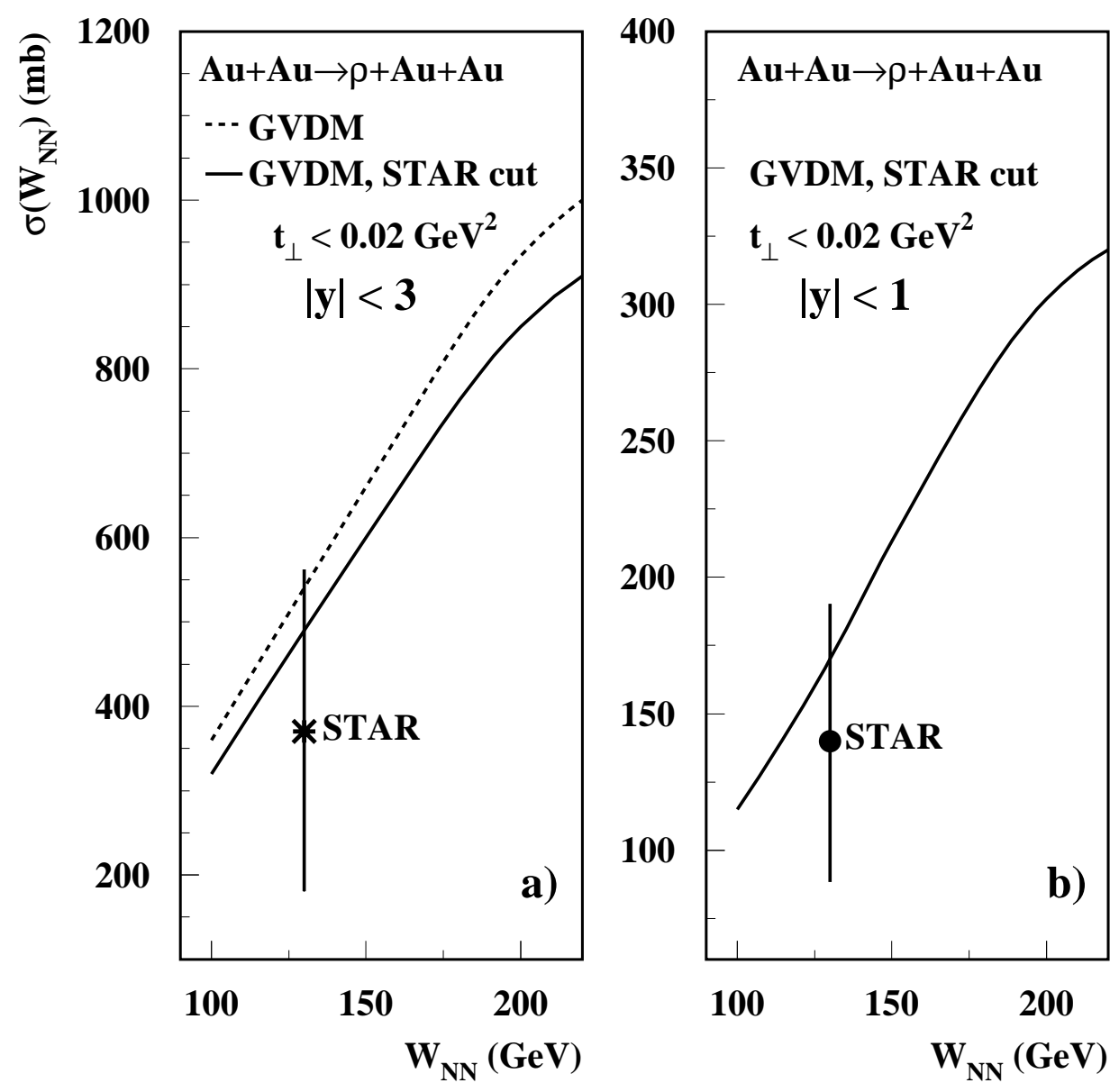

Figure 15. The energy dependence of the total coherent $\rho^{0}$ production cross section in ultraperipheral $\mathrm{Au}+\mathrm{Au}$ collisions, calculated in the GVDM [77]. Copyright 2003 by the American Physical Society (http://link.aps.org/abstract/PRC/v67/e034901).

ion collisions at the LHC would lead to significant coherent vector meson photoproduction rates, including $\Upsilon$. It is thus possible to study vector meson photoproduction in $\mathrm{Pb}+\mathrm{Pb}$ and $\mathrm{Ca}+\mathrm{Ca}$ collisions at the $\mathrm{LHC}$ with much higher energies than $W_{\gamma p} \leq 17.3$ $\mathrm{GeV}$, the range of fixed-target experiments at FNAL [39]. Even current experiments at RHIC, with $W_{\gamma p} \leq 25 \mathrm{GeV}$, also exceed the fixed-target limit. As indicated by the STAR study, coherent photoproduction, leaving both nuclei intact, can be reliably identified using veto triggering from the zero degree calorimeters (ZDCs). Selecting low $p_{T}$ quarkonia removes incoherent events where the residual nucleus is in the ground state.

Hadronic absorption should be moderate or small for heavy vector mesons. The production cross sections are, however, sensitive to gluon shadowing in the parton distribution functions. If two-gluon exchange is the dominant production mechanism $[46,79]$,

$$
\frac{d \sigma_{\gamma A \rightarrow V A} /\left.d t\right|_{t=0}}{d \sigma_{\gamma N \rightarrow V N} /\left.d t\right|_{t=0}}=\left[\frac{g_{A}\left(x, Q^{2}\right)}{g_{N}\left(x, Q^{2}\right)}\right]^{2}
$$

where $g_{A}$ and $g_{N}$ are the gluon distributions in the nucleus and nucleon, respectively. 

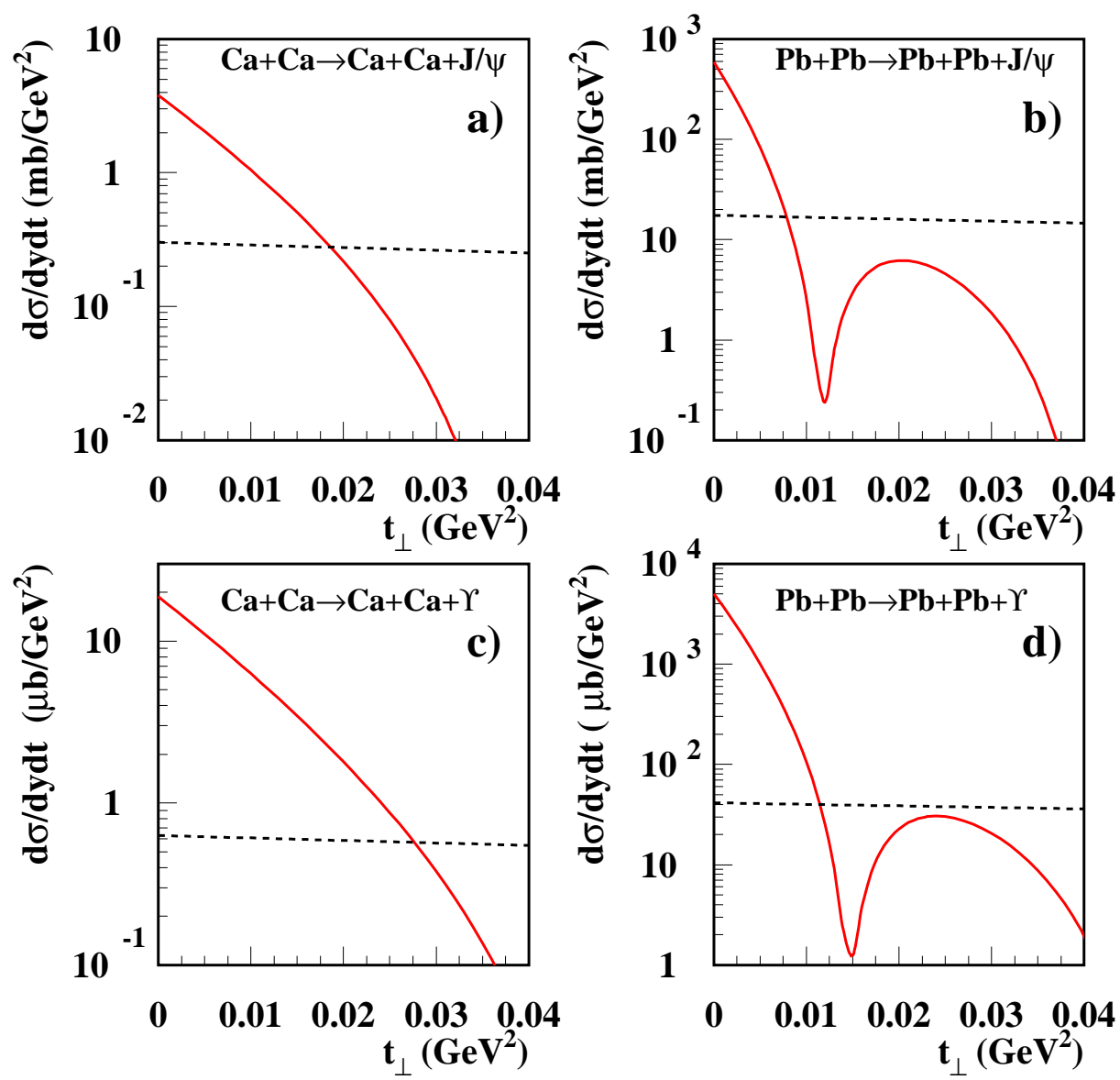

Figure 16. The $t_{\perp}$ distribution of coherent $J / \psi$ and $\Upsilon$ production in $\mathrm{Ca}+\mathrm{Ca}$ and $\mathrm{Pb}+\mathrm{Pb}$ UPCs at the LHC, including leading-twist shadowing but neglecting the photon $p_{T}$ spread. The dashed curves show the incoherent distributions. Reprinted from Ref. [70] with permission from Acta Physica Polonica.

The sensitivity of heavy quarkonia to the gluon distribution functions can be further illustrated by a model comparison. In Fig. 16, the $t_{\perp}$ distributions of coherent $J / \psi$ and $\Upsilon$ photoproduction, calculated with leading-twist shadowing, are compared to incoherent photoproduction. The spread in photon $p_{T}$ is again neglected. The maximum incoherent cross section is estimated to be the elementary cross section on a nucleon target scaled by $A$.

Figure 17 shows the coherent $J / \psi$ and $\Upsilon$ rapidity distributions calculated in the impulse approximation and with nuclear gluon shadowing. At central rapidities, $J / \psi$ production is suppressed by a factor of four (six) for $\mathrm{Ca}+\mathrm{Ca}(\mathrm{Pb}+\mathrm{Pb})$. For comparison, the $\rho^{0}, \phi$ and $J / \psi$ rapidity distributions calculated with the parametrization of Section 2.3.2 are presented in Fig. 18. While at RHIC energies the rapidity distributions have two peaks, corresponding to production off each of the two nuclei [72], the higher LHC energies largely remove the two-peak structure, as shown in Figs. 17 and 18.

The $J / \psi$ and $\Upsilon$ total cross sections are given in Table 2.3.4 for the impulse 

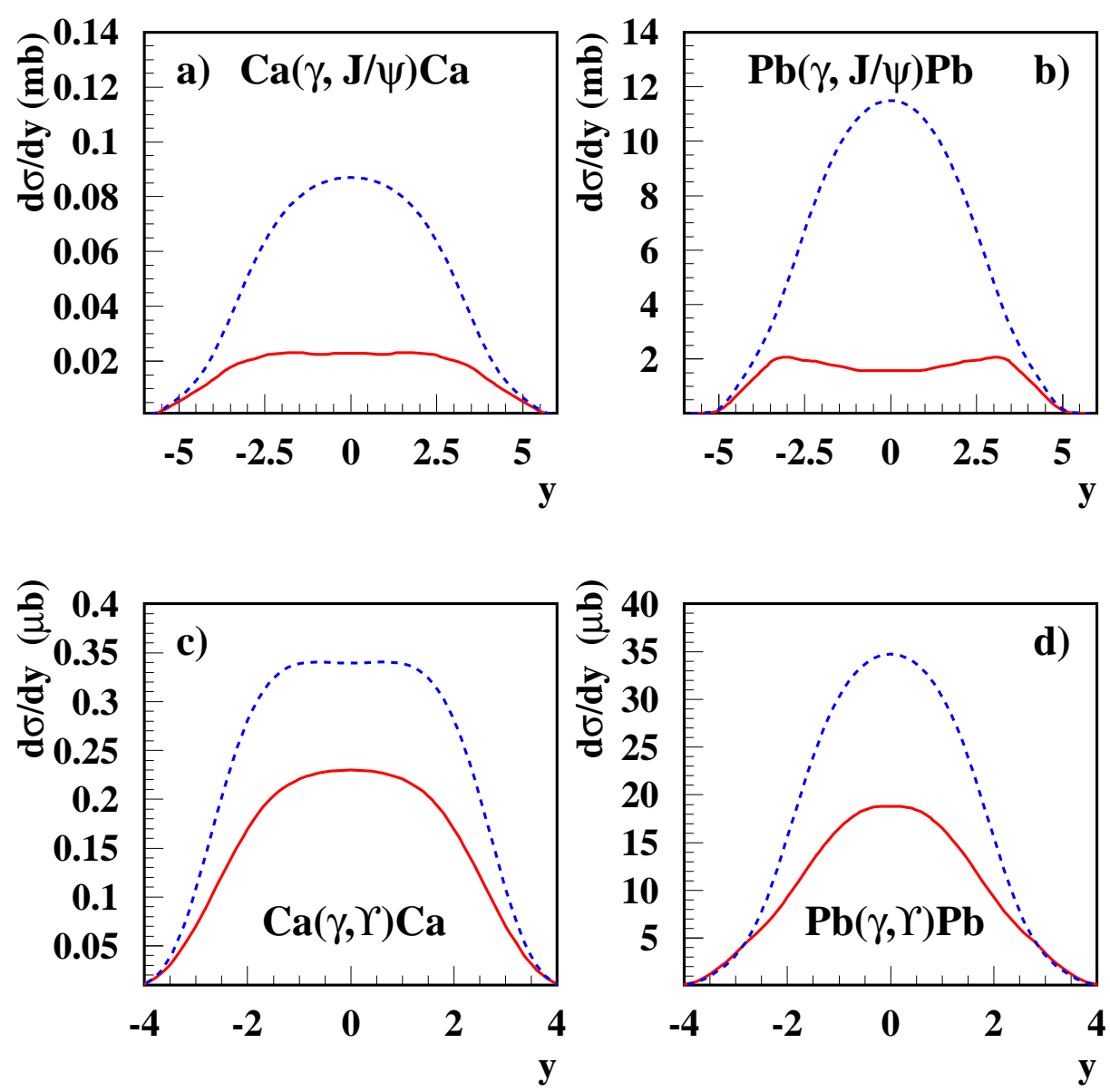

Figure 17. The coherent $J / \psi$ and $\Upsilon$ rapidity distributions in $\mathrm{Ca}+\mathrm{Ca}$ and $\mathrm{Pb}+\mathrm{Pb}$ UPCs at the LHC calculated in the impulse approximation (dashed) and including leading-twist shadowing based on the H1 gluon density parametrization (solid). Reprinted from Ref. [70] with permission from Acta Physica Polonica.

Table 2. Vector meson production cross sections in ultraperipheral $\mathrm{Au}+\mathrm{Au}$ interactions at $\mathrm{RHIC}$ and $\mathrm{Pb}+\mathrm{Pb}$ and $\mathrm{Ca}+\mathrm{Ca}$ interactions at the $\mathrm{LHC}$. The results are shown with the cross section parametrization of Ref. [72] (CP), the impulse approximation (IA) and the IA including leading-twist (LT) shadowing [80, 81].

\begin{tabular}{|c|c||c|c|c||c|c|c|}
\hline & $\mathrm{Au}+\mathrm{Au}$ & \multicolumn{3}{c||}{$\mathrm{Pb}+\mathrm{Pb}$} & \multicolumn{3}{c|}{$\mathrm{Ca}+\mathrm{Ca}$} \\
\hline $2-8 \mathrm{VM}$ & $\sigma_{\mathrm{CP}}(\mathrm{mb})$ & $\sigma_{\mathrm{CP}}(\mathrm{mb})$ & $\sigma_{\mathrm{IA}}(\mathrm{mb})$ & $\sigma_{\mathrm{LT}}(\mathrm{mb})$ & $\sigma_{\mathrm{CP}}(\mathrm{mb})$ & $\sigma_{\mathrm{IA}}(\mathrm{mb})$ & $\sigma_{\mathrm{LT}}(\mathrm{mb})$ \\
\hline$\rho^{0}$ & 590 & 5200 & & & 120 & & \\
$\omega$ & 59 & 490 & & & 12 & & \\
$\phi$ & 39 & 460 & & & 7.6 & & \\
$J / \psi$ & 0.29 & 32 & 70 & 15 & 0.39 & 0.6 & 0.2 \\
$\Upsilon(1 S)$ & $5.7 \times 10^{-6}$ & 0.17 & 0.133 & 0.078 & 0.0027 & 0.0018 & 0.0012 \\
\hline
\end{tabular}

approximation (IA) and including leading-twist shadowing (LT). Comparison of the LT and IA calculations shows that the $\Upsilon$ yield is predicted to be suppressed by a factor 

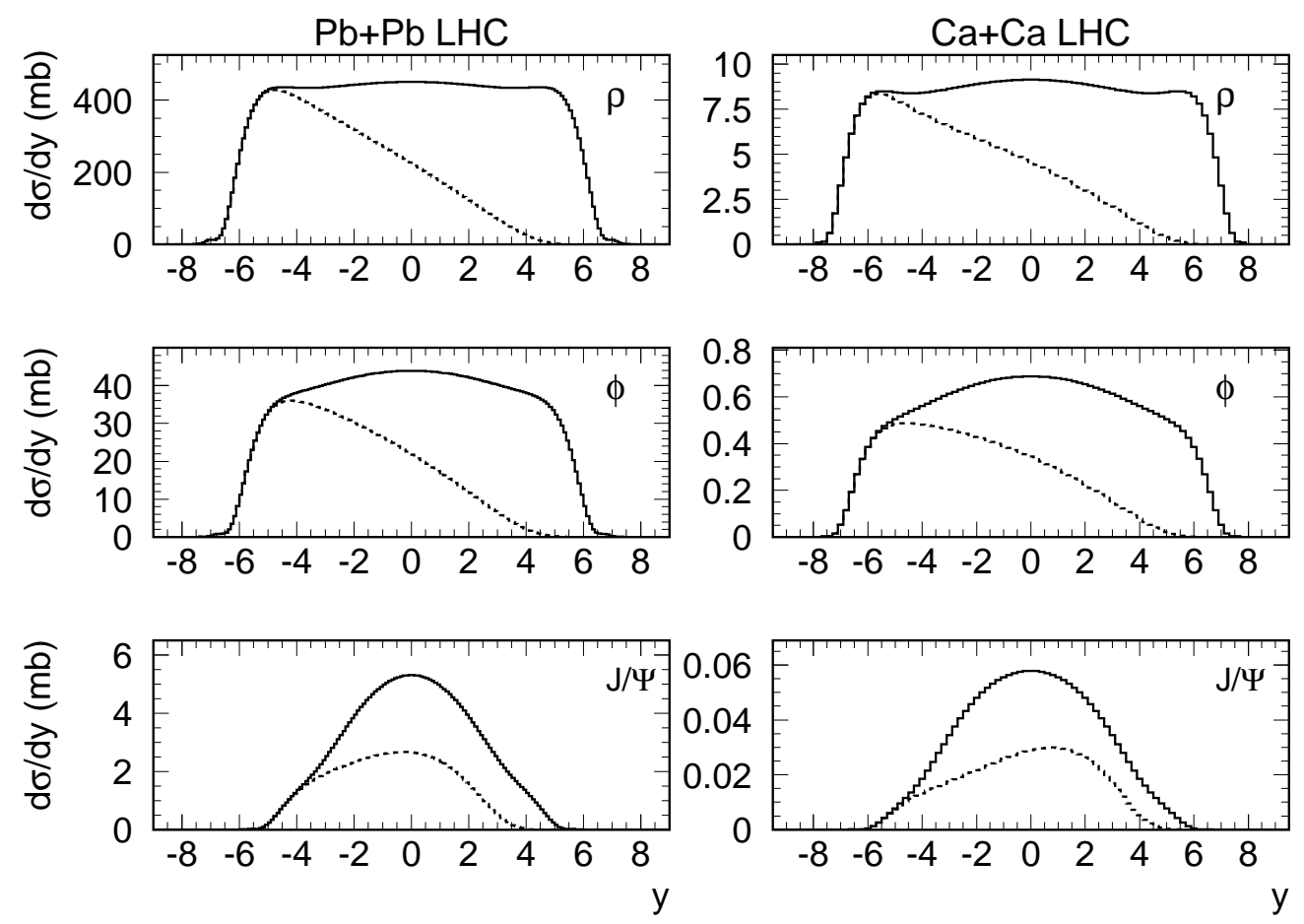

Figure 18. The rapidity distributions of coherent $\rho^{0}, \phi$ and $J / \psi$ production in $\mathrm{Pb}+\mathrm{Pb}$ and $\mathrm{Ca}+\mathrm{Ca}$ UPCs at the LHC. From Ref. [72]. The solid lines correspond to the total rate while the dashed lines are the contribution due to photon emission by the nucleus with negative rapidity. Copyright 1999 by the American Physical Society (http://link.aps.org/abstract/PRC/v60/e014903).

of $\sim 2$ due to leading-twist shadowing. The suppression factor is higher for the $J / \psi$. Hence, coherent quarkonium photoproduction at the LHC can probe shadowing effects on the nuclear gluon distributions in a kinematic regime that would be hard to probe at other facilities. For comparison, the cross sections calculated with the parametrization in Ref. [72] are also given in Table 2.3.4.

2.3.5. Cross sections at pp colliders The strong electromagnetic fields generated by high-energy protons may also lead to exclusive vector meson production in $p p$ and $\bar{p} p$ collisions $[74,75]$.

Although there is no coherent enhancement of the photon spectrum or the photonnucleon cross section, the LHC $p p$ luminosity is about seven orders of magnitude larger than in $\mathrm{Pb}+\mathrm{Pb}$ collisions. In addition, since protons are smaller than ions, photoproduction can occur at smaller impact parameters. Together, these factors more than compensate for the coherent enhancement in $\mathrm{Pb}+\mathrm{Pb}$ collisions. Also, due to the smaller proton size, the photon spectrum extends to higher energies, increasing the kinematic reach.

The calculations are similar to those discussed above for nuclei. The photon spectrum for relativistic protons is, however, different, since the impact parameter is 

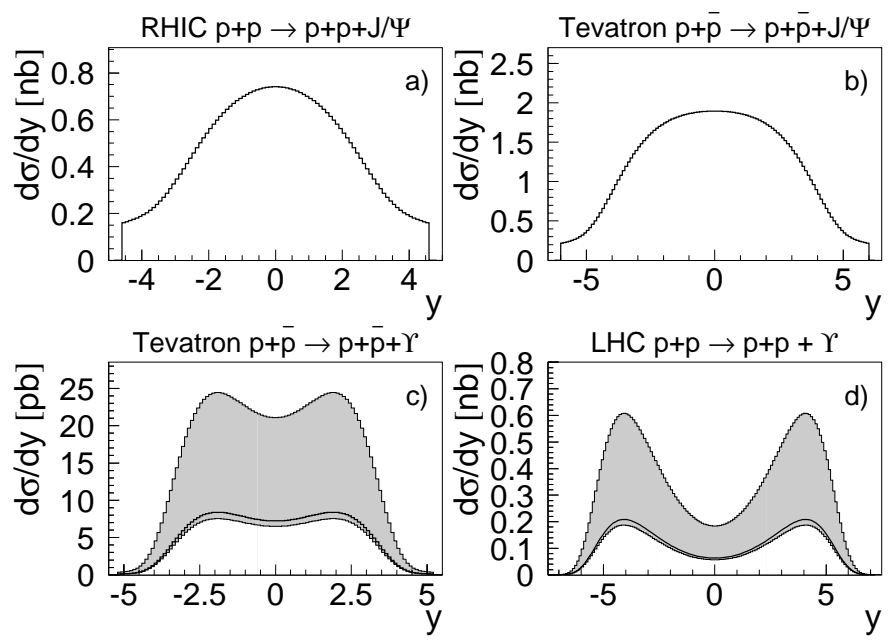

Figure 19. The $J / \psi$ (RHIC, Tevatron) and $\Upsilon$ (Tevatron, LHC) rapidity distributions are shown in $p p$ and $\bar{p} p$ interactions. The cross sections are calculated using $b_{\min }=1.4$ fm. The shaded area in the lower $\Upsilon$ plots due to the poorly known $\gamma p \rightarrow \Upsilon p$ cross section. From Refs. [74,75]. Copyright 2004 by the American Physical Society (http://link.aps.org/abstract/PRL/v92/e142003).

not always well defined for $p p$ collisions. Interference between production sources also differs in $\bar{p} p$ relative to $p p$ and $A A$ collisions due to the different $C P$ symmetry.

The proton photon spectrum, calculated using the dipole formula for the electric form factor, is [82]

$$
\frac{d N_{\gamma}}{d k}=\frac{\alpha}{2 \pi z}\left[1+(1-z)^{2}\right]\left(\ln D-\frac{11}{6}+\frac{3}{D}-\frac{3}{2 D^{2}}+\frac{1}{3 D^{3}}\right)
$$

where

$$
D=1+\frac{0.71 \mathrm{GeV}^{2}}{Q_{\min }^{2}},
$$

$z=W_{\gamma p}^{2} / s_{p p}$ and $Q_{\min }$ is the minimum momentum transfer needed to produce the vector meson.

The $J / \psi$ and $\Upsilon$ rapidity distributions in $p p$ collisions at RHIC and the LHC and in $\bar{p} p$ collisions at the Tevatron are shown in Fig. 19. The corresponding total cross sections are listed in Table 2.3.5.

2.3.6. Multiple vector meson production A unique feature of heavy-ion colliders, not accessible in ep or $e A$ interactions, is multiple vector meson production in a single event. This is a consequence of the extreme electromagnetic fields present in grazing ultrarelativistic heavy-ion collisions. since the photon spectrum in Eq. (5) is proportional to $Z^{2}$ and inversely proportional to $k$ and $b^{2}$. Thus the low energy photon density is large at small $b$. 
Table 3. The vector meson production cross sections in $p p$ and $p \bar{p}$ collisions at RHIC, the Tevatron and the LHC.

\begin{tabular}{|c|c|c|c|}
\hline & $p p$ RHIC & $\bar{p} p$ Tevatron & $p p$ LHC \\
\hline $2-4 \mathrm{VM}$ & $\sigma(500 \mathrm{GeV})(\mu \mathrm{b})$ & $\sigma(1.96 \mathrm{TeV})(\mu \mathrm{b})$ & $\sigma(14 \mathrm{TeV})(\mu \mathrm{b})$ \\
\hline$\rho^{0}$ & 5.1 & 8.6 & 17 \\
$J / \psi$ & 0.0043 & 0.15 & 0.075 \\
$\Upsilon$ & $5.2 \times 10^{-6}$ & $5.5 \times 10^{-5}$ & 0.0016 \\
\hline
\end{tabular}

By changing the order of integration in Eqs. (25) and (23), we can write the unnormalized interaction probability for single vector meson photoproduction as a function of impact parameter as

$$
P_{V}^{(1)}(b)=\frac{d \sigma}{d^{2} b}=\left.\int d k \frac{d^{2} N_{\gamma}}{d k d^{2} b} \frac{d \sigma_{\gamma A \rightarrow V A}}{d t}\right|_{t=0} \int_{t_{\min }}^{\infty} d t|F(t)|^{2}
$$

where $d^{2} N_{\gamma} / d k d^{2} b$ is the photon density of Eq. (5). The superscript '(1)' indicates a single photon exchange. At small $b, P_{V}^{(1)}(b)>1$ for photonuclear processes with low thresholds and/or high cross sections. Thus $P_{V}^{(1)}(b)$ cannot be interpreted as an ordinary probability but should be interpreted as a first-order amplitude. Unitarity can be restored by accounting for multiple exchanges where the probability of having exactly $N$ exchanges is given by a Poisson distribution,

$$
P_{V}^{(N)}(b)=\frac{\left[P_{V}^{(1)}(b)\right]^{N} \exp \left[-P_{V}^{(1)}(b)\right]}{N !} .
$$

The $\rho^{0}$ production probability, $P_{\rho^{0}}^{(1)}(b)$, in $\mathrm{Au}+\mathrm{Au}$ interactions at $\mathrm{RHIC}$ and $\mathrm{Pb}+\mathrm{Pb}$ interactions at the LHC is shown in Fig. 20. Since $\left.P_{V}^{(1)}(b) \ll 1, P_{V}(b) \approx P_{V}^{(1)}(b)\right)$.

Neglecting correlations, the cross section for producing a pair of identical mesons is then

$$
\sigma_{V V}=\frac{1}{2} \int d^{2} b\left[P_{V}(b)\right]^{2} .
$$

If the mesons are not identical,

$$
\sigma_{V_{1} V_{2}}=\frac{1}{2} \int d^{2} b\left[P_{V_{1}}(b)\right]\left[P_{V_{2}}(b)\right] .
$$

This gives $\sigma_{\rho^{0} \rho^{0}} \sim 9 \mathrm{mb}, \sigma_{\phi \phi} \approx \sigma_{\omega \omega} \approx 70 \mu \mathrm{b}$ and $\sigma_{\rho^{0} J / \psi} \sim 0.2 \mathrm{mb}$ in ultraperipheral $\mathrm{Pb}+\mathrm{Pb}$ collisions at the LHC [72].

Production of two vector mesons in a single $A A$ collision introduces angular correlations among the decay products. The linear polarization of the photon follows the electric field vector of the emitting ion so that, at the target, the photon polarization is parallel to $\vec{b}$ [84]. In the case of multiple vector meson production, the photon polarizations are either parallel or anti-parallel.

The photon polarizations affect the angular distribution of the decay products. In the rest frame of a vector meson making a two-body decay such as $\rho^{0} \rightarrow \pi \pi$ or 


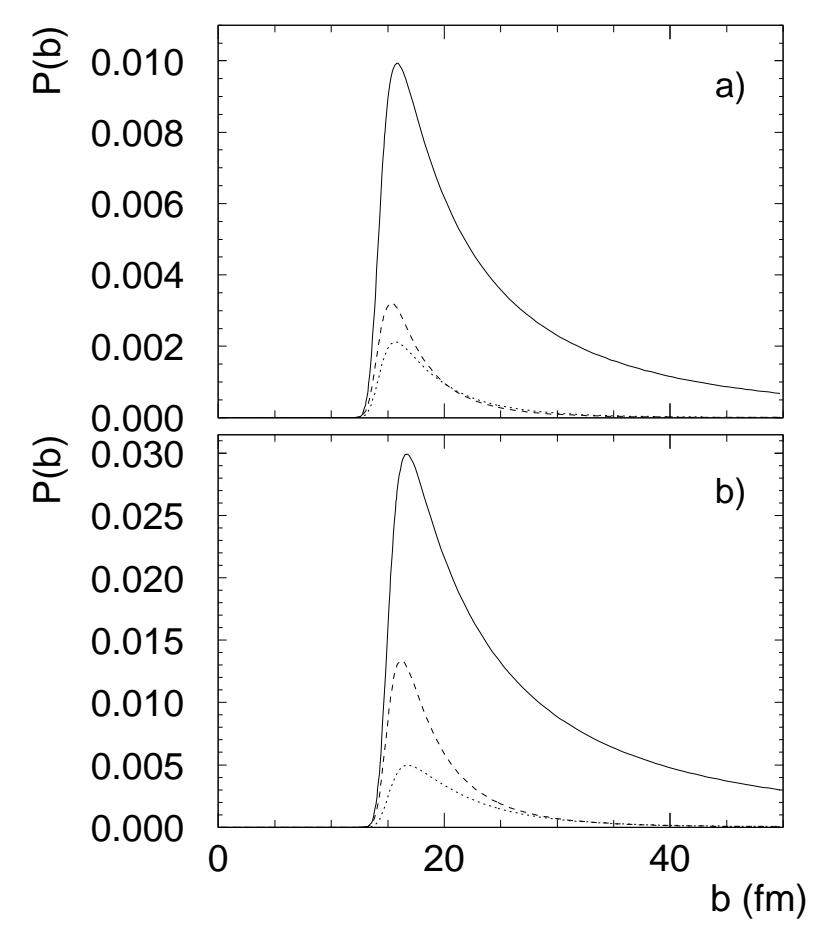

Figure 20. The interaction probability for $\rho^{0}$ production as a function of $b$ in a) $\mathrm{Au}+\mathrm{Au}$ interactions at $\mathrm{RHIC}$ and b) $\mathrm{Pb}+\mathrm{Pb}$ interactions at the LHC. The solid curve is exclusive production, the dashed and dotted curves are for $(X n, X n)$ and $(1 n, 1 n)$ neutron emission due to Coulomb breakup, as described in the text. The dotted curves have been scaled up by a factor of 10 [83]. Copyright 2002 by the American Physical Society (http://link.aps.org/abstract/PRL/v89/e012301).

$J / \psi \rightarrow e e$, the final-state decay particle angular distribution with respect to the photon polarization goes as $\cos \phi$ where $\phi$ is the azimuthal angle, perpendicular to the direction of motion, between the photon polarization and the decay particle direction. Although the polarization is not directly observable, a correlation function can be defined for double vector meson production,

$$
C(\Delta \phi)=1+\frac{1}{2} \cos (2 \Delta \phi),
$$

where $\Delta \phi=\phi_{1}-\phi_{2}$ is the azimuthal difference between the two positively (or negatively) charged decay particles.

Similar neutron correlations are expected in neutron emission in giant dipole resonances (GDRs) which typically decay by single neutron emission. The direction of the neutron $p_{T}$ follows the same azimuthal angle distribution as vector meson decays. For mutual Coulomb excitation to a double giant dipole resonance, the azimuthal separation between the two emitted neutrons should follow Eq. (36).

These angular correlations make UPC studies possible with linearly polarized photons. If the direction of the neutron $p_{T}$ can be measured, a single or mutual GDR excitation tag can be used to determine the polarization direction of any additional photons in the event, allowing studies of polarized proton collisions. The RHIC ZDCs 
have been upgraded to include position-sensitive shower-maximum detectors which can make directional measurements [85]. Similar detectors could be useful at the LHC.

These calculations neglect quantum statistics, addressed in the next section. The cross sections are large enough for multiple vector meson production to be observable, making correlation studies possible.

\subsubsection{Vector meson production in coincidence with nuclear breakup As discussed in the} previous section, strong fields in heavy-ion collisions may lead to large cross sections for interactions involving multiple photon exchanges. The additional photons may interact with the target nucleus in a number of ways. In particular, they may lead to the breakup of one or both of the interacting nuclei. The largest contribution comes from the nuclear excitation to a GDR [86]. About $83 \%$ of GDR decays are via single neutron emission [87].

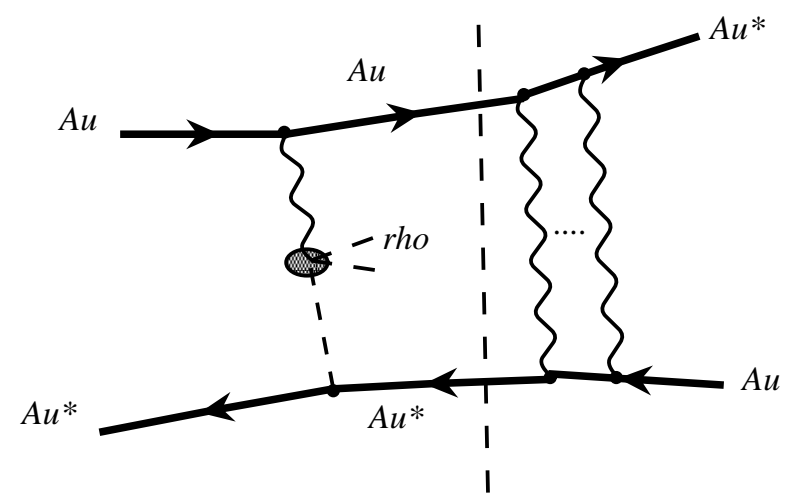

Figure 21. Diagram of nuclear excitation accompanied by $\rho^{0}$ production. From Ref. [83]. Copyright 2002 by the American Physical Society (http://link.aps.org/abstract/PRL/v89/e012301).

There is a $\sim 35 \%$ probability for mutual excitation of both ions in the same event by two-photon exchange in $\mathrm{Au}+\mathrm{Au}$ collisions with $b \sim 2 R_{A}$ at RHIC. The cross section for vector meson production in coincidence with mutual Coulomb breakup of the beam nuclei, see Fig. 21), was calculated in Ref. [83] based on parameterizations of measured photo-nuclear cross sections, $\sigma_{\gamma A \rightarrow A^{*}}$ [88]. The probability for Coulomb breakup of a single nucleus is

$$
P_{X n}(b)=\int d k \frac{d N_{\gamma}}{d k d b^{2}} \sigma_{\gamma A \rightarrow A^{*}}(k) .
$$

Assuming that Coulomb excitation and vector meson production are independent, the probabilities factorize so that

$$
\sigma_{A A \rightarrow A^{*} A^{*} V}=2 \int d b^{2} P_{V}(b) P_{(X n, X n)}(b) \exp \left(-P_{H}(b)\right)
$$

where $P_{(X n, X n)}=P_{X n} P_{X n}$ is the probability for mutual Coulomb excitation followed by the emission of an arbitrary number of neutrons in either direction, and $\exp \left(-P_{H}(b)\right)$ is 
the probability of no hadronic interaction. The cross sections for $\mathrm{Pb}+\mathrm{Pb}$ interactions are shown in Table 2.3.7 for the Coulomb breakup of a single nucleus with multiple neutron emission, $X n$, and for single and multiple neutron emission and Coulomb breakup of both nuclei, $(1 n, 1 n)$ and $(X n, X n)$ respectively, in addition to the total cross section.

Table 4. The cross sections and average impact parameters, $\langle b\rangle$, for vector meson production in $\mathrm{Pb}+\mathrm{Pb}$ interactions at the $\mathrm{LHC}$.

\begin{tabular}{|c|c|c|c|c|c|c|c|c|}
\hline \multirow{2}{*}{$\mathrm{VM}$} & \multicolumn{2}{|c|}{ total } & \multicolumn{2}{c|}{$X n$} & \multicolumn{2}{c|}{$(X n, X n)$} & \multicolumn{2}{c|}{$(1 n, 1 n)$} \\
\cline { 2 - 8 } & $\sigma(\mathrm{mb})$ & $\langle b\rangle(\mathrm{fm})$ & $\sigma(\mathrm{mb})$ & $\langle b\rangle(\mathrm{fm})$ & $\sigma(\mathrm{mb})$ & $\langle b\rangle(\mathrm{fm})$ & $\sigma(\mathrm{mb})$ & $\langle b\rangle(\mathrm{fm})$ \\
\hline$\rho^{0}$ & 5200 & 280 & 790 & 24 & 210 & 19 & 12 & 22 \\
$\omega$ & 490 & 290 & 73 & 24 & 19 & 19 & 1.1 & 22 \\
$\phi$ & 460 & 220 & 74 & 24 & 20 & 19 & 1.1 & 22 \\
$J / \psi$ & 32 & 68 & 8.7 & 23 & 2.5 & 19 & 0.14 & 21 \\
$\Upsilon(1 S)$ & 0.17 & 31 & 0.078 & 21 & 0.025 & 18 & 0.0013 & 20 \\
\hline
\end{tabular}

Vector meson production in coincidence with nuclear breakup is of experimental as well as theoretical interest. All the RHIC and LHC experiments are equipped with ZDCs. These detectors measure neutrons emitted at forward angles by the fragmenting nuclei and may be used for triggering and thus provide a UPC trigger for experiments that lack a low multiplicity trigger at midrapidity.

Requiring the vector mesons to be produced in coincidence with Coulomb excitation alters the impact parameter distribution compared with exclusive production. The probability for $\rho^{0}$ production for single and multiple neutron emission by both nuclei is shown in the dashed and dotted curves of Fig. 20. As seen in the figure and in Table 2.3.7, the mean impact parameter, $\langle b\rangle$, is dramatically reduced in interactions with Coulomb dissociation. The decreased average impact parameter changes the photon energy spectrum, enhancing the relative hard photon yield and modifying the rapidity distributions in interactions with breakup. As discussed in the next section, since both single and multiple neutron emission usually involves at least 3 photons, $\langle b\rangle$ is essentially independent of the photon energy. Thus $\langle b\rangle$ is similar for $(1 n, 1 n)$ and $(X n, X n)$ and is independent of vector meson mass. The change in $\langle b\rangle$ also affects interference between vector meson photoproduction on two nuclear targets, discussed in the next section.

2.3.8. Interference effects on the $p_{T}$ distribution One important feature of $A A$ and $p p$ collisions is that the two incoming particles are identical, i.e. indistinguishable. The initial state is completely symmetric and, because of the small momentum transfers in vector meson photoproduction, it is not possible to tell which nucleus emitted the photon and which was the target. Since the two processes (nucleus one as the photon emitter, nucleus two as the target and nucleus two as the emitter, one the target) are indistinguishable, the amplitudes must be added rather than the cross sections. 
Changing nucleus one from the photon target to the photon emitter is the equivalent of a parity transformation. Since vector mesons have negative parity, the amplitudes are subtracted. If the amplitudes for the two processes are $\mathcal{A}_{1}$ and $\mathcal{A}_{2}$, then

$$
\sigma=\left|\mathcal{A}_{1}-\mathcal{A}_{2} \exp \left[i\left(\vec{b} \cdot \vec{p}_{T}+\delta\right)\right]\right|^{2}
$$

where $\delta$ accounts for possible changes in $\rho^{0}$ photoproduction with photon energy. The exponential is a propagator accounting for the phase shift when nucleus one becomes the target. At midrapidity, $\mathcal{A}_{1}=\mathcal{A}_{2}=\mathcal{A}$ and the expression simplifies to

$$
\sigma=\mathcal{A}^{2}\left[1-\cos \left(\vec{b} \cdot \vec{p}_{T}\right)\right]
$$

Since the impact parameter is unknown, it is necessary to integrate over all $b$. There is significant suppression when

$$
p_{T}<\frac{\hbar}{\langle b\rangle} \text {. }
$$

Using Eq. (33), $\langle b\rangle=46 \mathrm{fm}$ for $\rho^{0}$ production in $\mathrm{Au}+\mathrm{Au} \mathrm{UPC}$ at $\mathrm{RHIC}$, rising to 290 $\mathrm{fm}$ in $\mathrm{Pb}+\mathrm{Pb}$ interactions at the LHC. There is thus significant suppression for $p_{T}<5$ $\mathrm{MeV} / c$ at RHIC and $p_{T}<1 \mathrm{MeV} / c$ at the LHC. At the LHC, observing this suppression may be challenging. A detailed study of the transverse momentum distribution within the framework of the semi-classical and Glauber approximations is done in Ref. [89]. The numerical results are rather similar to those in Ref. [78].

Multiple interactions in heavy-ion collisions makes observation of the interference effect easier since the more photons that are exchanged, the smaller the impact parameter. The average impact parameter is [84]

$$
\langle b\rangle=\frac{\int d^{2} b b P(b)}{\int d^{2} b P(b)}
$$

for any probability $P(b)$. In the case of vector meson production, as long as $\gamma_{L} / k>b>$ $2 R_{A}, P_{V}(b) \approx 1 / b^{2}$. For single photon exchange, $P_{V}(b)=P_{V}^{(1)}(b)$, and

$$
\langle b\rangle=\frac{b_{\max }-b_{\min }}{\ln \left(b_{\max } / b_{\min }\right)}
$$

where $b_{\min }=2 R_{A}$ and $b_{\max }=\gamma_{L} / k$. For $N$ photon exchanges, $N \geq 2$, and $b_{\max }>>b_{\min }$, the result is

$$
\left\langle b_{N}\right\rangle=\frac{2 N-2}{2 N-3} b_{\min }
$$

so that, for example, for vector meson production accompanied by mutual nuclear excitation $(N=3),\langle b\rangle=1.33 b_{\min } \approx 18 \mathrm{fm}$, almost independent of the details of the interaction. Since this independence does not change with beam energy, interference should significantly affect the cross section of mutual nuclear excitation for $p_{T}<11$ $\mathrm{MeV} / \mathrm{c}$ at both RHIC and the LHC. Interference has already been observed at RHIC $[90,91]$ for both single and multiple photon exchange. It should thus be equally observable at the LHC.

A detailed calculation of the $p_{T}$ spectrum requires consideration of both the photon $p_{T}$ and the Pomeron momentum transferred to the vector meson during scattering [78]. 
These two sources, shown in Fig. 22 for $\phi$ production, neglecting interference, must be added in quadrature. The photon $p_{T}$ is approximately peaked around $p_{T} \sim k / \gamma_{L}$. At midrapidity, $k=M_{V} / 2$, resulting in a peak at $M_{V} / 2 \gamma_{L} \sim 5 \mathrm{MeV} / c$ while the photon flux goes to zero as $p_{T} \rightarrow 0$. The Pomeron $p_{T}$ is peaked at zero with a width of $\sim \hbar c / R_{A} \approx 30 \mathrm{MeV} / c$ for heavy ions.

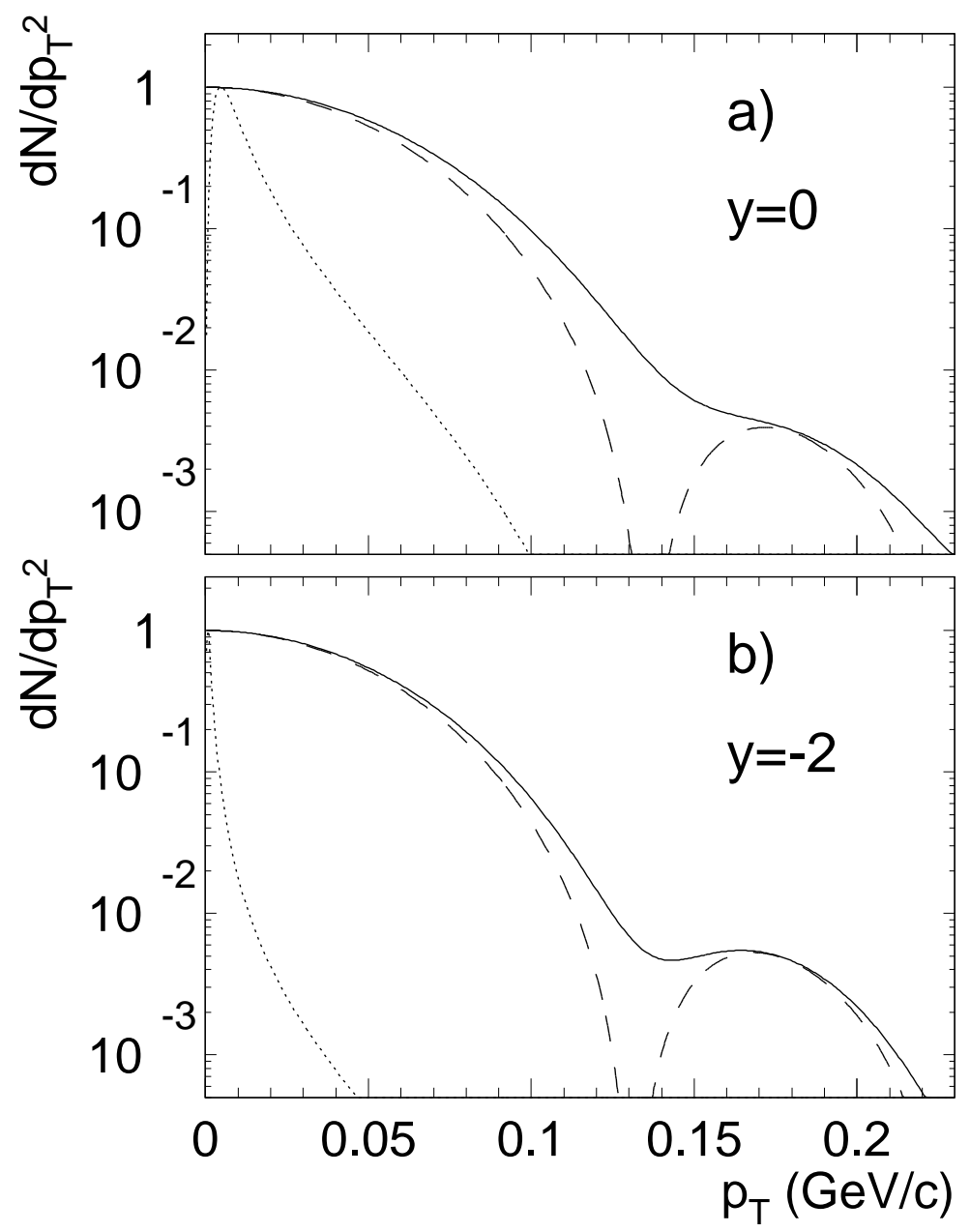

Figure 22. The $p_{T}$ distributions of exchanged photons (dotted) and Pomerons (dashed) as well as final state $\phi$ mesons (solid) in $\sqrt{s_{N N}}=200 \mathrm{GeV} \mathrm{Au}+\mathrm{Au}$ collisions at RHIC, at (a) $y=0$ and (b) $y=-2$. The curves are normalized to unity at $p_{T}=0$. Clear diffraction minima are visible in the Pomeron spectra but are largely washed in the final state. From Ref. [78]. Copyright 2000 by the American Physical Society (http://link.aps.org/abstract/PRL/v84/p2330).

If $P_{V}(b)$ is known, the interference may be calculated. Figure 23 shows $d N / d p_{T}^{2}$ for $\rho^{0}$ and $J / \psi$ production at RHIC and the LHC, both for exclusive vector meson production and vector mesons accompanied by mutual Coulomb excitation. The RHIC and LHC results are very different for exclusive production, and, at the LHC, production is only reduced for $p_{T}<5 \mathrm{MeV} / c$. While small, this $p_{T}$ is much larger than estimates using Eq. (41) because significant production occurs for $b \approx 2 R_{A}$. For vector mesons 
accompanied by mutual Coulomb dissociation, the RHIC and LHC curves are quite similar. Interference has been studied by the STAR Collaboration at RHIC. The results are presented in Section 9.2.4.

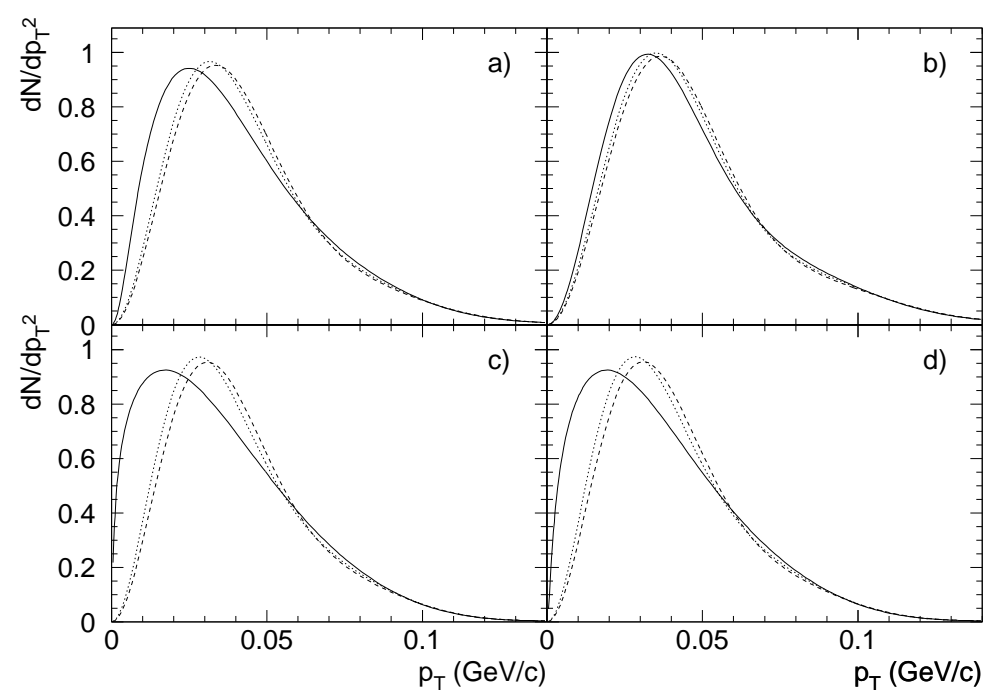

Figure 23. The $p_{T}$ distributions, $d N / d p_{T}^{2}$, for $\rho^{0}$ production at (a) RHIC and (c) the LHC and $J / \psi$ production at (b) RHIC and (d) the LHC. The solid curve shows exclusive vector meson production, the dashed, mutual Coulomb dissociation and the dotted, mutual Coulomb dissociation with single neutron emission in both directions $(1 n, 1 n)$. All the calculations include interference. The results in Fig. 22 are comparable $\phi$ calculations without interference. From Ref. [83]. Copyright 2002 by the American Physical Society (http://link.aps.org/abstract/PRL/v89/e012301).

Interference effects are also present in $p p$ and $\bar{p} p$ collisions. One complication for these smaller systems is that $b_{\min }$ is not easily defined. However, $b_{\min }=0.7 \mathrm{fm}$ is reasonable choice. Thus $\langle b\rangle$ is smaller than in $A A$ collisions, extending the $p_{T}$ scale of the interference to $p_{T} \sim 200 \mathrm{MeV} / c$.

In $p p$ and $\bar{p} p$ collisions the interference depends on the symmetry of the system. For $p p$ or $A A$ collisions, there is a parity transformation from the situation where hadron one emits a photon while hadron two is the photon target to the opposite situation where hadron two is the photon emitter. However, a charge-parity transformation is required for $p \bar{p}$ collisions. Since vector mesons have $C P=--$, the amplitudes from the two sources add so that

$$
\sigma=\left|\mathcal{A}_{1}+\mathcal{A}_{2} \exp \left[i\left(\vec{b} \cdot \vec{p}_{T}\right)\right]\right|^{2} .
$$

Thus the $p \bar{p}$ and $p p$ interference patterns are of opposite sign.

Figure 24 compares the $t$ distributions for $\Upsilon$ production at RHIC ( $p p$ collisions at $\sqrt{s}=500 \mathrm{GeV})$ and the Fermilab Tevatron $(\bar{p} p$ collisions at $\sqrt{s}=1.96 \mathrm{TeV})$. Interference makes the two spectra very different. At the LHC, the average impact parameters will be somewhat larger. Since the vector meson rates will also be much larger, it should be possible to study interference with the $\Upsilon$. 


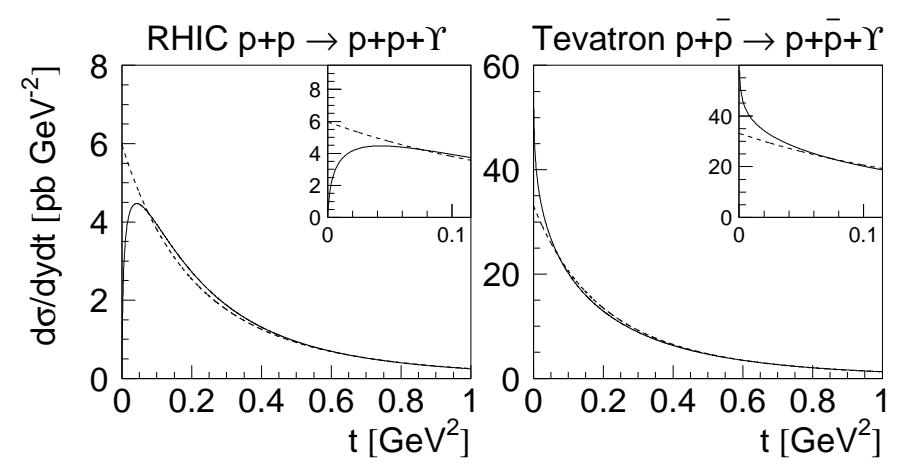

Figure 24. The cross sections, $d \sigma / d y d t$, at $y=0$ for $\Upsilon$ production in (a) $\sqrt{s}=500$ $\mathrm{GeV} p p$ collisions at RHIC and (b) $\sqrt{s}=1.96 \mathrm{TeV} \bar{p} p$ collisions at the Tevatron. The sign of the interference differs in the two cases. From Refs. [74,75]. Copyright 2004 by the American Physical Society (http://link.aps.org/abstract/PRL/v92/e142003).

2.3.9. Interferometry with short-lived particles Interference is especially interesting because the vector mesons have very short lifetimes. For example, the $\rho^{0}$ decays before it travels $1 \mathrm{fm}$ while interference typically occurs at $20<b<40 \mathrm{fm}$. Thus the $\rho^{0}$ photoproduction amplitudes cannot overlap before the $\rho^{0}$ decay. The conventional interpretation [92] is that the interference must occur at a later time, after the wavefunctions of the two sources overlap, thus involving the vector meson decay products. Since interference depends on the sum of the final-state momenta, it is a global quantity and involves non-local effects. In other words, the global final-state wavefunction, $|\Psi\rangle$, is not the product of the individual final-state particle wavefunctions, $\left|\Psi_{n}\right\rangle$,

$$
|\Psi\rangle \neq\left|\Psi_{1}\right\rangle\left|\Psi_{2}\right\rangle \cdots\left|\Psi_{n}\right\rangle,
$$

an example of the Einstein-Podolsky-Rosen paradox.

\subsection{Coherent vector meson production in ultraperipheral $p A$ collisions}

Contributed by: L. Frankfurt, M. Strikman and M. Zhalov

2.4.1. Introduction The $p A$ runs at the LHC will provide another means for studying photonuclear processes. Ultraperipheral vector meson production in $p A$ interactions originate predominantly from protons scattering off the photon field of the nucleus. Interactions where the nucleus scatters with a photon emitted by the proton give a smaller contribution, see Fig. 6 of the introduction.

The elementary reaction, $\gamma p \rightarrow V p$, is the only high-energy two-body reaction dominated by vacuum exchange which can readily be compared to elastic $p p$ scattering. 
Moreover, studying production of light to heavy vector mesons probes increasingly hard interactions.

UPC studies in $A A$ collisions have two limitations. In heavy-ion collisions, the photon can be emitted by either of the two nuclei, making it difficult to study coherent quarkonium production at $x<M_{V} / 2 E_{N}$ where $E_{N}$ is the ion energy per nucleon in the center-of-mass frame. Different systematic errors can also hinder the comparison of data taken at more than one facility such as $\gamma p$ data at HERA and $\gamma A$ data at the LHC.

Studies of UPCs in $p A$ collisions (or $\mathrm{d} A$ collisions, studied at RHIC for technical reasons) can circumvent these problems by measuring vector meson production over a much larger energy and momentum transfer range than at HERA [93]. Effective Pomeron trajectories for light vector meson production and elastic $p p$ scattering can be compared at similar energies, complementing the planned elastic $p p$ scattering studies by TOTEM [29] and possibly also ATLAS [94].

Calculations of the reaction

$$
p+A \rightarrow p+A+V
$$

are performed within the same formalism as vector meson production in ultraperipheral $A A$ collisions. The $k$ integrand in Eq. (23) for $A A$ collisions can be replaced by

$$
\frac{d \sigma_{p A \rightarrow p A V}}{d y d t}=\frac{d N_{\gamma}^{Z}(y)}{d k} \frac{d \sigma_{\gamma p \rightarrow V p}(y)}{d t}+\frac{d N_{\gamma}^{p}(-y)}{d k} \frac{d \sigma_{\gamma A \rightarrow V A}(-y)}{d t}
$$

where the rapidity of the produced vector meson is

$$
y=\frac{1}{2} \ln \frac{E_{V}-p_{z V}}{E_{V}+p_{z V}} .
$$

For large rapidities, the suppression of the finite- $t$ cross section is negligible. We have arbitrarily chosen the nuclear beam to be at positive rapidity and the proton to be at negative rapidity. The equivalent photon flux from the nucleus, $N_{\gamma}(y)$, corrected for absorption at small impact parameters is given by Eq. (6). The condition that the nucleus remains intact restricts scattering to large impact parameters, $b \geq R_{A}+r_{N}$, quantified by the nuclear thickness function, similar to $A A$ interactions. At LHC energies, the total nucleon-nucleon cross section is $\sim 100 \mathrm{mb}$. Therefore, interactions at $b<R_{A}+r_{N}$ give a negligible contribution. The transition region, $b \sim R_{A}+r_{N}$, where absorption, while incomplete, is still significant, gives a very small contribution. Thus inelastic screening corrections can be neglected. The photon flux from the proton is given in Eqs. (30) and (31). The squared vector meson-nucleon center-of-mass energy, $s_{\gamma p}$, is $s_{\gamma p}=2 E_{N}\left(E_{V}+p_{z V}\right)=2 E_{N} M_{V} \exp (y)$. Recall that we generally refer to the squared energy as $s_{\gamma p}$ and the energy itself as $W_{\gamma p}$. In Eq. (30), $z=W_{\gamma p} / \sqrt{s_{N N}}$ and $s_{N N}=4 \gamma_{L}^{p} \gamma_{L}^{A} m_{N}^{2}$ is the nucleon-nucleon center-of-mass energy while $\gamma_{L}^{p}$ and $\gamma_{L}^{A}$ are the proton and nuclear Lorentz factors in the lab frame of each beam.

2.4.2. Heavy quarkonium production We begin with heavy quarkonium photoproduction. A fit [95] to the data was used for $J / \psi$ photoproduction while for $\Upsilon$ production, 
we approximate the cross section as in Eq. (13), consistent with the limited HERA data. The energy dependence follows from the calculations in the leading $\log Q^{2}$ approximation [33], taking into account the inequality of the light-cone momentum fractions on the gluon ladder, see Eq. (8).
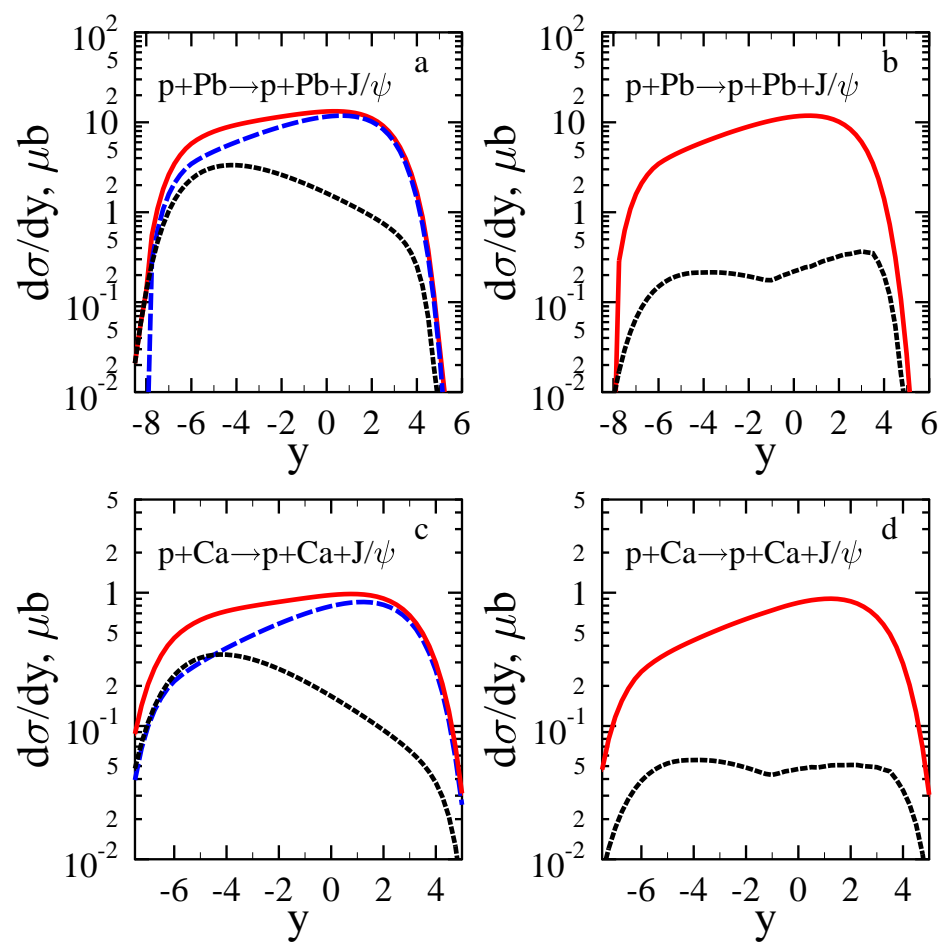

Figure 25. The $J / \psi$ rapidity distribution in $p \mathrm{~Pb},(\mathrm{a})$ and (b), and $p \mathrm{Ca}$, (c) and (d), UPCs at the LHC. The long-dashed curve is the $\gamma p$ contribution and the shortdashed curve is the $\gamma A$ contribution. The solid curve is the sum. Leading-twist nuclear shadowing is included in (b) and (d). Here the $\gamma p$ contribution is indistinguishable from the sum. Reprinted from Ref. [118] with permission from Elsevier.

The coherent quarkonium photoproduction cross section is calculated with leadingtwist nuclear shadowing, see Ref. [70] and Section 2.3. The QCD factorization theorem for exclusive meson photoproduction $[23,24]$ expresses the imaginary part of the forward amplitude for $\gamma A \rightarrow V A$ by convolution of the meson wavefunction at zero $q \bar{q}$ transverse separation, the hard scattering amplitude, and the generalized parton distribution of the target, $G\left(x_{1}, x_{2}, Q^{2}, t_{\min }\right)$, where $t_{\min } \approx-x^{2} m_{N}^{2}$. To a good approximation, $G_{A}\left(x_{1}, x_{2}, Q^{2}, t=0\right) \approx g_{A}\left(x, Q^{2}\right)$ where $x=\left(x_{1}+x_{2}\right) / 2[23,50]$. Hence, the amplitude for $\Upsilon$ photoproduction at $k_{T}^{2}=0$ is [48]

$$
\mathcal{M}(\gamma A \rightarrow \Upsilon A)=\mathcal{M}(\gamma N \rightarrow \Upsilon N) \frac{g_{A}\left(x, Q_{\text {eff }}^{2}\right)}{A g_{N}\left(x, Q_{\text {eff }}^{2}\right)} F_{A}\left(t_{\text {min }}\right)
$$

since the meson wavefunction cancels in the ratio. The nuclear form factor, $F_{A}$, is normalized so that $F_{A}(0)=A$, giving Eq. (29) for the cross section ratio at $t=0$. We use the same model of gluon shadowing as in Section 4.2. Current uncertainties 
in leading-twist gluon shadowing will be reduced after the recent H1 data on hard diffraction [96] is incorporated in the analysis.

In our calculations, we neglect quasi-elastic nuclear scattering since the probability is relatively small and is easily separated from coherent production using information from the ZDCs, see Section 2.5 in Ref. [95].

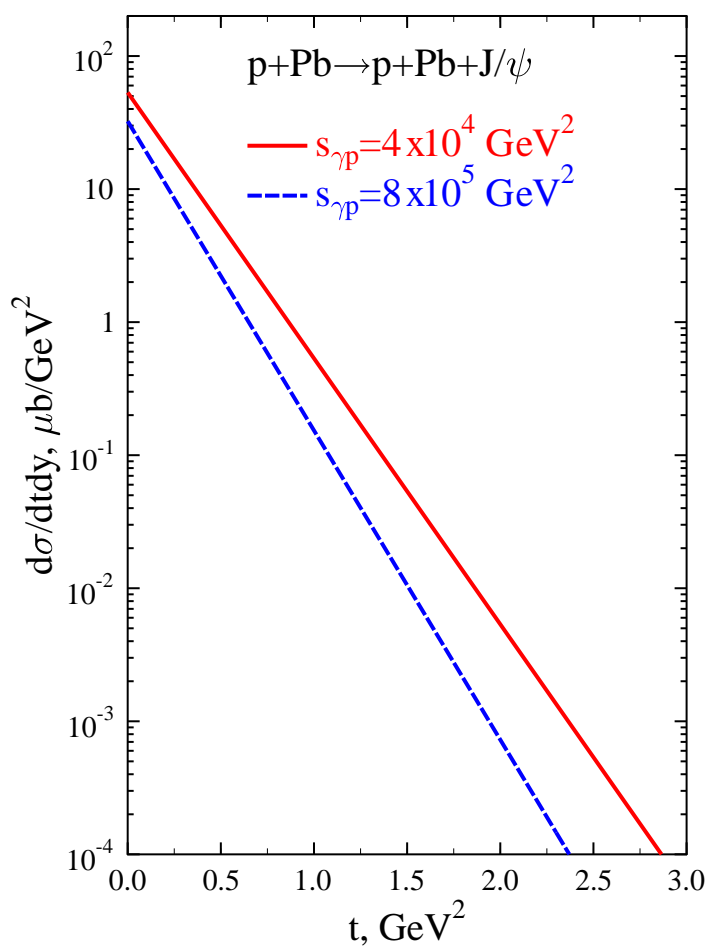

Figure 26. The $J / \psi t$ distribution in $p \mathrm{~Pb}$ UPCs. Reprinted from Ref. [118] with permission from Elsevier.

The $J / \psi$ results are presented in Figs. 25 and 26. The direction of the incoming nucleus corresponds to positive rapidities. One can see from Fig. 25 that the $\gamma p \rightarrow J / \psi p$ cross section in ultraperipheral $p A$ collisions is large enough to be measured in the interval $20<W_{\gamma p}<2 \times 10^{3} \mathrm{GeV}$. The minimum $W_{\gamma p}$ reflects the estimated maximum rapidity at which $J / \psi$ 's could be detected $\|$. The maximum $W_{\gamma p}$ corresponds to $x_{\text {eff }} \sim M_{J / \psi}^{2} / W_{\gamma p}^{2} \sim 2 \times 10^{-6}$, low enough to reach the domain where interaction of small dipoles contributing to the $J / \psi$ photoproduction amplitude already requires significant taming (see e.g. [98]).

For large $W_{\gamma p}$ (positive $y$ ), the coherent $\gamma A \rightarrow J / \psi A$ contribution to $d \sigma / d y$ is negligible. At negative $y, W_{\gamma p}$ is small and $W_{\gamma A}$ is large so that $\gamma A$ contribution becomes relevant. Nevertheless, it remains a correction to the total even without nuclear shadowing. The $t$-dependence of the coherent $\gamma A$ contribution is determined primarily by the nuclear matter form factor. On the other hand, the $t$-dependence of $\|$ In the case when the photon comes from the left, the ALICE detector is expected to have good $J / \psi$ acceptance to $y \sim 3.5$ and $\Upsilon$ acceptance to $y \sim 2$ as well for several smaller $|y|$ intervals [97]. 
the coherent $\gamma p$ contribution is due to the gluon transverse momentum distribution at the relevant $x$ value and is a much weaker function of $t$. Both these $t$-dependencies can be approximated by exponentials. Accordingly, the $\gamma A$ contribution can be determined by fitting $d \sigma / d t$ to a sum of two exponentials. The $\gamma A$ contribution to $J / \psi$ production could be effectively enhanced or reduced by introducing a $p_{T}$ cut, e.g. $p_{T}<300 \mathrm{MeV} / c$ to enhance the $\gamma A$ contribution or $p_{T} \geq 300 \mathrm{MeV} / c$ to reduce it. An observation of the $\gamma A$ contribution with the low $p_{T}$ cut would probe the small dipole interaction with nuclei at $x_{A} \sim 10^{-5}-10^{-6}$. If gluon shadowing is large, observing the $\gamma A$ contribution for such $x_{A}$ would require very good $p_{T}$ resolution, $p_{T} \leq 150 \mathrm{MeV} / c$ or better. It may be possible to eliminate/estimate the $\gamma p$ contribution by measuring recoil protons produced with $x_{\mathbb{P}}=M_{J / \psi}^{2} / W_{\gamma p}^{2}$. In the kinematic range $0.1 \leq x_{\mathbb{P}} \leq 0.001$, the proton could be detected, for example, by the TOTEM T1 and T2 trackers or by the Roman pot system proposed in Ref. [32].
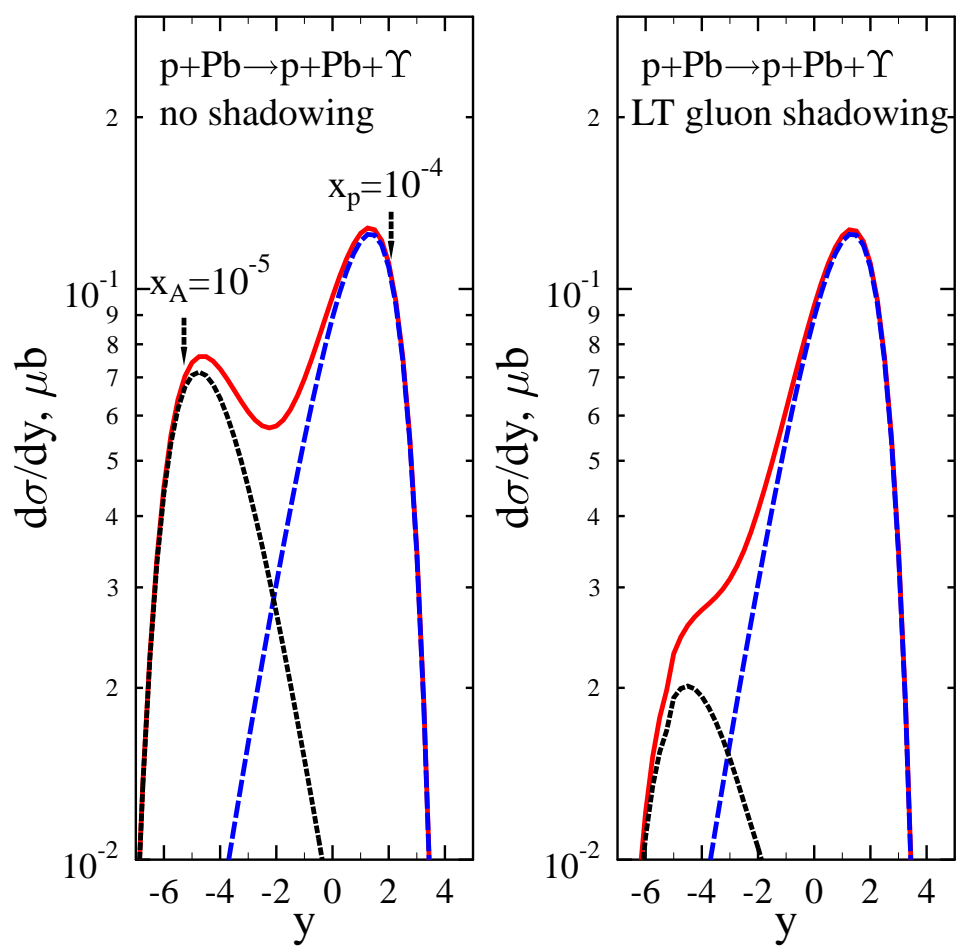

Figure 27. The $\Upsilon$ rapidity distribution in $p \mathrm{~Pb}$ UPCs at the LHC without (left-hand side) and with (right-hand side) leading-twist nuclear shadowing. The $\gamma p$ contribution is given by the long-dashed lines, $\gamma A$ the short-dashed lines and the solid curves are the sum. Reprinted from Ref. [118] with permission from Elsevier.

The $J / \psi$ production cross section will be sufficiently large to measure the $t$ dependence of the $\gamma p$ contribution, shown in Fig. 26, up to $-t \sim 2 \mathrm{GeV}^{2}$, if the contribution from proton dissociation can be suppressed. This measurement provides a unique opportunity to study the $t$ dependence of the hard Pomeron trajectory since the power of $s_{\gamma p} / s_{0}$ in e.g. Eq. (13) can be written as $2\left(\alpha_{\mathbb{P}}^{\text {hard }}(t)-1\right)$. This study will complement measurements of $\alpha_{\mathbb{P}}^{\text {hard }}(t)$ in vector meson production with rapidity gaps, 
discussed in Section 3.2

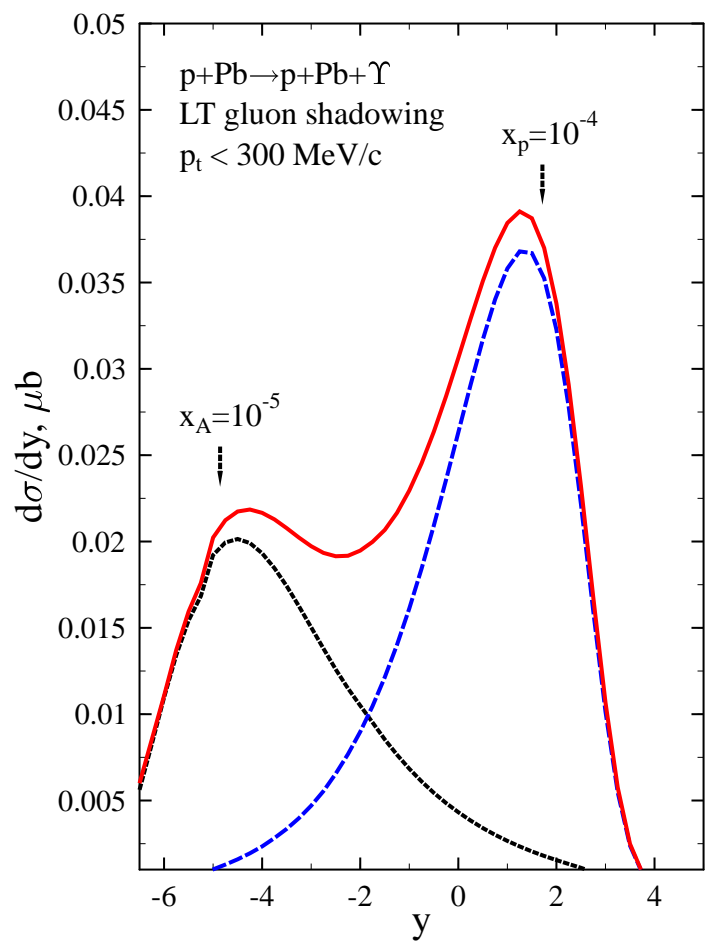

Figure 28. The $\Upsilon$ rapidity distribution in $p \mathrm{~Pb}$ UPCs including leading-twist shadowing and a $p_{T}$ cut on the $\Upsilon, p_{T}<300 \mathrm{MeV} / c$. The curves are the same as in Fig. 27. Reprinted from Ref. [118] with permission from Elsevier.

For $\Upsilon$ production, Fig. 27, the $\gamma p$ contribution can be studied for $10^{2} \leq W_{\gamma p} \leq 10^{3}$ $\mathrm{GeV}$. The $W_{\gamma p}$ interval is smaller due to the strong drop of the cross section with $W_{\gamma p}$, not compensated by the larger photon flux at small $W_{\gamma p}$. Still, this interval is sufficient for a study of the $\Upsilon$ energy dependence since the cross section is expected to increase with $W_{\gamma p}$ by a factor of $\sim 30$ as $W_{\gamma p}$ increases from $100 \mathrm{GeV}$ to $1 \mathrm{TeV}$. The statistics will also be sufficient to determine the slope of the $t$-dependence. Since the $\Upsilon$ is the smallest dipole, this would provide a valuable addition to the measurement of the transverse gluon distribution.

The relative $\gamma A$ contribution is much larger for $\Upsilon$ production. Without nuclear shadowing, $\gamma A$ would dominate at rapidities corresponding to $x_{A} \sim 10^{-5}$, as shown in Fig. 27(a). Even if nuclear shadowing reduces the $\gamma A$ cross section by a factor of $3-4$, Fig. 27(b), the cross section is still dominated by the nuclear contribution at negative rapidities. If the $p_{T}<300 \mathrm{MeV} / c$ cut is applied, Fig. 28, the background could be suppressed further. Hence $p A$ scattering can probe the interaction of $\sim 0.1 \mathrm{fm}$ dipoles with nuclei at very small $x$, virtually impossible in any other process available in the next decade.

The estimates of Ref. [93] indicate that $J / \psi$ photoproduction measurements would also be feasible in high luminosity $p A / \mathrm{d} A$ runs at RHIC. Thus we find that the LHC $p A$ runs will significantly add to quarkonium photoproduction studies in $A A$ collisions by 
new studies of the elementary reaction $\gamma p \rightarrow V p$ in an energy substantially exceeding that of HERA. In addition, ultraperipheral $p A$ collisions provide independent $\Upsilon$ and possibly also $J / \psi$ photoproduction at very small $x_{A}$ in $\gamma A$ interactions.

2.4.3. Light vector meson production The Pomeron hypothesis of universal strong interactions has provided a good description of $p p$ and $\bar{p} p$ interactions at collider energies [99]. The total and elastic hadron scattering cross sections are hypothesized to proceed via single Pomeron exchange. The cross section can be written as

$$
\frac{d \sigma_{h_{1} h_{2} \rightarrow h_{1} h_{2}}}{d t}=f_{h_{1} h_{2}}(t)\left(\frac{s_{h_{1} h_{2}}}{s_{0}}\right)^{2 \alpha_{\mathbb{P}}(t)-2}
$$

where $f_{h_{1} h_{2}}(t)$ parametrizes the $t$ dependence of the cross section, $s_{h_{1} h_{2}}$ is the square of the $h_{1} h_{2}$ center-of-mass energy and $s_{0} \sim 1 \mathrm{GeV}^{2}$. Here $\alpha_{\mathbb{P}}(t)$ is the Pomeron trajectory. At small $t$, as in coherent production,

$$
\alpha_{\mathbb{P}}(t)=\alpha_{0}+\alpha^{\prime} t
$$

The $p p$ and $\bar{p} p$ total and elastic cross sections can be well described with [99]

$$
\alpha_{0}=1.0808, \quad \alpha^{\prime}=0.25 \mathrm{GeV}^{-2} .
$$

Checking the universality hypothesis at fixed-target energies is hampered by exchanges from non-Pomeron sources that die out at high energies. However, significant deviations from universality cannot be ruled out. For example, studies of the total $\Sigma^{-} N$ cross section [100] are consistent with Lipkin's prediction of $\alpha_{0}=1.13$ for this reaction [101].

Vector meson photo/electroproduction plays a unique role in strong interaction studies. Light vector meson photoproduction is the only practical way to check the accuracy of the universality hypothesis for soft interactions at collider energies. The exclusive photoproduction is predicted to be

$$
\frac{d \sigma(\gamma p \rightarrow V p)}{d t}=f_{\gamma p}(t)\left(\frac{s_{\gamma p}}{s_{0}}\right)^{2 \alpha_{\mathbb{P}}(t)-2} .
$$

There are several mechanisms which could cause the predicted universality to break down. In soft interactions there are non-universal multi-Pomeron exchanges which are generally more important at large $t$. The $\rho^{0}$ data are consistent with Eq. (54) for the universal Pomeron trajectory in Eq. (52) with the parameters of Eq. (53) [102]. The very recent $\mathrm{H} 1$ results [103] for $\alpha_{\mathbb{P}}(t)$ using Eq. (54), assuming a linear trajectory lead, to

$$
\alpha_{\mathbb{P}}(t)=1.093 \pm 0.003_{-0.007}^{+0.008}+\left(0.116 \pm 0.027_{-0.046}^{+0.036} \mathrm{GeV}^{-2}\right) t
$$

This result agrees well with the previous ZEUS analysis based on a comparison with fixed-target data [104], seemingly contradicting the universality of $\alpha^{\prime}$. However, the data allow another interpretation: a significant $t$-dependence with $\alpha^{\prime}(t) \sim 0.25 \mathrm{GeV}^{-2}$ for $-t \leq 0.2 \mathrm{GeV}^{2}$.

Thus new questions about soft dynamics arise from the HERA studies of light vector meson photoproduction: 
- To what accuracy is the Pomeron trajectory linear?

- Is $\phi$ production purely soft or will a larger $\alpha_{0}$ be observed, as in $J / \psi$ photoproduction?

- Does $\alpha^{\prime}$ decrease with increasing vector meson mass as expected in pQCD or it is the same for $M \leq M_{J / \psi}$ as the current HERA data may suggest?

- Are nonlinearities in the effective Pomeron trajectories, where $\alpha^{\prime}$ is not constant, the same for all vector mesons?

To address these questions, it is necessary to measure $\rho^{0}$ and $\phi$ photoproduction over the largest possible interval of $W_{\gamma p}$ and $t$. To determine the feasibility of this program in ultraperipheral $p A$ interactions, we used the Donnachie-Landshoff parametrization of the elementary cross section [102],

$$
\frac{d \sigma_{\gamma p \rightarrow V p}}{d t}=\left|T_{S}\left(s_{\gamma p}, t\right)+T_{H}\left(s_{\gamma p}, t\right)\right|^{2},
$$

where $T_{S}\left(s_{\gamma p}, t\right)$ is the amplitude for soft Pomeron and Reggeon exchange and $T_{H}\left(s_{\gamma p}, t\right)$ is the hard Pomeron amplitude. Two Regge trajectories were used [102] to parameterize $T_{S}: \alpha_{\mathbb{P}_{1}}(t)=1.08+\alpha_{\mathbb{P}_{1}}^{\prime} t, \alpha_{\mathbb{P}_{1}}^{\prime}=0.25 \mathrm{GeV}^{-2}$ for soft Pomeron exchange and $\alpha_{R}(t)=$ $0.55+\alpha_{R}^{\prime} t, \alpha_{R}^{\prime}=0.93 \mathrm{GeV}^{-2}$ for Reggeon exchange. The Regge trajectory for the hard Pomeron also uses a linear parametrization: $\alpha_{\mathbb{P}_{0}}=1.44+\alpha_{\mathbb{P}_{0}}^{\prime} t, \alpha_{\mathbb{P}_{0}}^{\prime}=0.1 \mathrm{GeV}^{-2}$.
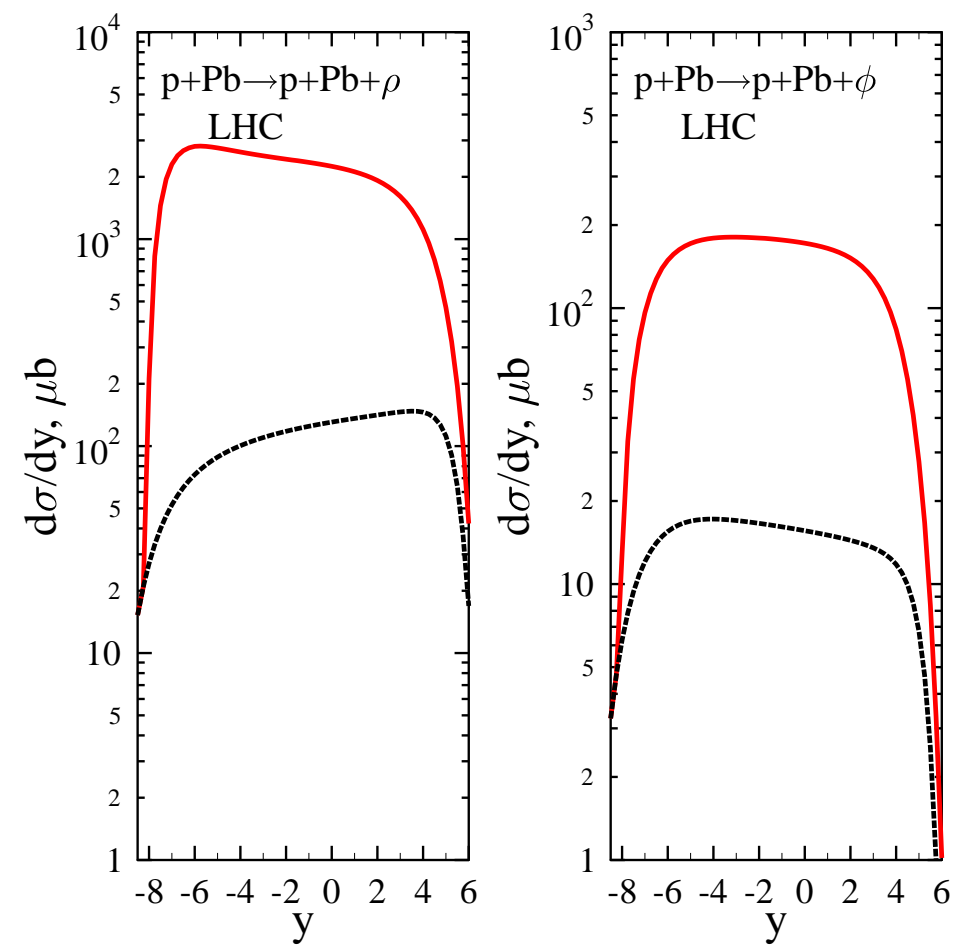

Figure 29. The $\rho^{0}$ and $\phi$ rapidity distributions in $p \mathrm{~Pb}$ UPCs at the LHC. The short-dashed lines are the $\gamma A$ contribution while the solid curves are the total, indistinguishable from the $\gamma p$ contribution. Reprinted from Ref. [118] with permission from Elsevier. 
The coherent light vector meson production cross section for $\gamma A$ interactions was calculated using the vector dominance model combined with Glauber-Gribov multiple scattering. The final-state interaction is determined by the total $V N$ cross sections. The $\rho^{0}$ cross section was calculated using vector dominance with the Donnachie-Landshoff parameterizations for the $\gamma p \rightarrow \rho^{0} p$ amplitude. The energy dependence of the $\phi N$ total cross section was assumed to be $\sigma_{\phi N}=9.5\left(s_{\phi N} / 1 \mathrm{GeV}^{2}\right)^{0.11} \mathrm{mb}$, taken from a fit to the data. The $t$-integrated results are presented in Fig. 29. The rates at the expected LHC $p A$ luminosities are very large, even for $W_{\gamma p}=2 \mathrm{TeV}$. The $t$-dependence is shown in Fig. 30, demonstrating that the rates at $L_{p \mathrm{~Pb}} \approx 1.4 \times 10^{30} \mathrm{~cm}^{-2} \mathrm{~s}^{-1}$ are sufficient for studying the differential cross sections from $|t| \geq 2 \mathrm{GeV}^{2}$ up to $\sqrt{s_{\gamma N}} \approx 1 \mathrm{TeV}$.
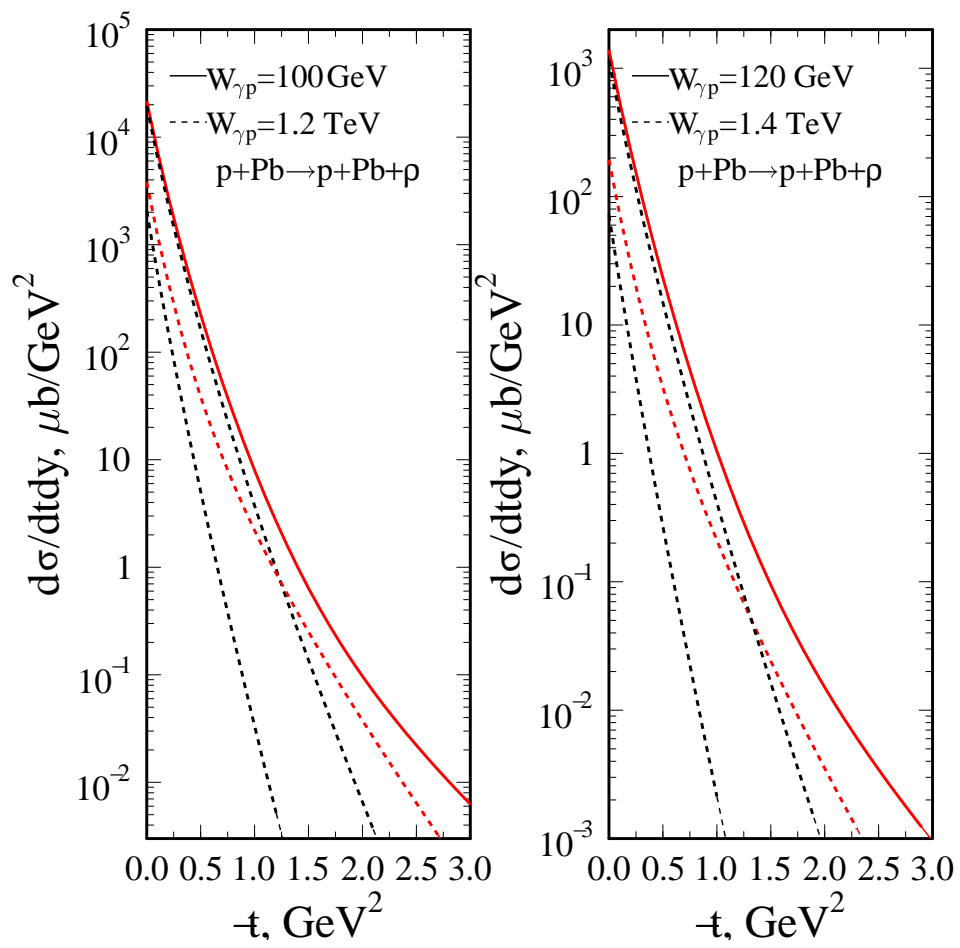

Figure 30. The $\rho^{0}$ and $\phi t$ distributions in $p \mathrm{~Pb}$ UPCs. The solid and long-dashed lines are the results of Eq. (56) for two different values of $W_{\gamma p}$. The short-dashed lines are the same results without the contribution from $T_{H}$. Reprinted from Ref. [118] with permission from Elsevier.

Measurements of the $t$-dependence over two orders of magnitude in $W_{\gamma p}$ in the same experiment would allow precision measurements of $\alpha^{\prime}$ for $\rho^{0}$ and $\phi$ production. For example, if the $t$ dependence of $f_{h_{1} h_{2}}(t)$ is parametrized as $\exp \left[B_{0} t\right]$ and $\left(s_{\gamma p} / s_{0}\right)^{[2(\alpha(t)-1)]}=\left(s_{\gamma p} / s_{0}\right)^{\left[2 \alpha^{\prime} t\right]}$ is reformulated as $\exp \left[2 \alpha^{\prime} t \ln \left(s_{\gamma p} / s_{0}\right)\right]$, then, in general, the cross section is proportional to $\exp [B t]$ where $B=B_{0}+2 \alpha^{\prime} \ln \left(s_{\gamma p} / s_{0}\right)$. Thus, if $\alpha^{\prime}=0.25 \mathrm{GeV}^{-2}$, the change in slope is $\Delta B=B-B_{0} \sim 4.6 \mathrm{GeV}^{-2}$, a $\sim 50 \%$ change. The data should then be sensitive to any nonlinearities in the Pomeron trajectory. It therefore appears that light meson production studies will substantially contribute to the understanding of the interplay between soft and hard dynamics. 
Thus UPC studies in $p A$ collisions at the LHC will provide unique new information about diffractive $\gamma p$ collisions, both in the hard regime, down to $x \sim 10^{-6}$, and in the soft regime.

\subsection{Neutron tagging of quasi-elastic $J / \psi$ and $\Upsilon$ photoproduction}

Contributed by: M. Strikman, M. G. Tverskoy and M. B. Zhalov

In Section 2.4 we argued that ultraperipheral heavy-ion collisions could study coherent vector meson production up to $s_{\gamma N}=2 M_{V} E_{N}$. Although coherent events can be easily identified by selecting vector mesons with sufficiently small transverse momentum, $p_{T} \leq \sqrt{3} / R_{A}$, it is very difficult to determine whether the left or right moving nucleus was the source of the photon that converted into a vector meson. Since the photon flux strongly decreases with increasing photon energy, lower energy photons are the dominant contribution at $y \neq 0$.

Another vector meson production process is governed by similar dynamics and comparable cross section: quasi-elastic production, $\gamma+A \rightarrow V+A^{\prime}$. It is as sensitive to the dynamics of the vector meson interaction in the nuclear medium as coherent processes. The $A$ dependence of this process varies from $\propto A$ for weak absorption to $A^{1 / 3}$ for strong absorption since only scattering off the nuclear rim contributes. Thus, the sensitivity to the change of the interaction regime from color transparency to the black disk regime is up to $\propto A^{2 / 3}$, as is the case for coherent processes where the $t$-integrated cross section is $\propto \sigma_{\text {tot }}^{2}(V A) / R_{A}^{2}$ and changes from $\propto A^{4 / 3}$ to $\propto A^{2 / 3}$. Thus the ratio of quasi-elastic to coherent cross sections should be a weak function of the $Q \bar{Q}$ dipole interaction strength in the medium. This expectation is consistent with Glauber-model estimates where the ratio of quasi-elastic to coherent $J / \psi$ and $\Upsilon$ production cross sections is $0.3-0.2$ over the entire energy range from color transparency (impulse approximation) to the BDR. The $\mu^{+} \mu^{-}$continuum, an important background, for coherent vector meson production, see Sections 2.6 and 2.7, is reduced in incoherent production.

The QCD factorization theorem for quarkonium leads to color transparency for moderate energies, $s_{\gamma N} \leq M_{V}^{2} / x_{0}$ where $x_{0} \sim 0.01$ is a minimum scale where little absorption is expected. As $x$ decreases and $s_{\gamma N}$ increases, color transparency gives way to leading-twist nuclear shadowing and, ultimately, the BDR, violating the factorization theorem.

In most of the LHC detectors, it is much easier to trigger on vector meson production if it is accompanied by the breakup of at least one of the nuclei, resulting in one or more neutrons with the per nucleon beam energy, $\sim E_{N} \approx 0.5 \sqrt{s_{N N}}$, hitting one of the ZDCs. Current measurements and numerical estimates indicate that, at RHIC, given coherent $J / \psi$ production, there is a $50-60 \%$ probability for excitation. The probability will be somewhat larger at the LHC [83]. The removal of a nucleon from a heavy nucleus in the quasi-elastic process should also lead to significant nuclear breakup, resulting in the emission of several neutrons with a probability of order one. Hence, the 
detection rates for quasi-elastic and coherent processes in UPCs at RHIC and the LHC should be comparable.

Here we summarize the first study [95] of the characteristics of quasi-elastic processes relevant for their identification in UPCs. As a starting point, we use $J / \psi$ photoproduction at RHIC and $\Upsilon$ production at the LHC. In both cases, the effective cross sections for the $Q \bar{Q}$ pair interaction with the medium are rather small.

We then use the impulse approximation to model the neutron yields with quarkonium production. Data shows that the $t$ dependence of the $\gamma+N \rightarrow J / \psi+N$ cross section is rather flat, $B_{J / \psi} \sim 4-5 \mathrm{GeV}^{-2}$, in the RHIC and LHC energy range. The $\Upsilon$ slope, $B_{\Upsilon}$, is expected to be even smaller, $\sim 3.5 \mathrm{GeV}^{-2}$. The effective $t$ range in quasielastic production can be rather large, up to $\sim 1 \mathrm{GeV}^{2}$, relative to coherent quarkonium photoproduction where $|t| \leq 0.015 \mathrm{GeV}^{2}$ since higher $t$ is suppressed by the nuclear form factor. The ejected nucleons have average momenta $p_{N} \approx \sqrt{|t|} \approx 1 / B_{V} \sim 0.3$ $\mathrm{GeV}$, large enough for strong reinteraction in the nucleus, making the probability for the nucleus to break up when a nucleon is emitted of order one.

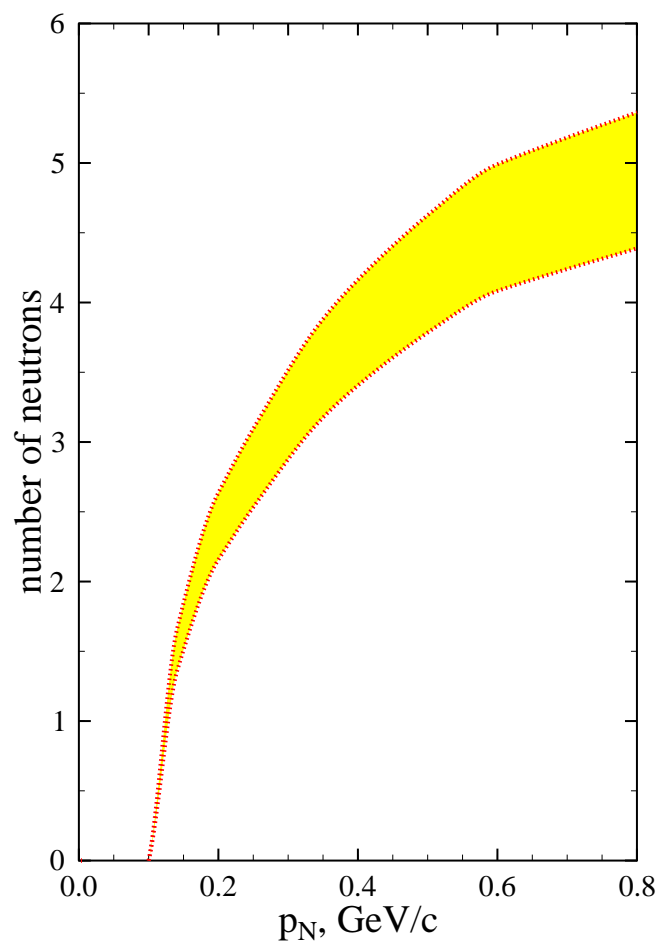

Figure 31. The average number of neutrons emitted in incoherent $J / \psi$ production in $\mathrm{Au}+\mathrm{Au}$ UPCs at RHIC and $\Upsilon$ production in $\mathrm{Pb}+\mathrm{Pb}$ UPCs at the LHC as a function of the recoil nucleon momentum, $p_{N}=\sqrt{|t|}$. The band indicates the estimated uncertainties of the Monte Carlo. Reprinted from Ref. [95] with permission from Elsevier.

To characterize the interaction of the recoil nucleon with the residual nucleus in the reaction, $N+(A-1) \rightarrow C_{i}+k n$, we introduce the excitation function, $\Phi_{C_{i}, k n}\left(p_{N}\right)$, the probability to emit $k$ neutrons with $C_{i}$ charged fragments. The excitation function 
was calculated including the nucleon cascade within the nuclear medium followed by the evaporation of nucleons and nuclear fragments from the nucleus. In Ref. [105], the same Monte Carlo was used to analyze neutron production in the E665 fixed-target experiment at Fermilab which studied soft neutron production in $\mu \mathrm{Pb}$ DIS. A a good description of these data [106], as well as other intermediate energy neutron production data in $p A$ interactions, was obtained. The dependence of the average number of emitted neutrons on the recoil nucleon momentum is shown in Fig. 31. For typical quasi-elastic $J / \psi$ or $\Upsilon$ production, $p_{T} \sim B_{J / \psi}^{-1 / 2} \sim 0.5 \mathrm{GeV} / c$, about four neutrons are emitted per event.

In Ref. [95], a more realistic estimate of the absolute $J / \psi$ production rate at $\mathrm{RHIC}$ was obtained, including absorption of the $c \bar{c}$ in the nuclear medium. An effective $c \bar{c}$ interaction cross section, $\sigma_{\text {eff }}(x \geq 0.015)=3 \mathrm{mb}$, was used, based on Ref. [107]. In these kinematics, the contribution of double elastic scattering can be neglected since $\sigma_{\text {el }} / \sigma_{\text {in }}$ is very small for quarkonium interactions. Thus a simple Glauber-type model approximation can be used to obtain the probability for exactly one elastic rescattering and no inelastic interactions,

$$
\sigma_{\gamma A \rightarrow J / \psi A^{\prime}}^{\text {incoh }}=2 \pi \sigma_{\gamma N \rightarrow J / \psi N} \int_{0}^{\infty} d b b \int_{-\infty}^{\infty} d z \rho_{A}(b, z) \exp \left[-\sigma_{\text {tot }}^{J / \psi N} T_{A}(b)\right] .
$$

Here $\sigma_{\text {tot }}^{J / \psi N}$ is the effective quarkonium-nucleon total cross section, $\sim 3 \mathrm{mb}$.

The coherent and incoherent $J / \psi$ photoproduction cross sections in UPCs, integrated over rapidity and momentum transfer in the RHIC kinematics, are given in Table 5. The table also shows the quasi-elastic $J / \psi$ partial cross sections without any emitted neutrons, $(0 n, 0 n)$, and with the breakup of one nucleus, $(0 n, X n)$, where $X \geq 1$. The ratios $(0 n, 0 n) /$ total and $(0 n, X n) /$ total should be similar for $\Upsilon$ production at $y=0$ at the LHC.

Table 5. The total coherent and incoherent $J / \psi$ photoproduction cross sections calculated in the impulse approximation (IA) and the Glauber approach in $\mathrm{Au}+\mathrm{Au}$ UPCs at RHIC.

\begin{tabular}{|c|c|c|c|c|}
\hline & $\sigma_{\text {coh }}(\mu \mathrm{b})$ & $\sigma_{\text {incoh }}(\mu \mathrm{b})$ & $\sigma_{\text {incoh }}^{(0 n, 0 n)}(\mu \mathrm{b})$ & $\sigma_{\text {incoh }}^{(0 n, X n)}(\mu \mathrm{b})$ \\
\hline IA & 212 & 264 & 38 & 215 \\
Glauber & 168 & 177 & 25.5 & 144 \\
\hline
\end{tabular}

The coherent and quasi-elastic $J / \psi$ rapidity distributions, integrated over $t$, are shown in Fig. 32 for several values of $\sigma_{\text {tot }}^{J / \psi N}$ to illustrate their sensitivity to the $J / \psi N$ interaction strength. The coherent distribution is narrower because it is suppressed by the nuclear form factor in the region where the longitudinal momentum transfer, $p_{z}=M_{J / \psi}^{2} m_{N} / s_{\gamma N}$, is still significant. The predictions of Ref. [95] at $y=0$ agrees with the preliminary PHENIX data, see Section 9.3. 


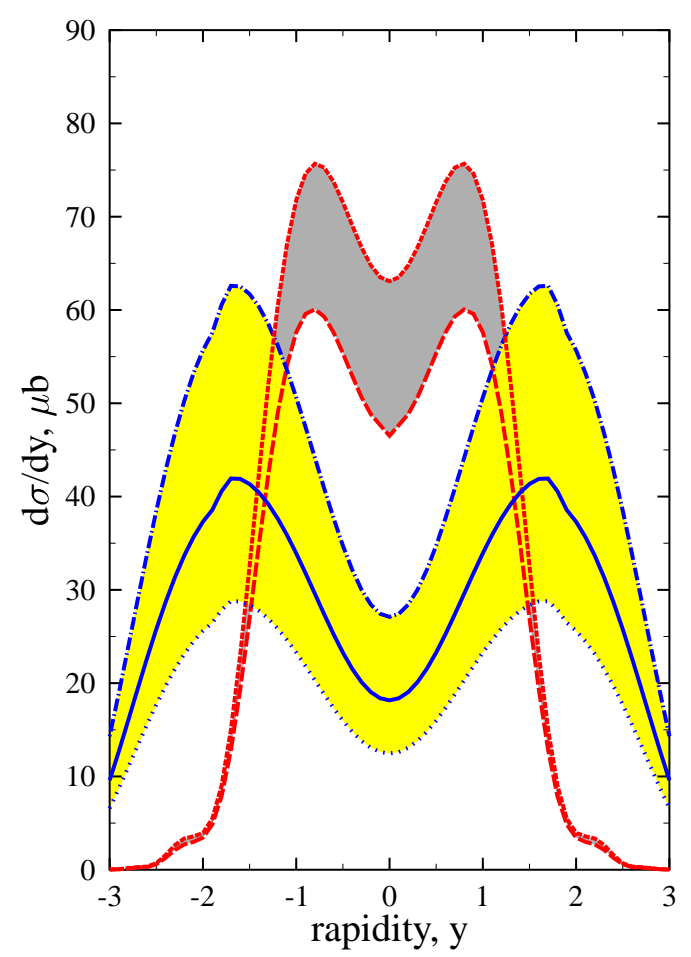

Figure 32. The $t$-integrated rapidity distributions for coherent $J / \psi$ photoproduction in $\mathrm{Au}+\mathrm{Au}$ UPCs at RHIC calculated in the impulse approximation (short-dashed line) and with $\sigma_{\text {tot }}^{J / \psi N}=3 \mathrm{mb}$ (long-dashed line). The incoherent $J / \psi$ cross section calculated in the Glauber model for $\sigma_{\text {tot }}^{J / \psi N}=0$ (dot-dashed line), 3 (solid) and 6 (dotted) mb. Reprinted from Ref. [95] with permission from Elsevier.

The $t$ dependence of the rapidity-integrated cross sections is shown in Fig. 33. It is easy to discriminate between the coherent and quasi-elastic events by selecting different t. At $t \leq 0.01 \mathrm{GeV}^{2}$ the quasi-elastic contribution (dashed line) is small while it is dominant at higher $t$. The shaded histogram shows incoherent $J / \psi$ photoproduction accompanied by neutron emission due to final-state interactions with the recoil nucleon. Quasi-elastic $J / \psi$ production accompanied by neutron emission has a probability of almost unity. The only exception is the region of very small $t$ where the recoil energy is insufficient for nucleon removal. In gold, the minimum separation energy is $\sim 5 \mathrm{MeV}$. Generally, the ratio of the incoherent cross section with emission of one or more neutrons is about $80 \%$ of the total incoherent cross section. The dependence of the incoherent cross section, integrated over rapidity and $t$, on the number of emitted neutrons is presented in Fig. 34. The distribution has a pronounced peak for $k=2$ with a long tail up to $k=14$. The average number of emitted neutrons is $\langle k\rangle \approx 4.5$ with a standard deviation of $\approx 2.5$. Single neutron emission is strongly suppressed due to the low probability of the decay of the hole produced by knock-out nucleon into a single neutron. The probability for the knock-out nucleon to emit a neutron while propagating through the nucleus is greater than $50 \%$.

Neutron tagging of incoherent quarkonium photoproduction can determine which 


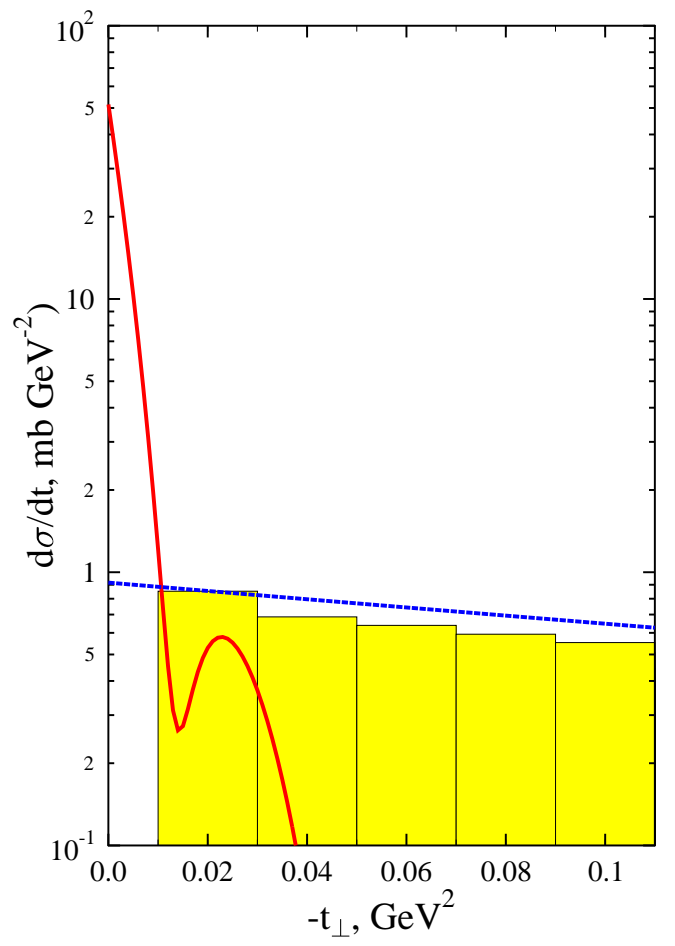

Figure 33. The $t$ distribution integrated over $-3 \leq y \leq 3$ for coherent (solid line) and incoherent (dashed line) $J / \psi$ photoproduction in UPCs at RHIC. The shaded histogram shows the incoherent cross section with neutron emission. Reprinted from Ref. [95] with permission from Elsevier.

nucleus was the photon target since the neutrons are emitted by the target. It is then possible to resolve the ambiguity between photon-emitter and photon-target for a given rapidity, not possible for coherent production on an event-by-event basis.

To a first approximation, neutron emission due to electromagnetic dissociation does not depend on the quarkonium $p_{T}$. Hence, this mechanism can be quantified in coherent production at small $t_{\perp}$ and folded into quasi-elastic $J / \psi$ production at larger $t$.

The pattern of neutron emission we find in quasi-elastic $J / \psi$ production is qualitatively different from electromagnetic excitation. Reference [83] predicts that $\sim 50-70 \%$ of RHIC collisions occur without electromagnetic excitation. The largest partial channel is one-neutron emission, $1 n$, followed by two-neutron emission, $2 n$, about $35 \%$ of $1 n$ events, and a long tail with a broad and falling distribution [108111]. On the other hand, two-neutron emission is most probable for the quasi-elastic mechanism. In addition, the correlation between emitted neutrons in the quasi-elastic and electromagnetic mechanisms is different. In the quasi-elastic case, neutrons are emitted in only one of two directions while simultaneous emission in both directions is possible in the electromagnetic case.

At the LHC, electromagnetic neutron emission is more important than at RHIC. The probability of nuclear dissociation is close to $50 \%$ [112]. Most likely, only one neutron is emitted, see Fig. 35, calculated with RELDIS [109,113]. It is possible to either 


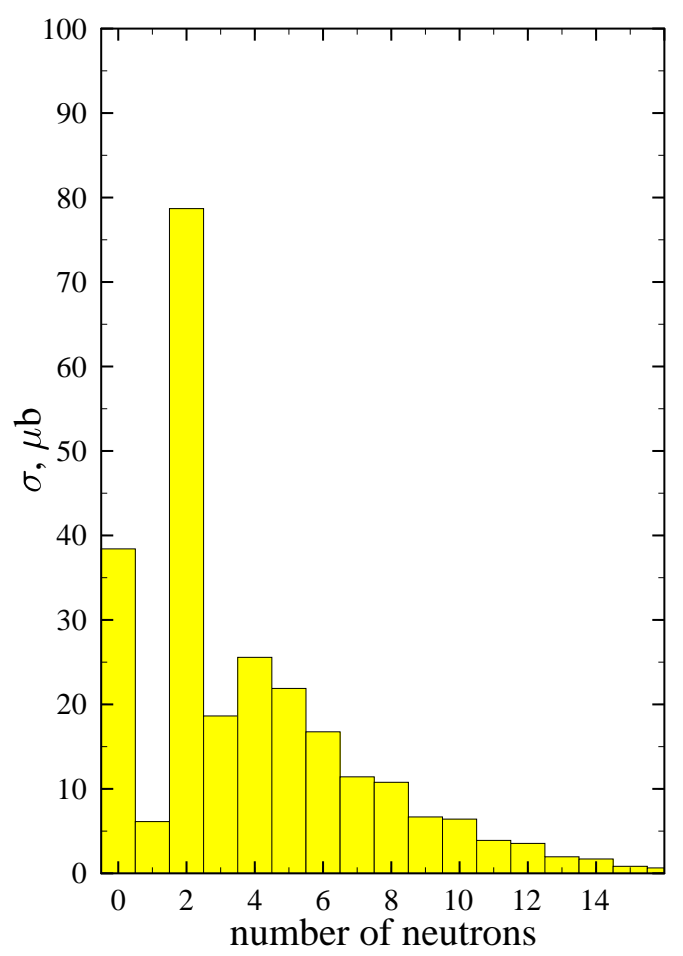

Figure 34. The incoherent $J / \psi$ cross section in $\mathrm{Au}+\mathrm{Au}$ UPCs at RHIC as a function of the number of emitted neutrons. A similar dependence is expected for $\Upsilon$ production at the LHC. Reprinted from Ref. [95] with permission from Elsevier.

select only events where one nucleus did not dissociate or use a deconvolution procedure to separate events where neutron emission is due to electromagnetic excitation rather than nuclear dissociation. For example, the difference between the number of neutrons emitted by two nuclear decays could be studied. A more detailed analysis, including both electromagnetic and quasi-elastic neutron emission in quarkonium photoproduction in UPCs will be presented elsewhere.

We have neglected diffractive quarkonium production with nucleon breakup, $\gamma+p \rightarrow$ $J / \psi(\Upsilon)+M_{X}$. For relatively small $M_{X}$, the dissociation products will be not detected in the central detector and the process would be identified as quasi-elastic. At HERA, the ratio of quasi-elastic to elastic channels at $t=0,\left(d \sigma_{\gamma p \rightarrow J / \psi M_{X}} / d t\right) /\left(d \sigma_{\gamma p \rightarrow J / \psi p} / d t\right) \approx 0.2$ and increases with $t$. Hence, although this process will be a small correction to low $t$ quasi-elastic scattering, it will dominate at $|t| \geq 0.5 \mathrm{GeV}^{2}$. It will thus further enhance the quasi-elastic signal. In principle, diffractive production could be separated using the t-dependence of quasi-elastic quarkonium production with the neutron signal.

In summary, neutron tagging of incoherent quarkonium photoproduction in ultraperipheral heavy-ion collisions may provide reliable event selection for quarkonium production by high energy photons. Precision measurements of quasi-elastic processes, combined with improved $\gamma p$ measurements, described in Section 2.4, could improve the understanding of $Q \bar{Q}$ propagation through the nuclear medium. 


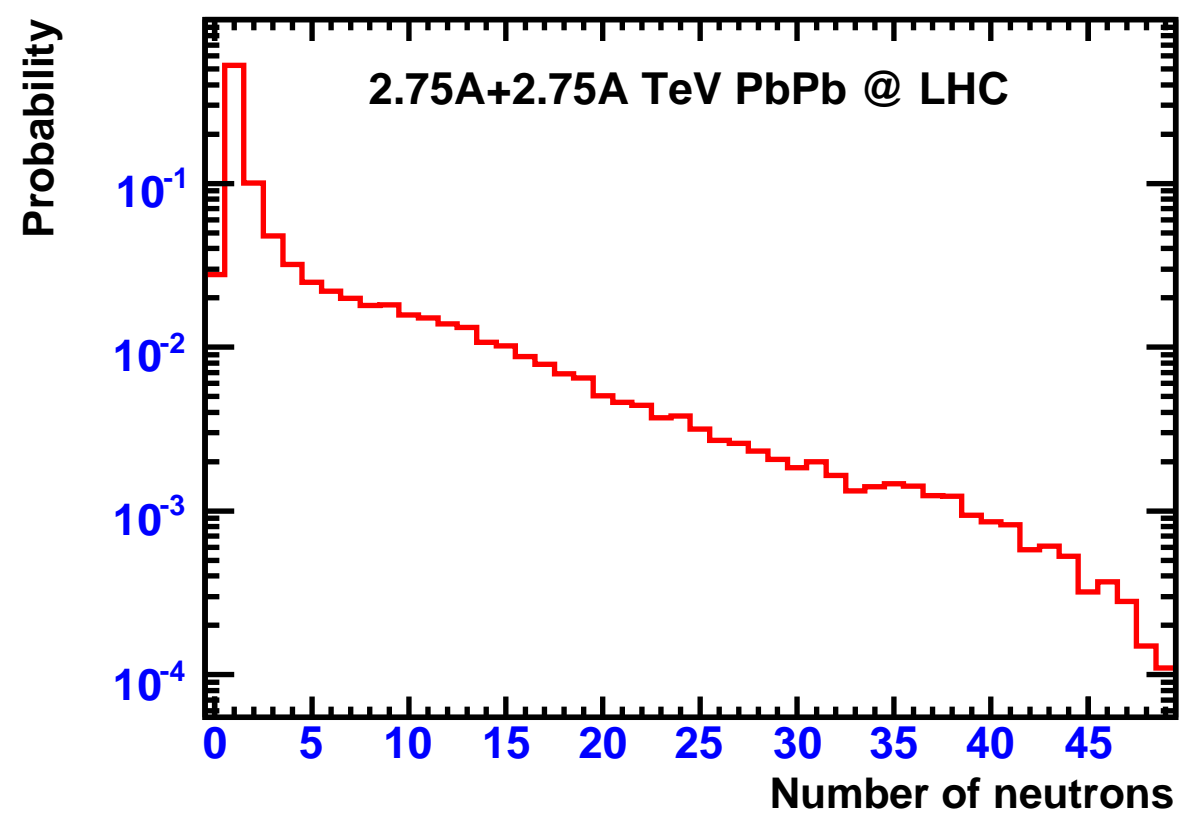

Figure 35. The neutron production probability in mutual electromagnetic dissociation in $\mathrm{Pb}+\mathrm{Pb}$ collisions at the $\mathrm{LHC}$.

\subsection{Quarkonium photoproduction in ALICE}

Contributed by: V. Nikulin and M. Zhalov

The quarkonium cross sections have been calculated in both the impulse and leading-twist approximations, IA and LTA respectively, see Refs. [80,81]. A more detailed discussion and further references can be found in Ref. [97]. Estimates of the total cross sections are presented in Table 2.6.

Table 6. The $J / \psi$ and $\Upsilon$ total cross sections in ultraperipheral collisions at the LHC.

\begin{tabular}{|c|c|c||c|c|}
\hline \multirow{2}{*}{} & \multicolumn{2}{|c||}{$\mathrm{Ca}+\mathrm{Ca}$} & \multicolumn{2}{c|}{$\mathrm{Pb}+\mathrm{Pb}$} \\
\cline { 2 - 5 } & $\sigma_{\text {tot }}^{\text {J/ }}(\mathrm{mb})$ & $\sigma_{\text {tot }}^{\Upsilon}(\mu \mathrm{b})$ & $\sigma_{\text {tot }}^{\text {Jw }}(\mathrm{mb})$ & $\sigma_{\text {tot }}^{\Upsilon}(\mu \mathrm{b})$ \\
\hline IA & 0.6 & 1.8 & 70 & 133 \\
LTA & 0.2 & 1.2 & 15 & 78 \\
\hline
\end{tabular}

The coherent $J / \psi$ and $\Upsilon t$ distributions for $\mathrm{Ca}$ and $\mathrm{Pb}$ beams at $y=0$ are shown in Fig. 16 while the rapidity distributions are presented in Fig. 17. The incoherent contribution in the impulse approximation, the upper limit of the expected cross section, was estimated in Refs. [80, 81].

Comparison of the LTA and IA results shows that leading-twist shadowing suppresses the $\Upsilon$ yield by a factor of two at central rapidity. The $J / \psi$ suppression 
is a factor of $4-6$ larger. In principle, multiple eikonal-type rescatterings due to gluon exchanges could also suppress vector meson production. This mechanism predicts up to a factor of two less suppression than leading-twist shadowing, at least for $x \leq 0.001$.

Coherent quarkonium photoproduction in ultraperipheral $A A$ collisions has a clear signature: a single muon pair in the detector. Since the ions remain in the ground state, there should be no ZDC signal. Any hadronic interaction would show ZDC activity. The ZDC inefficiency is expected to be very low. Further improvement could be achieved, if necessary, with a veto from the outer rings of the V0 detectors, see Section 10.2

The standard ALICE Level-0 trigger, L0, was not well suited for ultraperipheral studies since it did not cover the barrel rapidity region, $|y|<1$. However, the recently proposed inner tracking system (ITS) pixel L0 trigger [114] might improve the situation. Additional studies are still required to determine its utility for UPCs. Therefore events with two muons in the barrel are not considered here. The photon detector (PHOS) L0 trigger has recently been introduced. It covers a relatively small area, about $10 \%$ of the barrel solid angle. A fast veto from PHOS or the future electromagnetic calorimeter can suppress more central events.

The dimuon trigger [115], covering $-4<\eta<-2.5$, together with the PHOS veto could select very low multiplicity events accompanied by fast muons. In addition, low multiplicity selection could be applied at Level 1, L1. The dimuon L0 processor can produce three kinds of triggers. The minimal- $p_{T}$ trigger, initially intended for monitoring and testing, fires when a single muon passes a loose cut of $p_{T}>0.5 \mathrm{GeV} / c$. The low- $p_{T}$ trigger, used predominantly to select two-muon events with $p_{T}>1 \mathrm{GeV} / c$, is designed to tag $J / \psi$ decays. The high- $p_{T}$ trigger selects heavy resonances $\left(\Upsilon, \Upsilon^{\prime}\right)$ by tagging muon pairs with $p_{T}>2 \mathrm{GeV} / c$. The minimal trigger rate is expected to be at the level of $8 \mathrm{kHz}$ for $\mathrm{Pb}+\mathrm{Pb}$ interactions. The two last, tighter, triggers are intended to reduce the dimuon rate to $1 \mathrm{kHz}$ or less.

We have studied which trigger configurations may be most useful for studies of quarkonium photoproduction in ALICE. At L0, only the muon trigger is used. The minimal- $p_{T}$ muon trigger is vetoed by activity in PHOS. It selects events with at least one muon in the muon spectrometer, including events with one muon in the spectrometer and a second muon in the barrel. If the trigger rates are too high, the low- $p_{T}$ and/or the high$p_{T}$ triggers will be utilized. Here, only events with two muons in the spectrometer can be triggered. At L1, ZDC information will be used to perform additional selection of very peripheral events. The Level-2 trigger performs standard TPC past-future protection while the high level trigger checks that the event contains only a few tracks.

Thus the proposed trigger enables the study of a class of events with "abnormally" low multiplicity tagged by a muon. Among the reactions that could be measured are coherent and incoherent quarkonium photoproduction and lepton pair production in $\gamma \gamma$ interactions. Such a trigger could be integrated into the standard ALICE running conditions.

The expected rates were estimated using the ALICE simulation code AliRoot [116]. In the simulation, a muon which traverses ten tracking and four trigger chambers of the 
muon spectrometer or produces hits in both the ITS and TPC is considered detected. The LTA distributions were used for the analysis, resulting in a $\sim 5 \%$ acceptance for the $J / \psi$ and $\sim 2 \%$ for the $\Upsilon$. These acceptances correspond to $\sim 1000$ muon pairs/day from $J / \psi$ decays and about 3 pairs/day from $\Upsilon$ decays detected in the muon spectrometer. The machine-induced (beam-gas) background is expected to be negligible.

The physical background due to coherent quarkonium production in coherent and incoherent diffractive (Pomeron-Pomeron) interactions is expected to be small. This contribution still needs to be evaluated. Another source of physical background is muon pair production in $\gamma \gamma$ interactions. The total number of triggers could be significant since the background was underestimated in Ref. [97]. The degradation of the mass resolution due to the uncertainty in the interaction point and the far forward peaked muon angular distribution should be taken into account. The ratio of the coherent signal, $S$, to background events below the signal peak, $B$, the signal-to-background ratio, $S / B$, is of order unity for the $J / \psi$ (Table 7 ) and $\sim 0.5$ for the $\Upsilon$ (Table 8 ).

The statistical significance, $S / \sqrt{S+B}$, of data collected during a $10^{6} \mathrm{~s}$ run is estimated to be $\sim 100$ for the $J / \psi$ (Table 7 ) and 3-4 for the $\Upsilon$ (Table 8 ). A significance of $\sim 100$ is sufficient for study of the differential distributions. The LTA $J / \psi$ and $\Upsilon$ rates expected in a $10^{6} \mathrm{~s} \mathrm{~Pb}+\mathrm{Pb}$ run are given in Table 7 and 8 , respectively, along with the signal-to-background ratios and the significance. In Table 7, the suppression of the rate due to LT shadowing is given by the ratio IA/LTA. The corresponding $\mathrm{Ar}+\mathrm{Ar}$ rates are also shown. The mass bin, $\Delta M$, used is approximately three times the detector mass resolution at the quarkonium mass. The interaction point resolution is also taken into account. The resolution is better if one muon goes to the barrel and the other to the muon spectrometer than if both muons go to the spectrometer. Since the $J / \psi$ resolution is not noticeably affected, the mass bin $\Delta M=0.2 \mathrm{GeV}$ has been used in both cases. For the $\Upsilon, \Delta M=0.3 \mathrm{GeV}$ is used when one muon is in the spectrometer and the other in the barrel while $\Delta M=0.4 \mathrm{GeV}$ is used when both muons are accepted in the spectrometer.

Table 7. The expected $J / \psi$ photoproduction rates in a $10^{6} \mathrm{~s}$ run for $\mathrm{Pb}+\mathrm{Pb}$ and $\mathrm{Ar}+\mathrm{Ar}$ collisions. The Ar+Ar luminosity assumed is $4 \times 10^{28} \mathrm{~cm}^{-2} \mathrm{~s}^{-1}$.

\begin{tabular}{|l|c|c|c|c||c|}
\hline & \multicolumn{4}{|c||}{$\mathrm{Pb}+\mathrm{Pb}$} & $\mathrm{Ar}+\mathrm{Ar}$ \\
\cline { 2 - 6 } & LTA & IA/LTA & $S / B$ & Significance & LTA \\
\hline Muon Arm & 25,000 & 2.28 & 6 & 150 & 25,000 \\
Barrel & 21,400 & 6.19 & 0.7 & 90 & 13,000 \\
\hline
\end{tabular}

The dimuon invariant mass and $p_{T}$ will be reconstructed offline. Since coherent events are peaked at $p_{T} \sim 0$, it is possible to estimate the incoherent contribution and reconstruct the coherent cross section. The Monte Carlo acceptance will be used for reconstruction of the coherent cross section.

The muon spectrometer and barrel measurements are complementary in their 
Table 8. The expected $\Upsilon$ photoproduction rates in a $10^{6} \mathrm{~s}$ run for $\mathrm{Pb}+\mathrm{Pb}$ and $\mathrm{Ar}+\mathrm{Ar}$ collisions.

\begin{tabular}{|l|c|c|c||c|}
\hline & \multicolumn{3}{|c||}{$\mathrm{Pb}+\mathrm{Pb}$} & $\mathrm{Ar}+\mathrm{Ar}$ \\
\cline { 2 - 5 } & LTA & $S / B$ & Significance & LTA \\
\hline Muon Arm & 25 & 0.7 & 3 & 33 \\
Barrel & 60 & 0.26 & 4 & 72 \\
\hline
\end{tabular}

rapidity coverage. When one muon is detected in the barrel and the other in the spectrometer, $-2.5<y<-1$ for the vector meson, the measurement is more central. Effects related to the reaction mechanism are dominant and IA/LTA $\sim 6.2$. When both muons are detected in the spectrometer, $-4<y<-2.5$ for the vector meson, IA/LTA $\sim 2.2$ and the forward cross sections are more sensitive to the gluon density.

Comparison of $J / \psi$ and $\psi^{\prime}$ yields from different collision systems $(\mathrm{Pb}+\mathrm{Pb}, \mathrm{Ar}+\mathrm{Ar}$ and $p A$ ) may provide further information about the gluon density at $x$ values as yet unexplored.

\subsection{Detection and reconstruction of vector mesons in $C M S$}

Contributed by: D. d'Enterria and P. Yepes

In this section, we present the CMS capabilities for diffractive photoproduction measurements of light $\left(\rho^{0}\right)$ and heavy $(\Upsilon)$ vector mesons as well as two photon production of high-mass dileptons $\left(M_{l^{+} l^{-}}>5 \mathrm{GeV} / c^{2}\right)$, part of the $\Upsilon$ photoproduction background. On one hand, $\rho^{0}$ photoproduction studies extend the HERA measurements [117] and provide new information about the interplay of soft and hard physics in diffraction [93,118]. A clean signature with a low $\pi^{+} \pi^{-}$invariant mass background makes this measurement relatively straightforward in UPCs, as demonstrated in $\mathrm{Au}+\mathrm{Au}$ collisions at RHIC [62]. On the other hand, heavy quarkonium $(J / \psi, \Upsilon)$ production provides valuable information on the nuclear gluon density, $x g_{A}\left(x, Q^{2}\right)$ [22], and extends studies at RHIC [63] into a previously unexplored $x$ and $Q^{2}$ range, see Fig. 37.

Table 9 lists the expected $\rho^{0}, J / \psi$ and $\Upsilon$ photoproduction cross sections in UPCs at the LHC, as given by STARLIGHT $[72,74,83,119]$. which satisfactorily reproduces the present RHIC UPC $\rho^{0}[62]$ and $J / \psi$ [63] data as well as the low [120] and high mass [63] dielectron data. For comparison, we note that the calculated $\Upsilon$ cross section in inelastic $p p$ collisions at $5.5 \mathrm{TeV}$ is $\sim 600$ times smaller, $\sigma_{p p \rightarrow \Upsilon X} \approx 0.3 \mu \mathrm{b}$ [121], while the inelastic minimum bias $\mathrm{Pb}+\mathrm{Pb} \Upsilon$ cross section is $\sim 100$ times larger, $\sigma_{\mathrm{PbPb} \rightarrow \Upsilon X}=A^{2} \sigma_{p p \rightarrow \Upsilon X} \approx 13 \mathrm{mb}$.

The most significant physical background for these measurements is coherent lepton pair production in two-photon processes, shown on the right-hand side of Fig. 36. Table 10 lists the expected dilepton cross sections in the mass range relevant for quarkonium measurements. The fraction of the continuum cross sections accompanied 
Table 9. Exclusive vector meson photoproduction cross sections predicted by STARLIGHT $[72,83,119]$ in ultraperipheral $\mathrm{Pb}+\mathrm{Pb}$ interactions at $5.5 \mathrm{TeV}$ accompanied by neutron emission in single $(X n)$ or double $(X n \mid X n)$ dissociation of the lead nuclei, shown on the left-hand side of Fig. 36. (Note that $\sigma_{X n}$ includes $\sigma_{X n \mid X n}$ ).

\begin{tabular}{|c|c|c|c|}
\hline Vector Meson & $\sigma_{\text {tot }}(\mathrm{mb})$ & $\sigma_{X n}(\mathrm{mb})$ & $\sigma_{X n \mid X n}(\mathrm{mb})$ \\
\hline$\rho^{0}$ & 5200 & 790 & 210 \\
$J / \psi$ & 32 & 8.7 & 2.5 \\
$\Upsilon(1 S)$ & 0.173 & 0.078 & 0.025 \\
\hline
\end{tabular}

by nuclear breakup with neutron emission is expected to be the same as for quarkonia photoproduction, on the order of $\sim 50 \%$ for high-mass dileptons.

Table 10. Dilepton production cross sections predicted by STARLight $[72,83,119]$ for two-photon interactions in ultraperipheral $\mathrm{Pb}+\mathrm{Pb}$ interactions at $5.5 \mathrm{TeV}$, see the right-hand side of Fig. 36. The results are given in the mass regions of interest for $J / \psi$ and $\Upsilon$ production, $M>1.5 \mathrm{GeV}$ and $M>6 \mathrm{GeV}$ respectively.

\begin{tabular}{|l|c|c|}
\hline Mass & $\sigma_{\gamma \gamma \rightarrow e^{+} e^{-}}(\mathrm{mb})$ & $\sigma_{\gamma \gamma \rightarrow \mu^{+} \mu^{-}}(\mathrm{mb})$ \\
\hline$M>1.5 \mathrm{GeV} / c^{2}$ & 139 & 45 \\
$M>6.0 \mathrm{GeV} / c^{2}$ & 2.8 & 1.2 \\
\hline
\end{tabular}

2.7.1. Trigger considerations Ultraperipheral collisions are mediated by photon exchange with small momentum transfer and are characterized by a large rapidity gap between the produced system and the beam rapidity. After the interaction, the nuclei either remain essentially intact or in a low excited state. Thus UPCs can be considered 'photon-diffractive' processes sharing many characteristics with 'hadrondiffractive' (Pomeron-mediated) collisions. An optimum UPC trigger is thus usually defined based on these typical signatures.

UPCs are characterized by a large rapidity gap between the produced state and the interacting nuclei accompanied by forward neutron emission from the de-excitation of one or both nuclei. Single or mutual Coulomb excitation, indicated by the soft photon exchange in Fig. 36, occurs in about $50 \%$ of UPCs. The Coulomb excitation generates a Giant-Dipole Resonance (GDR) in the nucleus which subsequently decays via neutron emission. Since the global multiplicity is very low, the central detector is virtually empty apart from the few tracks/clusters originating from the produced system. The resulting rapidity distribution is relatively narrow, becoming narrower with increasing mass of the produced system, $M_{X}$, and centered at midrapidity. Note that although the energies of the $\gamma$ and the "target" nucleus are very different and the produced final state is boosted 
in the direction of the latter, since each of the nuclei can act as both "emitter" and "target", the sum of their rapidity distributions is symmetric around $y=0$.

Given these general properties of UPC events and based upon our previous experience with the $J / \psi$ in $\mathrm{Au}+\mathrm{Au}$ UPCs at RHIC [63], we devised the following CMS Level-1 primitives for the ultraperipheral trigger.

To ensure a large rapidity gap in one or in both hemispheres, we reject events with signals in the forward hadron calorimeters towers, $3<|\eta|<5$, above the default energy threshold for triggering on minimum-bias nuclear interactions ( $\overline{\mathrm{HF}+}$.OR. $\overline{\mathrm{HF}-}$ ). Although pure $\gamma \mathrm{Pb}$ coherent events have rapidity gaps in both hemispheres, we are also interested in triggering on "incoherent" $\gamma N$ photoproduction which usually breaks the target nucleus, partially filling one of the hemispheres with particles.

To tag $\mathrm{Pb}^{*}$ Coulomb breakup by GDR neutron de-excitation, we require energy deposition in the Zero Degree Calorimeters [122] (ZDC + .OR.ZDC-) above the default threshold in normal $\mathrm{Pb}+\mathrm{Pb}$ running. The availability of the $\mathrm{ZDC}$ signals in the $\mathrm{L} 1$ trigger decision is an advantage of CMS.

\subsubsection{Light meson reconstruction Contributed by: P. Yepes}

Here we present a feasibility study of light meson analysis in UPCs with CMS. Triggering on reactions without nuclear breakup in CMS is difficult because the detector is designed to trigger on transverse energy rather than multiplicity. The mesons considered here, with masses less than a few $\mathrm{GeV} / c^{2}$, will deposit little energy in the calorimeters. However, even for low mass particles, triggering on reactions with nuclear breakup should be feasible using the CMS ZDCs. The $\rho^{0}$ is used as a test case. We show that, despite the $4 \mathrm{~T}$ magnetic field of CMS and a tracker designed for high $p_{T}$ particles, acceptable reconstruction efficiencies are achieved.

A set of $1000 \rho^{0}$ s produced in ultraperipheral $\mathrm{Pb}+\mathrm{Pb}$ collisions was generated $[72,83]$ and run through the detailed GEANT-3 based CMS simulation package, CMSIM 125, using a silicon pixel detector with three layers. Events were then passed through the digitization packages using version 7.1 .1 of the ORCA reconstruction program. Only information from the silicon pixels was used. The performance of the reconstruction algorithm does not significantly improve with one or two additional silicon layers. The

$\rho^{0}$ candidates are reconstructed by combining opposite-sign tracks. The same-sign background was negligible. The overall reconstruction efficiency is $\epsilon=35 \%$. For central rapidities, $|\eta|<1, \epsilon=42 \%$, while for more forward rapidities, $1<\eta<1.8, \epsilon=16 \%$. Therefore, we conclude that light mesons produced in UPCs with nuclear breakup can be reconstructed in CMS if they are triggered with the ZDCs.

\subsection{3. $\Upsilon$ Detection in CMS Contributed by: D. d'Enterria}

At leading order, diffractive $\gamma A \rightarrow J / \psi(\Upsilon)$ proceeds through a colorless two-gluon (Pomeron) exchange, see the left-hand side of Fig. 36. After the scattering, both nuclei 
remain intact, or at a low level of excitation, and separated from the produced state by a rapidity gap. Such hard diffractive processes are thus valuable probes of the gluon density since their cross sections are proportional to the square of the gluon density, $\left.\left(d \sigma_{\gamma p, A \rightarrow V p, A} / d t\right)\right|_{t=0} \propto\left[x g\left(x, Q^{2}\right)\right]^{2}$ where $Q^{2} \approx M_{V}^{2} / 4$ and $x=M_{V}^{2} / W_{\gamma p, A}^{2}$, see Eq. (9). At $y=0, x \sim 2 \times 10^{-3}$ in $\gamma A \rightarrow \Upsilon A$ interactions at the LHC. The $x$ values can vary by an order of magnitude in the range $|y| \leq 2.5$, thus probing the nuclear PDFs in an $x$ and $Q^{2}$ range so far unexplored in nuclear DIS or in lower energy $A A$ collisions, see Fig. 37. Photoproduction measurements thus help constrain the low- $x$ behavior of the nuclear gluon distribution in a range where saturation effects due to nonlinear evolution of the PDFs are expected to set in $[30,31]$.
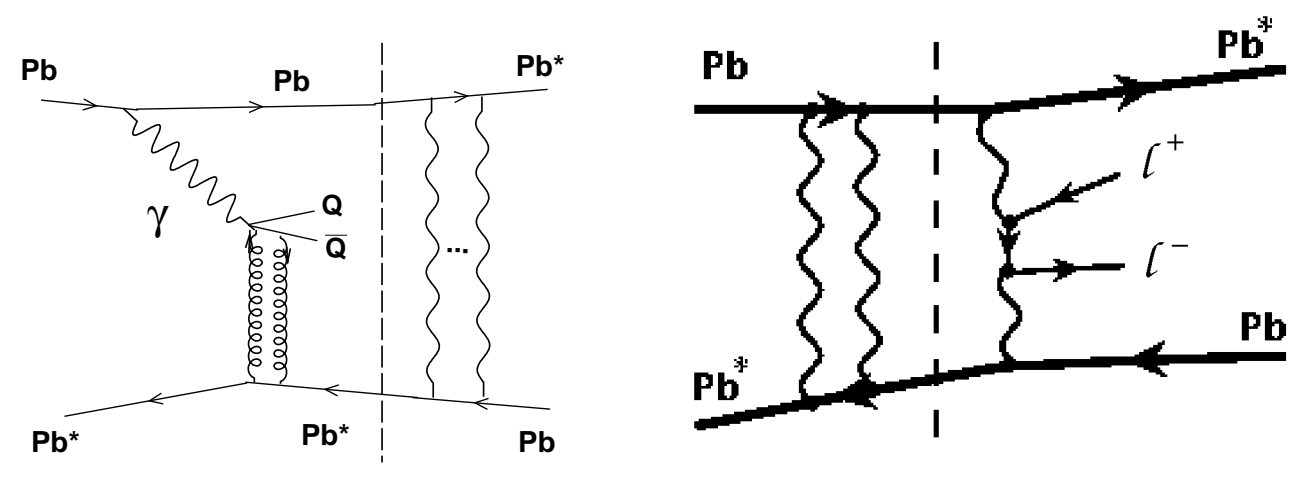

Figure 36. The leading order diagrams for $\Upsilon$ (left) and lepton pair [123] (right) production in $\gamma A$ and $\gamma \gamma$ processes accompanied by Coulomb excitation in ultraperipheral $\mathrm{Pb}+\mathrm{Pb}$ collisions.

Expected cross sections The expected $J / \psi$ and $\Upsilon$ photoproduction cross sections in ultraperipheral $\mathrm{Pb}+\mathrm{Pb}$ collisions at the LHC given by STARLIGHT $[72,83,119]$ are listed in Table 9. The $\gamma \mathrm{Pb}$ cross sections do not include the $\sim 10-20 \%$ feeddown contributions from excited $S$ states. They also do not include contributions from incoherent $\gamma N$ processes which should increase the $J / \psi$ and $\Upsilon$ yields by $\sim 50 \%$ [95]. Other $\gamma \mathrm{Pb} \rightarrow \Upsilon$ predictions for LHC energies, e.g. $\sigma_{\Upsilon}=135 \mu \mathrm{b}$ [80], give cross sections comparable to Table 9 . Including leading-twist shadowing reduces the $\Upsilon$ yield by up to a factor of $\sim 2$ to $78 \mu \mathrm{b}$ [80], see Table 2.3.4. Even larger reductions are expected when saturation effects, see Section 5.2, are included [124]. Our motivation is to precisely pin down the differences between the lead and proton PDFs at low $x$ and relatively large $Q^{2}, \approx 40$ $\mathrm{GeV}^{2}$.

Roughly $50 \%$ of the UPCs resulting in $\Upsilon$ production are accompanied by Coulomb excitation of one or both nuclei due to soft photon exchange, as shown in Fig. 36. The excitations can lead to nuclear breakup with neutron emission at very forward rapidities, covered by the ZDCs. This dissociation, primarily due to the excitation and decay of giant dipole resonances, provides a crucial UPC trigger, as discussed in the next section. 


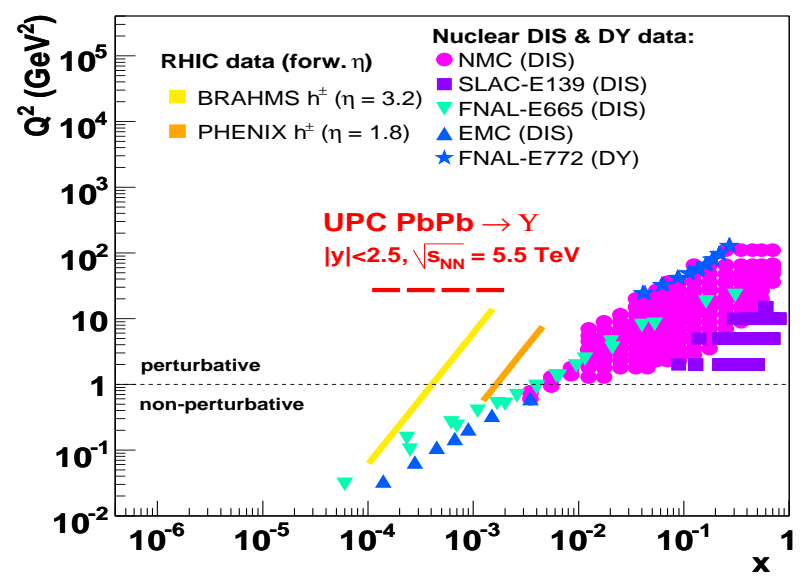

Figure 37. Measurements in the $\left(x, Q^{2}\right)$ plane used to constrain the nuclear PDFs. The approximate $\left(x, Q^{2}\right)$ range covered by ultraperipheral $\Upsilon$ photoproduction in $\mathrm{Pb}+\mathrm{Pb}$ collisions at $\sqrt{s_{N N}}=5.5 \mathrm{TeV}$ in $|\eta|<2.5$ is indicated. Reprinted from Ref. [125] with permission from the Institute of Physics.

The coherent photon fields generated by the ultrarelativistic nuclei have very small virtualities, $p_{T}<2 \hbar c / R_{A} \approx 50 \mathrm{MeV} / c$. Coherent production thus results in very low $p_{T} J / \psi \mathrm{s}$ so that the $p_{T}$ of the decay leptons, $\sim m_{J / \psi} / 2$, is too low to reach the detectors due to the large CMS magnetic field. We thus concentrate on the $\Upsilon$ since the decay lepton energies are $\sim 5 \mathrm{GeV}$ and can, therefore, reach the electromagnetic calorimeter (ECAL) and the muon chambers to be detected. In particular, this analysis focuses on $\Upsilon$ measurements in the CMS barrel and endcap regions, $|\eta|<2.5$, for:

(1) $\gamma \mathrm{Pb} \rightarrow \Upsilon+\mathrm{Pb}^{\star} \mathrm{Pb}^{(\star)}, \Upsilon \rightarrow e^{+} e^{-}$measured in the ECAL;

(2) $\gamma \mathrm{Pb} \rightarrow \Upsilon+\mathrm{Pb}^{\star} \mathrm{Pb}^{(\star)}, \Upsilon \rightarrow \mu^{+} \mu^{-}$measured in the muon chambers.

The $\star$ superscript indicates that one or both lead nuclei may be excited. Here and below, the presence of the lead nucleus that emits the photon is implied but not explicitly shown.

The most significant background source is coherent dilepton production in twophoton processes, shown on the right-hand side of Fig. 36:

(1) $\gamma \gamma \rightarrow \mathrm{Pb}^{\star} \mathrm{Pb}^{(\star)}+e^{+} e^{-}$, measured in the ECAL;

(2) $\gamma \gamma \rightarrow \mathrm{Pb}^{\star} \mathrm{Pb}^{(\star)}+\mu^{+} \mu^{-}$, measured in the muon chambers.

These are interesting pure QED processes and have been proposed as a luminosity monitor in $p p$ and $A A$ collisions at the LHC [126, 127]. As such, they may be used to normalize the absolute cross section of this and other heavy-ion measurements. Table 10 lists the expected dilepton cross sections in the mass ranges relevant for the quarkonia measurements. The fraction of the continuum cross sections accompanied by nuclear breakup with neutron emission is of the order of $\sim 50 \%$, as is the case for quarkonium photoproduction. 


\section{Level-1 trigger}

Given the general considerations discussed in Section 2.7.1 and experience with $J / \psi$ photoproduction studies in ultraperipheral $\mathrm{Au}+\mathrm{Au}$ collisions at $\mathrm{RHIC}$ [63], we propose to use several CMS Level-1 (L1) primitives as part of the ultraperipheral trigger.

We require a large rapidity gap in one or both hemispheres (forward/backward of midrapidity). Thus we veto signals in the forward hadron (HF) calorimeters, $3<|\eta|<5$, above the default minimum bias energy threshold, $\overline{\mathrm{HF}+} . \mathrm{OR} . \overline{\mathrm{HF}}-$, where the \pm refers to the forward/backward region and the bar over the HF signifies veto. We do not make an AND veto to require the absence of a signal in both $\mathrm{HF}$ towers, a signal of coherent $\gamma \mathrm{Pb}$ events with gaps in both hemispheres, because we also want to trigger on incoherent photoproduction $(\gamma N)$ where the target nucleus breaks up, partially populating the hemisphere on that side. There should be one or more neutrons, $X n$, in at least one ZDC, ZDC + .OR.ZDC-, to tag Coulomb breakup of the excited lead nucleus due to deexcitation of the GDR by neutron emission.

Leptons from $\Upsilon$ decays have energy $E_{l} \gtrsim m_{\Upsilon} / 2 \sim 4.6 \mathrm{GeV}$. Electrons and muons from these decays are triggered in two different ways. Electrons from $\Upsilon$ decays are selected by energy deposition in an isolated ECAL trigger tower with threshold energy greater than $3 \mathrm{GeV}$. Muons can be selected by hits in the muon resistive plate chambers (RPCs), $|\eta|<2.1$, or cathode strip chambers (CSCs), $0.8<|\eta|<2.4$. No track momentum threshold is required since the material budget in front of the chambers effectively reduces any muon background below $\sim 4 \mathrm{GeV}$.

The following two dedicated L1 UPC triggers are thus proposed:

$$
\begin{aligned}
& \mathrm{UPC}-\mathrm{mu}-\mathrm{L} 1=(\mathrm{ZDC}+. \mathrm{OR} \cdot \mathrm{ZDC}-) \cdot \mathrm{AND} \cdot(\overline{\mathrm{HF}+} \cdot \mathrm{OR} \cdot \overline{\mathrm{HF}-}) \cdot \mathrm{AND} \cdot(\text { muonRPC } \cdot \mathrm{OR} \cdot \mathrm{mu} \text { onCSC }) \\
& \mathrm{UPC}-\mathrm{e}-\mathrm{L} 1=(\mathrm{ZDC}+. \mathrm{OR} \cdot \mathrm{ZDC}-) \cdot \mathrm{AND} \cdot(\overline{\mathrm{HF}+} \cdot \mathrm{OR} \cdot \overline{\mathrm{HF}-}) \cdot \mathrm{AND} \cdot \text { ECALtower }(E>2.5 \mathrm{GeV})
\end{aligned}
$$

\section{Expected L1 trigger rates}

The coherent $\Upsilon \rightarrow l^{+} l^{-}$photoproduction rate, $N_{\Upsilon}$, assuming a perfect trigger, full acceptance and no efficiency losses at the nominal $\mathrm{Pb}+\mathrm{Pb}$ luminosity, $L_{\mathrm{PbPb}}=0.5$ $\mathrm{mb}^{-1} \mathrm{~s}^{-1}$, is

$$
\begin{aligned}
N_{\Upsilon} & =L_{\mathrm{PbPb}} B\left(\Upsilon \rightarrow l^{+} l^{-}\right) \sigma_{\Upsilon} \\
& =0.5 \mathrm{mb}^{-1} \mathrm{~s}^{-1} \times 0.024 \times 0.078 \mathrm{mb}=0.001 \mathrm{~Hz}
\end{aligned}
$$

or $1000 \Upsilon(1 S)$ dilepton decays in a $10^{6}$ s run.

There will be several sources of background that will also satisfy the UPC-L1 triggers defined above. For the purpose of estimating the trigger rates, we consider sources of physical and "non-physical" backgrounds which have characteristics similar to a UPC event and, therefore, can potentially fulfill UPC-L1 trigger criteria. All these processes give a ZDC signal.

Beam-gas and beam-halo collisions do not have a good vertex. They have a comparatively large multiplicity with an asymmetric $d N / d y$ and relatively low transverse 
energy, $E_{T}$. However, this process will be suppressed by the rapidity gap requirement, $(\overline{\mathrm{HF}+}$.OR. $\overline{\mathrm{HF}-})$, and will not be discussed further.

High L1 background rates may be generated by the coincidence of cosmic-ray muons with electromagnetic nuclear dissociation (ED) and peripheral nuclear collisions. Cosmic rays in coincidence with $\mathrm{ED}, \gamma A \rightarrow \mathrm{Pb}^{\star}+\mathrm{Pb}^{(\star)}$ with $X n$ neutrons hitting the ZDC, will have large net $p_{T}$ tracks in the muon chambers alone but no collision vertex. The ZDC signal is from lead dissociation. Peripheral nuclear collisions, $A A \rightarrow X$ also have a ZDC signal but have relatively large hadron multiplicities with large $p_{T}$.

A background process with an almost indistinguishable signal at L1 arises from two-photon production of dileptons, $\gamma \gamma \rightarrow l^{+} l^{-}$. We thus discuss this process further as a possible reference process in the remainder of this section. This background can be significantly reduced by an asymmetry cut on the lepton pair while the residual contribution below the $\Upsilon$ mass can be statistically subtracted in the offline analysis, see Section 2.7.3.

Finally, some background arises from interesting low rate processes that can be studied offline as a byproduct of the UPC trigger. These include hadronic diffraction, hard diffractive photoproduction and two-photon hadronic production. Hadronic diffractive collisions, $\mathbb{P P b}, \mathbb{P} \mathbb{P} \rightarrow X$, have larger multiplicities than diffractive photoproduction and predominantly produce pions rather than vector states. A likesign subtraction can remove the pion background. The $p_{T}$ is also larger for diffractive processes, $p_{T}(\mathbb{P} \mathbb{P})>p_{T}(\gamma \mathbb{P})>p_{T}(\gamma \gamma)$. Hard diffraction, e.g. dijet and $Q \bar{Q}$ production, is also characterized by larger multiplicities. These background events can be removed offline using standard subtraction techniques.

The single and mutual electromagnetic lead dissociation cross section at the crossing point of the two beams is $\sigma^{\mathrm{S}(\mathrm{M}) \mathrm{ED}}=215 \mathrm{~b}$ [109], the main limitation on the $\mathrm{Pb}+\mathrm{Pb}$ luminosity achievable at the LHC. Such large cross sections translate into very large rates, $N^{\mathrm{S}(\mathrm{M}) \mathrm{ED}}=L_{\mathrm{PbPb}} \sigma^{\mathrm{S}(\mathrm{M}) \mathrm{ED}}=10^{5} \mathrm{~Hz}$. Thus accidental coincidences with cosmic ray muons traversing the muon chamber and activating the UPC-mu-L1 trigger are possible. The typical cosmic ray muon rate at ground level is about $60 \mathrm{~Hz} / \mathrm{m}^{2}$ with $\left\langle E_{\mu}\right\rangle \approx 4$ $\mathrm{GeV}$ [128]. At the IP5 cavern, $\sim 80 \mathrm{~m}$ underground, the rate is reduced to $\sim 6 \mathrm{~Hz} / \mathrm{m}^{2}$. Muons which traverse the rock overburden above CMS typically have an energy at the surface of at least $10 \mathrm{GeV}$. Since the surface area of the muon chambers is $\sim 20 \times 15$ $\mathrm{m}^{2}$, the total rate of cosmic ray muons entering the chambers is $N_{\text {cosmic }} \approx 2 \mathrm{kHz}$. The accidental coincidence rate for two detectors with counting rates $N_{1}$ and $N_{2}$ in a trigger time window $\Delta t_{\text {trig }}$ is $N_{\text {acc }}=2 N_{1} N_{2} \Delta t_{\text {trig. }}$. If $\Delta t_{\text {trig }}=10$ ns around the nominal bunch crossing time of $25 \mathrm{~ns}$, we have

$$
\begin{aligned}
N_{\text {cosmic }}^{\mathrm{S}(\mathrm{M}) \mathrm{ED}} & =2 N^{\mathrm{S}(\mathrm{M}) \mathrm{ED}} N_{\text {cosmic }} \Delta t_{\mathrm{trig}} \\
& \approx 2 \times 10^{5} \mathrm{~Hz} \times 2000 \mathrm{~Hz} \times 10^{-8} \mathrm{~Hz}^{-1} \approx 4 \mathrm{~Hz} .
\end{aligned}
$$

However, very few cosmic ray muons pass the trigger if we require the tracks to be pointing to the vertex. There is a factor of 2500 reduction when we require $z_{\text {hit }}<60$ $\mathrm{cm}$ and $R_{\text {hit }}<20 \mathrm{~cm}$. In the high-level trigger (HLT), this background can be reduced 
by requiring vertex reconstruction.

At RHIC energies, usually $\epsilon_{\text {periph }} \approx 5 \%$ of the most peripheral nuclear $A A$ interactions $(95-100 \%$ of the total $A A$ cross section) do not generate activity within $3<|\eta|<4$ but still produce a signal in the ZDC [129]. Assuming that the same fraction of $\mathrm{Pb}+\mathrm{Pb}$ collisions at the LHC will be accepted by the virtually identical L1 condition

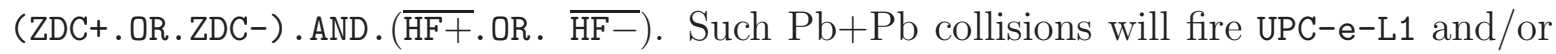
UPC-mu-L1 provided that these reactions also produce a lepton of sufficient energy. An analysis of a few hundred thousand minimum-bias $p p$ events at $5.5 \mathrm{TeV}$ generated using PYтнiA 6.4 [130], $\epsilon_{l} \approx 1 \%$ of the collisions generate at least one lepton within $|\eta|<2.5$ above the UPC-L1 energy thresholds. We assume the same relative fraction will hold for peripheral $\mathrm{Pb}+\mathrm{Pb}$ interactions. The corresponding rate for this background is

$$
\begin{aligned}
N_{\text {had }} & =L_{\mathrm{PbPb}} \sigma_{\text {tot }} \epsilon_{\text {periph }} \epsilon_{l} \\
& =0.5 \mathrm{mb}^{-1} \mathrm{~s}^{-1} \times 8000 \mathrm{mb} \times 0.05 \times 0.01 \approx 2 \mathrm{~Hz} .
\end{aligned}
$$

If the $\mathrm{HF}$ veto is insufficient to reduce these background rates at L1, an additional L1 primitive may be considered such as the total energy in the ECAL-HCAL system, requiring an energy deposition only a few $\mathrm{GeV}$ above the calorimeter noise level. This will suppress peripheral hadronic interactions since their multiplicity is much larger than ultraperipheral events.

We now discuss the $\gamma \gamma \rightarrow e^{+} e^{-}$background in more detail. The known $\gamma \gamma \rightarrow e^{+} e^{-}$ QED cross sections are given in Table 10. Simulations have shown that the fraction of these events producing an electron within $|\eta|<2.5$ and above the $3 \mathrm{GeV}$ ECAL trigger threshold and potentially firing UPC-e-L1, is only $\epsilon_{e} \sim 5 \%$. Since the corresponding fraction of muons triggering UPC-mu-L1 is even lower, we do not include it here. There is a $50 \%$ probability for neutron emission, $P_{n}$, incorporated into the rate. Thus, the expected rate for this background is

$$
\begin{aligned}
N_{\gamma \gamma} & =L_{\mathrm{PbPb}} \sigma_{\gamma \gamma \rightarrow e^{+} e^{-}} P_{n} \epsilon_{e} \\
& =0.5 \mathrm{mb}^{-1} \mathrm{~s}^{-1} \times 139 \mathrm{mb} \times 0.5 \times 0.05=1.7 \mathrm{~Hz} .
\end{aligned}
$$

The conservative background sum, $N_{L 1}=N_{\text {cosmic }}^{\mathrm{S}(\mathrm{M}) \mathrm{ED}}+N_{\text {had }}+N_{\gamma \gamma}+$ others $\sim 5-7$ $\mathrm{Hz}$, is a factor of $\sim 5000-7000$ smaller than the $\Upsilon$ rate in Eq. (59). It is therefore important to not have any significant trigger dead-time and not to remove good events in the high-level trigger selection.

\section{High Level Trigger}

The CMS L1 trigger can pass all selected $\mathrm{Pb}+\mathrm{Pb}$ events, $\sim 3 \mathrm{kHz}$ on average, and send them to the HLT without reduction $[131,384]$. The UPC trigger bandwidth allocated in the HLT is $2.25 \mathrm{MByte} / \mathrm{s}$ (1\% of the total rate) or $\sim 1-2 \mathrm{~Hz}$ for an ultraperipheral event of $1-2 \mathrm{MB}$. The estimated event size of a very peripheral $\mathrm{Pb}+\mathrm{Pb}$ hadronic interaction with $b>12 \mathrm{fm}$ is $0.3 \mathrm{MB}$ plus a conservative $1 \mathrm{MB}$ "noise" overhead. Since events triggering the UPC-L1 trigger have, by design, very low multiplicities, they will be below 2 MB already at L1. 
Recording UPC-HLT rates at the allocated $1-2 \mathrm{~Hz}$ rate requires a factor of $2.5-7$ reduction relative to the expected UPC-L1 rates. To do so, we will need to apply one or more simple algorithms at the HLT level to match the allocated bandwidth. First, the L1 electron/muon candidates should be verified with a L1-improved software check to remove fake triggers. Next, the event vertex should be within $z<15 \mathrm{~cm}$ of $(0,0,0)$. The inherent low track/cluster multiplicity of UPC events results in a rather wide vertex distribution. An even looser cut, $z<60 \mathrm{~cm}$, is expected to reduce the cosmic ray background by a factor of 2500, as well as any remaining beam-gas or beamhalo events. Finally, two $p_{T}$ cuts can be applied. The total $p_{T}$ of all particles should be low. This can be checked by making a rough determination of the net $p_{T}$ of all muon/electron HLT candidates in the event. Hadrons emitted in peripheral hadronic events at $\sqrt{s_{N N}}=5.5 \mathrm{TeV}$ have $\left\langle p_{T}\right\rangle \approx 600 \mathrm{MeV} / c$, much larger than the $\left\langle p_{T}\right\rangle \approx 70$ $\mathrm{MeV} / c$ expected for coherent photoproduction events. Thus this cut should significantly reduce the peripheral $A A$ background. However, we may also want to study other hard photoproduction events with larger $p_{T}$ which satisfy the UPC-L1 trigger. Therefore it is probably more appropriate to select back-to-back dileptons, part of the global calorimeter and muon triggers. All these considerations can be taken into account when setting the final L1 thresholds and HLT algorithms and do not affect the quantitative conclusions about the $\Upsilon$ measurement described here.

Input Monte Carlo Event samples for the $\Upsilon \rightarrow l^{+} l^{-}$signal and the dilepton continuum are generated with the starlight Monte Carlo [72, 83, 119]. The input Monte Carlo $p_{T}$, rapidity and lepton pair invariant mass distributions for the signal and background are shown in Figs. 38 and 39.

The most significant characteristic of coherent particle production in UPCs is the extremely soft $p_{T}$ distribution. The $\Upsilon$ and the lepton pairs are produced almost at rest. The $\Upsilon p_{T}$ distribution is also sensitive to the nuclear form factor for lead. Figure 38 shows a diffractive pattern with several diminishing local maxima. The dilepton mass distribution decreases locally like an exponential or power law, shown in the top left plot of Fig. 39. The signal and background rapidity distributions are peaked at $y=0$. The continuum distribution is broader because it also includes lower mass pairs. Interestingly, the rapidity distributions of the single decay leptons are much narrower for the $\Upsilon$ (Fig. 38, right) than the $l^{+} l^{-}$continuum (Fig. 39, bottom right). One or both leptons from the continuum is often emitted outside the CMS rapidity coverage and, therefore, will not affect the $\Upsilon$ invariant mass reconstruction.

$\Upsilon \rightarrow l^{+} l^{-}$acceptance and reconstruction efficiency Figure 40 shows the convolution of efficiency with acceptance for CMS as a function of the $\Upsilon$ rapidity and transverse momentum respectively in the $\mu^{+} \mu^{-}$(dashed) and $e^{+} e^{-}$(solid) analyses, obtained by taking the ratio of reconstructed relative to input spectra. Note that although the 

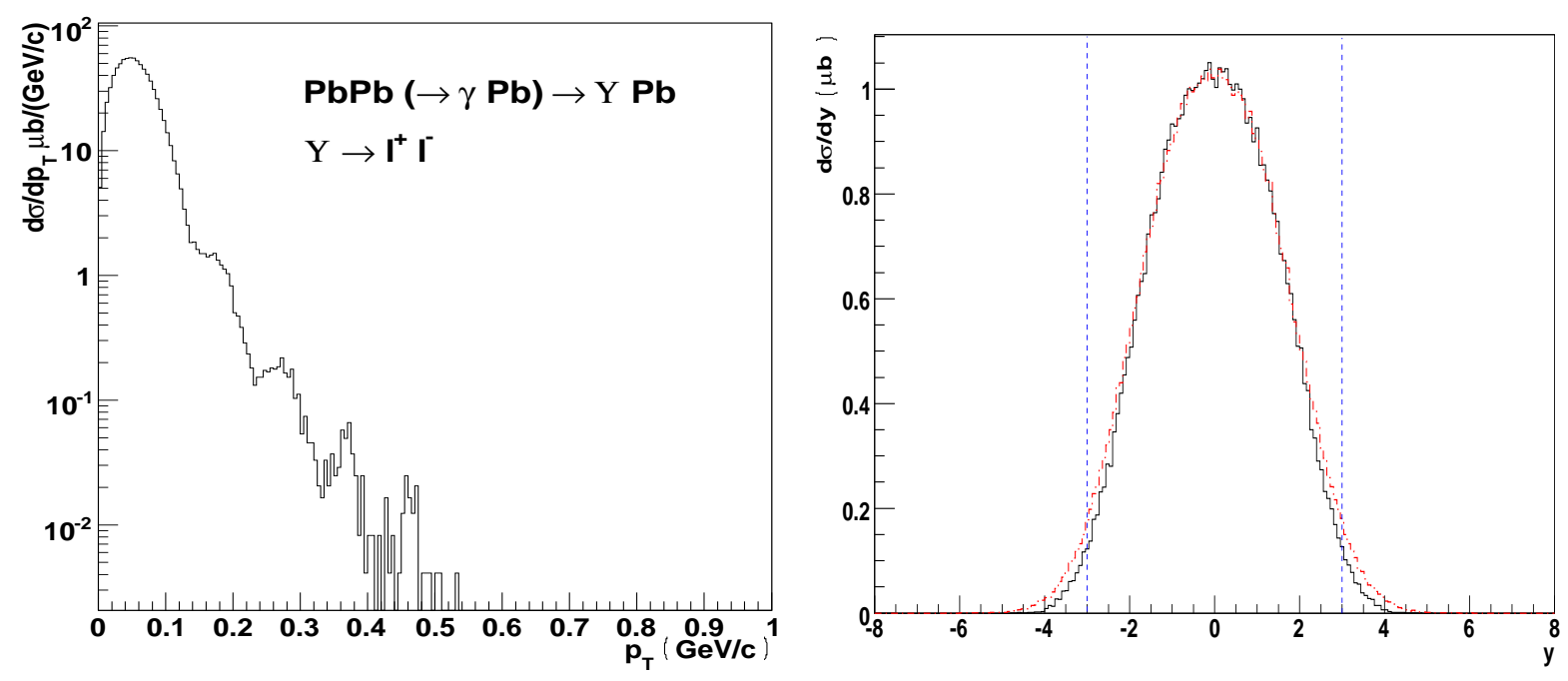

Figure 38. The Starlight $p_{T}$ (left-hand side) and $y$ (right-hand side) distributions for coherent $\Upsilon$ photoproduction in ultraperipheral $\mathrm{Pb}+\mathrm{Pb}$ collisions at $\sqrt{s_{N N}}=5.5$ $\mathrm{TeV}$ [132]. Note the diffractive-like peaks in the $p_{T}$ distribution. The rapidity distribution of single leptons from $\Upsilon$ decays, dot-dashed curve, is also shown on the right-hand side. The vertical dashed lines indicate the approximate CMS acceptance.

rapidity acceptances of both analyses are very different and complementary - the muon efficiency is peaked around $|y|=2$ and the electron efficiency at $|y|<1$ - the $p_{T}$ efficiencies are very similar. The efficiency is about $8 \%$ for $\Upsilon$ produced at rest. At the expected coherent production peak, $p_{T} \approx 40-80 \mathrm{MeV} / c$, the average efficiency is $\sim 10 \%$, increasing with $p_{T}$ thereafter. The reconstructed spectrum is higher than the generated one for $p_{T} \geq 130 \mathrm{MeV} / c$. This 'artifact' is due to the combination of a steeply-falling spectrum and a reconstruction yielding larger $p_{T} \Upsilon$ than the inputs.

The integrated combination of the geometric acceptance with the reconstruction efficiency in both analyses is $26 \%$ for $e^{+} e^{-}$and $21 \%$ for $\mu^{+} \mu^{-}$.

Invariant mass distributions and continuum subtraction To determine the $\Upsilon$ invariant mass distribution, it is necessary to include the lepton pair continuum in the mass background. Any residual combinatorial background can be removed from the measured $d N / d M$ distributions by subtracting the like-sign, $l^{ \pm} l^{ \pm}$, background from the oppositesign, $l^{ \pm} l^{\mp}$, signal. In this simulation, the like-sign background is negligible because we reconstruct only the opposite-sign pairs.

The generated $\Upsilon$ signal and lepton pair continuum, $6<M<12 \mathrm{GeV} / c^{2}$, events are mixed according to their relative cross sections in Tables 9 and 10, taking the $\Upsilon$ branching ratio, $B\left(\Upsilon \rightarrow l^{+} l^{-}\right) \sim 2.4 \%$, into account. The input signal-to-background ratio integrated over all phase space is rather low,

$$
\frac{N_{S}}{N_{B}}=\frac{B\left(\Upsilon \rightarrow l^{+} l^{-}\right) \sigma_{\Upsilon}}{\sigma_{l^{+} l^{-}}\left(6<M<12 \mathrm{GeV} / c^{2}\right)} \approx 0.35 \%\left(\mu^{+} \mu^{-}\right) ; 0.15 \%\left(e^{+} e^{-}\right)
$$



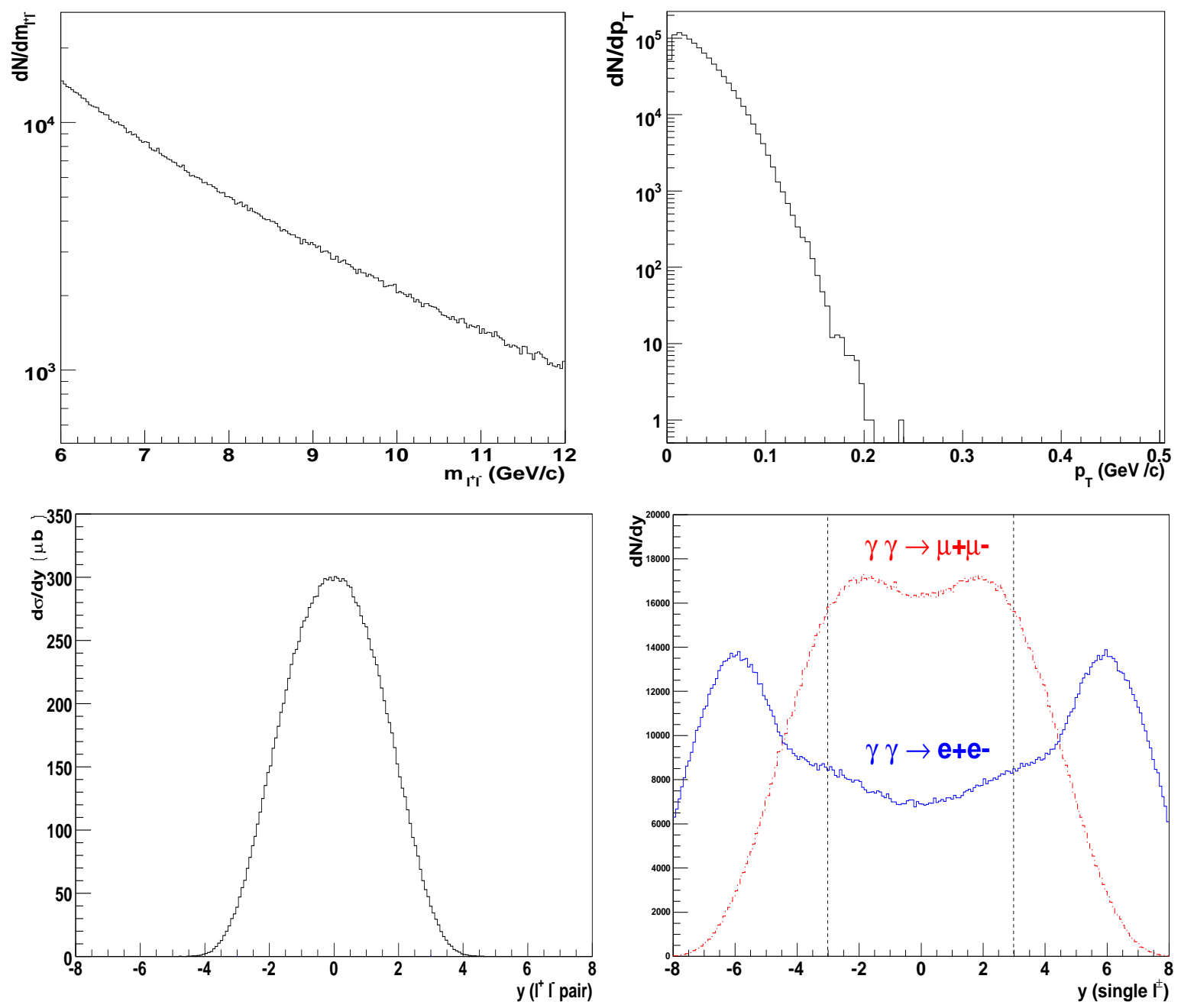

Figure 39. The StARLight dilepton $\left(e^{ \pm}, \mu^{ \pm}\right)$invariant mass (top left), pair $p_{T}$ (top right), pair rapidity (bottom left) and single lepton rapidity (bottom right) distributions in ultraperipheral $\mathrm{Pb}+\mathrm{Pb}$ collisions at $\sqrt{s_{N N}}=5.5 \mathrm{TeV}[132]$. The single muon (dashed) and electron (solid) rapidity distributions are shown separately in the bottom right plot. The vertical dashed lines indicate the CMS acceptance.

However, coherent lepton pair production is asymmetric and more forward than $\Upsilon \rightarrow$ $l^{+} l^{-}$so that single leptons from continuum pairs often fall outside the CMS acceptance, $|\eta|<2.5$. In practice, more electrons than muons miss the central CMS region, see Fig. 39, making the ratio $N_{S} / N_{B}$ very similar for the $e^{+} e^{-}$and $\mu^{+} \mu^{-}$analyses if the different detector responses are not included.

Figure 41 shows the combined signal+background mass spectra in the dielectron and dimuon channels. We find $N_{S} / N_{B} \sim 1$ for both cases. The combined reconstructed mass spectra are fitted to a Gaussian for the $\Upsilon$ peak plus an exponential for the continuum. The exponential fit to the continuum is subtracted from the signal+background entries. The resulting background-subtracted $\Upsilon$ mass distributions fitted to a Gaussian alone are shown in Fig. 42. The final $\Upsilon$ masses and widths are 

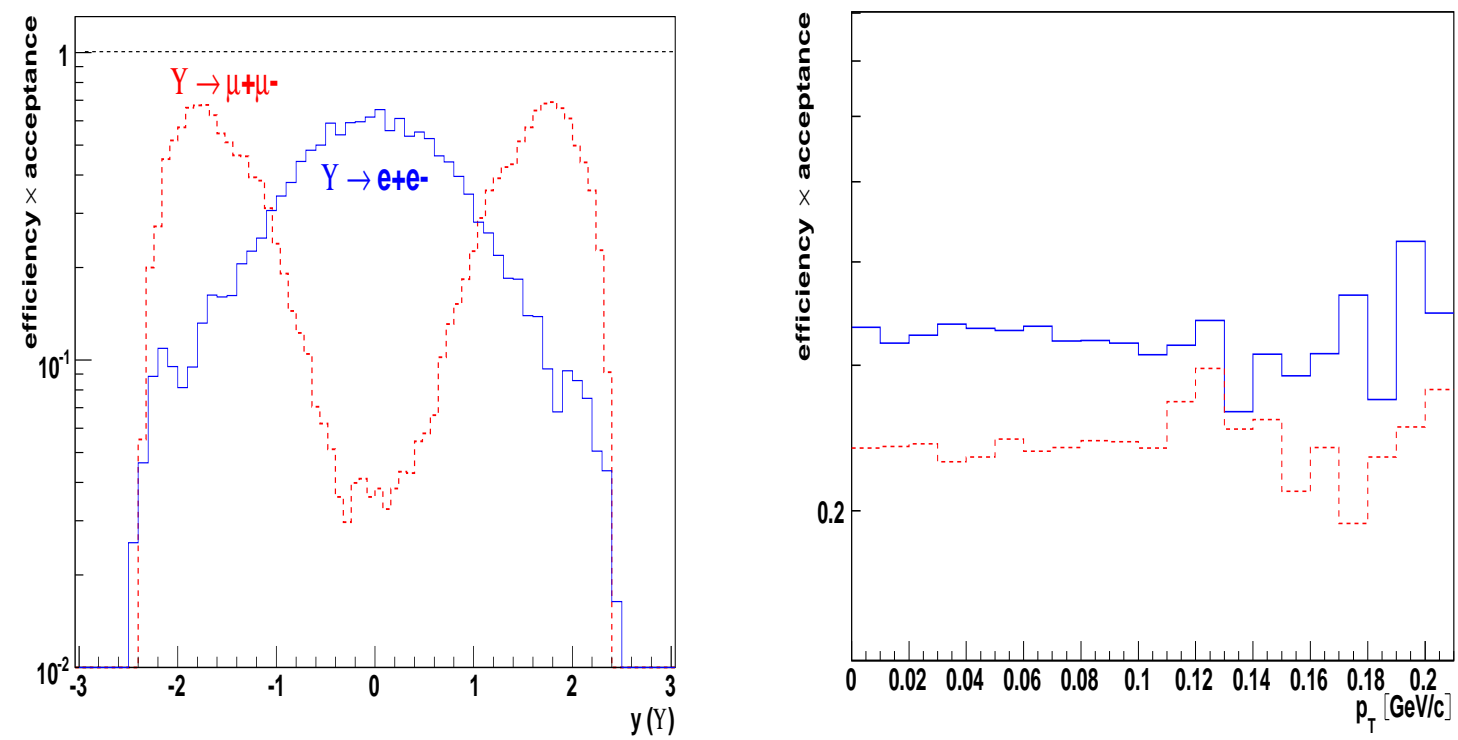

Figure 40. The combined efficiency and acceptance for $\Upsilon$ decays to $\mu^{+} \mu^{-}$(dashed) and $e^{+} e^{-}$(solid) obtained from a full CMS simulation and reconstruction of the input STARLIGHT distributions as a function of the $\Upsilon$ rapidity (left-hand side) and $p_{T}$ (righthand side) [132].
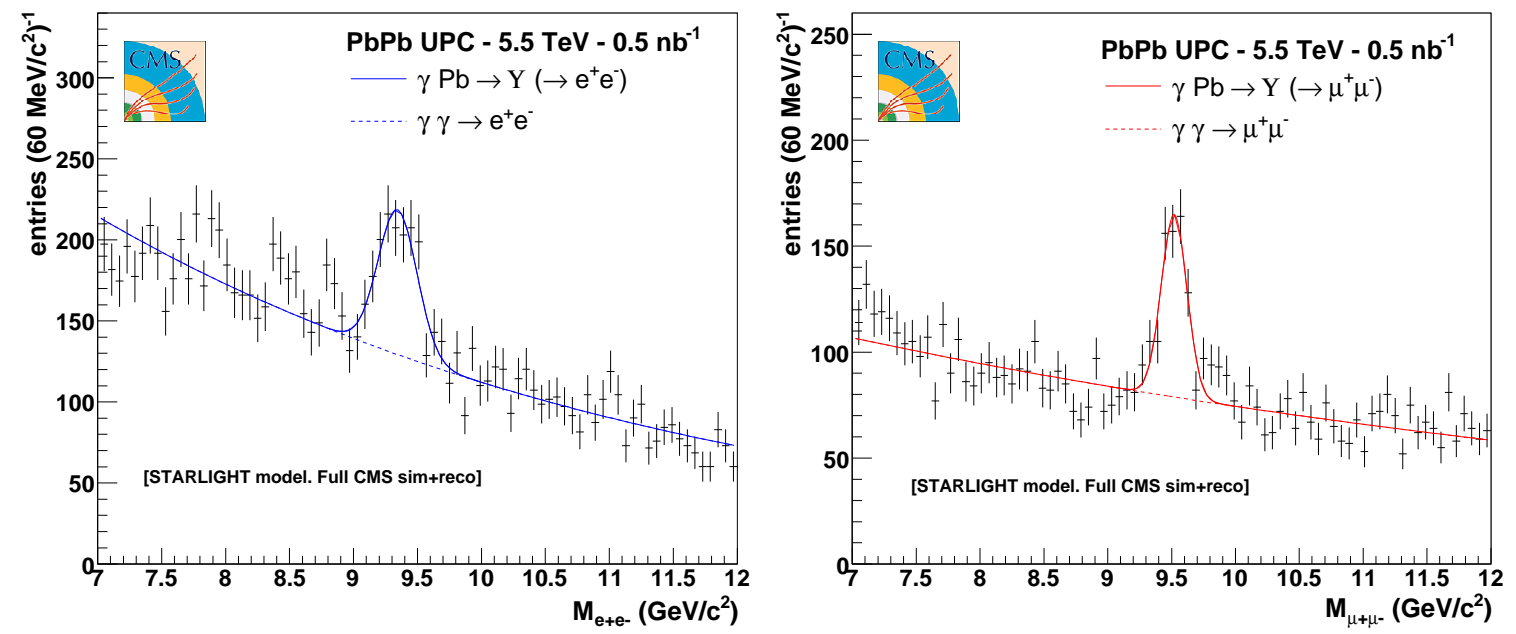

Figure 41. The $e^{+} e^{-}$(left) and $\mu^{+} \mu^{-}$(right) mass distributions for the $\Upsilon$ signal and dilepton continuum. Reprinted from Ref. [133] with permission from the Institute of Physics.

$M=9.52 \mathrm{GeV} / c^{2}$ and $\sigma_{\text {res }}=0.090 \mathrm{GeV} / c^{2}$ for the $\mu^{+} \mu^{-}$channel and $M=9.35$ $\mathrm{GeV} / c^{2}$ and $\sigma_{\text {res }}=0.16 \mathrm{GeV} / c^{2}$ for the $e^{+} e^{-}$channel, very close to the nominal $\Upsilon$ mass, $M=9.46 \mathrm{GeV} / c^{2}[128]$. In the dimuon channel, the mass resolution is sufficient to allow clean separation of the higher $\Upsilon S$ states, $\Upsilon^{\prime}\left(10.02 \mathrm{GeV} / c^{2}\right)$ and $\Upsilon^{\prime \prime}\left(10.36 \mathrm{GeV} / c^{2}\right)$, which can also be produced coherently but were not included in the current simulation. 

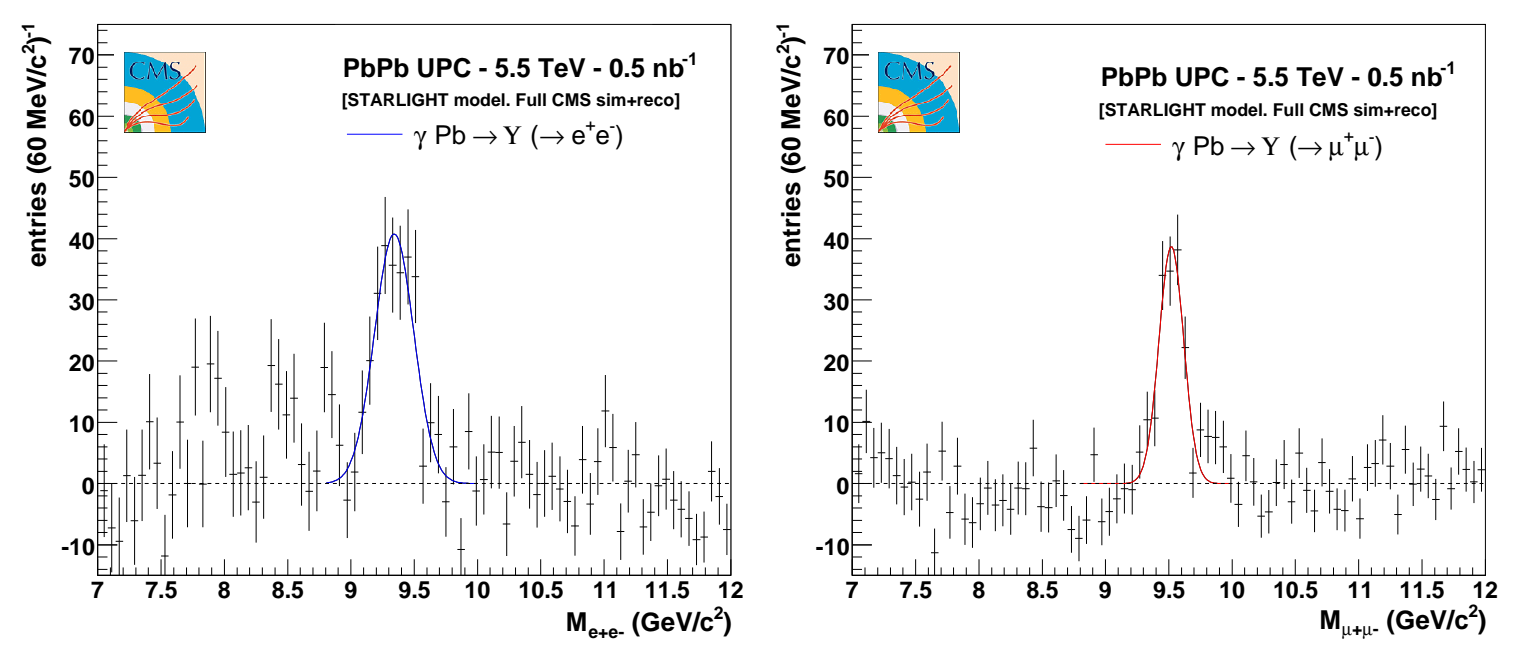

Figure 42. The $e^{+} e^{-}$(left) and $\mu^{+} \mu^{-}$(right) mass distributions for the $\Upsilon$ signal after background subtraction. Reprinted from Ref. [133] with permission from the Institute of Physics.

Total rates The extracted yields, integrating the counts within $3 \sigma_{l^{+} l^{-}}$around the $\Upsilon$ peak after continuum background subtraction, are computed for both decay modes. The efficiency of the yield extraction procedure is $\epsilon_{\text {extract }}=85 \%$ for the $e^{+} e^{-}$and $90 \%$ for the $\mu^{+} \mu^{-}$analysis. The efficiency is lower in the dielectron channel due to the larger background. The total $\Upsilon$ production yields expected with an integrated design $\mathrm{Pb}+\mathrm{Pb}$ luminosity of $0.5 \mathrm{nb}^{-1}$ are $N_{\Upsilon \rightarrow e^{+} e^{-}} \approx 220 \pm 15$ (stat) and $N_{\Upsilon \rightarrow \mu^{+} \mu^{-}} \approx 180 \pm 14$ (stat). Systematic uncertainties are estimated to be $\sim 10 \%$ by using different functional forms for the continuum and the method of $\Upsilon$ yield extraction. The uncertainty in the luminosity normalization will be $\sim 5 \%$ since the concurrent continuum measurement provides a direct calibration scale for the QED calculations [127,134]. Combining the statistics from both channels, the $\Upsilon y$ and $p_{T}$ spectra will test theoretical predictions of low- $x$ saturation in the nuclear PDFs. Even reducing the $\Upsilon$ yields by a factor of 4 , as predicted by calculations of nonlinear parton evolution at small $x$, would still provide a statistically significant sample to compare with theory.

\section{Inclusive photonuclear processes}

\subsection{Large mass diffraction in photon-induced processes}

Contributed by L. Frankfurt, V. Guzey, M. Strikman and M. Zhalov

Studies of inelastic diffraction at small $t$ through the $A$ dependence of hadronnucleus scattering provide information about fluctuations in the interaction strength $[59,135]$. The total cross section of inelastic diffraction has been calculated and used to study the $A$ dependence in two ways. First, assuming that the $A$ dependence of a particular diffractive channel is the same as the $A$ dependence of the total cross section, the calculations were compared to diffractive $p A \rightarrow p \pi A$ and $\pi A \rightarrow \pi \pi \pi A$ 
scattering. Second, the total cross section of inelastic diffraction has been measured in $p A$ interactions for $A={ }^{4} \mathrm{He}$ and emulsion at $p_{\text {lab }}=200$ and $400 \mathrm{GeV}$ [49]. Since the $N N$ cross section increases with energy, fluctuations in the elementary amplitudes lead to much smaller fluctuations in absorption in scattering off heavy nuclei. As a result, a much weaker $A$ dependence is expected for the diffractive cross section [136] at colliders. In particular, $\sigma_{p A \rightarrow X A}^{\text {diff }} \propto A^{0.25}$ at LHC energies [18] relative to $\sigma_{p A \rightarrow X A}^{\text {diff }} \propto A^{0.7}$ at fixedtarget energies. For high-mass diffraction, this suppression can also be understood by using the $t$ channel picture of Pomeron exchange due to the stronger screening of the triple Pomeron exchange [137].

Diffraction in deep-inelastic scattering corresponds to the transition of the (virtual) photon into its hadronic components, leaving the nucleus intact. Hence diffractive DIS has more in common with elastic hadron-nucleus scattering than inelastic diffractive hadron-nucleus scattering. The approach of the elastic cross section to half the total cross section is a direct indication of the proximity of the interaction regime to the BDR. Correspondingly, the most direct information on the proximity of hard interactions, such as $c \bar{c}$ photoproduction, to the BDR can be obtained if the diffractive fraction of the total cross section can be measured.

In the following, leading-twist diffraction and diffraction in the BDR will be discussed and applied to the analysis of diffractive UPCs.

3.1.1. Nuclear diffractive parton densities The key ingredient in calculations of hard diffractive processes in photon-nucleus scattering is nuclear diffractive PDFs (NDPDFs). In the photon case, the NDPDFs can be determined from direct photon studies, such as photon-gluon fusion or large angle Compton scattering, $\gamma q \rightarrow \gamma q$. Since the leadingtwist NDPDFs satisfy the factorization theorem, they can be analyzed on the basis of diffraction in DIS.

There is a deep connection between shadowing and diffractive scattering off nuclei. The simplest way to investigate this connection is to apply the AGK cutting rules [138]. Several processes contribute to nuclear diffraction: coherent diffraction where the nucleus remains intact; nuclear breakup without hadron production in the nuclear fragmentation region; and rapidity gap events with hadron production in the nuclear fragmentation region. For $x \leq 3 \times 10^{-3}$ and $Q^{2} \sim 4 \mathrm{GeV}^{2}$, the fraction of DIS events with rapidity gaps reaches $\sim 30-40 \%$ for heavy nuclei, rapidly decreasing with $A[139]$.

The effective cross section, $\sigma_{\text {eff }}$, in Eq. (11) which describes diffractive hard interactions of quark-gluon configurations with a nucleon can be used to estimate the probability of diffractive interactions in nuclei for a number of hard triggers beginning at resolution scale $Q_{0}^{2}$. The $\sigma_{\text {eff }}$ dependence of the fraction of events attributable to coherent diffraction and diffraction with nuclear breakup was considered, neglecting fluctuations in the interaction strength. For realistic values of $\sigma_{\text {eff }}$, the probability of coherent diffraction is quite large. The probability increases slowly with $\sigma_{\text {eff }}$ and does not approach $50 \%$ even for very large $\sigma_{\text {eff }}$, reflecting a significant diffuse nuclear surface, even for large $A$, see Fig. 43. Thus, the probability is not sensitive to fluctuations in $\sigma_{\text {eff }}$. 
In the quasi-eikonal approximation, the ratios $R=\sigma_{\mathrm{qel}} / \sigma_{\mathrm{el}}$ describe the dependence of the quasi-elastic to elastic scattering ratio on $\sigma_{\text {eff }}$.

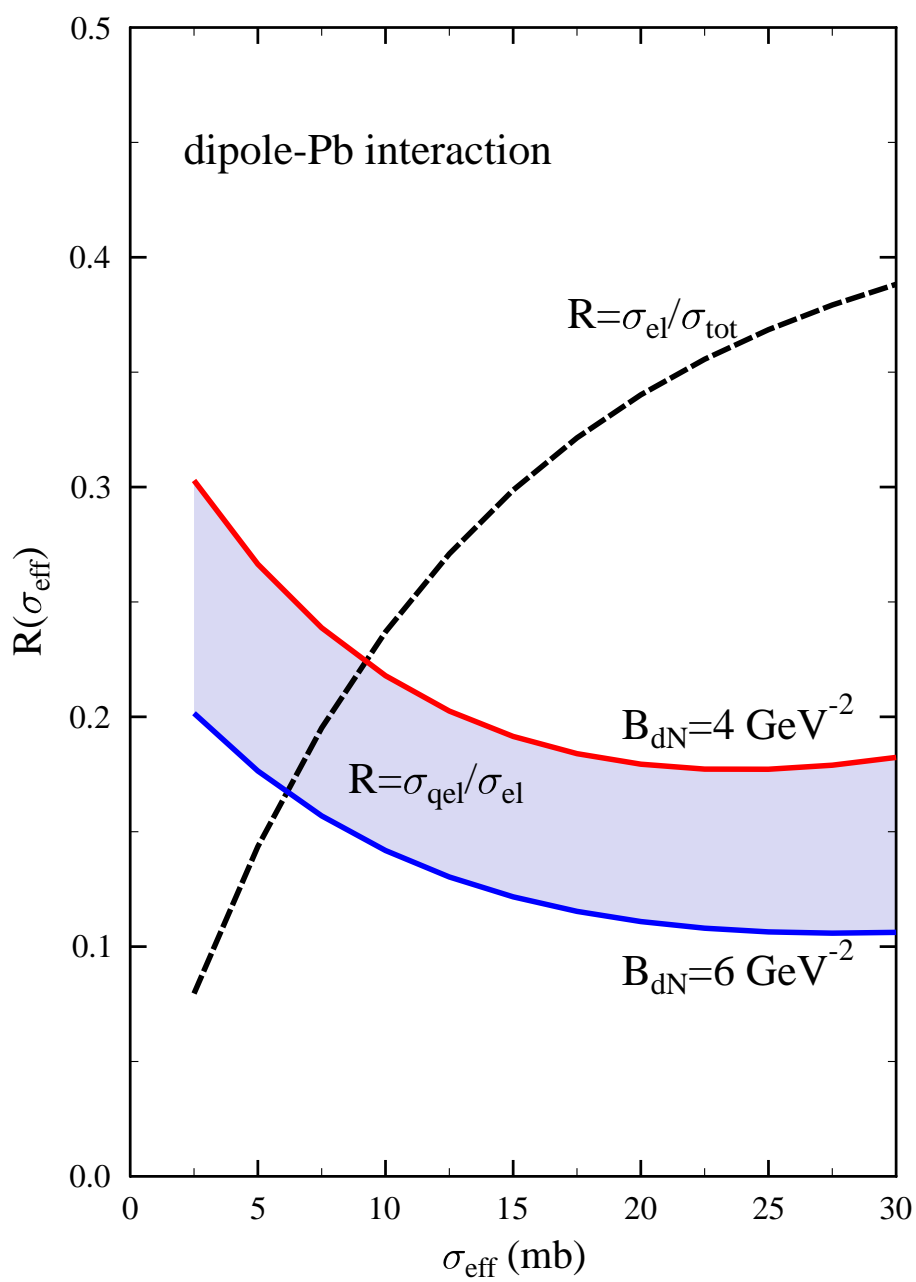

Figure 43. The dashed curve is the ratio of the coherent to total dipole-lead cross sections as a function of the effective dipole-nucleon cross section. The solid lines are the quasi-elastic to coherent dipole-nucleus cross section ratios for two different values of the slope, $B$, of the elastic dipole-nucleon $t$ distribution.

The diffractive parton densities were calculated by extending the leading-twist theory of nuclear shadowing on the total cross sections to the case of diffractive scattering [140],

$$
\begin{aligned}
& x f_{j / A}^{D(3)}\left(x, Q_{0}^{2},\right.\left.x_{\mathbb{P}}\right)=4 \pi \beta f_{j / N}^{D(4)}\left(x, Q_{0}^{2}, x_{\mathbb{P}}, t_{\mathrm{min}}\right) \int d^{2} b \\
& \times \mid \int_{-\infty}^{\infty} d z \rho_{A}(b, z) e^{i x_{\mathbb{P}} m_{N} z} e^{-\left.\frac{1-i \eta}{2} \sigma_{\mathrm{eff}}^{j}\left(x, Q_{0}^{2}\right) \int_{z}^{\infty} d z^{\prime} \rho_{A}\left(b, z^{\prime}\right)\right|^{2} .}
\end{aligned}
$$

The $2006 \mathrm{H} 1$ Fit B $[56,57]$ to the nucleon diffractive PDFs was used in the analysis of Eq. (63). The superscripts (3) and (4) denote the dependence of the diffractive PDFs on three and four variables, respectively. Equation (63) is presented for the $t$ integrated nuclear DPDFs since it is more compact and since it is not feasible to measure 
$t$ in diffraction off nuclei in colliders. In deriving Eq. (63) any possible dependence of $\sigma_{\text {eff }}^{j}\left(x, Q^{2}\right)$ on $\beta=x / x_{\mathbb{P}}$ in the exponential factor was neglected and an average value of $\sigma_{\text {eff }}^{j}$ was employed. Note that any suppression of small $\beta$ diffraction in interactions with nuclei in the soft regime is neglected since there are only elastic components for heavy nuclei (inelastic diffraction is zero). Hence the soft contribution at $Q_{0}^{2}$ due to triple Pomeron exchange is strongly suppressed [139]. As a result, the small $\beta$ nuclear DPDFs are suppressed by a factor $\propto A^{1 / 3}$ at $Q_{0}^{2}$. This suppression will be less pronounced at large $Q^{2}$ due to $\mathrm{QCD}$ evolution.

The nucleon DPDFs are well approximated by the factorized product of two functions, one dependent on $x_{\mathbb{P}}$ and $t$ and the other dependent on $\beta$ and $Q^{2}$. However, it is clear from Eq. (63) that the factorization approximation is not valid for the nuclear DPDFs. At fixed $x_{\mathbb{P}}$, the right-hand side of Eq. (63) depends not only on $\beta$ but also on Bjorken $x$ since the screening factor depends on $\sigma_{\text {eff }}^{j}$, a function of $x$. Equation (63) also depends on $A$ since nuclear shadowing increases with $A$. The breakdown of factorization results from the increase of the nuclear shadowing effects with incident energy and $A$. The resulting nuclear DPDFs are evolved to higher $Q^{2}$ using the NLO leading-twist (DGLAP) evolution equations.

3.1.2. Numerical results It is convenient for our discussion to quantify the nucleon and nuclear diffractive PDFs by introducing $P_{\text {diff }}^{j}$, the probability of diffraction for a given parton flavor $j$,

$$
P_{\mathrm{diff}}^{j}=\frac{\int_{x}^{x_{\mathbb{P}}^{0}} d x_{\mathbb{P}} x f_{j}^{D(3)}\left(x, Q^{2}, x_{\mathbb{P}}\right)}{x f_{j}\left(x, Q^{2}\right)} .
$$

First we discuss nucleon diffractive PDFs. Figure 44 presents the nucleon $P_{\text {diff }}^{j}$ as a function of $x$ for $Q^{2}=4,10$ and $100 \mathrm{GeV}^{2}$ for $u$ quarks and gluons. At low $Q^{2}$, $P_{\text {diff }}^{g}>P_{\text {diff }}^{u}$. Note also that $P_{\text {eff }}^{g}$ is very close to the unitarity limit, $P_{\text {diff,max }}^{j}=1 / 2$. The larger probability of diffraction for gluons is related to the larger gluon color dipole cross section in the $\mathbf{8} \times \mathbf{8}$ representation relative to the triplet $q \bar{q}$ dipole.

Next, we turn to hard diffraction with nuclear targets. Figure 45 presents $P_{\text {diff }}^{j}$ for ${ }^{40} \mathrm{Ca}$ and ${ }^{208} \mathrm{~Pb}$ at $Q^{2}=4 \mathrm{GeV}^{2}$ as a function of $x$ for $u$ quarks and gluons. The $A$ dependence of $P_{\text {diff }}^{j}$ is rather weak for $A \geq 40$ because at large $A$ and small $b$, the interaction is almost completely absorptive (black) with a small contribution from the opaque nuclear edge. The $A$ dependence for gluons is somewhat weaker since gluon interactions are closer to the black disk regime.

At small $x$, the $A$ dependence of $P_{\text {diff }}^{j}$ is qualitatively different for quarks and gluons. While the $A$ dependence of $P_{\text {diff }}^{g}$ is expected to be very weak $\boldsymbol{q}, P_{\text {diff }}^{q}$ is expected to grow with $A$ since the diffractive probability for quarks, shown in Fig. 44, is rather far from the BDR and thus can increase.

I The probability $P_{\text {diff }}^{g}$ for nuclei increases faster than for nucleons with decreasing $x$ since the nuclear center is like a black disk. However, scattering off nucleons near the edge of the nucleus slows the increase of $P_{\text {diff }}^{g}$ for nuclei as the ratio $\sigma_{\text {diff }} / \sigma_{\text {tot }}$ approaches 0.5 . 

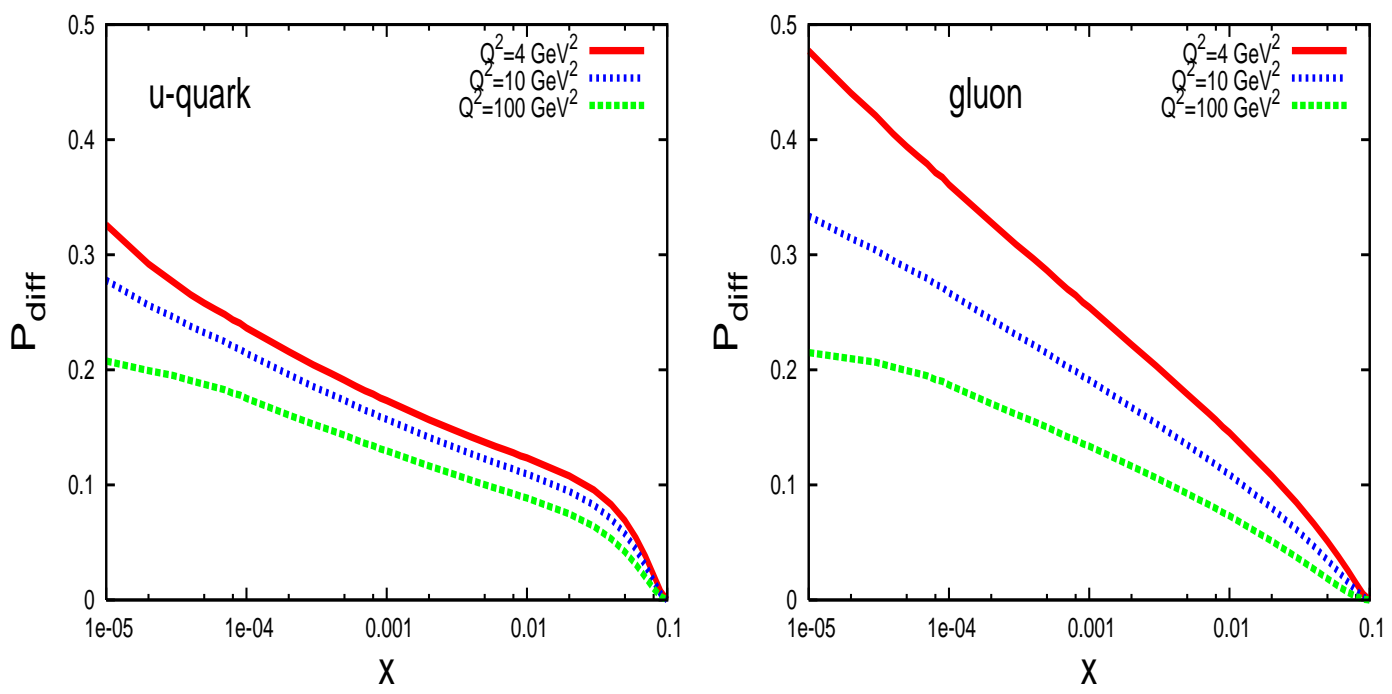

Figure 44. The probability of hard diffraction on the nucleon, $P_{\text {diff }}^{j}$, defined in Eq. (64), as a function of $x$ and $Q^{2}$ for $u$ quarks (left) and gluons (right).
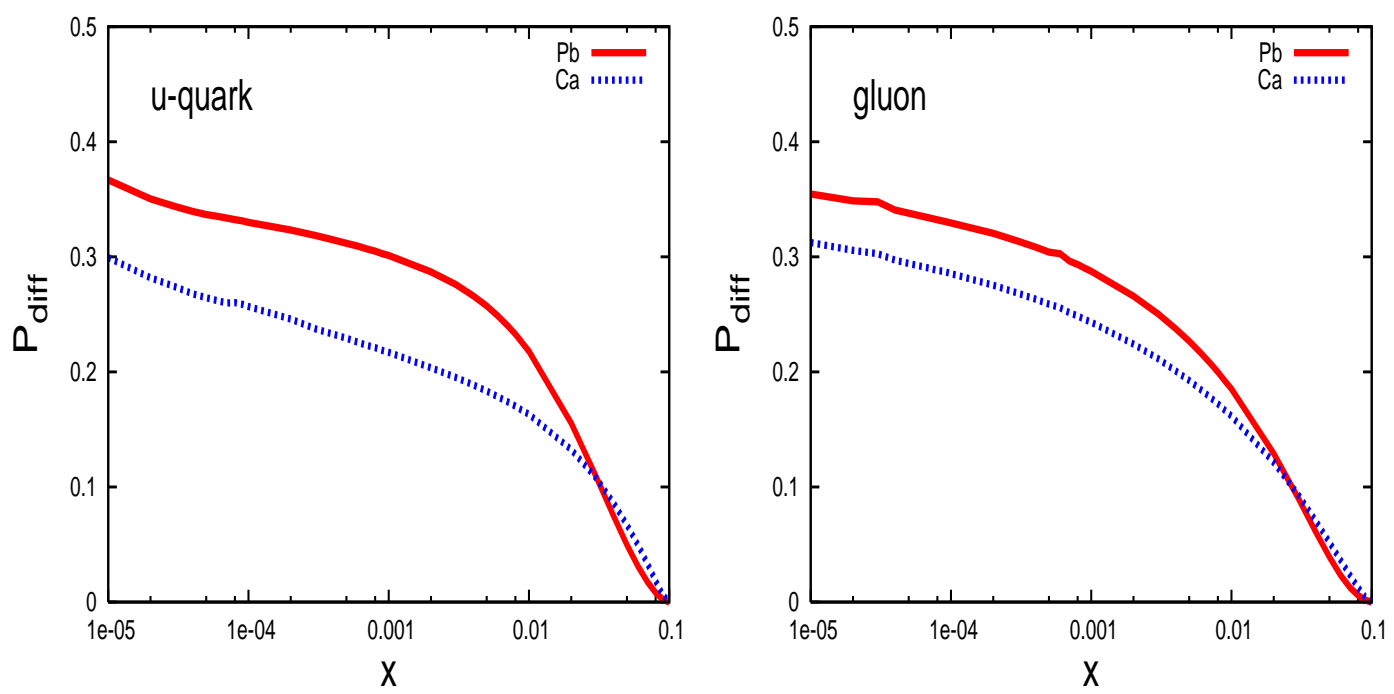

Figure 45. The probability of hard diffraction, $P_{\text {diff }}^{j}$, on ${ }^{40} \mathrm{Ca}$ and ${ }^{208} \mathrm{~Pb}$, at $Q^{2}=4$ $\mathrm{GeV}^{2}$ as a function of $x$ for $u$ quarks (left) and gluons (right).

We now turn to the $Q^{2}$ dependence of $P_{\text {diff }}^{j}$. For both nucleons and nuclei, $P_{\text {diff }}^{q}$ changes weakly with $Q^{2}$ and is $\sim 20-30 \%$ at small $x$, in good agreement with early estimates [139]. While $P_{\text {diff }}^{g}$ decreases faster with increasing $Q^{2}$, the probability is still $\sim 15-20 \%$ at $Q^{2}=100 \mathrm{GeV}^{2}$, making e.g. heavy flavor studies feasible in UPCs at the LHC, similar to inclusive production, considered in Ref. [141]. Dijet production is another alternative, studied by ZEUS [142] and H1 [96] using protons ${ }^{+}$.

+ The recent HERA data seem to indicate that the factorization theorem for direct photoproduction holds at lower transverse momentum for charm production than typical dijet production. 
The discussion presented here is relevant for hard processes produced in direct proton interactions. Spectator parton interactions will suppress the probability of diffraction for resolved photons. Estimates [143] indicate that spectator interactions will decrease the probability of nuclear diffraction by at least a factor of two for $A \sim 200$. Thus, the $A$ dependence of diffraction with resolved photons will also be interesting since it will measure the interaction strength of the spectator system with the media, providing another handle on the photon wavefunction.

3.1.3. Large mass diffraction in the black disk regime One striking feature of the BDR is the orthogonality of the Fock components of the photon wavefunction [64]. Thus there can be no transitions between non-diagonal components, e.g. $\langle q \bar{q} \mid q \bar{q} g\rangle \equiv 0$. Since the dominant contribution to coherent diffraction in the BDR originates from a 'shadow' of fully-absorptive interactions for $b \leq R_{A}$, the orthogonality argument is applicable. The orthonormality condition is used to derive the BDR expression for the differential cross section of the process $\gamma A \rightarrow X A$ where $X$ is a final state of invariant mass $M$ [44]. In the real photon case,

$$
\frac{d \sigma_{\gamma A \rightarrow X A}}{d t d M^{2}}=\frac{\alpha}{3 \pi} \frac{\left(2 \pi R_{A}^{2}\right)^{2}}{16 \pi} \frac{\rho\left(M^{2}\right)}{M^{2}} \frac{4\left|J_{1}\left(\sqrt{-t} R_{A}\right)\right|^{2}}{-t R_{A}^{2}}
$$

where $\rho\left(M^{2}\right)=\sigma_{e^{+} e^{-} \rightarrow \text { hadrons }} / \sigma_{e^{+} e^{-} \rightarrow \mu^{+} \mu^{-}}$at $s=M^{2}$. Diffractive measurements of states with a range of masses would determine the blackness of the photon wavefunction as a function of mass by comparing to the BDR results in Eq. (65). A similar equation for production of specific final states is valid in the BDR in the case of coherent nuclear recoil. It is then possible to determine the components of the photon wavefunction which interacts with the BDR strength in the coherent processes.

The onset of the BDR limit for hard processes should also reveal itself in a faster increase of the photoproduction cross sections of radial excited states with energy relative to the ground state cross section. Utilizing both an intermediate and a heavy nuclear beam, such as $\mathrm{Ar}$ and $\mathrm{Pb}$, would make it possible to remove edge effects as well as maximize the path length through nuclear matter, about $10 \mathrm{fm}$ in a large nucleus.

One especially interesting process is exclusive diffractive dijet production by real photons. For the $\gamma A$ energies available at the Electron Ion Collider [6,7] or in UPCs at the LHC, the BDR would be a good approximation for $M \sim$ few $\mathrm{GeV}$ in the photon wavefunction, the domain described by perturbative QCD for $x \sim 10^{-3}$ with proton targets, and larger $x$ for nuclei. The condition of large longitudinal distances, a small longitudinal momentum transfer, will be applicable up to quite large values of the produced diffractive mass $M$. In the BDR, the dominant channel for large mass diffraction is dijet production with a total cross section given by Eq. (65) and characteristic center-of-mass angular distribution $\left(1+\cos ^{2} \theta\right)$ [44]. In contrast, except for charm, diffractive dijet production is strongly suppressed in the perturbative QCD limit $[144,145]$. The suppression is due to the coupling of the $q \bar{q}$ component of the photon wavefunction to two gluons, calculated to lowest order in $\alpha_{s}$. As a result, 
for real photons, hard diffraction with light quarks is connected to the production of $q \bar{q} g$ and higher states. The mass distribution of diffractively-produced jets thus provides an important test of the onset of the BDR. In the DGLAP/color transparency regime, forward diffractive dijet production cross sections should should be $\propto 1 / M^{8}$ and dominated by charm and bottom jet production, strikingly different from the BDR expressions of Ref. [44].

Thus, dijet photoproduction should be very sensitive to the onset of the BDR. The $q \bar{q}$ component of the photon light-cone wavefunction can be measured using three independent diffractive phenomena: in the BDR off protons and heavy nuclei and in the color transparency regime where the wavefunction can be measured as a function of the inter-quark distance [26]. A competing process for dijet photoproduction off heavy nuclei is the process $\gamma \gamma \rightarrow$ dijets where the second photon comes from the Coulomb field of the opposite nucleus. Dijets produced in $\gamma \gamma$ collisions have positive $C$ parity. Thus this amplitude does not interfere with dijet production in $\gamma \mathbb{P}$ interactions with negative $C$-parity. Therefore $\gamma \gamma \rightarrow$ dijets are a small background over a wide range of energies [76].

3.1.4. High mass diffraction in UPCs The large predicted hard diffraction probability can be checked in UPCs at the LHC. For example, $\gamma A \rightarrow$ jet $_{1}+$ jet $_{2}+X+A$ can be studied in the kinematics where the direct photon process, $\gamma g \rightarrow q \bar{q}$, dominates. In this case, for $p_{T} \sim 10 \mathrm{GeV} / c$ and $Q^{2} \sim 100 \mathrm{GeV}^{2}, \sim 20 \%$ of the events will be diffractive. The hadroproduction background originates from glancing collisions where two nucleons interact through the double diffractive process $p p \rightarrow p p X$ where $X$ contains jets. The probability of hard processes with two gaps is very small at collider energies, even smaller than the probability of single diffractive hard processes [146]. Therefore, the relative backgrounds in the diffractive case are expected to be at least as good as in the inclusive case [141]. Thus, it would be rather straightforward to extract coherent diffraction by simply using anti-coincidence with the forward neutron detector, especially for heavy nuclei [105]. As a result, it would be possible to measure the nuclear DPDFs with high statistical accuracy. In contrast to diffractive vector meson production, it would be possible to determine the energy of the photon which induced the reaction on an event-by-event basis since the photon rapidity is close to the rapidities of the two jets. It would be possible to measure large rapidities by selecting photoproduction events with the highest kinematically allowed energies of the produced particles in the rapidity interval $y_{1}<y<y_{2}$ and determine the DPDFs for rather small $x$.

There are two contributions to dijet photoproduction, direct and resolved. In the direct process, the entire photon energy contributes to the hard process. In the resolved process, only a fraction of the photon energy, $z_{\gamma}$, is involved. HERA studies indicate that the requirement $z_{\gamma} \geq 0.8$ eliminates the resolved photon contribution. However, at higher $Q^{2}$, DGLAP evolution increases the relative importance of the resolved component. 
In $A A$ collisions, there are two possible contributions since the photon can come from either nucleus. It is thus more convenient to refer to the $x$ of the photon and the Pomeron. The values of $x$ can be reconstructed from the kinematics of the diffractive state, $X$, with mass $M_{X}$, produced in the reaction $A A \rightarrow A A X$. The light cone momentum fractions of the two nuclei, $x_{1}$ and $x_{2}$ are normalized to $A$ and satisfy the kinematic relation

$$
x_{1} x_{2} s_{N N}=M_{X}^{2} .
$$

One $x$ is carried by the photon and the other by the Pomeron. (Here Pomeron is used to define the kinematics of the process without specifying a particular dynamical mechanism). When no high $p_{T}$ jets are produced, the values of $x$ are related to the rapidity range of the produced system. In a symmetric $A A$ interaction, the convention is to define $y_{1}=y_{A}-\ln \left(1 / x_{1}\right)$ and $y_{2}=-y_{A}+\ln \left(1 / x_{2}\right)$.) The cross section for the production of state $X$ is

$$
\begin{aligned}
\frac{d \sigma_{A A \rightarrow X A A}}{d x_{1} d x_{2}}= & \frac{d N_{\gamma}\left(x_{1}\right)}{d k} \frac{d \sigma_{\gamma A \rightarrow X A}\left(\tilde{s}=x_{1} s_{N N}, x_{\mathbb{P}}=x_{2}\right)}{d x_{\mathbb{P}}} \\
& +\frac{d N_{\gamma}\left(x_{2}\right)}{d k} \frac{d \sigma_{\gamma A \rightarrow X A}\left(\tilde{s}=x_{2} s_{N N}, x_{\mathbb{P}}=x_{1}\right)}{d x_{\mathbb{P}}} .
\end{aligned}
$$

The direct photoproduction cross section for a hard process such as dijet or heavy quark production is given by the standard pQCD convolution formulas over the nuclear DPDFs and the photon flux. In the resolved case, $z_{\gamma} \ll 1$, diffraction should be suppressed by interaction of spectators in the photon wavefunction with the target, increasing the multiplicity and reducing the rapidity gap. Though these processes appear to be negligible for protons, they are likely to reduce the diffractive cross section considerably, see Section 3.1.2.

There is a potential problem specific to diffractive events: determining which nucleus emitted the photon and which emitted the "Pomeron". Such an event is shown schematically in Fig. 46. The photon source can generally be identified by comparing the invariant mass of the entire produced system, the dijet and the accompanying soft hadrons, the diffractive mass $M_{X}$, to that of the dijet alone, $M_{\text {dijet }}$. For most events, the diffractive mass is much larger than the dijet mass, $M_{X} \gg M_{\text {dijet }}$, and the gap between the dijet and the photon-emitting nucleus is larger than that on the Pomeronemitter side, making identification of the photon source possible. In the rare cases where $M_{X} \sim M_{\text {dijet }}$, fewer accompanying hadrons are produced in a limited rapidity range and the gaps on both sides of the produced system are nearly the same, making identification of the photon source impossible. In this case, the $x$ range is more restricted.

\subsection{Large t diffractive $\rho^{0}$ photoproduction with target dissociation}

Contributed by: L. Frankfurt, M. Strikman and M. Zhalov

3.2.1. Introduction An important feature of small $x$ processes is the nontrivial interplay between evolution in $\ln \left(x_{0} / x\right)$ and $\ln \left(Q^{2} / Q_{0}^{2}\right)$ on the perturbative ladder. Large $t$ 


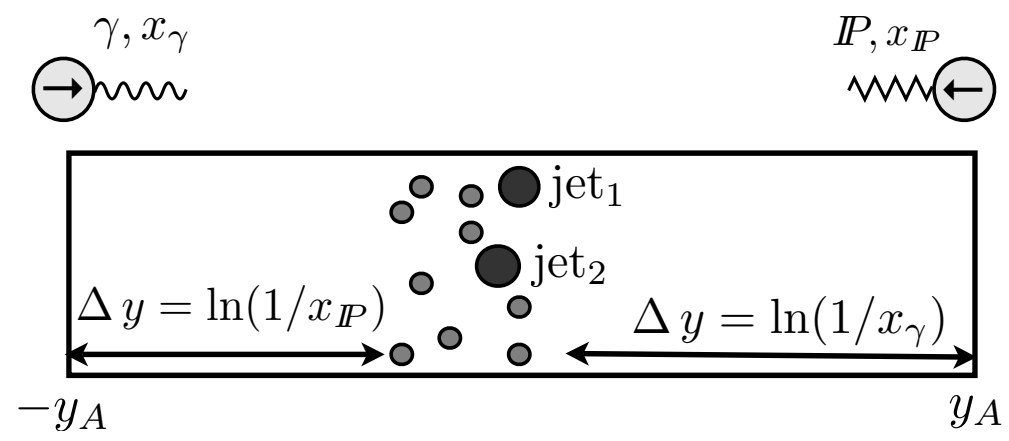

Figure 46. A schematic lego plot of a diffractive photoproduction event showing the gap between the photon-emitter nucleus and the produced dijet system on the right-hand side and the additional gap between the Pomeron-emitter nucleus and the dijet system on the left-hand side. The dijet is accompanied by fewer soft hadrons than in inclusive photoproduction where the nucleus that emits the parton breaks up. From Ref. [31]. Copyright 2006 by the American Physical Society (http://link.aps.org/abstract/PRL/v96/e082001).

processes accompanied by a large rapidity gap ensure that QCD evolution is suppressed as a function of $Q^{2}$ at small coupling. As a result, it is possible to investigate $\ln \left(x_{0} / x\right)$ and $\ln \left(Q^{2} / Q_{0}^{2}\right)$ evolution separately. Such phenomena include the transition from color transparency to color opacity in nuclei. Though color transparency is experimentally established $[38,39]$, further studies are necessary to determine the range of energies and virtualities at which the phenomenon occurs. There are a number of indirect indications for the color opacity regime although direct evidence is limited [147]. The rapidity gap processes we discuss here will provide additional means of addressing these questions.

A number of small $x$ processes which originate due to elastic parton scattering with small color-singlet $q \bar{q}$ dipoles (referred to as dipoles in the remainder of this section) at large momentum transfer and at high energies have been suggested including hard diffraction in $p p \rightarrow p X$ at large $t$. Jet studies include two jet production accompanied by a rapidity gap, the 'coherent Pomeron' [45], and enhanced production of back-toback dijets separated by a large rapidity gap [148] relative to the dijet rate in the same kinematics without a gap $[149,150]$. Dijet production accompanied by a gap was studied at the Tevatron [151]. In addition, high $t$ vector meson photo- and electroproduction with a rapidity gap has also been proposed [152-154]. Over the last decade, theoretical and experimental vector meson studies were focused on interactions with protons. HERA measured the relevant cross sections [117,155-158] in the $\gamma p$ center-of-mass range $20 \leq W_{\gamma p} \leq 200 \mathrm{GeV}$. The HERA data agree well with most predictions of QCD-motivated models [117], several of which use the LO BFKL approximation [159].

It would clearly be beneficial to extend these studies to higher $W_{\gamma p}$ and over a larger range of rapidity gap, $\Delta y$, to investigate the $s_{(q \bar{q}) j}$ and $t$ dependencies of dipole-parton scattering where $j$ is the interacting parton. Here we summarize feasibility studies $[93,118]$ for probing these processes in UPCs at the LHC.

We focus on $\rho^{0}$ photoproduction at large $t$ with a rapidity gap, $\Delta y$, between the $\rho^{0}$ 
and the hadronic system $X$ produced in ultraperipheral $p A$ and $A A$ collisions,

$$
\gamma+p(A) \rightarrow \rho^{0}+\Delta y+X .
$$

We consider the kinematics where $\Delta y \geq 4$, sufficiently large to suppress the fragmentation contribution. Related investigations include diffractive charm or dijet production where the hard final state is separated from the nucleon fragmentation region by large $\Delta y$. For example, studies of the $A$ dependence of dijet production in e.g. $\gamma A \rightarrow\left(\right.$ jet $\left.+M_{1}\right)+\Delta y+\left(\right.$ jet $\left.+M_{2}\right)$ can probe color transparency effects on gap survival in hard-photon induced processes [143]. CMS and ATLAS are well suited for such observations since they cover large rapidity intervals.

The main variables are the mass of the system produced in the proton dissociation, $M_{X}$, the square of momentum transfer $-t \equiv Q^{2}=-\left(p_{\gamma}-p_{V}\right)^{2}$, and the square of the $q \bar{q}$-parton elastic scattering energy

$$
s_{(q \bar{q}) j}=x W_{\gamma p}^{2}=x s_{\gamma p} .
$$

Here

$$
x=\frac{-t}{\left(-t+M_{X}^{2}-m_{N}^{2}\right)}
$$

is the minimum fraction of the proton momentum carried by the elastically-scattered parton for a given $M_{X}$ and $t$. At large $t$ and $W_{\gamma p}$, the gap, $\Delta y$, between the rapidity of the produced vector meson and the final-state parton, at the leading edge of the rapidity range of the hadronic system $X$, is

$$
\Delta y=\ln \frac{x W_{\gamma p}^{2}}{\sqrt{(-t)\left(M_{V}^{2}-t\right)}} .
$$

It is rather difficult to measure $M_{X}$ or $x$ directly. However, they can be adequately determined by studying the leading hadrons close to the rapidity gap; full reconstruction is not required.

Generally, large $t$ scattering with a rapidity gap can be described as an incoherent sum of terms describing elastic quark and gluon scattering. Each term is the product of the quasi-elastic large $t$ cross section of $p(A) j \rightarrow V j$ and the density of parton $j$ in the target $[45,152,153]$. Large $t$ ensures two important simplifications: the parton ladder mediating quasi-elastic scattering is attached to the projectile via two gluons while the attachment of the dipole ladder to more than one target parton is strongly suppressed. The gluon elastic-scattering cross section is enhanced by $81 / 16$ relative to quark scattering. Gluon scattering dominates over a wide $x$ range, constituting $\sim 80 \%$ (70\%) of the cross section at $x=0.1(0.3)$. The $t$ dependence can be parametrized as a power law where the power is twice the number of constituents in the hadron vertex, $1 / t^{6}$ for three quarks [45] and $1 / t^{4}$ for the $q \bar{q}$ system [153].

The cross section for vector meson photoproduction with target dissociation in the range $-t \gg 1 / r_{V}^{2}>\Lambda_{\mathrm{QCD}}^{2}$ where $r_{V}$ is the vector meson radius; $W_{\gamma p}^{2} \gg M_{X}^{2} \gg m_{N}^{2}$; and fixed $x(x<1)$ is [153]

$$
\frac{d \sigma_{\gamma p \rightarrow V X}}{d t d x}=\frac{d \sigma_{\gamma q \rightarrow V q}}{d t}\left[\frac{81}{16} g_{p}(x, t)+\sum_{i}\left[q_{p}^{i}(x, t)+\bar{q}_{p}^{i}(x, t)\right]\right] .
$$


Here $g_{p}(x, t), q_{p}^{i}(x, t)$ and $\bar{q}_{p}^{i}(x, t)$ are the gluon, quark and antiquark distributions in the proton. The $\gamma q \rightarrow V q$ amplitude, $f_{q}\left(s_{(q \bar{q}) q}, t\right)$, is dominated by quasi-elastic scattering of the small $q \bar{q}$ dipole configuration of the photon that transitions into the final-state vector meson.

Diffractive vector meson photoproduction from hadron and nuclear targets is a special case where evolution in $x$ is separated from evolution in the hard scale, see Ref. [45, 149, 152, 153]. Since $t$ is the same on all rungs of the ladder mediating quasielastic scattering, the amplitude $f_{q}\left(s_{(q \bar{q}) j}, Q^{2}=-t\right)$ probes evolution in $\ln (1 / x)$ at fixed $Q^{2}$. Because the momentum transfer is shared between two gluons, the characteristic virtuality of $t$-channel gluons on the ladder is $\approx Q^{2} / 4$ while the hard scale in the target parton density is $\approx Q^{2}$.

To lowest order in $\ln (1 / x)$, the amplitude, $f_{q}\left(s_{(q \bar{q}) j}, t\right)$, is independent of $W_{\gamma p}$ for fixed $t$. Higher order terms in $\ln (1 / x)$ were incorporated in the leading and next-toleading $\log$ approximations, including both $\ln Q^{2}$ and $\ln x$ effects so that $f_{q}$ increases with energy as a power of $\exp (\Delta y)$ in Eq. (71) with a weak $t$ dependence,

$$
f_{q}\left(s_{(q \bar{q}) j}, t\right) \propto\left(\frac{s_{(q \bar{q}) j}}{|t|}\right)^{\delta(t)}
$$

for $|t| \gg M_{V}^{2}$. Within NLO BFKL this dependence is not obvious since the solution may be given by a different saddle point at higher $Q^{2}[160,161]$. The value of $\delta(0)$ changes significantly between LO and NLO BFKL, $\delta(0) \sim 0.6$ at LO and $\delta(0) \sim 0.1$ at NLO. The difference between NLO and resummed BFKL is smaller since $\delta(0) \sim 0.2-0.25$ in resummed BFKL over a wide range of $Q^{2}[162]$. Hence we treat $\delta(t)$ as a free parameter and generally assume it weakly depends on $t$.

Similar small $t$ processes could be described by the triple Pomeron approximation of the amplitude,

$$
f_{q} \propto\left(\frac{W_{\gamma p}^{2}}{M_{X}^{2}}\right)^{\alpha_{\mathbb{P}}^{\mathrm{soft}}(t)-1},
$$

where the soft Pomeron trajectory is

$$
\alpha_{\mathbb{P}}^{\mathrm{soft}}(t)=\alpha_{0}+\alpha^{\prime} t
$$

with $\alpha_{0} \sim 1.08$ and $\alpha^{\prime} \sim 0.25 \mathrm{GeV}^{-2}$. The amplitude decreases with energy for $-t \geq 0.4$ $\mathrm{GeV}^{2}$.

We first use a simple parametrization of the HERA data based on a hard reaction mechanism [93] to estimate the $\gamma p \rightarrow \rho^{0}+\Delta y+X$ rates in $p A$ collisions at the LHC. We find that it will be possible to extend the HERA energy range by a factor of 10 . We then analyze the $A$ dependence of the process and show that it provides a critical test of the interplay between hard and soft dynamics as well as probes the onset of the hard black disk regime. We find that it will be possible to study this process to $W_{\gamma p} \sim 1 \mathrm{TeV}$ and study hard dynamics up to $x s_{\gamma p} / Q^{2} \sim 10^{5}$, corresponding to an rapidity interval of $\sim 12$ units for gluon emission. Hence the emission of several gluons in the ladder kinematics (when the rapidity interval between two gluons on the ladder is larger than one) is possible. 
3.2.2. Rapidity gap processes from ep at HERA to $p A$ at the $L H C$ The HERA experiments report cross sections integrated over $M_{X}$ from $M_{X}=m_{N}$ up to an experimentally-fixed upper limit, $\widehat{M}$. At fixed $t$, this corresponds to the cross section integrated over $x$ from the $x_{\text {min }}$ determined in Eq. (70) at $M_{X}=\widehat{M}$ to $x=1$.

We described these data using the following expression, based on Eq. (72),

$$
\frac{d \sigma_{\gamma p \rightarrow \rho^{0} X}}{d t}=\frac{C}{\left(1-t / t_{0}\right)^{4}}\left(\frac{s_{\gamma p}}{M_{V}^{2}-t}\right)^{2 \delta(t)} I\left(x_{\min }, t\right),
$$

where $\left|t_{0}\right|=1 \mathrm{GeV}^{2}$. The cross section, $d \sigma_{\gamma q \rightarrow V q} / d t$ is factorized into a component accounting for the $\gamma \rightarrow V$ transition, $C /\left(1-t / t_{0}\right)^{4}$, and the dipole-parton scattering amplitude, $f_{q}$. The amplitude has been modified to account for the virtuality of the recoiling parton, on the order of the soft scale, $\left|t_{0}\right|$ The factor $I\left(x_{\min }, t\right)$, is obtained by integrating over the parton densities,

$$
I\left(x_{\min }, t\right)=\int_{x_{\min }}^{1} d x x^{2 \delta(t)}\left[\frac{81}{16} g_{p}(x, t)+\sum_{i}\left[q_{p}^{i}(x, t)+\bar{q}_{p}^{i}(x, t)\right]\right]
$$

where the CTEQ6M PDFs [163] have been employed. The function $\delta(t)$ is parametrized as $\delta(t)=\delta_{0}+\delta^{\prime} t$. The values of $\delta_{0}, \delta^{\prime}$ and $C$ were adjusted to provide a reasonable description of the HERA $\rho^{0}$ data* ${ }^{*}$ The $t$ dependence was measured by H1 and ZEUS for different $M_{X}$ cuts over a rather narrow interval of $W_{\gamma p}$. As a result, these data cannot unambiguously fix the energy dependence of the dipole-parton amplitude in $\delta(t)$. We obtain a reasonable description of the data assuming both a relatively weak energy dependence, $\delta(t)=0.1(C=40)$, and a stronger energy dependence, $\delta(t)=0.2$ $(C=14)$, for hard processes. These values of $\delta(t)$ are significantly larger than those resulting from extrapolation of the soft Pomeron trajectory in Eq. (75) to higher $t$, even if a nonlinear term is introduced in the trajectory [164]. This can be seen by equating the exponents in Eqs. (73) and (74) at $-t \geq 0.4 \mathrm{GeV}^{2}, \delta(t)=\alpha_{\mathbb{P}}^{\text {soft }}(t)-1 \approx 0.08+0.25 t$. Our results are consistent with a rather weak $t$ dependence of $\delta(t)$, hence we take $\delta^{\prime}=0$. A very small negative value, $\delta^{\prime}=-0.01 \mathrm{GeV}^{-2}$, improves agreement with the H1 data at $-t>5 \mathrm{GeV}^{2}$ with $\delta_{0}=0.2$.

As mentioned previously, in the hard regime the energy dependence of the amplitude should be a weak function of $t$. In $\rho^{0}$ photoproduction with a rapidity gap, large $t$ is necessary for the hard mechanism to dominate. However, for exclusive quarkonium photo/electroproduction or light vector meson electroproduction at large $Q^{2}$, the hard mechanism is expected to dominate at $t \sim 0$. Hence $\delta(t)$ should be similar to the energy dependence of the exclusive $\gamma^{*} p \rightarrow V p$ amplitude. At HERA, the highest virtualities are reached in exclusive $J / \psi$ electroproduction and correspond to $\delta \sim 0.2$ for $t=0$ and $\delta \sim 0.1$ for $t \sim 1 \mathrm{GeV}^{2}$ [165]. The observation that a similar value of $\delta$ can describe the large- $t$ rapidity-gap data supports the interpretation of the data as due to hard elastic $q \bar{q}$ dipole-parton scattering.

* There is a relatively small rapidity interval available for gluon emission in the color singlet ladder, $\ln \left(x s_{\gamma p} / Q^{2}\right) \leq 5$, in the HERA data. Since only single gluon emission is allowed in the ladder kinematics, it is very difficult to apply a BFKL-type approximation. 

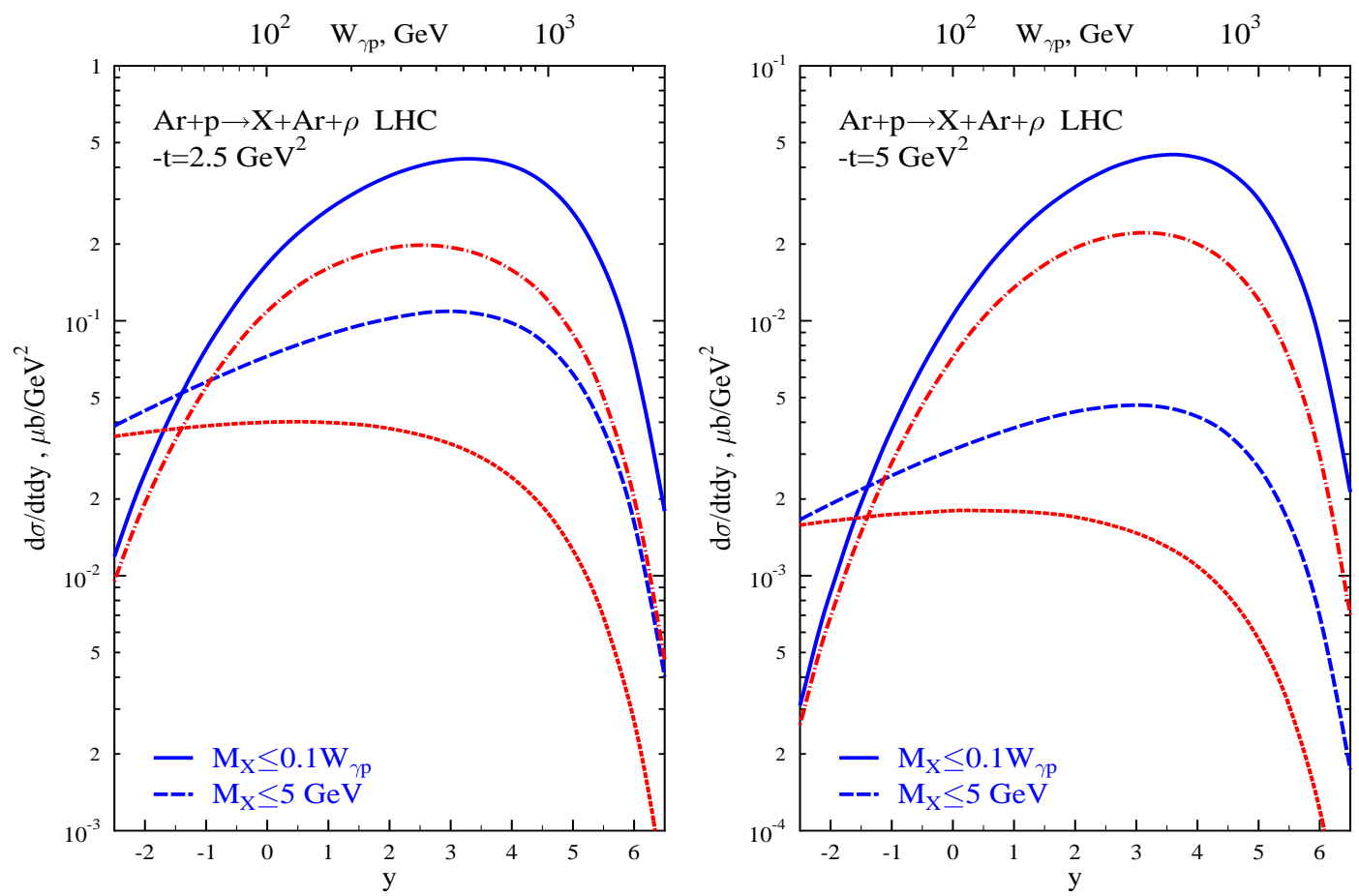

Figure 47. The $\rho^{0}$ rapidity distributions in ultraperipheral $p$ Ar collisions at the LHC for two different $M_{X}$ cuts at the indicated values of $t$ [93]. The solid and dashed lines are calculations with $\delta=0.2$ while the dot-dashed and short-dashed curves employ $\delta=0.1$.
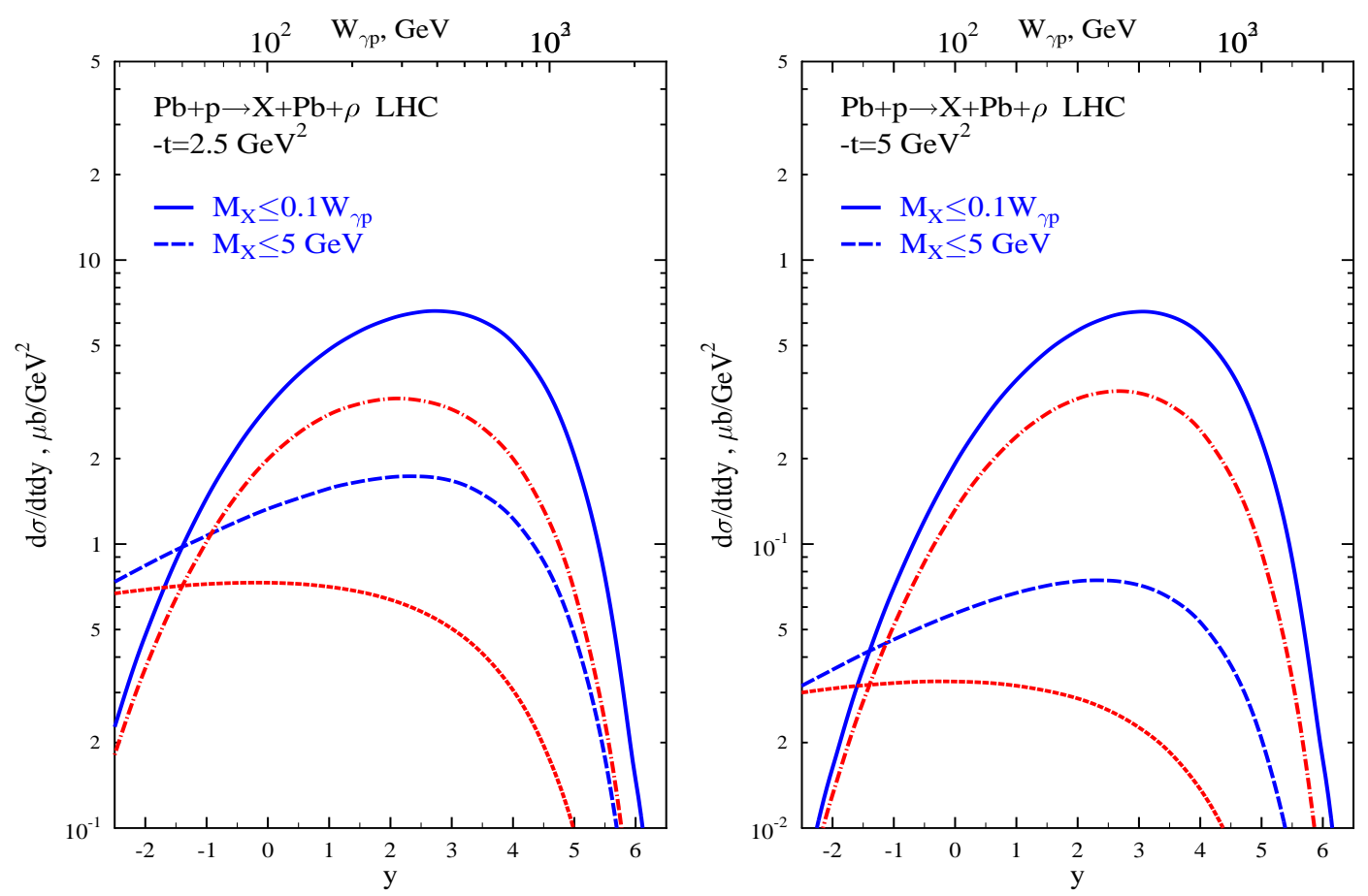

Figure 48. The same as for Fig. 47 for $p \mathrm{~Pb}$ collisions [93]. 
It is unlikely that further HERA studies will cover a sufficiently wide range of $W_{\gamma p}$ and $\Delta y$ to study the energy dependence of the large- $t$ elastic dipole-parton scattering amplitude. On the other hand, at the LHC, CMS and ATLAS will have sufficient rapidity coverage to study the process in Eq. (68) in ultraperipheral $p A$ collisions. Hence we use the parametrization of the $\gamma p \rightarrow X \rho^{0}$ cross section in Eqs. (76) and (77) to estimate the large- $t$ rapidity-gap $\rho^{0}$ cross section in ultraperipheral $p A$ and, later in $A A$ collisions at the LHC. We do not address the $p A$ contribution from $\gamma A \rightarrow \rho^{0} X$ since it is very small and can easily be separated experimentally. The large- $t$ nucleon-dissociation cross section is then

$$
\frac{d \sigma_{p A \rightarrow \rho^{0} X A}}{d t d y}=\frac{d N_{\gamma}^{Z}(y)}{d k} \frac{d \sigma_{\gamma N \rightarrow \rho^{0} X}(y)}{d t}
$$

where $d N_{\gamma}^{Z}(y) / d k$ is the photon flux generated by the ion with energy $k=$ $\left(M_{\rho^{0}} / 2\right) \exp (y)$. We consider intermediate and large momentum transfer in UPCs at the LHC, analogous to those studied at HERA.

The cross section can be studied at fixed $t$ as a function of the $\rho^{0}$ rapidity with the restriction $M_{X} \leq 5 \mathrm{GeV}$ to determine the energy dependence of the dipole-parton amplitude and thus $\delta(t)$. In this case, $x_{\min }$ does not depend on $W_{\gamma p}$ and the dipoleparton elastic scattering amplitude varies with $W_{\gamma p}$ due to the increase of the rapidity gap with $y$.

We also study the cross section when $M_{X} \propto W_{\gamma p}$, specifically $M_{X} \leq 0.1 W_{\gamma p}$. This cut corresponds to fixing $\Delta y$ and changing $x_{\text {min }}$. Such studies could test the parton distribution functions and the reaction mechanism by extracting $I\left(x_{\min }, t\right)$ from the data in different $x_{\min }$ and $t$ bins.

We do not consider $W_{\gamma p}<20 \mathrm{GeV}$ where our HERA-based parametrization, Eqs. (76) and (77), are unreliable, particularly for $M_{X} \leq 5 \mathrm{GeV}$. In any case, the data indicate that the cross section is very small if $M_{X} \leq 2 \mathrm{GeV}$.

The rapidity distribution of diffractive $\rho^{0}$ photoproduction accompanied by a rapidity gap between the $\rho^{0}$ and the system $X$ produced by the target proton break up is shown in Figs. 47 and 48 for $p \mathrm{Ar}$ and $p \mathrm{~Pb}$ collisions respectively. The distributions are shown for two fixed values of $t:-t=2.5$ and $5 \mathrm{GeV}^{2}$. We use the same sets of cuts as those employed in the HERA experiments. The cut $M_{X} \leq 5 \mathrm{GeV}$ corresponds to a fixed rapidity interval occupied by the hadrons in system $X$. The energy-based cut, $M_{X} \leq 0.1 W_{\gamma p}$, corresponds to the same minimum $\Delta y$ between the vector meson and the produced hadrons.

The choice $M_{X} \leq 5 \mathrm{GeV}$ gives a flatter and broader rapidity distribution since $x_{\text {min }}$ is independent of $W_{\gamma p}$ and not very small. When $M_{X} \leq 0.1 W_{\gamma p}$, smaller values of $x_{\text {min }}$ are reached for the same $-t$, giving a larger cross section over most of the rapidity range, particularly for $-t=5 \mathrm{GeV}^{2}$. The two choices exhibit the same behavior at large forward rapidity due to the steep decrease of the photon flux. Results are also shown for two assumptions of $\delta(t)$ : 0.2 and 0.1 . The assumption $\delta(t)=0.1$ narrows the rapidity distribution, as does going to a higher $-t$. The rates, which can be obtained by 
multiplying the cross sections by luminosities of $6 \mu \mathrm{b}^{-1}$ for $p \mathrm{Ar}$ and $p \mathrm{~Pb}$ respectively, are high.

The $t$-dependence of the cross section in Eq. (76) should decrease more slowly than the asymptotic behavior of the $(q \bar{q})+j \rightarrow V+j$ cross section, $\propto 1 / t^{4}$. As a result, the rate for $|t|>\left|t_{\min }\right| \geq 2.5 \mathrm{GeV}^{2}$ drop rather slowly with $t_{\min }$ (more slowly than $1 / t_{\min }^{3}$ ). With the expected LHC $p A$ luminosities, the rates remain high up to rather large $t$. The rates for $-t>10 \mathrm{GeV}^{2}$ are only a factor of 10 smaller than for $-t>5 \mathrm{GeV}^{2}$. The $J / \psi$ production rates would also be significant. Although the rates are smaller than for $\rho^{0}$ production at fixed $t$, it would be possible to use $-t \geq 1 \mathrm{GeV}^{2}$ in the analysis where the rates are larger than for the exclusive diffractive reaction, $\gamma p \rightarrow J / \psi p$.

Most events in these kinematics correspond to $x \geq 0.01$. Thus we can primarily infer the energy dependence of the elastic $(q \bar{q}) j$ amplitude at different $Q^{2}$. Some events will also probe as low as $x \sim 10^{-3}$. However, it will be probably very difficult to reach the $x$ range where quark scattering is larger than gluon scattering, $x \geq 0.4$. Overall, the energy range, $s_{\max } / s_{\min } \geq 4 \times 10^{3}$, is large enough for precision measurements of the energy dependence of the amplitude. If $\delta(t) \approx 0.2$, the elastic cross section should increase by a factor of $\sim 30$ in the energy range.

3.2.3. A dependence of rapidity gap production in $A A$ collisions Since large $t$ rapidity gap processes, $\gamma\left(\gamma^{*}\right) N \rightarrow V X$, are dominated by elastic $q \bar{q}$-parton scattering, these processes provide a novel way to investigate small dipole interactions in the nuclear medium.

Ultraperipheral $A A$ collisions at the LHC will provide the first opportunity to investigate the new QCD regime of strong interactions at small coupling as well as large target thickness. Further studies will be possible at a future $e A$ collider. Ultraperipheral $A A$ collisions differ from $p A$ collisions since vector mesons can be produced by photons emitted from either nucleus. The cross section is the sum of the two contributions,

$$
\frac{d \sigma_{A A \rightarrow \rho^{0} X A A^{\prime}}}{d y d t}=\frac{d N_{\gamma}^{Z}(y)}{d k} \frac{d \sigma_{\gamma A \rightarrow \rho^{0} X A^{\prime}}(y)}{d t}+\frac{N_{\gamma}^{Z}(-y)}{d k} \frac{d \sigma_{\gamma A \rightarrow \rho^{0} X A^{\prime}}(-y)}{d t} .
$$

Here $\sigma_{\gamma A \rightarrow \rho^{0} X A^{\prime}}$ is the $\rho^{0}$ photoproduction cross section with dissociation, the system $X$ results from diffractive dissociation of a nucleon and $A^{\prime}$ is the residual nucleus. Several neutrons will be produced in the electromagnetic excitation of $A^{\prime}$ by the photon-emitting nucleus, $A$, in Eq. (68).

The system $X$ should be similar to that produced in nuclear DIS at similar $x$ and $Q^{2} \sim-t$ except that here the system can be produced by both quark and gluon scattering. The hadron spectrum is obtained from quark and gluon fragmentation in the proportion of parton production given by Eq. (72). These hadrons should balance the vector meson transverse momentum. The leading hadron momenta in the nuclear rest frame are $\sim-t /\left(2 m_{N} x\right)$. Hence, based on EMC measurements [166], we expect that, at large $t$ and $x \leq 0.05$, leading hadron absorption is small. Nevertheless, a few neutrons will be produced in the nuclear fragmentation region by final-state hadronic 
interactions [105]. Therefore, either one or both ZDCs will detect several neutrons. Detecting the hadrons in $X$ can determine which nucleus emitted the photon, leading to the determination of the invariant $\gamma A$ energy.

Studies of the $A$ dependence of $\gamma A \rightarrow \rho^{0} X A^{\prime}$ at large $t$ can reveal the dynamics of the $(q \bar{q}) A$ interaction. Before discussing the predicted $A$ dependence in these kinematics, we estimate the $A$ dependence at small $t$. At high energies, the photon is in an average configuration which interacts inelastically with a strength comparable to that of the $\rho^{0}$. In this case, fluctuations in the interaction strength are rather small and the photoproduction cross section can be calculated in the Gribov-Glauber approximation for high-energy incoherent processes,

$$
\frac{d \sigma_{\gamma A \rightarrow \rho^{0} X A}}{d t}=A_{\mathrm{eff}} \frac{d \sigma_{\gamma p \rightarrow \rho^{0} X}}{d t} .
$$

The effective number of nucleons, $A_{\text {eff }}$, determines the rapidity gap survival probability,

$$
\frac{A_{\text {eff }}}{A}=\frac{1}{A} \int d^{2} b T_{A}(b) \exp \left[-\sigma_{\text {in }}^{\rho^{0} N} T_{A}(b)\right] .
$$

In the high energy regime, the growth of $\sigma_{\text {in }}^{\rho^{0} N}$ is significant. Thus the suppression becomes quite large, $A_{\text {eff }} / A \sim A^{-\frac{2}{3}}$, emphasizing the peripheral nature of the process.

At large $t$, the dominant component of the photon wavefunction responsible for vector meson photoproduction with nucleon dissociation is a $q \bar{q}$ dipole characterized by size $d \propto 1 / \sqrt{|t|}$. Leading and higher-twist nuclear shadowing should decrease with $t$ due to color transparency. The contribution of planar (eikonal/Glauber rescattering) diagrams to the high-energy amplitude is canceled in a quantum field theory $[167,168]$. This result has recently been generalized to pQCD for the interaction of a small dipole with a large color singlet dipole by $g g$ ladder exchanges: either of two color octet ladders [169] or of multiple color singlet ladders [170]. The primary distinction between a quantum-mechanical description of scattering and a quantum field theory like QCD is that a field theory allows fluctuations in the number of constituents in a given dipole configuration, all of which can scatter in the target [22,170], while quantum mechanics involves the interaction of systems with fixed number of constituents. Each constituent in a particular configuration can interact only once with a target parton through a $t$ channel amplitude with vacuum quantum numbers. Multiple scattering thus arises when the interaction partners are viewed as collections of partons, leading to a Gribov-Glauber type picture with causality and energy-momentum conservation.

In the case of dipole-nucleus scattering, the first rescattering is given by the pQCD cross section for the interaction of the $q \bar{q}$ dipole of transverse size $d$. At leading order, the cross section can be written as $[26,27,171]$,

$$
\sigma_{\text {in }}^{(q \bar{q}) N}\left(\tilde{x}, d^{2}\right)=\frac{\pi^{2}}{4} C_{F} d^{2} \alpha_{s}\left(Q_{\text {eff }}^{2}\right) \tilde{x} g\left(\tilde{x}, Q_{\text {eff }}^{2}\right) .
$$

where, similar to Eq. (7), $C_{F}=4 / 3, d$ is the transverse size of the dipole, $Q_{\text {eff }}^{2} \propto 1 / d^{2}$ is the effective dipole virtuality, $\tilde{x}=Q_{\text {eff }}^{2} / W_{\gamma p}^{2}$ and $g\left(\tilde{x}, Q_{\text {eff }}^{2}\right)$ is the inclusive gluon density of the target. Since the dipole size scales as $1 / \sqrt{|t|}$, at sufficiently large $t$ and fixed 
$W_{\gamma p}, \sigma_{\text {in }}^{(q \bar{q}) N}$ becomes small enough for interactions with more than three nucleons to be negligible. The rapidity gap survival probability then simplifies to

$$
\frac{A_{\mathrm{eff}}}{A}=1-\frac{\sigma_{\mathrm{in}}^{(q \bar{q}) N}}{A} \int d^{2} b T_{A}^{2}(b) .
$$

At fixed $t, \sigma_{\text {in }}^{(q \bar{q}) N}$ increases with $W_{\gamma p}$ due to the growth of the small $x$ gluon density, $\tilde{x} g_{T}\left(\tilde{x}, Q_{\text {eff }}^{2}\right) \propto\left(W_{\gamma p}^{2} / Q_{\text {eff }}^{2}\right)^{n}, n \geq 0.2$. At large $W_{\gamma p}$, Eq. (83) breaks down and higher-order rescatterings involving the interaction of more than three nucleons with configurations containing three or more partons ( $q \bar{q} g$ or higher) must be taken into account. The cross sections for such configurations should be larger than $\sigma_{\text {in }}^{(q \bar{q}) N}$ in Eq. (82) because the projectile has a non-negligible probability to consist of several dipoles with sizes comparable to the initial dipole. Therefore, in the following, we refer instead to an effective cross section, $\sigma_{\text {eff }}$, a parameter to model the average dipolenucleon interaction strength. Although the eikonal-type expansion in the number of rescatterings, based on the average interaction strength, will somewhat overestimate the absorption, it is still reasonable to use the eikonal approximation to estimate the suppression.

Figure 49 shows $A_{\text {eff }} / A$, calculated using Eq. (81), as a function of $\sigma_{\text {eff }}$. The accuracy of the calculated $A_{\text {eff }} / A$ should increase both in the limit of small $\sigma_{\text {eff }}$ where more than two scatterings is a small correction, $\sigma_{\text {eff }} \leq 3 \mathrm{mb}$ for $A \sim 200$, color transparency, and large $\sigma_{\text {eff }}$, close to the color opacity or black disk regime.

Increasing $t$ at fixed $W_{\gamma p}$ leads to $A_{\text {eff }} / A \rightarrow 1$, the onset of color transparency. When $W_{\gamma p} \sim 100 \mathrm{GeV}$, a typical energy for UPCs at the LHC and the upper range of HERA energies, a $d=0.2 \mathrm{fm}$ dipole results in $\sigma_{\text {eff }} \approx 5 \mathrm{mb}$. However, $A_{\text {eff }} / A$ is considerably less than unity even for such a relatively small value of $\sigma_{\text {eff }}$, see Fig. 49 . At these values of $\sigma_{\text {eff }}$, the difference between Eqs. (81) and Eq. (83) is substantial. since with $\sigma_{\text {eff }} \approx 5 \mathrm{mb}$ and $A=200, A_{\text {eff }} / A$ calculated with Eq. (83) is a factor of 1.6 smaller than that of Eq. (81). The difference increases with $\sigma_{\text {eff }}$. Hence either larger $t$ or smaller $W_{\gamma p}$ is needed for complete color transparency as described in Eq. (84).

Thus increasing $W_{\gamma p}$ at fixed $t$ is expected to lead to the onset of the BDR for dipole interactions with the nuclear medium. Vector meson photoproduction would then be strongly suppressed at central impact parameters so that the peripheral process dominates with a cross section proportional to $A^{1 / 3}$. The suppression of the $\rho^{0}$ yield would then be comparable to the soft regime estimated employing Eq. (81).

Higher-twist effects in these kinematics would also be manifested in the structure of the final state. Since the higher-twist mechanism is more peripheral, a large suppression in the nuclear medium would be combined with the emission of fewer neutrons. The suppression could be determined by neutron multiplicity studies in the ZDC.

In the leading-twist approximation, the cross section is given by Eq. (72) where the nucleon parton distributions are replaced by the nuclear parton densities $g_{A}, q_{A}$ and $\bar{q}_{A}$,

$$
\frac{d \sigma_{\gamma A \rightarrow V X A^{\prime}}}{d t d x}=\frac{d \sigma_{\gamma q \rightarrow V q}}{d t}\left[\frac{81}{16} x g_{A}(x, t)+\sum_{i}\left(x q_{A}(x, t)+x \bar{q}_{A}(x, t)\right)\right] .
$$




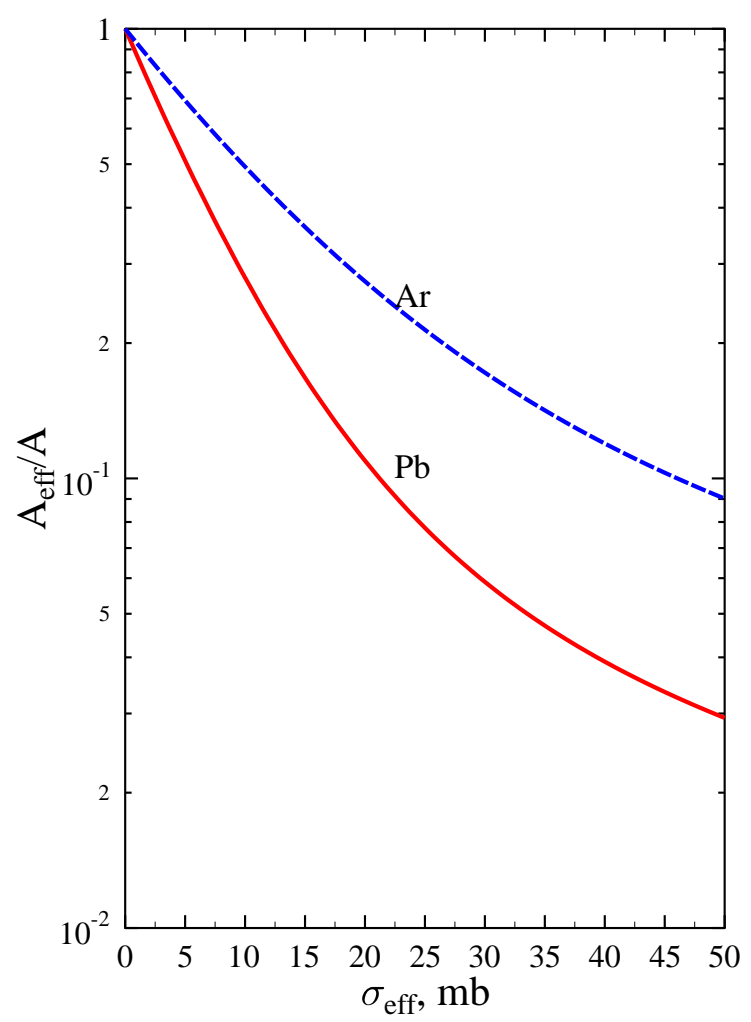

Figure 49. The rapidity gap survival probability as a function of $\sigma_{\text {eff }}[93]$.

The quark distributions do not deviate more than $10 \%$ from a linear $A$ dependence for $0.05<x<0.5$. Current models of the nuclear gluon density, which dominates Eq. (84), predict an enhancement of up to $20 \%$ for $x \sim 0.1$ with perhaps some suppression at $x \geq 0.4$. Hence the leading-twist approximation, Eq. (84), predicts the onset of color transparency with increasing $t$, characterized by strong suppression of the dipole interaction with the nuclear medium. The upper limit of the photoproduction cross section in the impulse approximation is

$$
\frac{d \sigma_{\gamma A \rightarrow \rho^{0} X A}}{d t}=A \frac{d \sigma_{\gamma p \rightarrow \rho^{0} X}}{d t} .
$$

The reasonable agreement of the predicted behavior with the major features of large- $t$ rapidity-gap processes at HERA in the kinematics corresponding to dipoleparton scattering at $x \geq 0.05$ suggests that it is possible to trigger on high-energy small $q \bar{q}$ dipole scattering without requiring small $x$. If the kinematics where $M_{X}$ corresponds to $x \leq 0.01$ could be reached, where leading-twist gluon shadowing is important [58], a further decrease of $A_{\text {eff }} / A$ is possible. On the other hand, elastic quarkonium photo/electroproduction is naturally at small $x$. Thus $\rho^{0}$ production with nucleon dissociation provides a complementary, clean way to study interactions with the nuclear medium. Hence, when $x \ll 10^{-2}$, both leading and higher-twist effects in the dipole-parton and dipole-nucleus interactions are addressed.

Numerical estimates were made for two scenarios at the LHC: the impulse 

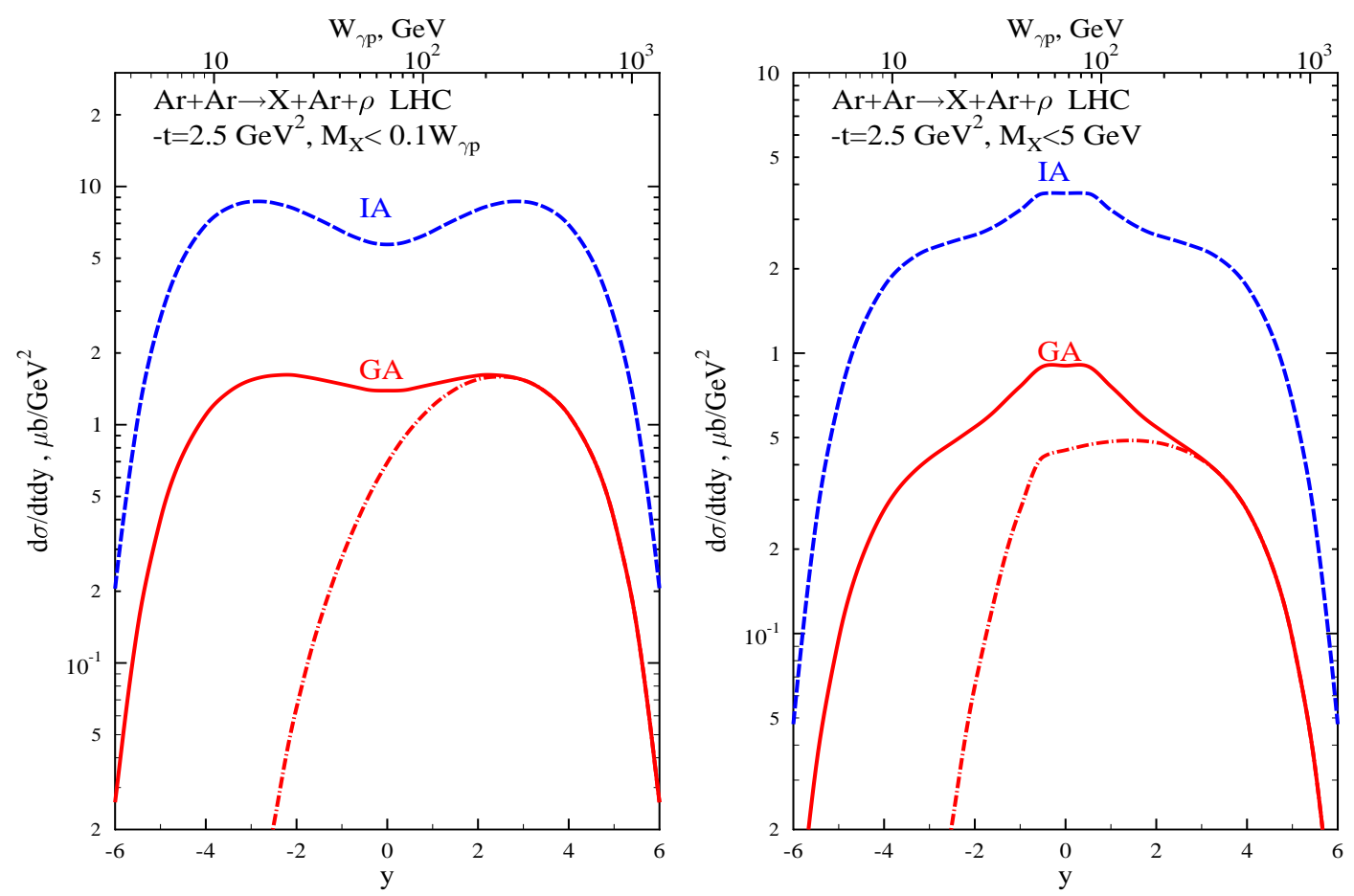

Figure 50. The rapidity distribution of $\rho^{0}$ production with nucleon dissociation in Ar+Ar collisions at $-t=2.5 \mathrm{GeV}^{2}[93]$. The left-hand figure takes $M_{X} \leq 0.1 W_{\gamma p}$ while the upper limit in the right-hand figure is fixed by restriction $M_{X} \leq 5 \mathrm{GeV}$. The dashed curves are the impulse approximation while the solid curves include GlauberGribov screening, neglecting the small nuclear shadowing correction. The lower dashed curves show the contribution from a single nucleus only.

approximation (IA) in Eq. (85) where the cross section is proportional to $A$ and strong screening due to Glauber-Gribov multiple scattering (GA), implemented using Eq. (81). The GA result gives a lower limit on the rate while the IA is an upper limit. We assume that absorption cross section for a small dipole should not be larger than the cross section for a hadron with the same valence quarks. Thus $\sigma_{\mathrm{in}}^{\rho^{0} N}$ in Eq. (81) is based on an elastic $\rho^{0} p$ scattering fit [102] and the vector dominance model.

Since the photon that produces the $\rho^{0}$ can come from either nucleus, the $M_{X}$ cuts described for $p A$ must be modified. We again use the cut $M_{X}<0.1 W_{\gamma p}$ but changes are needed for a fixed upper limit on $M_{X}$. In $p A$ interactions, our parametrization is reasonable for both cuts as long as $W_{\gamma p}>30 \mathrm{GeV}$. In lower energy $A A$ collisions, a large scattering energy in one nucleus corresponds to low energy in the second nucleus. The region $W_{\gamma p}<20 \mathrm{GeV}$ is then reached and the fit becomes inapplicable for $M_{X}<5$ $\mathrm{GeV}$. Thus, instead of a fixed upper limit of $M_{X} \leq 5 \mathrm{GeV}$ for all $W_{\gamma p}$, at $W_{\gamma p}<50$ $\mathrm{GeV}$, we change from the fixed upper limit to a $W_{\gamma p}$-dependent cut, $M_{X} \leq 0.1 W_{\gamma p}$.

The $\rho^{0}$ rapidity distributions with nuclear breakup for $\mathrm{Ar}+\mathrm{Ar}$ and $\mathrm{Pb}+\mathrm{Pb}$ collisions are shown in Figs. 50-53. Figures 50 and 52 show results for $-t=2.5 \mathrm{GeV}^{2}$ while Figs. 51 and 53 are for $-t=5 \mathrm{GeV}^{2}$. The two $M_{X}$ cuts are shown for each value of $-t$ with the 

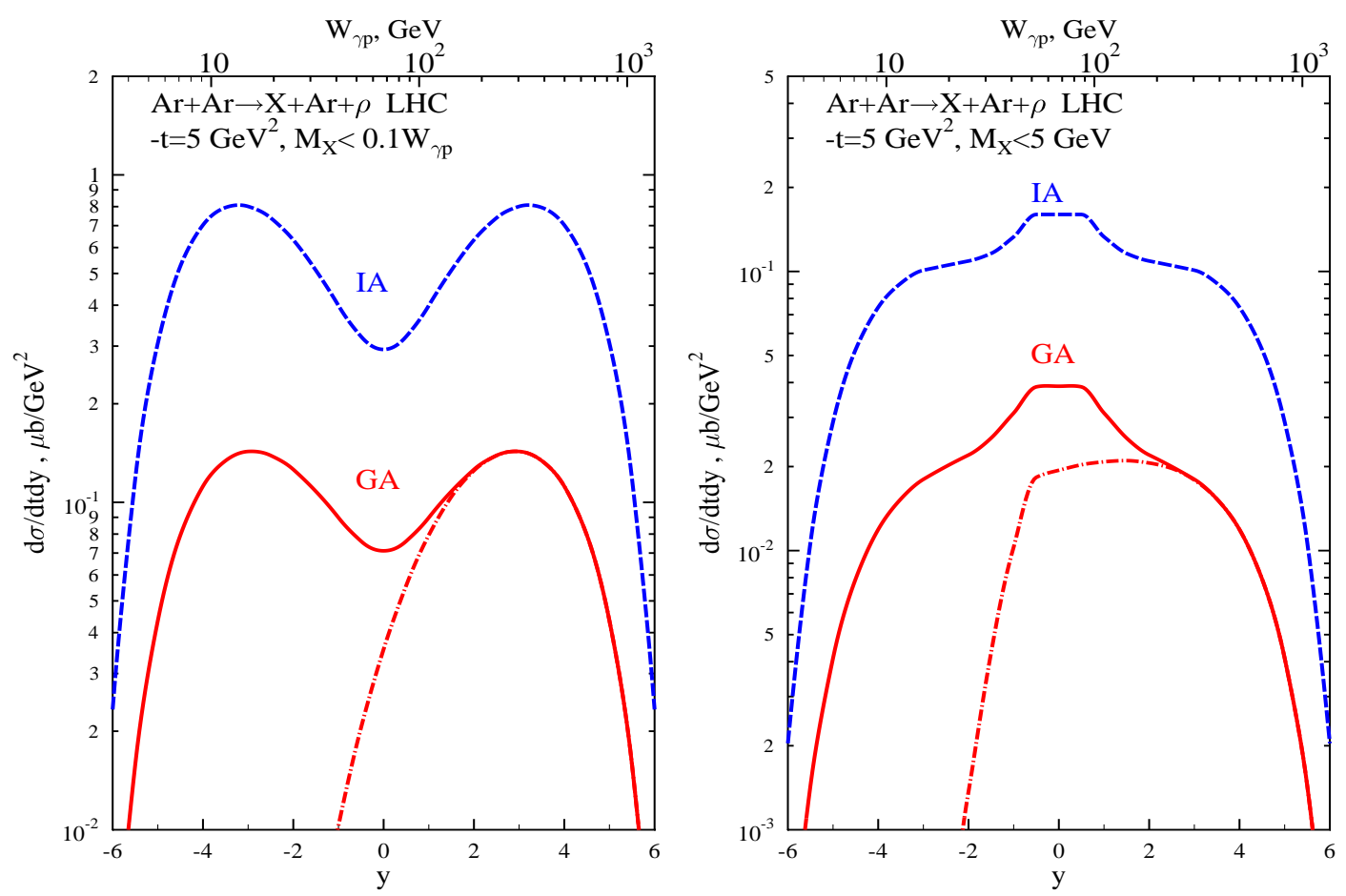

Figure 51. The same as Fig. 50 at $-t=5 \mathrm{GeV}^{2}$ [93].

energy dependent cut, reaching lower $x$, in the right part of each figure. Recall that the two cuts become equivalent for $W_{\gamma p} \leq 50 \mathrm{GeV}$. Each figure shows two $A A$ curves for each cut. The upper limit on the cross section, obtained in the impulse approximation, see Eq. (85), is shown in the dashed curves. The results obtained with Glauber-Gribov screening employing $\sigma_{\text {eff }}=\sigma_{\mathrm{in}}^{\rho^{0} N}\left(W_{\gamma N}\right)$, an effective lower limit, are shown in the solid curves. Recall, however, that the survival probability for the rapidity gap, shown in Fig. 49, is a strong function of $\sigma_{\text {eff }}$ and is thus sensitive to higher-twist effects.

The curves corresponding to a single nuclear target with the same energy and $\sigma_{\text {eff }}$ are shown in the dot-dashed curves for one side of the collision. These single-side curves are not exactly equivalent to the $p A$ curves in Figs. 47 and 48 since the $A A$ energy is lower than the $p A$ energy, narrowing the rapidity distributions. The behavior of the single side distribution near midrapidity explains the shape of the $A A$ results. The smooth decrease of the single-side result for $M_{X}<0.1 W_{\gamma p}$ at $y<0$ leads to an $A A$ result that is either flat at midrapidity $\left(-t=2.5 \mathrm{GeV}^{2}\right)$ or has a dip in the middle $\left(-t=5 \mathrm{GeV}^{2}\right)$. On the other hand, the flatter single side behavior with the fixed upper limit of $M_{X}$, corresponding to fixed $x_{\min }$, makes the $A A$ result increase at midrapidity.

The rapidity-integrated rates are shown in Fig. 54. The rates decrease more rapidly for $M_{X}$ independent of energy. This is not surprising since the average momentum fraction is larger. The shaded bands indicate the uncertainty between the IA (dashed curves) and GA calculations with $\sigma_{\text {eff }}=\sigma_{\text {in }}^{\rho^{0} N}\left(W_{\gamma N}\right)$ (solid curves). The larger suppression for $\mathrm{Pb}$ is demonstrated by the broader band. When the run time 

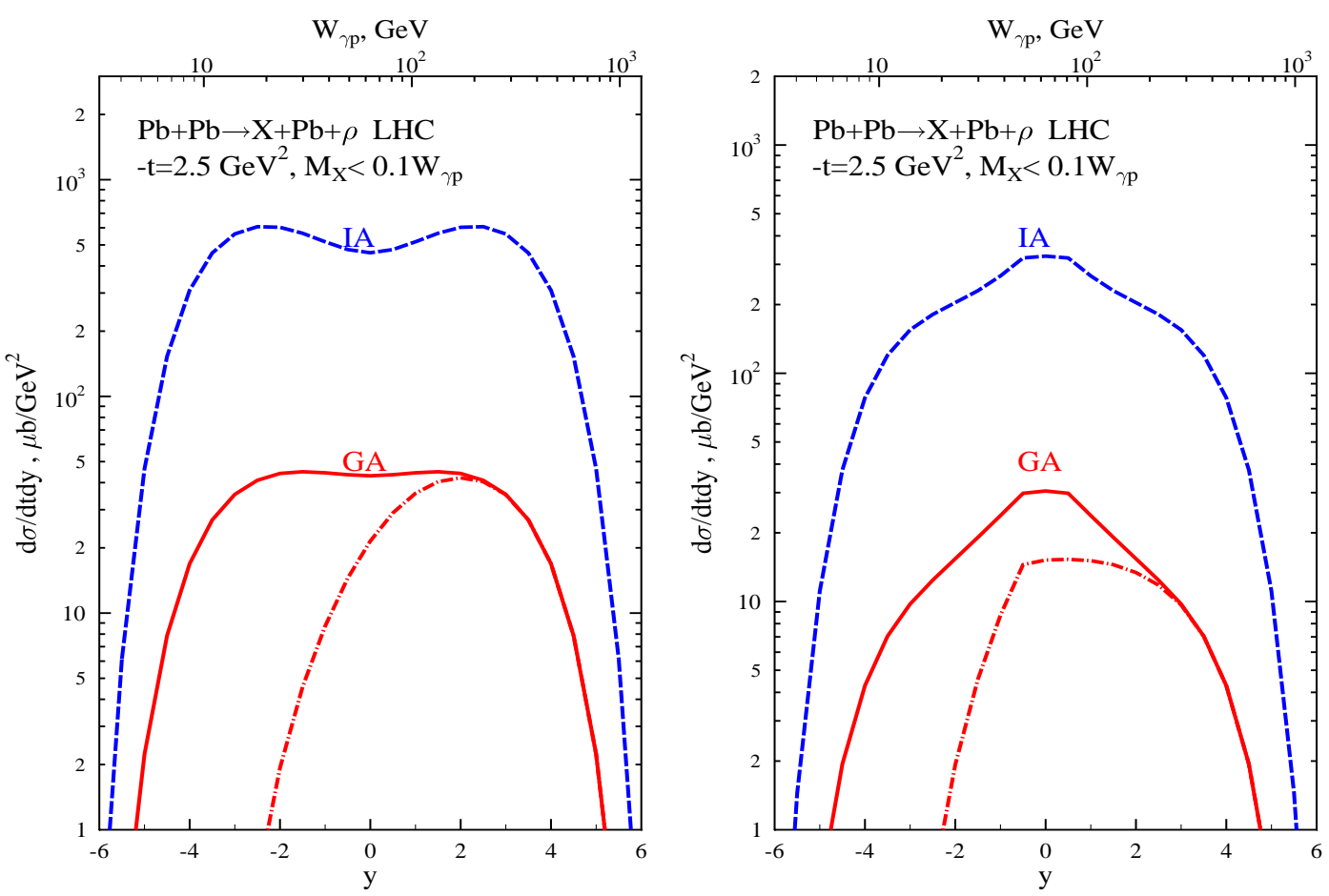

Figure 52. The same as Fig. 50 for $\mathrm{Pb}+\mathrm{Pb}$ collisions. The rates can be estimated using the expected $\mathrm{Pb}+\mathrm{Pb}$ luminosity, $L_{\mathrm{PbPb}}=10^{-3} \mu \mathrm{b}^{-1} \mathrm{~s}^{-1}$. [93]
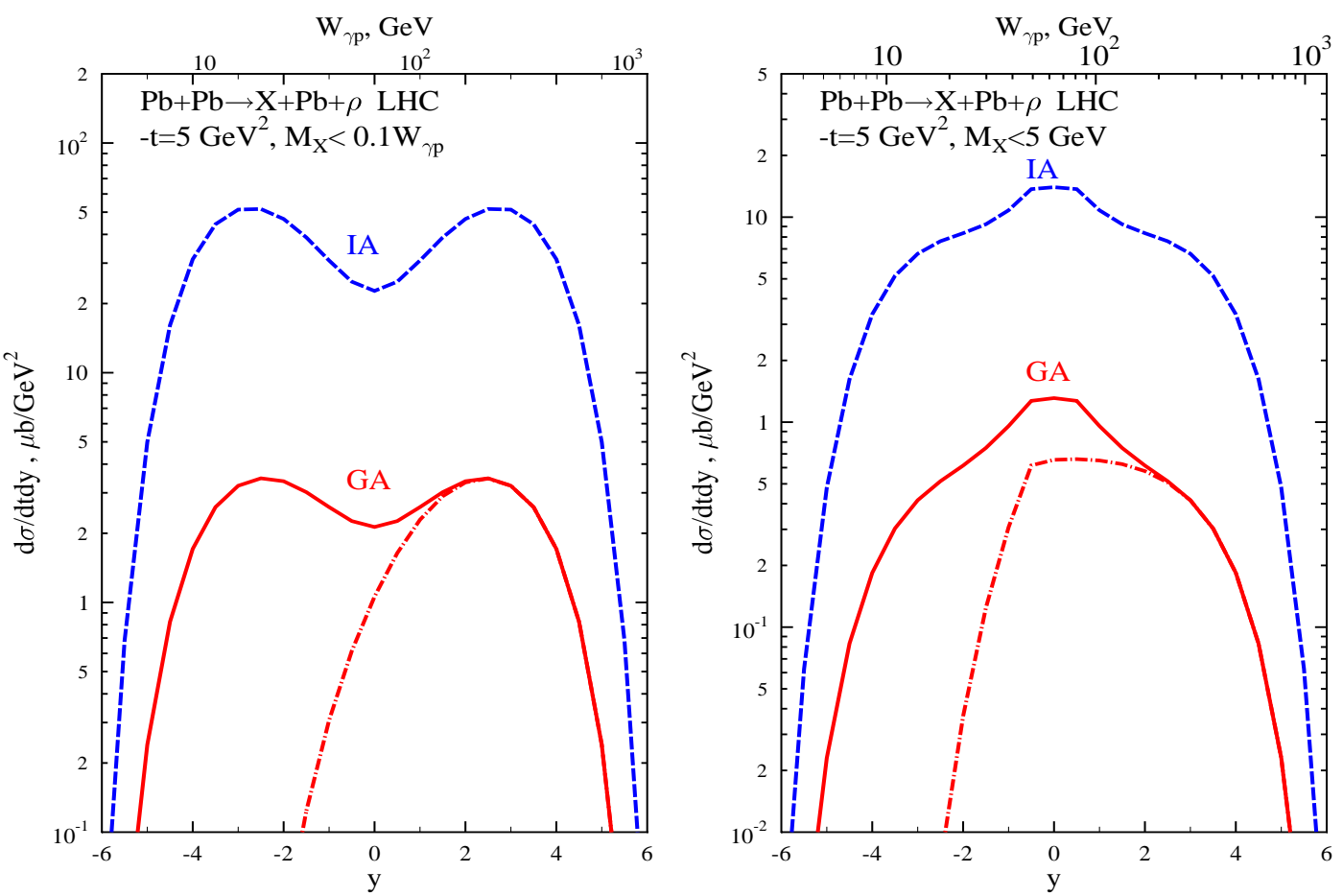

Figure 53. The same as in Fig. 52 at $-t=5 \mathrm{GeV}^{2}$ [93]. 

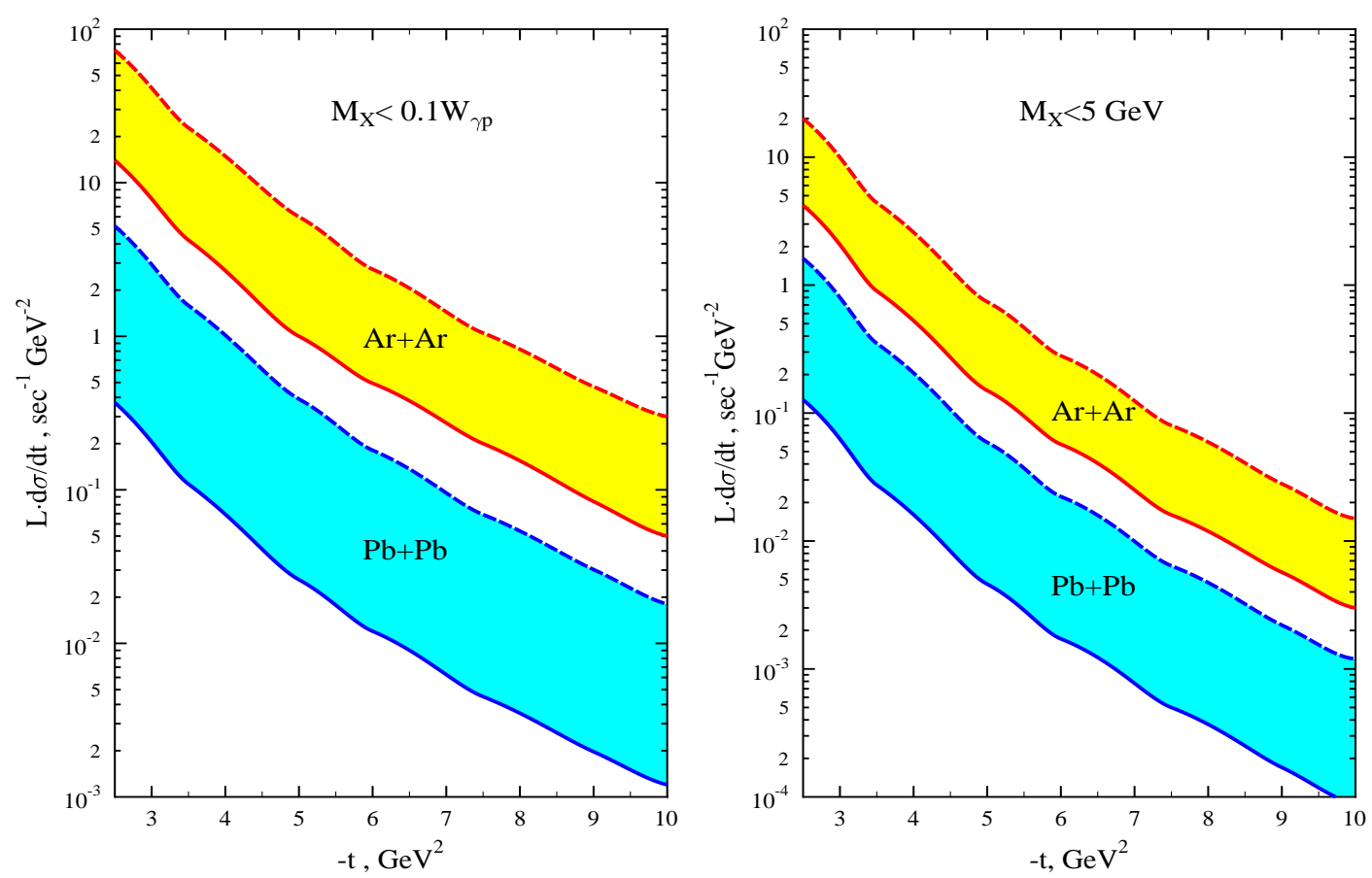

Figure 54. The rapidity-integrated rates for $\rho^{0}$ photoproduction with a rapidity gap in $\mathrm{Ar}+\mathrm{Ar}$ and $\mathrm{Pb}+\mathrm{Pb}$ UPCs as a function of $-t$ [93]. The lower bound of the bands correspond to the Gribov-Glauber approach while the upper bound is the result in the impulse approximation.

is taken into account, it is clear that the rates will be sufficiently high for meaningful measurements out to $-t=10 \mathrm{GeV}^{2}$.

3.2.4. Conclusions Studies of rapidity gap processes in UPCs at the LHC will directly measure the energy dependence of the large- $t$ elastic amplitude of dipole-parton scattering. The $\rho^{0}$ measurements will investigate the evolution of the $A$ dependence over the transition between several QCD regimes: from soft physics to color transparency with increasing $t$ for fixed $W_{\gamma p}$ and from color transparency to color opacity for fixed $t$ and increasing $W_{\gamma p}$. These measurements will also study the interplay of leading and higher-twist effects, a nontrivial function of $\Delta y$. Altogether, these studies provide a new, powerful tool for studying small dipole interactions with the medium.

\section{Determining the nuclear parton distributions}

Contributed by: R. Vogt 


\subsection{Introduction}

Here we discuss three possible avenues for measuring the nuclear parton distributions through ultraperipheral heavy-ion collisions: heavy quark, dijet and $\gamma+$ jet photoproduction. Photoproduction occurs by "direct" and "resolved" production. We will discuss both processes and compare the heavy quark, dijet and $\gamma+$ jet production rates from each one.

"Direct" photoproduction occurs when a photon emitted from one nucleus interacts with a parton from the other nucleus, forming the final state. There is only one leading order direct $Q \bar{Q}$ production process, $\gamma g \rightarrow Q \bar{Q}$. Thus $Q \bar{Q}$ production is a rather clean probe of the nuclear gluon distribution. Dijet production also proceeds via an initialstate gluon, $\gamma g \rightarrow q \bar{q}$. However, there is an additional dijet production process, $\gamma q \rightarrow g q$, the QCD Compton process. In the case of massive quarks, the heavy quark mass, $m_{Q}$, makes the $p_{T}$ distribution finite as $p_{T} \rightarrow 0$. Since the final state partons are massless in jet production, $p_{T}$ is the only scale. Thus some minimum $p_{T}, p_{T_{\min }}$, is chosen to regulate the cross sections. Finally, $\gamma+$ jet production proceeds via Compton scattering, $\gamma q \rightarrow \gamma q$. Thus $\gamma+$ jet production is a direct probe of the nuclear quark and antiquark distributions. This high $Q^{2}$ probe complements the nuclear deep-inelastic scattering measurements of the charged parton distributions in the nucleus made at lower $Q^{2}$.

A generic direct photoproduction cross section for ultraperipheral $A A$ collisions is obtained by convoluting the partonic photoproduction cross section, $d^{2} \sigma_{\gamma i} / d t_{1} d u_{1}$, with the photon flux from one nucleus, $d N_{\gamma} / d k$, and the parton distribution in the opposite nucleus, $F_{i}^{A}\left(x_{2}, Q^{2}\right)$,

$$
\begin{aligned}
s_{N N}^{2} \frac{d^{2} \sigma_{\text {dir }}^{\gamma A}}{d t_{1_{N N}} d u_{1_{N N}}}= & 2 \int_{k_{\min }}^{\infty} d k \frac{d N_{\gamma}}{d k} \int_{x_{2_{\min }}}^{1} \frac{d x_{2}}{x_{2}} \\
& \times\left[\sum_{i=q, \bar{q}, g} F_{i}^{A}\left(x_{2}, Q^{2}\right) s^{2} \frac{d^{2} \sigma_{\gamma i}}{d t_{1} d u_{1}}\right] .
\end{aligned}
$$

When the final state is a $Q \bar{Q}$ pair, $i=g$. For dijet production, $i=g, q$ and $\bar{q}$. Finally, in the Compton scattering process, $i=q$ and $\bar{q}$. The partonic and hadronic Mandelstam invariants are $s, t_{1}, u_{1}$ and $s_{N N}, t_{1_{N N}}, u_{1_{N N}}$ respectively, defined later. The fractional momentum of the nucleon carried by the gluon is $x_{2}$. The minimum possible $x_{2}$, determined from the nucleon-nucleon invariants using four-momentum conservation, is $x_{2_{\min }}=-u_{1_{N N}} /\left(s_{N N}+t_{1_{N N}}\right)$. The photon momentum is denoted by $k$. The minimum photon momentum needed to produce the final state is $k_{\min }$. The spatial coordinates are $b$, the impact parameter, and $z$, the longitudinal coordinate. The factor of two in Eq. (86) arises because both nuclei emit photons and thus serve as targets. For $p A$ collisions, this factor is not included. The incoherence of heavy quark and jet production eliminates interference between the two production sources [172].

The photon can also fluctuate into states with multiple $q \bar{q}$ pairs and gluons, i.e. $|n(q \bar{q}) m(g)\rangle, n q \bar{q}$ pairs and $m$ gluons, the combination of which remains a color singlet with zero flavor and baryon number. One of these photon components can interact 
with a quark or gluon from the target nucleus ("resolved" production) [173]. The photon components are described by parton densities similar to those used for protons except that no useful momentum sum rule applies to the photon [174]. The quark and gluon constituents of the photon open up more channels for heavy quark and jet photoproduction and could, in principle, lead to larger rates for resolved production in certain regions of phase space.

The generic cross section for resolved photoproduction is

$$
\begin{aligned}
& s_{N N}^{2} \frac{d^{2} \sigma_{\text {res }}^{\gamma A}}{d t_{1_{N N}} d u_{1_{N N}}}=2 \int_{k_{\min }}^{\infty} \frac{d k}{k} \frac{d N_{\gamma}}{d k} \int_{k_{\min } / k}^{1} \frac{d x}{x} \int_{x_{2_{\min }}}^{1} \frac{d x_{2}}{x_{2}} \\
& \times\left[\sum_{i, j=q, \bar{q}, g}\left\{F_{i}^{\gamma}\left(x, Q^{2}\right) F_{j}^{A}\left(x_{2}, Q^{2}\right)+F_{j}^{\gamma}\left(x, Q^{2}\right) F_{i}^{A}\left(x_{2}, Q^{2}\right)\right\} \hat{s}^{2} \frac{d^{2} \sigma_{i j}}{d \hat{t}_{1} d \hat{u}_{1}}\right]
\end{aligned}
$$

Since $k$ is typically larger in resolved than direct photoproduction, the average photon flux is lower in the resolved contribution. In heavy quark production, $i j=q \bar{q}$ and $g g$. In dijet production, $i j=q q, q q^{\prime}, q \bar{q}, q g, g g \cdots$. Finally, in $\gamma+$ jet production, $i j=q \bar{q}, q g$ and $\bar{q} g$. Since the photon has no valence quarks, the $q$ and $\bar{q}$ distributions in the photon are identical. Again, the factor of two accounts for the possibility of photon emission from each nucleus.

The total photoproduction cross section is the sum of the direct and resolved contributions [175],

$$
s_{N N}^{2} \frac{d^{2} \sigma_{\text {tot }}^{\gamma A}}{d t_{1_{N N}} d u_{1_{N N}}}=s_{N N}^{2} \frac{d^{2} \sigma_{\text {dir }}^{\gamma A}}{d t_{1_{N N}} d u_{1_{N N}}}+s_{N N}^{2} \frac{d^{2} \sigma_{\text {res }}^{\gamma A}}{d t_{1_{N N}} d u_{1_{N N}}} .
$$

In the remainder of this introduction, we will discuss the common ingredients of these calculations. We first discuss the calculation of the photon flux and the relevant kinematics. We then turn to the expected modifications of the nuclear parton distributions relative to those of the free proton. Finally, we present the photon parton distribution functions. The next two subsections deal with heavy quark and jet photoproduction.

The photon flux is calculated using Eqs. (5). The maximum center-of-mass energy, $\sqrt{s_{\gamma N}} \approx \sqrt{2 E_{\max } m_{p}}$, for single photon interactions with protons, $\gamma p \rightarrow Q \bar{Q}$ [176], at the LHC is given in Table 1. At the LHC, the energies are high enough for $t \bar{t}$ photoproduction [177]. The total photon flux striking the target nucleus must be calculated numerically. The numerical calculations are used for $A A$ interactions but the analytic flux in Eq. (6) is used for $p A$ interactions. The difference between the numerical and analytic expressions is typically less than $15 \%$, except for photon energies near the cutoff.

The nuclear parton densities $F_{i}^{A}\left(x, Q^{2}\right)$ in Eqs. (86) and (87) can be factorized into nucleon parton densities, $f_{i}^{N}\left(x, Q^{2}\right)$, and a shadowing function $S^{i}\left(A, x, Q^{2}\right)$ that describes the modification of the nuclear parton distributions in position and momentum space

$$
F_{i}^{A}\left(x, Q^{2}\right)=S^{i}\left(A, x, Q^{2}\right) f_{i}^{N}\left(x, Q^{2}\right)
$$


where $f_{i}^{N}\left(x, Q^{2}\right)$ is the parton density in the nucleon. In the absence of nuclear modifications, $S^{i} \equiv 1$. While we have previously treated the spatial dependence of shadowing, [178-182], we do not include it here. We use the MRST LO parton distributions [183]. For $Q \bar{Q}$ production, we evaluate the nucleon parton densities at $Q^{2}=\left(a m_{T}\right)^{2}$ where $m_{T}^{2}=p_{T}^{2}+m_{Q}^{2}, a=2$ for charm and 1 for bottom. The appropriate scale for jet production is $Q^{2}=\left(a p_{T}\right)^{2}$ where we take $a=1$.

We have chosen two recent parameterizations of the nuclear shadowing effect which cover extremes of gluon shadowing at low $x$. The Eskola et al. parametrization, EKS98, $[184,185]$ is based on the GRV LO [186] parton densities. At the minimum scale, $Q_{0}$, valence quark shadowing is identical for $u$ and $d$ quarks. Likewise, the shadowing of $\bar{u}$ and $\bar{d}$ quarks are identical at $Q_{0}$. Although the light quark shadowing ratios are not constrained to be equal at higher scales, the differences between them are small. Shadowing of the heavier flavor sea, $\bar{s}$ and higher, is calculated separately at $Q_{0}$. The shadowing ratios for each parton type are evolved to LO for $1.5<Q<100 \mathrm{GeV}$ and are valid for $x \geq 10^{-6}[184,185]$. Interpolation in nuclear mass number allows results to be obtained for any input $A$. The parametrization by Frankfurt, Guzey and Strikman, denoted FGS here, combines Gribov theory with hard diffraction [58]. It is based on the CTEQ5M [187] parton densities and evolves each parton species separately to NLO for $2<Q<100 \mathrm{GeV}$. Although the given $x$ range is $10^{-5}<x<0.95$, the sea quark and gluon ratios are unity for $x>0.2$. The EKS98 valence quark shadowing ratios are used as input since Gribov theory does not predict valence shadowing. The parametrization is available for $A=16,40,110$ and 206. Figure 55 compares the two parameterizations for $A \approx 200$ and $Q=2 m_{c}=2.4 \mathrm{GeV}$. We take the EKS98 parametrization [184, 185], as a default but we also compare it to the FGS [58] results in some cases.

We now turn to the photon parton distributions. There are a few photon parton distributions available [188-194]. The data $[195,196]$ cannot definitively rule out any of these parton densities. As expected, $F_{q}^{\gamma}\left(x, Q^{2}\right)=F_{\bar{q}}^{\gamma}\left(x, Q^{2}\right)$ flavor by flavor because there are no "valence" quarks in the photon. The gluon distribution in the photon is less well known. We compare results with the GRV-G LO set $[188,189]$, with a gluon distribution is similar to most of the other available sets [190,192-194], to the LAC1 set [191] where the low $x$ gluon density is up to an order of magnitude higher. The differences in the two photon parton densities are most important for heavy quark production.

The GRV-G LO photon parton densities are shown in Fig. 56 for scales equal to $2 m_{c}, m_{b}$ and $m_{t}$ where $m_{c}=1.2 \mathrm{GeV}, m_{b}=4.75 \mathrm{GeV}$ and $m_{t}=175 \mathrm{GeV}$. This set has a minimum $x$ of $10^{-5}$ and $0.25 \leq Q^{2} \leq 10^{6} \mathrm{GeV}^{2}$. At low $x$, the $u, d$ and $s$ distributions are identical. They diverge around $x \sim 10^{-3}$ with the $u$ and $d$ distributions increasing with $x$ while the $s$ distribution decreases until $x>0.1$ where it turns up again. As $x \rightarrow 1$ the quark distributions become larger than the gluon distributions.

The LAC1 LO photon parton densities are shown in Fig. 57 for the same scales. This set has a minimum $x$ of $10^{-4}$ and covers the range $4 \leq Q^{2} \leq 10^{5}$. All the densities are somewhat higher than those of GRV LO but they are less regular in shape, particularly 

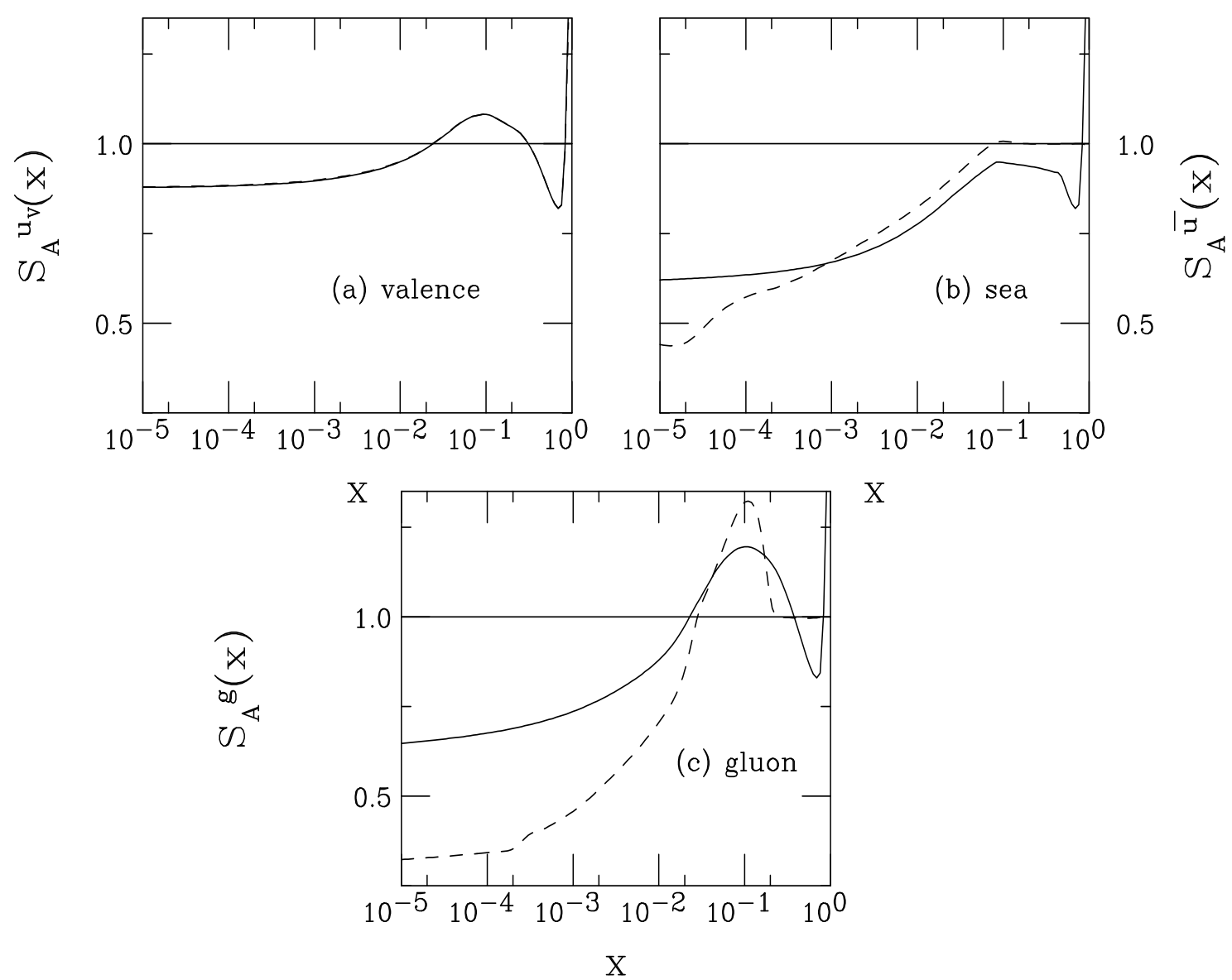

Figure 55. The EKS98 and FGS shadowing parameterizations are compared at the scale $Q=2 m_{c}=2.4 \mathrm{GeV}$. The solid curves are the EKS98 parametrization, the dashed, FGS.

the $s$ distribution when $Q^{2}=4 m_{c}^{2}$, possibly because this scale is rather close to $Q_{0}$. The gluon distributions are also rather irregular, particularly at high $x$.

The LAC1 densities are generally higher at low $x$ but the GRV-G gluon density is higher at $x>0.1$. The LAC1 and GRV-G quark distributions are also similar in this $x$ region. Thus if relatively low $x$ values are reached, the LAC1 resolved results will be larger. In the high $x$ region, the two densities will give either similar results or the GRV-G densities may give a larger resolved component. In any case, it is clear that, in certain kinematic regions, the difference in the resolved yields due to the choice of photon parton density could be significant.

With these ingredients, we turn to the specific final-state processes under consideration. We first discuss heavy quark photoproduction in Section 4.2. Here the rates are high and the nuclear gluon distribution should be rather directly accessible. We then show expected results for direct and resolved jet photoproduction in Section 4.3. The additional channels for resolved jet photoproduction could potentially enhance this contribution over the direct contribution, obscuring the nuclear gluon distribution. However, as we will discuss, it might then be possible to examine the nuclear quark 

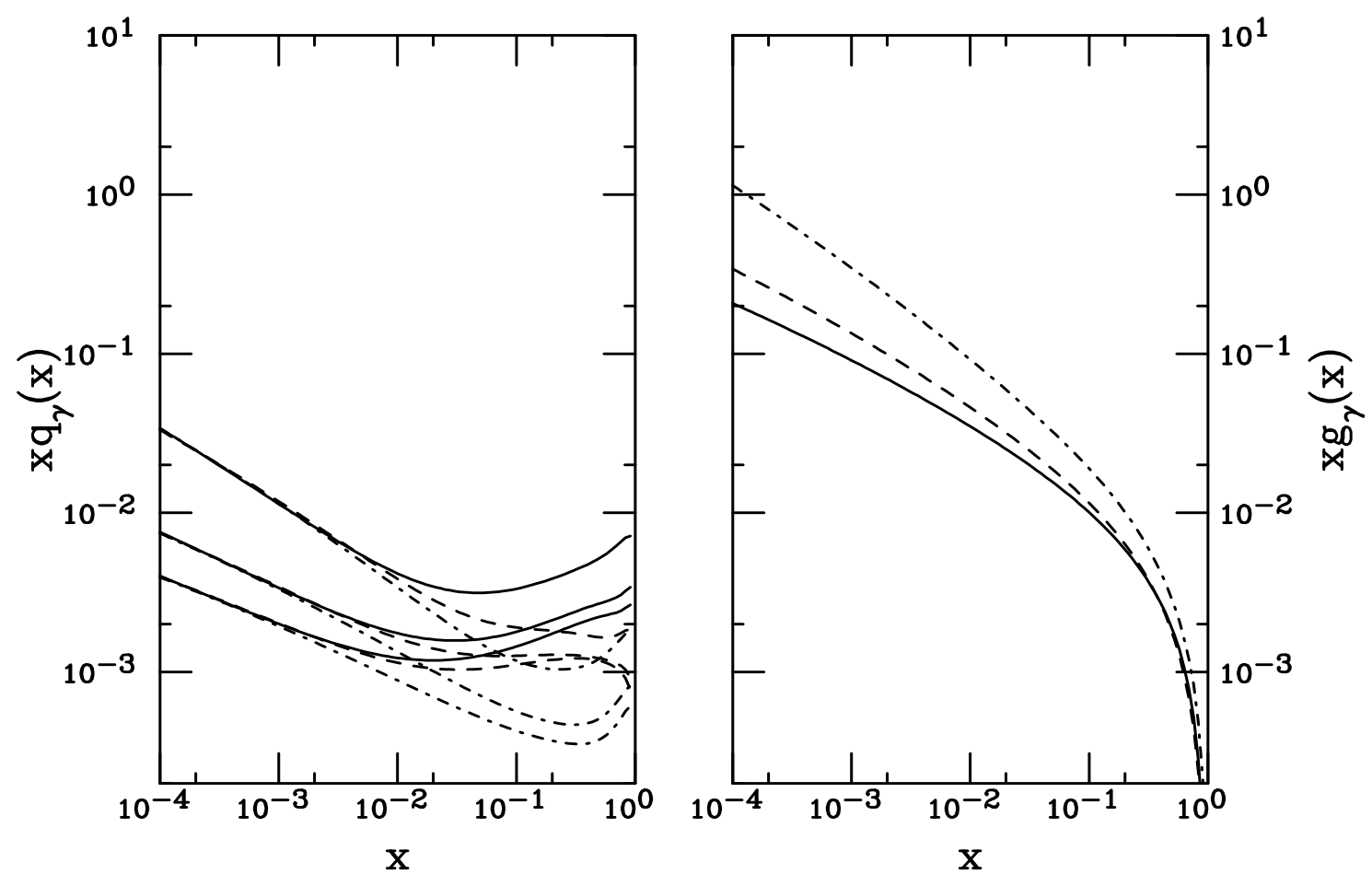

Figure 56. The GRV-G LO quark (a) and gluon (b) distributions of the photon. In (a) the up (solid), down (dashed) and strange (dot-dashed) distributions are evaluated at $2 m_{c}$ (lower curves), $2 m_{b}$ (middle curves) and $2 m_{t}$ (upper curves). In (b) the gluon distributions are shown at $2 m_{c}$ (solid), $2 m_{b}$ (dashed) and $2 m_{t}$ (dot-dashed).

distribution. Finally, we discuss how to distinguish between photoproduction and hadroproduction at the LHC in Section 4.5.

\subsection{Heavy quark photoproduction}

Contributed by: S. R. Klein, J. Nystrand and R. Vogt

In this subsection we discuss photoproduction of massive $Q \bar{Q}$ pairs at the LHC [141]. We also discuss the dependence of the resolved results on the photon parton density, comparing results from with the GRV-G set [188, 189] (Ref. [141]) to those with the LAC1 set [191]. We work to leading order in the strong coupling constant $\alpha_{s}$.

We include all $Q \bar{Q}$ pairs in the total cross sections and rates even though some of these pairs have masses below the $H \bar{H}$ threshold where $H \bar{H} \equiv D \bar{D}$ and $B \bar{B}$ for $c$ and $b$ quarks respectively. No such distinctions exist for top since it decays before hadronization. Photoproduction is an inclusive process; accompanying particles can combine with the $Q$ and $\bar{Q}$, allowing the pairs with $M<2 m_{H}$ to hadronize. We assume the hadronization process does not affect the rate.

Direct $Q \bar{Q}$ pairs are produced in the reaction $\gamma(k)+N\left(P_{2}\right) \rightarrow Q\left(p_{1}\right)+\bar{Q}\left(p_{2}\right)+X$ where $k$ is the four momentum of the photon emitted from the virtual photon field of the projectile nucleus, $P_{2}$ is the four momentum of the interacting nucleon $N$ in ion $A$, and $p_{1}$ and $p_{2}$ are the four momenta of the produced $Q$ and $\bar{Q}$. 

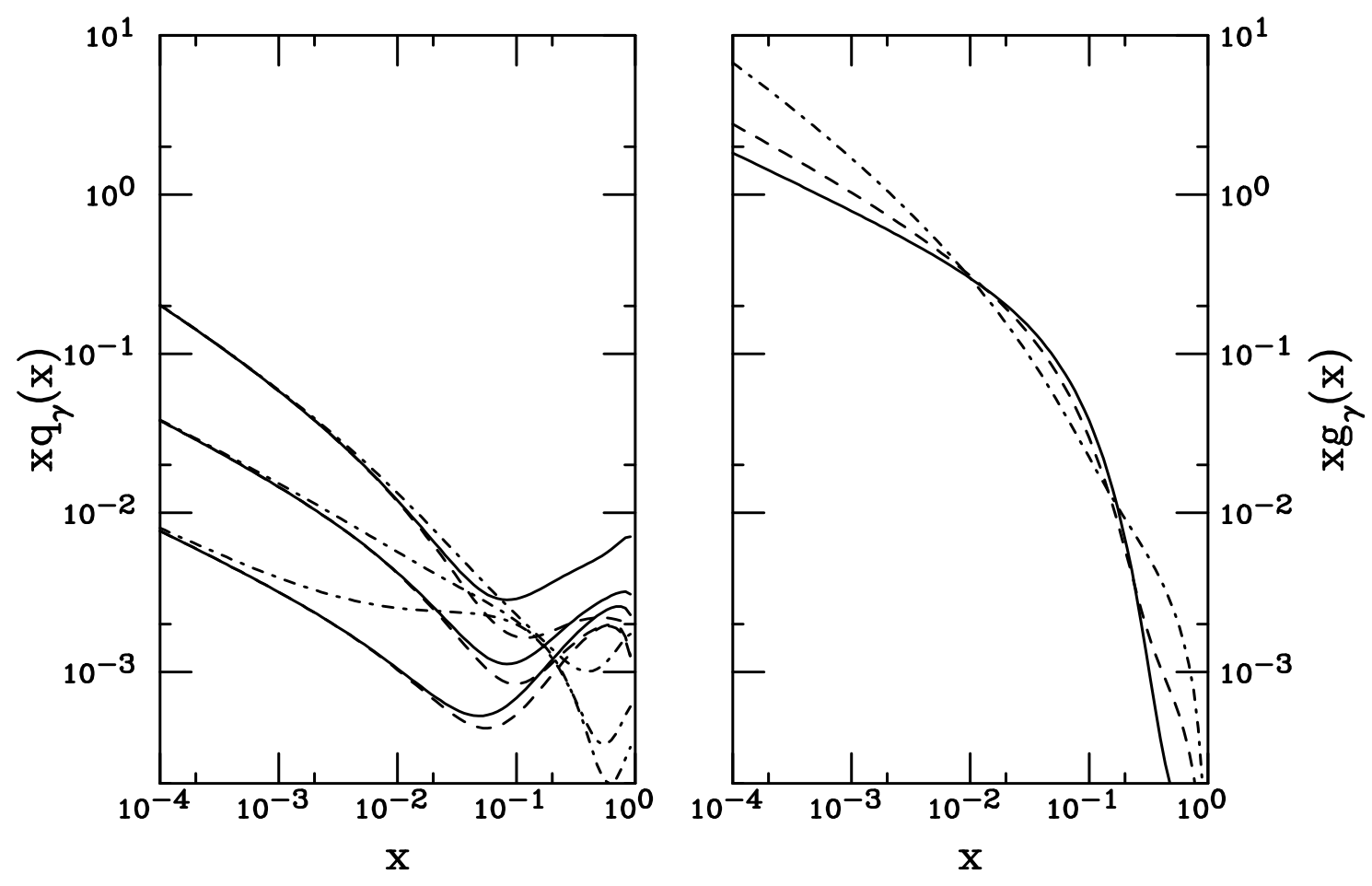

Figure 57. The LAC1 LO quark (a) and gluon (b) distributions of the photon. In (a) the up (solid), down (dashed) and strange (dot-dashed) distributions are evaluated at $2 m_{c}$ (lower curves), $2 m_{b}$ (middle curves) and $2 m_{t}$ (upper curves). In (b) the gluon distributions are shown at $2 m_{c}$ (solid), $2 m_{b}$ (dashed) and $2 m_{t}$ (dot-dashed).

On the parton level, the photon-gluon fusion reaction is $\gamma(k)+g\left(x_{2} P_{2}\right) \rightarrow Q\left(p_{1}\right)+$ $\bar{Q}\left(p_{2}\right)$ where $x_{2}$ is the fraction of the target momentum carried by the gluon. The LO $Q \bar{Q}$ photoproduction cross section for quarks with mass $m_{Q}$ is [197]

$$
s^{2} \frac{d^{2} \sigma_{\gamma g}}{d t_{1} d u_{1}}=\pi \alpha_{s}\left(Q^{2}\right) \alpha e_{Q}^{2} B_{\mathrm{QED}}\left(s, t_{1}, u_{1}\right) \delta\left(s+t_{1}+u_{1}\right)
$$

where

$$
B_{\mathrm{QED}}\left(s, t_{1}, u_{1}\right)=\frac{t_{1}}{u_{1}}+\frac{u_{1}}{t_{1}}+\frac{4 m_{Q}^{2} s}{t_{1} u_{1}}\left[1-\frac{m_{Q}^{2} s}{t_{1} u_{1}}\right] .
$$

At leading order (LO), the partonic cross section of the direct contribution is proportional to $\alpha \alpha_{s}\left(Q^{2}\right) e_{Q}^{2}$, where $\alpha_{s}\left(Q^{2}\right)$ is the strong coupling constant, $\alpha=e^{2} / \hbar c$ is the electromagnetic coupling constant, and $e_{Q}$ is the quark charge, $e_{c}=e_{t}=2 / 3$ and $e_{b}=-1 / 3$. Here $\alpha_{s}\left(Q^{2}\right)$ is evaluated to one loop at scale $Q^{2}$. The partonic invariants, $s, t_{1}$, and $u_{1}$, are defined as $s=\left(k+x_{2} P_{2}\right)^{2}, t_{1}=\left(k-p_{1}\right)^{2}-m_{Q}^{2}=\left(x_{2} P_{2}-p_{2}\right)^{2}-m_{Q}^{2}$, and $u_{1}=\left(x_{2} P_{2}-p_{1}\right)^{2}-m_{Q}^{2}=\left(k-p_{2}\right)^{2}-m_{Q}^{2}$. In this case, $s=4 k \gamma_{L} x_{2} m_{p}$ where $\gamma_{L}$ is the Lorentz boost of a single beam and $m_{p}$ is the proton mass. Since $k$ can be a continuum of energies up to $E_{\mathrm{beam}}=\gamma_{L} m_{p}$, we define $x_{1}=k / P_{1}$ analogous to the parton momentum fraction where $P_{1}$ is the nucleon four momentum. For a detected quark in a nucleon-nucleon collision, the hadronic invariants are then $s_{N N}=\left(P_{1}+P_{2}\right)^{2}$, $t_{1_{N N}}=\left(P_{2}-p_{1}\right)^{2}-m_{Q}^{2}$, and $u_{1_{N N}}=\left(P_{1}-p_{1}\right)^{2}-m_{Q}^{2}$. 
We label the quark rapidity as $y_{1}$ and the antiquark rapidity as $y_{2}$. The quark rapidity is related to the invariant $t_{1_{N N}}$ by $t_{1_{N N}}=-\sqrt{s_{N N}} m_{T} e^{-y_{1}}$. The invariant mass of the pair can be determined if both the $Q$ and $\bar{Q}$ are detected. The square of the invariant mass, $M^{2}=s=2 m_{T}^{2}\left(1+\cosh \left(y_{1}-y_{2}\right)\right)$, is the partonic center-of-mass energy squared. For $Q \bar{Q}$ pair production, $k_{\min }=M^{2} / 4 \gamma_{L} m_{p}$. At LO, $x_{1}=\left(m_{T} / \sqrt{s_{N N}}\right)\left(e^{y_{1}}+e^{y_{2}}\right)$ and $x_{2}=\left(m_{T} / \sqrt{s_{N N}}\right)\left(e^{-y_{1}}+e^{-y_{2}}\right)$. We calculate $x_{1}$ and $x_{2}$ as in an $N N$ collision and then determine the flux in the lab frame for $k=x_{1} \gamma_{L} m_{p}$, equivalent to the center-of-mass frame in a collider. The photon flux is exponentially suppressed for $k>\gamma_{L} \hbar c / R_{A}$, corresponding to a momentum fraction $x_{1}>\hbar c / m_{p} R_{A}$. The maximum $\gamma N$ center-ofmass energy, $\sqrt{s_{\gamma N}}$, is much lower than the hadronic $\sqrt{s_{N N}}$.

The cross section for direct photon-nucleon heavy quark photoproduction is obtained by inserting Eq. (90) into Eq. (86). The equivalent hadronic invariants can be defined for photon four momentum $k$ as $s_{\gamma N}=\left(k+P_{2}\right)^{2}, t_{1, \gamma N}=\left(P_{2}-p_{1}\right)^{2}-m_{Q}^{2}$, and $u_{1, \gamma N}=\left(k-p_{1}\right)^{2}-m_{Q}^{2}$ [198]. The partonic and equivalent hadronic invariants for fixed $k$ are related by $s=x_{2} s_{\gamma N}, t_{1}=u_{1, \gamma N}$, and $u_{1}=x_{2} t_{1, \gamma N}$.

The charm and bottom photoproduction distributions are shown in Fig. 58 for $\mathrm{Pb}+\mathrm{Pb}, \mathrm{Ar}+\mathrm{Ar}$ and $\mathrm{O}+\mathrm{O}$ collisions. The direct top photoproduction distributions for these three systems are given on the left-hand side of Fig. 59. There are three curves for each contribution, one without shadowing and two with homogeneous nuclear shadowing employing the EKS98 and FGS parameterizations. The photon comes from the left. Then $y_{1}<0$ corresponds to $k<\gamma_{L} x_{2} m_{p}$ in the center-of-mass (lab) frame. If the photon emitter and target nucleus are interchanged, the resulting unshadowed rapidity distribution, $S^{i}=1$, is the mirror image of these distributions around $y_{1}=0$. The $Q$ and $\bar{Q}$ distributions are asymmetric around $y_{1}=0$. The total heavy quark rapidity distributions are then the sum of the displayed results with their mirror images when both nuclei emit photons. This factor of two, shown in Eq. (86), is included in the transverse momentum and invariant mass distributions. Note that the peak in the rapidity distributions moves towards more negative $y_{1}$ and the distribution narrows as the quark mass increases. The $y_{1}$ phase space for a single top quark is $\approx 3.7$, a decrease of more than a factor of two relative to charm.

Since the distributions are shown on a logarithmic scale, shadowing appears to be a rather small effect over most of phase space. It is most prominent in the rapidity distributions and are otherwise is only distinguishable for charm production at low $p_{T}$ and low invariant mass. Shadowing is largest at forward rapidities where low momentum fractions in the nucleus are reached.

The total cross sections for direct $Q \bar{Q}$ photoproduction are given in Table $11 \not$. The EKS98 shadowing parametrization has a $10-20 \%$ effect on the total $c \bar{c}$ cross section. The effect is smallest for $\mathrm{O}+\mathrm{O}$ collisions, due to the small $A$, even though the energy is higher and the effective $x$ values probed are smaller. The stronger shadowing of the FGS parametrization gives a $23-46 \%$ reduction of the $c \bar{c}$ cross sections. Both the $x$

\# A typo in the direct cross section code caused the cross sections in Refs. $[141,177]$ to be somewhat overestimated. The results given here are correct. 

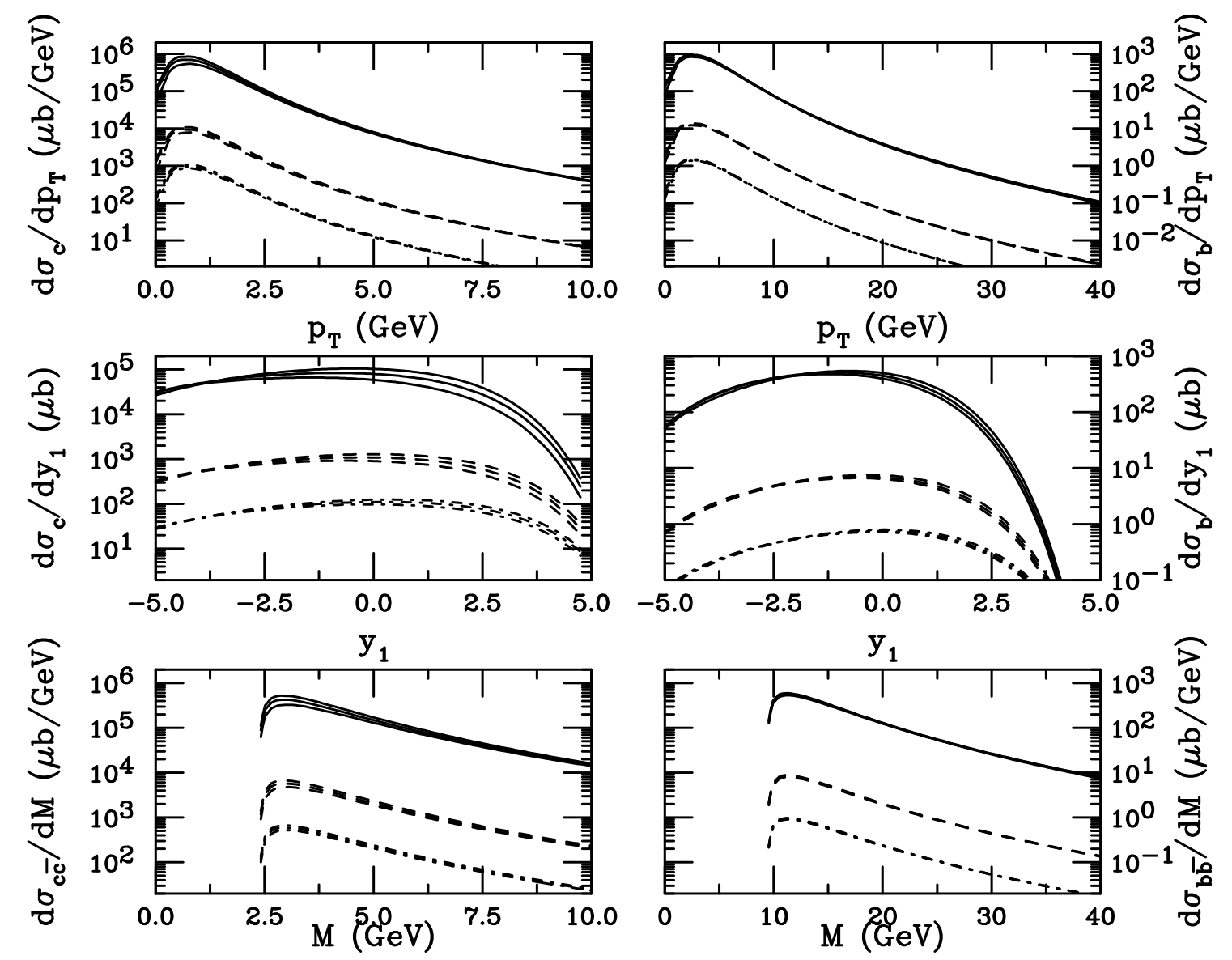

Figure 58. Direct $Q \bar{Q}$ photoproduction in peripheral $A A$ collisions. The left-hand side is for charm while the right-hand side is for bottom. The single $Q p_{T}$ (upper) and rapidity (middle) distributions are shown along with the $Q \bar{Q}$ pair invariant mass distributions (lower). The $\mathrm{O}+\mathrm{O}$ (dot-dashed), $\mathrm{Ar}+\mathrm{Ar}$ (dashed) and $\mathrm{Pb}+\mathrm{Pb}$ (solid) results are given. There are three curves for each contribution: no shadowing, EKS98 and FGS. At $y_{1}>0$, the highest curve is without shadowing, the middle curve with EKS98 and the lower curve with FGS. The photon is coming from the left.

and $Q$ values probed increase for $b \bar{b}$ production. Each of these increases reduces the overall effect of shadowing. The EKS98 parametrization results in only a $4 \%$ reduction of the $b \bar{b}$ total cross sections, independent of $A$, while the FGS parametrization gives an $8-10 \%$ effect. Although we include the $t \bar{t}$ cross sections with shadowing in Table 11 , we note that $m_{t}$ is larger than the maximum $Q$ for which the parameterizations may be considered reliable.

The integrated cross sections provide incomplete information about shadowing effects. To provide a more complete picture of the effects of shadowing on the distributions, in Fig. 60 we plot the ratio of the distributions with shadowing included to those without shadowing, denoted $R_{Q}$ for the single quark distributions and $R_{Q \bar{Q}}$ for the pair invariant mass distributions. The charm ratios are given on the left-hand side of Fig. 60 while the bottom ratios are on the right-hand side. The ratios are given for both the EKS98 (solid, dashed and dot-dashed curves for $\mathrm{Pb}+\mathrm{Pb}, \mathrm{Ar}+\mathrm{Ar}$, 

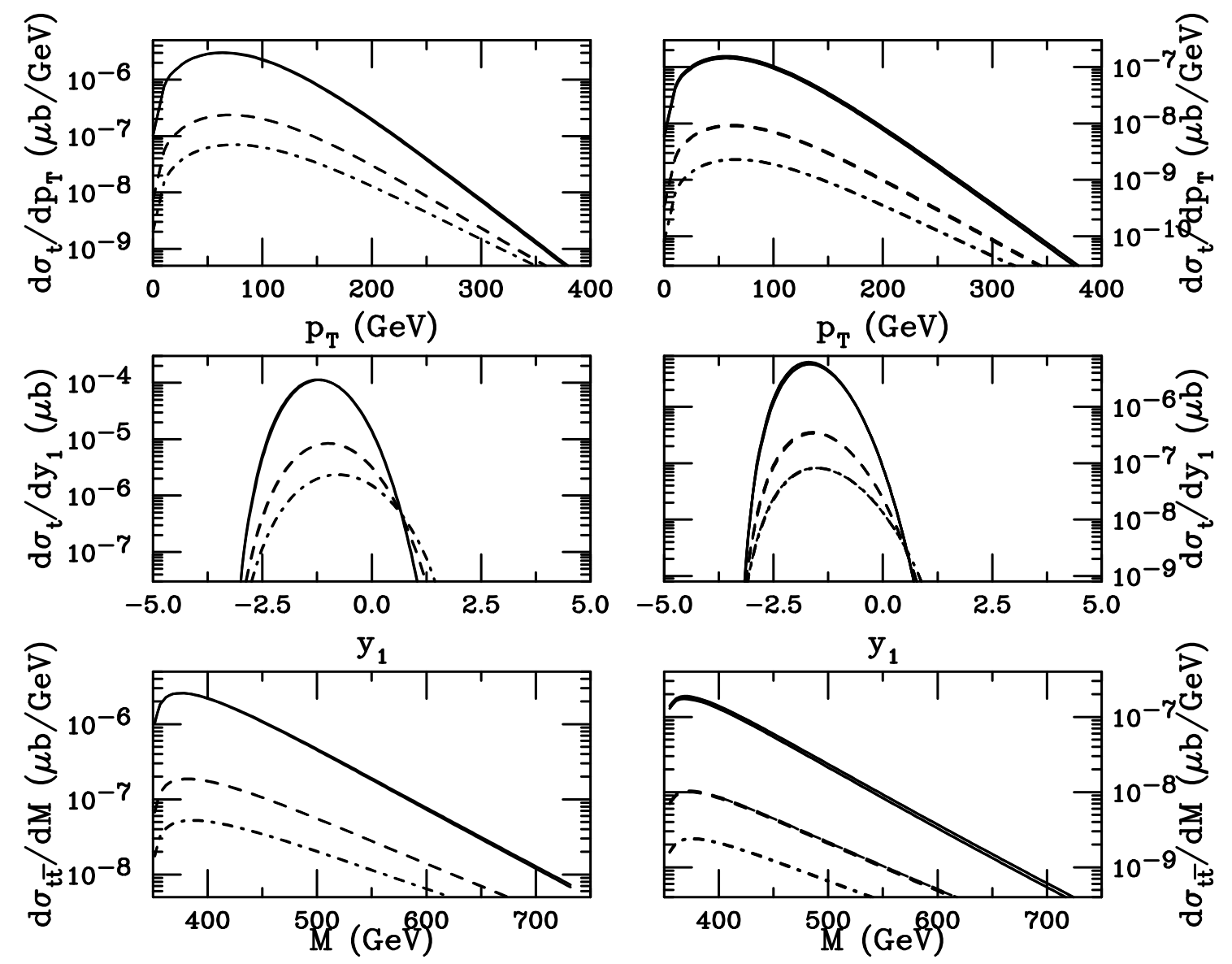

Figure 59. Direct (left) and resolved (right) $t \bar{t}$ photoproduction in peripheral $A A$ collisions. Note the different scales on the $y$-axes for the two production mechanisms. The single $t p_{T}$ (upper) and rapidity (middle) distributions are shown along with the $t \bar{t}$ pair invariant mass distributions (lower). The $\mathrm{O}+\mathrm{O}$ (dot-dashed), $\mathrm{Ar}+\mathrm{Ar}$ (dashed) and $\mathrm{Pb}+\mathrm{Pb}$ (solid) results are given. The photon is coming from the left.

and $\mathrm{O}+\mathrm{O}$ respectively) and the FGS (dash-dash-dash-dotted, dot-dot-dot-dashed and dotted curves for $\mathrm{Pb}+\mathrm{Pb}, \mathrm{Ar}+\mathrm{Ar}$ and $\mathrm{O}+\mathrm{O}$ respectively) shadowing parameterizations. The distributions employing the FGS parametrization are more strongly affected - the charm rapidity ratio for $\mathrm{Pb}+\mathrm{Pb}$ with EKS98 is similar to the $\mathrm{O}+\mathrm{O}$ ratio with FGS. Since the rapidity distributions are integrated over $p_{T}$, the largest weight goes to low $p_{T}$ where the $Q^{2}$ evolution of the shadowing parameterizations is still small, producing the largest shadowing effect at $Q \approx a\left\langle m_{T}\right\rangle$ where $a=1$ for bottom and 2 for charm and $\left\langle m_{T}\right\rangle \approx \sqrt{m_{Q}^{2}+\left\langle p_{T}^{2}\right\rangle}$.

The lowest $x$ values occur at the highest forward rapidities when the photon comes from the left, where the rapidity distribution begins to drop off. The lowest value of $R_{c}\left(p_{T}=0\right)$ corresponds to $R_{c}\left(y_{1}=y_{1 \max }\right)$ where $y_{1 \max }$ is the position of the peak of the rapidity distribution. At midrapidity, just forward of the peak in the distribution, $R_{c}\left(y_{1}=0\right) \sim 0.75$ for EKS98 and 0.55 for FGS with the $\mathrm{Pb}$ beam. The large difference between these midrapidity values and from $R_{c}\left(y_{1}\right)=1$ suggests that shadowing is 
Table 11. Direct $Q \bar{Q}$ photoproduction cross sections integrated over $b>2 R_{A}$ in peripheral $A A$ collisions.

\begin{tabular}{|c|c|c|c|}
\hline & \multicolumn{3}{|c|}{$\sigma^{\text {dir }}(\mathrm{mb})$} \\
\hline$A A$ & no shad & EKS98 & FGS \\
\hline \multicolumn{4}{|c|}{$c \bar{c}$} \\
\hline $\mathrm{O}+\mathrm{O}$ & 1.66 & 1.50 & 1.35 \\
$\mathrm{Ar}+\mathrm{Ar}$ & 16.3 & 14.3 & 12.3 \\
$\mathrm{~Pb}+\mathrm{Pb}$ & 1246 & 1051 & 850 \\
\hline \multicolumn{4}{|c|}{$b \bar{b}$} \\
\hline $\mathrm{O}+\mathrm{O}$ & 0.0081 & 0.0078 & 0.0075 \\
$\mathrm{Ar}+\mathrm{Ar}$ & 0.073 & 0.070 & 0.066 \\
$\mathrm{~Pb}+\mathrm{Pb}$ & 4.89 & 4.71 & 4.42 \\
\hline \multicolumn{5}{|c|}{$t \bar{t}$} \\
\hline $\mathrm{O}+\mathrm{O}$ & $9.13 \times 10^{-9}$ & $9.27 \times 10^{-9}$ & $9.31 \times 10^{-9}$ \\
$\mathrm{Ar}+\mathrm{Ar}$ & $2.86 \times 10^{-8}$ & $2.88 \times 10^{-8}$ & $2.87 \times 10^{-8}$ \\
$\mathrm{~Pb}+\mathrm{Pb}$ & $3.29 \times 10^{-7}$ & $3.21 \times 10^{-7}$ & $3.22 \times 10^{-7}$ \\
\hline
\end{tabular}

measurable in these interactions. While shadowing is reduced at the larger $x$ and $Q$ for bottom production, it is still significant enough for measurements to be feasible. The top cross section is too small for high statistics measurements.

The typical nucleon $x$ ranges for charm, bottom and top production are shown in Fig. 61 as a function of quark rapidity (left-hand side) and transverse momentum (righthand side). It is then clear how the rapidity and shadowing distributions in Fig. 55 map each other. At large negative rapidity, $\left\langle x_{c}\right\rangle \sim 0.1$ for charm, decreasing to $\left\langle x_{c}\right\rangle \sim 10^{-5}$ at $y_{1} \sim 5$. The average $x$ for bottom, $\left\langle x_{b}\right\rangle$, increases by $m_{b} / m_{c}$ relative to charm. For charm and bottom production, there is not much difference between curves at different values of $\sqrt{s_{N N}}$. Charm production is predominantly in the shadowing region over all $y_{1}$ while, at large negative rapidity, bottom production reaches the anti shadowing region. On the other hand, top production is in the 'EMC region', $\left\langle x_{t}\right\rangle>0.2$ for $y_{1}<0$.

Figure 61 also illustrates how, as a function of quark $p_{T}$, the average $x$ corresponds to the peak of the rapidity distribution. The average value of $x$ changes slowly with $p_{T}$. Some of this increase is due to the growing value of $\left\langle m_{T}\right\rangle$ entering in the calculation of $x$. However, the width of the rapidity distribution decreases with increasing $p_{T}$, an important effect, particularly for heavier flavors where phase space considerations are important.

We now turn to the resolved (hadronic) contribution to the photoproduction cross section. The hadronic reaction, $\gamma N \rightarrow Q \bar{Q} X$, is unchanged, but now, prior to the interaction with the nucleon, the photon splits into a color singlet state with some number of $q \bar{q}$ pairs and gluons. On the parton level, the resolved LO reactions are $g(x k)+g\left(x_{2} P_{2}\right) \rightarrow Q\left(p_{1}\right)+\bar{Q}\left(p_{2}\right)$ and $q(x k)+\bar{q}\left(x_{2} P_{2}\right) \rightarrow Q\left(p_{1}\right)+\bar{Q}\left(p_{2}\right)$ where $x$ is the 

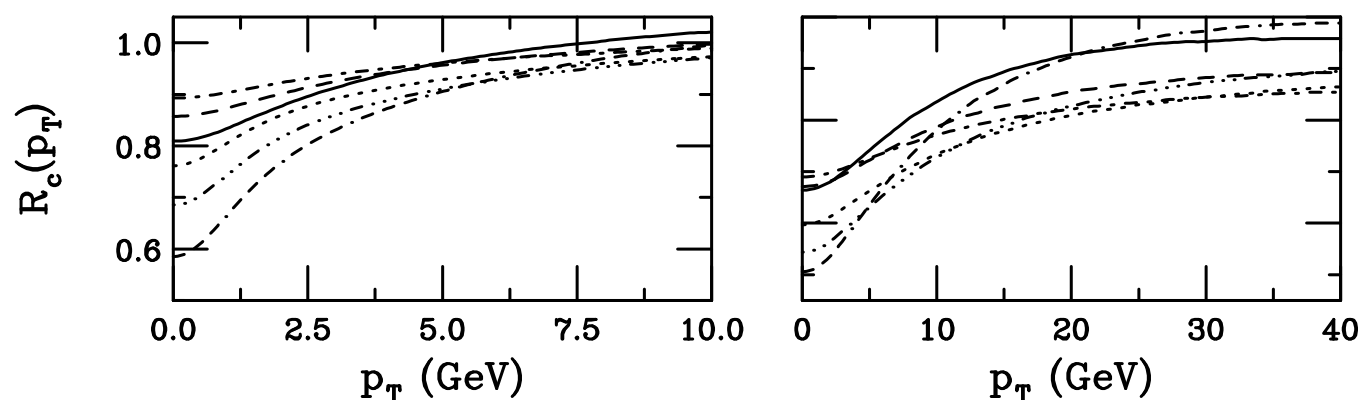

1.1
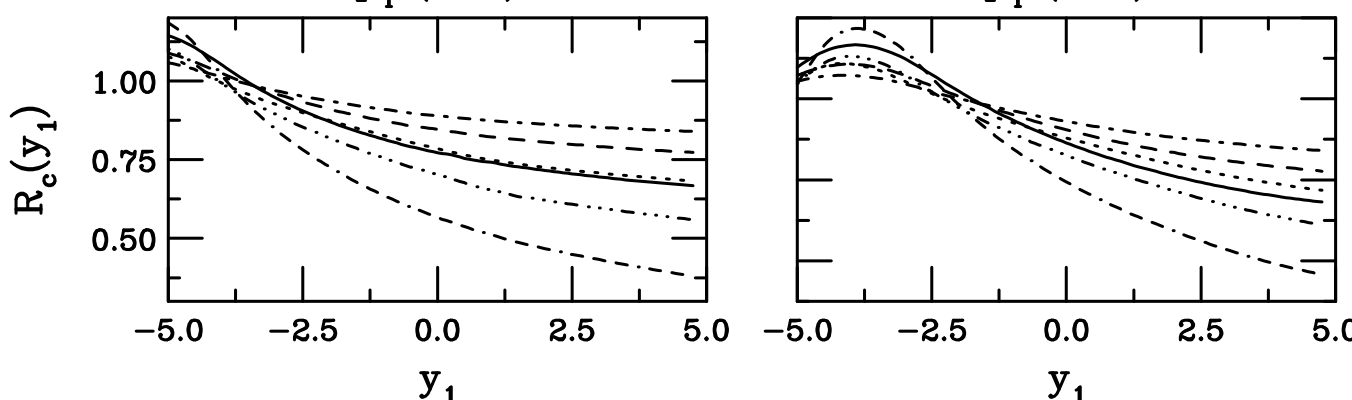

1.2
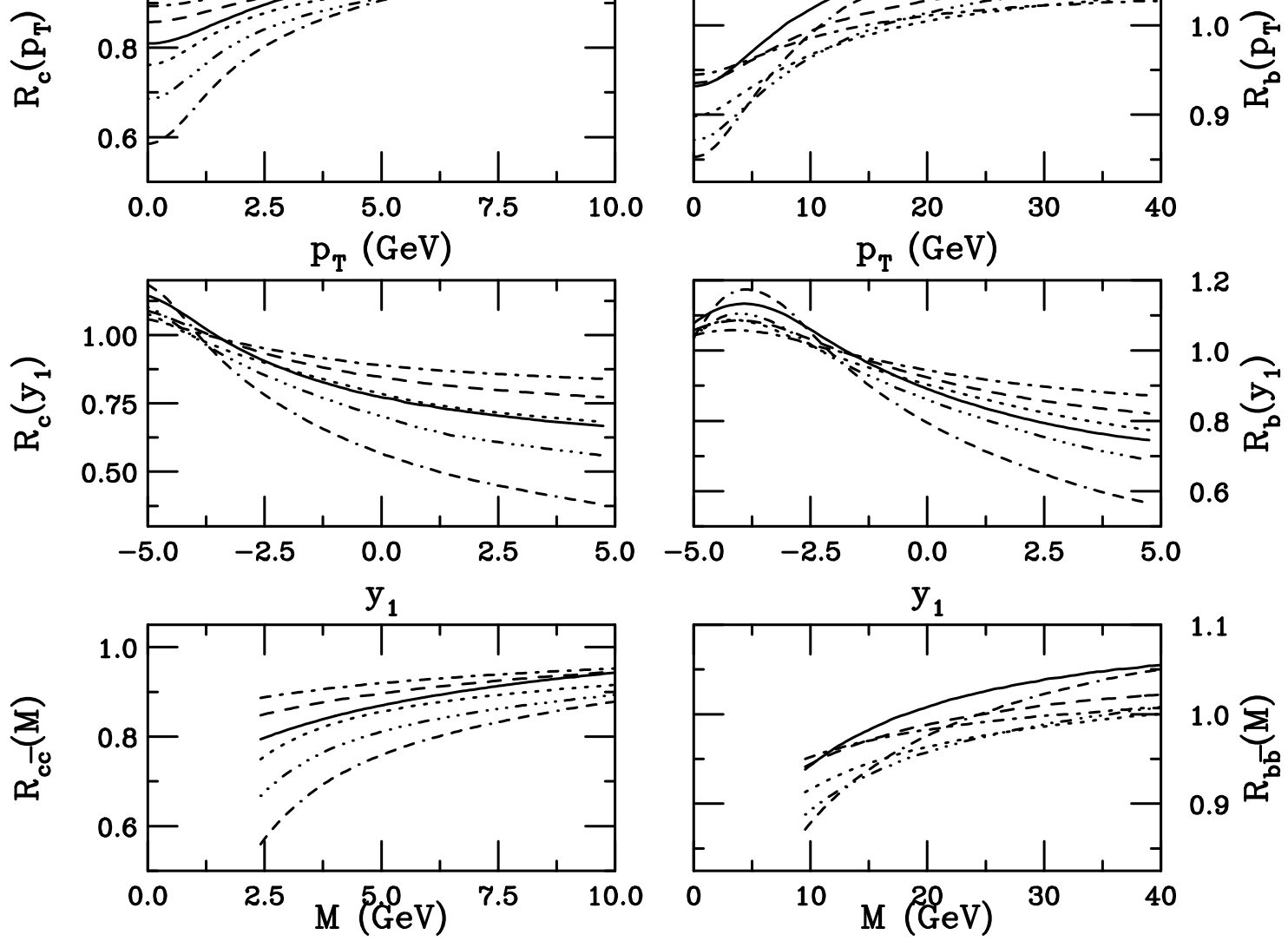

Figure 60. Shadowing in direct $Q \bar{Q}$ photoproduction in peripheral $A A$ collisions. The left-hand side shows the results for charm while the right-hand side gives the results for bottom. The single $Q p_{T}$ (upper) and rapidity (middle) ratios are shown along with the $Q \bar{Q}$ pair invariant mass ratios (lower). The results for the EKS98 (O+O (dot-dashed), $\mathrm{Ar}+\mathrm{Ar}$ (dashed) and $\mathrm{Pb}+\mathrm{Pb}($ solid)) and FGS $(\mathrm{O}+\mathrm{O}$ (dotted), Ar+Ar (dot-dot-dotdashed) and $\mathrm{Pb}+\mathrm{Pb}$ (dash-dash-dash-dotted)) shadowing parameterizations are given. The photon is coming from the left.

fraction of the photon momentum carried by the parton. The LO diagrams for resolved photoproduction are the same as for hadroproduction except that one parton source is a photon rather than a nucleon. The LO partonic cross sections are [199]

$$
\begin{aligned}
\hat{s}^{2} \frac{d^{2} \sigma_{q \bar{q}}}{d \hat{t}_{1} d \hat{u}_{1}}= & \pi \alpha_{s}^{2}\left(Q^{2}\right) \frac{4}{9}\left(\frac{\hat{t}_{1}^{2}+\hat{u}_{1}^{2}}{\hat{s}^{2}}+\frac{2 m_{Q}^{2}}{\hat{s}}\right) \delta\left(\hat{s}+\hat{t}_{1}+\hat{u}_{1}\right), \\
\hat{s}^{2} \frac{d^{2} \sigma_{g g}}{d \hat{t}_{1} d \hat{u}_{1}}= & \frac{\pi \alpha_{s}^{2}\left(Q^{2}\right)}{16} B_{\mathrm{QED}}\left(\hat{s}, \hat{t}_{1}, \hat{u}_{1}\right) \\
& \times\left[3\left(1-\frac{2 \hat{t}_{1} \hat{u}_{1}}{\hat{s}^{2}}\right)-\frac{1}{3}\right] \delta\left(\hat{s}+\hat{t}_{1}+\hat{u}_{1}\right),
\end{aligned}
$$

where $\hat{s}=\left(x k+x_{2} P_{2}\right)^{2}, \hat{t}_{1}=\left(x k-p_{1}\right)^{2}-m_{Q}^{2}$, and $\hat{u}_{1}=\left(x_{2} P_{2}-p_{1}\right)^{2}-m_{Q}^{2}$. The gg partonic cross section, Eq. (93), is proportional to the photon-gluon fusion cross section, Eq. (90), with an additional factor for the non-Abelian three-gluon vertex. 

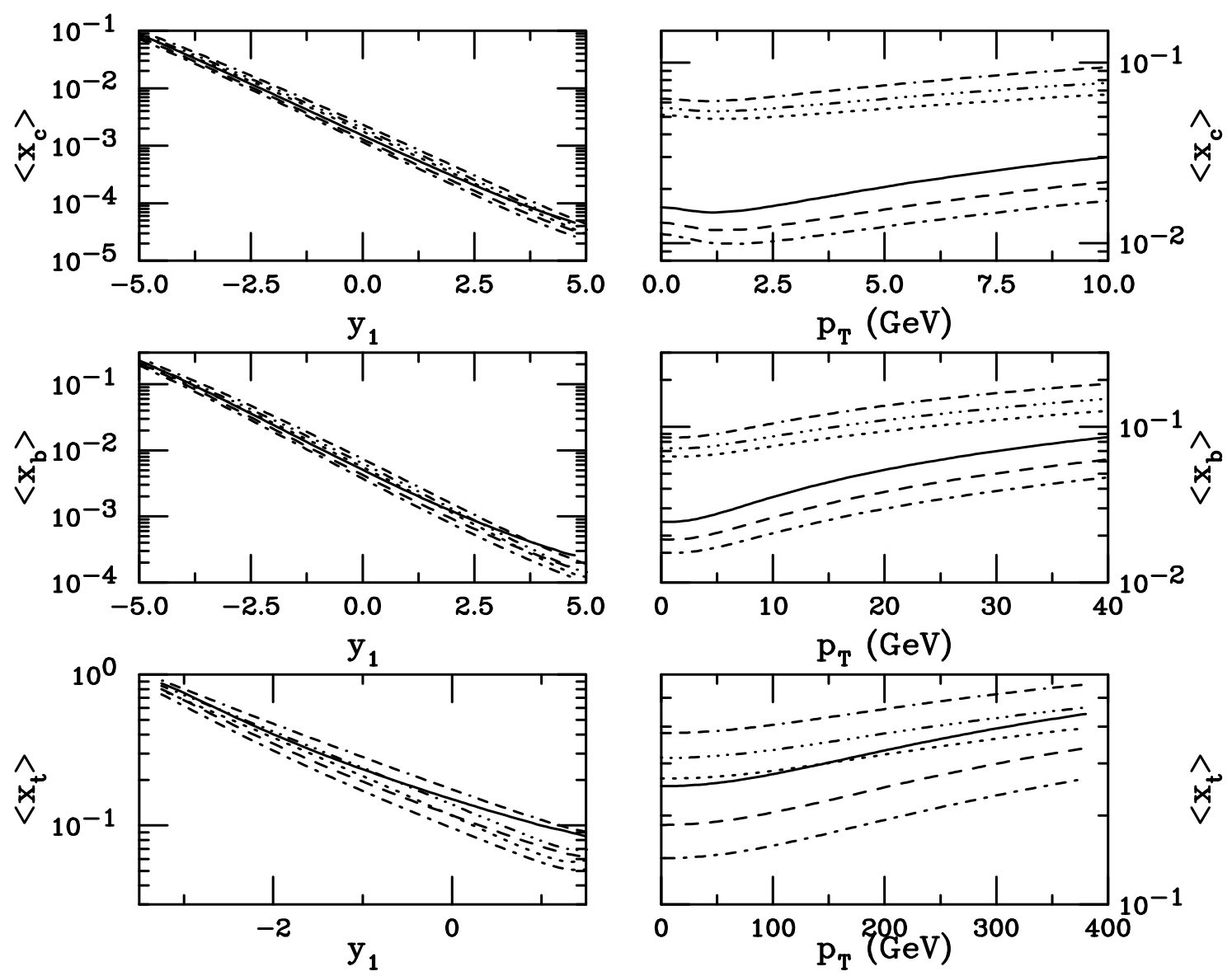

Figure 61. The average value of the nucleon parton momentum fraction $x$ as a function of quark rapidity (left-hand side) and transverse momentum (right-hand side). The results are given for charm (upper), bottom (middle) and top (lower). The direct values are given for $\mathrm{O}+\mathrm{O}$ (dot-dashed), $\mathrm{Ar}+\mathrm{Ar}$ (dashed) and $\mathrm{Pb}+\mathrm{Pb}$ (solid) while the resolved values are given for $\mathrm{O}+\mathrm{O}$ (dotted), $\mathrm{Ar}+\mathrm{Ar}$ (dot-dot-dot-dashed) and $\mathrm{Pb}+\mathrm{Pb}$ (dash-dash-dash-dotted). (Resolved production is calculated with the GRV-G photon parton distributions.) The photon is coming from the left.

The $q \bar{q}$ annihilation cross section has a different structure because it is a $\hat{s}$-channel process with gluon exchange between the $q \bar{q}$ and $Q \bar{Q}$ vertices. Modulo the additional factor in the $g g$ cross section, the resolved partonic photoproduction cross sections are a factor $\alpha_{s}\left(Q^{2}\right) / \alpha e_{Q}^{2}$ larger than the direct, $\gamma g$, partonic photoproduction cross sections. The cross section for resolved $Q \bar{Q}$ photoproduction, using Eq. (87) with the $q \bar{q}$ and $g g$ channels, is

$$
\begin{aligned}
& s_{N N}^{2} \frac{d^{2} \sigma_{\gamma A \rightarrow Q \bar{Q} X}^{\mathrm{res}}}{d t_{1_{N N}} d u_{1_{N N}}}=2 \int_{k_{\min }}^{\infty} \frac{d k}{k} \frac{d N_{\gamma}}{d k} \int_{k_{\min } / k}^{1} \frac{d x}{x} \int_{x_{2} \min }^{1} \frac{d x_{2}}{x_{2}} \\
& \times\left[F_{g}^{\gamma}\left(x, Q^{2}\right) F_{g}^{A}\left(x_{2}, Q^{2}\right) \hat{s}^{2} \frac{d^{2} \sigma_{g g}}{d \hat{t}_{1} d \hat{u}_{1}}\right. \\
& \left.+\sum_{q=u, d, s} F_{q}^{\gamma}\left(x, Q^{2}\right)\left\{F_{q}^{A}\left(x_{2}, Q^{2}\right)+F_{\bar{q}}^{A}\left(x_{2}, Q^{2}\right)\right\} \hat{s}^{2} \frac{d^{2} \sigma_{q \bar{q}}}{d \hat{t}_{1} d \hat{u}_{1}}\right] .
\end{aligned}
$$



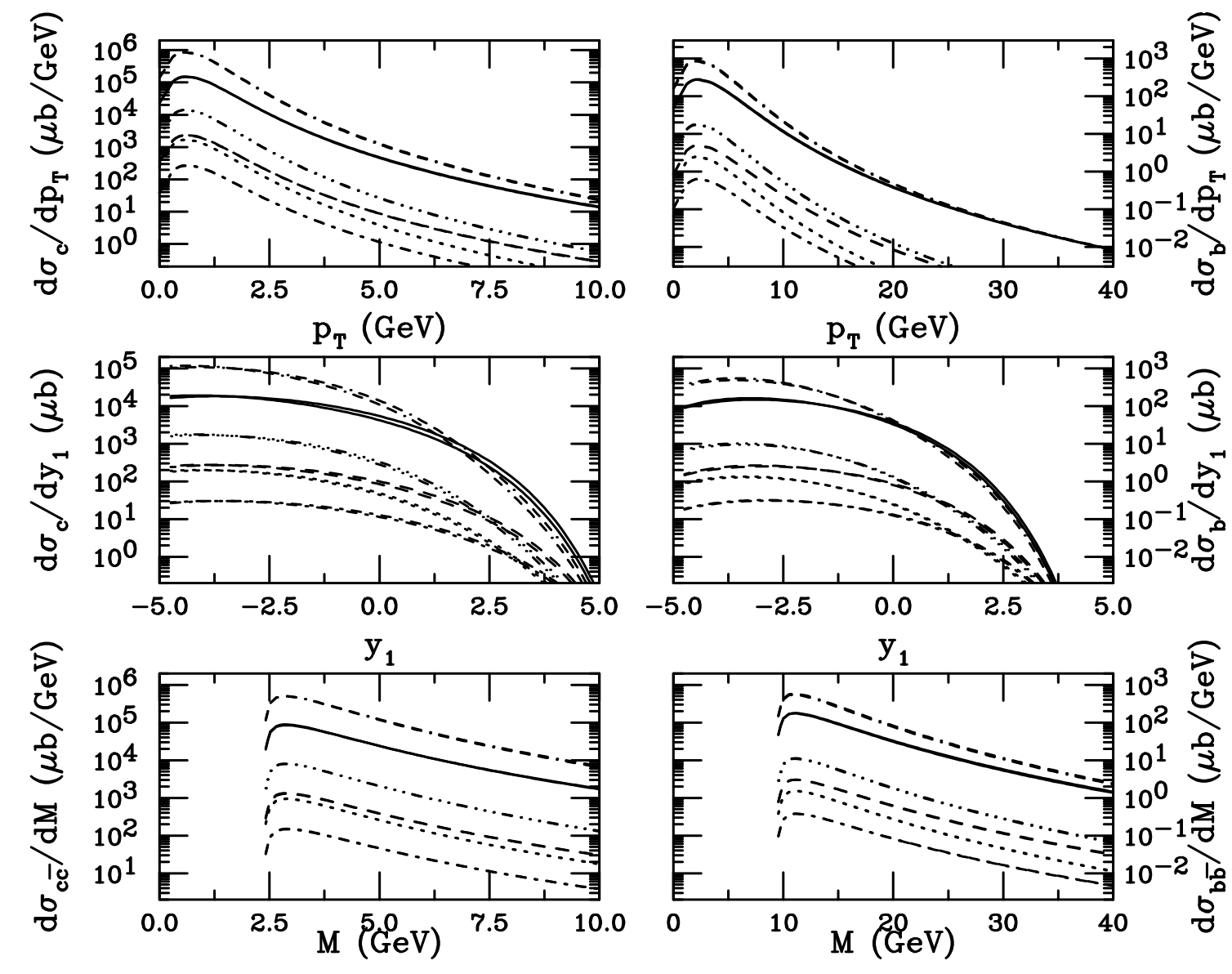

Figure 62. Resolved $Q \bar{Q}$ photoproduction in peripheral $A A$ collisions. The left-hand side shows the results for charm while the right-hand side gives the results for bottom. The single $Q p_{T}$ (upper) and rapidity (middle) distributions are shown along with the $Q \bar{Q}$ pair invariant mass distributions (lower). The results for the GRV-G $(\mathrm{O}+\mathrm{O}$ (dot-dashed), $\mathrm{Ar}+\mathrm{Ar}$ (dashed) and $\mathrm{Pb}+\mathrm{Pb}$ (solid)) and $\mathrm{LAC} 1(\mathrm{O}+\mathrm{O}$ (dotted), $\mathrm{Ar}+\mathrm{Ar}$ (dot-dot-dot-dashed) and $\mathrm{Pb}+\mathrm{Pb}$ (dash-dash-dash-dotted)) photon parton densities are given. There are two curves for each contribution: no shadowing and EKS98. At $y_{1}>0$, the highest curve is without shadowing. The photon is coming from the left.

Figure 62 shows the charm and bottom resolved photoproduction distributions in $\mathrm{Pb}+\mathrm{Pb}, \mathrm{Ar}+\mathrm{Ar}$ and $\mathrm{O}+\mathrm{O}$ collisions. The resolved top photoproduction distributions for these three systems are given on the right-hand side of Fig. 59. There are four curves for each contribution, two with the GRV-G set of photon parton distribution functions and two with the LAC1 set. One the two curves for each set of photon parton distributions is without shadowing while the other employs the EKS98 parametrization.

The difference between the two photon parton densities is quite large at negative rapidities where the parton $x$ entering the photon parton distribution is small. The LAC1 resolved cross sections are largest here. The GRV-G result is slightly larger at forward rapidities although the results for the two sets are similar. This crossover point occurs at larger forward rapidities for lighter nuclei where the energy is higher. For $c \bar{c}$ production, it occurs at $y_{1} \approx 1.75$ for $\mathrm{Pb}+\mathrm{Pb}, 2.5$ for $\mathrm{Ar}+\mathrm{Ar}$ and 3 for $\mathrm{O}+\mathrm{O}$. The 
larger $x$ of $b \bar{b}$ production moves the crossover point backwards to $y_{1} \approx 0.25$ for $\mathrm{Pb}+\mathrm{Pb}$, 1 for $\mathrm{Ar}+\mathrm{Ar}$ and 1.5 for $\mathrm{O}+\mathrm{O}$, a shift of around 1.5 units between charm and bottom production. At high $p_{T}$ and $M$, the GRV-G and LAC1 resolved distributions in $\mathrm{Pb}+\mathrm{Pb}$ collisions approach each other, showing that the differences in the two sets are reduced at high scales. The approach is more gradual for the higher energy light ion collisions. The same trend is seen in the mass distributions. The GRV-G and LAC1 results are indistinguishable for resolved top production.

The large difference in the resolved results will strongly influence whether the direct or resolved contribution is greater. This, in turn, directly affects the capability to clearly measure the nuclear gluon distribution. Thus Table 12 compares the total $Q \bar{Q}$ resolved cross sections. The LAC1 $c \bar{c}$ resolved cross sections are $5-6$ times higher than the GRV-G cross sections while the difference for $b \bar{b}$ production is a factor of $2.8-3.6$. In both cases, the smallest difference is for the heaviest ions, hence for the lowest energy and highest $x$ values. The difference in the $t \bar{t}$ resolved cross sections is on the few per cent level and is therefore negligible.

We may now compare these resolved cross sections to the direct cross sections in Table 11. With the GRV-G set, the resolved contributions are $\sim 15$ and $20 \%$ of the total charm and bottom photoproduction cross sections respectively, comparable to the shadowing effect on direct production. However, with the LAC1 set, the resolved contribution is equivalent to or larger than the direct. A measurement of the photon parton distributions at low $x$ and $Q$ will thus be important for a precision measurement of gluon shadowing.

Table 12. Resolved $Q \bar{Q}$ photoproduction cross sections integrated over $b>2 R_{A}$ in peripheral $A A$ collisions.

\begin{tabular}{|c|c|c|c|c|c|}
\hline & \multicolumn{5}{|c|}{$\sigma^{\text {res }}(\mathrm{mb})$} \\
\hline & GKS98 & FGS & no shad & EKS98 \\
\hline$A A$ & no shad & $c \bar{c}$ \\
\hline \multicolumn{7}{|c|}{ LAC1 } \\
\hline $\mathrm{O}+\mathrm{O}$ & 0.351 & 0.346 & 0.331 & 2.04 & 2.02 \\
$\mathrm{Ar}+\mathrm{Ar}$ & 3.00 & 2.93 & 2.77 & 16.6 & 16.6 \\
$\mathrm{~Pb}+\mathrm{Pb}$ & 190 & 187 & 174 & 987 & 1007 \\
\hline \multicolumn{7}{|c|}{$b \bar{b}$} \\
\hline $\mathrm{O}+\mathrm{O}$ & 0.0029 & 0.0029 & 0.0029 & 0.0105 & 0.0106 \\
$\mathrm{Ar}+\mathrm{Ar}$ & 0.0222 & 0.0226 & 0.0224 & 0.073 & 0.075 \\
$\mathrm{~Pb}+\mathrm{Pb}$ & 1.21 & 1.26 & 1.25 & 3.41 & 3.66 \\
\hline \multicolumn{7}{|c|}{$t \bar{t}$} \\
\hline $\mathrm{O}+\mathrm{O}$ & $2.81 \times 10^{-10}$ & $2.76 \times 10^{-10}$ & - & $2.92 \times 10^{-10}$ & $2.88 \times 10^{-10}$ \\
$\mathrm{Ar}+\mathrm{Ar}$ & $1.08 \times 10^{-9}$ & $1.04 \times 10^{-9}$ & - & $1.09 \times 10^{-9}$ & $1.05 \times 10^{-9}$ \\
$\mathrm{~Pb}+\mathrm{Pb}$ & $1.60 \times 10^{-8}$ & $1.48 \times 10^{-8}$ & - & $1.62 \times 10^{-8}$ & $1.49 \times 10^{-8}$ \\
\hline
\end{tabular}


Figure 63 shows the ratio of the resolved to direct charm and bottom production cross sections, $R_{Q}$, for the LAC1 and GRV-G photon parton densities. If $R_{Q}<1$, direct production dominates and the most direct information on the gluon distribution in the nucleus can be obtained. The middle plots show that even the GRV-G resolved contribution is equivalent to the direct one at large negative rapidity. However, direct production dominates at $y_{1}>0$ for both photon parton densities. In this region, the resolved rapidity distribution drops rather steeply with respect to the direct. Recall that the direct rapidity distribution is still rather broad at forward rapidities, see Fig. 58, particularly for charm. This is also the region where the nuclear gluon $x$ values are smallest. In addition, at sufficiently large $p_{T}$, direct production dominates. The upper plots of Fig. 63 show that $p_{T} \approx 2.5 \mathrm{GeV}$ should be sufficient for charm while 10 is needed for bottom if the LAC1 set is employed. There is no restriction on the $p_{T}$ range if the GRV-G set is more correct. Better measurements of the photon parton densities should help settle this issue.

Nuclear shadowing can also be studied in the resolved contribution although the contribution from the gluon is now only a portion of the total. In resolved photoproduction, the $q \bar{q}$ channel gives a larger contribution than in hadroproduction because the photon quark and antiquark distributions peak at large $x$. Indeed, the peak of the photon quark distribution is at higher $x$ than the valence quark distribution in the nucleon. Thus the $q \bar{q}$ contribution increases close to threshold. Although $c \bar{c}$ and $b \bar{b}$ resolved photoproduction is not very near threshold, the effective center-of-mass energies are reduced relative to $\sqrt{S_{\gamma N}}$ in Table 1 .

Shadowing effects on the resolved contributions are shown in Fig. 64 for the EKS98 parametrization. The direct and resolved ratios as a function of rapidity are remarkably similar, especially for charm, as seen in a comparison of the middle plots of Fig. 64 to those of Fig. 60. The similarity of the shadowing ratios may be expected since the rapidity distributions best reflect the $x$ values of the nuclear parton densities probed. The additional $q \bar{q}$ contribution to resolved $b \bar{b}$ production is larger than for charm, large enough to cause the small difference in $b \bar{b}$ shadowing between direct and resolved production. The basic kinematics of the partonic interactions are the same for the nuclear partons but the momentum entering the photon flux is effectively changed by the ratio $k / k_{\min }$. Thus a higher momentum photon is needed to produce the effective $x$ entering the photon parton momentum distributions. As seen in Fig. 61, there is little difference in $\left\langle x_{Q}\right\rangle$ of the nucleon between direct and resolved photoproduction.

The quark transverse momentum and pair mass distributions are, however, more strongly affected since they are integrated over all rapidity. The shift of the peak of the resolved rapidity distributions to more negative rapidities increases the average $x$ probed by the $p_{T}$ and $M$ distributions, decreasing the effects of shadowing on these distributions. This increase in $\left\langle x_{Q}\right\rangle$ as a function of $p_{T}$ due to the peak of the rapidity distribution is shown in Fig. 61 for the GRV-G photon parton distribution. Since the peak of the rapidity distribution with the LAC1 set is at even larger negative rapidity than the GRV-G, corresponding to larger nuclear momentum fractions, the shadowing 

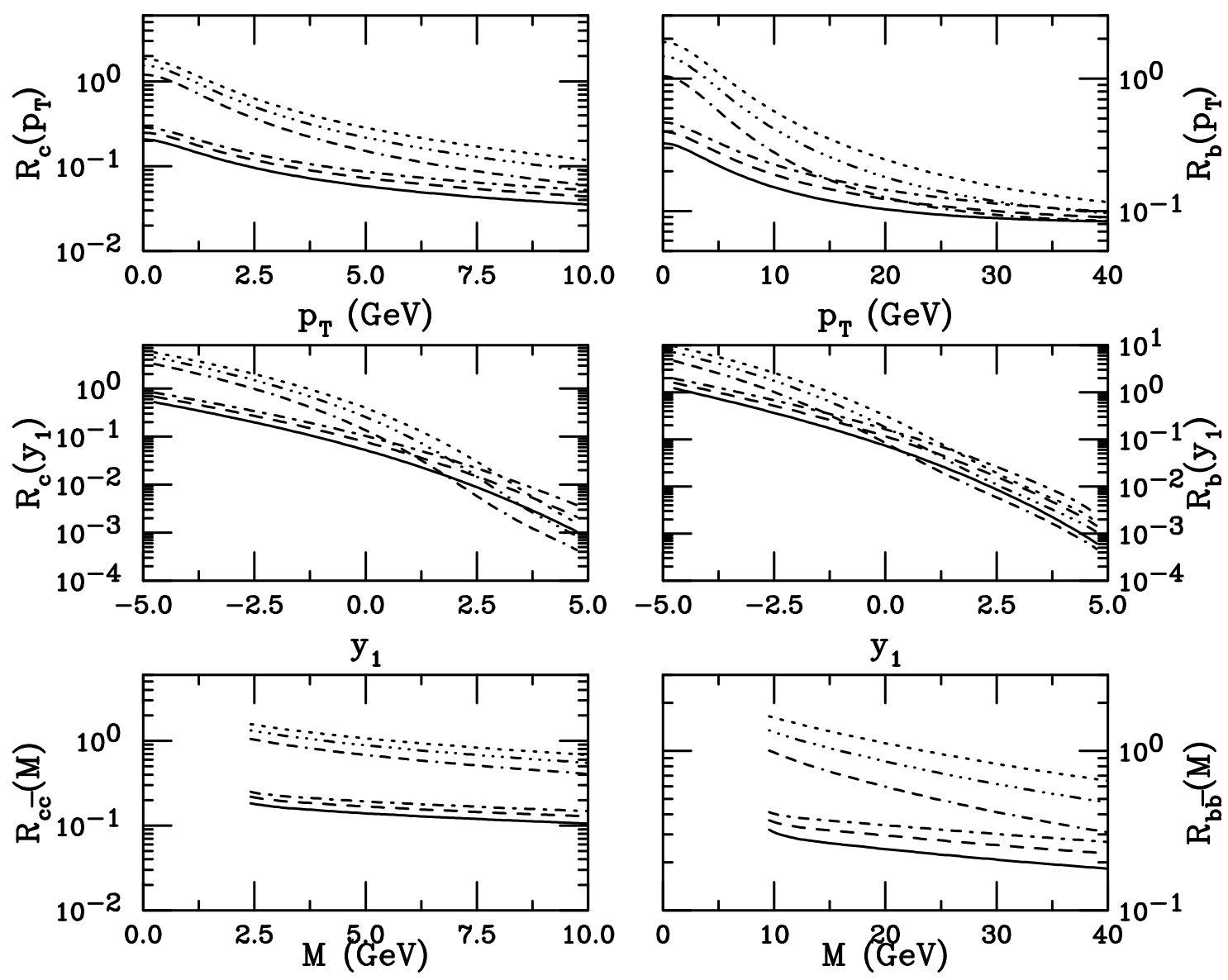

Figure 63. Resolved to direct $Q \bar{Q}$ photoproduction ratio in peripheral $A A$ collisions. The left-hand side shows the results for charm while the right-hand side gives the results for bottom. The EKS98 shadowing parametrization is used in both cases. The single $Q p_{T}$ (upper) and rapidity (middle) ratios are shown along with the $Q \bar{Q}$ pair invariant mass ratios (lower). The results for the GRV-G $(\mathrm{O}+\mathrm{O}$ (dot-dashed), $\mathrm{Ar}+\mathrm{Ar}$ (dashed) and $\mathrm{Pb}+\mathrm{Pb}$ (solid)) and LAC1 (O+O (dotted), $\mathrm{Ar}+\mathrm{Ar}$ (dot-dot-dotdashed) and $\mathrm{Pb}+\mathrm{Pb}$ (dash-dash-dash-dotted)) photon parton distributions are given. The photon is coming from the left.

ratios in this case are larger as well.

The total rates are given in Table 13 for all three collision systems, assuming a $10^{6} \mathrm{~s}$ run for each system. We have included the rates for both the GRV-G and LAC1 photon parton distribution sets. The difference in rate can be up to a factor of two when the LAC1 resolved cross sections are used. The top rates, particularly for the lighter nuclei, are lower than in Ref. [177], due to a revision in the expected LHC light ion luminosities. Thus using top quarks to measure the nuclear gluon distribution at high $Q^{2}$ seems unlikely.

These rates are based on total cross sections, without any acceptance cuts or detector requirements included. The largest difference between the GRV-G and LAC1 resolved cross sections lies at $y_{1}<-2.5$. Thus, for central detectors, the difference in the rates would be reduced. However, if heavy quark photoproduction is also studied 

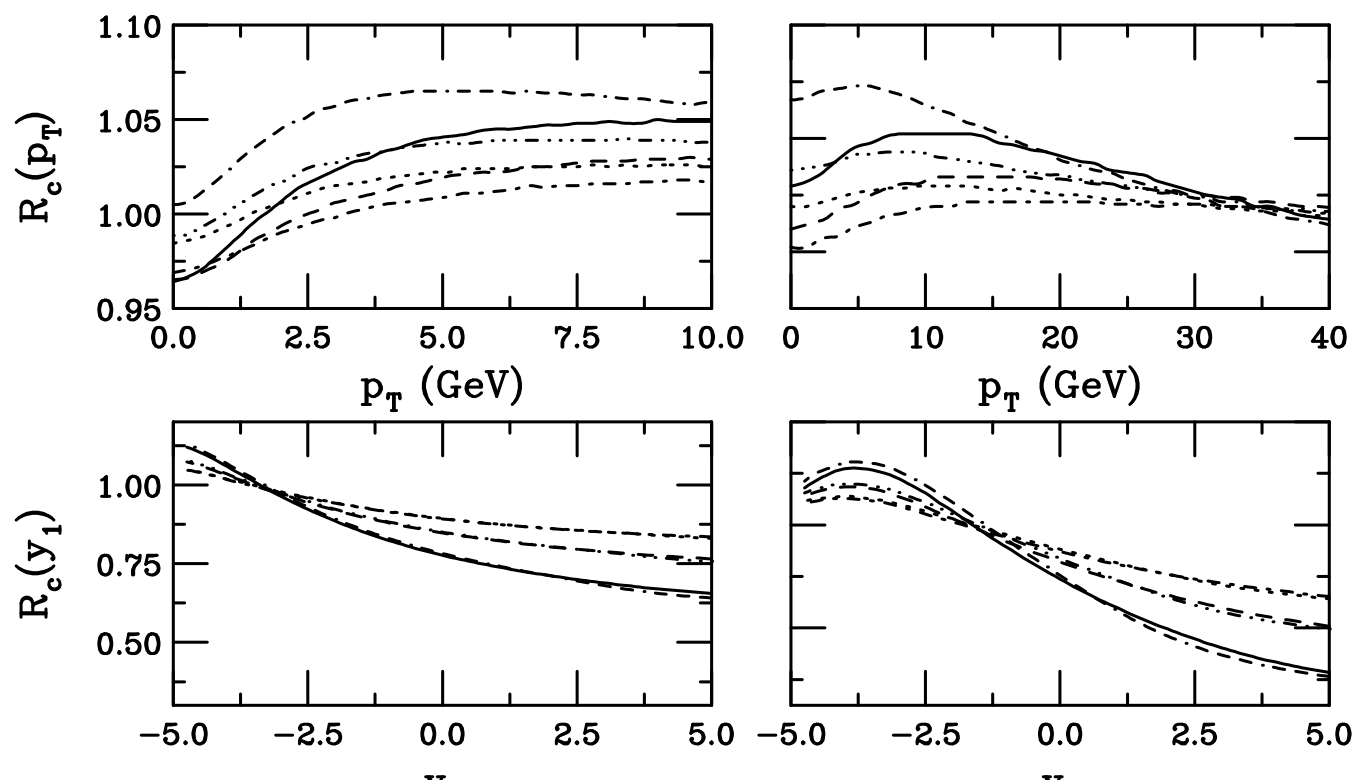

1.2

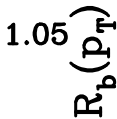

1.00
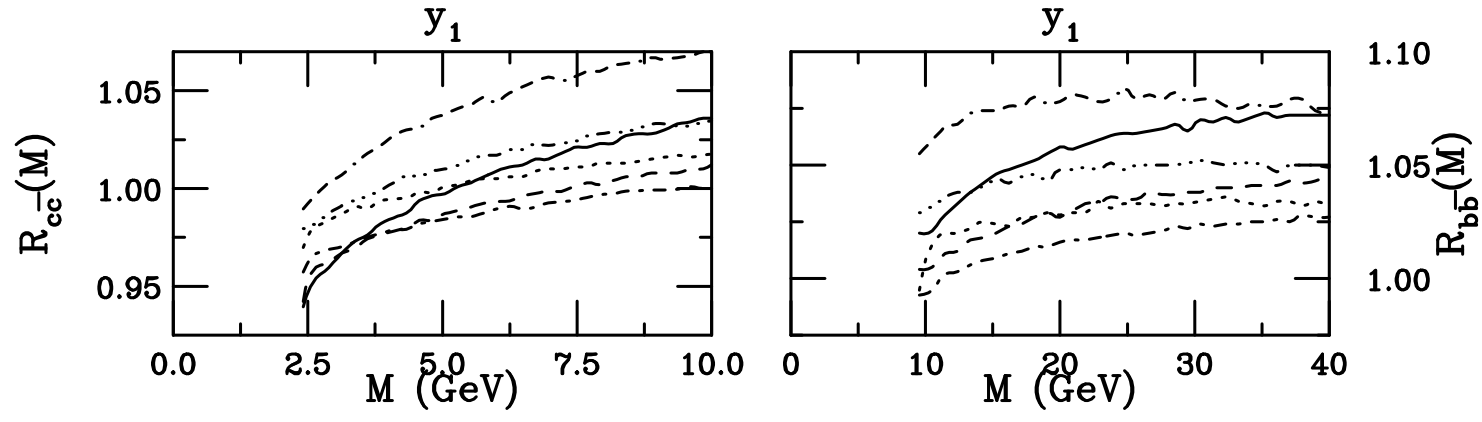

Figure 64. Shadowing in resolved $Q \bar{Q}$ photoproduction in peripheral $A A$ collisions. The left-hand side shows the results for charm while the right-hand side gives the results for bottom. The EKS98 shadowing parametrization is used in both cases. The single $Q p_{T}$ (upper) and rapidity (middle) ratios are shown along with the $Q \bar{Q}$ pair invariant mass ratios (lower). The results for the GRV-G (O+O (dot-dashed), $\mathrm{Ar}+\mathrm{Ar}$ (dashed) and $\mathrm{Pb}+\mathrm{Pb}($ solid)) and LAC1 $(\mathrm{O}+\mathrm{O}$ (dotted), Ar+Ar (dot-dot-dotdashed) and $\mathrm{Pb}+\mathrm{Pb}$ (dash-dash-dash-dotted)) photon parton distributions are given. The photon is coming from the left.

through its leptonic decays, the forward muon arm of ALICE, $2.4<y<4$, would be sensitive to the photon parton densities when the photon comes from the right-hand side, the mirror image of the plots.

The high $c \bar{c}$ and $b \bar{b}$ rates provide sufficient statistics to distinguish between shadowing parameterizations and perhaps the photon parton distributions as well, even with finite acceptance. The rates for the different systems are all of the same order of magnitude. The lower photon flux for light, smaller $Z$ ions is compensated by their higher luminosities. Unfortunately however, the top rates are discouragingly low, even for light ions.

There are a number of theoretical uncertainties in the calculations shown here aside from the obvious ones in the nuclear and photon parton densities and the photon flux. First, the calculation is to leading order only. Higher order corrections can be 
Table 13. Total $Q \bar{Q}$ photoproduction rates, integrated over $b>2 R_{A}$ in peripheral $A A$ collisions. The rates are based on Tables 11 and 12 for a $10^{6} \mathrm{~s}$ run.

\begin{tabular}{|c|c|c|c|c|c|}
\hline \multicolumn{6}{|c|}{$N_{Q \bar{Q}}$} \\
\hline & \multicolumn{3}{|c|}{ GRV-G } & \multicolumn{2}{|c|}{ LAC1 } \\
\hline$A A$ & no shad & EKS98 & FGS & no shad & EKS98 \\
\hline \multicolumn{6}{|c|}{$c \bar{c}$} \\
\hline $\mathrm{O}+\mathrm{O}$ & $3.20 \times 10^{8}$ & $2.96 \times 10^{8}$ & $2.69 \times 10^{8}$ & $5.92 \times 10^{8}$ & $5.63 \times 10^{8}$ \\
\hline $\mathrm{Ar}+\mathrm{Ar}$ & $8.30 \times 10^{8}$ & $7.40 \times 10^{8}$ & $6.49 \times 10^{8}$ & $1.41 \times 10^{9}$ & $1.33 \times 10^{9}$ \\
\hline $\mathrm{Pb}+\mathrm{Pb}$ & $6.03 \times 10^{8}$ & $5.20 \times 10^{8}$ & $4.30 \times 10^{8}$ & $9.38 \times 10^{8}$ & $8.64 \times 10^{8}$ \\
\hline \multicolumn{6}{|c|}{$b \bar{b}$} \\
\hline $\mathrm{O}+\mathrm{O}$ & $1.76 \times 10^{6}$ & $1.71 \times 10^{6}$ & $1.66 \times 10^{6}$ & $2.98 \times 10^{6}$ & $2.94 \times 10^{6}$ \\
\hline $\mathrm{Ar}+\mathrm{Ar}$ & $4.09 \times 10^{6}$ & $3.98 \times 10^{6}$ & $3.81 \times 10^{6}$ & $6.28 \times 10^{6}$ & $6.24 \times 10^{6}$ \\
\hline $\mathrm{Pb}+\mathrm{Pb}$ & $2.56 \times 10^{6}$ & $2.51 \times 10^{6}$ & $2.38 \times 10^{6}$ & $3.07 \times 10^{6}$ & $3.52 \times 10^{6}$ \\
\hline \multicolumn{6}{|c|}{$t \bar{t}$} \\
\hline $\mathrm{O}+\mathrm{O}$ & 1.53 & 1.53 & - & 1.53 & 1.53 \\
\hline $\mathrm{Ar}+\mathrm{Ar}$ & 1.28 & 1.28 & - & 1.28 & 1.28 \\
\hline $\mathrm{Pb}+\mathrm{Pb}$ & 0.14 & 0.14 & - & 0.14 & 0.14 \\
\hline
\end{tabular}

significant, with large theoretical $K$ factors, and can affect the shape of the distributions, particularly at large $p_{T}$, see Ref. [198] for a discussion of NLO photoproduction and Ref. [200] for discussions of the $K$ factors in heavy quark hadroproduction. There is also some uncertainty in the heavy quark mass and scale parameters. Variations in the mass generally cause larger changes in the cross sections than scale variations. Reference [141] has a more detailed discussion of the uncertainties.

One way to avoid some of these calculational uncertainties is to compare the $p A$ and $A A$ photoproduction cross sections at equal photon energies. The parameter dependence cancels in the direct $Q \bar{Q}$ production ratio $\sigma(A A) / \sigma(p A)$. In the equal speed system, equal photon energies correspond to the same final-state rapidities. In $p A$ collisions, the photon almost always comes from the nucleus due to its stronger photon field. Thus the $p A$ rates depend on the free proton gluon distribution. The photon fluxes are different for $p A$ and $A A$ because the minimum radii used to determine $\omega_{R}$ are different: $2 R_{A}$ in $A A$ rather than $R_{A}+r_{p}$ in $p A$. We use $r_{p} \approx 0.6 \mathrm{fm}$ for the proton radius [201] but our results do not depend strongly on $r_{p}$.

The $p A$ results are calculated in the equal-speed frame. At the LHC, the proton and nuclear beams must have the same magnetic rigidity and, hence, different velocities and per-nucleon energies. Thus the equal-speed frame is boosted with respect to the laboratory frame and the maximum $p A$ energy per nucleon is larger than the $A A$ energy. The $\gamma_{L}$ and $\sqrt{s_{N N}}$ for $p A$ at the LHC in Table 1 are those of the equal-speed system. The $p A$ total cross sections for $Q \bar{Q}$ production are given in Table 14 .

The total $p A$ cross sections are generally smaller than the $A A$ cross sections in 
Table 14. Direct and resolved $c \bar{c}$ and $b \bar{b}$ photoproduction cross sections integrated over $b>r_{p}+R_{A}$ in $p A$ collisions at the LHC.

\begin{tabular}{|c|c|c|c|}
\hline$p A$ & $\sigma^{\text {dir }}(\mu \mathrm{b})$ & $\sigma^{\text {res }}(\mathrm{GRV})(\mu \mathrm{b})$ & $\sigma^{\text {res }}(\mathrm{LAC} 1)(\mu \mathrm{b})$ \\
\hline \multicolumn{3}{|c|}{$c \bar{c}$} \\
\hline$p \mathrm{O}$ & 75.5 & 19.7 & 120.6 \\
$p \mathrm{Ar}$ & 335 & 81.1 & 486.3 \\
$p \mathrm{~Pb}$ & 5492 & 1160 & 6371 \\
\hline \multicolumn{3}{|c|}{$b \bar{b}$} \\
\hline$p \mathrm{O}$ & 0.419 & 0.190 & 0.773 \\
$p \mathrm{Ar}$ & 1.775 & 0.739 & 2.886 \\
$p \mathrm{~Pb}$ & 26.83 & 9.60 & 34.68 \\
\hline \multicolumn{3}{|c|}{$t \bar{t}$} \\
\hline$p \mathrm{O}$ & $1.54 \times 10^{-6}$ & $4.00 \times 10^{-8}$ & $4.00 \times 10^{-8}$ \\
$p \mathrm{Ar}$ & $4.40 \times 10^{-6}$ & $1.23 \times 10^{-7}$ & $1.24 \times 10^{-7}$ \\
$p \mathrm{~Pb}$ & $3.00 \times 10^{-5}$ & $9.74 \times 10^{-7}$ & $9.86 \times 10^{-7}$ \\
\hline
\end{tabular}

Tables 11 and 12 . Here the cross sections are given in $\mu \mathrm{b}$ while the $A A$ cross sections are in units of mb. In hadroproduction, without shadowing, at the same energies, the $A A / p A$ cross section ratio is $A$. In photoproduction, the corresponding ratio would be $2 A$ since there is only a single photon source in $p A$. Since the $A A$ and $p A$ results are given at different center-of-mass energies, the comparison is not so straightforward. However, even if the $p A$ energy is lowered to that of the $A A$ collisions, the $A A / p A$ ratio is larger than $2 A$. The difference in flux due to the change in the minimum impact parameter from $2 R_{A}$ in $A A$ to $r_{p}+R_{A}$ in $p A$ accounts for most of the difference, especially for lighter nuclei where the difference $R_{A}-r_{p}$ is smallest. Reducing the minimum impact parameter increases the photon flux [141].

Table 15. Total $Q \bar{Q}$ photoproduction rates in peripheral $p A$ collisions over a $10^{6} \mathrm{~s}$ run at the LHC. The rates are based on Table 14.

\begin{tabular}{|c|c|c|c|c|c|c|}
\hline & \multicolumn{2}{|c|}{$N_{c \bar{c}}$} & \multicolumn{2}{c|}{$N_{b \bar{b}}$} & \multicolumn{2}{c|}{$N_{t \bar{t}}$} \\
\hline$p A$ & GRV-G & LAC1 & GRV-G & LAC1 & GRV-G & LAC1 \\
\hline$p \mathrm{O}$ & $9.52 \times 10^{8}$ & $1.96 \times 10^{9}$ & $6.09 \times 10^{6}$ & $1.19 \times 10^{7}$ & 15.8 & 15.8 \\
$p \mathrm{Ar}$ & $2.41 \times 10^{9}$ & $4.76 \times 10^{9}$ & $1.46 \times 10^{7}$ & $2.70 \times 10^{7}$ & 33.4 & 33.4 \\
$p \mathrm{~Pb}$ & $4.92 \times 10^{9}$ & $8.78 \times 10^{9}$ & $2.79 \times 10^{7}$ & $4.55 \times 10^{7}$ & 23 & 23 \\
\hline
\end{tabular}

Clearly a $p A$ run at the same energy as $A A$ would reduce the uncertainties due to the energy difference. The same $x$ range of the proton and nuclear gluon distributions would then be probed. Such runs are possible but with a loss of luminosity, leading to a reduction in the $p A$ rates. However, the $p A$ rates shown in Table 15 are high-even 
higher than the $A A$ rates in the same $10^{6} \mathrm{~s}$ interval thanks to the higher maximum $p A$ luminosities. Including the reduced rates due to the lower energy, the $p A$ luminosity could be significantly reduced without lowering the $c \bar{c}$ and $b \bar{b}$ statistics. Then the only major uncertainty would be the difference in photon flux between $A A$ and $p A$ interactions. The uncertainties in the photon flux could be reduced by measurements of other baseline processes, allowing a cleaner comparison.

The relatively higher $t \bar{t}$ rate in $p A$ collisions suggests that the top quark charge of $2 / 3$ could be confirmed. While less than $100 t \bar{t}$ pairs are produced in a $10^{6} \mathrm{~s} p A$, run, essentially all the pairs fall into the $|y| \leq 2.5$ region. Thus, ideally, a difference in rate by a factor of four due to $e_{t}^{2}$ could be detected in a single $p A$ run although the combined results of several years of $p A$ runs would be better.

\subsection{Dijet photoproduction}

Contributed by: R. Vogt

We now consider jet photoproduction in peripheral $A A$ and $p A$ interactions. In central collisions at RHIC, leading particles in jets are easier to detect above the high charged particle multiplicity background than the jets themselves since these high $p_{T}$ particles can be tracked through the detector $[202,203]$. In peripheral collisions, especially at LHC energies, jets should be easier to isolate and may be observed directly using standard high energy jet detection techniques [204]. We thus discuss the leading order $p_{T}$ distributions of jets as well as leading particles. We work at LO to avoid any ambiguities such as jet reconstruction and cone size. Note, however that the $p_{T}$ distributions are likely harder at NLO even though the $K$ factor appears to be relatively constant at high $p_{T}$ [205]. We also discuss the fragmentation of jets and present the leading particle transverse momentum distributions, specifically charged pions, kaons and protons.

The hadronic reaction we study is $\gamma(k)+N\left(P_{2}\right) \rightarrow \operatorname{jet}\left(p_{1}\right)+\operatorname{jet}\left(p_{2}\right)+X$ where $k$ and $P_{2}$ are the photon and nucleon four-momenta. The two parton-level contributions to the jet yield in direct photoproduction are $\gamma(k)+g\left(x_{2} P_{2}\right) \rightarrow q\left(p_{1}\right)+\bar{q}\left(p_{2}\right)$ and $\gamma(k)+q\left(x_{2} P_{2}\right) \rightarrow g\left(p_{1}\right)+q\left(p_{2}\right)$ where also $q \rightarrow \bar{q}$. The produced partons are massless, requiring a minimum $p_{T}$ to keep the cross section finite. At LO, the jet yield is equivalent to the high $p_{T}$ parton yield. The jet $p_{T}$ distribution is modified for photoproduction from e.g. Refs. [181, 206, 207],

$$
\begin{aligned}
s_{N N}^{2} \frac{d^{2} \sigma_{\gamma A \rightarrow \text { jet }+ \text { jet }+X}^{\text {dir }}}{d t_{N N} d u_{N N}}= & 2 \int_{k_{\min }}^{\infty} d k \frac{d N_{\gamma}}{d k} \int_{x_{2} \min }^{1} \frac{d x_{2}}{x_{2}} \\
& \times\left[\sum_{i, j, l=q, \bar{q}, g} F_{i}^{A}\left(x_{2}, Q^{2}\right) s^{2} \frac{d^{2} \sigma_{\gamma i \rightarrow j l}}{d t d u}\right],
\end{aligned}
$$

where $x_{2}$ is the fraction of the initial hadron momentum carried by the interacting parton and $Q$ is the momentum scale of the interaction. The extra factor of two on the right-hand side of Eq. (95) arises because both nuclei can serve as photon sources in $A A$ 
collisions. The partonic cross sections are

$$
\begin{aligned}
& s^{2} \frac{d^{2} \sigma_{\gamma g \rightarrow q \bar{q}}}{d t d u}=\pi \alpha_{s}\left(Q^{2}\right) \alpha e_{Q}^{2}\left[\frac{t^{2}+u^{2}}{t u}\right] \delta(s+t+u), \\
& s^{2} \frac{d^{2} \sigma_{\gamma q \rightarrow g q}}{d t d u}=-\frac{8}{3} \pi \alpha_{s}\left(Q^{2}\right) \alpha e_{Q}^{2}\left[\frac{s^{2}+t^{2}}{s t}\right] \delta(s+t+u) .
\end{aligned}
$$

The first is the photon-gluon fusion cross section, the only contribution to massive $Q \bar{Q}$ photoproduction [141], while the second is the QCD Compton process. At LO, the partonic cross section is proportional to $\alpha \alpha_{s}\left(Q^{2}\right) e_{Q}^{2}$, where $\alpha_{s}\left(Q^{2}\right)$ is the strong coupling constant to one loop, $\alpha=e^{2} / \hbar c$ is the electromagnetic coupling constant, and $e_{Q}$ is the quark charge, $e_{u}=e_{c}=2 / 3$ and $e_{d}=e_{s}=-1 / 3$. The partonic invariants, $s, t$, and $u$, are defined as $s=\left(k+x_{2} P_{2}\right)^{2}, t=\left(k-p_{1}\right)^{2}=\left(x_{2} P_{2}-p_{2}\right)^{2}$, and $u=\left(x_{2} P_{2}-p_{1}\right)^{2}=\left(k-p_{2}\right)^{2}$. In this case, $s=4 k \gamma_{L} x_{2} m_{p}$ where $\gamma_{L}$ is the Lorentz boost of a single beam and $m_{p}$ is the proton mass. Since $k$ can be a continuum of energies up to $E_{\text {beam }}=\gamma_{L} m_{p}$, we define $x_{1}=k / P_{1}$, analogous to the parton momentum fraction in the nucleon where $P_{1}$ is the nucleon four momentum. For a detected parton in a nucleonnucleon collision, the hadronic invariants are then $s_{N N}=\left(P_{1}+P_{2}\right)^{2}, T=\left(P_{2}-p_{1}\right)^{2}$, and $U=\left(P_{1}-p_{1}\right)^{2}$.

The produced parton rapidities are $y_{1}$ and $y_{2}$. The parton rapidity is related to the invariant $t_{N N}$ by $t_{N N}=-\sqrt{s_{N N}} p_{T} e^{-y_{1}}$. At LO, $x_{1}=\left(p_{T} / \sqrt{s_{N N}}\right)\left(e^{y_{1}}+e^{y_{2}}\right)$ and $x_{2}=\left(p_{T} / \sqrt{s_{N N}}\right)\left(e^{-y_{1}}+e^{-y_{2}}\right)$. We calculate $x_{1}$ and $x_{2}$ as in an $N N$ collision and then determine the flux in the lab frame for $k=x_{1} \gamma_{L} m_{p}$, equivalent to the center-of-mass frame in a collider. The photon flux is exponentially suppressed for $k>\gamma_{L} \hbar c / R_{A}$, corresponding to a momentum fraction $x_{1}>\hbar c / m_{p} R_{A}$. The maximum $\gamma N$ center-of-mass energy, $\sqrt{S_{\gamma N}}$, is much lower than the hadronic $\sqrt{s_{N N}}$. The equivalent hadronic invariants can be defined for photon four momentum $k$ as $s_{\gamma N}=\left(k+P_{2}\right)^{2}$, $t_{\gamma N}=\left(P_{2}-p_{1}\right)^{2}$, and $u_{\gamma N}=\left(k-p_{1}\right)^{2}$ [198]. The partonic and equivalent hadronic invariants for fixed $k$ are related by $s=x_{2} s_{\gamma N}, t=u_{\gamma N}$, and $u=x_{2} t_{\gamma N}$.

The direct jet photoproduction $p_{T}$ distributions are given in Fig. 65 for $A A$ interactions at the LHC. For $\mathrm{Pb}+\mathrm{Pb}$ collisions at $\sqrt{s_{N N}}=5.5 \mathrm{TeV}$, we show the $p_{T}$ distributions of the produced quarks, antiquarks and gluons along with their sum. For $\mathrm{Ar}+\mathrm{Ar}$ collisions at $\sqrt{s_{N N}}=6.3 \mathrm{TeV}$ and $\mathrm{O}+\mathrm{O}$ collisions at $\sqrt{s_{N N}}=7 \mathrm{TeV}$, we show only the total $p_{T}$ distributions. All the results are shown in the rapidity interval $\left|y_{1}\right| \leq 1$. Extended rapidity coverage, corresponding to e.g. $\left|y_{1}\right| \leq 2.4$ for the CMS barrel and endcap systems, could increase the rates by a factor of $\approx 2$. (The increase in rate with rapidity acceptance is not linear in $\left|y_{1}\right|$ because the rapidity distributions are asymmetric around $y_{1}=0$ and increasing $p_{T}$ narrows the rapidity distribution. The effect of changing the $y_{1}$ cut is closer to linear at low $p_{T}$ and larger at high $p_{T}$ because the peak is at $y_{1}<-1$ for large $p_{T}$, as seen in the $y_{1}$ distributions on the right-hand side of Fig. 66.) At $p_{T} \approx 100 \mathrm{GeV}$, the cross sections are small.

There is a difference of $\approx 500$ in the $\mathrm{Pb}+\mathrm{Pb}$ and $\mathrm{O}+\mathrm{O}$ cross sections at $p_{T} \approx 10$ $\mathrm{GeV}$, decreasing to less than a factor of four at $\approx 400 \mathrm{GeV}$. The difference decreases with $p_{T}$ due to the larger phase space available at high $p_{T}$ for the higher $\sqrt{s_{N N}}$ systems. 
The rates are nearly the same for all systems because the higher luminosities and higher $\sqrt{s_{N N}}$ compensate for the lower $A$ in lighter systems.

The dijet jet hadroproduction cross sections are much higher because hard processes increase with the number of binary collisions in $A A$ collisions, a factor of $\approx A^{2}$ for the minimum bias cross section. (The relation is not exact due to shadowing.) Integration over all impact parameters leads to $\approx A^{2}$ scaling while there is only a factor of $A$ in dijet photoproduction since the photon flux is already integrated over impact parameter for $b>2 R_{A}$. This, combined with the lower effective energy and fewer channels considerably reduces the photoproduction rates relative to hadroproduction.

Quarks and antiquarks are produced in greatest abundance, with only a small difference at high $p_{T}$. Photon-gluon fusion alone produces equal numbers of quarks and antiquarks. The quark excess arises from the QCD Compton diagram which also produces the small final-state gluon contribution. The $\gamma(q+\bar{q})$ contribution grows with $p_{T}$ since the valence quark distributions eventually dominate production, as shown in Fig. 65(b) where the $\gamma g$ contribution is compared to the total. At low $p_{T}$, the $\gamma g$ contribution is $\approx 90 \%$ of the total, dropping to $10-30 \%$ at $p_{T} \approx 400 \mathrm{GeV}$. At the large values of $x$ needed for high $p_{T}$ jet production, $f_{p}^{u_{V}}>f_{p}^{g}$. Thus the QCD Compton process eventually dominates dijet production, albeit in a region of very low statistics. The $\gamma g$ contribution is larger for the lighter nuclei since the higher energies reduce the average $x$ values.

The direct dijet photoproduction cross section is significantly lower than the hadroproduction cross section. Some of this reduction is due to the different couplings. The photoproduction rate is reduced by a factor of $\alpha e_{Q}^{2} / \alpha_{s}, \approx 100$. There are also fewer diagrams for jet photoproduction relative to all $2 \rightarrow 2$ scatterings in hadroproduction. In addition, the $g g \rightarrow g g$ hadroproduction process, with its high parton luminosity, has no direct photoproduction equivalent.

Since the typical scales of jet production are large, the effects of shadowing, reflected in $R\left(p_{T}\right)=\left(d \sigma\left[S_{A}^{i}\right] / d p_{T}\right) /\left(d \sigma\left[S_{A}^{i}=1\right] / d p_{T}\right)$, are rather small, see Fig. $65(\mathrm{c})$ and $(\mathrm{d})$, because the average $x$ is high. The differences between the two shadowing parameterizations are on the few percent level. At low $p_{T}$, the produced quarks and antiquarks are mainly from gluons. The produced gluons only come from quarks. The peak for the produced quarks and antiquarks in $R\left(p_{T}\right)$ between $50 \leq p_{T} \leq 100 \mathrm{GeV}$ is due to gluon anti-shadowing. The total $R\left(p_{T}\right)$ for all produced partons in $\mathrm{Pb}+\mathrm{Pb}$ collisions is dominated by the $\gamma g$ contribution. The maximum value of $S_{A}^{i}$ in the antishadowing region is $\approx 1.07$ for EKS98 and $\approx 1.1$ for FGS, reflecting the high $Q^{2}$ behavior of the shadowing parameterizations.

The EKS98 ratios for the produced quarks and antiquarks in Fig. 65(c) follow $R\left(p_{T}\right)$ for the total rather closely over all $p_{T}$. The quark and antiquark ratios are slightly above the total at low $p_{T}$ due to the small $\gamma q$ contribution. They continue to follow the total at high $p_{T}$ since all the EKS98 ratios exhibit similar behavior at large $x$. The produced gluon ratio follows the quark ratios for $p_{T}>200 \mathrm{GeV}$. The large $p_{T}$ contribution arises from the valence quarks. Some antishadowing is observed at low $p_{T}$, due to the valence 

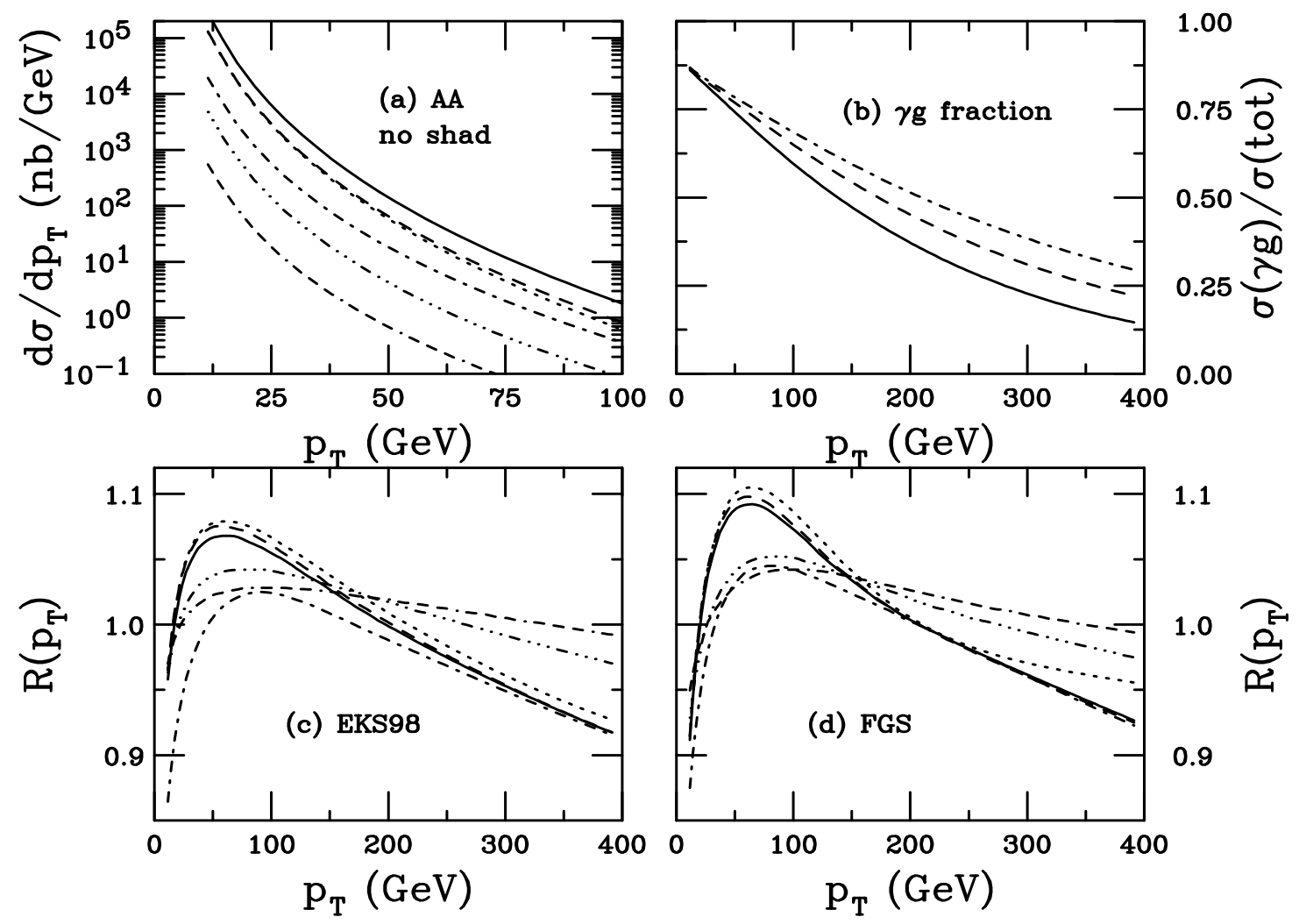

Figure 65. Direct dijet photoproduction in peripheral collisions. (a) The $p_{T}$ distributions for $\left|y_{1}\right| \leq 1$ are shown for $A A$ collisions. The solid curves is the total for $\mathrm{Pb}$ ions while the produced quarks (dashed), antiquarks (dotted) and gluons (dotdashed) are shown separately. The total production for Ar (dot-dot-dot-dashed) and $\mathrm{O}$ (dot-dash-dash-dashed) ions are also shown. (b) The fraction of gluon-initiated jets as a function of $p_{T}$ for $\mathrm{Pb}+\mathrm{Pb}$ (solid), $\mathrm{Ar}+\mathrm{Ar}$ (dashed) and $\mathrm{O}+\mathrm{O}$ (dot-dashed) interactions. (c) The EKS98 shadowing ratios for produced partons. The solid curve is the total for $\mathrm{Pb}$ ions while the ratios for produced quarks (dashed), antiquarks (dotted) and gluons (dot-dashed) are shown separately. The total ratios for Ar (dot-dot-dotdashed) and O (dot-dash-dash-dashed) ions are also shown. (d) The same as (c) for FGS.

quark contribution. The total ratios for the lighter ions are closer to unity for all $p_{T}$ due to their smaller $A$.

The results are similar for FGS, shown in Fig. 65(d), but with some subtle differences. The ratio $R\left(p_{T}\right)$ for produced gluons, arising from the $\gamma q$ contribution, exhibits a larger antishadowing effect on $R\left(p_{T}\right)$ because $S_{A}^{\bar{q}}$ is higher for this parametrization, see Fig. 55. The FGS antiquark shadowing ratio goes to unity for $x>0.2$, causing the flattening of $R\left(p_{T}\right)$ for antiquarks (dotted curve) due to the contribution from $\gamma \bar{q} \rightarrow g \bar{q}$. The FGS valence quark ratio is taken from EKS98, resulting in the similarity of $R\left(p_{T}\right)$ in Figs. 65(c) and (d) at high $p_{T}$.

Some care must be taken when applying these parameterizations to high $p_{T}$ since the upper limit of their fit range is $100 \mathrm{GeV}$. While no extraordinary effects are seen in their behavior beyond this scale, the results should be taken as indicative only. Finally, 
we remark that we have only considered the range $\left|y_{1}\right| \leq 1$. Including contributions from all rapidities would increase the effect of shadowing since large $\left|y_{1}\right|$ corresponds to smaller $x$ values.
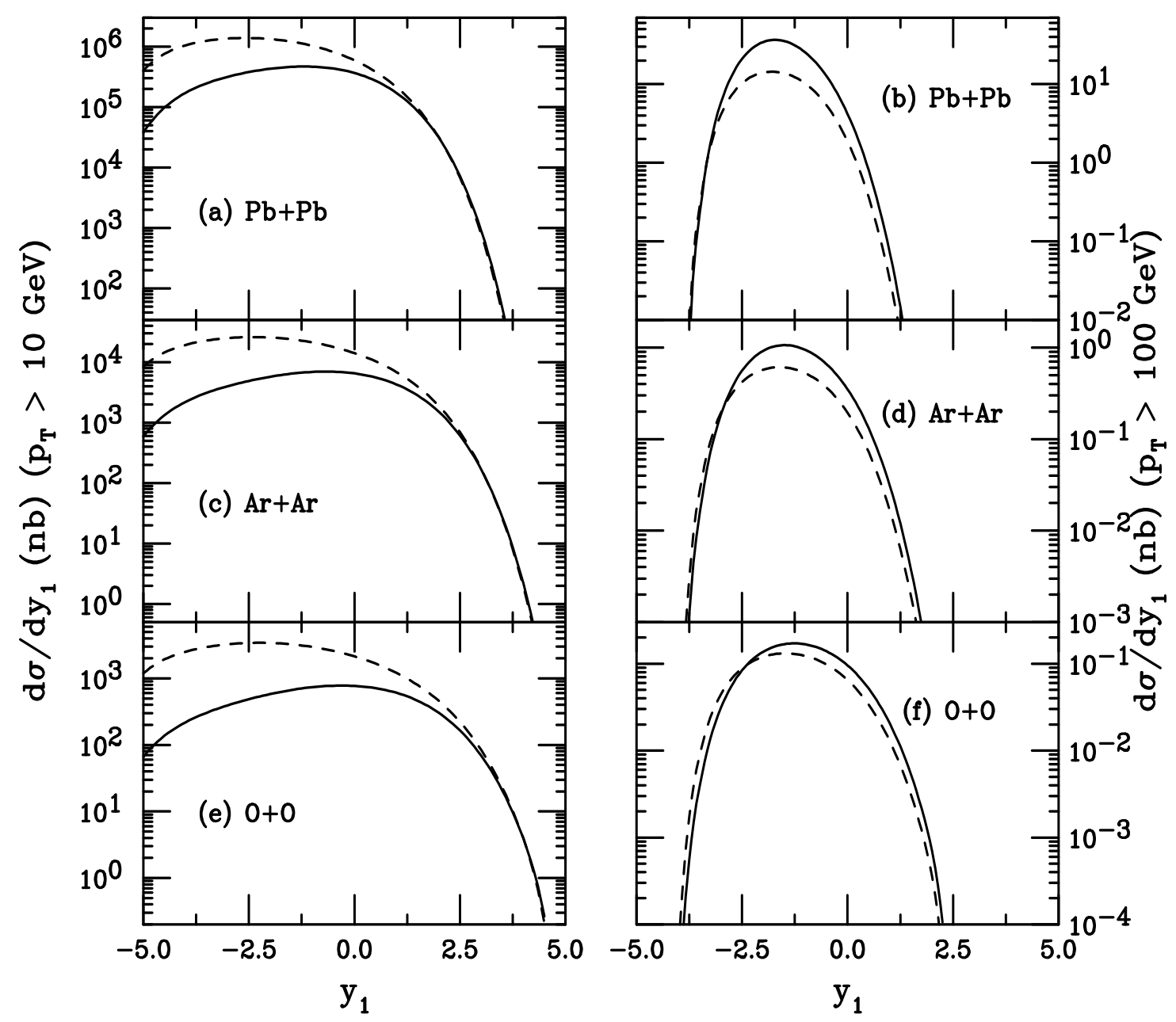

Figure 66. We compare the rapidity distributions of direct and resolved dijet production without shadowing in peripheral collisions. The left-hand side shows the results for $p_{T}>10 \mathrm{GeV}$ for (a) $\mathrm{Pb}+\mathrm{Pb}$, (c) $\mathrm{Ar}+\mathrm{Ar}$ and (e) $\mathrm{O}+\mathrm{O}$ collisions while the right-hand side is for $p_{T}>100 \mathrm{GeV}$ for (b) $\mathrm{Pb}+\mathrm{Pb}$, (d) $\mathrm{Ar}+\mathrm{Ar}$ and (f) $\mathrm{O}+\mathrm{O}$ collisions. The solid curves are the direct results while the dashed curves show the resolved results. The photon is coming from the left.

Figure 66 shows the rapidity distributions with two different values of the minimum $p_{T}, p_{T}>10 \mathrm{GeV}$ on the left-hand side and $p_{T}>100 \mathrm{GeV}$ on the right-hand side. The results, given by the solid curves, are shown without nuclear shadowing effects. In this case, the photon comes from the left. There is a symmetric case where the photon comes from the right, the factor of two on the $p_{T}$ distribution in Eq. (95). In this case, the $y_{1}$ distribution is reflected around $y_{1}=0$. With the $10 \mathrm{GeV}$ cut, the distributions are rather broad at negative $y_{1}$ where the photon has small momentum and, hence, large flux. At large $y_{1}>0$, corresponding to small $x$ for the nucleon momentum fractions and high photon momentum, the distributions fall rapidly since at high photon momenta, 
the photon flux is cut off as $k \rightarrow k_{\max }$. The distributions with the $100 \mathrm{GeV}$ cutoff are narrower because the edge of phase space is reached at lower values of $y_{1}$. The rapidity distributions are broader in general for the lighter systems due to the higher $\sqrt{S_{N N}}$.

Figure 67 gives the ratio $R\left(y_{1}\right)=\left(d \sigma\left[S_{A}^{i}\right] / d y_{1}\right) /\left(d \sigma\left[S_{A}^{i}=1\right] / d y_{1}\right)$ for the two $p_{T}$ cuts. The ratios reflect the direction of the photon, showing an antishadowing peak at $y_{1} \sim-3$, an EMC region at $y_{1}<-4$ and a shadowing region for $y_{1}>-0.5$ for $p_{T}>10$ $\mathrm{GeV}$, the left-hand side of Fig. 67. The shadowing effect is not large, $(20-25) \%$ at $y_{1} \sim 4$ for $\mathrm{Pb}+\mathrm{Pb}$ collisions and decreasing with $A$. The antishadowing peak is higher for FGS while its shadowing effect is larger at positive $y_{1}$, as also noted in the $p_{T^{-}}$ dependent ratios in Fig. 65. A comparison of the average effect around $\left|y_{1}\right| \leq 1$ with the $p_{T}$ ratios shown in Fig. 65, are in good agreement. Even though $x_{2}$ is smaller for the lighter systems, the shadowing effect is also reduced. Since shadowing also decreases with $Q^{2}$, the effect is even smaller for $p_{T}>100 \mathrm{GeV}$, shown on the right-hand side of Fig. 67. Here the rise at $y_{1}<-3.5$ is the Fermi motion as $x_{2} \rightarrow 1$. At $y_{1}>-1.2$, the antishadowing region is reached. The effect is rather small here, only $\sim 5 \%$ at $y_{1} \geq 0$.

We now turn to final-state particle production in the hadronization of jets. The particle with the highest $p_{T}$ is called the "leading" particle. The corresponding leading particle $p_{T}$ distribution is [208]

$$
\begin{aligned}
\frac{d \sigma_{\gamma A \rightarrow h X}^{\operatorname{dir}}}{d p_{T}}= & 4 p_{T} \int_{\theta_{\min }}^{\theta_{\max }} \frac{d \theta_{\mathrm{cm}}}{\sin \theta_{\mathrm{cm}}} \int d k \frac{d N_{\gamma}}{d k} \int \frac{d x_{2}}{x_{2}} \\
& \times\left[\sum_{i, l=q, \bar{q}, g} F_{i}^{A}\left(x_{2}, Q^{2}\right) \frac{d \sigma_{\gamma i \rightarrow l X^{\prime}}}{d t} \frac{D_{h / l}\left(z_{c}, Q^{2}\right)}{z_{c}}\right]
\end{aligned}
$$

where the $X$ on the left-hand side includes all final-state hadrons in addition to $h$ but $X^{\prime}$ on the right-hand side denotes the unobserved final-state parton. The subprocess cross sections, $d \sigma / d t$, are related to $s^{2} d \sigma / d t d u$ in Eq. (95) through the momentumconserving delta function $\delta(s+t+u)$ and division by $s^{2}$. The integrals over rapidity have been replaced by an integral over center-of-mass scattering angle, $\theta_{\min } \leq \theta_{\mathrm{cm}} \leq \theta_{\max }$, corresponding to a given rapidity cut. Here $\theta_{\min }=0$ and $\theta_{\max }=\pi$ covers the full rapidity range while $\theta_{\min }=\pi / 4$ and $\theta_{\max }=3 \pi / 4$ roughly corresponds to $\left|y_{1}\right| \leq 1$. The fraction of the final hadron momentum relative to that of the produced parton, $z_{c}$, appears in the fragmentation function, $D_{h / l}\left(z_{c}, Q^{2}\right)$, the probability to produce hadron $h$ from parton $l$. The fragmentation functions are assumed to be universal, independent of the initial state.

The produced partons are fragmented into charged pions, charged kaons and protons/antiprotons using LO fragmentation functions fit to $e^{+} e^{-}$data [209]. The final-state hadrons are assumed to be produced pairwise so that $\pi \equiv\left(\pi^{+}+\pi^{-}\right) / 2$, $K \equiv\left(K^{+}+K^{-}\right) / 2$, and $p \equiv(p+\bar{p}) / 2$. The equality of $p$ and $\bar{p}$ production obviously does not describe low energy hadroproduction well. As energy increases, this approximation may become more reasonable. The produced hadrons follow the parent parton direction. We have used the LO KKP fragmentation functions [209]. The KKP scale evolution is modeled using $e^{+} e^{-}$data at several different energies and compared to $p \bar{p}, \gamma p$ and $\gamma \gamma$ 

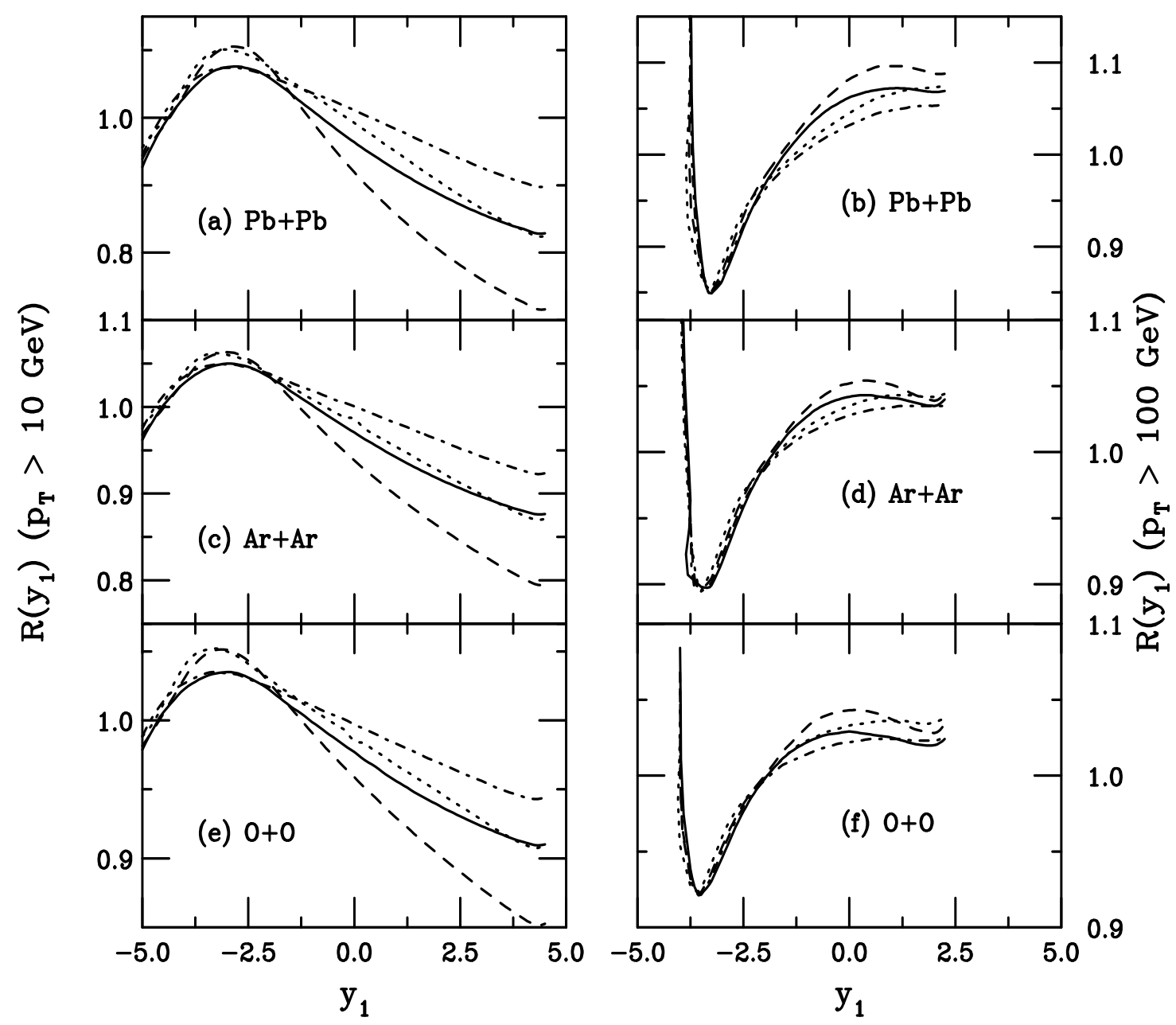

Figure 67. We compare shadowing ratios in direct and resolved dijet production in peripheral collisions. The left-hand side shows the results for $p_{T}>10 \mathrm{GeV}$ for (a) $\mathrm{Pb}+\mathrm{Pb},(\mathrm{c}) \mathrm{Ar}+\mathrm{Ar}$ and (e) $\mathrm{O}+\mathrm{O}$ collisions while the right-hand side is for $p_{T}>100$ $\mathrm{GeV}$ for (b) $\mathrm{Pb}+\mathrm{Pb}$, (d) $\mathrm{Ar}+\mathrm{Ar}$ and (f) $\mathrm{O}+\mathrm{O}$ collisions. The solid and dashed curves give the direct ratios for the EKS98 and FGS parameterizations respectively. The dot-dashed and dotted curves show the resolved ratios for the EKS98 and FGS parameterizations respectively. The photon comes from the left. Note the difference in the $y$-axis scales here.

data. After some slight scale modifications [210] all the $h^{-}$data could be fit. However, there are significant uncertainties in fragmentation when the leading hadron takes most of the parton momentum [211], as is the case here.

We assume the same scale in the parton densities and the fragmentation functions, $Q^{2}=p_{T}^{2}$. A larger scale, $p_{T}^{2} / z_{c}^{2}$, has sometimes been used in the parton densities. At high $p_{T}$, where $z_{c}$ is large, the difference in the results for the two scales is small. We have not included any intrinsic transverse momentum broadening in our calculations [212, 213]. This "Cronin" effect can be important when $p_{T}$ is small but becomes negligible for transverse momenta larger than a few $\mathrm{GeV}$.

The corresponding hadron distributions from direct jet photoproduction are shown 

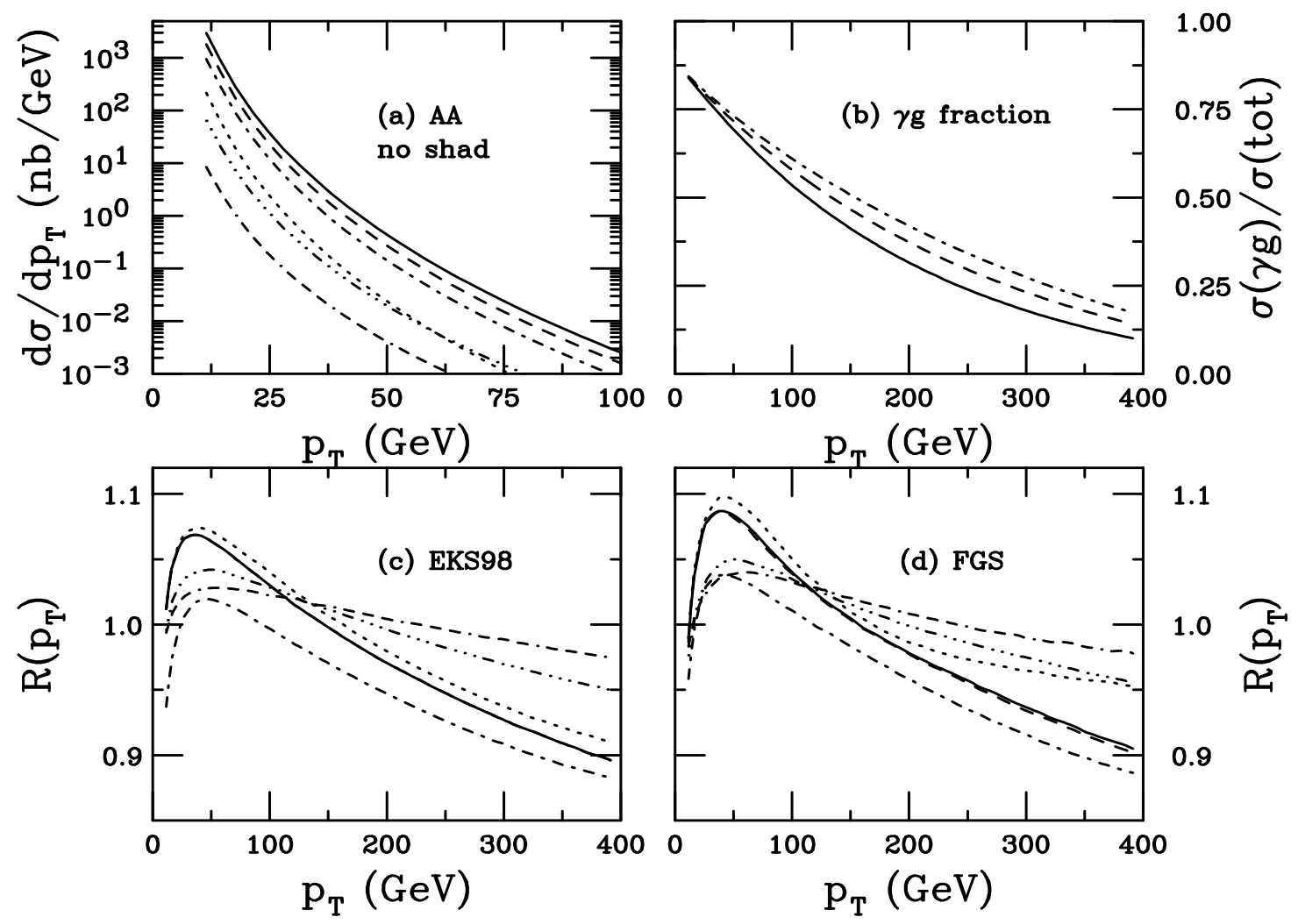

Figure 68. Direct photoproduction of leading hadrons in peripheral collisions. (a) The $p_{T}$ distributions for $\left|y_{1}\right| \leq 1$ are shown for $A A$ collisions. The solid curve is the total for $\mathrm{Pb}+\mathrm{Pb}$ while the produced pions (dashed), kaons (dot-dashed) and protons (dotted) are shown separately. The total production for $\mathrm{Ar}+\mathrm{Ar}$ (dot-dot-dot-dashed) and $\mathrm{O}+\mathrm{O}$ (dot-dash-dash-dashed) are also shown. (b) The fraction of gluon-initiated hadrons as a function of $p_{T}$. The curves are the same as in (a). (c) The EKS98 shadowing ratios for produced pions. The solid curve is the total for $\mathrm{Pb}+\mathrm{Pb}$ while the ratios for pions produced by quarks (dashed), antiquarks (dotted) and gluons (dotdashed) are shown separately. The total ratios for $\mathrm{Ar}+\mathrm{Ar}$ (dot-dot-dot-dashed) and $\mathrm{O}+\mathrm{O}$ (dot-dash-dash-dashed) are also shown. (d) The same as (c) for FGS.

in Fig. 68(a) for $A A$ collisions. The largest contribution to the total final-state charged particle production is charged pions, followed by kaons and protons. Note that the leading hadron cross sections are lower than the partonic jet cross sections, compare Figs. 65(a) and 68(a). Several factors can account for this. The maximum $\sqrt{s_{\gamma N}}$ is a factor of five or more less than $\sqrt{s_{N N}}$ for $A A$ collisions. The reduced number of processes available for direct dijet photoproduction is a significant contribution to the decrease. Note also that the $p_{T}$ distribution is steeper for leading hadrons than for the jets, as may be expected since the effective $p_{T}$ of the hadron is higher than than of the produced parton.

The average $z_{c}$ for direct photoproduction of high $p_{T}$ particles is $\approx 0.4$ for particles with $p_{T} \approx 10 \mathrm{GeV}$, increasing to $\left\langle z_{c}\right\rangle>0.45-0.55$ for $p_{T}>100 \mathrm{GeV}$. The lower $z_{c}$ values correspond to lighter ion collisions. In this $z_{c}$ region, the fragmentation 
functions are not very well known. As pointed out in Ref. [211], a small change in the fragmentation function fits can produce significant changes at large $z_{c}$. This region is not well constrained by the $e^{+} e^{-}$data used in the fits.

The effect of fragmentation on the production channels is shown in Fig. 68(b) where we present the fraction of leading hadron production from the $\gamma g$ channel for all charged hadrons. The ratios are rather similar to those of the partonic jets although the $\gamma g$ fraction is somewhat smaller due to the larger average $x$ of hadron production with respect to jets, as we discuss later.

The shadowing ratios for charged pions produced in $\mathrm{Pb}+\mathrm{Pb}$ collisions by quarks, antiquarks, gluons and the total from all partons, are shown for the EKS98 and FGS parameterizations in Fig. 68(c) and (d). The ratios for pion production in $\mathrm{Ar}+\mathrm{Ar}$ and $\mathrm{O}+\mathrm{O}$ collisions are also shown. The high $p_{T}$ FGS antiquark ratios flatten out relative to the EKS98 ratio because the $\gamma \bar{q}$ channel dominates gluon production at high $p_{T}$. The flattening behavior sets in earlier here because the $x$ for hadron production is higher than that for the jets. The ratio of pions arising from produced gluons follows the valence ratio, as expected. The ratios decrease with increasing $p_{T}$ due to the EMC effect for $x>0.2$ when $p_{T}>100 \mathrm{GeV}$.
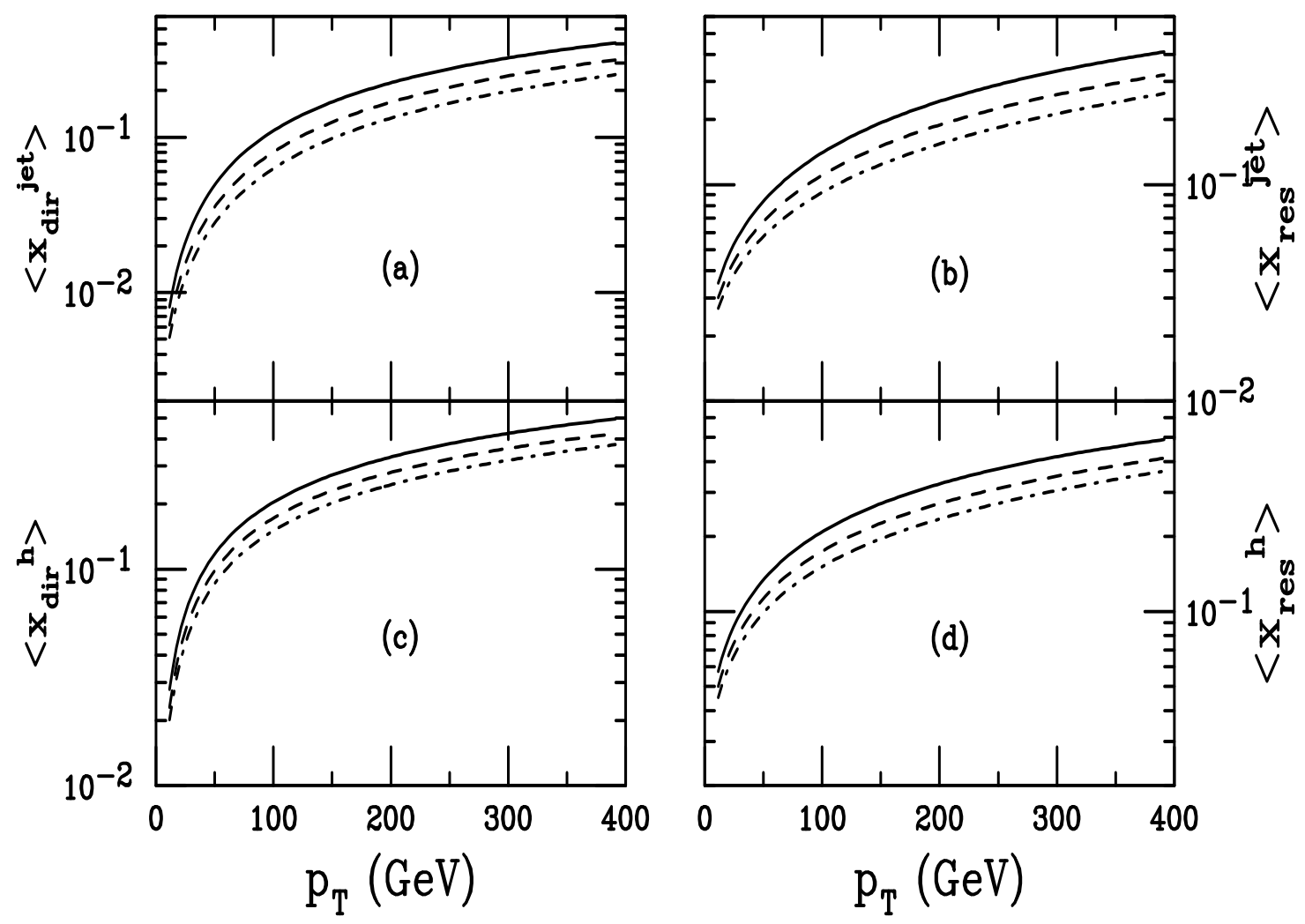

Figure 69. The average value of the nucleon parton momentum fraction $x$ as a function of transverse momentum. Results are given for (a) direct and (b) resolved gluon jet production and for (c) direct and (d) resolved pion production by gluons. The results are given for $\mathrm{O}+\mathrm{O}$ (dot-dashed), $\mathrm{Ar}+\mathrm{Ar}$ (dashed) and $\mathrm{Pb}+\mathrm{Pb}$ (solid) interactions. 
We now discuss the relative values of the nucleon momentum fraction, $x$ for parton and hadron production. On the left-hand side of Fig. 69, we compare the average $x$ values for produced gluon jets (upper plot) and for pions produced by these gluons (lower plot). We have chosen to compute the results for produced gluons alone to better compare with resolved jet photoproduction, discussed next. Since we are interested in produced gluons, we only consider the QCD Compton contribution, $\gamma q \rightarrow g q$. This channel is biased toward larger momentum fractions than $\gamma g \rightarrow q \bar{q}$ since the gluon distribution is largest at small $x$ while the valence quark distribution in the proton is peaked at $x \sim 0.2$. The average $x$ for a gluon jet is $\sim 0.005-0.008$ at $p_{T} \approx 10 \mathrm{GeV}$, increasing to $\sim 0.03-0.05$ at $50 \mathrm{GeV}$. The smallest $x$ is from the highest energy $\mathrm{O}+\mathrm{O}$ collisions. The average $x$ increases with $p_{T}$, to $\sim 0.25-0.4$ at $p_{T} \sim 400 \mathrm{GeV}$. When final state pions are considered, in the lower left-hand plot, at low $p_{T}$, the average $x$ is larger than for gluon jets. At $p_{T} \approx 10 \mathrm{GeV},\langle x\rangle \approx 0.02-0.03$ while at $50 \mathrm{GeV}$, $\langle x\rangle \approx 0.09-0.12$. At high $p_{T}$, however, the average $x$ becomes similar for jet and hadron production as $\left\langle z_{c}\right\rangle$ increases to $\approx 0.6-0.7$ at $p_{T} \sim 400 \mathrm{GeV}$.

We now turn to resolved production. The hadronic reaction, $\gamma N \rightarrow$ jet + jet $+X$, is unchanged, but in this case, prior to the interaction with the nucleon, the photon splits into a color singlet state of $q \bar{q}$ pairs and gluons. On the parton level, the resolved LO reactions are e.g. $g(x k)+g\left(x_{2} P_{2}\right) \rightarrow g\left(p_{1}\right)+g\left(p_{2}\right)$ where $x$ is the fraction of the photon momentum carried by the parton. The LO processes for resolved photoproduction are the same as those for LO $2 \rightarrow 2$ hadroproduction except that one parton source is a photon rather than a nucleon. The resolved jet photoproduction cross section for partons of flavor $f$ in the subprocess $i j \rightarrow k l$ in $A B$ collisions is, modified from Refs. [181, 206, 207],

$$
\begin{aligned}
s_{N N}^{2} \frac{d \sigma_{\gamma A \rightarrow \mathrm{jet}+\mathrm{jet}}^{\mathrm{res}}}{d t_{N N} d u_{N N}}= & 2 \int_{k_{\min }}^{\infty} \frac{d k}{k} \frac{d N_{\gamma}}{d k} \int_{k_{\min } / k}^{1} \frac{d x}{x} \int_{x_{2 \min }}^{1} \frac{d x_{2}}{x_{2}} \\
& \times \sum_{\substack{i j=\\
k k l\rangle}}\left\{F_{i}^{\gamma}\left(x, Q^{2}\right) F_{j}^{A}\left(x_{2}, Q^{2}\right)+F_{j}^{\gamma}\left(x, Q^{2}\right) F_{i}^{A}\left(x_{2}, Q^{2}\right)\right\} \\
& \times \frac{1}{1+\delta_{k l}}\left[\delta_{f k} \hat{s}^{2} \frac{d \sigma^{i j \rightarrow k l}}{d \hat{t} d \hat{u}}(\hat{t}, \hat{u})+\delta_{f l} \hat{s}^{2} \frac{d \sigma^{i j \rightarrow k l}}{d \hat{t} d \hat{u}}(\hat{u}, \hat{t})\right]
\end{aligned}
$$

where $\hat{s}=\left(x k+x_{2} P_{2}\right)^{2}, \hat{t}=\left(x k-p_{1}\right)^{2}$, and $\hat{u}=\left(x_{2} P_{2}-p_{1}\right)^{2}$. The $2 \rightarrow 2$ minijet subprocess cross sections, $d \sigma / d \hat{t}$, given in the Ref. [214], are related to $d \sigma / d \hat{t} d \hat{u}$ through the momentum-conserving delta function $\delta(\hat{s}+\hat{t}+\hat{u})$. The sum over initial states includes all combinations of two parton species with three flavors while the final state includes all pairs without a mutual exchange and four flavors (including charm). The factor $1 /\left(1+\delta_{k l}\right)$ accounts for identical particles in the final state.

The resolved jet results, shown in Fig. 70, are independent of the photon parton densities for $p_{T}>10 \mathrm{GeV}$. Along with the total quark, antiquark and gluon cross sections in $\mathrm{Pb}+\mathrm{Pb}$ collisions, we also show the individual partonic contributions to the jet $p_{T}$ distributions. The produced gluon contribution dominates for $p_{T}<25 \mathrm{GeV}$ but, by $50 \mathrm{GeV}$, quark and antiquark production becomes larger due to the increase 


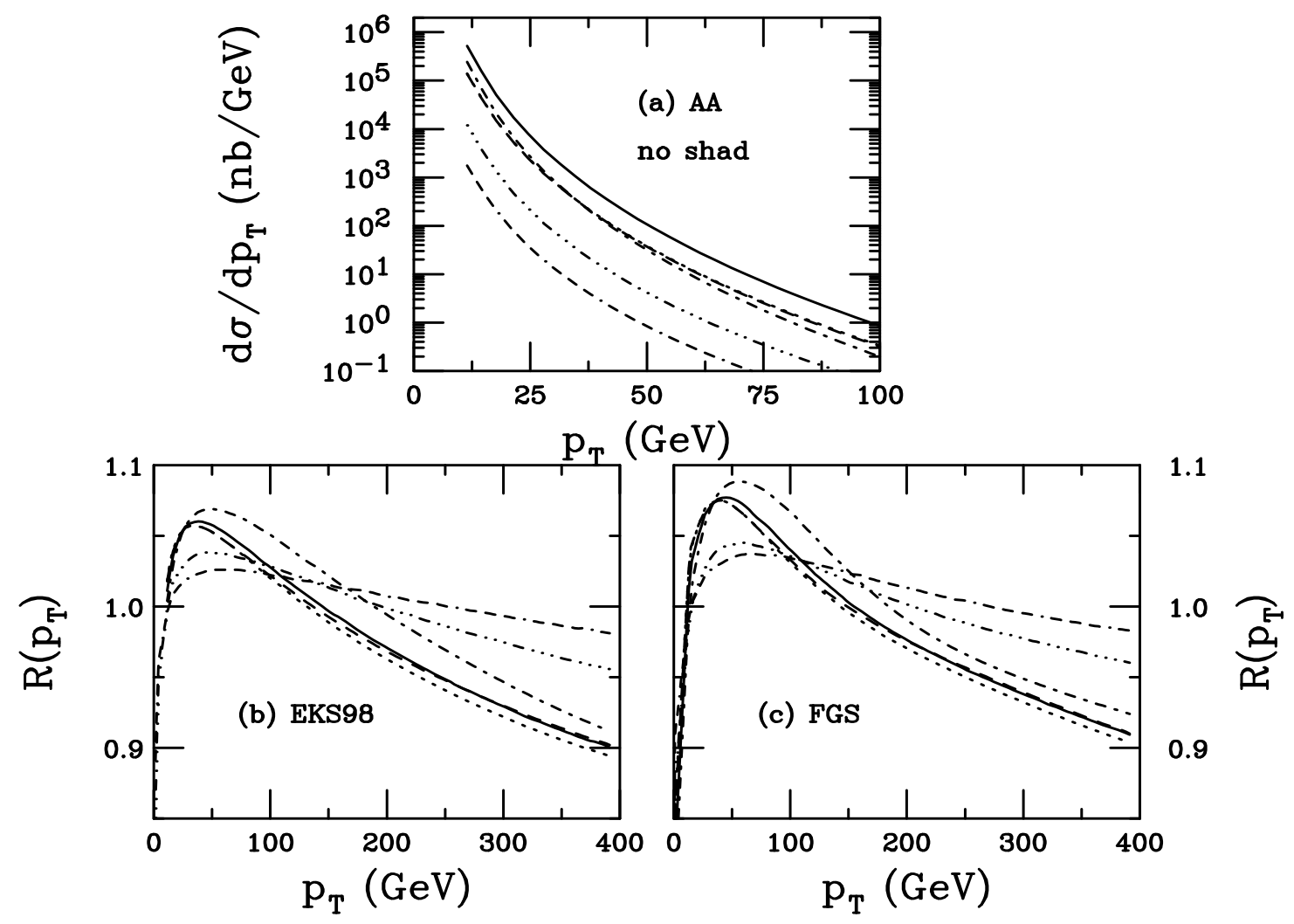

Figure 70. Resolved dijet photoproduction in peripheral $A A$ collisions. (a) The $\mathrm{Pb}+\mathrm{Pb}$ jet $p_{T}$ distributions with $\left|y_{1}\right| \leq 1$ are shown for quarks (dashed), antiquarks (dotted), gluons (dot-dashed) and the total (solid). We also show the total jet $p_{T}$ distributions in $\mathrm{Ar}+\mathrm{Ar}$ (dot-dot-dot-dashed) and $\mathrm{O}+\mathrm{O}$ (dash-dash-dash-dotted) collisions. (b) The relative EKS98 shadowing contributions from quarks (dashed), antiquarks (dotted) and gluons (dot-dashed) as well as the total (solid) are shown for $\mathrm{Pb}+\mathrm{Pb}$ collisions. The totals are also shown for $\mathrm{Ar}+\mathrm{Ar}$ (dot-dot-dot-dashed) and $\mathrm{O}+\mathrm{O}$ (dash-dash-dash-dotted) interactions. (c) The same as (b) for FGS.

of the $q g \rightarrow q g$ channel relative to the $g g \rightarrow g g$ channel. We also show the total $p_{T}$ distributions for $\mathrm{Ar}+\mathrm{Ar}$ and $\mathrm{O}+\mathrm{O}$ collisions. For lighter nuclei, the crossover between gluon and quark/antiquark dominance occurs at higher $p_{T}$ due to the higher collision energy.

The resolved dijet photoproduction contribution is two to three times larger than the direct for $p_{T}<50 \mathrm{GeV}$, despite the lower effective center-of-mass energy of resolved production. The largest increase is for the lightest nuclei since the lowest $x$ values are probed. However, with increasing $p_{T}$, the phase space is reduced. The average photon momentum is increased and, at large photon momentum, the flux drops faster. The average momentum fractions probed by the nuclear parton densities grows large and only valence quarks contribute. The lower effective energy of resolved relative to direct photoproduction reduces the high $p_{T}$ phase space for resolved production. Thus, at the highest $p_{T}$, the resolved rate is reduced relative to the direct by a factor of $4-9$. The smallest decrease is for the lightest system due to the higher effective $\sqrt{s_{N N}}$. 
Since resolved production has a narrower rapidity distribution than direct production, increasing the rapidity coverage would not increase the rate as much as for direct photoproduction.

Resolved production opens up many more channels through the parton components of the photon. Indeed, now all the $2 \rightarrow 2$ channels for LO jet hadroproduction are available. In addition, the quark and antiquark distributions in the photon are the same. These distributions are large at high momentum fractions, higher than the quark and antiquark distributions in the proton. Thus the quark and antiquark channels are enhanced relative to hadroproduction. The largest difference between the quark and antiquark production rates is due to the difference between the valence and sea quark distributions in the nucleus. Where the valence and sea quark contributions are similar, as for $\left|y_{1}\right| \leq 1$, the difference is rather small. If all rapidities were included, the relative quark and antiquark rates could differ more.

The direct and resolved rapidity distributions are compared in Fig. 66 for the two $p_{T}$ cuts, 10 and $100 \mathrm{GeV}$. While the $\left|y_{1}\right| \leq 1$ resolved contribution is a factor of two to three larger than the direct at $p_{T}<50 \mathrm{GeV}$, a comparison of the $y_{1}$ distributions over all rapidities shows that the resolved contribution can be considerably larger, a factor of $\sim 5-10$ at $y_{1}<-3.5$ for $p_{T}>10 \mathrm{GeV}$. At $p_{T}>100 \mathrm{GeV}$, the resolved contribution is still equivalent to or slightly larger than the direct at $y_{1}<-3$ but drops below at larger rapidities. Thus, going to higher $p_{T}$ can separate direct from resolved production, especially at forward rapidities. Recall that the produced gluons dominate resolved production at $p_{T}<25 \mathrm{GeV}$ while they are only a small contribution to direct production. The largest gluon production channels are typically $g g \rightarrow g g$ and $g q \rightarrow g q$. As $y_{1}$ becomes large and negative, the photon $x$ decreases while $x_{2}$ of the nucleon decreases, leading to the dominance of the $g q$ channel. The photon gluon distribution is largest as $x$ decreases. The valence quark distribution of the proton is also important at high $p_{T}$, causing the resolved to direct ratio to flatten for $y_{1}>-2.5$ when $p_{T}>100$ $\mathrm{GeV}$.

In Fig. 67, we compare the direct and resolved shadowing ratios $R\left(y_{1}\right)$. Shadowing is smaller for the resolved component due to the larger $x_{2}$ for resolved production. The difference in the direct and resolved shadowing ratios is reduced for larger $p_{T}$.

To directly measure the nuclear parton densities, direct production should be dominant. However, Fig. 71 shows that a $p_{T}$ cut is not very effective for dijet production, even at forward rapidity. The resolved to direct production ratios are all larger than unity for $p_{T}>10 \mathrm{GeV}$, even for large, positive $y_{1}$. While the ratio is less than 1 for $y_{1}>-2.5$ and $p_{T}>100 \mathrm{GeV}$, it is only $\sim 0.5$ for $\mathrm{Pb}+\mathrm{Pb}$, increasing to 0.8 for $\mathrm{O}+\mathrm{O}$.

Thus, although clean separation is possible at $p_{T}>100 \mathrm{GeV}$, precision parton density measurements are not possible at these $p_{T}$ 's due to the low rate. Other means of separation must then be found. Resolved processes will not be as clean as direct in the direction of the photon due to the breakup of the partonic state of the photon. The multiplicity in the photon fragmentation region will be higher than in direct production where the nucleus should remain intact. A cut on multiplicity in the photon direction 

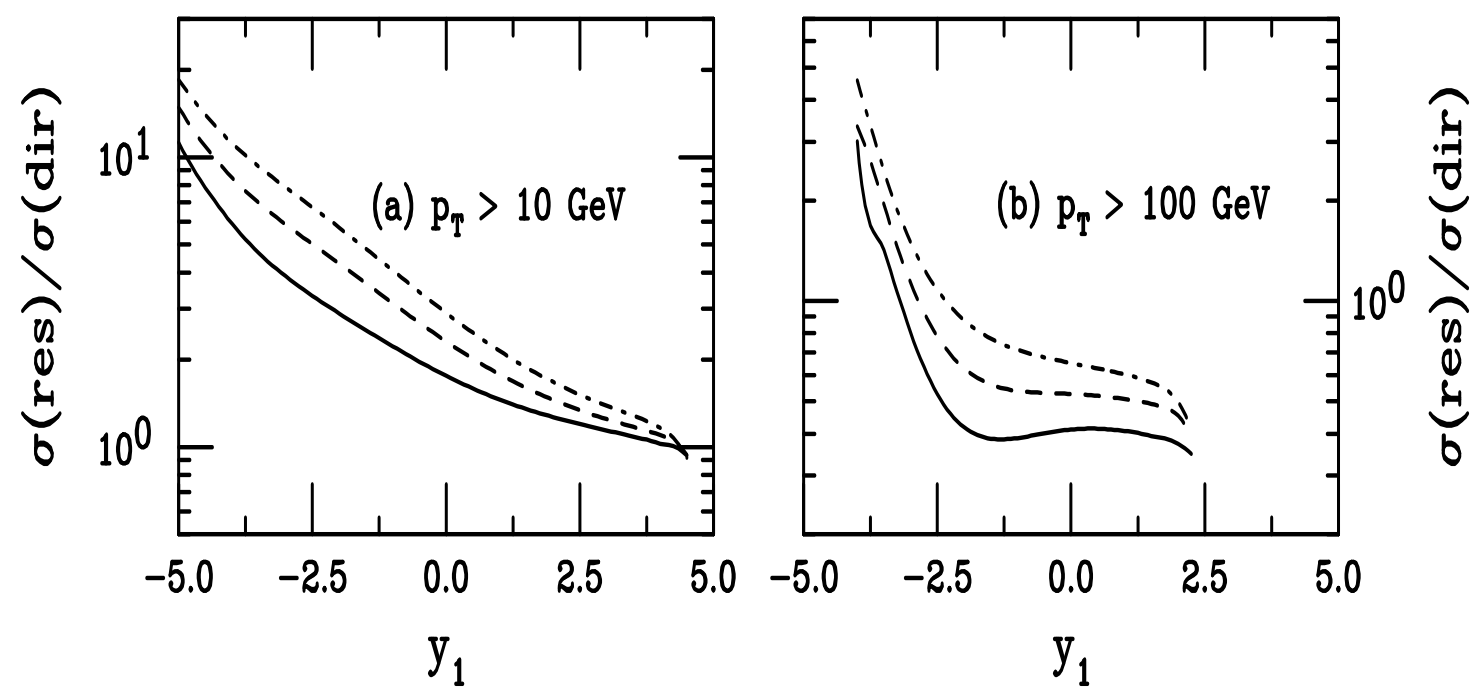

Figure 71. We present the resolved/direct dijet production ratios as a function of rapidity. In (a) we show the results for $p_{T}>10 \mathrm{GeV}$ while in (b) we show $p_{T}>100$ $\mathrm{GeV}$. The curves are $\mathrm{Pb}+\mathrm{Pb}$ (solid), $\mathrm{Ar}+\mathrm{Ar}$ (dashed) and $\mathrm{O}+\mathrm{O}$ (dot-dashed). The photon comes from the left.

may help separate the two so that, although there should be a rapidity gap for both direct and resolved photoproduction, the gap may be less prominent for resolved production.

Figure 70(b) shows the individual partonic shadowing ratios for $\mathrm{Pb}+\mathrm{Pb}$ collisions with the EKS98 parametrization. The quark and antiquark shadowing ratios are very similar although the quark ratio becomes larger for higher $p_{T}$ (higher $x$ ) values of $x$ due to the valence distribution. Now the gluon ratio shows larger antishadowing since gluon production is now through the $g g$ and $q g$ channels rather than $\gamma q$ in direct production, compare Fig. 65. The FGS parametrization gives similar results, Fig. 70(c). However, since the small FGS gluon antishadowing is stronger, $R\left(p_{T}\right)$ is larger for $p_{T}<150 \mathrm{GeV}$.

The leading particle $p_{T}$ distributions from resolved dijet photoproduction are

$$
\begin{aligned}
\frac{d \sigma_{\gamma A \rightarrow h X}^{\mathrm{res}}}{d p_{T}}= & 4 p_{T} \int_{\theta_{\min }}^{\theta_{\max }} \frac{d \theta_{\mathrm{cm}}}{\sin \theta_{\mathrm{cm}}} \int_{k_{\min }}^{\infty} \frac{d k}{k} \frac{d N_{\gamma}}{d k} \int_{k_{\min } / k}^{1} \frac{d x}{x} \int_{x_{2} \min }^{1} \frac{d x_{2}}{x_{2}} \\
& \times \sum_{\substack{i j=\\
\langle k l\rangle}}\left\{F_{i}^{\gamma}\left(x, Q^{2}\right) F_{j}^{A}\left(x_{2}, Q^{2}\right)+F_{j}^{\gamma}\left(x, Q^{2}\right) F_{i}^{A}\left(x_{2}, Q^{2}\right)\right\} \\
& \times \frac{1}{1+\delta_{k l}}\left[\delta_{f k} \frac{d \sigma^{i j \rightarrow k l}}{d \hat{t}}(\hat{t}, \hat{u})+\delta_{f l} \frac{d \sigma^{i j \rightarrow k l}}{d \hat{t}}(\hat{u}, \hat{t})\right] \frac{D_{h / k}\left(z_{c}, Q^{2}\right)}{z_{c}} .
\end{aligned}
$$

The subprocess cross sections, $d \sigma / d \hat{t}$, are related to $\hat{s}^{2} d \sigma / d \hat{t} d \hat{u}$ in Eq. (99) through the momentum-conserving delta function $\delta(\hat{s}+\hat{t}+\hat{u})$ and division by $\hat{s}^{2}$. The drop in rate between jets and high $p_{T}$ hadrons is similar to that in direct photoproduction, as can be seen by comparison of Figs. 70 and 72 relative to Figs. 65 and 68. Now that gluon fragmentation is also possible, the relative pion contribution is larger than in direct photoproduction while the relative proton contribution is significantly reduced. The smaller effective center-of-mass energy for resolved photoproduction lowers the phase 
space available for fragmentation. Baryon production is then reduced compared to light mesons.

The reduction in phase space for leading hadrons relative to fast partons can be seen in the comparison of the average $x$ values for resolved photoproduction of jets and leading hadrons, shown on the right-hand side of Fig. 69 for gluons and pions from gluons respectively. At low $p_{T}$, the average $x$ of the gluon jet is $0.03-0.04$, increasing to $0.16-0.24$ at $p_{T} \approx 200 \mathrm{GeV}$, higher than for direct photoproduction, as expected. The $x$ values for hadron production are larger still, $\approx 0.06$ at low $p_{T}$ while $x \approx 0.23-0.33$ at $p_{T} \approx 200 \mathrm{GeV}$.

The shadowing ratios in Fig. 72 also reflect the increasing $x$. Now the antishadowing peak is shifted to $p_{T} \approx 30 \mathrm{GeV}$ since the average $x$ values are in the EMC region, even at low $p_{T}$. The values of $R\left(p_{T}\right)$ at high $p_{T}$ are somewhat lower than for direct production due to the higher $x$. The average $z_{c}$ of the fragmentation functions is also somewhat larger for resolved production, $0.7-0.8$ at $p_{T} \approx 400 \mathrm{GeV}$.

Since the resolved jet cross section is larger than the direct at low $p_{T}$, it is more difficult to make clean measurements of the nuclear gluon distribution unless the two contributions can be separated by other methods. Instead, the large valence quark contribution at high $p_{T}$ suggests that jet photoproduction can probe the nuclear valence quark distributions at larger $Q^{2}$ than previously possible. At $p_{T}>100 \mathrm{GeV}$, more than half of direct jet production is through the $\gamma q$ channel. Unfortunately, the rates are low here, making high precision measurements unlikely. However, the events should be very clean.

\section{4. $\gamma+$ jet production}

\section{Contributed by: R. Vogt}

A clean method of determining the quark distribution in the nucleus at lower $p_{T}$ is the process where a jet is produced opposite a photon in the final state, Compton scattering in direct production. The cross sections are reduced relative to the jet+jet process since the coupling is $\alpha^{2} e_{Q}^{4}$ in the coupling rather than $\alpha \alpha_{s} e_{Q}^{2}$, as in dijet production. In addition, the quark distributions are lower than the gluon, also reducing the rate.

We now discuss the jet and leading particle distributions for direct and resolved $\gamma+$ jet photoproduction. Now, the hadronic process is $\gamma(k)+N\left(P_{2}\right) \rightarrow \gamma\left(p_{1}\right)+$ jet $\left(p_{2}\right)+$ $X$. The only partonic contribution to the $\gamma+$ jet yield in direct photoproduction is $\gamma(k)+q\left(x_{2} P_{2}\right) \rightarrow \gamma\left(p_{1}\right)+q\left(p_{2}\right)$ (or $q \rightarrow \bar{q}$ ) where the produced quark is massless. We now have

$$
\begin{aligned}
s_{N N}^{2} \frac{d^{2} \sigma_{\gamma A \rightarrow \gamma+\text { jet }+X}^{\operatorname{dir}}}{d t_{N N} d u_{N N}}= & 2 \int d z \int_{k_{\min }}^{\infty} d k \frac{d N_{\gamma}}{d k} \int_{x_{2_{\min }}}^{1} \frac{d x_{2}}{x_{2}} \\
& \times\left[\sum_{i=q, \bar{q}} F_{i}^{A}\left(x_{2}, Q^{2}\right) s^{2} \frac{d^{2} \sigma_{\gamma i \rightarrow \gamma i}}{d t d u}\right] .
\end{aligned}
$$




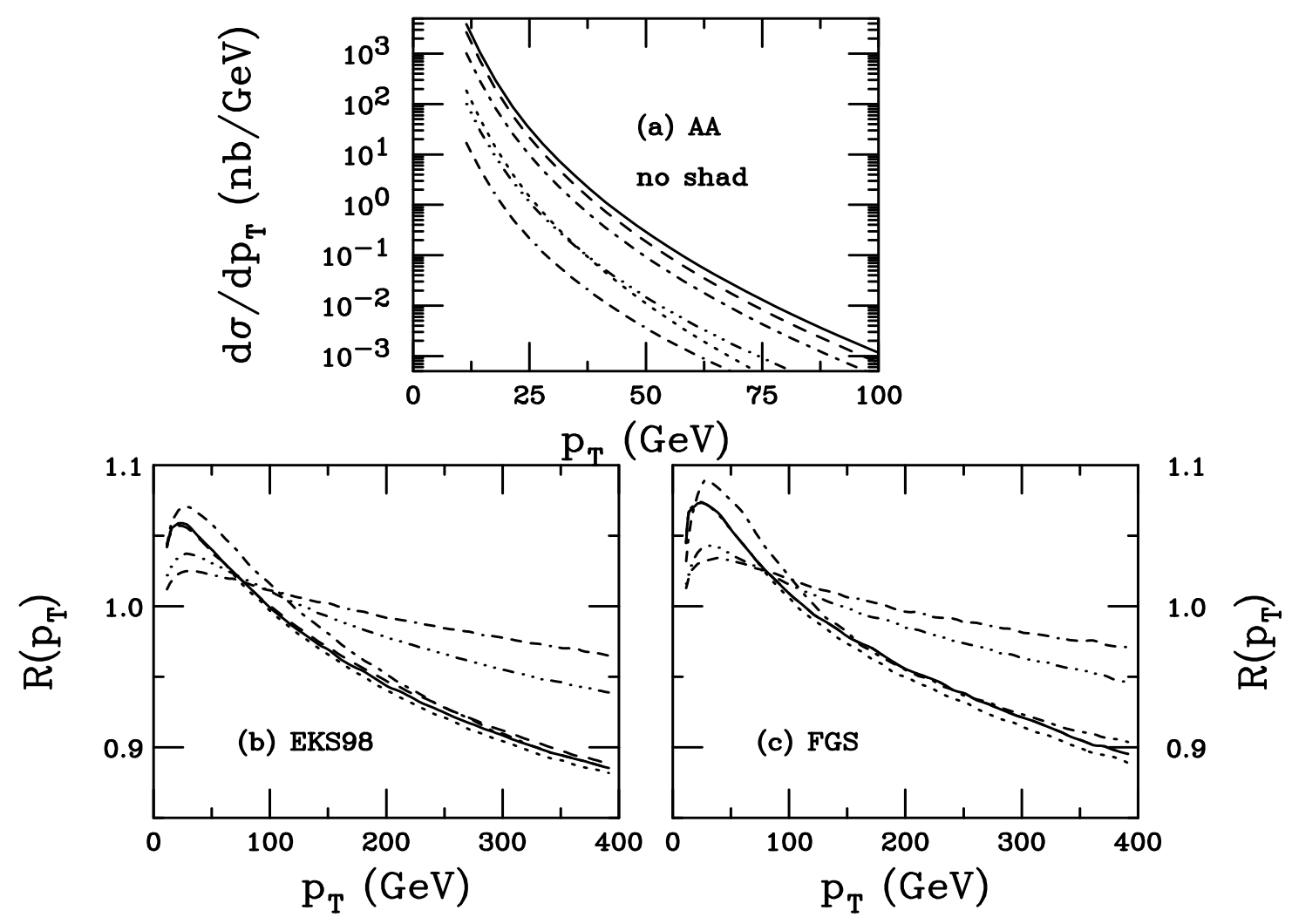

Figure 72. Resolved leading hadrons from dijet photoproduction in peripheral collisions. (a) The $p_{T}$ distributions for $\left|y_{1}\right| \leq 1$ are shown for $A A$ collisions. The $\mathrm{Pb}+\mathrm{Pb}$ results are shown for charged pions (dashed), kaons (dot-dashed), protons (dotted) and the sum of all charged hadrons (solid). The charged hadron $p_{T}$ distributions are also shown for $\mathrm{Ar}+\mathrm{Ar}$ (dot-dot-dot-dashed) and $\mathrm{O}+\mathrm{O}$ (dot-dashdash-dashed) collisions. (b) The EKS98 shadowing ratios for produced pions. For $\mathrm{Pb}+\mathrm{Pb}$ collisions, we show the ratios for pions produced by quarks (dashed), antiquarks (dotted), gluons (dot-dashed) and the total (solid) separately. The ratios for pions produced by all partons are also shown for $\mathrm{Ar}+\mathrm{Ar}$ (dot-dot-dot-dashed) and $\mathrm{O}+\mathrm{O}$ (dot-dash-dash-dashed) collisions. (c) The same as (b) for FGS.

The partonic cross section for the Compton process is

$$
s^{2} \frac{d^{2} \sigma_{\gamma q \rightarrow \gamma q}}{d t d u}=-\frac{2}{3} \pi \alpha^{2} e_{Q}^{4}\left[\frac{s^{2}+u^{2}}{s u}\right] \delta(s+t+u) .
$$

The extra factor of two on the right-hand side of Eq. (101) again arises because both nuclei can serve as photon sources in $A A$ collisions. The kinematics are the same as in jet+jet photoproduction, described in the previous section.

The direct $\gamma+$ jet photoproduction results are given in Fig. 73 for $A A$ interactions at the LHC. We show the transverse momentum, $p_{T}$, distributions for all produced quarks and antiquarks in $\mathrm{Pb}+\mathrm{Pb}, \mathrm{Ar}+\mathrm{Ar}$ and $\mathrm{O}+\mathrm{O}$ collisions for $\left|y_{1}\right| \leq 1$. The cross sections are lower than those for $\gamma+$ jet hadroproduction. Direct $\gamma+$ jet photoproduction proceeds through fewer channels than hadroproduction where the LO channels are $g q \rightarrow \gamma q$ and $q \bar{q} \rightarrow g \gamma$, the same diagrams for resolved $\gamma+$ jet photoproduction. This, along with the lower effective energy and correspondingly higher $x$, reduces the photoproduction 
cross sections relative to hadroproduction. The lower $A$ scaling for photoproduction also restricts the high $p_{T}$ photoproduction rate.
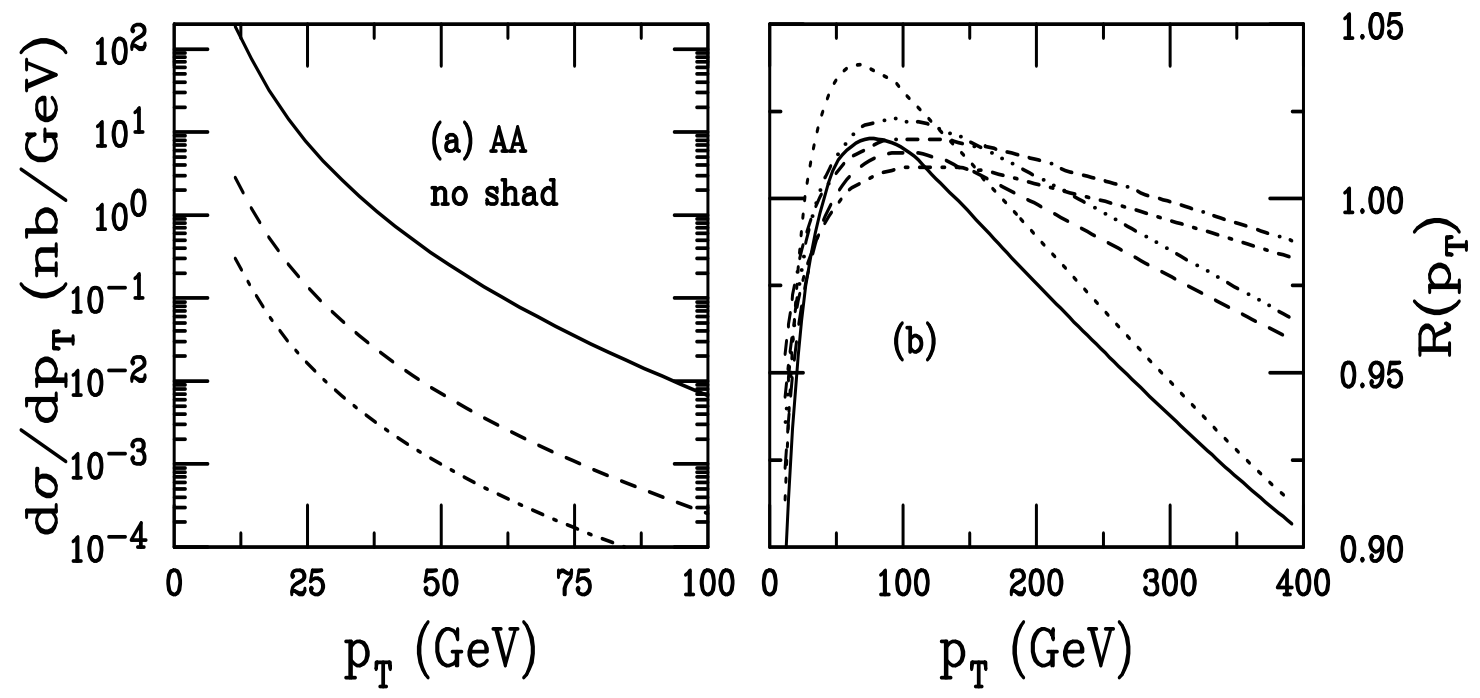

Figure 73. Direct $\gamma+$ jet photoproduction in peripheral collisions. (a) The $p_{T}$ distributions for $\left|y_{1}\right| \leq 1$ are shown for $\mathrm{Pb}+\mathrm{Pb}$ (solid), $\mathrm{Ar}+\mathrm{Ar}$ (dashed) and $\mathrm{O}+\mathrm{O}$ (dot-dashed) collisions. (b) The EKS98 shadowing ratios are shown for $\mathrm{Pb}+\mathrm{Pb}$ (solid), $\mathrm{Ar}+\mathrm{Ar}$ (dashed) and $\mathrm{O}+\mathrm{O}$ (dot-dashed) while the corresponding FGS ratios are shown for $\mathrm{Pb}+\mathrm{Pb}$ (dotted), $\mathrm{Ar}+\mathrm{Ar}$ (dot-dot-dot-dashed) and $\mathrm{O}+\mathrm{O}$ (dot-dash-dash-dashed) collisions. The photon comes from the left.

There is a drop of nearly three orders of magnitude between the dijet cross sections in Fig. 65 and the $\gamma+$ jet cross sections in Fig. 73. Most of this difference comes from the relative couplings, reduced by $\alpha_{s} / \alpha e_{Q}^{2}$ relative to dijet photoproduction. The rest is due to the reduced number of channels available for direct $\gamma+$ jet production since more than half of all directly produced are gluon-initiated for $p_{T}<100 \mathrm{GeV}$, see Fig. 65(b).

We have not distinguished between the quark and antiquark initiated jets. However, the quark-initiated jet rate will always be somewhat higher due to the valence contribution. When $p_{T}<100 \mathrm{GeV}$, the quark and antiquark jet rates are very similar since $x$ is still relatively low. At higher $p_{T}$, the valence contribution increases so that when $p_{T}=400 \mathrm{GeV}$, the quark rate is 1.5 times the antiquark rate. Since the initial kinematics are the same for $\gamma+$ jet and jet + jet final states, the average momentum fractions for $\gamma+$ jet production are similar to those shown for the $\gamma q \rightarrow g q$ channel in Fig. 69.

The shadowing ratios shown in Fig. 73(b) are dominated by valence quarks for $p_{T}>100 \mathrm{GeV}$. The FGS ratio is slightly higher because the EKS98 parametrization includes sea quark shadowing. The effect is similar to the produced gluon ratios, at the same values of $x$ in Fig. 65(c) and (d), since the final-state gluons can only come from quark and antiquark induced processes.

We next present the rapidity distributions for the same two $p_{T}$ cuts used for dijet photoproduction in Fig. 74. Note that the rapidity distribution for $p_{T}>10 \mathrm{GeV}$ 

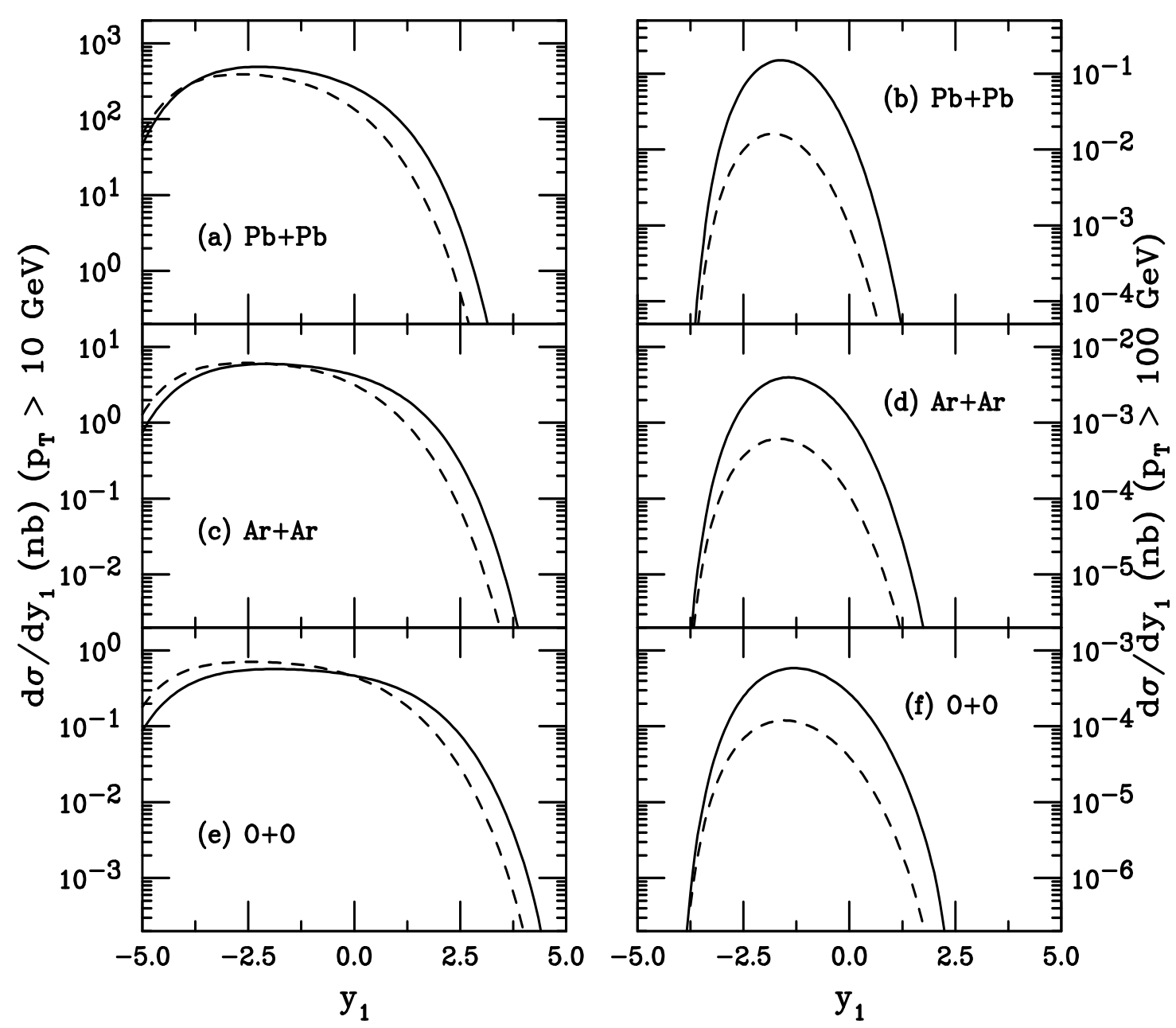

Figure 74. The rapidity distributions of direct and resolved $\gamma+$ jet photoproduction in peripheral collisions. The left-hand side shows the results for $p_{T}>10 \mathrm{GeV}$ for (a) $\mathrm{Pb}+\mathrm{Pb},(\mathrm{c}) \mathrm{Ar}+\mathrm{Ar}$ and (e) $\mathrm{O}+\mathrm{O}$ collisions while the right-hand side is for $p_{T}>100$ $\mathrm{GeV}$ for (b) $\mathrm{Pb}+\mathrm{Pb}$, (d) $\mathrm{Ar}+\mathrm{Ar}$ and (f) $\mathrm{O}+\mathrm{O}$ collisions. The solid curves are the direct results while the dashed curves show the resolved results. The photon comes from the left. Note the different scales on the $y$-axes.

is broader at negative $y_{1}$ than the dijet distributions in Fig. 66 because direct dijet production is dominated by $\gamma g \rightarrow q \bar{q}$ at these $p_{T}$ while the valence distribution entering the $\gamma q \rightarrow \gamma q$ does not drop as rapidly at large $x_{2}$ as the gluon distribution. When the turnover at large negative $y_{1}$ occurs, it is steeper than for the dijets. However, it drops even more steeply at forward $y_{1}$ because the quark distribution is smaller than the gluon at low $x_{2}$. When $p_{T}>100 \mathrm{GeV}$, the $\gamma+$ jet $y_{1}$ distribution is narrower than the dijets since the quark distributions drop faster with increasing $x_{2}$ at high $p_{T}$.

The shadowing ratios as a function of $y_{1}$ are shown in Fig. 75. They exhibit some interesting differences from their dijet counterparts in Fig. 67 because of the different production channels. At $p_{T}>10 \mathrm{GeV}$, the antishadowing peak is lower at $y_{1} \sim-2.5$ and the shadowing is larger at $y_{1}>0$. Although this may seem counter-intuitive, comparing the valence and sea quark shadowing ratios in Fig. 55 can explain this effect. Valence 

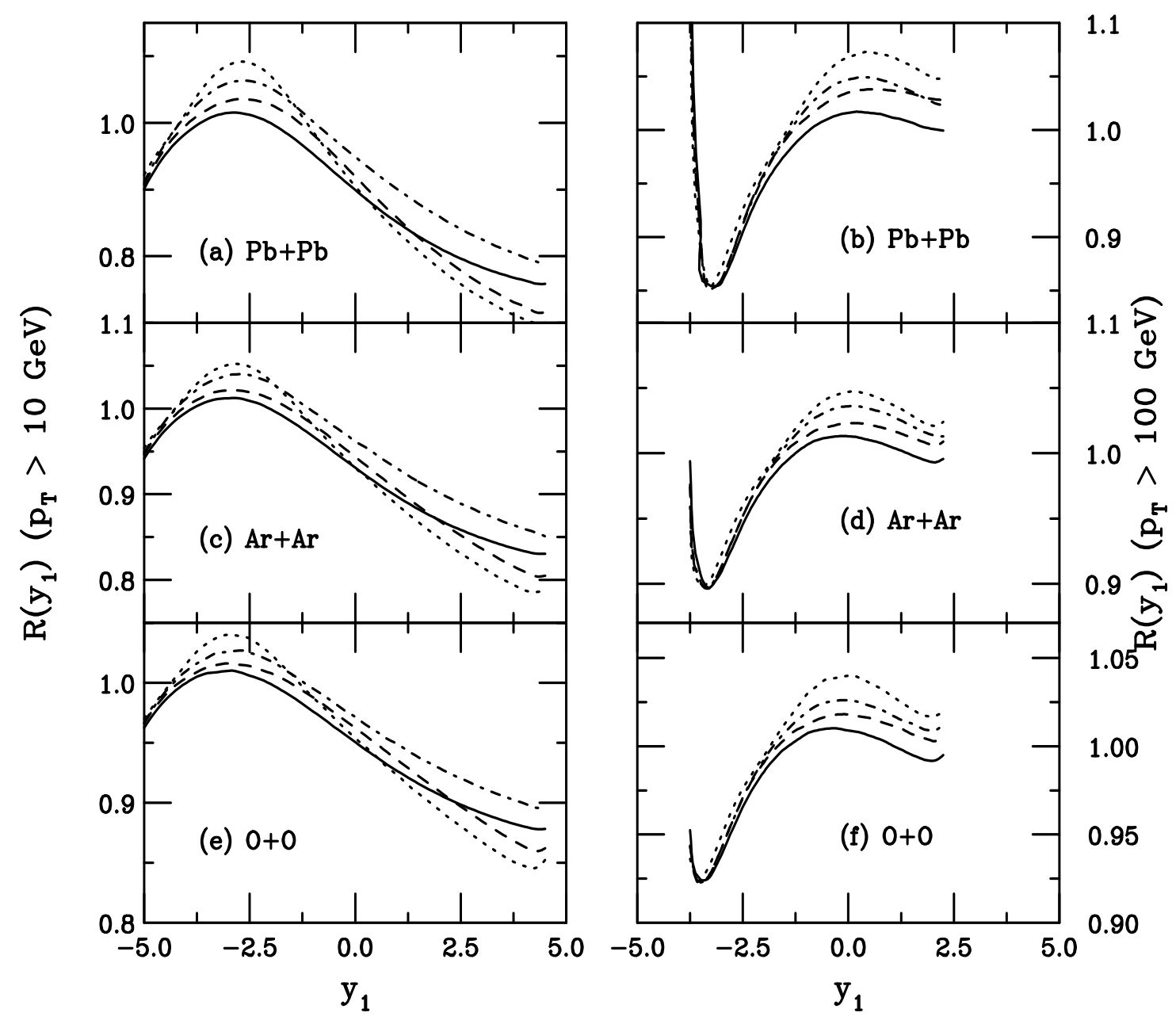

Figure 75. We compare shadowing ratios in direct and resolved $\gamma+$ jet production in peripheral collisions. The left-hand side shows the results for $p_{T}>10 \mathrm{GeV}$ for (a) $\mathrm{Pb}+\mathrm{Pb},(\mathrm{c}) \mathrm{Ar}+\mathrm{Ar}$ and (e) $\mathrm{O}+\mathrm{O}$ collisions while the right-hand side is for $p_{T}>100$ $\mathrm{GeV}$ for (b) $\mathrm{Pb}+\mathrm{Pb}$, (d) $\mathrm{Ar}+\mathrm{Ar}$ and (f) $\mathrm{O}+\mathrm{O}$ collisions. The solid and dashed curves give the direct ratios for the EKS98 and FGS parameterizations respectively. The dot-dashed and dotted curves show the resolved ratios for the EKS98 and FGS parameterizations respectively.

antishadowing, the same for EKS98 and FGS, is lower than that of the gluon. The sea quarks have either no antishadowing (EKS98) or a smaller effect than the valence ratios (FGS). Thus antishadowing is reduced for direct $\gamma+$ jet production. At large $y_{1}$, the $x_{2}$ values, while smaller than those shown in Fig. 67(a) for $\left|y_{1}\right| \leq 1$, are still moderate. Since the evolution of the gluon distribution is faster with $Q^{2}$, sea quark shadowing is actually stronger than gluon shadowing at $p_{T}>10 \mathrm{GeV}$ and low $x_{2}$. When $p_{T}>100$ $\mathrm{GeV}$, the Fermi momentum peak is not as prominent because the sharp increase in the valence and sea shadowing ratios appears at higher $x_{2}$ than for the gluons, muting the effect, particularly for the lighter systems.

We now turn to a description of final-state hadron production opposite a photon. 
The leading particle $p_{T}$ distribution is [208]

$$
\begin{aligned}
\frac{d \sigma_{\gamma A \rightarrow h X}^{\mathrm{dir}}}{d p_{T}}= & 4 p_{T} \int_{\theta_{\min }}^{\theta_{\max }} \frac{d \theta_{\mathrm{cm}}}{\sin \theta_{\mathrm{cm}}} \int d k \frac{d N_{\gamma}}{d k} \int \frac{d x_{2}}{x_{2}} \\
& \times\left[\sum_{i=q, \bar{q}} F_{i}^{A}\left(x_{2}, Q^{2}\right) \frac{d \sigma_{\gamma i \rightarrow \gamma i}}{d t} \frac{D_{h / i}\left(z_{c}, Q^{2}\right)}{z_{c}}\right]
\end{aligned}
$$

where $X$ on the left-hand side includes the final-state gluon. On the partonic level, both the initial and final state partons are identical so that parton $i$ fragments into hadron $h$ according to the fragmentation function, $D_{h / i}\left(z_{c}, Q^{2}\right)$. The subprocess cross sections, $d \sigma / d t$, are related to $s^{2} d \sigma / d t d u$ in Eq. (101) through the momentum-conserving delta function $\delta(s+t+u)$ and division by $s^{2}$. Our results, shown in Fig. 76, are presented in the interval $\left|y_{1}\right| \leq 1$.

The cross section for $\gamma+$ hadron production are, again, several orders of magnitude lower than the dijet calculations shown in Fig. 68(a). At the values of $z_{c}$ and $x$ important for dijet production, the final state is dominated by quarks and antiquarks which fragment more frequently into charged hadrons than do gluons. While $\gamma g \rightarrow q \bar{q}$ produces quarks and antiquarks with identical distributions, the contribution from the $\gamma q \rightarrow q g$ channel makes e.g. pion production by quarks and antiquarks asymmetric. For $p_{T}<100 \mathrm{GeV}, 60 \%$ of the dijet final state particles are pions, $\approx 33 \% \mathrm{kaons}$ and $\approx 7 \%$ protons. As $p_{T}$ increases, the pion and proton contributions decrease slightly while the kaon fraction increases. In the case of $\gamma+$ hadron final states, there is no initial state gluon channel. Thus the valence quarks dominate hadron production and the relative fraction of produced pions increases to $66 \%$. The kaon and proton fractions are subsequently decreased to $\approx 28 \%$ and $\approx 6 \%$ respectively.

The shadowing ratios, shown in Fig. 76(b) and (c) for produced pions, kaons and protons separately for $\mathrm{Pb}+\mathrm{Pb}$ as well as the total ratios for $\mathrm{Ar}+\mathrm{Ar}$ and $\mathrm{O}+\mathrm{O}$ collisions, reflect the quark-initiated processes. We show the results for all charged hadrons here since we do not differentiate between quark and antiquark production. The ratios are almost identical for produced pions, kaons and charged hadrons, are quite different from the ratios shown for pion production by quarks and antiquarks in Fig. 68(c) and (d) since these pions originate from initial-state gluons and thus exhibit antishadowing. The results are similar to pions from gluon jets in Fig. 68. However, in this case the ratios are slightly higher due to the relative couplings. The proton ratios are lower than those for pions and kaons due to the nuclear isospin. The dominance of $d$ valence quarks in nuclei reduces the proton production rate since $d$ quarks are only half as effective at producing protons as $u$ quarks in the KKP fragmentation scheme [209]. This lower weight in the final state reduces the effectiveness of proton production by the initial state, decreasing the produced proton shadowing ratios relative to pions and kaons. Valence quarks dominate the observed final state shadowing at these larger values of $x$, as in Fig. 69.

Now we turn to resolved production of $\gamma+$ jet final states. The resolved jet photoproduction cross section for partons of flavor $f$ in the subprocess $i j \rightarrow k \gamma$ in 


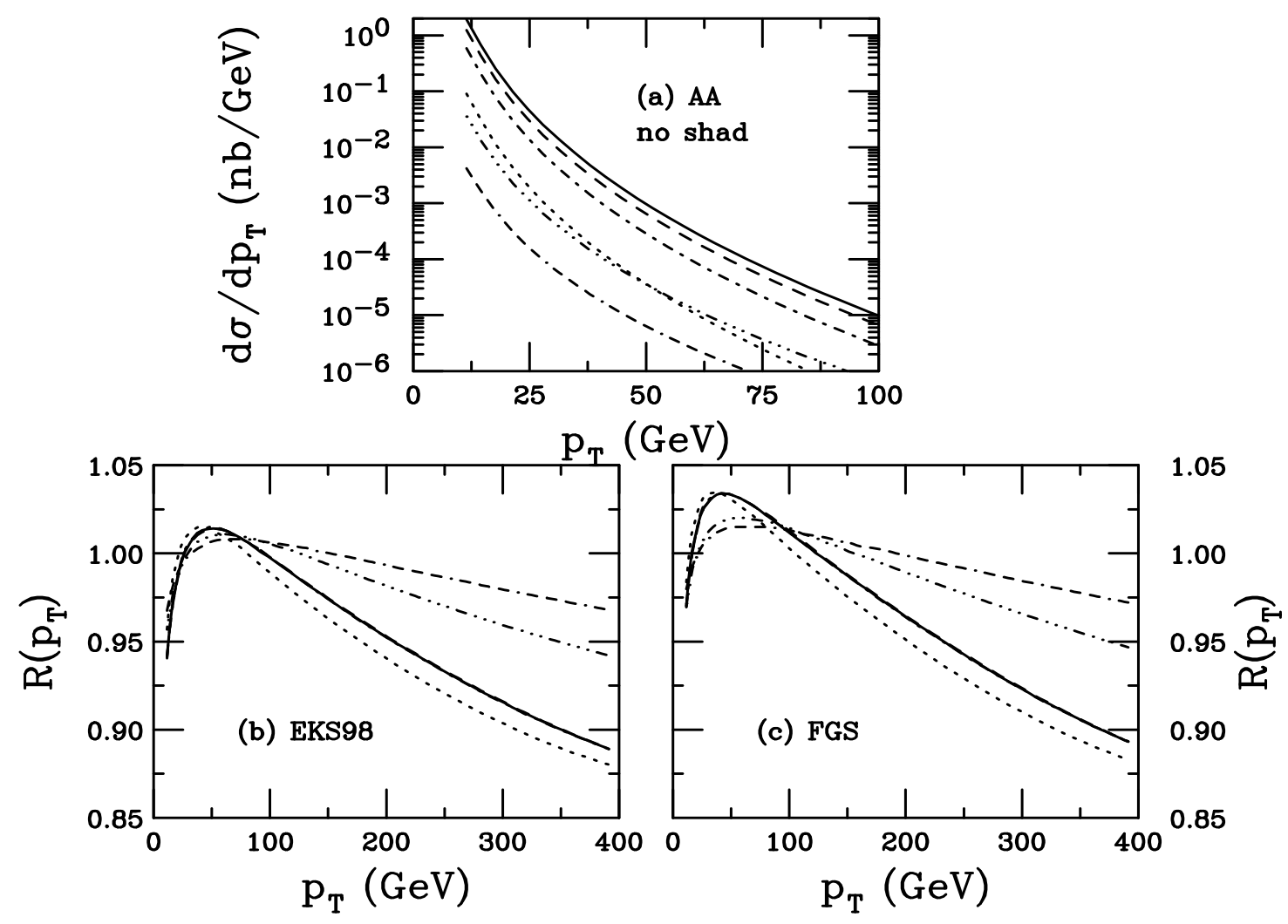

Figure 76. Direct leading hadrons from $\gamma+$ jet photoproduction in peripheral collisions. (a) The $p_{T}$ distributions for $\left|y_{1}\right| \leq 1$ are shown for $A A$ collisions. The $\mathrm{Pb}+\mathrm{Pb}$ results are shown for charged pions (dashed), kaons (dot-dashed), protons (dotted) and the sum of all charged hadrons (solid). The charged hadron $p_{T}$ distributions are also shown for $\mathrm{Ar}+\mathrm{Ar}$ (dot-dot-dot-dashed) and $\mathrm{O}+\mathrm{O}$ (dot-dashdash-dashed) collisions. (b) The EKS98 shadowing ratios for produced hadrons. The results for pions, kaons and the charged hadron total (solid) are nearly identical. The proton result (dotted) is lower. The total charged hadron ratios for $\mathrm{Ar}+\mathrm{Ar}$ (dot-dotdot-dashed) and $\mathrm{O}+\mathrm{O}$ (dot-dash-dash-dashed) collisions are also shown. (c) The same as (b) for FGS.

$A B$ collisions is modified from Eq. (99) so that

$$
\begin{aligned}
s_{N N}^{2} \frac{d \sigma_{\gamma A \rightarrow \gamma+\operatorname{jet} X}^{\text {res }}}{d t_{N N} d u_{N N}}= & 2 \int d z \int_{k_{\min }}^{\infty} \frac{d k}{k} \frac{d N_{\gamma}}{d k} \int_{k_{\min } / k}^{1} \frac{d x}{x} \int_{x_{2} \min }^{1} \frac{d x_{2}}{x_{2}} \\
& \times \sum_{\substack{i j=\\
\langle k l\rangle}}\left\{F_{i}^{\gamma}\left(x, Q^{2}\right) F_{j}^{A}\left(x_{2}, Q^{2}\right)+F_{j}^{\gamma}\left(x, Q^{2}\right) F_{i}^{A}\left(x_{2}, Q^{2}\right)\right\} \\
& \times \delta_{f k}\left[\hat{s}^{2} \frac{d \sigma^{i j \rightarrow k \gamma}}{d \hat{t} d \hat{u}}(\hat{t}, \hat{u})+\hat{s}^{2} \frac{d \sigma^{i j \rightarrow k \gamma}}{d \hat{t} d \hat{u}}(\hat{u}, \hat{t})\right]
\end{aligned}
$$

The resolved diagrams are those for hadroproduction of direct photons, $q g \rightarrow q \gamma$ and $q \bar{q} \rightarrow q g$. The $2 \rightarrow 2$ minijet subprocess cross sections are [214]

$$
\hat{s}^{2} \frac{d^{2} \sigma_{q g}}{d \hat{t} d \hat{u}}=-\frac{1}{3} \pi \alpha_{s} \alpha e_{Q}^{2}\left[\frac{\hat{s}^{2}+\hat{u}^{2}}{\hat{s} \hat{u}}\right] \delta(\hat{s}+\hat{t}+\hat{u})
$$




$$
\hat{s}^{2} \frac{d^{2} \sigma_{q \bar{q}}}{d \hat{t} d \hat{u}}=\frac{8}{9} \pi \alpha_{s} \alpha e_{Q}^{2}\left[\frac{\hat{t}^{2}+\hat{u}^{2}}{\hat{t} \hat{u}}\right] \delta(\hat{s}+\hat{t}+\hat{u}) .
$$

Note that there is no factor $1 /\left(1+\delta_{k l}\right)$, as in Eq. (99), since there are no identical particles in the final state.
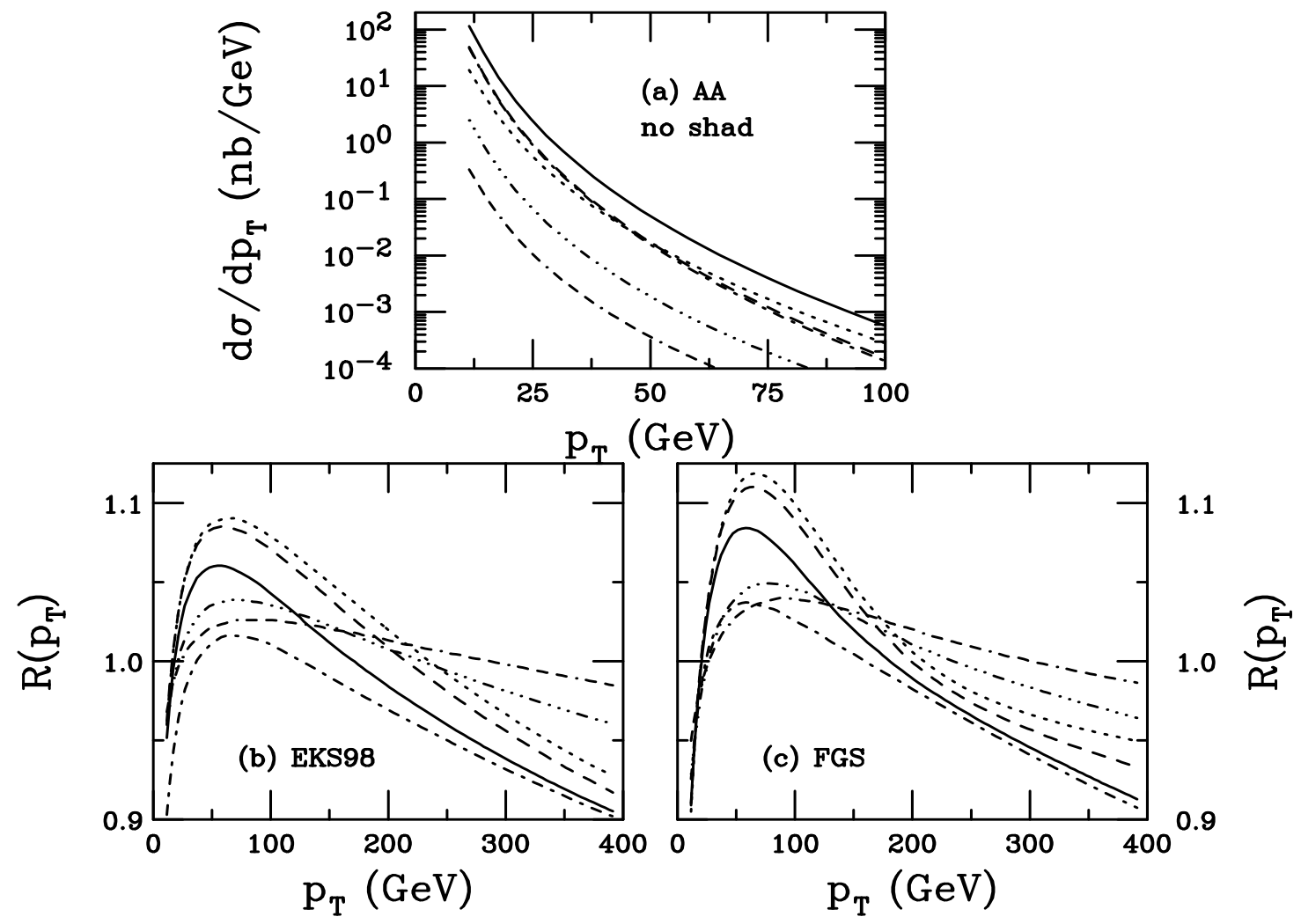

Figure 77. Resolved $\gamma+$ jet photoproduction in peripheral $A A$ collisions. (a) The $\mathrm{Pb}+\mathrm{Pb}$ jet $p_{T}$ distributions with $\left|y_{1}\right| \leq 1$ are shown for quarks (dashed), antiquarks (dot-dashed), gluons (dotted) and the total (solid). We also show the total jet $p_{T}$ distributions in $\mathrm{Ar}+\mathrm{Ar}$ (dot-dot-dot-dashed) and $\mathrm{O}+\mathrm{O}$ (dash-dash-dash-dotted) collisions. (b) The relative EKS98 shadowing contributions from quarks (dashed), antiquarks (dotted) and gluons (dot-dashed) as well as the total (solid) are shown for $\mathrm{Pb}+\mathrm{Pb}$ collisions. The totals are also shown for $\mathrm{Ar}+\mathrm{Ar}$ (dot-dot-dot-dashed) and $\mathrm{O}+\mathrm{O}$ (dash-dash-dash-dotted) interactions.

The resolved jet results are shown in Fig. 77 using the GRV LO photon parton densities. Along with the total partonic rates in $\mathrm{Pb}+\mathrm{Pb}$ collisions, we also show the individual partonic contributions to the jet $p_{T}$ distributions in Fig. 77(a). The total yields are slightly higher for the resolved than the direct contribution where only one channel is open and the coupling is smaller. Quark and antiquark production by the $q g$ process is dominant for $p_{T}<40 \mathrm{GeV}$ but, at higher $p_{T}$, gluon production dominates from the $q \bar{q}$ channel. The large values of $x$ again makes the valence quark contribution dominant at higher $p_{T}$. The total $p_{T}$ distributions for $\mathrm{Ar}+\mathrm{Ar}$ and $\mathrm{O}+\mathrm{O}$ collisions are also shown.

The strong antishadowing in the produced quark and antiquark ratios in Fig. 77(b) 
and (c) is due to the $q g$ channel. The antiquark ratio is higher because the $q g$ parton luminosity peaks at higher $x$ than the $\bar{q} g$ luminosity and at lower $x$ the gluon antishadowing ratio is larger. The difference between the quark and antiquark ratios increases with $p_{T}$ since the average $x$ and thus the valence quark contribution also grow with $p_{T}$. At high $p_{T}$, the flattening of the FGS quark and antiquark ratios is due to the flattening of the gluon parametrization at $x>0.2$.

The final-state gluon ratio shows little antishadowing since it arises from the $q \bar{q}$ channel. The antishadowing in the EKS98 ratio is due to the valence quarks while the higher ratio for FGS reflects the fact that the antiquark ratios also show antishadowing for $x<0.2$. The ratio for the total is essentially the average of the three contributions at low $p_{T}$, where they are similar, while at high $p_{T}$, where the $q \bar{q}$ channel dominates, the total ratio approximates the produced gluon ratio in both cases.

The resolved rapidity distributions are also shown in Fig. 74 for the two $p_{T}$ cuts. The resolved distribution is not as broad at negative $y_{1}$ as that of the dijet process in Fig. 66 due to the smaller relative gluon contribution and the reduced number of channels available for the $\gamma+$ jet process. Note that the relative resolved to direct production is reduced here and the direct process is actually dominant at positive $y_{1}>0$ for $p_{T}>10$ $\mathrm{GeV}$ and for all $y_{1}$ at $p_{T}>100 \mathrm{GeV}$. The antishadowing peak is higher for resolved production, shown in Fig. 75, thanks to the gluon contribution to resolved production.
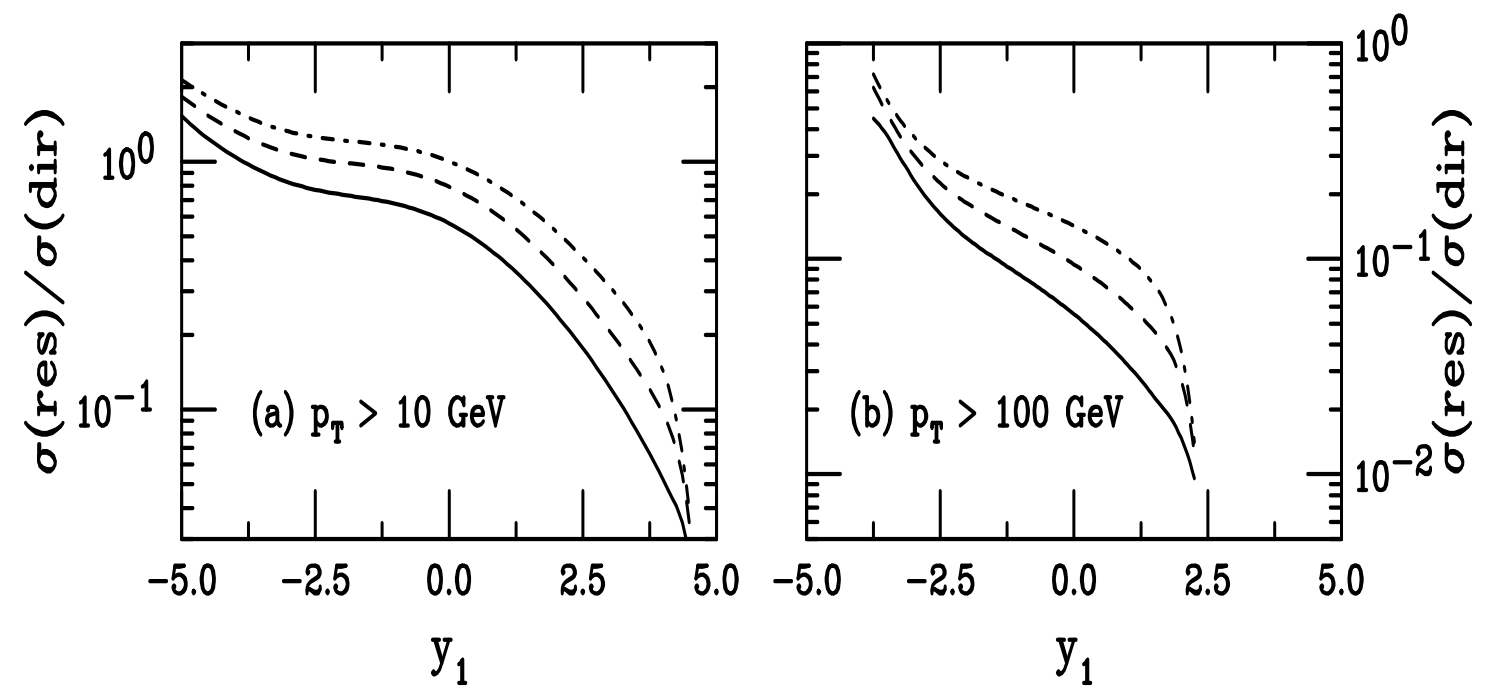

Figure 78. The resolved/direct $\gamma+$ jet production ratios as a function of rapidity. The left-hand side shows the results for $p_{T}>10 \mathrm{GeV}$ while the right-hand side is for $p_{T}>100 \mathrm{GeV}$. The curves are $\mathrm{Pb}+\mathrm{Pb}$ (solid), $\mathrm{Ar}+\mathrm{Ar}$ (dashed) and $\mathrm{O}+\mathrm{O}$ (dotdashed). The photon comes from the left.

Finally, we show the resolved to direct ratio in Fig. 78. The direct rate alone should be observable at $y_{1}>-4$ for $\mathrm{Pb}+\mathrm{Pb}, y_{1} \sim-2.5$ for $\mathrm{Ar}+\mathrm{Ar}$ and 0 for $\mathrm{O}+\mathrm{O}$ and $p_{T}>10$ $\mathrm{GeV}$. Direct production dominates over all $y_{1}$ by a large factor when $p_{T}>100 \mathrm{GeV}$. Although the rates are lower than the dijet, the dominance of direct $\gamma+$ jet production implies than the nuclear quark distribution can be cleanly studied. 
The leading particle $p_{T}$ distributions of jets from $\gamma+$ jet production are

$$
\begin{aligned}
\frac{d \sigma_{\gamma A \rightarrow \gamma+h X}^{\mathrm{res}}}{d p_{T}}= & 4 p_{T} \int_{\theta_{\min }}^{\theta_{\max }} \frac{d \theta_{\mathrm{cm}}}{\sin \theta_{\mathrm{cm}}} \int_{k_{\min }}^{\infty} \frac{d k}{k} \frac{d N_{\gamma}}{d k} \int_{k_{\min } / k}^{1} \frac{d x}{x} \int_{x_{2_{\min }}}^{1} \frac{d x_{2}}{x_{2}} \\
& \times \sum_{\substack{i j=\\
\langle k l\rangle}}\left\{F_{i}^{\gamma}\left(x, Q^{2}\right) F_{j}^{A}\left(x_{2}, Q^{2}\right)+F_{j}^{\gamma}\left(x, Q^{2}\right) F_{i}^{A}\left(x_{2}, Q^{2}\right)\right\} \\
& \left.\times \delta_{f k}\left[{\frac{d \sigma^{i j \rightarrow k \gamma}}{d \hat{t}}}^{i j}, \hat{u}\right)+\frac{d \sigma^{i j \rightarrow k \gamma}}{d \hat{t}}(\hat{u}, \hat{t})\right] \frac{D_{h / k}\left(z_{c}, Q^{2}\right)}{z_{c}} .
\end{aligned}
$$

The subprocess cross sections, $d \sigma / d \hat{t}$, are related to $\hat{s}^{2} d \sigma / d \hat{t} d \hat{u}$ in Eq. (104) through the momentum-conserving delta function $\delta(\hat{s}+\hat{t}+\hat{u})$ and division by $\hat{s}^{2}$.

The resolved $p_{T}$ distributions for hadrons are shown in Fig. 79(a). Note that the resolved cross section for leading hadron production is similar to direct production, shown in Fig. 76(a). The same effect is seen for dijet production in Figs. 72 and 68.

The shadowing ratios are shown in Fig. 79. The difference between the shadowing ratios for pions produced by quarks and antiquarks is rather large and reflects both gluon antishadowing at low $p_{T}$ as well as the relative valence to sea contributions for quark and antiquark production through $q(\bar{q}) g \rightarrow q(\bar{q}) \gamma$. In the FGS calculations, the antiquark ratio reflects the flattening of the antiquark and gluon ratios at $x>0.2$. Since pions produced by gluons come from the $q \bar{q} \rightarrow \gamma g$ channel alone, only a small effect is seen, primarily in the EMC region. Now the total pion rates follow those for quark and antiquark producing final-state pions than gluon.

Although our $p_{T}$-dependent calculations have focused on the midrapidity region of $\left|y_{1}\right| \leq 1$, we have shown that extending the rapidity coverage could lead to greater sensitivity to the small $x_{2}$ region and larger contributions from direct photoproduction, especially at low $p_{T}$.

Thus $\gamma+$ jet production is a good way to measure the nuclear quark distribution functions. Direct photoproduction is dominant at central rapidities for moderate values of $p_{T}$. Final-state hadron production is somewhat larger for direct production so that, even if the rates are low, the results will be relatively clean.

\subsection{Uncertainties}

There are a number of uncertainties in our results. All our calculations are at leading order so that there is some uncertainty in the total rate, see Refs. [141,200]. Some uncertainty also arises from the scale dependence, both in the parton densities and in the fragmentation functions. The fragmentation functions at large $z_{c}$ also introduce uncontrollable uncertainties in the rates. Hopefully more data will bring the parton densities in the photon, proton and nucleus under better control before the LHC begins operation. The data from RHIC also promises to bring the fragmentation functions under better control in the near future.

While the photon flux is also an uncertainty, it can be determined experimentally. The hadronic interaction probability near the minimum radius depends on the matter distribution in the nucleus. Our calculations use Woods-Saxon distributions with 


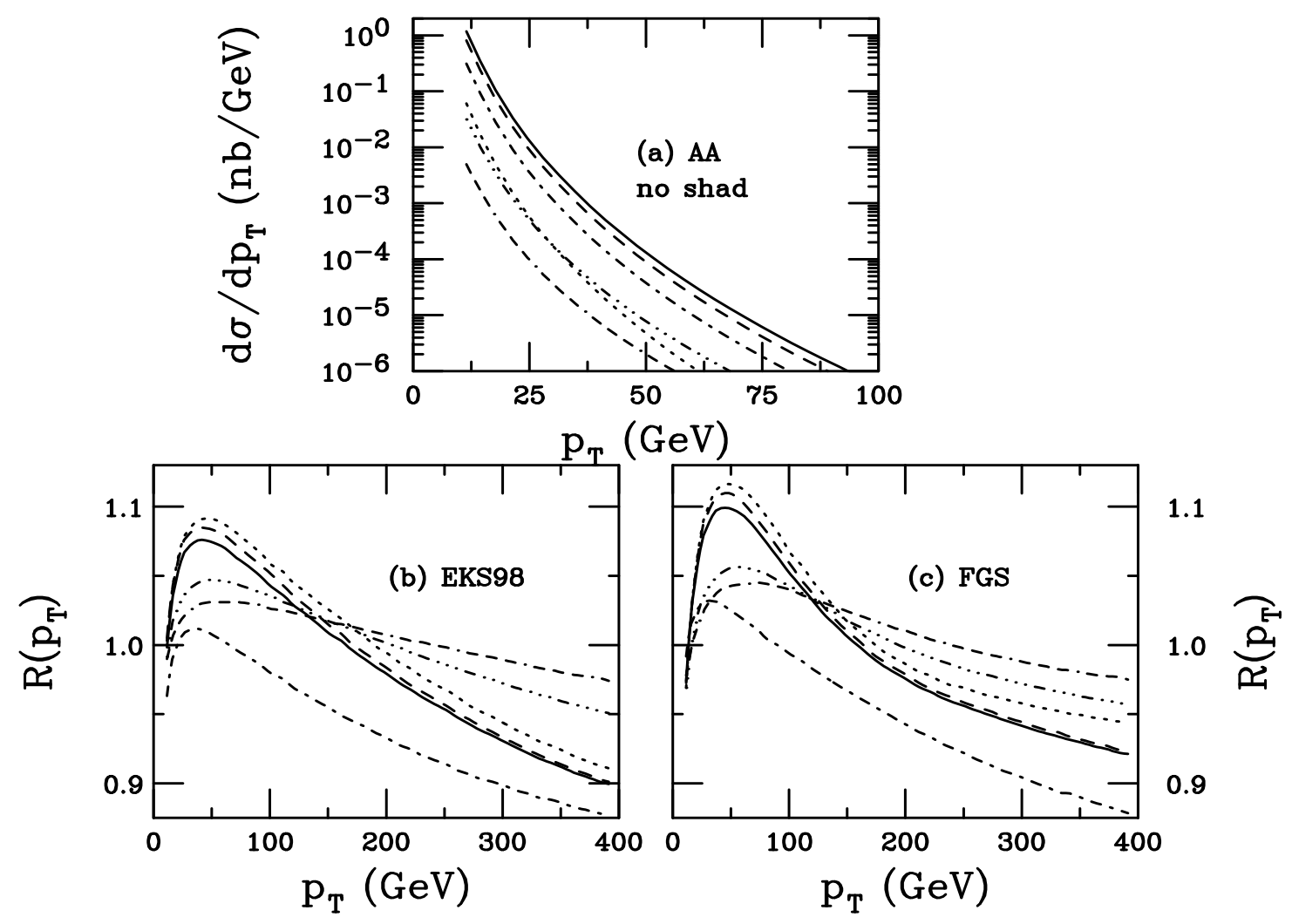

Figure 79. Resolved leading hadrons from $\gamma+$ jet photoproduction in peripheral collisions. (a) The $p_{T}$ distributions for $\left|y_{1}\right| \leq 1$ are shown for $A A$ collisions. The $\mathrm{Pb}+\mathrm{Pb}$ results are shown for charged pions (dashed), kaons (dot-dashed), protons (dotted) and the sum of all charged hadrons (solid). The charged hadron $p_{T}$ distributions are also shown for $\mathrm{Ar}+\mathrm{Ar}$ (dot-dot-dot-dashed) and $\mathrm{O}+\mathrm{O}$ (dot-dashdash-dashed) collisions. (b) The EKS98 shadowing ratios for produced pions. For $\mathrm{Pb}+\mathrm{Pb}$ collisions, we show the ratios for pions produced by quarks (dashed), antiquarks (dotted), gluons (dot-dashed) and the total (solid) separately. The ratios for pions produced by all partons are also shown for $\mathrm{Ar}+\mathrm{Ar}$ (dot-dot-dot-dashed) and $\mathrm{O}+\mathrm{O}$ (dot-dash-dash-dashed) collisions. (c) The same as (b) for FGS.

parameters fit to electron scattering data. This data is quite accurate. However, electron scattering is only sensitive to the charge distribution in the nucleus. Recent measurements indicate that the neutron and proton distributions differ in nuclei [215]. This uncertainty in the matter distribution is likely to limit the photon flux determination.

The uncertainty in the photon flux can be reduced by calibrating it with other measurements such as vector meson production, $\gamma A \rightarrow V A$. Studies of well known twophoton processes, like lepton production, can also help refine the determination of the photon flux. With such checks, it should be possible to understand the photon flux in $p A$ relative to $A A$ to better than $10 \%$, good enough for a useful shadowing measurement. 


\section{Small $x$ physics in UPCs}

\subsection{Identification of the $Q C D$ black disc regime}

Contributed by: L. Frankfurt and M. Strikman

\subsubsection{The black disk regime of nuclear scattering A number of new, challenging QCD} phenomena are related to the rapid increase of the gluon densities with decreasing $x$. As a result, the total inelastic cross section of the interaction of a small color singlet dipole with the target, given by Eq. (7) for LO pQCD, rapidly increases with incident energy. The increase in gluon density was directly observed in $J / \psi$ photo/electroproduction at HERA which found, as predicted by pQCD, $\sigma_{\text {in }}^{q \bar{q} N}\left(s_{(q \bar{q}) N}, d \sim 0.3 \mathrm{fm}\right) \propto s_{(\bar{q} \bar{q}) N}^{0.2}$ where $d \sim 0.3 \mathrm{fm}$ is a typical dipole size for $J / \psi$ production. In addition, the proton structure function, evaluated to NLO in a resummed series in $\alpha_{s} \ln \left(x_{0} / x\right)$ where $x_{0}$ is the starting point for evolution in $x$, increases similar to NLO DGLAP evolution for the energies studied so far [162]. Thus pQCD predicts that the hard cross section should increase rapidly with energy.

The increase in $\sigma_{\text {in }}$ must be reduced at sufficiently high energies to prevent the elastic cross section, proportional to $\sigma_{\text {tot }}^{2} / R_{A}^{2}$, from exceeding $\sigma_{\text {tot }}[48,152]$. Quantitative analyses show that the BDR should be reached in the ladder kinematics where the rapidity interval between gluon rungs on the ladder is large (multi-Regge kinematics) so that NLL calculations are sufficient in pQCD. The relatively rapid onset of the BDR follows primarily from the large input hadron (nucleus) gluon distribution at the nonperturbative starting scale for QCD evolution, a consequence of spontaneously broken chiral symmetry and confinement. The predicted increase of the cross section with energy leads to complete absorption of the $q \bar{q}$ components of the photon wavefunction at small impact parameters. The components of the photon wavefunction with $b>R_{A}$ produce a diffractive final state, calculable in the strongly-absorptive, small-coupling QCD regime. The absolute values and forms of these amplitudes naturally follow from the complete absorption in the BDR.

A variety of experimental observables with unambiguous predictions in the BDR [44] will be discussed below. One example is the structure functions in the limit $x \rightarrow 0$ : $F_{2}^{h}\left(x, Q^{2}\right)=c Q^{2} \ln ^{3}\left(x_{0} / x\right)$ where $c$ should be identical for hadrons and nuclei [216]. Note that BDR contribution is parametrically larger at high $Q^{2}$ than both the nonperturbative QCD result and the regime where pQCD evolution is valid with $F_{2}^{p} \propto Q$. The dominance of the BDR contribution explains why it is possible to evaluate the structure functions in the BDR without a quantitative understanding of the nonperturbative contributions at $Q \sim \Lambda_{\mathrm{QCD}}$. At realistic energies, the universality of the structure functions may only be achieved at small impact parameters.

Another BDR prediction is the increase of the photo-absorption cross section with energy as $c \ln ^{3}\left(s_{(q \bar{q}) N} / s_{0}\right)$ where $c$ is calculable in QCD. Thus QCD predicts a stronger energy dependence of the photo-absorption cross section than that of the Froissart bound 
for hadronic interactions. Other model-independent phenomena in the BDR kinematics, such as diffractive electroproduction of vector mesons and dijets on a nuclear target, will be discussed below. The theory of the BDR onset for high $p_{T}$ and hard phenomena with scales exceeding the BDR scale has been described in the context of a number of models [217].

The requirement of probability conservation (unitarity of the time-evolution operator of the quark-gluon wave packet) determines the kinematic region where the BDR may be accessible in hard interactions. The simplest approach is to consider the elastic-scattering dipole amplitude, $\Gamma\left(s_{(q \bar{q}) N}, b\right)$, in the impact parameter representation. The total, elastic and inelastic dipole-hadron cross sections can be written as

$$
\left.\begin{array}{l}
\sigma_{\text {tot }}\left(s_{(q \bar{q}) N}\right) \\
\sigma_{\text {el }}\left(s_{(q \bar{q}) N}\right) \\
\sigma_{\text {in }}\left(s_{(q \bar{q}) N}\right)
\end{array}\right\}=\int d^{2} b\left\{\begin{array}{l}
2 \operatorname{Re} \Gamma\left(s_{(q \bar{q}) N}, b\right) \\
\left|\Gamma\left(s_{(q \bar{q}) N}, b\right)\right|^{2} \\
1-\left|1-\Gamma\left(s_{(q \bar{q}) N}, b\right)\right|^{2}
\end{array}\right.
$$

When elastic scattering is the non-absorptive complement of inelastic scattering, the amplitude at a given impact parameter is restricted such that $\left|\Gamma\left(s_{(q \bar{q}) N}, b\right)\right| \leq 1$ where $\Gamma\left(s_{(q \bar{q}) N}, b\right)=1$ corresponds to complete absorption, the BDR.

The proximity of $\Gamma\left(s_{(q \bar{q}) N}, b\right)$ to unity is an important measure of the dipole-nucleon interaction strength. When $\Gamma\left(s_{(q \bar{q}) N}, b\right) \geq 0.5$, the probability for an inelastic dipole interaction, $\left|1-\Gamma\left(s_{(q \bar{q}) N}, b\right)\right|^{2}$, exceeds 0.75 , close to unity.

Assuming that the growth of $\Gamma\left(s_{(q \bar{q}) N}, b\right)$ is proportional to the nuclear thickness function, $T_{A}(b)$, given by pQCD for $\Gamma\left(s_{(q \bar{q}) N}, b\right) \leq 1 / 2$, it is straightforward to estimate the highest $p_{T}$ at which the BDR remains valid, $p_{T}^{\mathrm{BDR}}[22,218]$. Figure 80 shows $\left[p_{T}^{\mathrm{BDR}}\left(s_{(q \bar{q}) N}, b=0\right)\right]^{2}$ for gluon interactions with both a proton and a nucleus with $A \sim 208$. The value of $p_{T}^{\mathrm{BDR}}$ is determined by the $p_{T}$ at which a single gluon would be completely absorbed by the target like a colorless dipole of size $d=\pi / Q \sim \pi /\left(2 p_{T}\right)$. At $x \sim 10^{-4}$, the interaction scale for which a colorless gluon dipole at the edge of the BDR is $Q^{2} \sim 4 p_{T}^{\mathrm{BDR}} \sim$ few $\mathrm{GeV}^{2}$, corresponding to $1-3$ gluon rungs on the ladder in multiRegge kinematics. $b \sim 0$. This same kinematic region will also be covered in UPCs at the LHC. The $Q^{2}$ at which the BDR is reached for $q \bar{q}$ dipoles is about a factor of two smaller than for gluons at the same energy. This new strongly-interacting, small-coupling QCD regime is thus fundamentally different from the leading-twist approximation in NLO pQCD.

Here we outline the basic features of hard production in the BDR which can distinguish it from competing phenomena.

\subsubsection{Manifestations of the BDR for inclusive phenomena}

\section{Nuclear structure functions and parton densities}

One distinct feature of the QCD Lagrangian is its conformal invariance in the limit where the bare quark masses can be neglected. Conformal invariance is violated in QCD by 


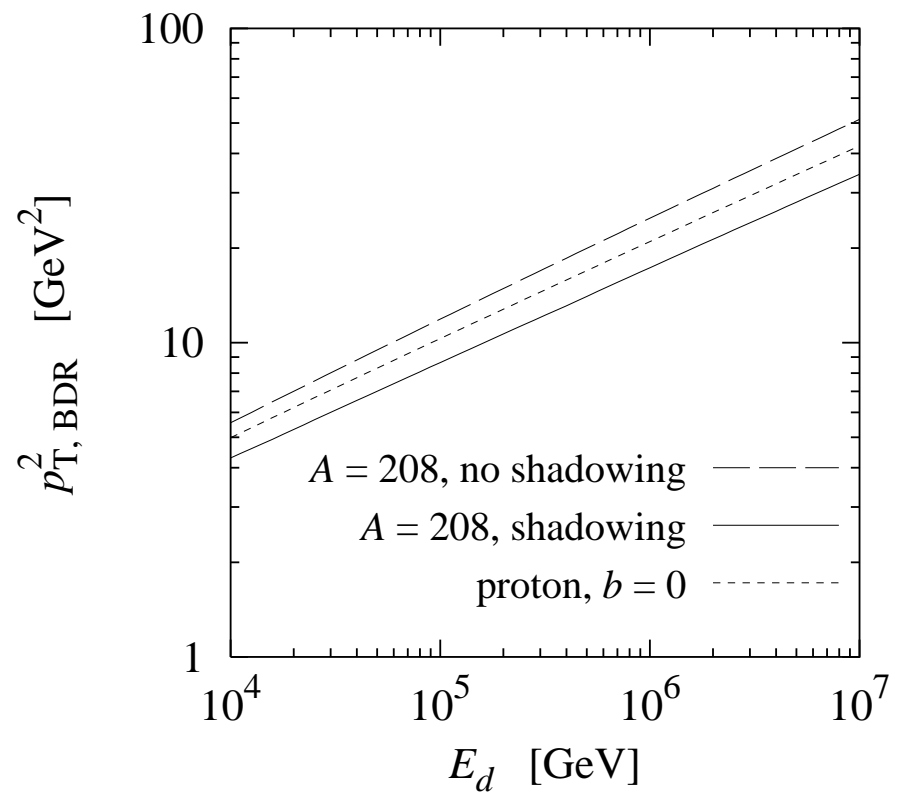

Figure 80. The dependence of $\left[p_{T}^{\mathrm{BDR}}\right]^{2}$ for gluon interactions with a proton at $b \sim 0$ (dotted line) and a lead nucleus without (dashed line) and with (solid) leading-twist nuclear shadowing as a function of the incident gluon dipole energy in the rest frame of the target. Note that for an incident quark, $\left[p_{T}^{\mathrm{BDR}}\right]^{2}$ is a factor of two smaller.

spontaneously broken chiral symmetry. Since the quark masses are typically neglected in hard scattering amplitudes, these amplitudes are conformally invariant except for effects due to the running of the coupling constant. Conformal invariance of the moments of the structure functions leads to approximate Bjorken scaling up to corrections due to the $Q^{2}$ evolution. It is often assumed that $p_{T}$ diffusion is unimportant after NLO effects are included in the BFKL approximation. This assumption is supported by numerical analysis of NLO BFKL approximation [219].

In contrast, at sufficiently small $x$ where the BDR is reached and the pQCD series diverges, conformal invariance is grossly violated: approximate Bjorken scaling disappears. At the small $x$ values in the BDR probed at the LHC the structure function of a heavy nucleus with $R_{A}=1.2 A^{1 / 3} \mathrm{fm}$ has the form

$$
F_{2}^{A}\left(x, Q^{2}\right)=\sum_{q} \frac{e_{q}^{2}}{12 \pi^{2}} 2 \pi R_{A}^{2} Q^{2}\left[\frac{1}{3} \ln A+\lambda \ln \left(\frac{x_{0}}{x}\right)\right] \theta\left(x_{0}-x\right)
$$

where $x_{0}$ does not depend on $A$. The sum is over the number of active flavors with charge $e_{q}$. Since the DIS cross section is $\propto F_{2} / Q^{2}$, in this limit the cross section becomes independent of $Q^{2}$. The parameter $\lambda \approx 0.2$ characterizes the increase of the hard amplitudes with energy for moderate $Q^{2}$. The first term in Eq. (109) is overestimated since LT nuclear shadowing is neglected. The result follows from the calculation of the nuclear structure function in terms of the polarization operator of the photon [64] 
modified to include color transparency. The structure function increase should change at asymptotically large energies where the interaction radius significantly exceeds $R_{A}$,

$$
F_{2}^{A}\left(x, Q^{2}\right)=\sum_{q} \frac{e_{q}^{2}}{12 \pi^{2}} 2 \pi R_{A}^{2} Q^{2} \ln \left(\frac{x_{0}}{x}\right) \theta\left(x_{0}-x\right) .
$$

The nuclear gluon density in the BDR, where the LT approximation breaks down, can be defined from the Higgs-hadron scattering cross section because the Higgs locally couples to two gluons. In the kinematics where the gluon-dipole interaction is in the BDR, at moderately small $x$, the gluon distribution is [152]

$$
x g_{A}\left(x, Q^{2}\right)=\frac{1}{12 \pi^{2}} 2 \pi R_{A}^{2} Q^{2}\left[\frac{1}{3} \ln A+\lambda \ln \left(\frac{x_{0}}{x}\right)\right] \theta\left(x_{0}-x\right) .
$$

Although the effect of color transparency was not taken into account in Ref. [152], it is included in Eq. (111).

\section{Nucleon structure functions and the total $\gamma N$ cross section}

In a nucleon, the onset of the BDR is accompanied by a fast increase of the interaction radius due to the steep decrease of nucleon density with impact parameter. As a result,

$$
R_{N}^{2}(\mathrm{eff})=R_{N}^{2}+c \ln ^{2}\left(x_{0} / x\right),
$$

leading to

$$
F_{2}^{N} \propto \ln ^{3}\left(\frac{x_{0}}{x}\right), \quad \sigma_{\gamma N} \propto \ln ^{3}\left(\frac{s_{\gamma N}}{s_{0}}\right) .
$$

A similar phenomenon occurs only at extremely high energies in nuclei. The calculation of $c$ in Eq. (112) remains model dependent except at ultrahigh energies where $R_{A}$ and $R_{N}$ are determined by pion exchange. Hence the total $\gamma N$ cross section should grow faster with energy than the $N N$ cross section. The same is true for nuclei. Since the energy increase is faster for a nucleon target, the ratio $\sigma_{\text {tot }}^{\gamma A} / A \sigma_{\text {tot }}^{\gamma N}$, characterizing nuclear shadowing, should decrease with energy. The fraction of the cross section due to heavy flavor production should then increase, asymptotically reaching the SU(4)/SU(5) limit.

\section{Inclusive jet and hadron production}

Since partons with $p_{T} \leq p_{T}^{\mathrm{BDR}}$ cannot propagate through nuclei without inelastic interactions, losing a significant fraction of their initial energy and broadening the $p_{T}$ distribution [147], we expect leading-hadron suppression, similar to that observed in $\mathrm{d}+\mathrm{Au}$ interactions at RHIC [220]. The suppression strongly enhances scattering off the nuclear edge, resulting in back-to-back correlations between high $p_{T}$ particles at central and forward rapidities. To study the $b$ dependence of this correlation, a centrality trigger is necessary, along with the inclusive asymmetry observables defined in Ref. [147]. The suppression of the correlation is small if the rapidity difference between the two jets is large [147]. It is also possible to study similar effects for leading charm production since $p_{T}^{\mathrm{BDR}} \geq m_{c}$. 
The rise of the dijet cross section is expected to slow for $p_{T} \leq p_{T}^{\text {BDR }}$. A similar decrease should be observed for back-to-back pions. As shown in Ref. [31] and in Section 4 , such studies will be feasible for $5 \times 10^{-5} \leq x \leq 10^{-2}$.

\subsubsection{Diffractive phenomena}

\section{Inclusive diffraction}

Diffraction in the BDR emerges from the complementary components of the photon wavefunction that are not fully absorbed at $b \leq R_{A}$. Thus it directly reflects the photon wavefunction at the BDR resolution scale. The diffractive cross section should constitute about half the total cross section. The difference from this limit is due to nuclear edge effects. Gribov's orthogonality argument for the derivation of the total cross section can be used to derive Eq. (65), the BDR expression for the real photon cross section as a function of invariant mass $M$ [44], qualitatively different from pQCD.

Dijet production dominates diffraction in the BDR. Corrections arise from three jet production as in $e^{+} e^{-} \rightarrow q \bar{q} g$. Dijet production is also strongly suppressed within the LT approximation where the cross section is proportional to $1 / p_{T}^{8}$. Within the BDR, the jet cross section is proportional to $A^{2 / 3}$ and decreases as $1 /(-t)=1 / p_{T}^{2}$, as shown in Eq. (65).

\section{Vector meson production}

The same approach gives the vector meson production cross section in the BDR, corresponding to diagonal vector meson dominance with a total cross section of $2 \pi R_{A}^{2}$,

$$
\frac{d \sigma_{\gamma A \rightarrow V A}}{d t}=\frac{3 \Gamma_{V \rightarrow e^{+} e^{-}}}{\alpha M_{V}} \frac{\left(2 \pi R_{A}^{2}\right)^{2}}{16 \pi} \frac{4\left|J_{1}\left(\sqrt{-t} R_{A}\right)\right|^{2}}{-t R_{A}^{2}}
$$

where the first factor is equivalent to $\left|C_{V}\right|^{2}$ in Eq. (16). The vector meson cross section in Eq. (114) decreases as $1 / M_{V}^{4}$ with $-t \sim M_{V}^{2}$ since $\Gamma_{V \rightarrow e^{+} e^{-}} \sim 1 / M_{V}$ while in the DGLAP regime the cross section decreases more rapidly as $M_{V}^{-8}$. The different $M_{V}$ dependencies are reminiscent of the change in the $Q^{2}$ dependence of coherent vector meson production from $\sigma_{L} \propto 1 / Q^{6}, \sigma_{T} \propto 1 / Q^{8}$ to $\sigma_{L} \propto 1 / Q^{2}, \sigma_{T} \propto 1 / Q^{4}$ in the BDR [44]. The $A$ dependence of the $t$-integrated cross section also changes from $A^{4 / 3}$ to $A^{2 / 3}$, see Eq. (114).

As discussed in Section 2.5, it will be difficult to push measurements of the coherent vector meson cross sections in $A A$ collisions to $s_{\gamma N} \geq 2 E_{N} M_{V}$ at $y=0$ because it is impossible to distinguish which nucleus emitted the photon. However, in $p A$ interactions, the $\gamma p$ contribution is much bigger than the $\gamma A$, making identification simpler, see Section 2.4.

There are two other ways to study the interaction of small dipoles up to $W_{\gamma N} \sim 1$ $\mathrm{TeV}$ in the BDR. One is vector meson production in incoherent diffraction which should change from $\sigma \propto A$ to $\sigma \propto A^{1 / 3}$. Another is high $t$ vector meson production 
in rapidity-gap events where a transition from the linear $A$ dependence of color transparency to the $A^{1 / 3}$ dependence in the BDR is expected. The slope of the $t$ dependence of hard diffractive production by nucleons should rapidly increase with energy, $B=B_{0}+c \ln ^{2}(1 / x)$ in the BDR kinematics.

\subsection{Testing saturation physics in ultraperipheral collisions}

Contributed by: F. Gelis and U. A. Wiedemann

Parton saturation is a phenomenon generically expected in hadronic collisions at sufficiently high center-of-mass energy. Within perturbative QCD, the linear evolution equation derived by Balitsky, Fadin, Kuraev and Lipatov [221,222] describes the growth of the unintegrated gluon distribution in a hadron as it is boosted towards higher rapidities. This BFKL evolution formalizes the picture that large- $x$ partons in a hadronic wavefunction are sources for small- $x$ partons. In the BFKL evolution, these small- $x$ contributions are generated by splitting processes such as $g \rightarrow g g$ which radiate into the phase space region newly opened up by the boost. This linear evolution leads to untamed growth of the parton density with $\log x$. It also leads to a power-like growth of hadronic cross sections with $\sqrt{s}$, known to violate unitarity at ultra-high $\sqrt{s}$.

As first noted by Gribov, Levin and Ryskin [223], at sufficiently high parton density, nonlinear recombination processes such as $g g \rightarrow g$ cannot be neglected. These processes tame further growth of the parton distributions: a saturation mechanism of some kind must set in. Treating the partons as ordinary particles, it is possible to make a crude estimate of the onset of saturation from a simple mean-free path argument. The recombination cross section for a gluon with transverse momentum $Q$ is

$$
\sigma \sim \frac{\alpha_{s}\left(Q^{2}\right)}{Q^{2}}
$$

while the number of gluons per unit transverse area is given by

$$
\rho \sim \frac{x g\left(x, Q^{2}\right)}{\pi r_{h}^{2}},
$$

where $r_{h}$ is the radius of the hadron and $x$ the momentum fraction of the gluons. Saturation sets in when $\rho \sigma \sim 1$, or equivalently for:

$$
Q^{2}=Q_{s}^{2} \sim \alpha_{s}\left(Q_{s}^{2}\right) \rho \sim \alpha_{s}\left(Q_{s}^{2}\right) \frac{x g\left(x, Q_{s}^{2}\right)}{\pi R_{A}^{2}} .
$$

The momentum scale that characterizes this new regime, $Q_{s}$, is called the saturation momentum [224]. Partons with transverse momentum $Q>Q_{s}$ are in a dilute regime; those with $Q<Q_{s}$ are in the saturated regime. Most generally, $Q_{s}$ characterizes the scale at which nonlinear QCD effects become important. In the high energy limit, contributions from different nucleons in a nucleus act coherently. For large nuclei, one thus expects $Q_{s}^{2} \propto \alpha_{s}\left(Q_{s}^{2}\right) A^{1 / 3}$. Another important parametric characterization of the saturated region is obtained by estimating the number of partons occupying a small disk of radius $1 / Q_{s}$ in the transverse plane. Combining Eqs. (116) and (117) shows that the number is proportional to $1 / \alpha_{s}$. This is the parametrically large occupation number 
of a classical field, supporting the idea that classical background field methods become relevant for describing nuclear wavefunctions at small $x$.

Within the last two decades, the qualitative arguments given above have been significantly substantiated. A more refined argument for the onset of saturation was given in Ref. [225] where recombination is associated with a higher-twist correction to the DGLAP equation. Early estimates of $Q_{s}$ in nucleus-nucleus collisions [226] do not differ much from more modern ones [227]. Finally, over the last decade, nonlinear equations have been obtained which follow the evolution of the partonic systems from the dilute regime to the dense, saturated, regime. These take different, equivalent, forms, generically referred to as the JIMWLK equation. The resulting calculational framework is also referred to as the color glass condensate (CGC) formalism.

5.2.1. The JIMWLK equation In the original McLerran and Venugopalan model [41, 228, 229], the fast partons are frozen, Lorentz-contracted color sources flying along the light-cone, constituting a color charge density $\rho\left(\mathbf{x}_{T}\right)$. Conversely, the low $x$ partons are described by classical gauge fields, $A^{\mu}(x)$, determined by solving the Yang-Mills equations with the source given by the frozen partonic configuration. An average over all acceptable configurations must be performed.

The weight of a given configuration is a functional $W_{x_{0}}[\rho]$ of the density $\rho$ which depends on the separation scale $x_{0}$ between the modes which are described as frozen sources and the modes which are described as dynamical fields. As one lowers this separation scale, more and more modes are included among the frozen sources. Therefore the functional $W_{x_{0}}$ evolves with $x_{0}$ according to a renormalization group equation [230239].

The evolution equation for $W_{x_{0}}[\rho]$, the so-called JIMWLK equation, derived in Refs. [230-239], is

$$
\frac{\partial W_{x_{0}}[\rho]}{\partial \ln \left(1 / x_{0}\right)}=\frac{1}{2} \int d^{2} x_{T} d^{2} y_{T} \frac{\delta}{\delta \rho_{a}\left(\vec{x}_{T}\right)}\left[\chi_{a b}\left(\vec{x}_{T}, \vec{y}_{T}\right) \frac{\delta W_{x_{0}}[\rho]}{\delta \rho_{b}\left(\vec{y}_{T}\right)}\right] .
$$

The kernel, $\chi_{a b}\left(\vec{x}_{T}, \vec{y}_{T}\right)$, only depends on $\rho$ via Wilson lines,

$$
U\left(\vec{x}_{T}\right) \equiv \mathcal{P} \exp \left[-i g \int_{-\infty}^{+\infty} d z^{-} A^{+}\left(z^{-}, \vec{x}_{T}\right)\right]
$$

where $\mathcal{P}$ denotes path ordering along the $x^{-}$axis and $A^{+}$is the classical color field of the hadron moving close to the speed of light in the $+z$ direction. The field $A^{+}$depends implicitly on the frozen sources, i.e. on $\rho\left(\vec{x}_{T}\right)$.

The JIMWLK equation can be rewritten as an infinite hierarchy of equations for $\rho$, or equivalently $U$ correlation functions. For example, the correlator $\operatorname{Tr}\left\langle U^{\dagger}\left(\vec{x}_{T}\right) U\left(\vec{y}_{T}\right)\right\rangle$ of two Wilson lines has an evolution equation that involves a correlator of four Wilson lines. If this 4-point correlator is assumed to be factorisable into the product of two 2-point functions, a closed equation for the 2-point function, the Balitsky-Kovchegov 
(BK) [232, 234] equation, is obtained,

$$
\begin{aligned}
& \frac{\partial \operatorname{Tr}\left\langle U^{\dagger}\left(\vec{x}_{T}\right) U\left(\vec{y}_{T}\right)\right\rangle_{x_{0}}}{\partial \ln \left(1 / x_{0}\right)}=-\frac{\alpha_{s}}{2 \pi^{2}} \int \frac{d^{2} z_{T}\left(\vec{x}_{T}-\vec{y}_{T}\right)^{2}}{\left(\vec{x}_{T}-\vec{z}_{T}\right)^{2}\left(\vec{y}_{T}-\vec{z}_{T}\right)^{2}} \\
& \times\left[N_{c} \operatorname{Tr}\left\langle U^{\dagger}\left(\vec{x}_{T}\right) U\left(\vec{y}_{T}\right)\right\rangle_{x_{0}}-\operatorname{Tr}\left\langle U^{\dagger}\left(\vec{x}_{T}\right) U\left(\vec{z}_{T}\right)\right\rangle_{x_{0}} \operatorname{Tr}\left\langle U^{\dagger}\left(\vec{z}_{T}\right) U\left(\vec{y}_{T}\right)\right\rangle_{x_{0}}\right] .
\end{aligned}
$$

The traces in Eq. (120) are performed over color indices.

When the color charge density is small, the Wilson line, $U$, can be expanded in powers of $\rho$. Equation (120) then becomes a linear evolution equation for the correlator $\left\langle\rho\left(\vec{x}_{T}\right) \rho\left(\vec{y}_{T}\right)\right\rangle_{x_{0}}$ or, equivalently, for the unintegrated gluon density, the BFKL equation. The same is true of Eq. (118) because, in this limit, the kernel $\chi_{a b}$ becomes quadratic in $\rho$.

Similar to the BFKL or DGLAP evolution equations, the initial condition is a nonperturbative input which can, in principle, be modeled, adjusting the parameters to fit experimental data. A simple input is the McLerran and Venugopalan (MV) model with a local Gaussian for the initial $W_{x_{0}}[\rho]$,

$$
W_{x_{0}}[\rho]=\exp \left[-\int d^{2} x_{T} \frac{\rho\left(\vec{x}_{T}\right) \rho\left(\vec{x}_{T}\right)}{\mu^{2}}\right] .
$$

Here, we stress that testing the predictions of the $\mathrm{CGC}$ requires testing both the evolution with rapidity and the initial conditions.

The MV model requires an infrared cutoff at the scale $\Lambda_{\mathrm{QCD}}$ because assuming a local Gaussian distribution ignores the fact that color neutralization occurs on distance scales smaller than the nucleon size $\left(\sim \Lambda_{\mathrm{QCD}}^{-1}\right)$ : two color densities can only be uncorrelated if they are transversely separated by at least the distance scale of color neutralization. Note that the sensitivity to this infrared cutoff gradually disappears as one lowers the separation scale $x_{0}$ in the JIMWLK equation. Indeed, in the saturated regime, color neutralization occurs on distance scales of the order of $Q_{s}^{-1}\left(x_{0}\right)$ [240], the physical origin of the universality of the saturated regime.

5.2.2. Saturation in photon-nucleus collisions High parton density effects can be tested in photo-nuclear UPCs. Quite generically, the cross section for the process $A A \rightarrow F X$, where $F$ denotes a specific produced final state and $X$ unidentified debris from the nucleus, is

$$
\sigma_{A A \rightarrow F X}\left(\sqrt{s_{N N}}\right)=\int_{k_{\min }}^{+\infty} d k \frac{d N_{\gamma}}{d k} \sigma_{\gamma A \rightarrow F X}\left(s_{\gamma N}=2 k \sqrt{s_{N N}}\right) .
$$

In this formula, $s_{\gamma N}=2 k \sqrt{S_{N N}}$ is the square of the center-of-mass energy of the $\gamma N$ system. The minimum photon energy for production of $F, k_{\min }$, is determined from the invariant mass squared, $M^{2}$, of $F$,

$$
k_{\min }=\frac{M^{2}}{2 \sqrt{s_{N N}}} .
$$

In Eq. (122), gluon saturation effects are included in the $\gamma A$ cross section in the integral. In the next subsection, we discuss the effects of gluon saturation on open $Q \bar{Q}$ production (detected as $D$ or $B$ mesons). 
5.2.3. Heavy quark production Heavy quark production has been proposed as a UPC observable sensitive to saturation effects. Calculations which support this statement treat the nucleus as a collection of classical color sources that acts via its color field. These sources produce a color field with which the $Q \bar{Q}$ pair interacts. For a nucleus moving in the $+z$ direction, this color field, expressed here in the Lorenz gauge, $\partial_{\mu} A^{\mu}=0$, is

$$
A^{\mu}(x)=-g \delta^{\mu+} \delta\left(x^{-}\right) \frac{1}{\nabla_{T}^{2}} \rho\left(\vec{x}_{T}\right)
$$

where $\rho\left(\vec{x}_{T}\right)$ is the number density of color charges as a function of the transverse position in the nucleus. The scattering matrix for a quark traveling through this color field is

$$
T(p, q)=2 \pi \gamma^{-} \delta\left(p^{-}-q^{-}\right) \epsilon \int d^{2} x_{T} e^{i\left(\vec{q}_{T}-\vec{p}_{T}\right) \cdot \vec{x}_{T}}\left[U^{\epsilon}\left(\vec{x}_{T}\right)-1\right]
$$

where $p(q)$ is the incoming (outgoing) four-momentum of the quark and $\epsilon \equiv \operatorname{sign}\left(p^{-}\right) \dagger \dagger$. The Wilson line in the fundamental representation of $\mathrm{SU}(3)$ that resums all multiple scatterings of the quark on the color field of Eq. (124) is defined as

$$
U\left(\vec{x}_{T}\right) \equiv T_{-} \exp \left[i g \int_{-\infty}^{+\infty} d z^{-} A_{a}^{+}\left(z^{-}, \vec{x}_{T}\right) t^{a}\right]
$$

where $T_{-}$denotes ordering in the variable $z^{-}$with the fields with the largest value of $z^{-}$placed on the left.

From this starting point, the cross section for $\gamma A \rightarrow Q \bar{Q} X$ can be derived [241]. At leading order in electromagnetic interactions, the three diagrams in Fig. 81 must be evaluated. The black dot represents the scattering matrix defined in Eq. (125). After
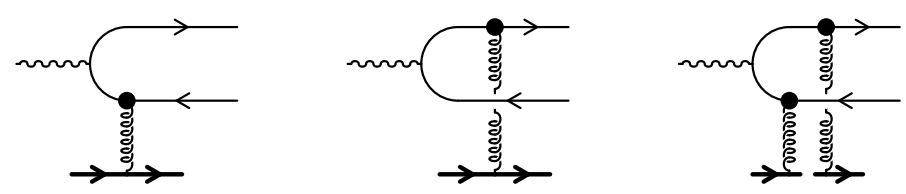

Figure 81. The three diagrams that contribute to the production of a $Q \bar{Q}$ pair in the interaction of a photon with the color field of the nucleus.

summing these three diagrams, we obtain the amplitude

$$
\begin{aligned}
& \mathcal{M}^{\mu}(\vec{k} \mid \vec{q}, \vec{p})=\frac{i e_{q}}{2} \int \frac{d^{2} \vec{l}_{T}}{(2 \pi)^{2}} \int d^{2} x_{1 T} d^{2} x_{2 T} \\
& \quad \times e^{i \vec{l}_{T} \cdot \vec{x}_{1 T}} e^{i\left(\vec{p}_{T}+\vec{q}_{T}-\vec{k}_{T}-\vec{l}_{T}\right) \cdot \vec{x}_{2 T}}\left(U\left(\vec{x}_{1 T}\right) U^{\dagger}\left(\vec{x}_{2 T}\right)-1\right) \bar{u}(\vec{q}) \Gamma^{\mu} v(\vec{p})
\end{aligned}
$$

where $l$ is the four-momentum transfer between the quark and the nucleus, $e_{q}$ is the electric charge of the produced quark and

$$
\Gamma^{\mu} \equiv \frac{\gamma^{-}(\not d-\not /+m) \gamma^{\mu}(\not d-\not k-\not /+m) \gamma^{-}}{p^{-}\left[\left(\vec{q}_{T}-\vec{l}_{T}\right)^{2}+m^{2}-2 q^{-} k^{+}\right]+q^{-}\left[\left(\vec{q}_{T}-\vec{k}_{T}-\vec{l}_{T}\right)^{2}+m^{2}\right]} .
$$

†† When $\epsilon=+1, U^{\epsilon}=U$ and when $\epsilon=-1, U^{\epsilon}=U^{\dagger}$. 
Here $\gamma^{-}$is the - component of the Dirac matrices. In these formulas, $k, q$ and $p$ are the four-momenta of the photon, quark and antiquark respectively. The cross section is obtained from this amplitude by

$$
\begin{aligned}
d \sigma_{\gamma A \rightarrow Q \bar{Q} X} & =\frac{d^{3} q}{(2 \pi)^{2} 2 q_{0}} \frac{d^{3} p}{(2 \pi)^{3} 2 p_{0}} \frac{1}{2 k^{-}} 2 \pi \delta\left(k^{-}-p^{-}-q^{-}\right) \\
& \times\left\langle\mathcal{M}^{\mu}(\vec{k} \mid \vec{q}, \vec{p}) \mathcal{M}^{\nu *}(\vec{k} \mid \vec{q}, \vec{p})\right\rangle_{\rho} \epsilon_{\mu}(k) \epsilon_{\nu}^{*}(k)
\end{aligned}
$$

where $\epsilon_{\mu}(k)$ is the polarization vector of the photon (all possible polarizations should in

principle be summed) and $\langle\cdots\rangle_{\rho}$ denotes the average over all the possible configurations of the distribution of color sources in the nucleus, $\rho\left(\vec{x}_{T}\right)$, weighted by the functional $W[\rho]$ defined in Section 5.2.

\section{Inclusive cross section}

After integrating over the phase space of the produced quark and antiquark, the total cross section is $[241,242]$

$$
\begin{aligned}
\sigma_{\gamma A \rightarrow Q \bar{Q} X}=\frac{\alpha e_{Q}^{2}}{2 \pi^{2}} & \int d l_{T}^{2}\left[\pi R_{A}^{2} C\left(x, l_{T}\right)\right] \\
& \times\left[1+\frac{4\left(l_{T}^{2}-m^{2}\right)}{l_{T} \sqrt{l_{T}^{2}+4 m^{2}}} \operatorname{arcth} \frac{l_{T}}{\sqrt{l_{T}^{2}+4 m^{2}}}\right],
\end{aligned}
$$

with

$$
C\left(x, l_{T}\right) \equiv \int d^{2} x_{T} e^{i \vec{l}_{T} \cdot \vec{x}_{T}}\left\langle U\left(\vec{x}_{T}\right) U^{\dagger}(0)\right\rangle_{\rho} .
$$

Note that Eq. (130) depends on the modulus of $\left|\vec{l}_{T}\right|$. The momentum fraction $x$ is given by $x=k_{\min } / k=4 m^{2} / s_{\gamma N}$, Eqs. (122) and (123) with $M=2 m$. We emphasize that the $x$ dependence of $C\left(x, l_{T}\right)$ comes entirely from the $x$-evolution of the distribution $W[\rho]$ of the classical color sources in the nucleus. Therefore, the $x$ dependence of this cross section tests some predictions of the $W[\rho]$ evolution equations, Eq. (118), or the simpler BK equation for the evolution of the correlator $\left\langle U\left(\vec{x}_{T}\right) U^{\dagger}(0)\right\rangle_{\rho}$, Eq. (120).

After manipulation of Eq. (129), $\sigma_{\gamma A \rightarrow Q \bar{Q} X}$ can alternatively be expressed in terms of the dipole cross section [243]

$$
\sigma_{\gamma A \rightarrow Q \bar{Q} X}=\int_{0}^{1} d z \int d^{2} r_{T}\left|\Psi\left(k \mid z, \vec{r}_{T}\right)\right|^{2} \sigma_{\operatorname{dip}}\left(x, \vec{r}_{T}\right) .
$$

In this formula, the "photon wavefunction", $\Psi\left(k \mid z, \vec{r}_{T}\right)$, denotes the $Q \bar{Q}$ Fock component of the virtual photon light-cone wavefunction that corresponds to a quark-antiquark dipole of transverse size $\vec{r}_{T}$. The square of the wavefunction is

$$
\begin{aligned}
\left|\Psi\left(k \mid z, \vec{r}_{T}\right)\right|^{2} \equiv & \frac{N_{c} \epsilon_{\mu}(k) \epsilon_{\nu}^{*}(k)}{64 \pi k_{-}^{2} z(1-z)} \int \frac{d^{2} l_{T}}{(2 \pi)^{2}} \frac{d^{2} l_{T}^{\prime}}{(2 \pi)^{2}} e^{i\left(\vec{l}_{T}-\vec{l}_{T}^{\prime}\right) \cdot \vec{r}_{T}} \\
& \times \operatorname{Tr}_{d}\left((\not 1+m) \Gamma^{\mu}(\not p-m) \Gamma^{\nu / \dagger}\right)
\end{aligned}
$$


where $\operatorname{Tr}_{d}$ indicates a trace over Dirac indices rather than a color trace. The longitudinal momentum fraction, $z$, is defined as $z=q^{-} / k^{-}$. The dipole cross section, an important quantity in saturation physics, can be defined in terms of a Wilson line correlator,

$$
\sigma_{\operatorname{dip}}\left(\vec{r}_{T}\right)=2 \int d^{2} b\left[1-S\left(\vec{b}, \vec{r}_{T}\right)\right]
$$

with

$$
S\left(\vec{b}, \vec{r}_{T}\right) \equiv \frac{1}{N_{c}} \operatorname{Tr}\left\langle U\left(\vec{b}+\frac{\vec{r}_{T}}{2}\right) U^{\dagger}\left(\vec{b}-\frac{\vec{r}_{T}}{2}\right)\right\rangle_{\rho} .
$$

The above expressions are valid for both $\gamma A$ and $\gamma p$ interactions. The only difference is that the averages are performed over the color field of a nucleus or a proton respectively.

Several models of the dipole cross section have been used to fit the HERA $\gamma p$ data. Golec-Biernat and Wüsthoff used a very simple parametrization [244, 245],

$$
\sigma_{\text {dip }}\left(x, \vec{r}_{T}\right)=\sigma_{0}\left[1-e^{-\frac{1}{4} Q_{s}^{2}(x) r_{T}^{2}}\right],
$$

which shows good agreement with the data at $x<10^{-2}$ and moderate $Q^{2}$. In this formula, the scale $Q_{s}(x)$ has the $x$-dependent form

$$
Q_{s}^{2}(x)=Q_{0}^{2}\left(\frac{x_{0}}{x}\right)^{\lambda}
$$

A fit of HERA $F_{2}$ data suggests $\lambda \approx 0.29$. The parameter $Q_{0}$ is set to $1 \mathrm{GeV}$ with $x_{0} \approx 3 \times 10^{-4}$ for a proton. In the nucleus $Q_{0}^{2}$ must be scaled by $A^{1 / 3}$. However, this model fails at large $Q^{2}$. The high $Q^{2}$ behavior was improved in Ref. [246] where the dipole cross section was parametrized to reproduce pQCD for small dipoles. Even if these approaches are inspired by saturation physics, they do not derive the dipole cross section from first principles. Recently, Iancu, Itakura and Munier [247] derived an expression of the dipole cross section from the color glass condensate framework and obtained a good fit of the HERA data with $\sigma_{0}, \lambda$ and $Q_{0}\left(\left(x_{0}\right)\right)$ as free parameters. An equally good fit was obtained by Gotsman, Levin, Lublinsky and Maor who derived the $x$ dependence of the dipole cross section by numerically solving the BK equation, including DGLAP corrections [248].

\section{Diffractive cross section}

Starting from the dipole cross section, Eq. (134), the elastic dipole cross section is [249]

$$
\sigma_{\text {dip }}^{\text {elastic }}\left(\vec{r}_{T}\right)=\int d^{2} b\left[1-S\left(\vec{b}, \vec{r}_{T}\right)\right]^{2} .
$$

If diffractive $Q \bar{Q}$ production is viewed as a sum of elastic dipole scatterings [250,251],

$$
\begin{array}{rl}
\sigma_{\gamma A \rightarrow Q \bar{Q} X}^{\operatorname{diff}}=\int d^{2} b \int_{0}^{1} & d z \int d^{2} r_{T}\left|\Psi\left(k \mid z, \vec{r}_{T}\right)\right|^{2} \\
\times & \left.\times 1-\frac{1}{N_{c}} \operatorname{Tr}\left\langle U\left(\vec{b}+\frac{\vec{r}_{T}}{2}\right) U^{\dagger}\left(\vec{b}-\frac{\vec{r}_{T}}{2}\right)\right\rangle\right]_{\rho}^{2} .
\end{array}
$$

Therefore, to simultaneously predict the inclusive and diffractive cross sections, a description of the source distribution, $W[\rho]$, that contains some information about the transverse profile of the nucleus is needed. If only the inclusive cross section is calculated, a model of the impact-parameter integrated total dipole cross section is sufficient. 


\section{Example results}

Several models, with various assumptions and degrees of sophistication, exist in the literature $[242,251,252]$. The dipole cross section on a proton is calculated with Eqs. (136) and (137) employing $\lambda \approx 0.29, x_{0} \approx 3.04 \times 10^{-4}$ and $\sigma_{0} \approx 23.03 \mathrm{mb}[244,245]$. The dipole cross section for a nucleus is obtained using Glauber scattering,

$$
\sigma_{\text {dip }}\left(x, \vec{r}_{T}\right)=2 \int d^{2} b\left[1-\exp \left(-\frac{1}{2} T_{A}(b) \sigma_{\text {dip }}^{p}\left(x, \vec{r}_{T}\right)\right)\right] .
$$

Although the average dipole size decreases with $x$ [251], the effect is not very significant. More importantly, if all other parameters are kept fixed, the dipole is larger in the diffractive cross section than in the inclusive cross section [251].

Figure 82 shows the results of Ref. [251] for inclusive $c \bar{c}$ and $b \bar{b}$ production. The cross sections are given for protons and deuterons as well as calcium and lead nuclei. The proton case is compared to ep data. Similar results for the diffractive cross section

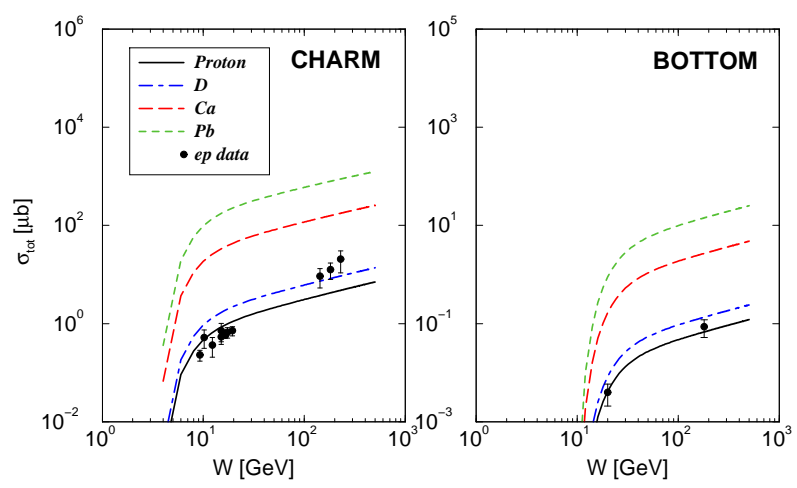

Figure 82. The inclusive $\gamma A \rightarrow Q \bar{Q} X$ cross section for (left-hand side) charm and (right-hand side) bottom as a function of $W$, the center-of-mass energy in the $\gamma A$ system. Reprinted from Ref. [251] with permission from Springer-Verlag.

are displayed in Fig. 83 for deuterons, calcium and lead. The diffractive cross section is about a factor of ten lower than the inclusive cross section.

\section{Two-photon physics at the LHC}

Since photons couple to all charged particles, two-photon processes involve a wide range of reactions. The large ion charge leads to high two-photon rates. In this section, we discuss some of the available physics processes that can be studied at the LHC due to these high rates. We begin with lepton-pair production in strong fields in Section 6.1 and then consider hadronic final states in Section 6.2. Finally, Section 6.3 discusses the observation of two-photon proceses at the LHC. 


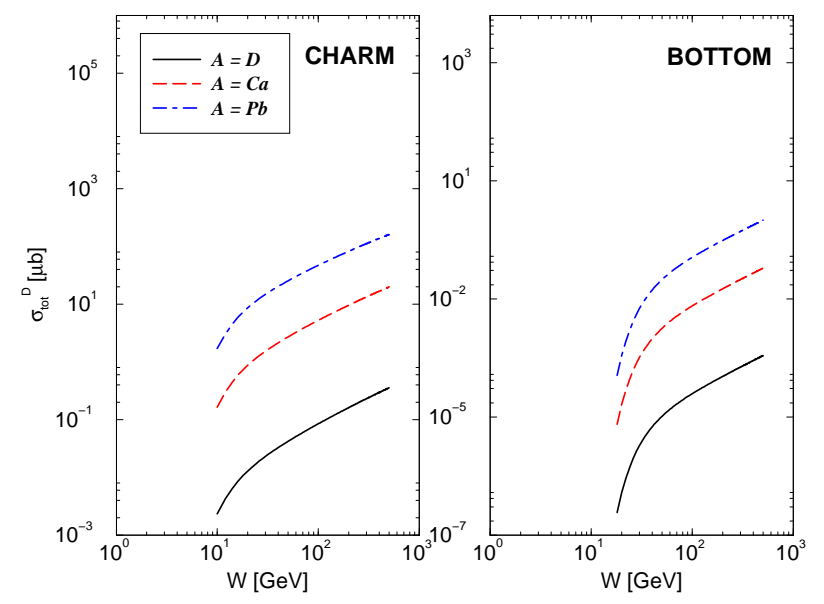

Figure 83. The diffractive $\gamma A \rightarrow Q \bar{Q} X$ cross section for (left-hand side) charm and (right-hand side) bottom as a function of $W$, as a function of the center-of-mass energy in the $\gamma A$ system, for various nuclei. Reprinted from Ref. [251] with permission from Springer-Verlag.

\subsection{Pure QED processes}

The lepton pair production cross section in heavy-ion collisions is extremely high, about $2 \times 10^{5} \mathrm{~b}$ for $e^{+} e^{-}$production by lead beams at the LHC. The large coupling, proportional to $Z^{4} \alpha$ invites discussion of nonperturbative effects. One particularly interesting process is the production of antihydrogen via positron capture by an antiproton [253]. Multiple lepton pair production by a single heavy-ion event is also interesting, as discussed in Section 6.1.2. In addition, Section 6.1.1 considers a special case of pair production, bound-free pairs, where an $e^{+} e^{-}$pair is produced so that the $e^{-}$is bound to one of the incident ions. Although the bound-free pair production cross section is smaller than the total lepton-pair cross section, it has a number of important implications for the LHC. Pair production has been studied at RHIC [120] and the Fermilab Tevatron [254], in addition to fixed-target experiments [255-257].

\subsubsection{Bound-free pair production Contributed by: S. R. Klein}

Bound-free pair production (BFPP) occurs when an $e^{+} e^{-}$pair is produced and the $e^{-}$becomes bound to one of the incident nuclei. This process has a large cross section, about $280 \mathrm{~b}$ for lead at the LHC [36]. BFPP is important because it limits the LHC luminosity with heavy-ion beams. The reaction changes the charge, $Z$, of a beam ion while leaving its momentum essentially unchanged. In essence, the process produces a beam of single-electron ions. Since the ions have an altered charge to mass ratio, they follow a different trajectory from the bare ion beam, eventually striking the LHC beam pipe. With lead beams, the change in charge is $1 / Z \approx 0.012$ and the ion strikes the beam pipe several hundred meters downstream from the interaction point. 
At the maximum design luminosity for lead, $10^{27} \mathrm{~cm}^{-2} \mathrm{~s}^{-1}$, the single-electron beam carries about 280,000 particles/s, dumping about 25 Watts of power into a relatively small section of the beam pipe, enough to overwhelm the LHC magnet cooling systems and causing the struck magnet to quench [258-261]. It is necessary to keep the LHC luminosity low enough to prevent this from happening.

Several different BFPP calculations have been made. One such calculation for capture into a $K$-shell orbital [262],

$$
\sigma_{A B \rightarrow A e^{-}+B+e^{+}}=Z_{A}^{5} Z_{B}^{2}\left[a \log \left(\gamma_{L \mathrm{CM}}\right)+b^{\prime}\right],
$$

shows the scaling with beam species and energy. Here, $Z_{A}$ is the charge of nucleus $A$ that the electron is bound to, $Z_{B}$ is the charge of $B, \gamma_{L}$ CM the Lorentz boost of a single beam in the center-of-mass frame and $a$ and $b^{\prime}$ are constants fit to lower energy data. This approach was used to obtain the $280 \mathrm{~b}$ cross section given above. Capture to higher $s$ orbitals decreases by a factor of $\sim 1 / n^{3}$, a factor of 8 reduction for the $L$-shell. The net effect of all the higher orbitals is to increase the cross section by about $20 \%$. Some earlier calculations have obtained BFPP cross section of $\sim 100 \mathrm{~b}$ but the value of $280 \mathrm{~b}$ is also in agreement with other calculations [263]. In addition, BFPP was measured at the CERN SPS for $158 \mathrm{GeV} /$ nucleon lead beams on a number of fixed targets [264,265]. The data are in reasonable agreement with the calculations of Ref. [262].

Bound-free pair production has been observed during the 2005 RHIC run with copper beams [36]. Although the cross section for bound-free pair production of ${ }^{+28} \mathrm{Cu}$ is small, only $150 \mathrm{mb}$, the change in $Z / A$ due to electron capture is larger than for heavier ions. The single-electron ${ }^{+28} \mathrm{Cu}$ ions struck the beam pipe about $136 \mathrm{~m}$ downstream from the interaction point, producing hadronic showers in the beam pipe and accelerator magnets. The ionization caused by the hadronic showers, with a rate of about $10 \mathrm{~Hz}$, consistent with theory predictions, was detected by small PIN diodes.

\subsubsection{Strong field effects in lepton pair production: Coulomb corrections and multiple} pair production Contributed by: A. J. Baltz, K. Hencken, and G. Baur

In this section we discuss the strong photon-ion coupling constant and how the nonperturbative QED effects arising from its strength might be observed in lepton pair production at the LHC. While the role of higher-order QED in electromagnetic heavyion reactions is interesting in itself, it is also useful as a simpler model for investigating aspects of nonperturbative QCD. Though the primary heavy-ion program involves the technically more challenging quantitative understanding of nonperturbative QCD, the more tractable theoretical treatment of higher-order QED should be experimentally verified. At present, both the experimental and theoretical state-of-the art of higherorder QED is unsatisfactory in UPCs. As we discuss, although some theoretical questions remain, more experimental data is greatly needed. Although there was sufficient interest during the planning stages of RHIC [266], no definitive experimental 
tests of higher-order QED have yet been performed. Here we review the theoretical and experimental situation and discuss some experimental probes of nonperturbative QED effects at the LHC.

Leading-order calculations of charged-particle induced lepton pair production date back to the work of Landau and Lifshitz [267] and Racah [268]. The 1937 Racah formula for the total cross section is remarkably accurate when compared with more recent Monte Carlo calculations [269-271] of $e^{+} e^{-}$production. However, for lead or gold beams, $Z \alpha \sim 0.6$ is not small. Higher-order effects may then be nonnegligible. In addition, strong-field QED effects are expected to be more pronounced at small impact parameters which can be well defined in heavy-ion collisions, making it possible to test this expectation. Calculations have suggested large nonperturbative enhancements relative to perturbative results at low energies on one hand and, on the other, significant reductions at ultrarelativistic energies. Neither effect has yet been verified experimentally.

Coupled-channel calculations of $e^{+} e^{-}$production have been performed at low kinetic energies, 1-2 GeV per nucleon [272-274]. A significant increase over perturbation theory was found. An enhancement relative to perturbation theory was also obtained in coupled-channel calculations of $b=0$ fixed-target $\mathrm{Pb}+\mathrm{Pb}$ interactions at 200 $\mathrm{GeV} /$ nucleon [275].

These calculated enhancements were obtained from large cancellations of positive and negative time contributions to the pair creation probability, with some contributions orders of magnitude larger than the signal. The coupled-channel basis is necessarily incomplete. This limitation, combined with other approximations, may render the method impractical. For example, a factor of 50 enhancement was found in a calculation of bound-free pair production in central $\mathrm{Pb}+\mathrm{Pb}$ fixed-target interactions at $1.2 \mathrm{GeV} /$ nucleon $\left(\gamma_{L}=2.3\right)[272,274]$. When the basis was expanded to include a $70 \%$ larger pertubative cross section [276], the higher-order result decreased the enhancement to a factor of nine even though the basis increased.

There are two interesting higher-order strong field effects: Coulomb corrections and multi-photon exchanges from either one or both ions. In this treatment, only one $e^{+} e^{-}$ pair is assumed to be present at any intermediate time step. Retarded propagators can then be utilized to calculate higher-order Coulomb effects on the total cross section and uncorrelated final electron or positron states [277-281].

The exact solution of the Dirac equation for an electron in the field of the two nuclei has been studied in the limit $\gamma_{L} \rightarrow \infty$. An all-order summation can be made in the high-energy limit in the related problem of bound-free pair production [282]. The summation can be done analytically for free pair production [283, 284]. After integration over $b$, the total cross section is identical to the leading-order result [284,285]. The CERN SPS pair production data [286] also showed perturbative scaling [285]. These data, obtained from $160 \mathrm{GeV} /$ nucleon $\mathrm{Pb}$ and $200 \mathrm{GeV} /$ nucleon $\mathrm{S}$ beams on $\mathrm{C}, \mathrm{Al}, \mathrm{Pa}$, and $\mathrm{Au}$ targets, are the only available ultrarelativistic $e^{+} e^{-}$data spanning a large part of the total cross section. They showed that the cross sections scale as the product of the 
squares of the projectile and target charges, $\left(Z_{A} Z_{B}\right)^{2}$ [286], in contrast to predictions of $e^{+} e^{-}$photoproduction on a heavy target, which shows a negative (Coulomb) correction, proportional to $Z^{2}$, well described by Bethe-Maximon theory [287, 288].

Subsequently, it was argued [289-291] that a more careful regularization of the propagator than that of Refs. [284,285] was needed. Negative Coulomb corrections then reappeared, in agreement with Bethe-Maximon theory. This result was confirmed by numerical calculations with a properly regularized propagator. The exact semi-classical total cross section for $e^{+} e^{-}$production with $A \sim 200$ is reduced by $28 \%$ at the SPS, $17 \%$ at RHIC and $11 \%$ at the LHC [292]. These calculations are in apparent disagreement with the SPS data. However, the coupled-channel treatment of the same basic reaction [275] finds an enhancement of the pair production probability at $b \sim 0$. The difficulties in the method have been previously noted.

At RHIC, the first experimental observation of $e^{+} e^{-}$pairs accompanied by nuclear dissociation was made by STAR [120]. As discussed in Section 2.3.8 and Ref. [84], this corresponds to pair production with $\langle b\rangle \sim 20 \mathrm{fm}$. Comparison with perturbative QED calculations set a limit on higher-order corrections of $-0.5 \sigma_{\mathrm{QED}}<\Delta \sigma<0.2 \sigma_{\mathrm{QED}}$, at a $90 \%$ confidence level. Detailed leading-order QED calculations are carried out in Ref. [293]. The electromagnetic excitation of both ions is included in the semi-classical approach according to Ref. [84].

A comparison to calculations without dissociation in the STAR acceptance gives an indication of the relative difference between the perturbative and higher-order results. Within the STAR acceptance, the calculated exact result is $17 \%$ lower than the perturbative one [292], $\Delta \sigma=-0.17 \sigma_{\mathrm{QED}}$, not excluded by STAR. On the other hand, the small impact parameter should enhance higher-order processes.

A sample numerical calculation has been performed using the same method for $e^{+} e^{-}$production by $\mathrm{Pb}+\mathrm{Pb}$ ions with cuts in a possible detector setup in the forward region [126] at the LHC. For electron and positron energy $E$ and angle $\theta$ such that $3<E<20 \mathrm{GeV}$ and $0.00223<\theta<0.00817$ radians, the perturbative cross section of $2.88 \mathrm{~b}$ without a form factor is reduced by $18 \%$, to $2.36 \mathrm{~b}$, in an exact numerical calculation. If forward $e^{+} e^{-}$pairs are employed for luminosity measurements at LHC, it seems necessary to consider the Coulomb corrections to the predicted cross sections.

Section 6.3.2 discusses $\mu^{+} \mu^{-}$pairs as a $\gamma \gamma$ luminosity monitor. While it is straightforward to calculate the perturbative $\mu^{+} \mu^{-}$pair production rate in heavyion collisions, the importance of Coulomb corrections is somewhat less clear than for $e^{+} e^{-}$. Analytic arguments suggest that Coulomb corrections are small for $\mu^{+} \mu^{-}$ production $[294,295]$. On the other hand, numerical calculations of the total $\mu^{+} \mu^{-}$ cross sections, employing the same method as the exact $e^{+} e^{-}$calculations for RHIC and LHC mentioned previously, find larger relative reductions with respect to perturbation theory, $22 \%$ for RHIC and $14 \%$ for LHC.

A second higher-order effect is multiple pair production in a single collision which restores unitarity, violated at leading order if only single pair production is assumed. The leading order single pair production probability is interpreted as the average number of 
pairs produced in a single collision. Integration of this probability over impact parameter gives the total multiple-pair production cross section. The matrix element for multiplepair production can be factorized into an antisymmetrized product of pair production amplitudes. Calculating the total multiple-pair production probability, neglecting the antisymmetrization of the amplitude, recovers the Poisson distribution $[3,296]$. There are also multi-particle corrections to single pair production which contribute up to $5 \%$ of the probability [3]. Studies of multiple-pair production for ALICE [293] found that about $10 \%$ of the produced pairs detected in the inner tracker come from multiple-pair production.

Lighter ion runs at the same $\gamma_{L}$ and with the same lepton pair acceptance could provide experimental verification of the predicted Coulomb corrections, observable through deviations from the predicted $Z^{4}$ scaling for $A=B$, so far unobserved at RHIC or the SPS. Asymmetric collisions, with $Z_{A} \neq Z_{B}$, could also help separate higher-order corrections from multi-photon exchange with only one or with both ions.

\subsection{Physics potential of two-photon and electroweak processes}

Contributed by: K. Hencken, S. R. Klein, M. Strikman and R. Vogt

This section briefly describes some processes accessible through two-photon interactions, including vector meson pair production and heavy flavor meson spectroscopy. We also briefly discuss tagging two-photon processes through forward proton scattering as a way to enhance searches for electroweak final states. Finally, we mention the possibility of using $\gamma \gamma \rightarrow e^{+} e^{-}$as a luminosity monitor at colliders.

Double vector meson production, $\gamma \gamma \rightarrow V V$ : Double vector meson production in $p A$ and $A A$ collisions is hadronically forbidden for kinematics where both the rapidity difference between one vector meson and the initial hadron and the rapidity difference between the two vector mesons is large. The negative $C$-parity of the vector mesons forbids the process to proceed via vacuum exchange. Accordingly, two-photon processes are the dominant contribution. Studies of final states where one of the vector mesons is heavy, such as $J / \psi \rho^{0}$, can measure the two-gluon form factor of the vector meson for the first time to determine the transverse size of the gluon in the vector meson. It is expected that the $t$-dependence of $\gamma \gamma \rightarrow J / \psi V$ is very broad with more than $30 \%$ of the events at $p_{T} \geq 1 \mathrm{GeV} / c$. In the case when both pairs are heavy, e.g. $J / \psi J / \psi$, the BFKL regime can be probed. On the other hand, if both mesons are light, e.g. $\rho^{0} \rho^{0}$ or $\rho^{0} \phi$, the Gribov-Pomeranchuk factorization theorem can be tested in a novel way. For a more extensive discussion and rate estimates in $A A$ collisions, similar to those in $p A$, see Ref. [18].

Heavy flavor meson spectroscopy: While single vector meson production is forbidden in two-photon processes, it is possible to study heavy $Q \bar{Q}$ pair production. The $\gamma \gamma \rightarrow Q \bar{Q}$ production rate is directly proportional to the two-photon decay width, $\Gamma_{\gamma \gamma}$. The two-photon luminosity is about three orders of magnitude larger than that at LEP. Thus it may be possible to distinguish between quark and gluon-dominated resonances, 
"glueballs". For the production rates in $A A$ collisions, see Refs. [3, 18, 297-299].

One important background to meson production in two-photon processes is vector meson photoproduction followed by radiative decay. For example, in ultraperipheral $\mathrm{Pb}+\mathrm{Pb}$ collisions at the $\mathrm{LHC}$, the $J / \psi$ photoproduction rate followed by the decay $J / \psi \rightarrow \gamma \eta_{c}$ is about 2.5 per minute, much higher than the $\gamma \gamma \rightarrow \eta_{c}$ rate [17]. The two channels have similar kinematics, complicating any measurement of the two-photon coupling.

Two-photon tagging and electroweak processes: Tagging two-photon interactions would enhance the detection capability of electroweak processes such as $W^{+} W^{-}$pairs, $H^{0}$ and $t \bar{t}$ final states. Detection of far-forward scattered protons has been routinely used at $p p$ and $e p$ colliders to select diffractive events. It not only suppresses backgrounds, allowing more efficient event selection, but also improves event reconstruction by employing the measured proton momentum.

At the LHC, forward proton detectors can also be used to measure photoproduction $[29,134]$. The acceptance of the detectors recently proposed by TOTEM and ATLAS at about $\pm 220 \mathrm{~m}$ from the interaction point is determined primarily by the strong dipole fields of the LHC beam line. Protons can be then detected if their fractional energy loss, $\xi$, as a result of photon or Pomeron exchange, is significant. For high luminosity running, the $\xi$ acceptance at $\pm 220 \mathrm{~m}$ is $0.01<\xi<0.1$. Unfortunately, this acceptance does not match the typical fractional energy loss of ions in UPCs. However, newly proposed detectors at $\approx \pm 420 \mathrm{~m}$ from the interaction point will extend the acceptance down to $\xi \sim 2 \times 10^{-3}$ [32]. With such detectors, a fraction of two-photon interactions in $p A$ and $A A$ collisions employing light ions such as Ar or Ca can be double tagged: both the forward scattered proton and ion (or both ions) are detected. Of course, detectors at both $\pm 220 \mathrm{~m}$ and $\pm 420 \mathrm{~m}$ can be used to tag diffractive scattering since larger values of $\xi$ are usually involved.

Thus forward proton detectors provide unique and powerful capabilities for tagging UPCs at the LHC. This tagging would allow selection and measurement of electroweak processes with small cross sections. For example, in a one month $p \mathrm{~Pb}$ run, 10 $\gamma \gamma \rightarrow W^{+} W^{-}$events are expected. These $W^{+} W^{-}$pairs are sensitive to the quartic gauge couplings and would be characterized by a small pair $p_{T}$. Single $W$ bosons will be produced at high $p_{T}$ in $\gamma A$ and $\gamma p$ interactions with much higher statistics, similar to previous studies at HERA [300,301]. In addition, photoproduction of $t \bar{t}$ pairs could provide a measure of the top quark charge [177]. In all these examples, measuring the forward proton improves background suppression as well as reconstruction of the event kinematics. Forward proton measurements can also be used to extend and cross-check other techniques, such as large rapidity gap signatures, which will be exclusively used in heavy-ion collisions.

Coherent $W^{+}$photoproduction, $\gamma p \rightarrow W^{+} n$, is a way to measure the $W^{+}$ electromagnetic coupling [302]. Detection of a single neutron in the ZDCs without additional hadrons could serve as a trigger. Coherent photoproduction was studied in both $p p$ and $p A$ collisions, along with incoherent production in $p p$ collisions, accompanied 
by proton breakup. The coherent and incoherent $p p$ production rates were found to be comparable with a few $W^{+}$produced per year.

\subsection{Photon-photon processes with ALICE}

Contributed by: Yu. Kharlov and S. Sadovsky

6.3.1. $e^{+} e^{-}$pairs in the ALICE forward detectors Multiple $e^{+} e^{-}$pair production in ultraperipheral heavy-ion collisions mainly affects the inner and forward ALICE detectors, located a short radial distance away from the beam axis. These detectors are the Inner Tracking System (ITS), the T0 and V0 detectors and the Forward Multiplicity Detector (FMD), see Section 10.2 for details. The load of the T0 and V0 detectors is of particular importance because these detectors provide the Level-0 ALICE trigger signals.

A full simulation of an electron or position track from $e^{+} e^{-}$pair production through the T0, V0 and FMD detectors in $\mathrm{Pb}+\mathrm{Pb}$ collisions was performed. The software package for ALICE simulation and reconstruction, ALIROOT [116], was used. An event generator for $e^{+} e^{-}$pair production, EPEMGEN [303], was incorporated into ALIRoOT. This generator simulates the lepton $p_{T}$ and $y$ distributions according to the five-dimensional differential cross section $d^{5} \sigma / d p_{T+} d y_{+} d p_{T-} d y_{-} d \phi_{+-}$calculated in Ref. [271].

Only the ITS, T0, V0, and FMD detectors and the beam pipe were taken into account. Three magnetic fields, $B=0.2,0.4$ and $0.5 \mathrm{~T}$, were simulated. The cross sections and detection rates for at least one $e^{ \pm}$in the detectors in $\mathrm{Pb}+\mathrm{Pb}$ collisions at $L=10^{6} \mathrm{~kb}^{-1} \mathrm{~s}^{-1}$ are shown in Table 16 .

The single electron cross sections rapidity distribution in multiple $e^{+} e^{-}$pair production is very flat over a wide rapidity range, giving relatively large cross sections even at forward rapidity. However, the rapidity acceptance is not the only factor determining the cross sections in Table 16. They also strongly depend on the inner radii of the detectors, representing an effective low $p_{T}$ cut. The left and right rapidity coverage of the T0 detectors are very similar and the inner radii are the same, resulting in nearly the same rates on the left and right-hand sides. On the other hand, while the right V0 detector has larger $\eta$ coverage, its larger inner radius reduces the cross section

so that the left V0 detector has a higher cross section. The right-hand FMD covers twice the $\eta$ range of the left-hand detector. Since the two detectors have the same inner radii, the right-hand cross section is twice as large. The forward detector load due to $e^{+} e^{-}$pair production is sufficiently high to be an important background, especially for $B=0.2 \mathrm{~T}$. The load should be compared to the maximum L0 trigger rate, $\sim 200 \mathrm{kHz}$.

\subsubsection{Detection of $\gamma \gamma$ processes in $A L I C E$}



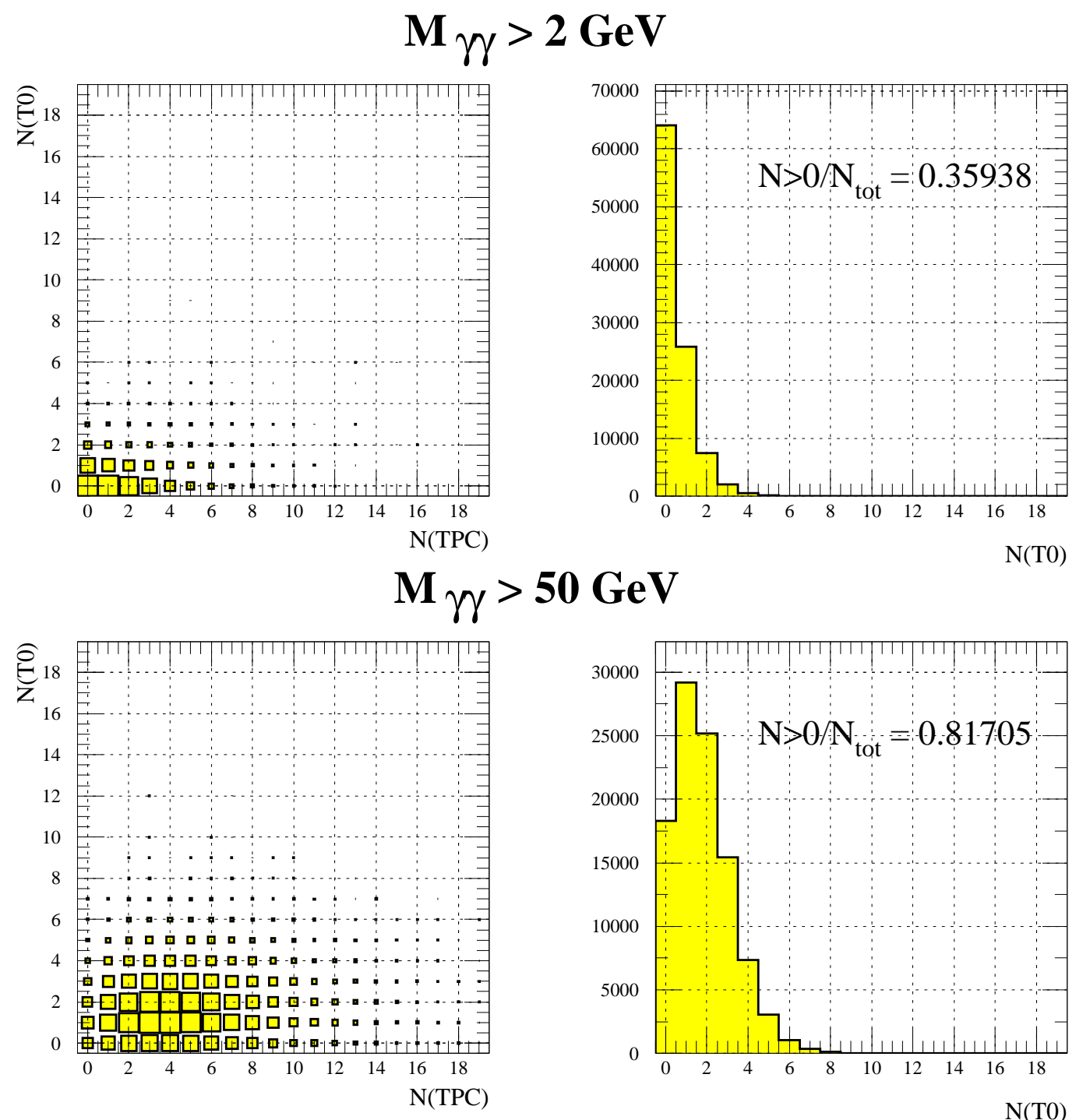

\section{$\mathrm{M}_{\gamma \gamma}>100 \mathrm{GeV}$}
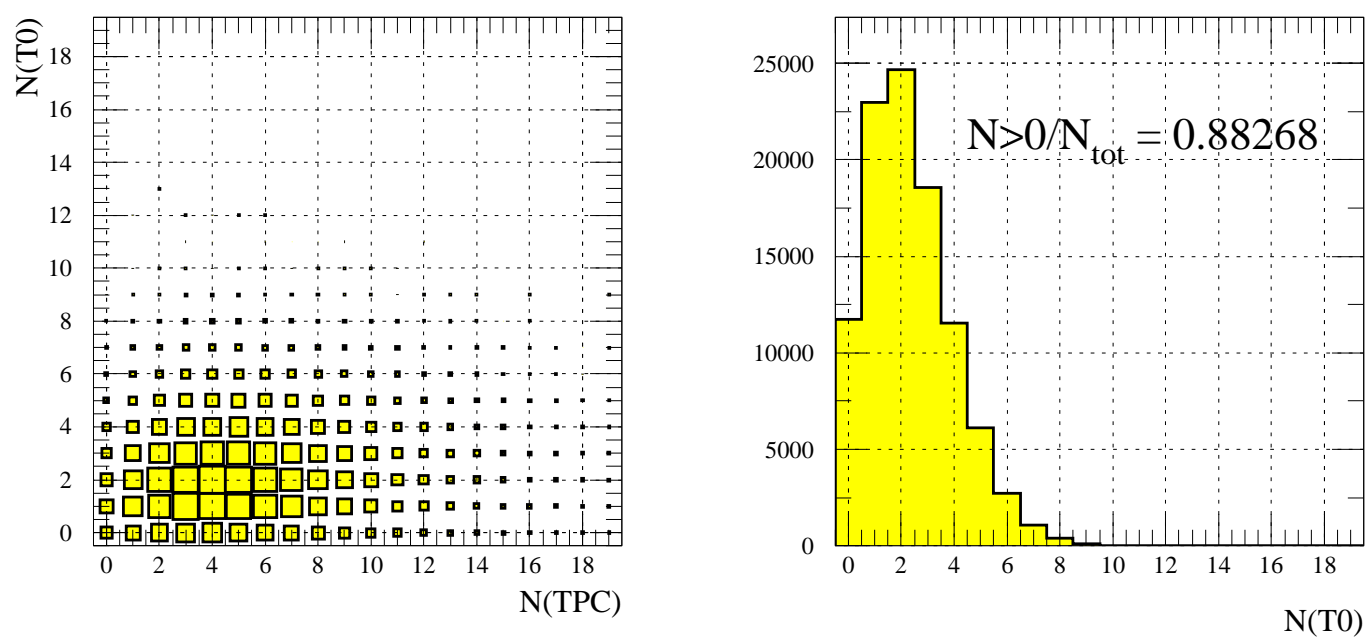

Figure 84. Charged track multiplicities in the TPC and T0 detectors in minimum bias events. 
Table 16. The electron cross sections and detection rates in the T0, V0 and FMD detectors in ALIROOT for $B=0.2,0.4$ and $0.5 \mathrm{~T}$.

\begin{tabular}{|c|c|c|c|c|c|}
\hline \multirow{2}{*}{ Detector } & \multirow{2}{*}{$B(\mathrm{~T})$} & \multicolumn{2}{|c|}{ Right } & \multicolumn{2}{|c|}{ Left } \\
\cline { 3 - 6 } & & $\sigma(\mathrm{kb})$ & Rate $(\mathrm{MHz})$ & $\sigma(\mathrm{kb})$ & Rate $(\mathrm{MHz})$ \\
\hline T0 & 0.2 & 1.7 & 1.7 & 1.9 & 1.9 \\
Right: $-5<\eta<-4.5$ & 0.4 & 0.7 & 0.7 & 0.7 & 0.7 \\
Left: $2.9<\eta<3.3$ & 0.5 & 0.4 & 0.4 & 0.5 & 0.5 \\
\hline V0 & 0.2 & 1.7 & 1.7 & 3.8 & 3.8 \\
Right: $-5.1<\eta<-2.5$ & 0.4 & 0.6 & 0.6 & 2.0 & 2.0 \\
Left: $1.7<\eta<3.8$ & 0.5 & 0.4 & 0.4 & 1.2 & 1.2 \\
\hline FMD & 0.2 & 7.9 & 7.9 & 3.8 & 3.8 \\
Right: $-5.1<\eta<-1.7$ & 0.4 & 3.1 & 3.1 & 1.8 & 1.8 \\
Left: $1.7<\eta<3.4$ & 0.5 & 2.2 & 2.2 & 1.1 & 1.1 \\
\hline
\end{tabular}

$\gamma \gamma \rightarrow X$

We now consider detection of $\gamma \gamma \rightarrow X$ in the ALICE central detectors. Charged particles with $p_{T}$ larger than $100 \mathrm{MeV}$ which pass the SPD2 or SSD2 trigger can be detected in the TPC with full azimuthal coverage and $|\eta|<0.9$ [304]. Photons and electrons with energies greater than $100 \mathrm{MeV}$ in $|\eta|<0.12$ and $\Delta \phi=100^{\circ}$ can be detected by PHOS [305].

To detect two-photon minimum bias events in ALICE, it is important to have hits in the T0 detector since T0 defines the event timing and starts a pre-trigger [306]. Figure 84 shows the correlation between charged track multiplicities in T0 and the TPC in $\gamma \gamma \rightarrow X$ events for three $\gamma \gamma$ invariant mass ranges: $M_{\gamma \gamma}>2,50$ and $100 \mathrm{GeV}$. These correlations demonstrate that the detection efficiency for low invariant mass $\gamma \gamma$ pairs in minimum bias events is small but the cross section is high. On the other hand, the small cross section at higher $\gamma \gamma$ invariant mass is compensated by higher detection efficiency.

The charged track multiplicity in $\gamma \gamma$ collisions is similar to that in hadronic collisions at the same center-of-mass energy because the main contribution to $\gamma \gamma$ interactions comes from strong interactions of vector mesons [307]. At high multiplicities, the $\gamma \gamma$ events cannot be exclusively detected in a restricted acceptance like that of the TPC. The charged particle multiplicity in the TPC pseudorapidity range predicted by PYTHIA [130] is shown in Fig. 85 as a function of $M_{\gamma \gamma}$.

Because particles escape in the forward region, the detected invariant mass is less than the true $M_{\gamma \gamma}$. In Fig. 86 the correlation between the invariant mass of the reconstructed event and the true invariant mass predicted by the event generator TPHIC [297] are shown. The vertical error bars show the width of the measured mass distribution. Up to $80 \%$ of the total $\gamma \gamma$ mass can be lost. A mass-unfolding procedure to reconstruct the true collision energy, similar to that used by the L3 collaboration to 


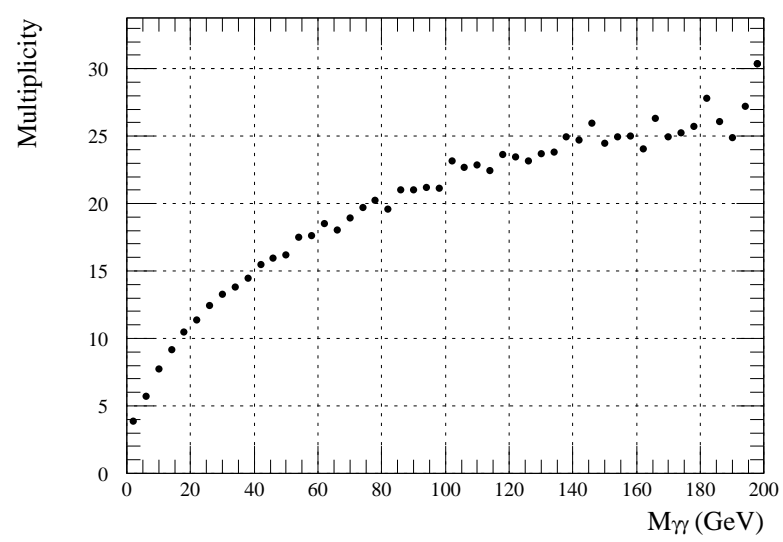

Figure 85. The average charged particle multiplicity in $\gamma \gamma$ interactions as a function of the $\gamma \gamma$ invariant mass predicted by PYTHIA [130]. Reprinted from Ref. [3] with permission from Elsevier.

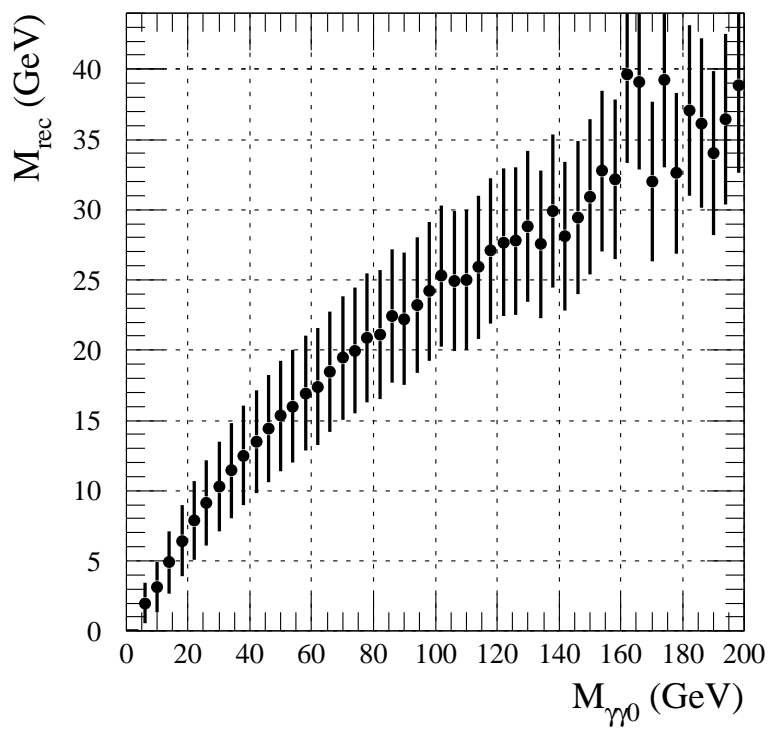

Figure 86. The correlation between the reconstructed invariant mass and the true $\gamma \gamma$ invariant mass.

measure the total $\gamma \gamma$ interaction cross section [308], can be applied.

On the left-hand side of Fig. 87, the detected $\gamma \gamma \rightarrow X$ cross sections (left axis) and event rates (right axis) in $\mathrm{Pb}+\mathrm{Pb}$ collisions are shown. The event rate is calculated for an average luminosity of $0.42 \mathrm{mb}^{-1} \mathrm{~s}^{-1}$ [309]. An event is assumed to be detected if it is selected by the SPD2 multiplicity trigger and if secondary particles come into the acceptance of the TPC and PHOS. A total $\gamma \gamma \rightarrow X$ cross section of $52 \mathrm{mb}$ for at $M_{\gamma \gamma}>2.3 \mathrm{GeV}$ in $\mathrm{Pb}+\mathrm{Pb}$ collisions at $\sqrt{s_{N N}}=5.5 \mathrm{TeV}$ is used. The reconstructed $\gamma \gamma$ cross section is $25 \mathrm{mb}$ with an integrated rate of $10 \mathrm{~Hz}$. 

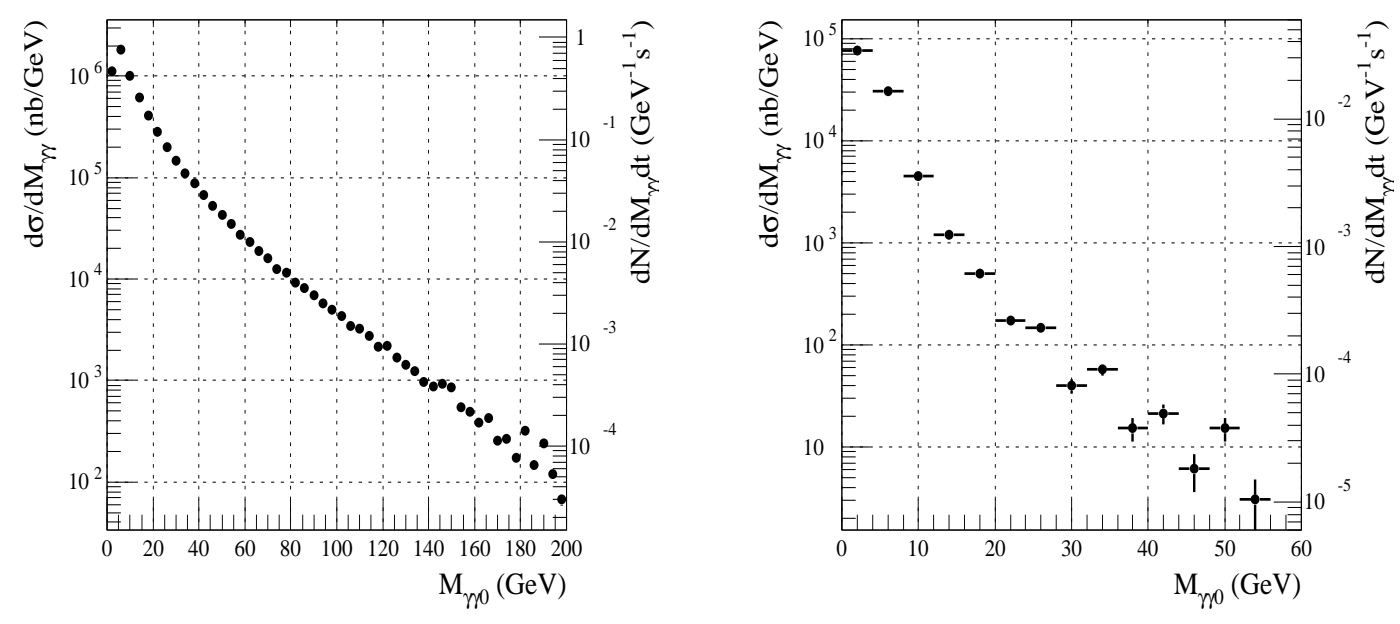

Figure 87. The differential cross section (left axis) and event rate (right axis) for reconstructed $\gamma \gamma$ invariant mass from $\gamma \gamma \rightarrow X$ (left-hand side) and $\gamma \gamma \rightarrow \mu^{+} \mu^{-}$ (right-hand side) in $\mathrm{Pb}+\mathrm{Pb}$ collisions.

$\gamma \gamma \rightarrow \mu^{+} \mu^{-}$

Muon pair production must be measured exclusively since both muons have to be detected. Therefore the SPD2 trigger cannot select these events since at least three charged particles are required in SPD2. An SSD2 trigger can select $\gamma \gamma \rightarrow \mu^{+} \mu^{-}$. The right-hand side of Fig. 87 shows the cross section for events selected by an SSD2 multiplicity trigger. The $\mathrm{Pb}+\mathrm{Pb}$ event rate for $L=0.42 \mathrm{mb}^{-1} \mathrm{~s}^{-1}$ is also shown. The geometric efficiency is about $4 \%$. The low efficiency is due to the dependence of the lepton pair production cross section on scattering angle, $\theta$, in the $\gamma \gamma$ center-of-mass frame [310],

$$
\frac{d \sigma_{\gamma \gamma \rightarrow l^{+} l^{-}}}{d \cos \theta} \propto \frac{1+\cos ^{2} \theta}{1-\beta^{2} \cos ^{2} \theta} .
$$

These lepton pairs can be used as a $\gamma \gamma$ luminosity monitor since they are easy to detect and simple to calculate. Since they are detected exclusively, the background is very small $[311,312]$.

\section{Quarkonium production}

Two-photon collisions can be used to study $C$-even charmonium and bottomonium states $\left(\eta_{c}, \chi_{c 0}, \chi_{c 2}, \eta_{b}, \chi_{b 0}, \chi_{b 2}\right)$. The two-photon widths of $C$-even charmonia were determined from $\gamma \gamma$ processes in $e^{+} e^{-}$collisions at LEP [313],, BELLE [314] and CLEO [315]. The corresponding properties of the bottomonium states still remain unknown. Predictions for the unknown quarkonia two-photon widths are given in Ref. [3], following Refs. [316,317]. The production cross sections are high enough for millions of $\eta_{c}$ and $\chi_{c}$ states to be produced in a $10^{6} \mathrm{~s} \mathrm{Ca}+\mathrm{Ca}$ run while $\sim 1000$ bottomonium states can be produced. The production cross sections and rates are shown in Table 17. 
Table 17. Cross sections and quarkonia production rates in $10^{6} \mathrm{~s} \mathrm{~Pb}+\mathrm{Pb}$ and $\mathrm{Ca}+\mathrm{Ca}$ LHC runs.

\begin{tabular}{|c|c|c|c|c|c|c|}
\hline & & \multirow{2}{*}{ State $(R)$} & \multicolumn{2}{|c|}{$\sigma(A A \rightarrow A A R)(\mu \mathrm{b})$} & \multicolumn{2}{|c|}{ Rate $\left(\right.$ per $\left.10^{6} \mathrm{~s}\right)$} \\
\cline { 4 - 7 } & $M(\mathrm{GeV})$ & $\Gamma_{\gamma \gamma}(\mathrm{keV})$ & $\mathrm{Pb}+\mathrm{Pb}$ & $\mathrm{Ca}+\mathrm{Ca}$ & $\mathrm{Pb}+\mathrm{Pb}$ & $\mathrm{Ca}+\mathrm{Ca}$ \\
\hline$\eta_{c}$ & 2.979 & 7.4 & 540 & 3.7 & $5.4 \times 10^{4}$ & $1.6 \times 10^{7}$ \\
$\chi_{c 0}$ & 3.415 & 4.0 & 170 & 1.2 & $1.7 \times 10^{4}$ & $4.8 \times 10^{6}$ \\
$\chi_{c 2}$ & 3.556 & 0.46 & 85 & 0.59 & $8.5 \times 10^{4}$ & $2.4 \times 10^{6}$ \\
$\eta_{b}$ & 9.366 & 0.43 & 0.32 & 0.0028 & 32 & $1.1 \times 10^{3}$ \\
$\chi_{b 0}$ & 9.860 & $2.5 \times 10^{-2}$ & 0.015 & $1.5 \times 10^{-4}$ & 1.5 & 600 \\
$\chi_{b 2}$ & 9.913 & $6.7 \times 10^{-3}$ & 0.020 & $1.8 \times 10^{-4}$ & 2.0 & 720 \\
\hline
\end{tabular}

The quarkonium states need to be detected exclusively. As an example, we discuss charmonium measurements in $\mathrm{Ca}+\mathrm{Ca}$ interactions. We restrict the event to 2,4 or 6 charged tracks with the sum of the charges in the TPC equal to zero and not more than 2 photons in PHOS. Since no particle identification is assumed for the charged tracks, all charged particles are assigned to be pions. The rates in Table 17 were simulated the TPHIC generator [297]. Further selection criteria were applied to restrict the sum of the transverse momenta of the detected particles to $\sum p_{T}<50 \mathrm{MeV} / c$. The main source of simulated background, $\gamma \gamma \rightarrow X$, had to pass the same selection criteria as the charmonium signal. The $\gamma \gamma \rightarrow X$ cross section in Ca+Ca collisions with $W_{\gamma \gamma}>2.3 \mathrm{GeV}$ is $0.38 \mathrm{mb}$, corresponding to $1.5 \times 10^{9}$ events in a $10^{6} \mathrm{~s}$ run.

Figure 88 shows the number of events as a function of invariant mass in the central ALICE detectors with (left-hand side) and without (right-hand side) the background. The peaks at the $\eta_{c}, \chi_{c 0}$ and $\chi_{c 2}$ masses are visible. The mass spectrum after background subtraction, fitted by an exponential, is shown on the right-hand side of Fig. 88 . During one run, $\sim 7000 \eta_{c}, 1200 \chi_{c 0}$, and $700 \chi_{c 2}$ can be detected. The non-resonant background, as well as additional peaks to the left of the charmonium states are explained by misidentification of charged tracks which spreads the invariant mass of the detected system and shifts it to lower masses. Note that the background from $\gamma \mathbb{P} \rightarrow J / \psi \rightarrow \gamma \eta_{c}$, larger than the $\gamma \gamma \rightarrow \eta_{c}$ rate, has not been included. These events may swamp the signal if the soft photon is not identified.

The bottomonium states are much harder to detect. The main decay channel for $C$-even bottomonium is to two gluons. Due to the high mass, the number of hadrons produced by gluon fragmentation is rather large. The average multiplicity of the $\eta_{b}$ decay products is predicted to be 18 . There are many $\pi^{0} \mathrm{~s}$ and strange mesons among the final-state particles. Due to the restricted ALICE acceptance, especially the small aperture of PHOS, the probability of detecting all the bottomonium decay products is very low. We simulated $10^{5} \eta_{b}$ events, $\sim 100$ times higher than the production rate, and reconstructed none of them. Therefore $\eta_{b}$ and $\chi_{b}$ detection in ALICE remains an open question. 

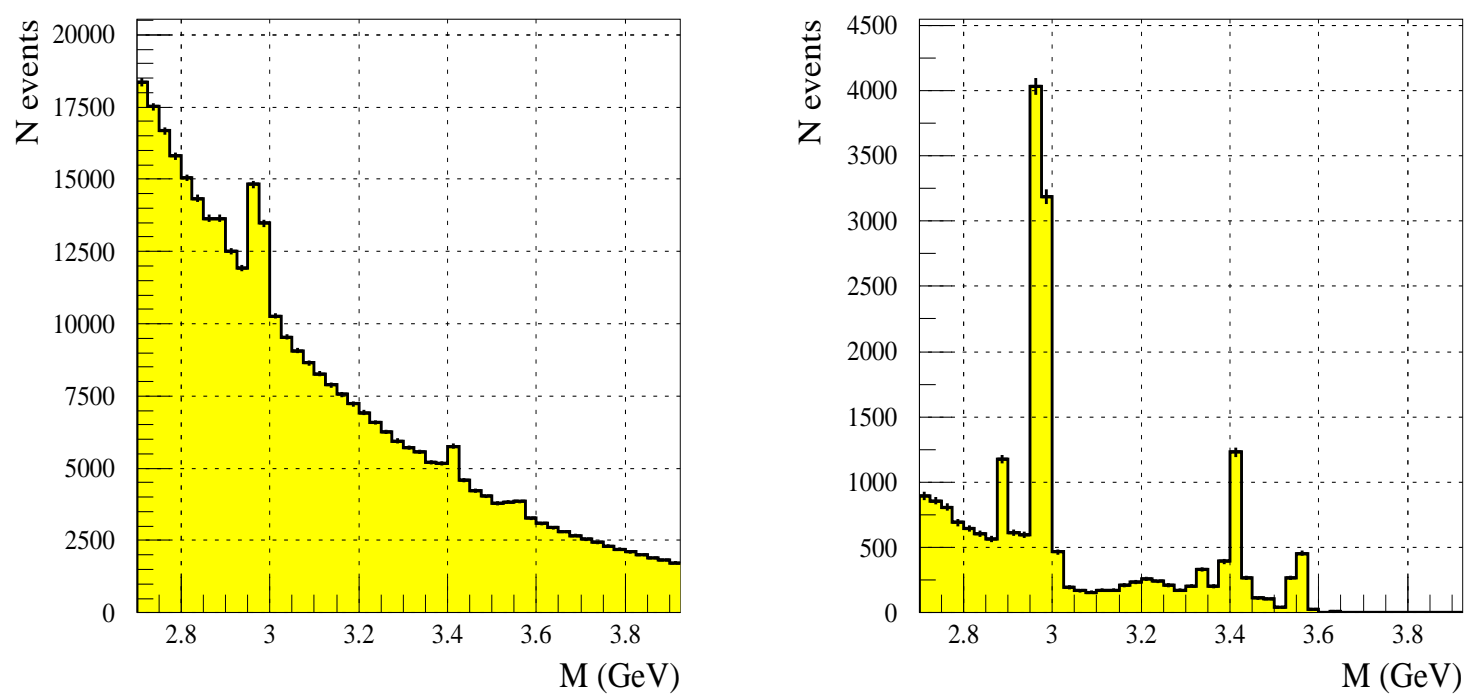

Figure 88. The $\gamma \gamma$ invariant mass distribution with $\eta_{c}, \chi_{c 0}$ and $\chi_{c 2}$ peaks in a $10^{6}$ s Ca+Ca run before (left) and after (right) background subtraction. Reprinted from Ref. [318] with permission from Institute of Physics.

Expected rates in the central barrel

The expected lepton pair yields in two-photon interactions were estimated from the geometrical acceptance of the ALICE central barrel and muon arm. Events were generated based on Refs. $[72,83,119,298]$. The rates were calculated for a $\mathrm{Pb}+\mathrm{Pb}$ luminosity of $5 \times 10^{26} \mathrm{~cm}^{-2} \mathrm{~s}^{-1}$.

The geometrical acceptance of the ALICE central barrel is defined as $|\eta|<0.9$ and $p_{T}>0.15 \mathrm{GeV} / c$ while, for the muon arm, $2.5 \leq \eta \leq 4.0$ and $p_{T}>1 \mathrm{GeV} / c$ is used. Both track are required to be within the acceptance cuts for the event to be reconstructed. In the TRD, a trigger cut of $p_{T}>3.0 \mathrm{GeV} / c$ will be necessary in central collisions. It is not clear if this is also necessary for ultraperipheral events. The rates for $e^{+} e^{-}$pairs are calculated for both $p_{T}>0.15 \mathrm{GeV} / c$ and $p_{T}>3 \mathrm{GeV} / c$.

The lepton pair rates for pairs with $M>1.5 \mathrm{GeV} / c^{2}$ are given in Table 18 . The expected $e^{+} e^{-}$yields in the central barrel are shown in Fig. 89 for $M>1.5 \mathrm{GeV} / c^{2}$ in a $2 \times 10^{4}$ s run (left) and for $M>6.0 \mathrm{GeV} / c^{2}$ in a $2 \times 10^{6} \mathrm{~s}$ run (right). The approximate quarkonium $1 S$ rates are also shown. Higher-lying $S$ states are not included

\section{Measuring beam luminosity with UPCs}

Contributed by: A. J. Baltz, S. N. White and I.A. Pshenichnov

\subsection{Introduction}

The determination of the absolute luminosity at the LHC is the responsibility of the individual experiments. The usual procedure is to select a physical process, a luminosity monitor, that is very stable with respect to luminosity. The yield of the luminosity 

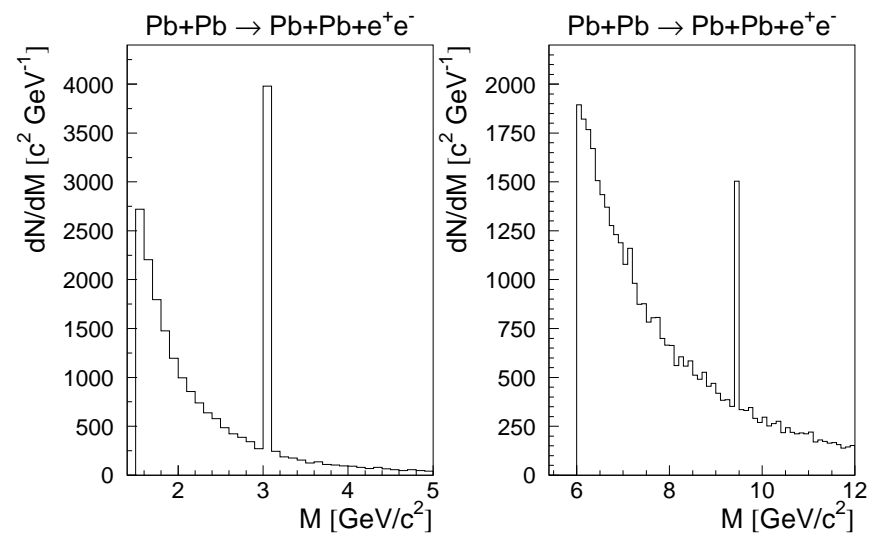

Figure 89. Invariant mass distributions for $\gamma \gamma \rightarrow e^{+} e^{-}$. Both leptons are within the geometrical acceptance of the central barrel. The left-hand side shows the expected yield for $M>1.5 \mathrm{GeV} / c^{2}$ in $2 \times 10^{4} \mathrm{~s}$ at design luminosity (an integrated $\mathrm{Pb}+\mathrm{Pb}$ luminosity of $10 \mu \mathrm{b}^{-1}$ ). The right-hand side shows the yield for $M>6 \mathrm{GeV} / c^{2}$ in $2 \times 10^{6} \mathrm{~s}\left(1 \mathrm{nb}^{-1}\right)$. Only the natural widths of the $1 S$ vector mesons have been included.

Table 18. Expected lepton pair yields for $M>1.5 \mathrm{GeV}$ within the ALICE geometrical acceptance.

\begin{tabular}{|c|c|c|}
\hline Selection & Geometrical Acceptance & Rate (per 106 $\mathrm{s})$ \\
\hline \multicolumn{3}{|c|}{$e^{+} e^{-}$} \\
\hline All & $100 \%$ & $7 \times 10^{7}$ \\
$|\eta|<0.9, p_{T}>0.15 \mathrm{GeV} / c$ & $1.0 \%$ & $7 \times 10^{5}$ \\
$|\eta|<0.9, p_{T}>3 \mathrm{GeV} / c$ & $0.02 \%$ & $1.4 \times 10^{4}$ \\
\hline \multicolumn{2}{|c|}{$\mu^{+} \mu^{-}$} \\
\hline All & $100 \%$ & $2.2 \times 10^{7}$ \\
$2.2 \leq \eta \leq 4.0, p_{T}>1.0 \mathrm{GeV} / c$ & $0.26 \%$ & $6 \times 10^{4}$ \\
\hline
\end{tabular}

monitor is taken with the rest of the data and, at some point, the cross section of the monitor is calibrated and used for absolute normalization of the data.

For the purposes of accelerator operation, it is sufficient to have a stable luminosity monitor which can be used for commissioning and optimizing the setup of the machine. The monitor can typically be calibrated to an accuracy of $10 \%$ based on accelerator instrumentation which determines the intensity and distribution of the stored beams [319].

In the same way, luminosity monitors used by experiments can be calibrated to this $\sim 10 \%$ accuracy by comparing counting rates to delivered luminosity. Since the desired luminosity uncertainty is typically of order $2 \%$, the accelerator-based calibration alone is insufficient. Achieving the higher necessary precision requires accurate knowledge of the monitor cross section, if it is calculable, or direct comparison with another cross 
section.

There are electromagnetic processes which can be calculated to the required accuracy both for heavy-ion and proton beams at the LHC. Since lepton pair production depends primarily on the ion charge and only weakly on its internal structure, it may be a good ion monitor process. The new ion luminosity monitoring technique, described in the following sections, is usable during normal beam conditions and is a by-product of heavy-ion data taking.

Luminosity measurements in $p p$ collisions are more problematic than in the $\mathrm{Pb}+\mathrm{Pb}$ scenario described below since there are no large cross sections which are both calculable and free of detector modeling. Instead both ATLAS [94] and CMS/TOTEM [29] plan to measure small angle $p p$ elastic scattering during runs with special optics at relatively low luminosity. Elastic scattering data can yield an absolute luminosity measurement if it can be extended into the calculable pure Coulomb regime, as proposed by ATLAS. Alternatively, TOTEM has proposed using a luminosity-independent method for deriving the total cross section to normalize the elastic scattering data. In both cases, the expected uncertainty in the luminosity determination is roughly the desired $2 \%$.

In order to make effective use of the precision luminosity measurements in the elastic scattering runs, a stable monitor of the relative luminosity which can be employed both during the special runs and high luminosity runs is needed. It is critical that this monitor be relatively insensitive to machine-related background processes since the machine luminosities differ by a large factor. Although ATLAS has a system of counters designed for this purpose (LUCID), both ATLAS and CMS can use the ZDCs developed for heavy-ion runs in $p p$ monitoring. The ZDCs are stable monitors but need to be calibrated. The ZDC cross section is predicted to be $\sim 9 \%$ of the inelastic cross section. Thus $p p$ elastic scattering can then be used to calibrate the ZDC so that the ZDCs can be used to calibrate the accelerator-based measurements and calculate the luminosity in $p p$ and heavy-ion runs.

\subsection{Luminosity monitoring at $\mathrm{RHIC}$ and $\mathrm{LHC}$}

In spite of the significant differences between the four RHIC experiments, all experiments incorporated an identical minimum bias interaction trigger which served as a standard luminosity monitor. The Zero Degree Calorimeters, ZDCs, trigger events in which at least one neutron is emitted in each beam direction. The calorimeters planned for LHC, like those at RHIC, will be sensitive to beam neutrons with transverse momentum $p_{T} \leq 200 \mathrm{MeV} / c$. Measurements at SPS and RHIC confirmed that, over the full range of centralities, hadronic interactions of $\mathrm{Pb}$ or $\mathrm{Au}$ ions always result in neutron emission within the ZDC acceptance.

In addition to these collisions, the ZDC trigger is sensitive to ultraperipheral interactions resulting in mutual electromagnetic dissociation (MED). The MED calculation, used for absolute luminosity determination, is discussed below. At 
RHIC, data taken with the ZDC trigger were analyzed to determine the fraction of electromagnetic events based on event topology and particle multiplicity. The total cross section, including both hadronic and electromagnetic contributions, was calculated to $5 \%$ accuracy with the ZDC trigger.

The RHIC ZDC cross section, $\sigma_{\text {tot }}$, is $10.8 \mathrm{~b}$ for $\mathrm{Au}+\mathrm{Au}$ collisions at $\sqrt{s_{N N}}=130$ $\mathrm{GeV}$. A similar calculation predicts $14.8 \mathrm{~b}$ for $\mathrm{Pb}+\mathrm{Pb}$ collisions at $\sqrt{s_{N N}}=5.5 \mathrm{TeV}$. Further measurements at RHIC, which will improve the accuracy of the ZDC cross section, are expected to reduce the uncertainty in the LHC prediction to $\sim 2 \%$.

\subsection{Mutual electromagnetic dissociation as a luminosity monitor}

A method to measure and monitor beam luminosity in heavy-ion colliders was proposed in Ref. [88]. According to this method, the rate of mutual electromagnetic dissociation events, $R^{\mathrm{MED}}$, measured by the ZDCs provides the luminosity,

$$
L=\frac{R^{\mathrm{MED}}}{\sigma^{\mathrm{MED}}},
$$

if the mutual electromagnetic dissociation cross section, $\sigma^{\mathrm{MED}}$, is computed with sufficient accuracy. Simultaneous forward-backward single neutron emission from each beam is a clear signature of mutual electromagnetic dissociation which proceeds by mutual virtual photon absorption. The excitation and subsequent decay of the Giant Dipole Resonances (GDR) in both nuclei is responsible for the bulk of this process. In heavy nuclei, such as gold or lead, single neutron emission, $1 n$, is the main mechanism of GDR decay.

Measurements of neutron emission in mutual dissociation of gold nuclei recently performed at RHIC give some confidence in the ZDC technique [320], including the theoretical interpretation of the data necessary for the luminosity measurements [110].

Table 19, from Ref. [110], presents the measured ratios of the ZDC hadronic cross section, $\sigma_{\text {geom }}$, to the total ZDC cross section, $\sigma_{\text {tot }}$, including mutual electromagnetic dissociation. This ratio agrees well with both Weizsäcker-Williams calculations employing measured photodissociation cross sections as input [88] and with RELDIS calculations [109].

Figure 90 shows the energy spectrum obtained in one ZDC when the opposite ZDC measures only one neutron. Requiring only one neutron in one of the ZDCs provides "Coulomb" event selection. The total number of events in the spectrum of Fig. 90 after background subtraction corresponds to the cross section $\sigma(\ln X \mid \mathcal{D})$ for the $(1 n X \mid \mathcal{D})$ topology. Here $1 n$ signifies one neutron, $X$ denotes the undetected particles emitted along with the single neutron and $\mathcal{D}$ denotes an arbitrary dissociation mode for the other nucleus.

The decay topology $(\ln X \mid 1 n Y)$ corresponds to exactly one neutron in each ZDC accompanied by undetected particles $X$ and $Y$ respectively and gives rise to the highest peak in the energy spectrum shown in Fig. 90. The topology trigger with a single neutron in each ZDC is about $35 \%$ of the total $(1 n X \mid \mathcal{D})$ topology, as shown in Table 19. 
Table 19. Experimental and theoretical ratios of mutual dissociation cross sections [110]. See the text for an explanation of the notation. Copyright 2002 by the American Physical Society (http://link.aps.org/abstract/PRL/v89/e021302).

\begin{tabular}{|c|c|c|c|c|c|}
\hline & PHENIX & PHOBOS & BRAHMS & Ref. [88] & Ref. [109] \\
\hline$\sigma_{\text {tot }}(\mathrm{b})$ & - & - & - & $10.8 \pm 0.5$ & 11.2 \\
$\sigma_{\text {geom }}(\mathrm{b})$ & - & - & - & 7.1 & 7.3 \\
$\frac{\sigma_{\text {geom }}}{\sigma_{\text {tot }}}$ & $0.661 \pm 0.014$ & $0.658 \pm 0.028$ & $0.68 \pm 0.06$ & 0.67 & 0.659 \\
$\frac{\sigma(\ln X \mid \mathcal{D})}{\sigma_{\text {tot }}}$ & $0.117 \pm 0.004$ & $0.123 \pm 0.011$ & $0.121 \pm 0.009$ & 0.125 & 0.139 \\
$\frac{\sigma(1 n X \mid 1 n Y)}{\sigma(\ln X \mid \mathcal{D})}$ & $0.345 \pm 0.012$ & $0.341 \pm 0.015$ & $0.36 \pm 0.02$ & 0.329 & - \\
$\frac{\sigma(2 n X \mid \mathcal{D})}{\sigma(\ln X \mid \mathcal{D})}$ & $0.345 \pm 0.014$ & $0.337 \pm 0.015$ & $0.35 \pm 0.03$ & - & 0.327 \\
$\frac{\sigma(\ln X \mid 1 n Y)}{\sigma_{\text {tot }}}$ & $0.040 \pm 0.002$ & $0.042 \pm 0.003$ & $0.044 \pm 0.004$ & $0.041 \pm 0.002$ & - \\
\hline
\end{tabular}

The $(2 n X \mid 1 n Y)$ topology, with two neutrons in the left-hand ZDC, gives rise to the second peak in Fig. 90. Emission of a second neutron in the $(2 n X \mid \mathcal{D})$ topology is about $35 \%$ of the single neutron topology $(\ln X \mid \mathcal{D})$, see Table 19 . The table also shows the ratios of $\sigma(1 n X \mid 1 n Y)$ and $\sigma(1 n X \mid \mathcal{D})$ to the total ZDC cross section, $\sigma_{\text {tot }}$.

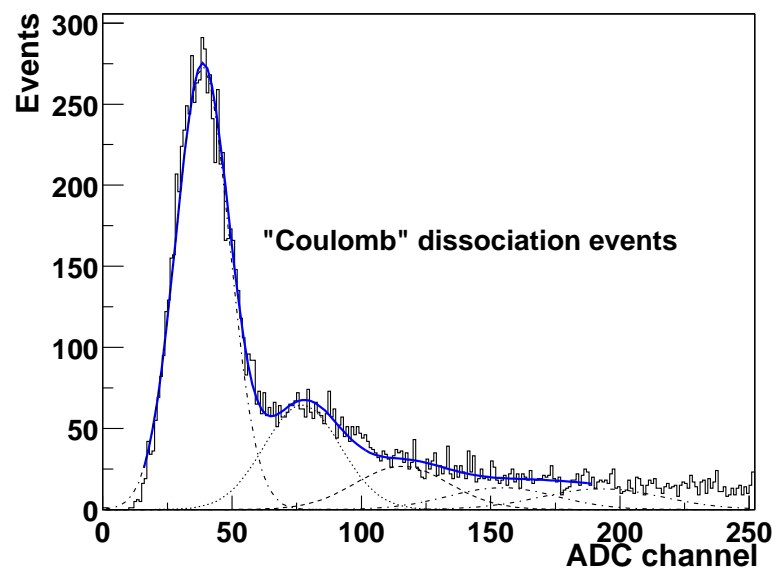

Figure 90. Energy spectrum for the left-hand ZDC with "Coulomb" selection for events with a single neutron in the right-hand ZDC [110]. Copyright 2002 by the American Physical Society (http://link.aps.org/abstract/PRL/v89/e012302). 
The $(\ln X \mid 1 n Y)$ topology is useful for luminosity measurements because contamination from hadronic events is small and the dependence on the nuclear radius is weak, as shown in Ref. [88]. Table 19 shows that the ZDC cross section ratios measured by three RHIC experiments (PHENIX, PHOBOS and BRAHMS) agree well with each other and with the calculations. The ZDC has also successfully tagged UPC events with $\rho^{0}$ production by virtual photons $[62,83]$.

In addition to employing the $(1 N N \mid 1 n Y)$ topology, it is also possible [113] to use the sum $\sigma(1 n X \mid 1 n Y)+\sigma(1 n X \mid 2 n Y)+\sigma(2 n X \mid 1 n Y)+\sigma(2 n X \mid 2 n Y)$ as a luminosity monitor, as explained below.

At the LHC, the advantage of the proposed methods $[88,113]$ is the use of the ZDCs, intended for centrality measurements in hadronic heavy-ion collisions [122, 321, 322]. Therefore, no additional instrumentation is needed for luminosity measurements. However, a key ingredient is an accurate calculation of the neutron emission cross sections in mutual electromagnetic dissociation $\sigma^{\mathrm{MED}}$, the subject of this chapter.

References [323,324] show that, due to the excitation of discrete nuclear states, there will be high energy photons in the forward direction which could be a signature of UPCs. It seems however, that there is presently no practical experimental means to use these high energy photons in the LHC experiments. A related process has been discussed in cosmic ray physics [325] where TeV gamma rays originate from the excitation and deexcitation of cosmic ray nuclei in the cosmic microwave background (CMB) radiation. Thus ultrarelativistic nuclei may be viewed as 'relativistic mirrors' which boost low energy photons from the $\mathrm{CMB}$ and the equivalent photon spectrum, respectively, to very high energies.

\subsection{Unique characteristics of mutual electromagnetic dissociation of heavy ions}

Since the first pioneering studies of the electromagnetic dissociation [326,327], the process has commonly been defined as disintegration of one of the nuclei involved in a UPC even though their nuclear densities do not overlap. Recent experiments [328, 329] have measured projectile or target dissociation, respectively.

Both at RHIC and the LHC, single electromagnetic dissociation, when only one of the nuclei is excited and dissociates, far exceeds the geometric cross section, $\sigma_{\text {geom }}$, due to direct nuclear overlap $[3,16,86,109]$. As a result, electromagnetic dissociation and $e^{+} e^{-}$pair production (when followed by electron capture) reduce the beam lifetime in colliders [86].

Both nuclei may be disintegrated in one event by the corresponding Coulomb fields

of their collision partners [109]. Here we focus on the mutual dissociation of lead ions at the LHC to monitor and measure luminosity by detection of forward-backward neutrons. Details can be found in Ref. [109, 113]. 


\subsection{Leading order mutual electromagnetic $\mathrm{Pb}+\mathrm{Pb}$ dissociation}

The Weizsäcker-Williams method $[3,16]$ treats the impact of the Lorentz-boosted Coulomb field of nucleus $A$ as the absorption of equivalent photons by nucleus $B$.

Figure 91 shows the leading and next-to-leading order processes contributing to mutual electromagnetic dissociation, with each order treated independently (see Refs. [84,109] for details). The open and closed circles on the diagrams denote elastic and inelastic vertices, respectively. Thus, at LO, a photon with energy $E_{1}$ is exchanged between $A$ and $B$, leaving $B$ in excited state $B^{*}$ after absorption of the photon. A photon with energy $E_{2}$ is exchanged between $B^{*}$ and $A$ and absorbed by $A$, exciting it to $A^{*}$. Both excited nuclei dissociate. There is no time ordering and, for calculational purposes, the photon emission spectrum does not depend on whether the nuclei are excited or not. The photon exchange between ground-state nuclei is the primary exchange while the photon exchange between an excited nucleus and a ground-state nucleus is a secondary photon exchange. There is a complementary diagram to $\mathrm{NLO}_{12}, \mathrm{NLO}_{21}$, where nucleus $B$ is excited by double photon absorption while $A$ is excited by single photon absorption.

The cross section for dissociation of $A$ and/or $B$ to final states $i$ and $j$ (single and mutual dissociation) respectively, is

$$
\sigma^{(\mathrm{S}, \mathrm{M}) \mathrm{ED}}(i \mid j)=2 \pi \int_{b_{c}}^{\infty} d b b P_{A}^{i}(b) P_{B}^{j}(b)
$$

where SED stands for dissociation of only one of the nuclei (single electromagnetic dissociation) and MED is for mutual electromagnetic dissociation. When only one nucleus is dissociated, the cross section includes only one probability factor, i.e. either $P_{A}^{i}(b)$ or $P_{B}^{j}(b)$ is unity. The lower limit of integration, $b_{c}$, is a sharp cutoff, approximately given by the sum of the nuclear radii, $b_{c} \approx R_{A}+R_{B}$, to separate the nuclear and electromagnetic interaction domains. The choice of the lower limit is discussed further in Sec. 7.7. In Eq. (143), probability for dissociation of $B$ at impact parameter $b$ is defined as

$$
P_{B}^{j}(b)=e^{-m_{B}(b)} \int_{E_{\min }}^{E_{\max }} d E_{1} \frac{d^{3} N_{\gamma A}}{d E_{1} d^{2} b} \sigma_{B}\left(E_{1}\right) f_{B}\left(E_{1}, j\right)
$$

where $m_{B}(b)$ is the mean number of photons absorbed by nucleus $B$,

$$
m_{B}(b)=\int_{E_{\min }}^{E_{\max }} d E_{1} \frac{d N_{\gamma A}^{3}}{d E_{1} d^{2} b} \sigma_{B}\left(E_{1}\right) .
$$

Here $d N_{\gamma A} / d E_{1} d^{2} b$ is the virtual photon spectra from nucleus $A$ at $b$ from Eq. (5), $\sigma_{B}\left(E_{1}\right)$ and $f_{B}\left(E_{1}, j\right)$ are the total photo-absorption cross section and the branching ratio for dissociation into final state $j$ due to absorption of a photon with energy $E_{1}$ by $B$ [109]. The expression for $P_{A}^{i}(b)$ is obtained by exchanging subscripts and taking $j \rightarrow i$. The neutron emission threshold is used for $E_{\min }$ while $E_{\max } \approx \gamma_{L} / R_{A, B}$. In the case of a collider, the Lorentz factor of the heavy-ion beam is boosted to the rest frame 
of the collision partner, $\gamma_{L}^{\text {rest }}=2 \gamma_{L}^{2}-1$. At the LHC, the Coulomb fields of the ions are extremely Lorentz-contracted with $\gamma_{L} \sim 1.7 \times 10^{7}$.
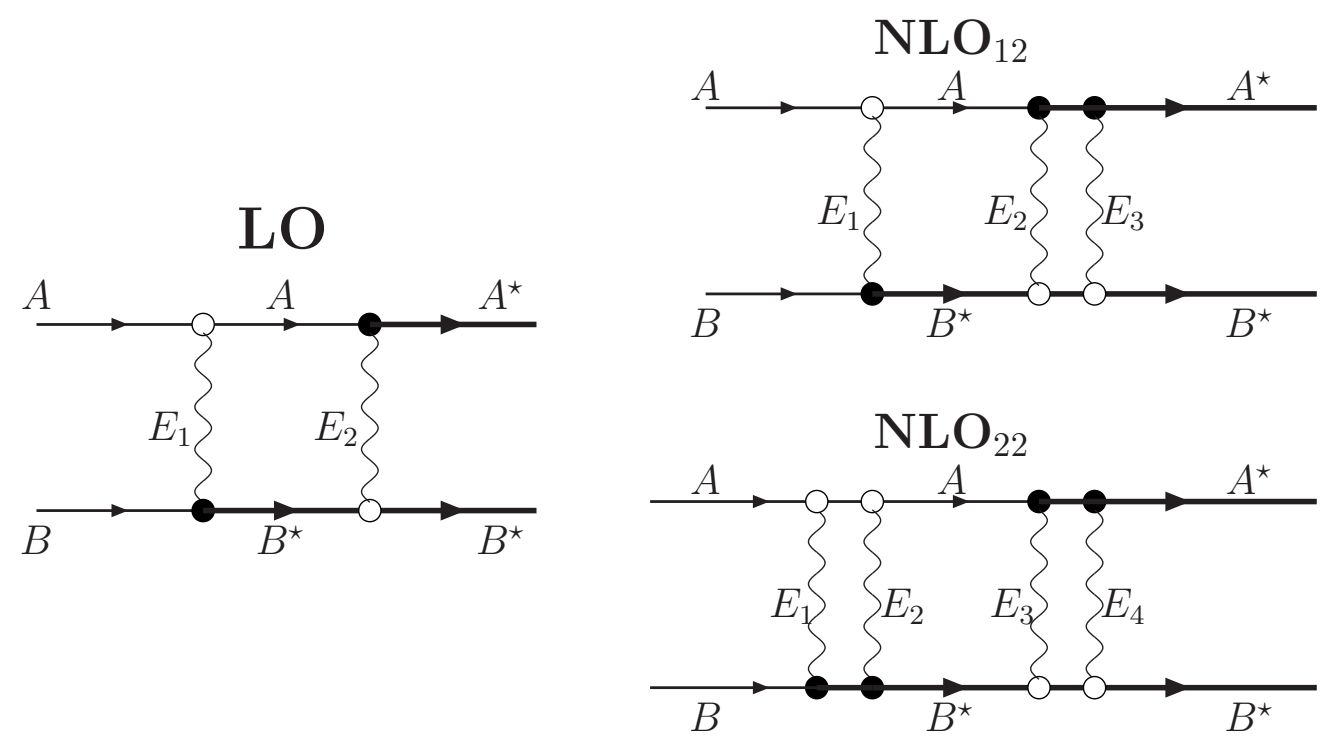

Figure 91. The electromagnetic excitation and mutual dissociation of relativistic nuclei. Open and closed circles denote elastic and inelastic vertices, respectively. The $\mathrm{LO}$ contribution is shown on the left-hand side. The NLO contributions with single and double photon exchange, $\mathrm{NLO}_{12}$, and with two double-photon exchange, $\mathrm{NLO}_{22}$, are shown on the right-hand side.

The total LO cross section for mutual electromagnetic dissociation by two-photon exchange, as shown in Fig. 91, is

$$
\begin{aligned}
\sigma_{\mathrm{LO}}^{\mathrm{MED}} & =2 \pi \int_{b_{c}}^{\infty} d b b\left[m_{A}(b) e^{-m_{A}(b)}\right]\left[m_{B}(b) e^{-m_{B}(b)}\right] \\
& =2 \pi \int_{b_{c}}^{\infty} d b b m_{A}^{2}(b) e^{-2 m_{A}(b)} .
\end{aligned}
$$

The last equality assumes $A=B$ and $Z_{A}=Z_{B}$. In the case of single dissociation, SED, only one factor of $m_{A}(b) \exp \left[-m_{A}(b)\right]$ is included. Note that we have taken $f_{B}(E, j)=f_{A}(E, i) \equiv 1$ in Eq. (144) since no final state is specified and the sum over branching ratios is by definition unity at each photon energy.

\subsection{Next-to-leading-order mutual electromagnetic dissociation}

In addition to the LO mutual dissociation process, a set of NLO processes with three or four photon exchanges can be considered. The total MED cross section for the three photon process, $\mathrm{NLO}_{12}$, shown in Fig. 91 is

$$
\sigma_{\mathrm{NLO}_{12}}^{\mathrm{MED}}=2 \pi \int_{b_{c}}^{\infty} d b b\left[m_{A}(b) e^{-m_{A}(b)}\right]\left[\frac{m_{B}^{2}(b)}{2} e^{-m_{B}(b)}\right]
$$




$$
=2 \pi \int_{b_{c}}^{\infty} d b b \frac{m_{A}^{3}(b)}{2} e^{-2 m_{A}(b)}
$$

where again $A=B$ is assumed in the last equality. The complementary process, $\mathrm{NLO}_{21}$, with the excitation of $B$ via double photon absorption is equally possible and has the same cross section. Likewise the total SED cross section for breakup of one nucleus by exchange of two photons is

$$
\sigma_{\mathrm{NLO}_{2}}^{\mathrm{SED}}=2 \pi \int_{b_{c}}^{\infty} d b b \frac{m_{A}^{2}(b)}{2} e^{-m_{A}(b)} .
$$

The MED cross section for four photon exchange, denoted $\mathrm{NLO}_{22}$ in Fig. 91, is

$$
\begin{aligned}
\sigma_{\mathrm{NLO}_{22}}^{\mathrm{MED}} & =2 \pi \int_{b_{c}}^{\infty} d b b\left[\frac{m_{A}^{2}(b)}{2} e^{-m_{A}(b)}\right]\left[\frac{m_{B}^{2}(b)}{2} e^{-m_{B}(b)}\right] \\
& =2 \pi \int_{b_{c}}^{\infty} d b b \frac{m_{A}^{4}(b)}{4} e^{-2 m_{A}(b)}
\end{aligned}
$$

when $A=B$ in the last equality. The SED cross section for exchange of three or more photons is the sum over the series $\left[m_{A}^{n}(b) / n\right.$ !] for $n \geq 3$,

$$
\sigma_{\mathrm{NLO}_{3+}}^{\mathrm{SED}}=2 \pi \int_{b_{c}}^{\infty} d b b e^{-m_{A}(b)} \sum_{n=3}^{\infty} \frac{m_{A}^{n}(b)}{n !} .
$$

In MED, the exchange of at least three photons on one side is referred to as triple excitation or $\mathrm{NLO}_{\mathrm{TR}}$.

One can calculate the sum of all contributions to single and mutual electromagnetic exchange using the prescription of Ref. [88]:

$$
\sigma_{\text {tot }}^{\mathrm{S}(\mathrm{M}) \mathrm{ED}}=2 \pi \int_{b_{c}}^{\infty} d b b\left[1-e^{-m_{A}(b)}\right]^{E}
$$

where $E=1$ for SED and 2 for MED. Since the collision probability for each ion without photon exchange is equal to $\exp \left[-m_{A}(b)\right]$, Eq. (151) is evident.

In a more detailed treatment, the character of the intermediate state would be considered since a given photon energy $E_{1}$ leads to a specific set of intermediate states. The excitation cross sections of the intermediate states differ somewhat relative to the unexcited nuclei introducing correlations between the photon energies which are not considered here.

\subsection{Hadronic nuclear dissociation in grazing collisions}

At grazing impact parameters, $b \sim R_{A}+R_{B}$, nuclei are partly transparent to each other. Hadronic nucleon-nucleon collisions may be absent in peripheral events with a weak overlap of diffuse nuclear surfaces while electromagnetic interactions may lead to electromagnetic dissociation. Both hadronic and electromagnetic interactions may occur in the same event. For example, a neutron-neutron collision in the density overlap 
zone may lead to neutron ejection accompanied by photon emission and absorption in electromagnetic interactions.

Therefore, a smooth transition from purely nuclear collisions at $b<R_{A}+R_{B}$ to electromagnetic collisions at $b>R_{A}+R_{B}$ takes place. Such a transition region was considered in the "soft-sphere" model of Ref. [330]. A similar approach was adopted in Ref. [88], where the cross section for nuclear or electromagnetic dissociation alone or for both together was written in an unexponentiated form as

$$
\sigma=2 \pi \int_{0}^{\infty} d b b\left(\mathcal{P}^{\mathrm{nuc}}(b)+\mathcal{P}^{\mathrm{ED}}(b)-\mathcal{P}^{\mathrm{nuc}}(b) \mathcal{P}^{\mathrm{ED}}(b)\right)
$$

where $\mathcal{P}^{\text {nuc }}(b)$ and $\mathcal{P}^{\mathrm{ED}}(b)$ are the probabilities of nuclear and electromagnetic dissociation at $b$. Including the limits of integration for each term separately, we have

$$
\begin{aligned}
\sigma= & 2 \pi \int_{0}^{b_{c}^{\mathrm{nuc}}} d b b \mathcal{P}^{\mathrm{nuc}}(b)+2 \pi \int_{b_{c}^{\mathrm{ED}}}^{\infty} d b b \mathcal{P}^{\mathrm{ED}}(b) \\
& -2 \pi \int_{b_{c}^{\mathrm{ED}}}^{b_{c}^{\mathrm{nuc}}} d b b \mathcal{P}^{\mathrm{nuc}}(b) \mathcal{P}^{\mathrm{ED}}(b) .
\end{aligned}
$$

Here individual impact parameter cutoff values, $b_{c}^{\text {nuc }}$ and $b_{c}^{\mathrm{ED}}$, were used for the nuclear and electromagnetic interactions. However, the simpler expression,

$$
\sigma=\sigma^{\mathrm{nuc}}+\sigma^{\mathrm{ED}}=2 \pi \int_{0}^{b_{c}} d b b \mathcal{P}^{\mathrm{nuc}}(b)+2 \pi \int_{b_{c}}^{\infty} d b b \mathcal{P}^{\mathrm{ED}}(b),
$$

is widely used with a single cutoff, $b_{c}$, chosen so that $b_{c}^{\mathrm{ED}}<b_{c}<b_{c}^{\text {nuc }}$. Using a single cutoff allows the first and second terms of Eq. (153) to be simplified while the third term is eliminated. Numerical results based on Eqs. (153) and (154) are similar, as shown for the "sharp-cutoff" and "soft-sphere" models of Ref. [330]. In the case of heavy nuclei, the difference between realistic values of $b_{c}^{\mathrm{ED}}, b_{c}^{\text {nuc }}$ and $b_{c}$ is less than $1 \mathrm{fm}$. As a result, the third term in Eq. (153) turns out to be small. Finally, the nuclear and electromagnetic contributions can be studied separately using Eq. (154). This separation is important for understanding nuclear and electromagnetic dissociation at ultrarelativistic colliders where the nuclear and electromagnetic interaction products populate different, nonoverlapping rapidity regions. In the widely-used BCV parametrization [331], $b_{c}$ is

$$
b_{c}=R_{\mathrm{BCV}}\left(A^{1 / 3}+B^{1 / 3}-X_{\mathrm{BCV}}\left(A^{-1 / 3}+B^{-1 / 3}\right)\right) .
$$

The parameters $R_{\mathrm{BCV}}=1.34 \mathrm{fm}$ and $X_{\mathrm{BCV}}=0.75$ are obtained by fitting Glauber-type calculations of the total nuclear reaction cross Sections [331]. The fragment angular distributions, very sensitive to $b_{c}$, can be described by the BCV parametrization [332].

Even when the nuclear densities partly overlap and only a few nucleon-nucleon collisions occur, intense hadron production is expected at LHC energies. These secondary hadrons will be produced at midrapidity while neutrons from electromagnetic dissociation are emitted close to beam rapidity. This difference can be used to disentangle hadronic and electromagnetic dissociation. 
The cross section for the removal (abrasion) of $a_{1}$ nucleons from projectile $A$ by interaction with target $B$ may be derived from Glauber multiple-scattering theory [333]

$$
\sigma^{\mathrm{nuc}}\left(a_{1}\right)=\left(\begin{array}{c}
A \\
a_{1}
\end{array}\right) 2 \pi \int_{0}^{\infty} d b b[1-P(b)]^{a_{1}}[P(b)]^{A-a_{1}} .
$$

Here $P(b)$ is the overlap of the projectile, $T_{A}(s)$, and target, $T_{B}(|\vec{b}-\vec{s}|)$, thickness functions at impact parameter $b$

$$
P(b)=\frac{1}{A} \int d^{2} s T_{A}(s) \exp \left[-\sigma_{N N} T_{B}(|\vec{b}-\vec{s}|)\right] .
$$

The nuclear densities are parametrized by Woods-Saxon distributions for heavy nuclei.

More details and numerical results for hadron-nuclear dissociation in $\mathrm{Pb}+\mathrm{Pb}$ collisions at the LHC can be found in Ref. [109]. Here we only give the expression for the dissociation cross section when no nucleons are ejected, due to partial transparency of surface nucleons,

$$
\sigma^{\mathrm{nuc}}(0)=2 \pi \int_{0}^{\infty} d b b[P(b)]^{A} .
$$

\subsection{Mutual electromagnetic excitation as a filter for close collisions}

In this section, we compare the probability distributions for single and mutual electromagnetic dissociation. In single electromagnetic dissociation, only one of the two nuclei dissociate. The cross section for the two processes can be written as

$$
\sigma^{(\mathrm{S}, \mathrm{M}) \mathrm{ED}}=2 \pi \int_{b_{c}}^{\infty} d b b \mathcal{P}^{(\mathrm{S}, \mathrm{M}) \mathrm{ED}}(b)
$$

where $\mathcal{P}^{\mathrm{SED}}(b)$ and $\mathcal{P}^{\mathrm{MED}}(b)$ are the probabilities for single and mutual electromagnetic dissociation. The expressions for $\mathcal{P}^{\mathrm{MED}}(b)$ can be taken from Eqs. (146), (147) and (149). The expressions for $\mathcal{P}^{\mathrm{SED}}$ follow from Eqs. (148) and (150). Equation (159) corresponds to the sharp-cutoff approximation. For comparison, in the soft-sphere model, the cross section without the sharp cutoff, $b_{c}$, can be written as

$$
\sigma^{(\mathrm{S}, \mathrm{M}) \mathrm{ED}}=2 \pi \int_{0}^{\infty} d b b[P(b)]^{A} \mathcal{P}^{(\mathrm{S}, \mathrm{M}) \mathrm{ED}}(b),
$$

as follows from Eq. (158) when no nucleons are ejected. The product $[P(b)]^{A} \mathcal{P}^{(\mathrm{S}, \mathrm{M}) \mathrm{ED}}(b)$ is presented in Fig. 92 for single and mutual electromagnetic dissociation for each of the LO and NLO processes discussed previously. Using the sharp-cutoff for heavy nuclei gives a few percent error on $\sigma^{(\mathrm{S}, \mathrm{M}) \mathrm{ED}}$, within the uncertainty introduced by the photonuclear cross section data used in calculations.

The largest contributions to the MED cross section comes from 'close' collisions with $b \sim b_{c}$, where the probability to absorb a virtual photon is large, and two or more photons can be absorbed by each nucleus. The MED probabilities decrease faster with 


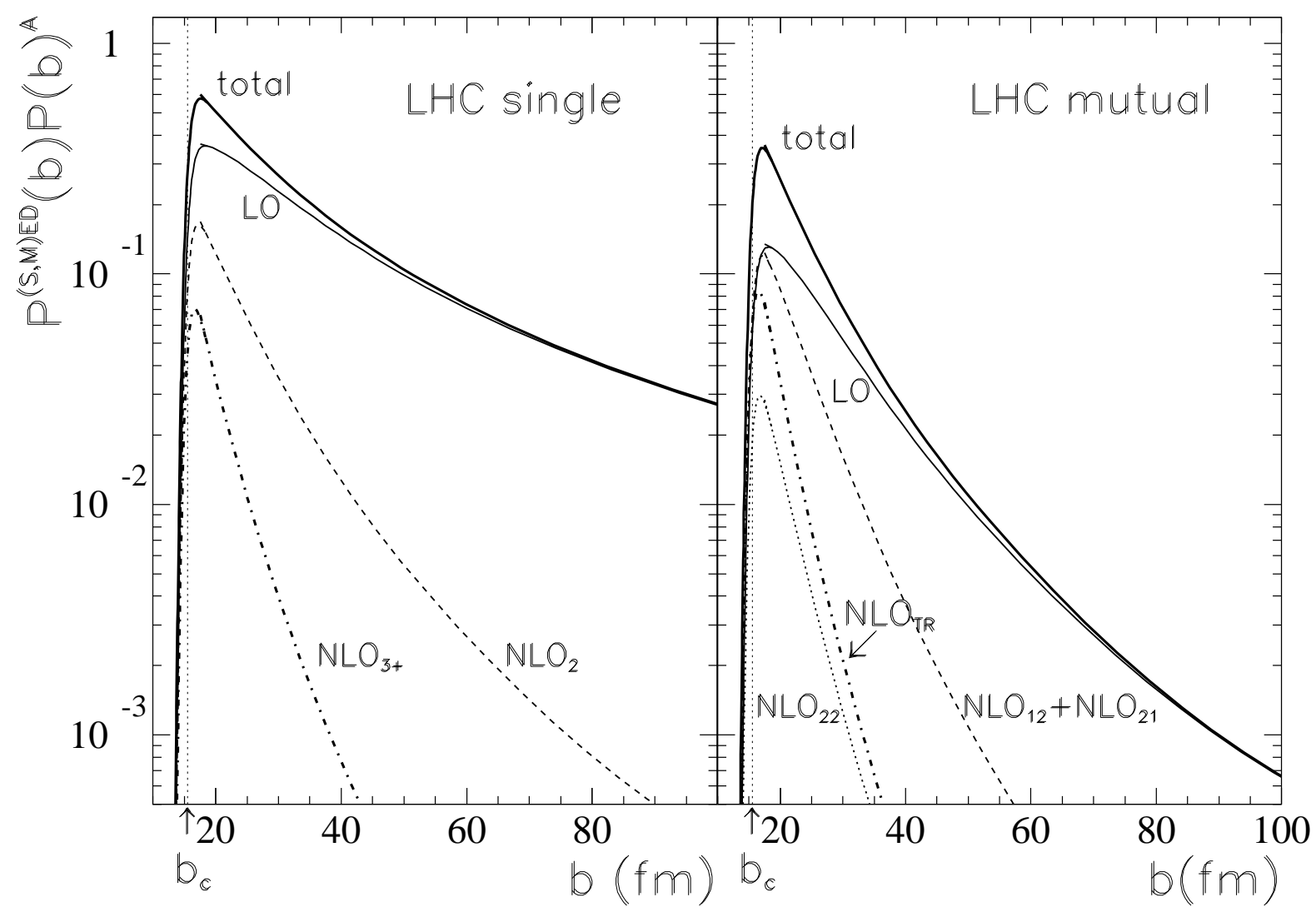

Figure 92. The probability of single (left panel) and mutual (right panel) electromagnetic dissociation to LO and NLO as a function of $b$ in $2.75 A+2.75 A$ $\mathrm{TeV} \mathrm{Pb}+\mathrm{Pb}$ collisions at the LHC predicted by the RELDIS and soft-sphere models. The thick solid lines give the sum of the LO and NLO contributions. The value $b_{c}=15.54 \mathrm{fm}$ is indicated.

$b$ than SED. Thus mutual dissociation can be used as a filter for selecting collisions with $b \sim b_{c}$.

The relative NLO contributions to MED are enhanced compared to SED, as shown in Fig. 92. The sum $\mathrm{NLO}_{12}+\mathrm{NLO}_{21}$ is similar to the $\mathrm{LO}$ contribution when $b \sim b_{c}$. In this region, the probability of triple excitations, $\mathrm{NLO}_{\mathrm{TR}}$, is also comparable to the LO contribution. However, all the NLO contributions decrease faster with $b$ than the LO contribution. Thus the NLO cross sections in Table 20 are lower than the LO cross sections.

Table 20. The total LO, individual NLO corrections and summed MED cross sections for $2.75 A+2.75 A \mathrm{TeV} \mathrm{Pb}+\mathrm{Pb}$ collisions at the $\mathrm{LHC}$ [109].

\begin{tabular}{|c|c|c|c|c|}
\hline$\sigma_{\mathrm{LO}}^{\mathrm{MED}}(\mathrm{b})$ & $\sigma_{\mathrm{NLO}_{12}}^{\mathrm{MED}}+\sigma_{\mathrm{NLO}}^{\mathrm{MED}}(\mathrm{b})$ & $\sigma_{\mathrm{NLO}}^{\mathrm{MED}}(\mathrm{b})$ & $\sigma_{\mathrm{NLO}}^{\mathrm{MED}}(\mathrm{b})$ & $\sigma_{\mathrm{tot}}^{\mathrm{MED}}(\mathrm{b})$ \\
\hline 3.92 & 1.50 & 0.23 & 0.56 & 6.21 \\
\hline
\end{tabular}


The MED cross sections presented here were calculated using the modified RELDIS code [109] employing a special simulation mode for MED events. Table 20 gives the inclusive $\mathrm{LO}, \mathrm{NLO}_{12}, \mathrm{NLO}_{22}, \mathrm{NLO}_{\mathrm{TR}}$ contributions and total cross sections with $f(E, i)=f(E, j)=1$ in $\sigma^{\mathrm{MED}}(i \mid j)$. The LO contribution is $\sim 63 \%$ of $\sigma^{\mathrm{MED}}$ (tot) at LHC energies. The sum of the NLO contributions gives an additional $\sim 28 \%$. Therefore, the remaining contribution, $\sim 9 \%$ of the total MED cross section, is due to exotic triple nuclear excitations with three or more photons absorbed by at least one nucleus.

Electromagnetic heavy-ion excitation is widely used to study nuclear structure, as demonstrated by fixed-target experiments at intermediate energies [334,335]. Experimental studies of MED at the LHC can provide valuable information about double and triple nuclear excitations in electromagnetic interactions, particularly for multiphonon resonances. Triple excitation data is very important for triple giant resonance excitations since there are currently no data on such extreme excitations. The first theoretical predictions for the energies and widths of such states are given in Ref. [336].

The number of forward neutrons emitted in the dissociation process and detected in the ZDCs can be used to study multiple excitations, even when the ZDC resolution cannot determine the exact number of neutrons in one of the ZDCs or for poor statistics. To demonstrate the utility of the dissociation process, we assume that the dissociation channel of one of the nuclei in MED is unknown. Then the inclusive MED cross sections, $\sigma^{\operatorname{MED}}(1 n X \mid \mathcal{D}), \sigma^{\operatorname{MED}}(2 n X \mid \mathcal{D})$ and $\sigma^{\operatorname{MED}}(3 n X \mid \mathcal{D})$ for emission of one, two and three neutrons, by one of the nuclei, respectively, can be considered. The $X$ indicates that neutron emission can be accompanied by some number of undetected particles. In the notation of Section 7.3, $\mathcal{D}$ denotes an arbitrary dissociation mode for the other nucleus so that $f(E, i) \equiv 1$.

Table 21. The MED cross sections for $2.75 A+2.75 A \mathrm{TeV} \mathrm{Pb}+\mathrm{Pb}$ collisions at the LHC where $X$ and $Y$ denote other particles emitted from the nucleus with the neutrons and $\mathcal{D}$ is an arbitrary dissociation channel for the other nucleus $(f(E, i)=1)$. Results are given at LO alone and with the sum of the NLO contributions included [109].

\begin{tabular}{|c|c|c|}
\hline Final State & $\sigma_{\mathrm{LO}}(\mathrm{mb})$ & $\sigma_{\mathrm{LO}}+\sigma_{\mathrm{NLO}_{12}}+\sigma_{\mathrm{NLO}_{21}}+\sigma_{\mathrm{NLO}_{22}}(\mathrm{mb})$ \\
\hline$(1 n X \mid 1 n Y)$ & 750 & 805 \\
$(1 n X \mid \mathcal{D})$ & 1698 & 2107 \\
$(2 n X \mid \mathcal{D})$ & 443 & 654 \\
$(3 n X \mid \mathcal{D})$ & 241 & 465 \\
\hline
\end{tabular}

The LHC cross sections for several MED channels are given in Table 21. The specific branching ratios for the final-state channels, $f(E, 1 n X), f(E, 2 n X)$ and $f(E, 3 n X)$, are calculating by simulating neutron emission from a lead nucleus following the absorption of photons with energy $E$. The probability $P_{A}$ in Eq. (144) is modified by the branching ratio in the integral over $E$ while the factor $\exp \left[-m_{A}(b)\right]$ remains the same for both nuclei when $A=B$. If one final-state neutron is required for both nuclei, the cross section 
$\sigma^{\mathrm{MED}}(\ln X \mid 1 n Y)$ is reduced relative to $\sigma^{\mathrm{MED}}(1 n X \mid \mathcal{D})$ since both branching ratios are included in the probabilities.

The relative NLO contributions are very different for one, two and three neutron emission. The NLO contribution to $\sigma^{\mathrm{MED}}(1 n X \mid 1 n Y)$ is small, $\sim 7 \%$. On the other hand, the NLO correction to $\sigma^{\mathrm{MED}}(3 n X \mid \mathcal{D})$ is almost a factor of two. This large increase is because the NLO processes shown in Fig. 91 include nuclear excitation due to double photon absorption, particularly double GDR excitation. Since the average GDR energy for gold and lead nuclei is $\sim 13-14 \mathrm{MeV}$, double GDR introduces, on average, $26-28 \mathrm{MeV}$ excitation, above the three neutron emission threshold. Thus the $1 n$ and $2 n$ emission cross sections have smaller NLO corrections than the $3 n$ cross sections. Measurements of the forward $3 n$ emission rates in ALICE may detect multiple GDR excitations in lead.

\subsection{Reliability of the RELDIS predictions}

The reliability of the RELDIS code was studied in Ref. [109] by examining its sensitivity to variations in the input data and parameters. A good description of the existing SED data on lead and gold nuclei at the CERN SPS Refs. [111, 328, 329, 337] was obtained. As shown in Sec. 7.3, good agreement with the first RHIC data on mutual dissociation [110] was also found.

The photonuclear cross sections for specific neutron emission channels $(f(E, i) \neq 1$, $f(E, j) \neq 1$ ) were calculated by two different models of photonuclear reactions, GNASH [338] and RELDIS, see Table 22 and Ref. [109] for details. In addition, two different values for the probability of direct neutron emission in the $1 n$ channel, $P_{n}^{\text {dir }}=0$ and 0.26 , were used in the RELDIS code.

At the LHC, secondary nuclei are produced by electromagnetic dissociation of beam nuclei induced by interactions with residual gas and collimator material. These nuclear fragments diverge from the primary beam because of their scattering angle and their different $Z / A$ relative to the primary beam. Since these fragments do not fall within the acceptance of the collimation system, they induce a significant heat load in the superconducting magnets when they hit the magnet vacuum chamber. The yields of specific nuclear fragments from SED, MED and fragmentation of beam nuclei were calculated using RELDIS and abrasion-ablation models to estimate the heat load at the LHC [339, 340].

The cross sections for one or two neutron emission are given in Table 22 for different maximum values of the photon energy, $E_{\gamma} \leq E_{\max }$, the upper limit in the energy integrals in Eqs. (144) and (145). Results are shown for the GDR region, $E_{\gamma} \leq 24 \mathrm{MeV}$, energies up to the quasi-deuteron absorption region, $E_{\gamma} \leq 140 \mathrm{MeV}$, and the full range. In addition to the specified one and two neutron emission channels, a cumulative value, the Low Multiplicity Neutron (LMN) emission cross section,

$$
\begin{aligned}
\sigma^{\mathrm{MED}}(\mathrm{LMN})= & \sigma^{\mathrm{MED}}(1 n X \mid 1 n Y)+\sigma^{\mathrm{MED}}(1 n X \mid 2 n Y) \\
& +\sigma^{\mathrm{MED}}(2 n X \mid 1 n Y)+\sigma^{\mathrm{MED}}(2 n X \mid 2 n Y),
\end{aligned}
$$


Table 22. The LO and NLO MED cross section are presented for the GNASH and RELDIS codes in $2.75 A+2.75 A \mathrm{TeV} \mathrm{Pb}+\mathrm{Pb}$ collisions[109]. The sensitivity of the MED cross sections in selected channels to the photon energy range, $E_{\gamma}$, the probability of direct single neutron emission, $P_{n}^{\text {dir }}$, and the input photonuclear cross sections is illustrated. The recommended values are shown in boldface. For comparison, the predicted value of $\sigma_{\mathrm{LO}}^{\mathrm{MED}}(1 n \mid 1 n)$ in the GDR region $\left(E_{\gamma} \leq 24 \mathrm{MeV}\right)$ calculated in Ref. [88] is $533 \mathrm{mb}$.

\begin{tabular}{|c|c|c|c|c|c|}
\hline \multirow[b]{2}{*}{ Channel } & \multirow{2}{*}{$\begin{array}{c}\text { GDR region } \\
E_{\gamma} \leq 24 \mathrm{MeV} \\
\sigma_{\mathrm{LO}}^{\mathrm{MED}}(\mathrm{mb}) \\
\text { RELDIS } \\
P_{n}^{\mathrm{dir}}=0 \\
\end{array}$} & \multicolumn{2}{|c|}{$\begin{array}{c}\text { quasi-deuteron region } \\
E_{\gamma} \leq 140 \mathrm{MeV} \\
\sigma_{\mathrm{LO}}^{\mathrm{MED}}(\mathrm{mb})\end{array}$} & \multicolumn{2}{|c|}{$\begin{array}{c}\text { all } E_{\gamma} \\
\sigma_{\mathrm{LO}}^{\mathrm{MED}}+\sigma_{\mathrm{NLO}}^{\mathrm{MED}}+ \\
\sigma_{\mathrm{NLO}_{21}}^{\mathrm{MED}}+\sigma_{\mathrm{NLO}_{22}}^{\mathrm{MED}}(\mathrm{mb})\end{array}$} \\
\hline & & GNASH & $\begin{array}{l}\text { RELDIS } \\
P_{n}^{\text {dir }}=0\end{array}$ & $\begin{array}{l}\text { RELDIS } \\
P_{n}^{\text {dir }}=0\end{array}$ & $\begin{array}{c}\text { RELDIS } \\
P_{n}^{\text {dir }}=0.26\end{array}$ \\
\hline$(1 n X \mid 1 n Y)$ & 519 & 488 & 544 & 727 & 805 \\
\hline$(1 n X \mid 2 n Y)+(2 n X \mid 1 n Y)$ & 154 & 220 & 217 & 525 & 496 \\
\hline$(2 n X \mid 2 n Y)$ & 11 & 24 & 22 & 96 & 77 \\
\hline LMN & 684 & 732 & 783 & 1348 & 1378 \\
\hline
\end{tabular}

is also shown.

Table 22 shows that there is a $\sim 10 \%$ ambiguity in $\sigma(\ln X \mid 1 n Y)$, mainly due to uncertainties in the photo-neutron cross sections measured in experiments with real photons. However, when the sum of the one and two neutron emission channels, $\sigma^{\mathrm{MED}}(\mathrm{LMN})$, is considered, the uncertainty is reduced to $\sim 2 \%$. The sum, $\sigma^{\mathrm{MED}}(\mathrm{LMN})$, is also more stable with respect to other parameters relative to the other cross sections in Table 22, as discussed in Ref. [109]. Therefore, $\sigma^{\mathrm{MED}}(\mathrm{LMN})$ serves as a cumulative neutron emission rate useful for luminosity measurements at heavy-ion colliders.

At collider energies, neutron emission in mutual electromagnetic dissociation is not entirely exhausted by the simultaneous excitation and giant resonance decays in both of the colliding nuclei. In addition to mutual GDR excitation, asymmetric processes, such as GDR excitation of one nucleus accompanied by a photonuclear reaction in the other nucleus, are very likely. The presence of such asymmetric dissociations is clear in Fig. 93 which shows the forward neutron energy distributions.

The ALICE ZDC has several advantages relative to the RHIC ZDCs. The forward neutron energy resolution is expected to be $\sim 10 \%$ at the LHC [321,322] while it is $\sim 20 \%$ at RHIC [320]. As a result, the $3 n$ and $4 n$ emission channels can be unambiguously identified by the ALICE ZDC, making it possible to study multiple GDR excitations.

\section{Hard photoproduction at HERA}

Contributed by: M. Klasen 

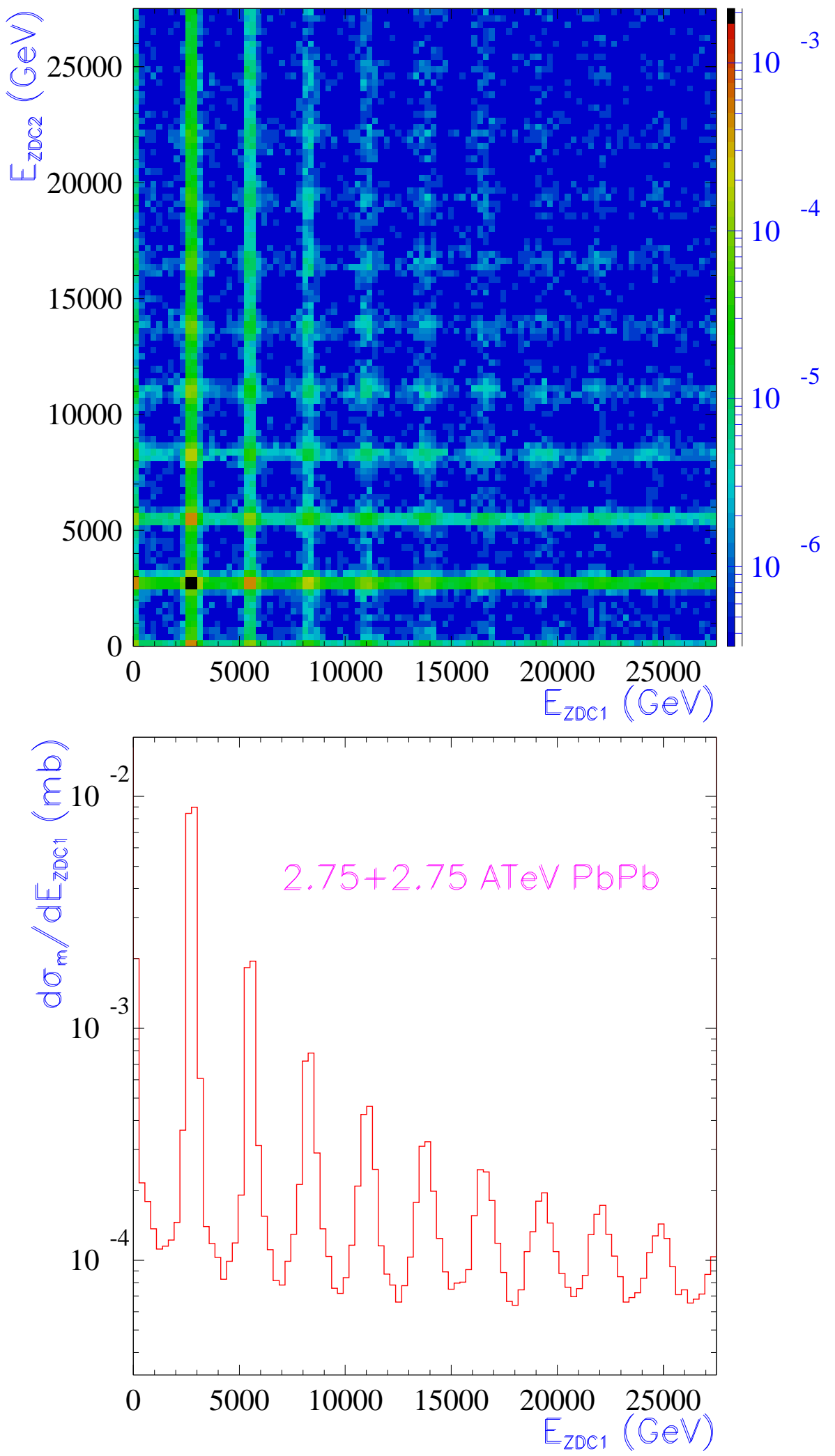

Figure 93. Top panel: The distribution of the total forward-backward neutron energy emitted in MED in $\mathrm{Pb}+\mathrm{Pb}$ collisions at the LHC. Bottom panel: The energy distribution in one ZDC obtained by projection of the top plot. The results are given for the LO process without including the ZDC energy resolution. From Ref. [109]. 


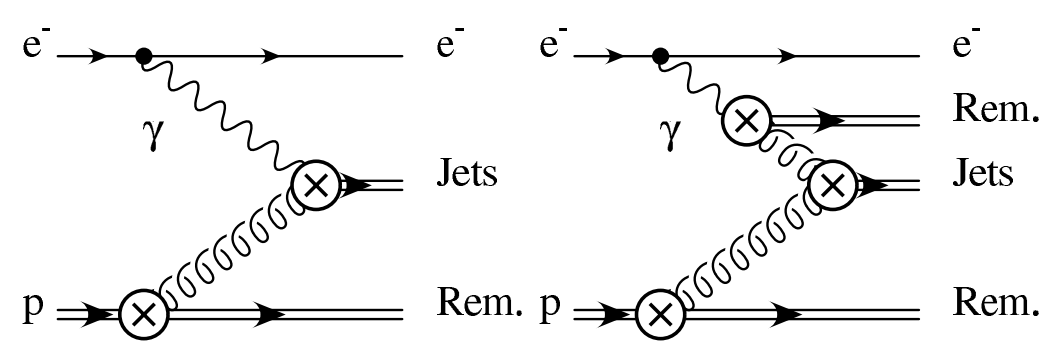

Figure 94. Factorization of direct (left) and resolved (right) photoproduction in the QCD-improved parton model [341]. Here Rem. indicates the proton and photon remnants. Copyright 2002 by the American Physical Society (http://link.aps.org/abstract/RPM/v74/p1221).

\subsection{Introduction}

In view of possible photoproduction studies in ultraperipheral heavy-ion collisions at the LHC, we briefly review the present theoretical understanding of photons and hard photoproduction processes at HERA, discussing the production of jets, light and heavy hadrons, quarkonia, and prompt photons. We address in particular the extraction of the strong coupling constant from photon structure function and inclusive jet measurements, the infrared safety and computing time of jet definitions, the sensitivity of dijet cross sections on the parton densities in the photon, factorization breaking in diffractive dijet production, the treatment of the heavy-quark mass in charm production, the relevance of the color-octet mechanism for quarkonium production, and isolation criteria for prompt photons.

Electron-proton scattering at HERA is dominated by the exchange of low-virtuality (almost real) photons [341]. If the electron is anti-tagged or tagged at small angles, the photon flux from the electron can be calculated in the Weizsäcker-Williams approximation, where the energy spectrum of the exchanged photons is given by

$$
f_{\gamma / e}^{\text {brems }}(x)=\frac{\alpha}{2 \pi}\left[\frac{1+(1-x)^{2}}{x} \ln \frac{Q_{\max }^{2}(1-x)}{m_{e}^{2} x^{2}}+2 m_{e}^{2} x\left(\frac{1}{Q_{\max }^{2}}-\frac{1-x}{m_{e}^{2} x^{2}}\right)\right]
$$

and the subleading non-logarithmic terms modify the cross section typically by $5 \%$ [342]. In the QCD-improved parton model, valid for hard scatterings, the photons can then interact either directly with the partons in the proton (Fig. 94, left) or resolve into a hadronic structure, so that their own partonic constituents interact with the partons in the proton (Fig. 94, right). While this separation is valid at leading order (LO) in QCD perturbation theory, the two processes are intimately linked at next-to-leading order (NLO) through the mandatory factorization of a collinear singularity that arises from the splitting of the photon into a quark-antiquark pair and induces a mutual logarithmic factorization scale dependence in both processes. In close analogy to deepinelastic electron-proton scattering, one can define a photon structure function

$$
F_{2}^{\gamma}\left(Q^{2}\right)=\sum_{q} 2 x e_{q}^{2}\left\{f_{q / \gamma}\left(Q^{2}\right)\right.
$$




$$
\left.+\frac{\alpha_{s}\left(Q^{2}\right)}{2 \pi}\left[C_{q} \otimes f_{q / \gamma}\left(Q^{2}\right)+C_{g} \otimes f_{g / \gamma}\left(Q^{2}\right)\right]+\frac{\alpha}{2 \pi} e_{q}^{2} C_{\gamma}\right\}
$$

that is related to the parton densities in the photon and has been measured in electronpositron collisions at LEP. Even the strong coupling constant $\alpha_{s}$ that appears in the expression above can be determined rather precisely in fits to these data [343]. A convenient modification of the $\overline{\mathrm{MS}}$ factorization scheme consists in absorbing the pointlike Wilson coefficient

$$
C_{\gamma}(x)=2 N_{C} C_{g}(x)=3\left[\left(x^{2}+(1-x)^{2}\right) \ln \frac{1-x}{x}+8 x(1-x)-1\right]
$$

in the Altarelli-Parisi splitting function $P_{q \leftarrow \gamma}^{\mathrm{DIS}}=P_{q \leftarrow \gamma}^{\overline{\mathrm{MS}}}-e_{q}^{2} P_{q \leftarrow q} \otimes C_{\gamma}[189]$.

\subsection{Inclusive and diffractive jet production}

While at LO hadronic jets are directly identified as final-state partons, their definition becomes subtle at higher orders, when several partons (or hadrons) can be combined to form a jet. According to the standardization of the 1990 Snowmass meeting, particles $i$ are added to a jet cone $J$ with radius $R$, if they are a distance $R_{i}=$ $\sqrt{\left(\eta_{i}-\eta_{J}\right)^{2}+\left(\phi_{i}-\phi_{J}\right)^{2}}<R$ from the cone center. However, these broad combined jets are difficult to find experimentally, so that several modifications (mid-points, additional seeds, iterations) have been successively applied by the various experiments. The deficiencies of the cone algorithm are remedied in the longitudinally invariant $k_{T^{-}}$ clustering algorithm, where one uses only the combination criterion $R_{i j}<1$ for any pair of particles $i$ and $j$. Unfortunately, this algorithm scales numerically with the cubic power of the number, $N$, of particles involved. Only recently a faster version has been developed making use of geometrical arguments and diagrammatic methods known from computational science [344]. The publicly available FastJet code scales only with $N \ln N$ and is now rapidly adopted, in particular for the LHC, where the particle multiplicity is high.

Single (inclusive) jets benefit from high statistics and the presence of a single (transverse) energy scale $E_{T}$, which makes them easily accessible experimentally and their prediction theoretically stable. The $E_{T}$-distribution of the single-jet cross section can then be used to determine e.g. the strong coupling constant from scaling violations, as shown in Fig. 95. However, the single-jet cross section,

$$
\frac{d^{2} \sigma}{d E_{T} d \eta}=\sum_{a, b} \int_{x_{a, \min }}^{1} d x_{a} x_{a} f_{a / A}\left(x_{a}, M_{a}^{2}\right) x_{b} f_{b / B}\left(x_{b}, M_{b}^{2}\right) \frac{4 E_{A} E_{T}}{2 x_{a} E_{A}-E_{T} e^{\eta}} \frac{d \sigma}{d t},
$$

includes a convolution over one of the longitudinal momentum fractions of the partons so that parton densities cannot be uniquely determined.

In addition to the transverse energy $E_{T}$ and pseudorapidity $\eta_{1}$ of the first jet, the inclusive dijet cross section

$$
\frac{d^{3} \sigma}{d E_{T}^{2} d \eta_{1} d \eta_{2}}=\sum_{a, b} x_{a} f_{a / A}\left(x_{a}, M_{a}^{2}\right) x_{b} f_{b / B}\left(x_{b}, M_{b}^{2}\right) \frac{d \sigma}{d t}
$$




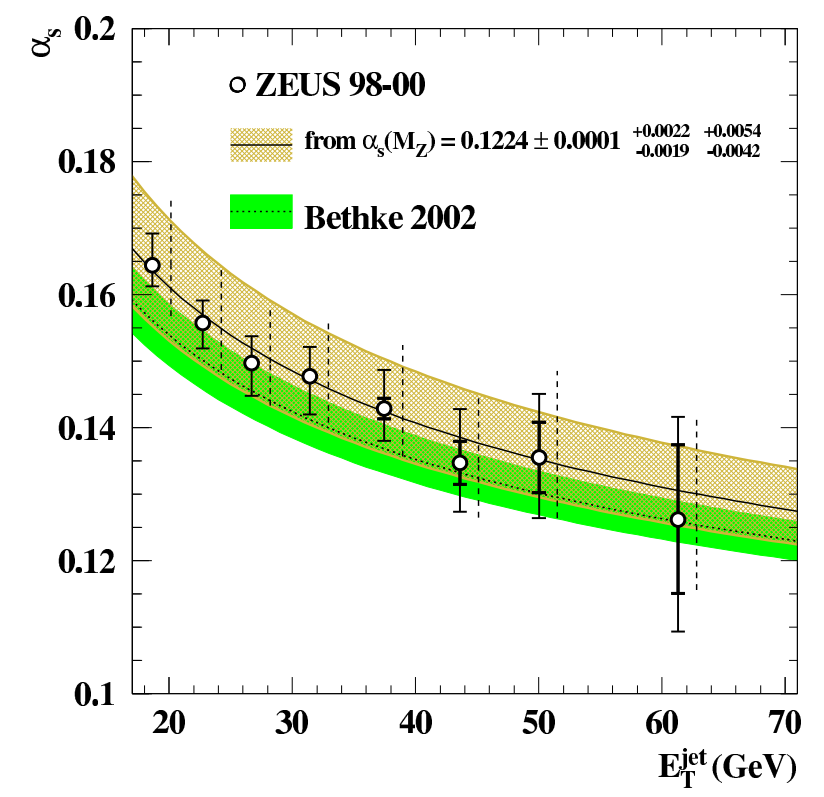

Figure 95. Strong coupling constant as measured from scaling violations in inclusive single-jet production at ZEUS. Reprinted from Ref. [345] with permission from Elsevier.
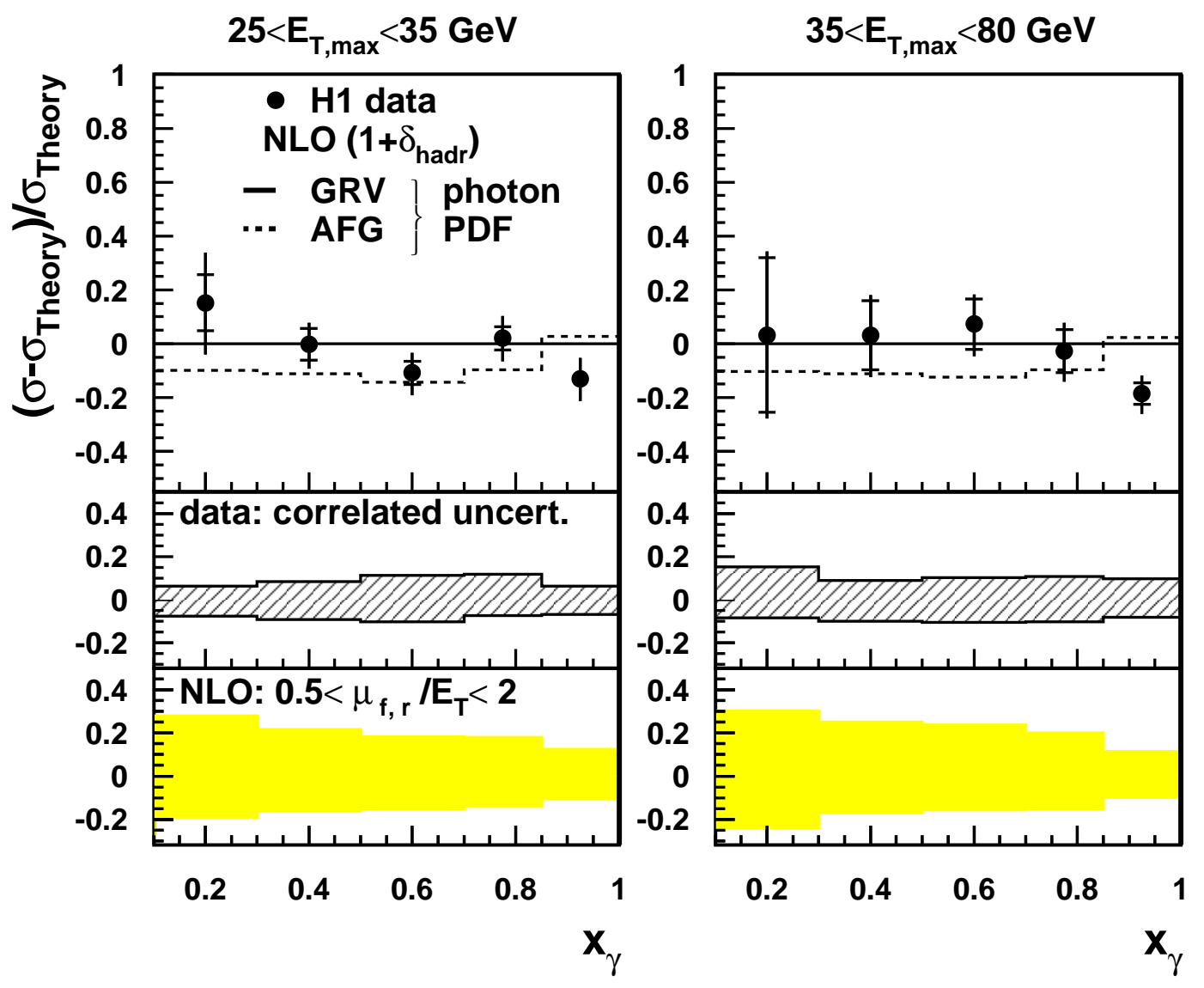

Figure 96. Sensitivity of the dijet photoproduction cross section as measured by H1 on the GRV and AFG parameterizations of the parton densities in the photon. Reprinted from Ref. [346] with permission from Springer-Verlag. 


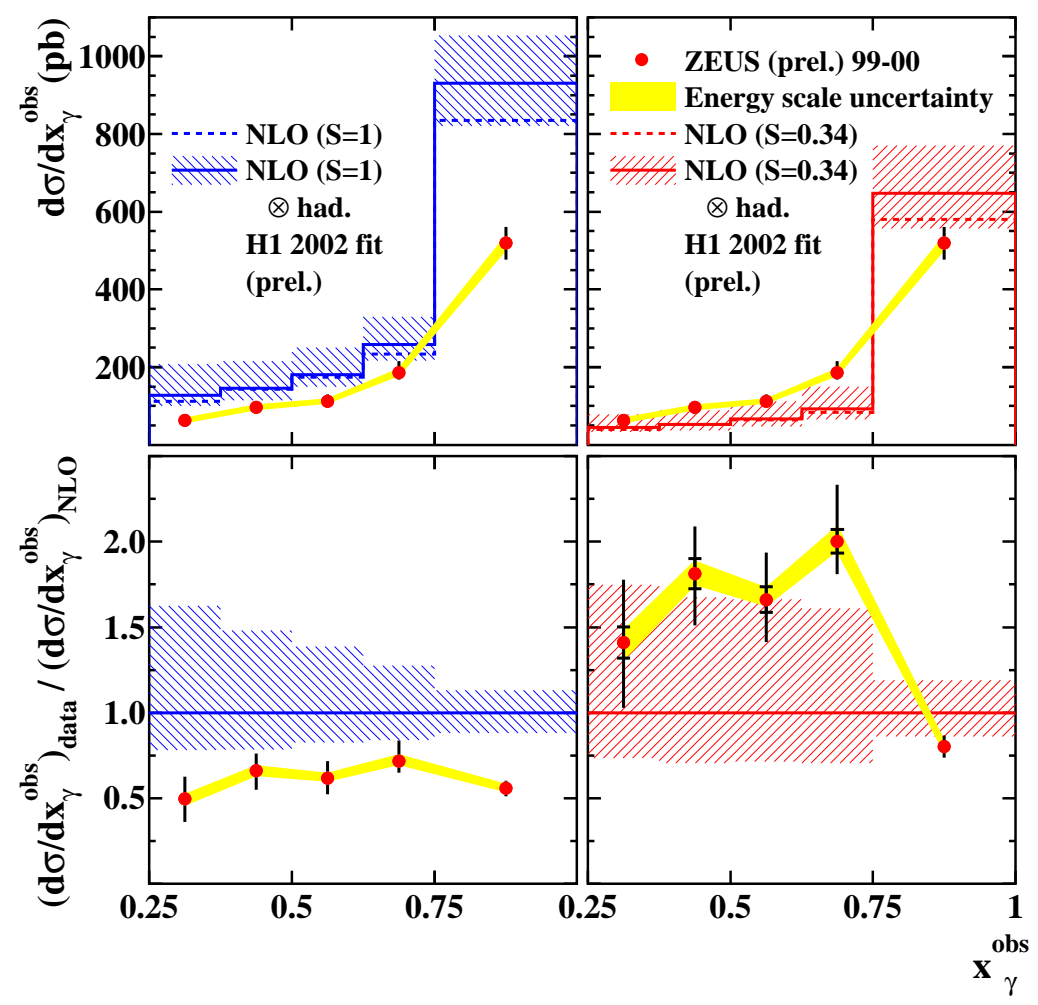

Figure 97. Dependence of the diffractive dijet cross section on the observed longitudinal momentum fraction of the scattered photon at ZEUS [347].

depends on the pseudorapidity of the second jet $\eta_{2}$. In LO only two jets with equal transverse energies can be produced and the observed momentum fractions of the partons in the initial electrons or hadrons $x_{a, b}^{\mathrm{obs}}=\sum_{i=1}^{2} E_{T_{i}} e^{ \pm \eta_{i}} /\left(2 E_{A, B}\right)$ equal the true momentum fractions $x_{a, b}$. If the energy transfer $y=E_{\gamma} / E_{e}$ is known, momentum fractions for the partons in photons $x_{\gamma}^{\text {obs }}=x_{a, b}^{\text {obs }} / y$ can be deduced. In NLO, where a third jet can be present, the observed momentum fractions are defined by the sums over the two jets with highest $E_{T}$ and they match the true momentum fractions only approximately. Furthermore, the transverse energies of the two hardest jets no longer need to be equal to each other. Even worse, for equal $E_{T}$ cuts and maximal azimuthal distance, $\Delta \phi=\phi_{1}-\phi_{2}=\pi$, the NLO prediction becomes sensitive to the method chosen for the integration of soft and collinear singularities. The theoretical cross section is then strongly scale dependent and thus unreliable. This sensitivity also propagates into the region of large observed momentum fractions. It is thus preferable to cut on the average $\bar{E}_{T}=\left(E_{T_{1}}+E_{T_{2}}\right) / 2$. The sensitivity of the dijet photoproduction cross section as measured by $\mathrm{H} 1$ on the GRV and AFG parameterizations of the parton densities in the photon is shown in Fig. 96.

In diffractive processes with a large rapidity gap between a leading proton [348], neutron [349] or some other low-mass hadronic state and a hard central system, QCD factorization is expected to hold for deep-inelastic scattering, so that diffractive parton densities can be extracted from experiment, but to break down for hadron-hadron 
scattering, where initial-state rescattering can occur. In photoproduction, these two factorization scenarios correspond to direct and resolved processes, which are, however, closely related, as noted in Section 8.1. It is thus interesting to investigate the breakdown of factorization in kinematic regimes where direct or resolved processes dominate. This can either be done by measuring the dependence on the photon virtuality $Q^{2}$ (transition from virtual to real photons) [350], $E_{T}$ (direct processes are harder than resolved photons), or $x_{\gamma}^{\text {obs }}$ (unity for direct processes at LO). The $x_{\gamma}^{\text {obs }}$ distribution is confronted with the hypothesis of no (or global) factorization breaking (left) and with a suppression factor $S$ of 0.34 [351] applied to resolved processes only (right) in Fig. 97. Note that the interdependence of direct and resolved processes requires the definition of a new factorization scheme with suppression of the scale-dependent logarithm also in the direct contribution [352]

$$
\begin{aligned}
M\left(Q^{2}, S\right)_{\overline{\mathrm{MS}}}= & {\left[-\frac{1}{2 N_{c}} P_{q_{i} \leftarrow \gamma}(z) \ln \left(\frac{M_{\gamma}^{2} z}{p_{T}^{* 2}(1-z)}\right)+\frac{Q_{i}^{2}}{2}\right] S } \\
& -\frac{1}{2 N_{c}} P_{q_{i} \leftarrow \gamma}(z) \ln \left(\frac{p_{T}^{* 2}}{z Q^{2}+y_{s} s}\right) .
\end{aligned}
$$

\subsection{Light and heavy hadron production}

If individual hadrons are experimentally identified, the cross sections above have to be modified to include convolutions over fragmentation functions $D(z)$. For light quarks and gluons, these non-perturbative, universal distributions must be fitted to $e^{+} e^{-}$data, but then produce successful predictions for HERA data at NLO. For heavy quarks, the fragmentation functions can in principle be calculated perturbatively, if the heavy-quark mass is kept finite ("fixed-order scheme"), although, for large $E_{T}$, they must be evolved using renormalization group equations (for example at "next-to-leading logarithm") [354]. An alternative method is to fit the fragmentation functions for $D$ - and $B$-mesons again to $e^{+} e^{-}$data at large $E_{T}$ ("variable flavor number scheme"). If, in addition, the finite mass terms are kept in the hard coefficient functions, one moves from a "zeromass scheme" to a "general-mass scheme" and can achieve a smooth transition from large to small $E_{T}$ [355]. A comparison of both theoretical approaches to recent $D^{*}+$ jet data from ZEUS is shown in Fig. 98. While the massive calculation with central scale choice clearly underestimates the data, the variable flavor number scheme allows not only for direct, but also for resolved-photon contributions and tends to give a better description of the data over the full rapidity range. Note that both predictions have been multiplied by hadronization corrections modeled with Monte Carlo simulations. While several calculations for inclusive single-hadron production with real photons are available, a theoretical investigation of the transition region to virtual photons and of the production of two hadrons, for example in the forward region, is still needed.

The production of heavy quark-antiquark bound states is still far from being understood theoretically. While color-singlet (CS) states are to some extent formed already during hard collisions, their contribution has been shown to be both theoretically 

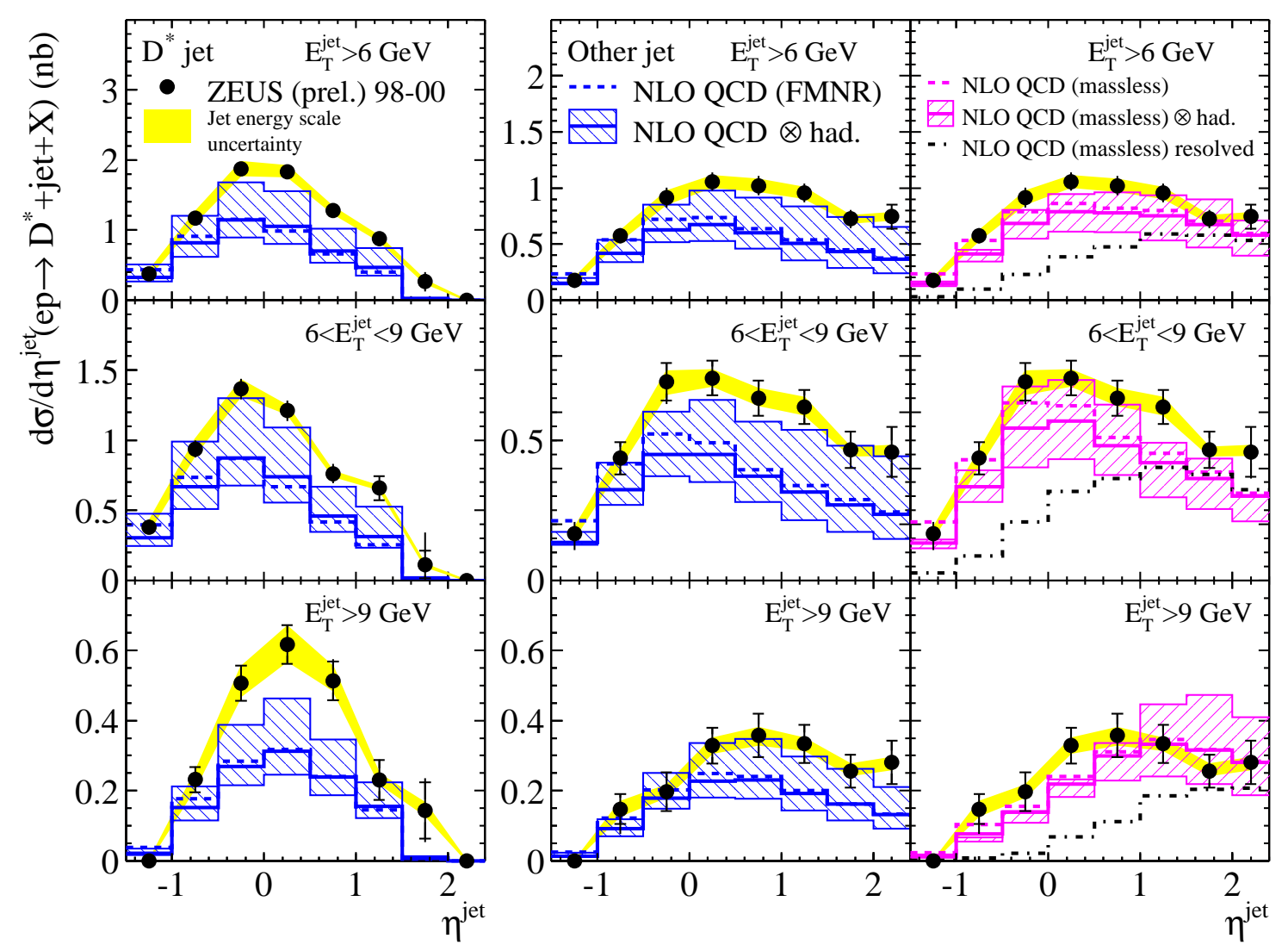

Figure 98. Rapidity distributions of $D^{*}$-mesons and associated jets as measured by ZEUS and compared to massive (fixed-order) and massless (variable flavor number scheme) calculations [353].

incomplete due to uncanceled infrared singularities as well as phenomenologically insufficient due to an order-of-magnitude discrepancy with the measured $\mathrm{J} / \psi p_{T^{-}}$ spectrum at the Tevatron. On the other hand, non-relativistic QCD (NRQCD) allows for a systematic expansion of the QCD Lagrangian in the relative quark-antiquark velocity and for additional color-octet $(\mathrm{CO})$ contributions with subsequent color neutralization through soft gluons. Then $J / \psi$-production in photon-photon collisions at LEP can be consistently described [357], as can be the photoproduction data from HERA in Fig. 99. At HERA, the color-octet contribution becomes important only at small momentumtransfer $z$ of the photon to the $J / \psi$. Unfortunately, recent CDF data do not support the prediction of transverse polarization of the produced $J / \psi$ at large $p_{T}$ as predicted from the on-shell fragmentation of final-state gluons within NRQCD. Further experimental and theoretical studies are thus urgently needed. 


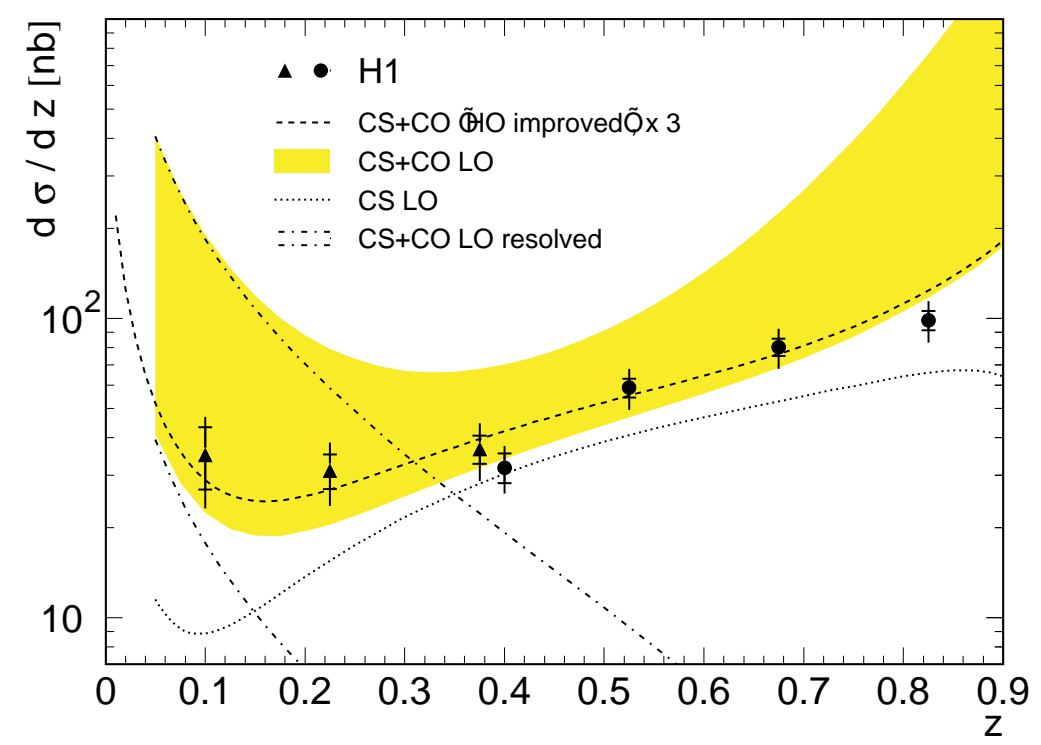

Figure 99. Direct and resolved contributions to the color-singlet and color-octet $J / \psi$ energy distribution in photoproduction at HERA [356]. Reprinted from Ref. [345] with permission from Springer-Verlag.

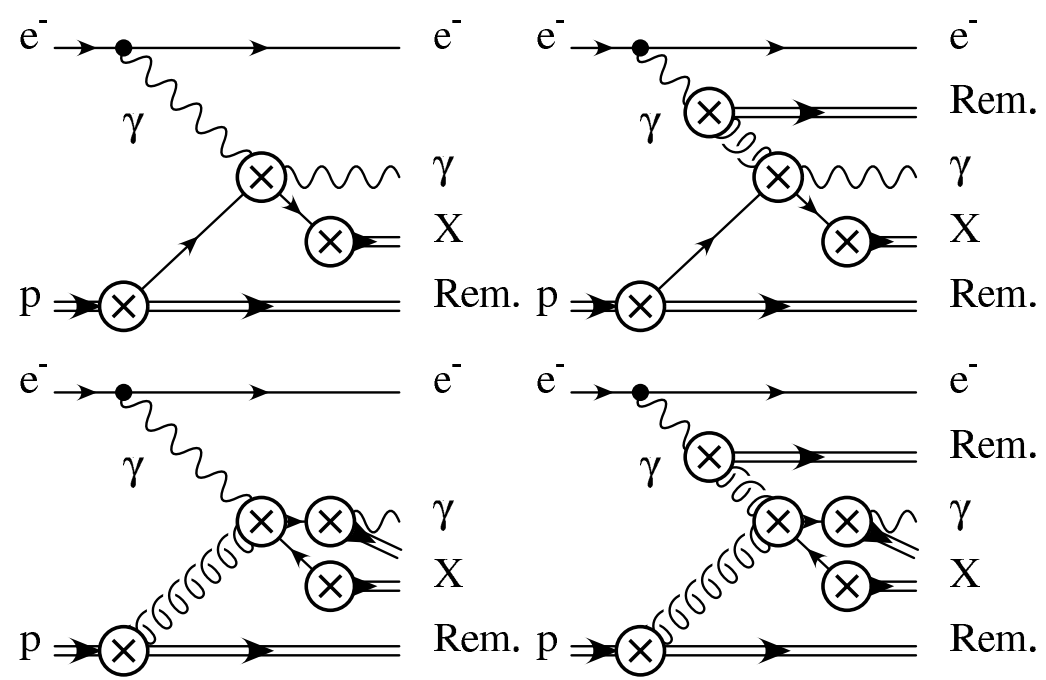

Figure 100. Factorization of prompt photon photoproduction [341]. Copyright 2002 by the American Physical Society (http://link.aps.org/abstract/RPM/v74/p1221).

\subsection{Prompt photon production}

The production of prompt photons in association with jets receives contributions from direct and resolved initial photons as well as direct and fragmentation contributions in the final state, as shown in Fig. 100. Photons produced via fragmentation usually lie inside hadronic jets while directly produced photons tend to be isolated from the final state hadrons. The theoretical uncertainty coming from the non-perturbative fragmentation function can therefore be reduced if the photon is isolated in phase space. At the same time the experimental uncertainty coming from photonic decays of $\pi^{0}, \eta$, 


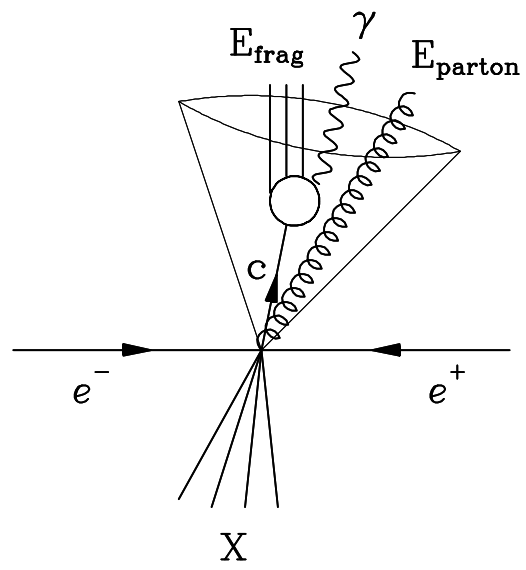

Figure 101. Illustration of an isolation cone containing a parton $c$ that fragments into a photon $\gamma$ plus hadronic energy $E_{\text {frag }}$ [341]. In addition, a gluon enters the cone and fragments giving hadronic energy $E_{\text {parton }}$. Copyright 2002 by the American Physical Society (http://link.aps.org/abstract/RPM/v74/p1221).

and $\omega$ mesons is considerably reduced. Photon isolation can be achieved by limiting the (transverse) hadronic energy $E_{(T)}^{\text {had }}$ inside a cone of size $R$ around the photon to

$$
E_{(T)}^{\text {had }}<\epsilon_{(T)} E_{(T), \gamma},
$$

illustrated in Fig. 101. Recently an improved photon isolation criterion

$$
\sum_{i} E_{(T), i}^{\mathrm{had}} \theta\left(\delta-R_{i}\right)<\epsilon E_{(T), \gamma}\left(\frac{1-\cos \delta}{1-\cos \delta_{0}}\right),
$$

has been proposed, where $\delta \leq \delta_{0}$ and $\delta_{0}$ is now the isolation cone [358]. This procedure allows the fragmentation contribution to vanish in an infrared safe way.

Photoproduction of prompt photons and jets has been measured by the $\mathrm{H} 1$ collaboration and compared with two QCD predictions, which differ in their inclusion of NLO corrections to the resolved and fragmentation contributions. Only after modeling hadronization corrections and multiple interactions with Monte Carlo generators, the measured distributions shown in Fig. 102 agree with the QCD predictions, showing the particular sensitivity of photon final states to hadronic uncertainties.

\subsection{Summary}

Photoproduction processes have been abundantly measured at HERA and stimulated many theoretical studies, ranging from the investigation of the foundations of QCD as inscribed in its factorization theorems, over the determination of its fundamental parameter, the strong coupling constant, to improvements in our understanding of proton and photon structure as well as light and heavy hadron formation.

With the shutdown of HERA on July 1, 2007, many questions, in particular in the diffractive and non-relativistic kinematic regimes, will remain unanswered for quite some time until the eventual construction of a new electron-hadron collider such as eRHIC or 

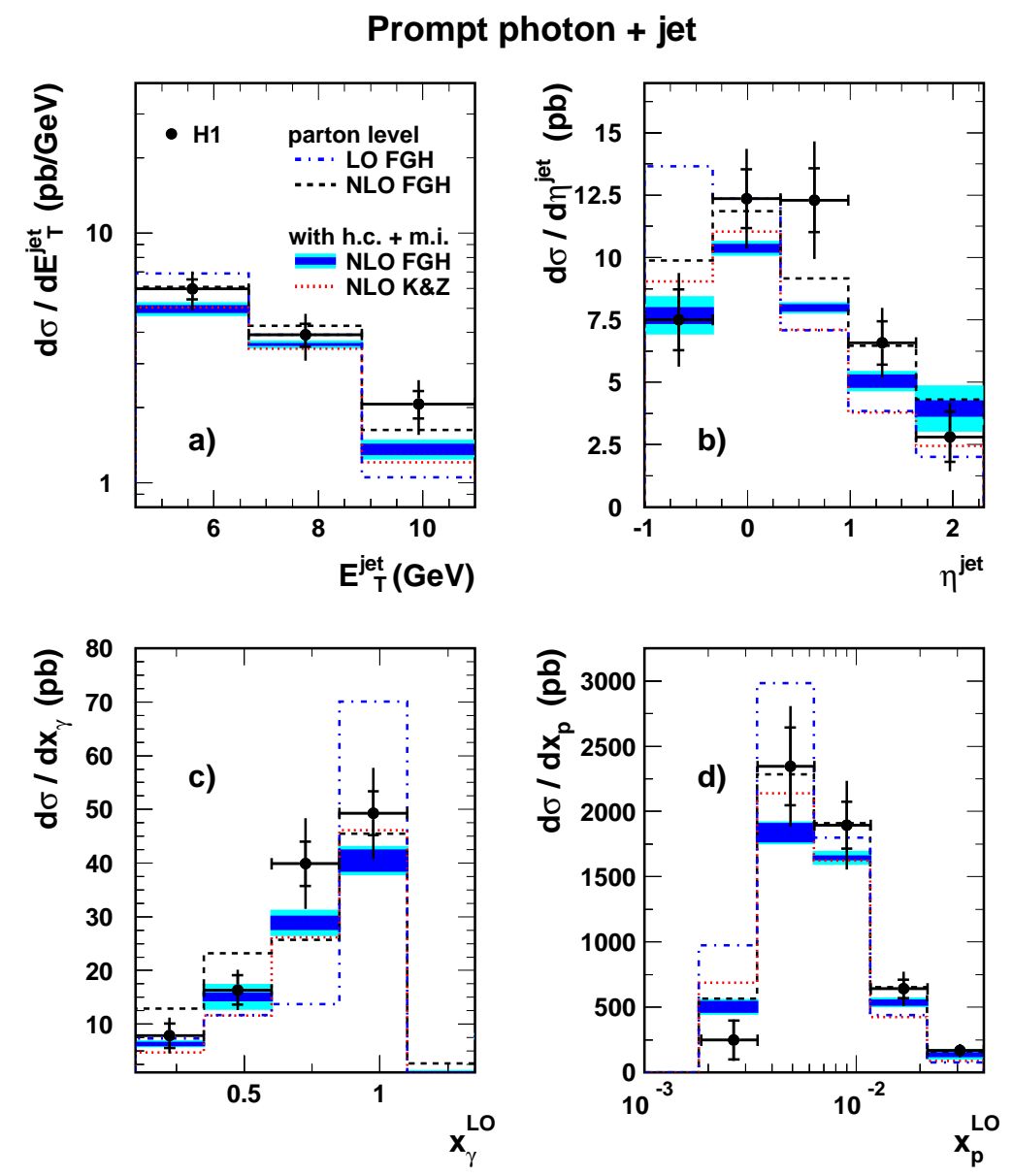

Figure 102. Various distributions of photoproduction of prompt photons in association with jets as measured by $\mathrm{H} 1$ and compared to two different QCD calculations. Reprinted from Ref. [359] with permission from Elsevier.

an International Linear Collider. Photon-induced processes in ultraperipheral heavy-ion collisions may offer a chance to continue investigations in this interesting field, opening in addition a window to nuclear structure, if these processes can be experimentally isolated.

\section{UPC lessons from RHIC}

Contributed by: D. d'Enterria, S. R. Klein, J. Seger and S. N. White

\subsection{RHIC}

Contributed by: J. Seger

In the Relativistic Heavy Ion Collider, counter-rotating beams of fully ionized nuclei collide head on at each of six locations around the 2.4 mile ring. Particle species ranging from protons to gold can be accelerated, stored and collided at RHIC. RHIC can study both "symmetric" collisions of equal ion species, such as $\mathrm{Au}+\mathrm{Au}$, and "asymmetric" 
collisions of unequal ion species, such as $\mathrm{d}+\mathrm{Au}$. Collisions of polarized protons can also be studied. The top energy for heavy-ion beams is $100 \mathrm{GeV} /$ nucleon while for protons it is $250 \mathrm{GeV}$. The design luminosity was $2 \times 10^{26} \mathrm{~cm}^{-2} \mathrm{~s}^{-1}$ [360]. During the initial run in the fall of 2000, $\mathrm{Au}+\mathrm{Au}$ collisions at $\sqrt{S_{N N}}=130 \mathrm{GeV}$ were studied at the target luminosity of $2 \times 10^{25} \mathrm{~cm}^{-2} \mathrm{~s}^{-1}, 10 \%$ design. In 2001, RHIC reached the design energy of $\sqrt{s_{N N}}=200 \mathrm{GeV}$ with a peak luminosity of $3.7 \times 10^{26} \mathrm{~cm}^{-2} \mathrm{~s}^{-1}$. The 2003 run consisted of 9 weeks of polarized $p p$ collisions and 11 weeks of $\mathrm{d}+\mathrm{Au}$ collisions while the 2004 run consisted of 12 weeks of $\mathrm{Au}+\mathrm{Au}$ collisions at $\sqrt{s_{N N}}=200 \mathrm{GeV}$ followed by 1 week of $\mathrm{Au}+\mathrm{Au}$ collisions at $\sqrt{s_{N N}}=62.4 \mathrm{GeV}$ and 5 weeks of polarized $p p$ collisions. The 2005 run included 9 weeks of $\mathrm{Cu}+\mathrm{Cu}$ collisions at $\sqrt{s_{N N}}=200 \mathrm{GeV}$ and 2 weeks at $\sqrt{s_{N N}}=62.4 \mathrm{GeV}$. The 2006 run was dedicated to $p p$ collisions. The 2007 run is another $\mathrm{Au}+\mathrm{Au}$ run. While peak luminosities have risen above design values, it was not until the 2004 run that the integrated luminosities finally outpaced the design projections, providing an integrated luminosity of $1270 \mu \mathrm{b}^{-1}$ to STAR in the 12 week $\mathrm{Au}+\mathrm{Au}$ run [361].

Studies of mutual Coulomb dissociation by photon-nucleus scattering were made with data from three RHIC experiments: PHENIX, PHOBOS and BRAHMS [110]. The cross section for mutual Coulomb dissociation was found to be comparable to the geometric cross section, in good agreement with Ref. [88]. This process may thus be useful for luminosity monitoring.

Ultraperipheral collisions occur with great frequency at RHIC. While the cross section for coherent interaction is large, these events typically produce fewer than 10 charged particles (often only two). This low multiplicity implies significant background, making triggering difficult. For RHIC, the experimental challenge is to not throw events away: effective triggers are critical for selecting a reasonable data set. The ability to trigger on either very low multiplicity events or on nuclei only mildly affected by collisions is crucial to successful UPC studies at RHIC.

\subsection{STAR results}

Contributed by: S. Klein and J. Seger

While all four RHIC experiments have shown interest in UPCs, most of the physics results published to date come from the Solenoidal Tracker at RHIC (STAR) experiment [362]. The STAR detector, shown in Fig. 103, tracks charged particles in a $4.2 \mathrm{~m}$ long Time Projection Chamber (TPC) [363] with an inner radius of $50 \mathrm{~cm}$ and an outer radius of $2 \mathrm{~m}$. A solenoidal magnet surrounds the TPC. In 2000, the TPC was operated in a $0.25 \mathrm{~T}$ magnetic field. In subsequent runs, the magnetic field was operated primarily at the design value of $0.5 \mathrm{~T}$ with small data sets taken at $0.25 \mathrm{~T}$. Tracks in the TPC are reconstructed in the pseudorapidity range $|\eta|<1.5$. Tracks with $p_{T}>100 \mathrm{MeV} / c$ are reconstructed with high efficiency. Tracks can be identified by their energy loss in the TPC. STAR also has two radial-drift forward TPCs, one on each side of the central TPC, to extend tracking capabilities into the range of $2.5<|\eta|<4.0$. 


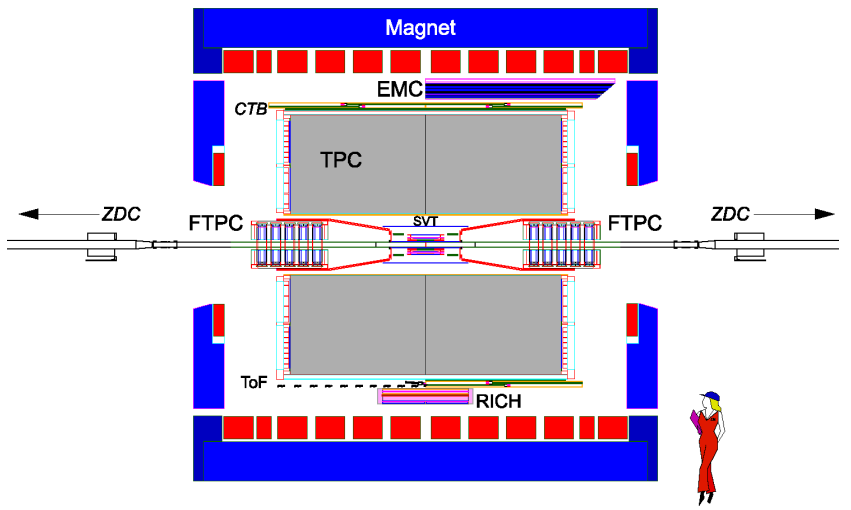

Figure 103. A schematic view of the STAR detector at RHIC. Reprinted from Ref. [364] with permission from Elsevier.

In addition, STAR is installing a barrel electromagnetic calorimeter covering $|\eta|<1$, and an endcap electromagnetic calorimeter on the west pole tip of the detector covering $1.086<\eta<2$.

Each RHIC detector includes two ZDCs, located at $z= \pm 18 \mathrm{~m}$ from the interaction point to detect undeflected neutrons from nuclear breakup. These calorimeters are sensitive to single neutrons and have an acceptance of close to $100 \%$ for neutrons from nuclear breakup [110,365].

Although each RHIC detector also includes beam-beam counters (BBCs), the implementation differs between the experiments. For STAR, the BBCs are a hexagonal scintillator array structure $\pm 3.5 \mathrm{~m}$ from the interaction point. They detect charged particles with $2<|\eta|<5$ with full coverage in $\phi$. Requiring coincidence between the two BBCs can reduce background contributions from beam-gas events. The STAR BBCs were partially implemented for the 2001 run and fully implemented for the 2003 run $[366]$.

9.2.1. Triggers The primary STAR trigger detector is a cylindrical Central Trigger Barrel (CTB) consisting of 240 scintillator slats surrounding the TPC. Each slat in the CTB covers a pseudorapidity interval of $\Delta \eta=0.5$ and a range in azimuthal angle $\phi$ of $\Delta \phi=\pi / 30$. The scintillator is sensitive to charged particles with $p_{T}>130 \mathrm{MeV} / c$ in the $0.25 \mathrm{~T}$ magnetic field. The acceptance for charged particles depends on where the particles are produced. For a vertex at the center of the TPC, the trigger barrel is sensitive only to charged particles with $|\eta|<1$. The finite acceptance of the CTB therefore limits its usefulness since many events of interest (such as $e^{+} e^{-}$pairs) produce tracks at high $|\eta|$.

Some ultraperipheral events with zero tracks reaching the CTB can be extracted from the STAR minimum-bias data. The STAR minimum-bias trigger requires coincident neutron signals in the East and West ZDCs. It therefore triggers only on the subset of ultraperipheral events that include mutual nuclear excitation. STAR 
found that the majority of such events deposited a single neutron in each ZDC. Very few events deposited more than three neutrons into either ZDC.

For those events with tracks that do reach the CTB, STAR can select events with a particular topology or multiplicity. STAR initially focused on selecting events with a two particle final state. The CTB was divided in four azimuthal quadrants for this 'topology' trigger [367]. Single hits were required in the opposite side quadrants while the top and bottom quadrants acted as vetoes to suppress cosmic rays. The topology trigger did not place any requirement on ZDC signals. Analysis of the ZDC signal in events selected by the topology trigger shows that, in almost all cases, both ZDC's are empty. Datasets with this trigger therefore consist primarily of events with no nuclear excitation. To extend the triggering capabilities to events with more than two tracks in the final state requires modification of the trigger algorithms.

9.2.2. $e^{+} e^{-}$production Exclusive $e^{+} e^{-}$pair production has been observed in ultraperipheral collisions at RHIC $[120,368]$. The two tracks are approximately back-toback in the transverse plane due to the small $p_{T}$ of the pair, $\sim 5 \mathrm{MeV} / c$. The maximum cross section is at low invariant mass with a peak at small forward angles. Many of the tracks have such low transverse momentum that, even in a $0.25 \mathrm{~T}$ field, they do not reach the CTB. Thus triggering is limited to the minimum bias trigger: $e^{+} e^{-}$pair production with mutual nuclear excitation.

The STAR data is compared to two different calculations of the pair production probability, $P_{e e}(b)$. The first calculation uses the equivalent photon approximation [3]. The photon flux is calculated from each nucleus using the Weizsäcker-Williams approach. The photons are treated as if they were real [369]. Then $e^{+} e^{-}$pair production is calculated to leading order [370]. The $p_{T}$ spectrum of a photon with energy $\omega$ is given by $[78,371]$

$$
\frac{d N_{\gamma}}{d^{2} p_{T} d \omega}=\frac{Z^{2} \alpha^{2}\left|p_{T}\right|^{2}}{\pi^{2}}\left[\frac{F\left(p_{T}^{2}+\omega^{2} / \gamma^{2}\right)}{p_{T}^{2}+\omega^{2} / \gamma^{2}}\right]^{2}
$$

where $\alpha$ is the electromagnetic coupling constant, $Z$ is the nuclear charge and $F$ is the nuclear form factor. The calculation of the form factor uses a Woods-Saxon nuclear density distribution with $R_{\mathrm{Au}}=6.38 \mathrm{fm}$ and skin thickness of $0.535 \mathrm{fm}$ [72].

The second calculation is a LO QED pair production calculation [271]. This calculation includes the photon virtuality. Within the measured kinematic range, the results differ mainly in the pair $p_{T}$ spectrum. Figure 104 compares the $p_{T}$ distributions of the two calculations with the data. The QED calculation is in much better agreement.

In the $\mathrm{AuAu} \rightarrow \mathrm{Au}^{*} \mathrm{Au}^{*} e^{+} e^{-}$analysis, STAR identifies $52 \mathrm{UPC} e^{+} e^{-}$pairs in an 800,000 event sample at $\sqrt{s_{N N}}=200 \mathrm{GeV}$ with the $0.25 \mathrm{~T}$ magnetic field setting. Within the limited kinematic range, STAR measures a cross section of $\sigma=1.6 \pm 0.2 \pm 0.3$ $\mathrm{mb}$ [120], $1.2 \sigma$ lower than the equivalent photon prediction of $2.1 \mathrm{mb}$ and close to the QED calculations $\sigma_{\mathrm{QED}}=1.9 \mathrm{mb}$. The $e^{+} e^{-}$measurement can be used to put limits on changes in the cross section due to higher order corrections. At a $90 \%$ 


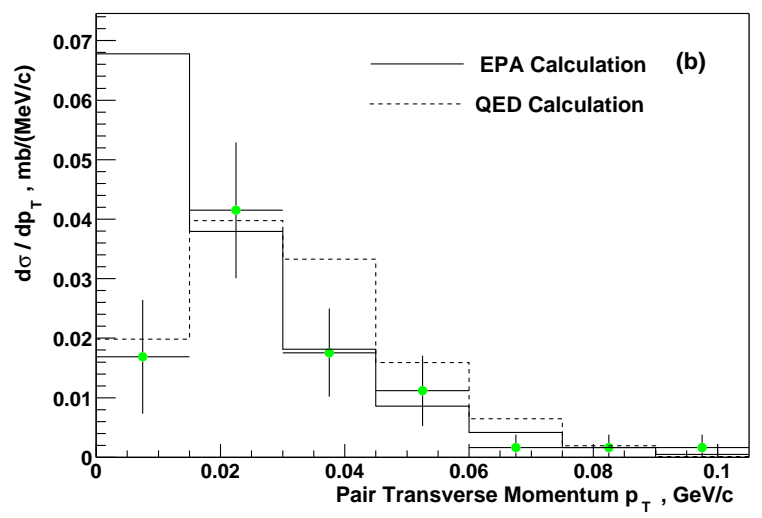

Figure 104. The $e^{+} e^{-}$pair $p_{T}$ distribution. The data (points) are compared with the EPA (solid histogram) and LO QED (dashed histogram) predictions. The error bars include both statistical and systematic errors [120]. Copyright 2004 by the American Physical Society (http://link.aps.org/abstract/PRC/v70/e301902).

confidence level, higher order corrections to the cross section must be within the range $-0.5 \sigma_{\mathrm{QED}}<\Delta \sigma<0.2 \sigma_{\mathrm{QED}}$.

A study of $e^{+} e^{-}$production in sulfur-induced fixed-target heavy-ion collisions at $\sqrt{s_{N N}}=20 \mathrm{GeV}$ found that the positrons had a higher average energy than the electrons [256]. This difference may be explained by Coulomb corrections [257] since electrons are attracted to nuclei while positrons are repelled. Calculations show that interference between the leading (two-photon) and the next-to-leading order (three-

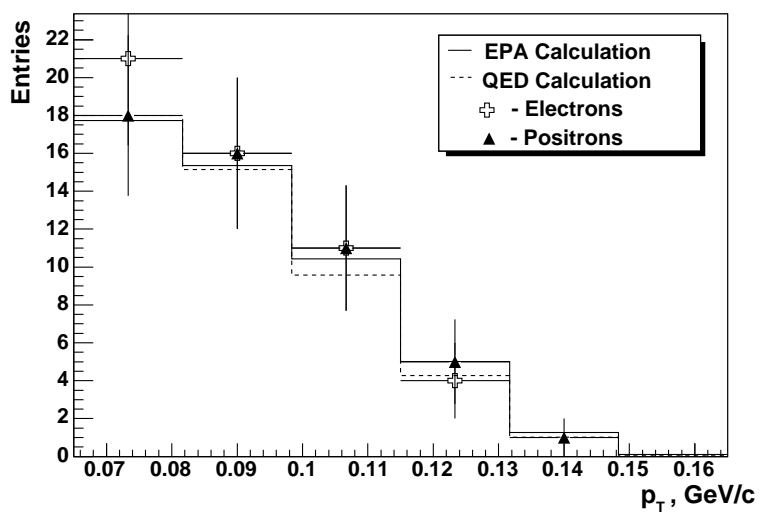

Figure 105. The $p_{T}$ spectra of produced electrons (open crosses) and positrons (solid triangles). The solid histogram shows the EPA calculation [120]. Copyright 2004 by the American Physical Society (http://link.aps.org/abstract/PRC/v70/e301902).

photon) channels can lead to 30-60\% asymmetries in some kinematic variables [144]. Figure 105 compares the $p_{T}$ spectra of produced electrons and positrons at RHIC. No large asymmetry is seen, indicating that it is not yet necessary to invoke higher-order terms. 
9.2.3. $\rho^{0}$ production Photonuclear $\rho^{0}$ production has also been observed at RHIC [62]. Events with no nuclear excitation are observed in the STAR topology-triggered data. Such events are labeled $(0 n, 0 n)$ to indicate that there are no neutrons in either ZDC. Events involving nuclear excitation in addition to $\rho^{0}$ production can be observed in the STAR minimum bias data. These events are labeled $(X n, X n)$ to indicate that at least one neutron was deposited into each ZDC.

Figure 106 shows the transverse momentum spectrum of $\pi^{+} \pi^{-}$pairs (points) in $\mathrm{Au}+\mathrm{Au}$ collisions at $\sqrt{s_{N N}}=130 \mathrm{GeV}$. A clear peak at $p_{T}<150 \mathrm{MeV} / c$, the signature for coherent coupling, can be observed. The like-sign combinatorial background (shaded histogram), normalized to the signal for $p_{T}>250 \mathrm{MeV} / c$, does not show such a peak. The open histogram is a Monte Carlo simulation [72] for coherent $\rho^{0}$ production accompanied by nuclear breakup superimposed on the background. The simulation, including not only the nuclear form factor but also the photon $p_{T}$ distribution and the interference of production amplitudes from both gold nuclei, matches the data reasonably well.
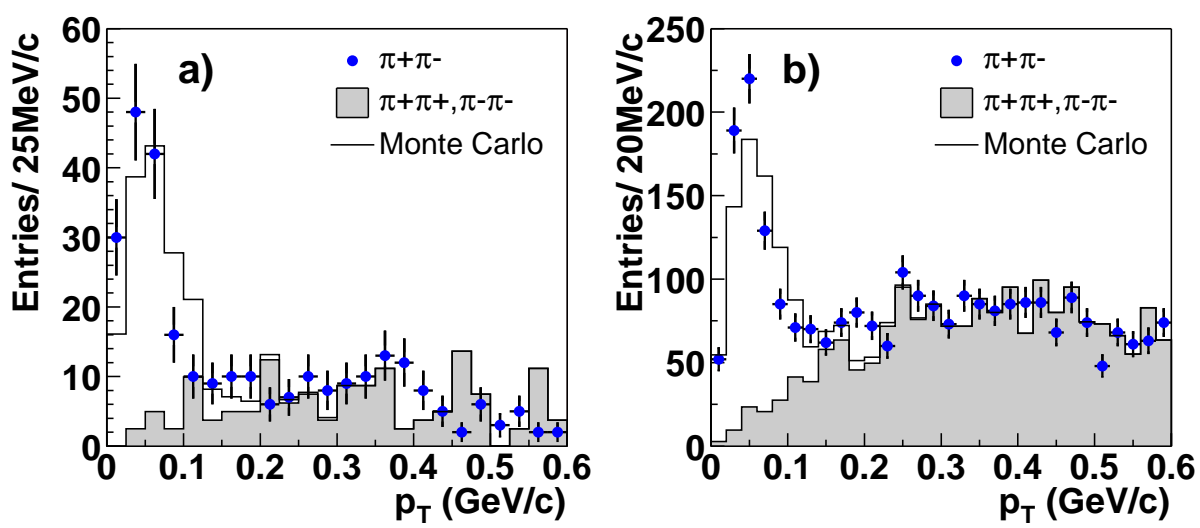

Figure 106. The $p_{T}$ spectra of pion pairs for two-track events selected by the STAR (a) topology $(0 n, 0 n)$ and (b) minimum bias $(X n, X n)$ triggers at $\sqrt{s_{N N}}=130$ $\mathrm{GeV}$. The points are $\pi^{+} \pi^{-}$pairs and the shaded histograms are the normalized likesign combinatorial background. The open histograms are the simulated $\rho^{0}$ spectra superimposed on the background [62]. Copyright 2002 by the American Physical Society (http://link.aps.org/abstract/PRL/v89/e272302).

The Monte Carlo simulation also closely matches the observed rapidity distribution, shown in Fig. 107 for the $\sqrt{s_{N N}}=200 \mathrm{GeV} \mathrm{Au+Au} \mathrm{data.} \mathrm{The} \mathrm{STAR} \mathrm{exclusive}$ $\rho^{0}$ acceptance is about $40 \%$ for $\left|y_{\rho}\right|<1$. Above $\left|y_{\rho}\right|=1$, the acceptance is small and this region is excluded from the analysis. The cross sections are extrapolated to the full $4 \pi$ by Monte Carlo. For coherent $\rho^{0}$ production at $\sqrt{s_{N N}}=130 \mathrm{GeV}$ accompanied by mutual nuclear break-up $(X n, X n)$, the measured cross section is $\sigma\left(\mathrm{AuAu} \rightarrow \mathrm{Au}_{X n}^{*} \mathrm{Au}_{X n}^{*} \rho^{0}\right)=28.3 \pm 2.0 \pm 6.3 \mathrm{mb}$. By selecting single neutron signals in both ZDCs, STAR obtains $\sigma\left(\mathrm{AuAu} \rightarrow \mathrm{Au}_{1 n}^{*} \mathrm{Au}_{1 n}^{*} \rho^{0}\right)=2.8 \pm 0.5 \pm 0.7 \mathrm{mb}$. These cross sections are in agreement with calculations [72,88]. 


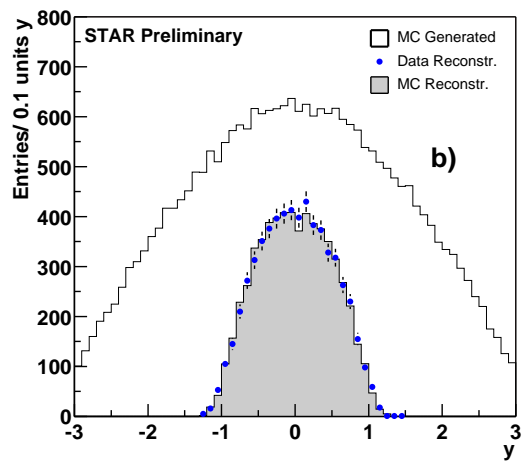

Figure 107. The STAR $\rho^{0}$ minimum bias $(X n, X n)$ rapidity distribution data (points) compared to the normalized reconstructed (shaded histogram) and generated (open histogram) simulated events at $\sqrt{s_{N N}}=200 \mathrm{GeV}$. Reprinted from Ref. [372] with permission from Elsevier.

Figure 108 shows the $200 \mathrm{GeV} d \sigma / d M_{\pi \pi}$ spectrum for events with $p_{T}<150 \mathrm{MeV} / c$ (points). The fit (solid curve) is the sum of a relativistic Breit-Wigner for $\rho^{0}$ production

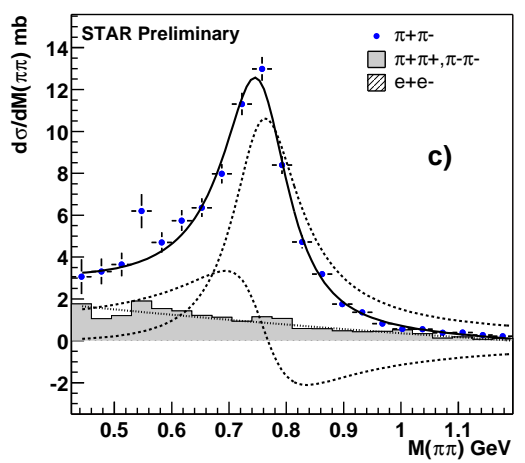

Figure 108. The $d \sigma_{\mathrm{Au} \mathrm{Au} \rightarrow \mathrm{Au}^{*} \mathrm{Au}^{*} \rho^{0}} / d M_{\pi \pi}$ spectrum for two-track $(X n, X n)$ events with $p_{T}<150 \mathrm{MeV} / c$ in the STAR minimum bias data. The shaded histogram is the combinatorial background. The hatched histogram includes an additional contribution from coherent $e^{+} e^{-}$pairs. The solid curve is the sum of a Breit-Wigner (dashed curve), a mass-independent contribution from direct $\pi^{+} \pi^{-}$production and interference (dotted curve), and a second order polynomial for the residual background (dot-dashed curve). Reprinted from Ref. [372] with permission from Elsevier.

and a Söding interference term for direct $\pi^{+} \pi^{-}$production [373]. A second-order polynomial (dash-dotted) describes the combinatorial background (shaded histogram) from grazing nuclear collisions and incoherent photon-nucleon interactions. The $\rho^{0}$ mass and width are consistent with accepted values [374]. Alternative parameterizations such as a modified Söding parametrization [201] and a phenomenological Ross-Stodolsky parametrization [375] yield similar results. Incoherent $\rho^{0}$ production, where a photon interacts with a single nucleon, yields high $p_{T} \rho^{0}$. The small number of $\rho_{0}$ that survive the low $p_{T}$ cut are indistinguishable from the coherent process. A coherent 
two-photon background, $\mathrm{Au} \mathrm{Au} \rightarrow \mathrm{Au}^{*} \mathrm{Au}^{*} l^{+} l^{-}$, contributes mainly at low invariant mass, $M_{\pi \pi}<0.5 \mathrm{GeV} / c^{2}$. A second order polynomial models these residual background processes.

The $2003 \mathrm{~d}+\mathrm{Au}$ run also yielded $\rho^{0}$ events. These asymmetric collisions involve two distinct processes, depending on whether the gold or the deuterium emits the photon: $\mathrm{Au} \rightarrow \gamma \mathrm{Au}$ followed by $\gamma \mathrm{d} \rightarrow \mathrm{d} \rho^{0}$ or $\mathrm{d} \rightarrow \gamma \mathrm{d}$ and $\gamma \mathrm{Au} \rightarrow \mathrm{Au} \rho^{0}$. Photon emission is much more likely from the gold nucleus. When a photon scatters from the gold scatters from off the deuteron, it may scatter coherently, leaving the deuterium intact or incoherently, dissociating the deuterium. About $10^{6} \mathrm{~d}+\mathrm{Au}$ collisions were recorded using the STAR topology trigger with a subsample that required a neutron signal in the ZDC. Requiring a neutron signal in the ZDC cleanly separates incoherent reactions in which the deuterium breaks up. Figure 109 shows a clear $\rho^{0}$ signal in the preliminary $M_{\pi \pi}$ invariant mass spectrum. Further analysis is underway.

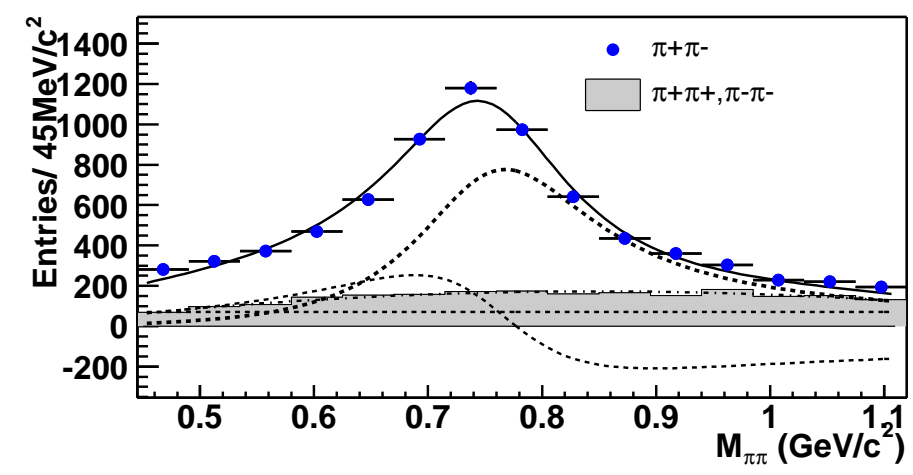

Figure 109. The $d N / d M_{\pi \pi}$ invariant mass distribution for two-track events in $\sqrt{s_{N N}}=200 \mathrm{GeV} \mathrm{d}+\mathrm{Au}$ collisions at STAR [372]. No $p_{T}$ cut is applied to the $\mathrm{d}+\mathrm{Au}$ data. Reprinted from Ref. [372] with permission from Elsevier.

STAR has also observed photoproduction of four-pion final states. Fig. 110 shows an excess of zero net charge four-prong final states such as $\pi^{+} \pi^{-} \pi^{+} \pi^{-}$at low $p_{T}$ in $\sqrt{s_{N N}}=200 \mathrm{GeV} \mathrm{Au}+\mathrm{Au}$ collisions, as expected for coherent photoproduction. Since no particle identification is applied the particles are assumed to be pions. No excess is seen for finite net-charged final states. The four-pion mass is peaked around $\sim 1.5$ $\mathrm{GeV} / c^{2}$, as also shown in Fig. 110, consistent with a $\rho^{0 \prime}$ decaying to four pions.

9.2.4. Interference Stringent event selection criteria were used to select a clean, lowbackground sample [90] from the STAR $200 \mathrm{GeV} \mathrm{Au}+\mathrm{Au}$ data to study $\rho^{0}$ interference. The magnitude of the interference depends on the ratio of the amplitudes for $\rho^{0}$ production from the two nuclei. Away from $y=0$, the amplitudes differ and the interference is reduced. Thus this analysis focuses on the midrapidity region. We neglect $\rho^{0}$ candidates with $|y|<0.1$ to avoid possible cosmic ray contamination. A Monte Carlo is used to calculate the expected interference for different rapidity ranges $[72,78]$. 

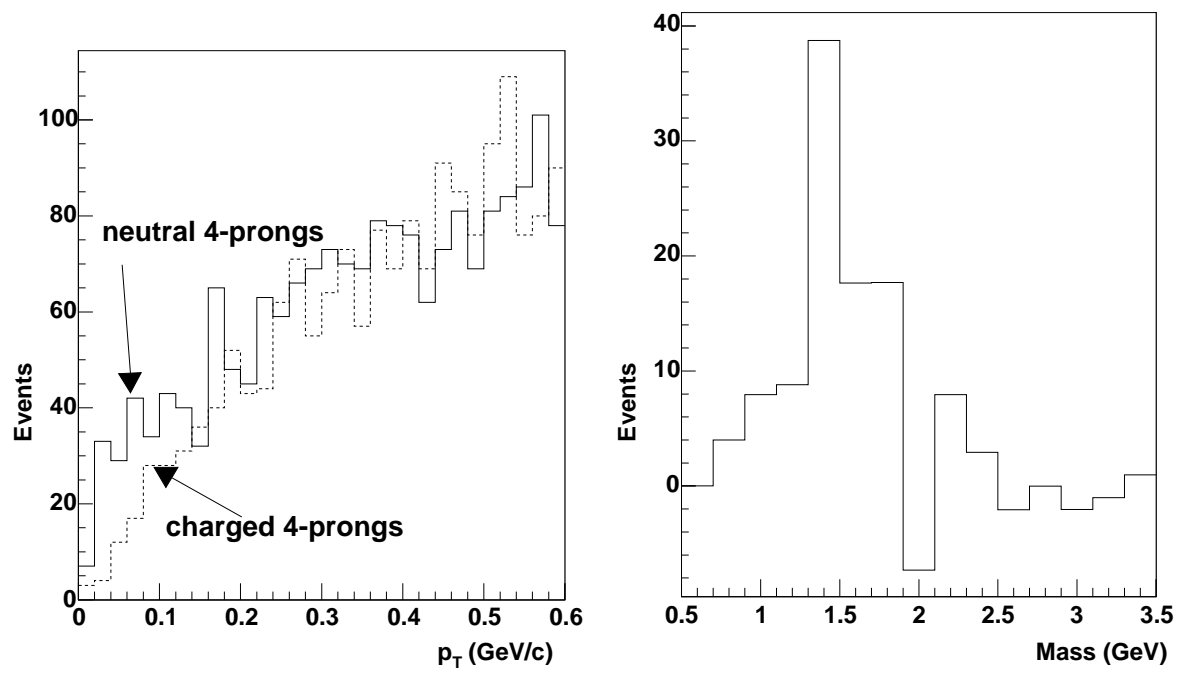

Figure 110. Left-hand side: The $p_{T}$ spectrum for 4-prong UPC final states. The solid histogram is for neutral combinations (e.g. $\pi^{+} \pi^{-} \pi^{+} \pi^{-}$) while the dashed histogram indicates charged combinations (e.g. $\left.\pi^{+} \pi^{+} \pi^{+} \pi^{-}\right)$. The difference is the net coherent photoproduction signal. Right-hand side: The background-subtracted mass spectrum of 4-prong coherent production, treating all charged particles as pions. From Ref. [376]. Copyright 2005 by the American Institute of Physics.

STAR studies the $p_{T}$ spectra using the variable $t_{\perp}=p_{T}^{2}$. At RHIC energies, the longitudinal component of the 4-momentum transfer is small so that $t \approx t_{\perp}$. Without interference, $d N / d t \propto \exp (-b t)[78,377]$. Figure 111 compares the uncorrected minimum bias data for $0.1<|\eta|<0.5$ with two simulations, with and without interference. Both simulations include the detector response. The data has a significant downturn for $t<0.001 \mathrm{GeV}^{2}$, consistent with $\langle b\rangle=18 \mathrm{fm}$ expected for a $\rho^{0}$ accompanied by mutual excitation [84]. These data match the calculation that includes interference but not the one without interference.

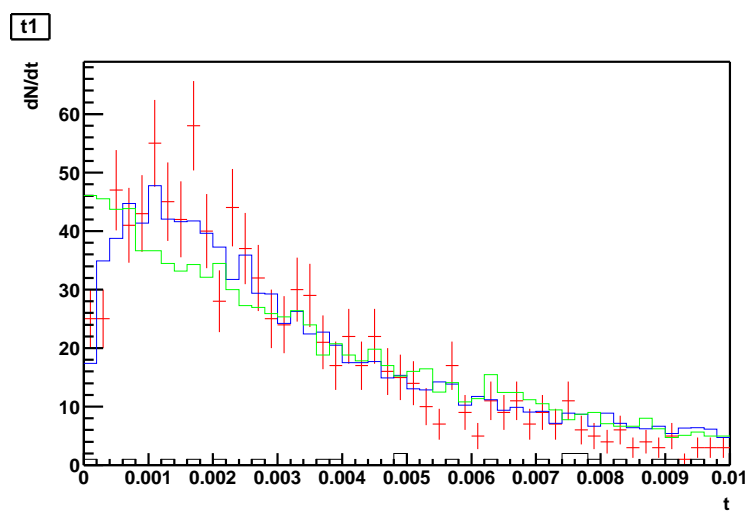

Figure 111. The raw (uncorrected) STAR $t_{\perp}$ spectrum for the $\rho^{0}$ topology sample in $0.1<|y|<0.5$. The points are the data. The dashed histogram is a simulation including interference while the dot-dashed histogram is a calculation without interference. The solid histogram with $d N / d t \sim 0$ is the like-sign background [90]. 
The efficiency-corrected data are shown in Fig. 112 [90]. The minimum bias and topology data are shown separately in two rapidity bins: $0.1<|y|<0.5$ and $0.5<|y|<1.0$. The data is fit with three parameter,

$$
\frac{d N}{d t}=a \exp (-B t)[1+c(R(t)-1)]
$$

where $R(t)=\operatorname{Int}(t) /$ Noint $(t)$ is the ratio of the Monte Carlo $t$ distribution with and without interference. The factor $a$ provides an overall normalization, $B$ is the slope and $c$ quantifies the interference effect: $c=0$ corresponds to no interference, while $c=1$ corresponds to the expected interference [78]. This functional form separates the interference effect $c$ from the nuclear form factor $B$.

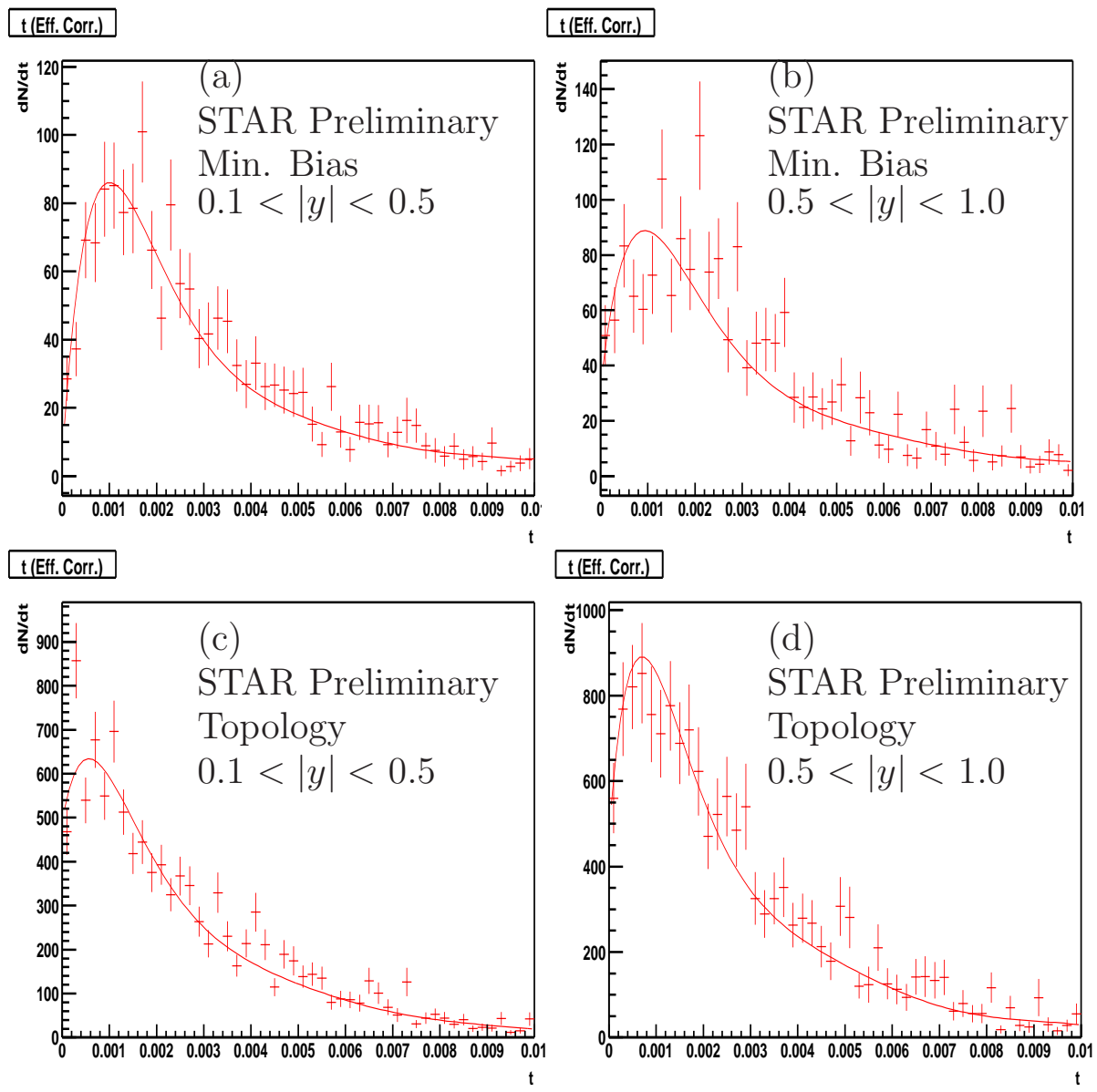

Figure 112. The efficiency-corrected $\rho^{0}$ distributions as a function of $t_{\perp}$ spectrum for $\rho^{0}$ from the minimum bias trigger with (a) $0.1<|y|<0.5$ and (b) $0.5<|y|<1.0$ and the topology trigger with (c) $0.1<|y|<0.5$ and (d) $0.5<|y|<1.0$. The points are the data while the curve is a fit to Eq. (171) [90].

Table 9.2.4 gives the fit results. At small rapidities the amplitudes are similar and the interference reduces the cross section at $p_{T}=0$ more than at larger rapidities. In the minimum bias data, the interference extends to higher $p_{T}$ because it has a smaller average slope, $B$, than the topology data. 
Table 23. The fit results for the two different triggers in two rapidity bins [90].

\begin{tabular}{|c|c|c|c|c|c|}
\hline Trigger & $\left|y_{\min }\right|$ & $\left|y_{\max }\right|$ & $B\left(\mathrm{GeV}^{-2}\right)$ & $c$ & $\chi^{2} / \mathrm{DOF}$ \\
\hline minimum bias & 0.1 & 0.5 & $301 \pm 14$ & $1.01 \pm 0.08$ & $50 / 47$ \\
minimum bias & 0.5 & 1.0 & $304 \pm 15$ & $0.78 \pm 0.13$ & $73 / 47$ \\
topology & 0.1 & 0.5 & $361 \pm 10$ & $0.71 \pm 0.16$ & $81 / 47$ \\
topology & 0.5 & 1.0 & $368 \pm 12$ & $1.22 \pm 0.21$ & $50 / 47$ \\
\hline
\end{tabular}

The fit values of $B$ for the minimum bias and topology $\rho^{0}$ data differ by $\sim 20 \%$. This difference may be attributed to the different impact parameter distributions caused by tagging the minimum bias data by nuclear breakup since the photon flux decreases as the inverse square of the impact parameter, $1 / b^{2}$. When $b$ is a few times $R_{A}$, the $\rho^{0} \mathrm{~s}$ are more likely to be produced on the side of the target near the photon emitter than on the far side. Thus $\rho^{0}$ production is concentrated on the near side, leading to a smaller effective production volume and a smaller $B$ in the minimum bias data since $B \propto 1 / b^{2}$.

The four values of $c$ are consistent within errors. The weighted average is $c=0.93 \pm 0.06$. The preliminary interference measured by STAR is $93 \pm 6$ (stat.) \pm 8 (syst.) \pm 15 (theory) $\%$ of the expected [90].

9.2.5. Proton-nucleus and deuteron-nucleus results In $\mathrm{Au}+\mathrm{Au}$ collisions, coherent vector meson photoproduction has a strong signature since most of the signal is at $p_{T}<$ few $\hbar / R_{A} \approx 100 \mathrm{MeV} / c$.

In contrast, the deuteron is small and has only two nucleons. Thus the coherent enhancement is limited and the $p_{T}$ constraint is not useful. Nevertheless, by taking advantage of the large solid angle coverage, a fairly clean $\rho^{0}$ sample can be isolated [378]. Figure 113 compares the $\rho^{0} p_{T}$ spectra from STAR in $\mathrm{Au}+\mathrm{Au}$ and $\mathrm{d}+\mathrm{Au}$ collisions. These events were selected using a trigger that required a low charged multiplicity in the region $|\eta|<1$ in coincidence with a ZDC signal that indicated deuteron dissociation. This trigger had a reasonably high selectivity but the deuteron breakup requirement reduced the $\rho^{0}$ cross sections considerably.

Data was also taken with a trigger that did not require a neutron signal from deuteron breakup. This trigger was sensitive to reactions where the deuteron remained intact and also where the neutron acquired a large $p_{T}$ through deuteron dissociation. A $\rho^{0}$ signal is also seen with this trigger. In addition to the $\rho^{0}$ at large $p_{T}$, a small peak is visible at very low $p_{T}$, consistent with photon emission from the deuteron. Both signals (with and without the neutron) had similar $\pi \pi$ invariant mass spectra. In addition to the $\rho^{0}$, direct $\pi^{+} \pi^{-}$production was measured. The proportion of direct $\pi^{+} \pi^{-}$to $\rho^{0}$ production was comparable to that observed in both $\mathrm{Au}+\mathrm{Au}$ collisions at RHIC and ep collisions at HERA. 

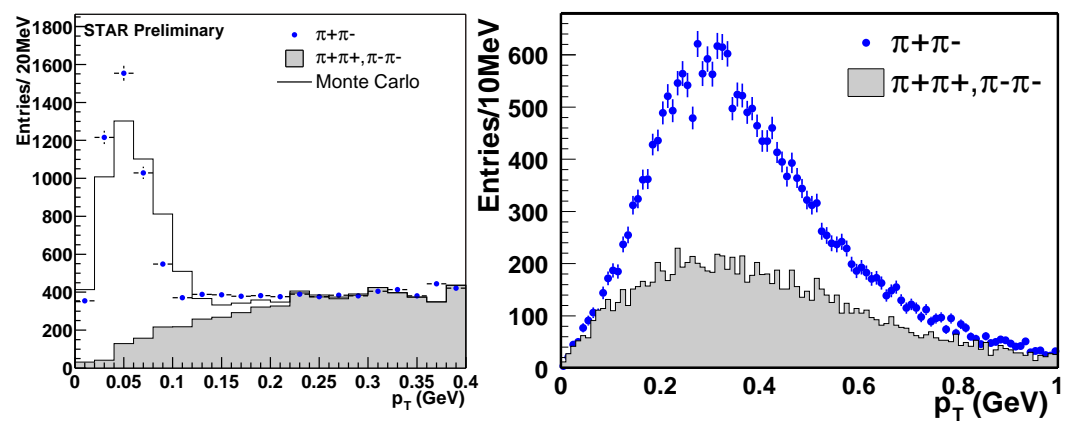

Figure 113. The $p_{T}$ spectrum of $\rho^{0}$ photoproduction in $\mathrm{Au}+\mathrm{Au}$ (left-hand side) relative to $\mathrm{d}+\mathrm{Au}$ (right-hand side) collisions [378]. The points are the data while the shaded histogram is the like-sign background. On the left-hand side, the like-sign background has been scaled to match the zero net-charge data for $p_{T}>150 \mathrm{MeV} / c$ to include the background from incoherent $\rho^{0}$ production as a background.

\subsection{PHENIX Results}

Contributed by: D. d'Enterria and S. N. White

9.3.1. Introduction During the $2003 \mathrm{RHIC} \mathrm{d}+\mathrm{Au}$ run at $\sqrt{s_{N N}}=200 \mathrm{GeV}$, PHENIX commissioned new detectors in the forward region. PHENIX installed a proton calorimeter (fCAL) on either side of the outgoing beam pipe near the ZDC ( $z= \pm 18$ $\mathrm{m}$ ) and embedded a shower maximum position-sensitive hodoscope (SMD) between the first and second ZDC modules [379]. The fCAL [379] detects protons from deuteron breakup. Since beam-energy protons have a lower rigidity than the deuteron beam, protons from deuteron breakup are deflected into the fCAL by an accelerator dipole (DX) located at $z= \pm 11 \mathrm{~m}$. The SMD recorded the transverse momentum of neutrons interacting in the ZDC by measuring the centroid of the shower secondaries.

The cross section for the deuteron dissociation reaction, $\mathrm{d}+\mathrm{Au} \rightarrow n+p+\mathrm{Au}$ has been calculated [380] and found to be $1.38 \mathrm{~b}( \pm 5 \%)$ with $0.14 \mathrm{~b}$ due to hadronic diffraction dissociation [381] and the rest due to electromagnetic dissociation in the field of the gold nucleus. PHENIX measured this dissociation cross section with a trigger sample requiring $E_{\mathrm{ZDC}} \geq 10 \mathrm{GeV}$ in either $\mathrm{ZDC}$ (in the gold or deuteron direction).

During the 2004 RHIC $\mathrm{Au}+\mathrm{Au}$ run at $\sqrt{s_{N N}}=200 \mathrm{GeV}$, PHENIX also commissioned a trigger to study high mass $e^{+} e^{-}$pair production in UPCs. Two sources of high mass $e^{+} e^{-}$pairs are relevant for this measurement. The high mass continuum from $\gamma \gamma \rightarrow e^{+} e^{-}$was measured for $M \geq 1.8 \mathrm{GeV}$, significantly above the range explored by STAR. A $J / \psi \rightarrow e^{+} e^{-}$sample was also observed in photoproduction off the Au target [63]. Coherent and incoherent $J / \psi$ production cross sections have been calculated $[72,83,95,119]$. The contributions to the $J / \psi p_{T}$ distribution from the two processes should be distinguishable due to their different shapes.

The high mass dilepton measurement is interesting because it demonstrates the 
feasibility of triggering on hard photoproduction processes with small cross section. Deuteron dissociation, the earliest calculation of diffractive dissociation to be found in the literature, has a large cross section and is both theoretically and experimentally clean. Thus PHENIX used this process to calibrate the cross sections of other processes produced in $\mathrm{d}+\mathrm{Au}$ interactions [382].

\subsubsection{Deuteron diffractive dissociation}

\section{$d+A u$ Cross Sections}

In addition to deuteron dissociation, the total $\mathrm{d}+\mathrm{Au}$ inelastic cross section interesting for the RHIC program. The inelastic cross section is sampled in the experiments, the "minimum-bias trigger", for use as a luminosity monitor. Once the minimum-bias cross section is known, the cross sections of other processes recorded during the same luminosity interval can also be calculated.

There are two approaches to this cross section normalization. In the first, it is derived from known, elementary, $N N$ inelastic cross sections using the Glauber model with a Woods-Saxon density distribution. The second approach, adopted by PHENIX, is to directly determine the minimum-bias trigger cross section by comparing to the reliably-calculated [380] deuteron dissociation process measured by PHENIX in 2003.

\section{Instrumentation}

The four RHIC experiments have midrapidity spectrometers with different characteristics but all share identical ZDCs located at $z= \pm 18 \mathrm{~m}$. The ZDCs cover $\pm 5 \mathrm{~cm}$ in $x$ and $y$ about the forward beam direction and have an energy resolution of $\sigma_{E} / E<21 \%$ for $100 \mathrm{GeV}$ neutrons within $x, y \leq 4.5 \mathrm{~cm}$ [379]. Almost all non-interacting spectator neutrons are detected in the ZDCs while charged particles are generally swept out of the ZDC region by strong $(16 \mathrm{Tm})$ accelerator dipoles at $z= \pm 11 \mathrm{~m}$. These dipoles sweep spectator protons from deuteron dissociation beyond the outgoing beam trajectory since they have twice the deuteron charge-to-mass ratio. In PHENIX, the spectator protons are detected in the fCal [379].

PHENIX used two additional hodoscopes (beam-beam counters or BBCs) [383], located at $z= \pm 1.5 \mathrm{~m}$ and covering $3.0 \leq|\eta| \leq 3.9$, as the main minimum-bias trigger. Events with one or more charged particles hitting both the $+z$ and $-z$ BBCs fired this trigger. Determining the $\mathrm{BBC}$ cross section, $\sigma_{\mathrm{BBC}}$, is equivalent to determining the luminosity for the PHENIX data. All $\mathrm{d}+\mathrm{Au}$ events occurring well within the $z$ interval between the BBCs fire this trigger with an $88 \pm 4 \%$ efficiency [383]. The efficiency decreases for $\left|z_{\text {vertex }}\right| \geq 40 \mathrm{~cm}$. Thus $\sigma_{\mathrm{BBC}}$ was determined using only events within the interval $\left|z_{\text {vertex }}\right|<40 \mathrm{~cm}$. A correction was then applied to the fraction of all RHIC events within this interval. The $z$ distribution of the data can be determined using timeof-flight measurements between the ZDCs for events with a north-south coincidence of the ZDCs (with single event resolution of $\sigma_{z} \sim 2 \mathrm{~cm}$ ). 


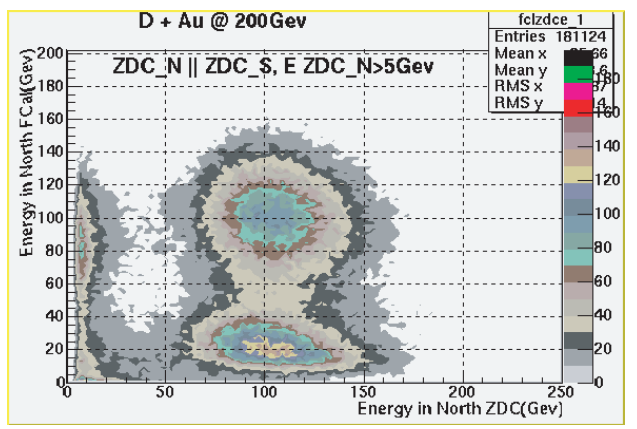

Figure 114. The energy deposition in the proton calorimeter (fCal) as a function of energy deposition in the ZDC (neutron calorimeter) for events with some activity in the ZDC in the deuteron beam direction [85]. This sample includes absorptive stripping as well as $\mathrm{d} \rightarrow n p$. Copyright 2005 by the American Institute of Physics.

\section{Data analysis}

Typical event rates were several $\mathrm{kHz}$ with $L \sim(1-4) \times 10^{28} \mathrm{~cm}^{-2} \mathrm{~s}^{-1}$ for all processes considered here. Therefore the analysis was based on a representative data sample with

$$
\begin{array}{ll}
N^{\text {trig }}(\mathrm{BBC}) & =2.3 \times 10^{5} \\
N^{\text {trig }}\left(\mathrm{ZDC}_{\mathrm{Au}} . \mathrm{OR} . \mathrm{ZDC}_{\mathrm{d}}\right) & =4.6 \times 10^{5}
\end{array}
$$

events where the energy deposited in the ZDC is greater than $10 \mathrm{GeV}$. The subscripts $\mathrm{Au}$ and $\mathrm{d}$ represent the $\mathrm{Au}$ or d directions. The second trigger is sensitive to deuteron dissociation, characterized by a $100 \mathrm{GeV}$ neutron in $\mathrm{ZDC}_{\mathrm{d}}$ and a $100 \mathrm{GeV}$ proton in the fCal with no activity at midrapidity.

Additional data samples were recorded with one of the RHIC beams intentionally displaced by up to $1 \mathrm{~mm}$ to measure the fraction of triggers due to $\mathrm{d}+\mathrm{Au}$ collisions relative to beam-gas background. The largest background was $\leq 3 \%$. The quoted rates have been corrected for the measured background. The BBC event yield, corrected for the accelerator interaction distribution is

$$
N^{\text {corr }}(\mathrm{BBC})=228634 \pm 0.5 \% \text {. }
$$

\section{Deuteron dissociation analysis}

As stated previously, deuteron dissociation events have a clear signature in PHENIX. This is illustrated in Fig. 114 which shows the forward (deuteron direction) energy deposited in the neutron and proton calorimeters in ZDC-triggered events.

The SMD distribution confirmed that neutrons have a small angular divergence. Consequently there is only a small correction for ZDC acceptance. Absorptive stripping events where one nucleon in the deuteron is absorbed in the target is the main potential background for the deuteron dissociation sample. PHENIX used an iterative procedure to extract the dissociation event yield, fitting the $\mathrm{ZDC}_{\mathrm{d}}+$ fCal total energy to the sum of $100+200 \mathrm{GeV}$ lineshapes and correcting for the calculated efficiency as 
successive cuts on activity in other detectors were applied. The first two iterations yield $N(\mathrm{~d} \rightarrow n+p)=157149$ and 156951 events, showing that the procedure is clearly stable.

The final result is :

$$
\begin{aligned}
\sigma_{\mathrm{BBC}} & =\frac{N^{\operatorname{corr}}(\mathrm{BBC})}{N(\mathrm{~d} \rightarrow n p)} \sigma_{\mathrm{d} \rightarrow n p}=\frac{228634}{158761} 1.38( \pm 0.5 \%) \mathrm{b} \\
& =1.99( \pm 1.6 \% \pm 5.0 \%) \mathrm{b},
\end{aligned}
$$

the quantity needed for the luminosity normalization. In order to compare with Glauber calculations in the literature, a correction was applied for the $88 \%$ BBC detector efficiency to obtain the inelastic d+Au cross section,

$$
\sigma_{\text {inel }}^{\mathrm{dAu}}=\frac{\sigma_{\mathrm{BBC}}}{0.88}=2.26 \pm 1.6 \% \pm 5.0 \% \pm 4.5 \% \mathrm{~b} .
$$

The last two errors reflect the theoretical uncertainty on $\sigma_{\mathrm{d} \rightarrow n p}$ and the BBC efficiency uncertainty. A similar analysis yields the $\mathrm{ZDC}_{\mathrm{Au}}$ cross section for $E>10 \mathrm{GeV}$, also used as a minimum bias trigger,

$$
\sigma_{\mathrm{ZDC}_{\mathrm{Au}}}=2.06 \pm 1.7 \% \pm 5.0 \% \mathrm{~b} .
$$

9.3.3. $J / \psi$ and high mass $e^{+} e^{-}$photoproduction Ultraperipheral electromagnetic interactions of nuclei can be calculated using the equivalent photon approximation with $b \geq 2 R_{A}$. As long as the photon squared momentum transfer is restricted to $Q^{2}<1 / R_{A}^{2}$, the photon spectrum is dominated by coherent emission with a $Z^{2}$ enhancement in the equivalent $\gamma A$ luminosity. Because the coupling strength, $Z^{2} \alpha$, is close to unity, additional low energy photon exchanges occur with high probability, particularly at $b \sim 2 R_{A}$ [84]. These low energy photons excite collective nuclear resonances, such as the GDR, very effectively. The excited nuclei return to their ground state predominantly by the emission of one or two neutrons. Neutron tagging is particularly useful for triggering UPC events and, when combined with a rapidity gap requirement on the side of the photon-emitting nucleus, is a powerful trigger selection criteria in heavy-ion collisions. The fraction of $J / \psi$ events with at least one neutron tag is calculated to be $60 \%$.

Several calculations can be found in the literature $[72,83,119]$ and an event generator, STARLIGHT, is used at RHIC to simulate both coherent vector meson production and $\gamma \gamma \rightarrow e^{+} e^{-}$. Recently [95] a calculation of incoherent $\gamma \mathrm{Au} \rightarrow J / \psi X$ production was also presented. This calculation considers the same coherent photon flux but instead of quasi-elastic $J / \psi$ production off the entire nucleus it considers the corresponding production off individual target nucleons. Signatures of the incoherent process are a broader $J / \psi p_{T}$ distribution and a higher neutron multiplicity due to interactions of the recoiling nucleon within the nucleus, see the discussion in Section 2.3.7.

\section{Trigger selection}

PHENIX has excellent capabilities for electron identification since it includes a high resolution electromagnetic calorimeter (EMCal) and Ring Imaging Čerenkov (RICH) 
counters. The RICH and EMCal cover the same rapidity acceptance, $|\eta| \leq 0.35$, as the PHENIX tracking system in two approximately back-to-back spectrometer arms covering $\Delta \phi=\pi$. In addition to the tracking coverage near $\eta=0$, PHENIX used the $\mathrm{BBC}$ hodoscopes to trigger on inelastic heavy-ion collisions. The ZDCs measure the number of neutrons from beam dissociation and can be used to trigger on one or more neutrons in either beam direction.

The PHENIX ultraperipheral dielectron trigger combined three of the trigger elements to select one or more beam dissociation neutrons in the ZDC, at least one electromagnetic cluster in the EMCal with energy and a rapidity gap signaled by no hits in one or the other of the BBC counters.

$$
\text { UPC Trigger }=(\text { EMCal } \geq 0.8 \mathrm{GeV}) \cdot \operatorname{AND} \cdot\left(\mathrm{ZDC}_{\mathrm{N}} \cdot \mathrm{OR} \cdot \mathrm{ZDC} \mathrm{S}_{\mathrm{S}}\right) \cdot \mathrm{AND} \cdot \overline{\mathrm{BBC}} \text {. }
$$

This very loose trigger yielded $8.5 \times 10^{6}$ events out of $1.12 \times 10^{9}$ recorded minimum bias interactions. Thus the UPC trigger comprised less than $0.5 \%$ of the inelastic cross section and a negligible part of the available trigger bandwidth.
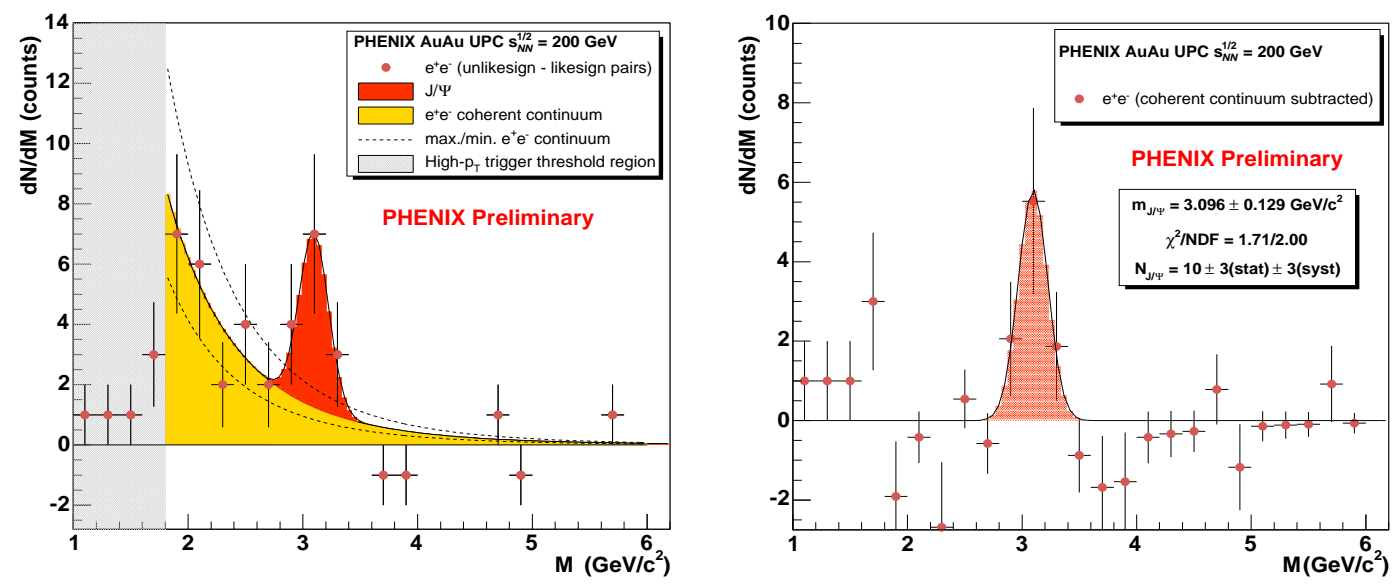

Figure 115. Preliminary invariant mass distribution of $e^{+} e^{-}$pairs measured by PHENIX in ultraperipheral $\mathrm{Au}+\mathrm{Au}$ collisions at $\sqrt{s_{N N}}=200 \mathrm{GeV}$. The left-hand plot shows a fit assuming an $e^{+} e^{-}$continuum and a $J / \psi$ signal. The two dashed curves indicate the continuum uncertainty. The right-hand plot shows the signal after continuum subtraction. From [63].

\section{Event selection}

The main features of dilepton photoproduction are small pair transverse momentum and low multiplicity tracks (both characteristic of diffractive processes). Coherent $J / \psi$ production is primarily at midrapidity, $|y| \leq 1$. For a charged particle track to be reconstructed in the tracking detectors, $n_{\text {track }} \leq 15$ and $\left|z_{\text {vertex }}\right| \leq 30 \mathrm{~cm}$ was required.

The integrated luminosity corresponding to this data sample was calculated after the vertex cut was applied and $21 \%$ of the data with different running conditions was removed. Using the number of minimum bias interaction triggers in the remaining 
sample and the $6.3 \pm 0.5 \mathrm{~b}$ minimum bias $\mathrm{Au}+\mathrm{Au}$ cross section [129], we find $\int d t L=$ $120 \pm 10 \mu \mathrm{b}^{-1}$.

The momentum of electron candidate tracks was measured using the deflection in the magnetic spectrometer. After defining electron candidate trajectories and momenta in the spectrometers, cuts consistent with electron response in the RICH and EMCal were imposed. At least two photomultipliers were required to have a Čerenkov signal in the correct region of the RICH and at least one electron was required to deposit greater than $1 \mathrm{GeV}$ energy in the EMCal. Finally, electron candidates had to occupy different spectrometer arms since low $p_{T} J / \psi$ s decay to back-to-back electrons.

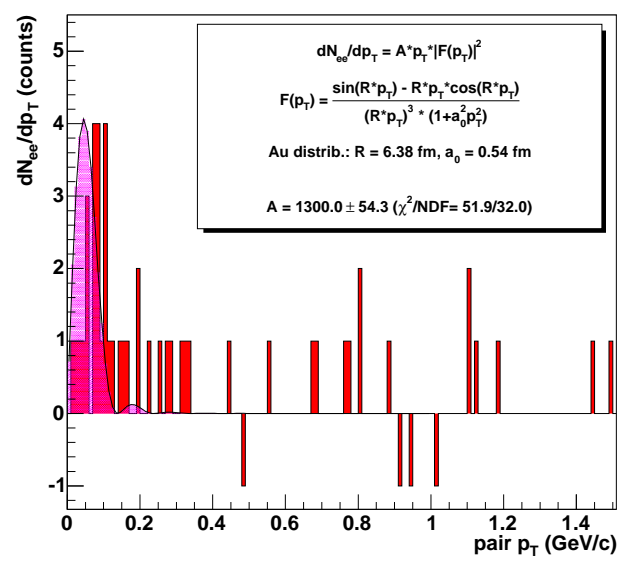

Figure 116. The $J / \psi \rightarrow e^{+} e^{-} p_{T}$ distribution from ultraperipheral $\mathrm{Au}+\mathrm{Au}$ collisions compared to a calculation of coherent photoproduction with a realistic nuclear form factor. From [63].

\section{Results}

The event selection cuts yielded $42 e^{+} e^{-}$signal candidates and $7 e^{ \pm} e^{ \pm}$candidates with $M \geq 1.8 \mathrm{GeV} / c^{2}$. A like-sign subtraction was performed to estimate the combinatorial background, resulting in the signal spectrum shown in Fig. 115.

To extract the $J / \psi$ signal, the continuum spectrum was fit to a power law (with the power determined from a full simulation). The number of events with $1.8<M<2.0$ $\mathrm{GeV} / \mathrm{c}^{2}$ was used to estimate the continuum, shown in Fig. 115. The extracted signal is $10 \pm 3$ (stat) \pm 3 (syst) events. The continuum subtraction dominates the systematic error.

Inclusive hadronic $J / \psi$ production in heavy-ion collisions has a broad $p_{T}$ distribution with an average $p_{T}$ of $\sim 1.5 \mathrm{GeV} / c$ [382]. This should be compared to the measured coherent photoproduction $p_{T}$ distribution peaked at $p_{T} \approx 80 \mathrm{MeV} / c$, as expected when all pairs with $M>1.8 \mathrm{GeV} / c^{2}$ are included, see Fig. 116. Figure 116 also shows the expected shape due to the $\mathrm{Au}$ form factor.

The $J / \psi$ photoproduction cross section was calculated, correcting for detector acceptance and cut efficiencies obtained by simulating $J / \psi$ production with the expected 
$p_{T}$ distribution. The geometrical acceptance and efficiencies reduce the $J / \psi$ yield in $|y| \leq 0.5$ by $5.0 \%$ and $56.4 \%$ respectively. The preliminary cross section at $y=0$ is

$$
\frac{B d \sigma_{J / \psi}}{d y}=48 \pm 14 \text { (stat) } \pm 16 \text { (syst) } \mu \mathrm{b},
$$

in good agreement with the $58 \mu \mathrm{b}$ STARLIGHT $[72,83,119]$ prediction. In future $\mathrm{Au}+\mathrm{Au}$ runs, PHENIX will see a 10-fold increase in event yield, making detailed studies of both coherent and quasi-elastic $J / \psi$ photoproduction possible. PHENIX will also commission a second trigger, sensitive to $J / \psi \rightarrow \mu^{+} \mu^{-}$at large rapidity where the quasi-elastic signal will dominate [95]. Nevertheless, the present low-statistics measurement clearly demonstrates the feasibility of small cross section diffractive measurements in heavy-ion collisions at RHIC and the LHC.

\section{LHC detector overview of UPC potential}

\subsection{Introduction}

Contributed by: P. Yepes

The ALICE, ATLAS and CMS collaborations plan to take data at the LHC with heavy-ion beams. ALICE was specifically designed for heavy-ion physics and intends to address both soft and hard physics. CMS and ATLAS were designed for hard physics and initially focused on proton-proton collisions. However their potential for heavy-ion physics was soon pointed out. In this chapter, a brief description of each detector is presented with special emphasis on those features most relevant for UPCs.

Table 24 shows the main features of the LHC detectors. All three detectors have complete azimuthal tracking coverage over different rapidity regions. ALICE is limited to $|\eta| \lesssim 1$ while ATLAS and CMS extend their coverage to $|\eta|<2.4$. The latter two detectors have tracking systems that can be read out at every beam crossing. The ALICE TPC provides excellent resolution, $\Delta p_{T} / p_{T}=1.5 \%$, for low momentum particles, $0.05<p_{T}<2 \mathrm{GeV} / c$. However, ALICE can only be read out with a rate on the order of $\mathrm{kHz}$. The ATLAS (CMS) momentum resolution is $\Delta p_{T} / p_{T} \approx 3 \%(<2 \%)$. ATLAS can reconstruct low momentum particles down to $p_{T}=0.5 \mathrm{GeV} / c$, while CMS measures tracks as low as $p_{T}=0.2 \mathrm{GeV} / c[384,385]$.

ALICE is equipped with a muon spectrometer with full azimuthal acceptance in the rapidity range $-4<\eta<-2.5$. ATLAS has large acceptance, $|\eta|<2.4$ for muons with $p_{T}>4.5 \mathrm{GeV} / c$. In CMS, muons with $p_{T}>3.5 \mathrm{GeV} / c$ will be detected in the central region, $|\eta|<1$, while the forward muon detector, $1<|\eta|<2.4$, has muon acceptance for $p_{T}>1.5 \mathrm{GeV} / c$.

ALICE is best designed for particle identification. It can separate pions from kaons in the range $0.1<p<3 \mathrm{GeV} / c$, kaons from protons over $0.2<p<5 \mathrm{GeV} / c$, and electrons from $\pi^{0}$ 's for $0.1<p<25 \mathrm{GeV} / c$. Studies in CMS indicate good low $p_{T}$ capabilities using the three layers of the silicon pixel tracker to achieve $\pi, K$ and $p$ separation within $0.4<p_{T}<1 \mathrm{GeV} / c$ [384,385]. In addition, a conservative range over which electrons can be separated from neutral pions is $2<p_{T}<20 \mathrm{GeV} / c$. 
Table 24. Summary of the main characteristics of the ALICE, ATLAS and CMS detectors.

\begin{tabular}{|c|c|c|c|c|c|}
\hline Component & & & ALICE [386] & ATLAS [94] & CMS $[123,385]$ \\
\hline \multirow[t]{4}{*}{ Tracking } & \multirow[t]{3}{*}{ Acceptance } & $\eta$ & $|\eta|<0.9$ & $|\eta|<2.4$ & $|\eta|<2.4$ \\
\hline & & $\phi$ & $0<\phi<2 \pi$ & $0<\phi<2 \pi$ & $0<\phi<2 \pi$ \\
\hline & & $p_{T}$ & $p_{T}>0.05 \mathrm{GeV} / c$ & $p_{T}>0.5 \mathrm{GeV} / c$ & $p_{T}>0.2 \mathrm{GeV} / c$ \\
\hline & Resolution & $\Delta p_{T} / p_{T}$ & $\begin{array}{l}1.5 \%\left(p_{T}<2 \mathrm{GeV} / c\right) \\
9.0 \%, p_{T}=100 \mathrm{GeV} / c\end{array}$ & $\approx 3 \%$ & $\begin{array}{l}<2 \%, \quad p_{T}<100 \\
\mathrm{GeV} / c\end{array}$ \\
\hline Muons & Acceptance & & $-4<\eta<-2.5$ & $\begin{array}{l}|\eta|<2.4 \\
p_{T}>4.5 \mathrm{GeV} / c\end{array}$ & $\begin{array}{l}|\eta|<1, p_{T}>3.5 \\
\mathrm{GeV} / c \\
|\eta|>1, p_{T}>1.5 \\
\mathrm{GeV} / c\end{array}$ \\
\hline \multirow[t]{3}{*}{ Particle ID } & $\pi / K$ & & $0.1<p<3 \mathrm{GeV} / c$ & TBD & $0.2<p_{T}<1 \mathrm{GeV} / c$ \\
\hline & $K / p$ & & $0.2<p<5 \mathrm{GeV} / c$ & TBD & $0.4<p_{T}<2 \mathrm{GeV} / c$ \\
\hline & $e / \pi$ & & $0.1<p<25 \mathrm{GeV} / c$ & $p>2 \mathrm{GeV} / c$ & $\begin{array}{l}0.1<p<0.2 \mathrm{GeV} / c \\
p>2 \mathrm{GeV} / c\end{array}$ \\
\hline \multirow{6}{*}{$\begin{array}{l}\text { Electromagnetic } \\
\text { Calorimetry }\end{array}$} & \multirow[t]{2}{*}{ Acceptance } & $\eta$ & $|\eta|<0.12$ & $|\eta|<3.1$ & $|\eta|<3$ \\
\hline & & $\phi$ & $1.22 \pi<\phi<1.78 \pi$ & $2 \pi$ & $2 \pi$ \\
\hline & Segmentation & $\Delta \phi \times \Delta \eta$ & $0.0048 \times 0.0048$ & $0.025 \times 0.003$ & $0.0175 \times 0.0175$ \\
\hline & & Longitudinal & No & Yes & No \\
\hline & Resolution $(E$ in $\mathrm{GeV})$ & $\Delta E / E$ & $\begin{array}{l}0.03 / \sqrt{E} \oplus 0.03 / E \\
\oplus 0.01\end{array}$ & $0.1 / \sqrt{E} \oplus 0.005$ & $0.027 / \sqrt{E} \oplus 0.0055$ \\
\hline & Technology & & $\mathrm{PbWO}_{4}$ crystals & Liquid $\operatorname{Ar}(\mathrm{LAr})$ & $\mathrm{PbWO}_{4}$ crystals \\
\hline \multirow{6}{*}{$\begin{array}{l}\text { Hadronic } \\
\text { Calorimetry }\end{array}$} & Acceptance & $\eta$ & NA & $|\eta|<3$ & $|\eta|<3$ \\
\hline & & $\phi$ & $\mathrm{NA}$ & $2 \pi$ & $2 \pi$ \\
\hline & Segmentation & $\Delta \phi \times \Delta \eta$ & $\mathrm{NA}$ & $0.1 \times 0.1$ & $0.087 \times 0.087$ \\
\hline & & Longitudinal & $\mathrm{NA}$ & Yes & Yes \\
\hline & Resolution & $\Delta E / E$ & $\mathrm{NA}$ & $0.5 / \sqrt{E} \oplus 0.02$ & $1.16 / \sqrt{E} \oplus 0.05$ \\
\hline & Technology & & NA & $\mathrm{Pb} \operatorname{Scint}(\mathrm{B})-\mathrm{LAr}(\mathrm{F})$ & Cu Scint \\
\hline \multirow{4}{*}{$\begin{array}{l}\text { Forward } \\
\text { Calorimetry }\end{array}$} & \multirow[t]{2}{*}{ Acceptance } & $\eta$ & NA & $3<|\eta|<4.9$ & $3<|\eta|<5$ \\
\hline & & $\phi$ & $\mathrm{NA}$ & $2 \pi$ & $2 \pi$ \\
\hline & Segmentation & $\Delta \phi \times \Delta \eta$ & $\mathrm{NA}$ & $0.1 \times 0.1$ & $0.087 \times 0.087$ \\
\hline & Technology & & $\mathrm{NA}$ & $\mathrm{Cu} / \mathrm{LAr}-\mathrm{W} / \mathrm{LAr}$ & $\mathrm{Fe} /$ quartz fibers \\
\hline \multirow{3}{*}{$\begin{array}{l}\text { Very } \\
\text { Forward } \\
\text { Calorimetry } \\
\end{array}$} & \multirow[t]{2}{*}{ Acceptance } & $\eta$ & NA & NA & $5.3<\eta<6.7$ \\
\hline & & $\phi$ & $\mathrm{NA}$ & $\mathrm{NA}$ & $2 \pi$ \\
\hline & Segmentation & $\Delta \phi \times \Delta \eta$ & $\mathrm{NA}$ & $\mathrm{NA}$ & $\pi / 8(\pi / 4)$ Had $(\mathrm{EM})$ \\
\hline \multirow{2}{*}{$\begin{array}{l}\text { Forward } \\
\text { Tracking (TOTEM) }\end{array}$} & \multirow[t]{2}{*}{ Acceptance } & $\eta$ & $\mathrm{NA}$ & $\mathrm{NA}$ & $5.3<\eta<6.7$ \\
\hline & & $\phi$ & NA & NA & $2 \pi$ \\
\hline \multirow{2}{*}{$\begin{array}{l}\text { Zero-Degree } \\
\text { Calorimeters (ZDC) }\end{array}$} & \multirow[t]{2}{*}{ Acceptance } & $|\eta|$ (neutrals) & $\gtrsim 8.6$ & $\gtrsim 8.3$ & $\gtrsim 8.3$ \\
\hline & & $\phi$ & $2 \pi$ & $2 \pi$ & $2 \pi$ \\
\hline
\end{tabular}


The features of the electromagnetic calorimeters are also given in Table 24. ALICE has a $\mathrm{PbWO}_{4}$ photon spectrometer with excellent spatial and energy resolution, albeit small acceptance, and a larger lead/scintillator electromagnetic calorimeter. ATLAS and CMS have hermetic calorimeters employing liquid argon and $\mathrm{PbWO}_{4}$ crystals, respectively, both covering $|\eta| \lesssim 3$. CMS has slightly better resolution while ATLAS provides additional information on the longitudinal shower shape.

Both ATLAS and CMS are equipped with large coverage, $|\eta|<5$, hadron calorimetry. The CMS copper-scintillator calorimeter has slightly finer transverse segmentation than ATLAS. However, ATLAS combines lead scintillators with liquid argon to achieve a factor of two better energy resolution than CMS. Both experiments also feature ZDCs $(|\eta| \gtrsim 8.5$ for neutrals), a basic tool for neutron tagging in ultraperipheral heavy-ion interactions. CMS has an additional electromagnetic/hadronic calorimeter, CASTOR $(5.3<|\eta|<6.7)$, and shares the

interaction point with the TOTEM experiment, providing two additional trackers at very forward rapidities, T1 $(3.1<|\eta|<4.7)$ and T2 $(5.5<|\eta|<6.6)[29]$.

\subsection{The ALICE detector}

\section{Contributed by: V. Nikulin, J. Nystrand, S. Sadovsky and E. Scapparone}

The ALICE detector [386], shown in Fig. 117, is designed to study the physics of strongly interacting matter at extreme energy densities where the formation of a new phase of matter, the quark-gluon plasma, is expected. The detector is designed to cope with up to 8000 particles per unit rapidity. It consists of a central part which measures hadrons, electrons and photons, a forward muon spectrometer and two zero degree calorimeters located up- and downstream from the detector [386].

The central (barrel) part, which covers the full azimuth over $|\eta|<0.9$ in pseudorapidity, is embedded in a large solenoid magnet. The barrel detectors include a silicon inner tracking system (ITS), a cylindrical time-projection chamber (TPC), three time-of-flight (TOF) arrays, ring-imaging Cerenkov (HMPID) and transition radiation (TRD) counters and a high resolution electromagnetic calorimeter (PHOS). Several smaller detectors (FMD, V0, T0) are located at small angles forward and backward of midrapidity. Note that the Forward Muon Spectrometer, shown on the right-hand side of Fig. 117, is actually at backward rapidity according to the LHC frame convention.

A solenoidal magnetic field of $0.2-0.5 \mathrm{~T}$ allows full tracking and particle identification down to $p_{T} \sim 100 \mathrm{MeV} / c$. The optimal field strength and volume is a compromise between momentum resolution, momentum acceptance and tracking efficiency.

The inner tracking system The ITS consists of six layers of high-resolution detectors. The innermost four layers are equipped with silicon pixel and drift detectors designed to handle the high multiplicities expected. The outer layers, at a $\sim 50 \mathrm{~cm}$ radius, are equipped with double-sided silicon micro-strip detectors. Four of the layers have analog 


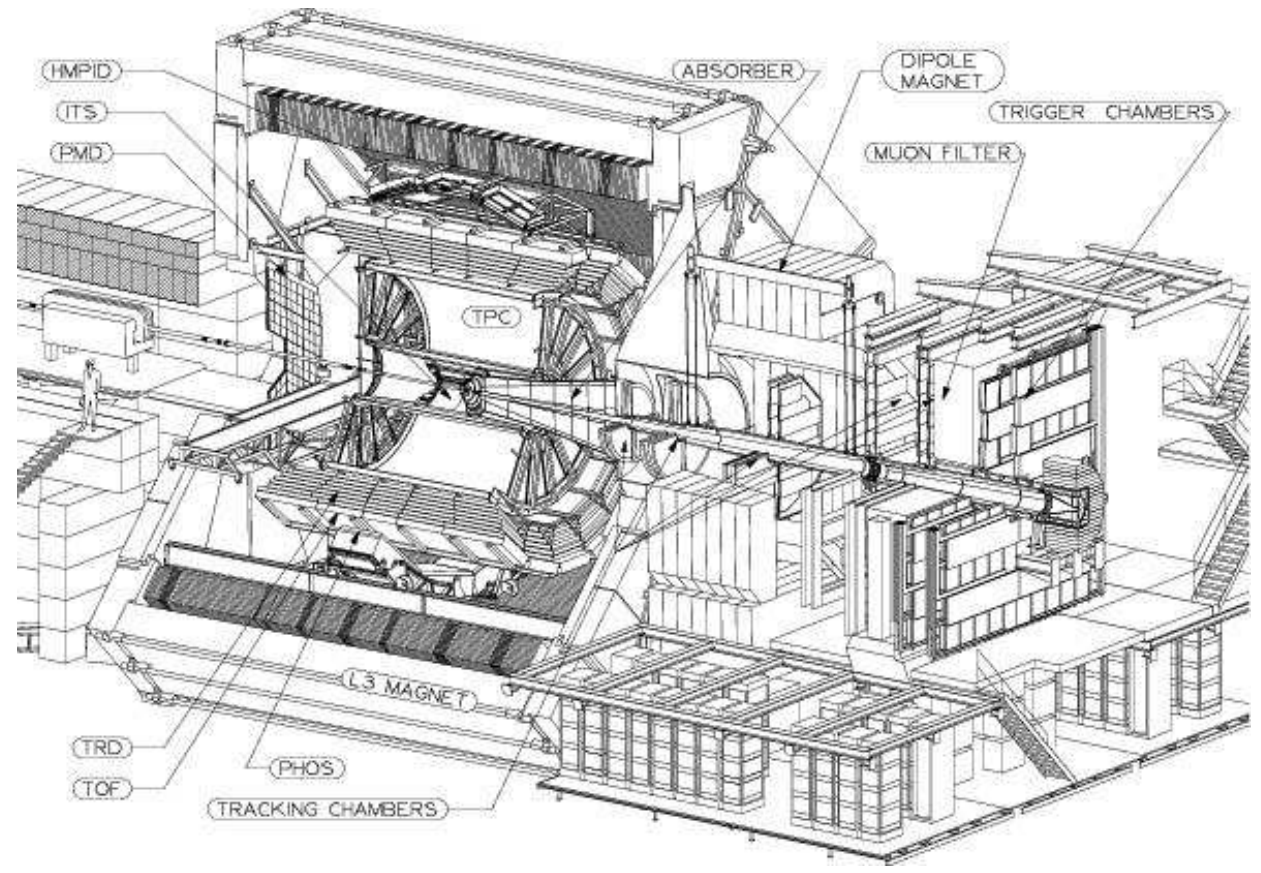

Figure 117. The ALICE Detector. The ZDCs, positioned more than $100 \mathrm{~m}$ from the interaction point, are not shown.

readout for independent particle identification via $d E / d x$ in the non-relativistic region, which gives the ITS standalone capability as a low $p_{T}$ particle spectrometer.

The Level-0 (L0) trigger for the pixel detectors is under development. The $10^{6}$ fast logical OR outputs are grouped into 1200 processor inputs by serialization/deserialization. The processor enables implementation of a very flexible decision algorithm. The possibilities of using this trigger for UPC events should be carefully studied.

The time projection chamber The inner radius of the TPC $(90 \mathrm{~cm})$ is fixed by the maximum acceptable hit density, $0.1 \mathrm{~cm}^{-2}$. The $250 \mathrm{~cm}$ outer radius is determined by the length required for the $d E / d x$ resolution to be better than $10 \%$. The large granularity, $\sim 5 \times 10^{8}$ pixels, together with an optimized gas choice, ensures good twotrack resolution.

Particle identification Good particle identification over a wide $p_{T}$ range is one of the strongest features of the ALICE detector. Several detector systems are used for particle identification: the ring-imaging Cerenkov detector (HMPID), the transition radiation detector (TRD) and the time-of-flight array (TOF). A short description of the functioning of these detectors in UPCs is given here.

The HMPID, covering $15 \%$ of the $(\eta, \phi)$ acceptance, is optimized for high $p_{T}$ particle detection. The six-layer TRD will identify electrons with momenta above $1 \mathrm{GeV} / c$. In order to reduce the large energy dissipation by the TRD front-end electronics, it remains in stand-by mode when nothing interesting is happening. A pre-trigger signal is required 
to wake up the electronics within $100 \mathrm{~ns}$ of the interaction. In hadronic interactions, the fast logical OR of the small angle T0 detector is used as a pre-trigger. Since the T0 detectors are only useful in a high-multiplicity environment and cannot be employed in UPCs, the OR from the TOF can be used as an alternate TRD pre-trigger.

The large TOF detector covers a cylindrical surface with polar angle acceptance of $45^{\circ}<\theta<135^{\circ}$. The TOF consists of 90 modules with $18 \phi$ sectors and $5 z$ segments. The TOF modules are made up of multi-gap resistive plate chambers (MRPCs) with an intrinsic timing resolution of $\sim 100$ ps. The 5 longitudinal segments have different numbers of strips, according to their position. There are $1674 \mathrm{MRPC}$ strips in the TOF, each with 96 pads, for a total of $\sim 1.6 \times 10^{5}$ readout pads. The TOF L0 trigger can provide the total hit multiplicity. A more sophisticated Level-1 (L1) trigger could be applied.

Photon detectors The photon spectrometer (PHOS) is intended to measure direct photons and high $p_{T}$ neutral mesons. PHOS is a single arm, high-resolution electromagnetic calorimeter, positioned $4.6 \mathrm{~m}$ below the interaction vertex. It covers 8 $\mathrm{m}^{2}, \sim 10 \%$ of the barrel solid angle, with $\sim 17,000$ channels of scintillating $\mathrm{PbWO}_{4}$ crystals. Thus PHOS could be used as veto counter to select events with "abnormally" low multiplicity.

The electromagnetic calorimeter (EMCAL) will be an ALICE upgrade. It will be a medium-resolution scintillation sandwich calorimeter. While the EMCAL is primarily for jet studies, the L0 EMCAL trigger could probably be adapted to UPC needs. Additional studies are required.

The photon multiplicity detector (PMD) is a pre-shower detector that measures the $(\eta, \phi)$ distribution of the photons in the region, $1.8<\eta<2.6$. The PMD consists of two identical planes of proportional honeycomb chambers with a 3 interaction length thick lead converter in between the chambers.

Small angle detectors ALICE has a number of smaller detector systems (ZDC, FMD, V0, and T0) positioned at small angles for triggering. Two ZDCs are located in the accelerator tunnels $100 \mathrm{~m}$ away from the interaction point. Their pseudorapidity acceptance is $8.6<|\eta|$. They measure the spectator nucleons (both neutrons and protons) in the collision.

The other small detectors are located asymmetrically with respect to the interaction point. The right-hand arrays are at negative rapidity while the left-hand detectors are at positive rapidity. The forward multiplicity detector (FMD) is a silicon strip ring counter with about 25000 channels. It measures charge particle production in the pseudorapidity ranges $-3.4<\eta<-1.7$ (right) and $1.7<\eta<5.1$ (left). The T0 (beambeam) detector, 12 Cerenkov radiators coupled to photomultiplier tubes, is located at $2.9<\eta<3.3$ (left) and $-5.0<\eta<-4.5$ (right). It produces fast signals with good timing resolution, $\sigma \sim 50 \mathrm{ps}$, allowing online reconstruction of the main vertex. The V0 detector, 72 plastic scintillators grouped in five rings, covers $-3.8<\eta<-1.7$ (right) 
and $2.8<\eta<5.1$ (left). It provides a minimum bias trigger for the central detectors and can be used for centrality determination in $A A$ collisions and to validate the trigger signal in the muon spectrometer in $p p$ collisions.

Forward muon spectrometer The forward muon spectrometer, covering the backward region $-4.0<\eta<-2.5$ in the $\mathrm{LHC}$ reference frame, will study quarkonium decays to dimuons. The expected mass resolution is $\sim 100 \mathrm{MeV}$ at $10 \mathrm{GeV}$, sufficient to distinguish between the $\Upsilon S$ states. The muon spectrometer consists of a composite absorber with high- $Z$ (small angles) and low- $Z$ (near the front) materials, located 90 $\mathrm{cm}$ behind the interaction point; a large dipole magnet with a $3 \mathrm{~T} \mathrm{~m}$ field integral; 10 planes of thin, high-granularity tracking chambers with $\sim 100 \mu \mathrm{m}$ spatial resolution; a $1.2 \mathrm{~m}$ iron muon filter; and four trigger chambers for the L0 trigger. The muon filter sits between the tracking and trigger chambers to reduce the trigger chamber background.

Trigger and data acquisition The ALICE trigger is especially important because the very large event size causes severe data acquisition and storage problems. ALICE features a complex and flexible trigger. Several detectors provide input to the different trigger levels to select signals such as centrality, high $p_{T}$ electrons, muons, or photons. Several trigger levels are foreseen.

- Level-0 (L0): This is the fast, minimum-bias interaction trigger, issued after $\sim 0.8$ $\mu \mathrm{s}$, used as strobe for some electronics. It includes various decisions from the T0 and the muon spectrometer as well as from other auxiliary sub-detectors such as the cosmic telescope. Recently, L0 triggers were developed for the ITS, PHOS and TOF detectors. The T0 decision is used as a TRD pre-trigger for hadronic interactions.

- Level-1 (L1): This trigger, with a latency of $\sim 6.5 \mu \mathrm{s}$, receives additional information from the ZDC, PHOS, TRD, FMD and PMD.

- Level-2 (L2): Relatively slow detector decisions are included with a delay of $\sim 88$ $\mu$ s so that it is possible to veto events where a second high multiplicity event occurs just before or soon after the trigger of interest (TPC past-future protection).

- High Level Trigger (HLT): The HLT is an on-line computing farm with several hundred commodity processors providing further event selection and event compression.

Most UPC events are characterized by very low multiplicity. Unfortunately, the low multiplicity background tends to be rather large. Therefore, L0 background suppression is necessary. UPC events with nuclear dissociation can be selected by combining low multiplicity with nuclear break-up using the ZDCs, greatly suppressing the background. However, the ALICE ZDCs are located too far from the main detector to be used at L0. Thus UPC events need to be triggered by the central barrel and/or the muon spectrometer.

UPC events are normally rejected by the standard ALICE L0 trigger. However, recent developments employing the ITS pixel trigger are quite encouraging. The trigger 
could apply $p_{T}$ cuts to the L0 signal, rejecting the low $p_{T}$ background due to $e^{+} e^{-}$pair production, $\sigma_{\text {tot }} \sim 200 \mathrm{~kb}$ [387], to study UPCs in the barrel. Detailed studies of this option are underway. The EMCAL, currently under construction, will be used as an additional dijet trigger.

The muon spectrometer trigger could be successfully used to detect UPCs with final-state muons. The PHOS can be used to efficiently veto high multiplicity events at L0, considerably reducing the UPC background for muon events.

The offline analysis, including ZDC information and reconstruction of the parent particle $p_{T}$ distributions, could select events of interest. Further studies are presented in section 6.3.

Barrel trigger strategies The 'elastic' and 'inelastic' UPC classes require different triggers. The identification of exclusive vector meson production is based on reconstruction of the entire event (the two tracks from the decay) and identifying coherent production through the low lepton pair $p_{T}$. On the other hand, $\gamma$-parton interactions must be identified by a rapidity gap between the photon-emitting nucleus and the produced particles.

The very different topology of ultraperipheral interactions relative to central nucleus-nucleus collisions leads to different trigger requirements. In the case of hadronic interactions, the forward detectors trigger large multiplicity events. This is not possible for UPCs since they are characterized by voids of produced particles, rapidity gaps, several units wide. To detect ultraperipheral events, it is necessary to have a low-level trigger sensitive to the production of a few charged particles around midrapidity [318].

In ALICE, the fast response, large pseudorapidity coverage, $|\eta|<1$, and high segmentation, of the TOF make it well suited for a L0 trigger in the central region. Since the T0 detectors are not used in UPCs, the fast OR TOF signal can be used as a pre-trigger for the TRD. The pad signals from each of the 90 modules are included in the TOF L0 trigger. They can provide information on event multiplicity and topology, important for the development of a UPC trigger. Hits in several TOF pads are required for the pre-trigger.

A possible trigger scheme for exclusive $\rho^{0}, J / \psi$ and $\Upsilon$ photoproduction is described below.

- L0: The TOF L0 multiplicity coupled with a suitable topology cut can provide a trigger for exclusive events with exactly two charged tracks in the central barrel. The forward detectors are available at L0 and can identify the presence of one more more rapidity gaps. For example, if only the V0 detectors (V0L, $2.8<\eta<5.1$, and V0R, $-1.7>\eta>3.8$ ) are available at L0, then if there is no signal in V0L but a signal in V0R, there is a rapidity gap of at least $-1.7>\eta>3.8$. Thus the ALICE trigger logic unit can carry the information on track multiplicity as well as rapidity gap.

- L1: The main trigger cut for $J / \psi \rightarrow e^{+} e^{-}$and $\Upsilon \rightarrow e^{+} e^{-}$decays at this level will 
be identification of one electron and one positron in the TRD. If a more accurate measurement of the central barrel multiplicity is available, it could be used to select events with exactly two charged tracks. Information from the ZDCs may be used to select events with or without Coulomb breakup.

- HLT: The HLT may be used to require exactly two opposite-sign tracks from the primary vertex in the TPC. Using the reconstructed momenta, a cut on the summed track $p_{T}$ can be applied. Such a cut is highly efficient for suppressing the incoherent background. Some of the pions from coherent $\rho^{0}$ decays could be misidentified as electrons at L1 in the TRD. Due to the extremely high $\rho^{0}$ rate, it may be necessary to apply an invariant mass cut in the HLT to scale down these events so that they do not occupy the full bandwidth.

A similar triggering scheme for other photonuclear events is given below.

- L0: At L0, there would be an asymmetric signal in the V0 counters: low or intermediate multiplicity on one side and no signal from the opposite side, supplemented by a low-multiplicity trigger in the central arm, such as from the TOF.

- L1: The ZDC on the same side as the rapidity gap should be empty. The signal in the ZDC on the opposite side should be low.

- HLT: The photonuclear event rate will be high but only a small fraction of these events will be interesting. In addition, the asymmetric signature at L0 and L1 are also caused by beam-gas interactions. The HLT will be needed to reject beam-gas events and select the interesting photonuclear events such as open charm production.

The expected vector meson and lepton pair yields from two-photon interactions were estimated using the geometrical acceptance of the ALICE central barrel and muon arm. Events were generated from a Monte Carlo model based on the calculations in Refs. [72,83,119,298]. The rates were calculated assuming a $\mathrm{Pb}+\mathrm{Pb}$ luminosity of $5 \times 10^{26} \mathrm{~cm}^{-2} \mathrm{~s}^{-1}$. The ALICE acceptance is defined as $|\eta|<0.9$ and $p_{T}>0.15 \mathrm{GeV} / c$ in the central barrel, $-4 \leq \eta \leq-2.5$ and $p_{T}>1.0 \mathrm{GeV} / c$ in the muon spectrometer. Both tracks are required to be within the acceptance for the event to be reconstructed. A trigger cut of $p_{T}>3.0 \mathrm{GeV} / c$ is necessary for central collisions in the TRD. The expected vector meson and lepton pair rates are shown in Table 10.2 [318].

ITS low multiplicity trigger A low level trigger for ultraperipheral processes in ALICE can be based on the charged track multiplicity in the central rapidity region. The ITS [388], useful for fast charged-multiplicity measurements, is considered as a trigger here. The ITS consists of six coaxial cylindrical detectors: two pixel detectors (SPD1 and SPD2); two drift detectors (SDD1 and SDD2); and two strip detectors (SSD1 and SSD2). Table 10.2 presents their main features.

The ALICE detector will typically operate in a solenoidal magnetic field of strength $0.2<B<0.5 \mathrm{~T}$. Although the nominal field value is $0.2 \mathrm{~T}$ [386], ALICE is likely to run 
Table 25. The expected yields from $\mathrm{Pb}+\mathrm{Pb}$ UPCs for several final states within the geometrical acceptance of the ALICE central barrel [318].

\begin{tabular}{|c|c|c|}
\hline Final State & Acceptance & Rate $/ 10^{6} \mathrm{~s}$ \\
\hline$\rho^{0} \rightarrow \pi^{+} \pi^{-}$ & central barrel & $2 \times 10^{8}$ \\
$J / \psi \rightarrow e^{+} e^{-}$ & central barrel & $1.50 \times 10^{5}$ \\
$\Upsilon(1 S) \rightarrow e^{+} e^{-}$ & central barrel & $400-1400$ \\
$e^{+} e^{-}, M>1.5 \mathrm{GeV} / c^{2}$ & central barrel, $p_{T}>0.15 \mathrm{GeV} / c$ & $7 \times 10^{5}$ \\
$e^{+} e^{-}, M>1.5 \mathrm{GeV} / c^{2}$ & central barrel, $p_{T}>3 \mathrm{GeV} / c$ & $1.4 \times 10^{4}$ \\
$\mu^{+} \mu^{-}, M>1.5 \mathrm{GeV} / c^{2}$ & muon spectrometer, $p_{T}>1 \mathrm{GeV} / c$ & $6 \times 10^{4}$ \\
\hline
\end{tabular}

Table 26. The elements of the ALICE inner tracker, their type and their radial distance from the beam pipe.

\begin{tabular}{|c|c|c|}
\hline Detector & Type & Radius $(\mathrm{cm})$ \\
\hline SPD1 & Pixel & 3.9 \\
SPD2 & Pixel & 7.6 \\
SDD1 & Drift & 14.0 \\
SDD2 & Drift & 24.0 \\
SSD1 & Strip & 40.0 \\
SSD2 & Strip & 45.0 \\
\hline
\end{tabular}

at the highest field value [389]. The magnetic field restricts the kinematic acceptance for charged particles. At $0.2 \mathrm{~T}$, the minimum transverse momenta, $p_{T}^{\min }$, necessary for charged particles to reach SPD1 and SPD2, the inner detectors, are $1.2 \mathrm{MeV} / c$ and $2.3 \mathrm{MeV} / c$ respectively. The minimum $p_{T}$ needed to reach the outermost detector, $\mathrm{SSD} 2$, is $13.5 \mathrm{MeV} / c$. (For higher field values, $p_{T}^{\min }$ increases linearly with $B$. Thus low fields are best for studying soft physics.) Particle absorption in the beam pipe and detector layers is not taken into account. The detector load is defined as the cross section for having $N_{e}$ charged hits from electrons or positrons in the detector acceptance. The load [390] and the corresponding UPC trigger rate estimates are presented in Table 10.2.

These rates have to be compared to the hadronic collision rates. The impactparameter integrated $\mathrm{Pb}+\mathrm{Pb}$ cross section is about $8 \mathrm{~b}$. Thus the ALICE L0 trigger rate should be at least comparable to this cross section, $\sim 8 \mathrm{kHz}$, up to a limitation of $0.4 \mathrm{MHz}$ due to the LHC clock. Thus the SPD2 trigger is required to select events with at least three charged particles while $p_{T}^{\min }$ is high enough for the SSD2 trigger rate to be able to function for $N_{e} \geq 1$. On the other hand, charge conservation in $\gamma \gamma$ processes requires the restriction $N_{e} \geq 2$. Note that at higher field values, the increased $p_{T}^{\min }$ would mean reduced event rates in all the detectors.

The L0 pixel trigger is under development. 
Table 27. The cross sections and rates of $N_{e} e^{+}$or $e^{-}$interacting in the SPD1, SPD2 and SSD2 layers of the ITS detectors in $\mathrm{Pb}+\mathrm{Pb}$ collisions with $L_{\mathrm{PbPb}}=10^{3} \mathrm{~b}^{-1} \mathrm{~s}^{-1}$ and $B=0.2 \mathrm{~T}$.

\begin{tabular}{|c|c|c|c|c|c|c|}
\hline \multirow{2}{*}{$N_{e}$} & \multicolumn{2}{|c|}{ SPD1 } & \multicolumn{2}{c|}{ SPD2 } & \multicolumn{2}{c|}{ SSD2 } \\
\cline { 2 - 7 } & $p_{T}>1.2 \mathrm{MeV} / c,|\eta|<1.5$ & \multicolumn{2}{c|}{$p_{T}>2.3 \mathrm{MeV} / c,|\eta|<1.5$} & \multicolumn{2}{c|}{$p_{T}>13.5 \mathrm{MeV} / c,|\eta|<1$} \\
\hline 1 & $\sigma_{N_{e}}(\mathrm{~b})$ & Rate $(\mathrm{Hz})$ & $\sigma_{N_{e}}(\mathrm{~b})$ & Rate $(\mathrm{Hz})$ & $\sigma_{N_{e}}(\mathrm{~b})$ & Rate $(\mathrm{Hz})$ \\
2 & 44000 & $1.3 \times 10^{7}$ & 4600 & $4.6 \times 10^{6}$ & 140 & $1.4 \times 10^{5}$ \\
3 & $8.4 \times 10^{6}$ & 1200 & $1.2 \times 10^{6}$ & 19 & $1.9 \times 10^{4}$ \\
4 & 20 & $8.7 \times 10^{4}$ & 8.4 & $8.4 \times 10^{3}$ & 0.3 & $3.0 \times 10^{2}$ \\
5 & 4.3 & $4.0 \times 10^{4}$ & 1.7 & $1.7 \times 10^{3}$ & $3.4 \times 10^{-2}$ & 34 \\
\hline
\end{tabular}

TOF Trigger backgrounds The UPC signal is characterized by a few tracks in an otherwise empty detector. The most important trigger background is the fake trigger rate (FTR) due to spurious hits in the TOF. The main source of FTR for the TOF L0 trigger is the MRPC noise, measured to be $0.5 \mathrm{~Hz} / \mathrm{cm}^{2}$. The MRPC noise is due to ionizing particles in the chamber. The fraction of MRPC noise from the front-end electronics is just few percent, measured by switching off the MRPC high voltage. The main source of background noise in the TOF during ALICE operation are beam-gas collisions, beam misinjection and neutrons from $\mathrm{Pb}+\mathrm{Pb}$ interactions. To be conservative, we assume an MRPC noise level of $2.5 \mathrm{~Hz} / \mathrm{cm}^{2}$, a factor 5 larger than the measured value.

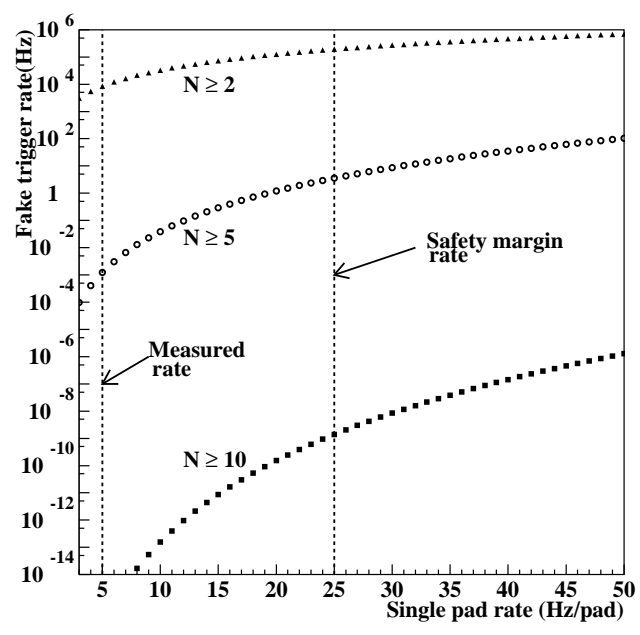

Figure 118. The L0 FTR as a function of the single pad rate. Reprinted from Ref. [318] with permission from Institute of Physics.

Figure 118 shows the FTR as a function of the rate in a single TOF pad. The FTR is $\simeq 1 \mathrm{~Hz}$ when five or more pads are fired, $N_{\text {pad }} \geq 5$ while the FTR for $N_{\text {pad }}=2$ is 200 


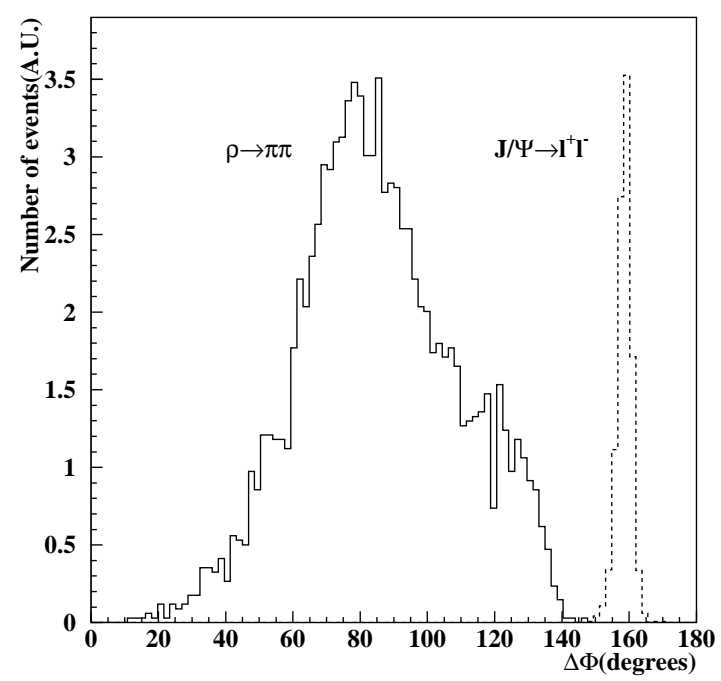

Figure 119. The azimuthal difference between the $J / \psi$ and $\rho^{0}$ decay products. Reprinted from Ref. [318] with permission from Institute of Physics.

$\mathrm{kHz}$. Such high rate, unmanageable at L0, can be further reduced by using the vector meson decay topology. The $J / \psi \rightarrow l^{+} l-$ and $\rho^{0} \rightarrow \pi \pi$ decays were simulated using STARLIGHT $[72,83,119]$. The decay products were tracked through the TPC volume in a $0.5 \mathrm{~T}$ magnetic field without any secondary interactions or multiple scattering effects on the track direction. Efficiencies for containing both decay products in the volume of $\epsilon_{\mathrm{cont}}^{J / \psi}=16.7 \%$ and $\epsilon_{\mathrm{cont}}^{\rho}=8.3 \%$ respectively were found. Figure 119 shows the distribution of the azimuthal opening between the two decay products, $\Delta \varphi$, in the plane orthogonal to the beam axis. Although smeared by the magnetic field, a clear topology is still evident. The FTR can be reduced by a factor $f_{\text {top }}$ using the $\Delta \varphi$ distribution, resulting in an additional "efficiency", $\epsilon_{\phi}$, for detecting the decay products. The $J / \psi$ decay FTR can be reduced by $f_{\text {top }}=18$ by selecting only pairs of pads (one for each decay product) within a $150^{\circ} \leq \Delta \varphi \leq 170^{\circ}$ window with $\epsilon_{\phi}=1$. The $\rho^{0}$ FTR can be reduced by $f_{\text {top }}=9$ by selecting only pairs of pads in a $70^{\circ} \leq \Delta \varphi \leq 110^{\circ}$ window with $\epsilon_{\phi}=0.6$ for the $\rho^{0}$ signal.

Further FTR reduction can be obtained for both decays by synchronizing the ITS readout with the beam bunches. This is possible because the LHC accelerator timing, trigger and control (TTC) system distributes fast timing signals from the RF generators and synchronous with the circulating beams to all experiments. The bunch clock frequency, $40 \mathrm{MHz}$, is the same in proton and ion modes even though the ion bunch spacing is $125 \mathrm{~ns}$. Since the ITS readout has a $20 \mathrm{~ns}$ duration, it can read out five times between bunches. By aligning the readout with the beam bunches and the bunch clock while vetoing the next four readout pulses, the noise can be reduced by an additional factor of five for each track, a factor of 25 reduction in the combinatorial background for $N_{\text {cell }}=2$. Then the L0 FTR is less than $200 / 25 f_{\text {top }} \mathrm{kHz}$ for the two 
vector meson decays so that

$$
\begin{aligned}
& J / \psi \mathrm{FTR}<440 \mathrm{~Hz} \\
& \rho^{0} \mathrm{FTR}<880 \mathrm{~Hz} .
\end{aligned}
$$

This L0 FTR should be compared to the $J / \psi$ and $\rho^{0}$ signal rates,

$$
\begin{aligned}
J / \psi \text { Rate } & =L_{\mathrm{PbPb}} B\left(J / \psi \rightarrow l^{+} l^{-}\right) \sigma_{J / \psi} \epsilon_{\mathrm{cont}}^{J / \psi} \\
& =0.5 \mathrm{mb}^{-1} \mathrm{~Hz} \times 0.12 \times 32 \mathrm{mb} \times 0.167=0.32 \mathrm{~Hz} \\
\rho^{0} \text { Rate } & =L_{\mathrm{PbPb}} \sigma_{\rho} \epsilon_{\mathrm{cont}}^{\rho} \epsilon_{\phi} \\
& =0.5 \mathrm{mb}^{-1} \mathrm{~Hz} \times 5200 \mathrm{mb} \times 0.083 \times 0.6=120 \mathrm{~Hz} .
\end{aligned}
$$

Note that the $J / \psi \rightarrow l^{+} l^{-}$branching ratio in the $J / \psi$ rate is the sum of the branching ratios in the electron and muon decay channels.

Thus the TOF can tag vector meson decays at L0. Detailed studies of varies ultraperipheral processes are underway.

TPC trigger backgrounds Experience from RHIC shows that coherent events can be identified with good signal to background ratios when the entire event is reconstructed and a cut is applied on the summed transverse momentum of the event. The incoherent background can be estimated by reconstructing events with two same-sign tracks, e.g. $\pi^{+} \pi^{+}$or $\pi^{-} \pi^{-}$for $\rho^{0} \rightarrow \pi^{+} \pi^{-}[62]$. The main heavy vector meson background will most likely be lepton pairs produced in two-photon interactions. Since these pairs are produced coherently, they are not rejected by a pair $p_{T}$ cut $[72,83,119]$.

The following TPC background sources have been investigated: peripheral $A A$ interactions; incoherent $\gamma A$ interactions and cosmic ray muons. These same sources were also considered in a STAR study [298].

The trigger contribution from cosmic ray muons was not negligible in STAR since the scintillator counters used in the central trigger barrel surrounded the TPC and covered a large area. Measurements from L3+Cosmics, which also used scintillators surrounding a large volume (the L3 magnet), observed a cosmic ray muon rate five times lower than that calculated for STAR because L3 is about $100 \mathrm{~m}$ underground. In STAR, the cosmic ray trigger rate was reduced by a topology cut on the zenith angle. If a silicon pixel detector is used for triggering, the area susceptible to cosmic ray muon triggers is greatly reduced since at least one of the tracks will point to the vertex.

Peripheral $A A$ interactions have been studied using 5000 events with $13<b<20$ fm generated by FRITIOF 7.02 [391]. The inelastic $\mathrm{Pb}+\mathrm{Pb}$ cross section for this range is $2.1 \mathrm{~b}$, corresponding to about $25 \%$ of the $8 \mathrm{~b}$ total inelastic cross section. Of these 5000 events, 435 (9\%) had between one and five charged tracks in the TPC. A subset of these, 97 events ( $2 \%$ of the original 5000) had two charged tracks in the TPC. The cross section for exactly two charged tracks in the TPC is then $0.02 \times 2.1 \mathrm{~b}=40 \mathrm{mb}$, an order of magnitude lower than the $\rho^{0}$ photoproduction cross section. In addition, the summed $p_{T}$ distribution for two charged tracks is peaked at higher values in the background events than in the signal events. 
Incoherent photonuclear interactions might be an important background at the trigger level, and at the analysis level for inclusive events. Direct $\gamma$-parton interactions are only a small fraction of the total $\gamma A$ cross section. The bulk of the vector meson cross section can be described by generalized vector meson dominance, see Section 2.3.1. Since the virtual photon energy spectrum has a peak much lower than the beam energy, these interactions resemble interactions between the beam nucleus and a hadron nearly at rest. However, these photonuclear events have a much broader $p_{T}$ distribution than that of coherent $\rho^{0}$ and $J / \psi$ production.

The total photonuclear cross section can be calculated by integrating over the virtual photon energy spectrum,

$$
\sigma_{\gamma A}^{\mathrm{tot}}=2 \int_{k_{\min }}^{\infty} d k \frac{d N_{\gamma}}{d k} \sigma_{\gamma A}(k) .
$$

The factor of two arises because each nucleus can act as both photon emitter and target. With a minimum photon energy cutoff of $k_{\text {min }}=10 \mathrm{GeV}$ in the rest frame of the target nucleus and assuming that $\sigma_{\gamma \mathrm{Pb}}(k)$ is independent of photon energy, $\sigma_{\gamma \mathrm{Pb}}=15 \mathrm{mb}$ [392], $\sigma_{\gamma A}^{\text {tot }}=44 \mathrm{~b}$. The DTUNUC 2.2 event generator [392] was used to simulate $50000 \gamma \mathrm{Pb}$ events. Of these, 1595 (3\%) left one to five charged tracks in the TPC, a cross section of $44 \mathrm{~b} \times 0.03=1.4 \mathrm{~b}$, larger than the peripheral $A A$ cross section fulfilling the same criteria.

Thus the TPC backgrounds appear to be under control and we conclude that $\rho^{0}$ and $J / \psi$ photoproduction can be triggered on without being swamped by background.

\subsection{The ATLAS detector}

\section{Contributed by: S. N. White}

The ATLAS detector is designed to study $14 \mathrm{TeV} p p$ collisions. The physics pursued by the collaboration is vast and includes Higgs boson and SUSY searches and other scenarios beyond the Standard Model. To achieve these goals at a full machine luminosity of $10^{34} \mathrm{~cm}^{-2} \mathrm{~s}^{-1}$, the calorimeter is designed to be as hermetic as possible and has extremely fine segmentation. The detector, shown in Fig. 120, is a combination of three subsystems: the inner tracking system, the electromagnetic and hadronic calorimeters and a full coverage muon detector. The inner tracker is composed of a finely-segmented silicon pixel detector; a semiconductor tracker (SCT) and the transition radiation tracker (TRT). The segmentation is optimized for $p p$ collisions at design luminosity.

The ATLAS calorimeters are divided into electromagnetic and hadronic sections and cover $|\eta|<4.9$. The EM calorimeter is an accordion liquid argon device, finely segmented longitudinally (lines of constant $\eta$ ) and transversely (in $\phi$ ) over $|\eta|<3.1$.

The ATLAS electromagnetic calorimeter has three longitudinally-segmented

sections. The first is closest to the beam pipe while the third is furthest away. The first longitudinally-segmented section has granularity $\Delta \eta \times \Delta \phi=0.003 \times 0.1$ in the barrel and is slightly coarser in the endcaps. Note that $\Delta \phi$ is larger in the first 
longitudinally-segmented section because the showers spread more in $\phi$ here. The second longitudinally-segmented section is composed of $\Delta \eta \times \Delta \phi=0.025 \times 0.025$ cells while the last segmented section has $\Delta \eta \times \Delta \phi=0.05 \times 0.05$ cells. In addition, a finely segmented, $\Delta \eta \times \Delta \phi=0.025 \times 0.1$, pre-sampler system is placed in front of the electromagnetic calorimeter. The overall energy resolution of the electromagnetic calorimeter is $10 \% / E^{1 / 2}+0.5 \%$. The calorimeter also has good pointing resolution, $60 / E^{1 / 2} \mathrm{mrad}$, for photons and better than $200 \mathrm{ps}$ timing resolution for showers with $E>20 \mathrm{GeV}$.

The hadronic calorimeter is also segmented longitudinally and transversely. The barrel calorimeter is a lead scintillator tile structure with a granularity of $\Delta \eta \times \Delta \phi=$ $0.1 \times 0.1$. In the endcaps, liquid argon technology is used for radiation hardness. The granularity in the endcaps is the same as in the barrel. The energy resolution is $50 \% / E^{1 / 2}+2 \%$ for pions.

The very forward region, up to $|\eta|<4.9$, is covered by the Forward Calorimeter, an axial-drift liquid argon calorimeter [393]. The ATLAS muon spectrometer is located behind the calorimeters, shielded from hadronic showers. The spectrometer uses several tracking devices and a toroidal magnet system. Most of the volume is covered by monitored drift tubes. In the forward region, where the rate is high, cathode strip chambers are used. The standalone muon momentum resolution is $\sim 2 \%$ for muons with $10<p_{T}<100 \mathrm{GeV} / c$. The performance of each subsystem is summarized in Ref. [94].

\subsection{The CMS detector}

Contributed by: D. d'Enterria and P. Yepes

The CMS detector is designed to identify and precisely measure muons, electrons, photons and jets over a large energy and rapidity range. A detailed description of detector elements can be found in the Technical Design Reports [123,394-397]. An overall view of one quadrant of the detector is shown in Fig. 121. The central element of CMS is the magnet, a $13 \mathrm{~m}$ long, $6 \mathrm{~m}$ diameter, high-field solenoid with an internal radius of $\sim 3 \mathrm{~m}$, providing a uniform $4 \mathrm{~T}$ magnetic field. The tracking system, electromagnetic, and hadronic calorimeters are positioned inside the magnet, while the muon detector is outside. The tracker covers the pseudorapidity region $|\eta|<2.4$ while the electromagnetic and hadronic calorimeters cover $|\eta|<3$ and $|\eta|<5$ respectively. The complete CMS geometry is included in the detailed GEANT-4 based simulation package, CMSSW [123].

Tracker Starting from the beam axis, the tracker $(|\eta|<2.4)$ is composed of two different types of detectors: pixel layers and silicon strip counters. The pixel detector is composed of 3 barrel layers which are located at $4.5 \mathrm{~cm}, 7.5 \mathrm{~cm}$ and $10 \mathrm{~cm}$ from the beam axis and 2 endcap disks in each of the forward and backward directions with a possibility of a third set of disks to be added later. The barrel layers, covering rapidities 


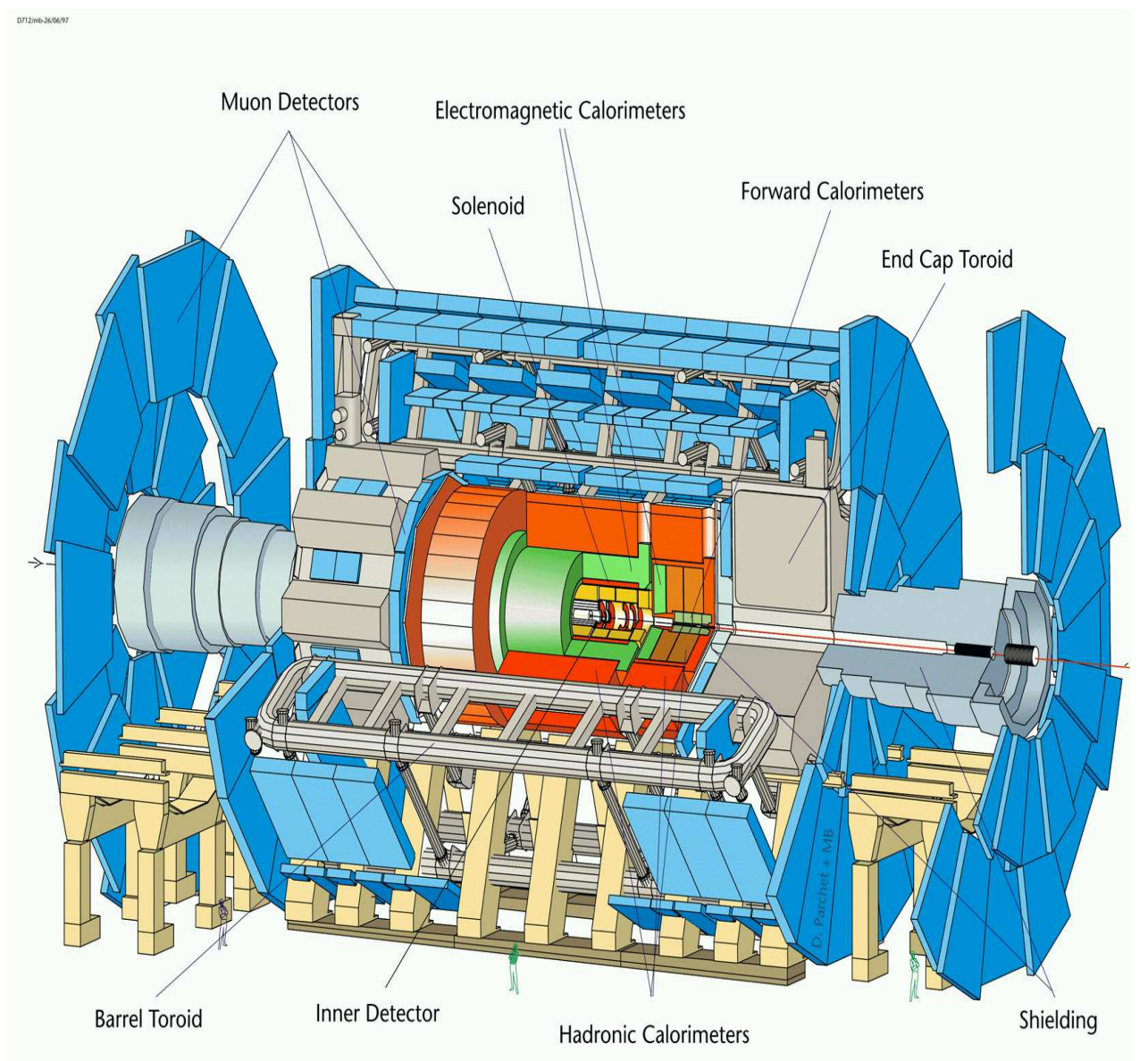

Figure 120. The ATLAS Detector.

up to $|\eta|=2.1$, are made of more than $9.6 \times 10^{6}, 1.6 \times 10^{7}$ and $2.24 \times 10^{7}$ pixels for the inner, middle and outer layers respectively, with pixel dimensions of $100 \times 150 \mu \mathrm{m}^{2}$. The inner Si strip counter consists of 4 cylindrical layers in the barrel and 3 mini-disks in the endcap. The detectors have $80 \mu \mathrm{m}$ pitch and a strip length of $6.1 \mathrm{~cm}$.

Electromagnetic Calorimeter The electromagnetic calorimeter, ECAL, is composed of $\sim 83000$ scintillating $\mathrm{PbWO}_{4}$ crystals. The barrel part of the ECAL covers the pseudorapidity range $|\eta|<1.479$. The front face of the crystals is at $1.29 \mathrm{~m}$ radius. Each crystal has a square cross section of $22 \times 22 \mathrm{~mm}^{2}$ and a length of $230 \mathrm{~mm}$, corresponding to $25.8 X_{0}$. The crystal cross section corresponds to $\Delta \eta \times \Delta \phi=0.0175 \times 0.0175$ in the barrel region. The endcap crystal calorimeter covers the pseudorapidity range $1.48<|\eta|<3$. A pre-shower is located in front of the endcap crystal calorimeter covering the pseudorapidity range $1.5<|\eta|<2.5$.

Hadronic Calorimeter The hadronic calorimeter has two parts: a central section, HB and HE, covering $|\eta|<3$ and a forward/backward section, HF, covering $3<|\eta|<5$. 


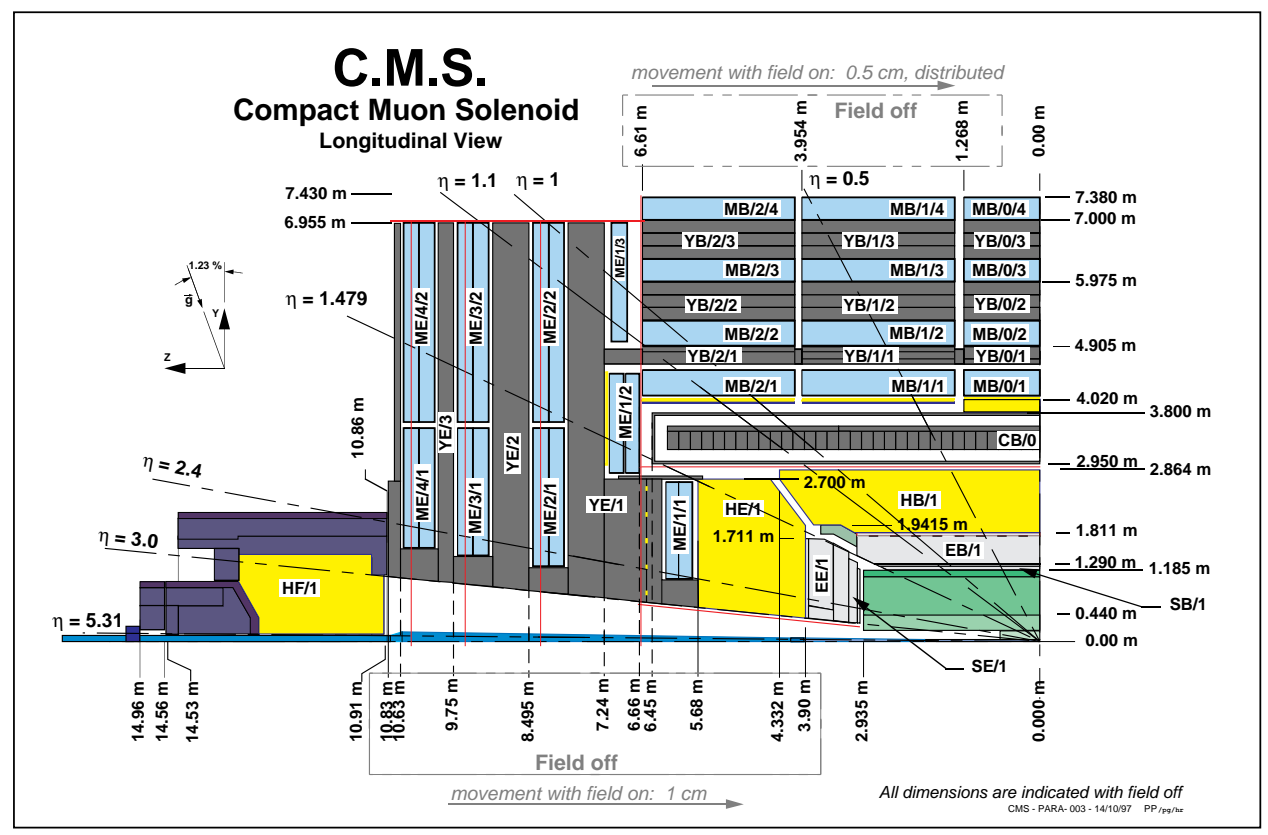

Figure 121. The CMS detector: a longitudinal view [123].

The central calorimeter consists of the hadron barrel, HB, and hadron endcap, HE, both located inside the CMS magnet cryostat. It is a sampling calorimeter made of scintillator/copper plates. The forward calorimeter is located $6 \mathrm{~m}$ downstream of the HE endcaps. The granularity is $\Delta \eta \times \Delta \phi \sim 0.087 \times 0.087$.

Muon system The muon system is composed of drift tubes in the barrel region, MB, $|\eta|<1.3$; cathode strip chambers in the endcap regions, ME, $0.9<|\eta|<2.4$ and resistive plate chambers in both barrel and endcap, covering $|\eta|<2.1$, dedicated to triggering. All the muon chambers are positioned approximately perpendicular to the muon trajectories and cover the pseudorapidity range $|\eta|<2.4$.

Trigger and Data Acquisition The trigger and data acquisition system of a collider experiment plays an important role because the collision frequency and overall data rates are much larger than the rate at which events can be written to mass storage. Events seen by the detector are inspected online and only those with potentially interesting physics are selected for further offline reconstruction.

In CMS, this online inspection of events is divided into two steps. The first step, the Level-1 Trigger (L1), brings the data flow below 100 GBytes/s, while the second step or High-Level Trigger, HLT, further reduces the data rate to 100 MBytes/s.

The L1 trigger is on raw quantities in the calorimeters and muon detectors. It can select jets, electron, photons, and muons with transverse momenta above certain thresholds. On the other hand, the HLT has access to information from all detectors, identical to that available in the offline analysis. 
Triggering in UPCs, characterized by very low particle multiplicities depositing a relatively small amount of energy in the central part of the detector, is done by requiring a neutron signal in one of the ZDCs in combination with at least one large rapidity gap between the produced system and the beam rapidity. Such a trigger will select UPCs with nuclear breakup. Even if the rates are high due to backgrounds, they can be reduced in the HLT where the full event information is available for detailed analysis, see Section 2.7.3.

\section{Conclusions}

Ultraperipheral collisions at the LHC can provide a new means of studying small $x$ physics, continuing along the road pioneered at HERA in the last decade. The rates and collision energies for many inclusive and diffractive hard phenomena will be high enough to extend the HERA studies to a factor of ten lower $x$ and, for the first time, explore hard phenomena at small $x$ with nuclei in the same $x$ range as the proton. This larger reach in $x$ will explore a kinematic range where nonlinear effects should be much larger than at HERA and the leading-twist approximation should break down. It would then be possible to test various theoretical predictions for the new high gluon density QCD regime such as parton saturation and the physics of the black disk regime. Except for measurements of the parton distributions, none of the information accessible in UPCs will be available from the other studies at the LHC. UPC photons allow smaller virtualities to be probed than hadronic collisions so that larger nonlinear effects on the parton distributions could be measured at the same $x$. In addition, photons are cleaner probes than hadrons, simplifying the interpretation of UPC data compared to partonparton interactions in hadron collisions.

UPC studies require good particle tracking over a large solid angle combined, for many analyses, with good particle identification and selective triggers able to select fewparticle final states with specified topologies. All of the LHC detectors are well suited to this task with large usable solid angles and various forms of particle identification. As amply demonstrated at RHIC, triggering is a bigger challenge. However, the particlephysics style, multi-level triggers seem up to this challenge. Triggers and analyses for several key benchmark processes were presented here, showing that the detectors are able to collect and analyze UPC events.

\section{Acknowledgments}

We thank C. Bertulani, D. Brandt, U. Dreyer, J. M. Jowett, V. Serbo, D. Trautmann and C. Weiss for helpful discussions. This work was supported in part by the US Department of Energy, Contract Numbers DE-AC02-05CH11231 (S. R. Klein and R. Vogt); W7405-Eng-48 (R. Vogt); DE-FG02-93ER40771 (M. Strikman); DE-AC02-98CH10886 (A. J. Baltz and S. N. White); and DE-FG03-93ER40772 (P. Yepes). The work of R. Vogt was also supported in part by the National Science Foundation Grant NSF PHY- 
0555660. D. d'Enterria acknowledges support by the 6th EU Framework Programme contract MEIF-CT-2005-025073. V. Nikulin and M. Zhalov would like to express their acknowledgment for support by CERN-INTAS grant no 05-103-7484 .

\section{References}

[1] Fermi E 1924 Nuovo Cimento 2 143, translated by Gallinaro M and White S N 2001, in proceedings of the Workshop on Electromagnetic Probes of Fundamental Physics, Erice, Sicily, 2001 Preprint hep-th/0205086; Fermi E 1924 Z. Physik 29315

[2] von Weizsäcker C 1934 Z. Physik 88 612; Williams E J 1934 Phys. Rev. 45729

[3] Baur G, Hencken K, Trautmann D, Sadovsky S and Kharlov Yu 2002 Phys. Rep. 364359

[4] Bertulani C A and Baur G 1988 Phys. Rep. 163299

[5] Khoze V A, Martin A D, and Ryskin M G 2002 Eur. Phys. J C 23311

[6] Holt R et al. 2002 Preprint BNL-68933

[7] Deshpande A et al. 2005 Ann. Rev. Nucl. Part. Sci. 55165

[8] Jackson J D 1975 Classical Electrodynamics 2nd Ed., John Wiley \& Sons, New York

[9] Cahn R N and Jackson J D 1990 Phys. Rev. D 423690

[10] Baur G and Ferreira Filho L G 1990 Nucl. Phys. A 518786

[11] Brüning et al 2004 Preprint CERN-2004-003-V1

[12] Jowett J M and Carli C 2006 EPAC06 550 Preprint CERN-LHC-PROJECT-REPORT-928

[13] Brandt D, Eggert K, and Morsch A 1994 Preprint CERN AT/94-05(DI)

[14] Ohnemus J, Walsh T F, and Zerwas P M 1994 Phys. Lett. B 328369

[15] Drees M, J. Ellis J, and Zeppenfeld D 1989 Phys. Lett. B 223454

[16] Krauss F, Greiner M and Soff G 1997 Prog. Nucl. Part. Phys. 39503

[17] Bertulani C, Klein S R and Nystrand J 2005 Ann. Rev. Nucl. Part. Sci. 55271

[18] Ageev A et al. 2002 J. Phys. G 28 R117

[19] Baur G et al 2002 Preprint hep-ex/0201034

[20] Marciano W and White S 2003 Electromagnetic Probes of Fundamental Physics World Scientific, Singapore

[21] Butterworth J M and Wing M 2005 Rept. Prog. Phys. 682773

[22] Frankfurt L, Strikman M and Weiss C 2005 Ann. Rev. Nucl. Part. Sci. 55403

[23] Brodsky S J, Frankfurt L, Gunion J F, Mueller A H and Strikman M 1994 Phys. Rev. D 503134

[24] Collins J C, Frankfurt L and Strikman M 1997 Phys. Rev. D 562982

[25] Blaettel B, Baym G, Frankfurt L and Strikman M 1993 Phys. Rev. Lett. 70896

[26] Frankfurt L, Miller G A and Strikman M 1993 Phys. Lett. B 3041

[27] Frankfurt L, Radyushkin A and Strikman M 1997 Phys. Rev. D 5598

[28] Arneodo M, Bialas A, Krasny M W, Sloan T and Strikman M 1996 Preprint hep-ph/9610423

[29] Albrow M et al. [CMS and TOTEM Collaborations] 2006 Preprint CERN/LHCC 2006-039/G-124

[30] d'Enterria D 2007 Eur. Phys. J. A 31816

[31] Strikman M, Vogt R and White S 2006 Phys. Rev. Lett. 96082001

[32] Albrow A G et al. 2005 Preprint CERN-LHCC-2005-025, LHCC-I-015

[33] Frankfurt L, McDermott M F and Strikman M 1999 JHEP 9902002

[34] Martin A D, Ryskin M G and Teubner T 1999 Phys. Lett. B 454339

[35] Alexopoulos T et al. 2003 Preprint H1-04-03-609

[36] Jowett J M, Bruce R, Gilardoni S S, Drees A, Fischer W, Tepikian S and Klein S 2006 Preprint CERN-AB-2006-046

[37] Beole $\mathrm{S}$ et al. [ALICE Collaboration], Preprint CERN-LHCC-98-19

[38] Aitala E M et al. [E791 Collaboration], 2001 Phys. Rev. Lett. 864773

[39] Sokoloff M D et al. 1986 Phys. Rev. Lett. 573003

[40] Frankfurt L and Strikman M 1999 Eur. Phys. J. A 5293 
[41] McLerran L D and Venugopalan R 1994 Phys. Rev. D 502225

[42] Mueller A H 1988 Nucl. Phys. B 30734

[43] Kovchegov Y V 1997 Phys. Rev. D 555445

[44] Frankfurt L, Guzey V, McDermott M and Strikman M 2001 Phys. Rev. Lett. 87192301

[45] Frankfurt L and Strikman M 1989 Phys. Rev. Lett. 63 1914; [Erratum ibid 199064 815]

[46] Ryskin M G 1993 Z. Phys. C 5789

[47] Low F E 1975 Phys. Rev. D 12163

[48] Frankfurt L, Koepf W and Strikman M 1996 Phys. Rev. D 54 3194; 199857512

[49] Frankfurt L, Guzey V and Strikman M 2001 J. Phys. G 27 R23

[50] Radyushkin A V 2001 Preprint hep-ph/0101225

[51] Freund A and McDermott M 2002 Phys. Rev. D 65074008 and references therein

[52] Gribov V N 1969 Sov. Phys. JETP 29483 [Zh. Eksp. Tor. Fiz. 56 892]

[53] Gribov V N 1969 Sov. J. Nucl. Phys. 9 369; 1970 Sov. Phys. JETP 30709

[54] Collins J C 1998 Phys. Rev. D 573051 [Erratum-ibid. 2000 D 61 019902]

[55] Adloff C et al. [H1 Collaboration] 2001 Eur. Phys. J. C 2029

[56] Aktas A et al. [H1 Collaboration] 2006 Preprint hep-ex/0606004

[57] Aktas A et al. [H1 Collaboration] 2006 Preprint hep-ex/0606003

[58] Frankfurt L, Guzey V and Strikman M 2005 Phys. Rev. D 71054001

[59] Feinberg E L and Pomeranchuk I 1956 Suppl. Nuovo Cimento III 562

[60] Frankfurt L, Guzey V and Strikman M 2003 Preprint hep-ph/0303022v1

[61] Frankfurt L and Strikman M 2002 Phys. Rev. D 66031502

[62] Adler C et al. [STAR Collaboration] 2002 Phys. Rev. Lett. 89272302

[63] d'Enterria D 2006 Preprint nucl-ex/0601001

[64] Gribov V N 1969 Zh. Eksp. Teor. Fiz. 571306

[65] Brodsky S J and Pumplin J 1969 Phys. Rev. 1821794

[66] Donnachie A and Shaw G 1978 Electromagnetic Interactions Of Hadrons vol 2 (Plenum Press) p 169

[67] Fraas H, Read B and Schildknecht D 1975 Nucl. Phys. B 86 346; Ditsas P and Shaw G 1976 Nucl. Phys. B 113246

[68] Bauer T H, Spital R D, Yennie D R and Pipkin F M 1978 Rev. Mod. Phys. 50261 [Erratum-ibid. 197951 407]

[69] Pautz A and Shaw G 1988 Phys. Rev. C 572648

[70] Frankfurt L, Strikman M and Zhalov M 2003 Acta Phys. Polon. B 343215

[71] Alvensleben H et al. 1970 Nucl. Phys. B 18 333; McClellan G et al. 1971 Phys. Rev. D 42683

[72] Klein S and Nystrand J 1999 Phys. Rev. C 60014903

[73] Drell S and Trefil J S 1966 Phys. Rev. Lett. 16552 [Erratum-ibid. 196616 832]

[74] Klein S R and Nystrand J 2004 Phys. Rev. Lett. 92142003

[75] Klein SR and Nystrand J 2003 Preprint hep-ph/0310223

[76] Frankfurt L, Strikman M and Zhalov M 2002 Phys. Lett. B 53751

[77] Frankfurt L, Strikman M and Zhalov M 2003 Phys. Rev. C 67034901

[78] Klein S R and Nystrand J 2000 Phys. Rev. Lett. 842330

[79] Ryskin M G, Roberts R G, Martin A D and Levin E M 1997 Z. Phys. C 76231

[80] Frankfurt L, Guzey V, Strikman M and Zhalov M 2003 JHEP 0308043

[81] Frankfurt L, Strikman M and Zhalov M 2002 Phys. Lett. B 540220

[82] Drees M and Zeppenfeld D 1989 Phys. Rev. D 392536

[83] Baltz A J, Klein S R and Nystrand J 2002 Phys. Rev. Lett. 89012301

[84] Baur G, Hencken K, Aste A, Trautmann D and Klein S R 2003 Nucl. Phys. A 729787

[85] White S N 2005 AIP Conf. Proc. 792 527, Preprint nucl-ex/0507023

[86] Baltz A J, Rhoades-Brown M J and Weneser J 1996 Phys. Rev. E 544233

[87] Veyssiere A et al. 1970 Nucl. Phys. A 159561

[88] Baltz A J, Chasman C and White S N 1998 Nucl. Instrum. Meth. A 4171 
[89] Hencken K, Baur G and Trautmann D 2006 Phys. Rev. Lett. 96012303

[90] Klein S R [STAR Collaboration] 2004 Preprint nucl-ex/0402007

[91] Klein S R [STAR Collaboration] 2003 Preprint nucl-ex/0310020

[92] Klein S R and Nystrand J 2003 Phys. Lett. A 308323

[93] Frankfurt L, Strikman M and Zhalov M Preprint hep-ph/0612072

[94] Individual Detector TDRs can be found at http://atlasinfo.cern.ch/ATLAS/internal/tdr.html

[95] Strikman M, Tverswkoy M and Zhalov M 2005 Phys. Lett. B 62672

[96] Aktas A et al [H1 Collaboration] 2006 Eur. Phys. J. C 48 715; 749; Preprint hep-ex/0610076

[97] Nikulin V and Zhalov M 2003 Preprint ALICE-INT-2003-043

[98] Rogers T, Guzey V, Strikman M and Zu X 2004 Phys. Rev. D 69074011

[99] Donnachie S, Dosch G, Nachtmann O and Landshoff P 2002 Camb. Monogr. Part. Phys. Nucl. Phys. Cosmol. 191

[100] Dersch U et al. [SELEX Collaboration] 2000 Nucl. Phys. B 579277

[101] Lipkin H 1975 Phys. Rev. D 111827

[102] Donnachie A and Landshoff P V 2000 Phys. Lett. B 478146

[103] Olsson J 2006 Preprint hep-ex/0610077

[104] Derrick M et al. [ZEUS Collaboration] 1995 Z. Phys. C 6939

[105] Strikman M, Tverskoy M G and Zhalov M B 1999 Phys. Lett. B 45937

[106] Adams M R et al. [E665 Collaboration] 1995 Phys. Rev. Lett. 745198 [Erratum-ibid. 199880 2020]

[107] Frankfurt L, Gerland L, Strikman M and Zhalov M 2003 Phys. Rev. C 68044602

[108] Vidovic M, Greiner M and Soff G 1993 Phys. Rev. C 482011

[109] Pshenichnov I A, Bondorf J P, Mishustin I N, Ventura A and Masetti S 2001 Phys. Rev. C 64 024903

[110] Chiu M, Denisov A, Garcia E, Katzy J and White S 2002 Phys. Rev. Lett. 89012302

[111] Golubeva M B et al. 2005 Phys. Rev. C 71024905

[112] Pshenichnov I 2003 Preprint nucl-th/0306016

[113] Pshenichnov I A, Bondorf J P, Kurepin A B, Mishustin I N, Ventura A 2002 Preprint ALICEINT-2002-07

[114] http://agenda.cern.ch/fullAgenda.php?ida=a021989

[115] ALICE Collaboration 1999 Preprint CERN LHCC 99-22, ALICE TDR 5; 2000 Preprint CERN LHCC 2000-046, Addendum 1 to ALICE TDR 5

[116] AliRoot http://www1.cern.ch/ALICE/Projects/offline/aliroot/Welcome.html

[117] Aktas A et al. [H1 Collaboration] 2006 Phys. Lett. B 638422

[118] Frankfurt L, Strikman M and Zhalov 2006 Phys. Lett. B 640162

[119] Nystrand J 2005 Nucl. Phys. A 752 470c

[120] Adams J et al. [STAR Collaboration] 2004 Phys. Rev. C 70031902

[121] Vogt R 2003 Heavy Ion Phys. 1811

[122] Grachov O A et al. 2006 Preprint nucl-ex/0608052

[123] CMS Collaboration 2006 "CMS Physics Technical Design Report: Detector performance and software" Preprint CERN-LHCC-2006-001

[124] Goncalves V P and Machado M V T 2006 Preprint hep-ph/0601131

[125] d'Enterria D 2004 J. Phys. G 30 S767

[126] Bocian D and Piotrzkowski K 2004 Acta Phys. Polon. B 352417

[127] Krasny M W, Chwastowski J and Slowikowski K 2006 Preprint hep-ex/0610052

[128] Yao W M et al. [Particle Data Group] 2006 J. Phys. G 331

[129] Adler S S et al. [PHENIX Collaboration] 2003 Phys. Rev. Lett. 91072301

[130] Sjöstrand T 1994 Computer Phys. Commun. 8274

[131] Ballintijn M, Loizides C and Roland G Preprint CMS AN-2006/099

[132] d'Enterria D and Hees A 2006 Preprint CMS AN-2006/107

[133] d'Enterria D 2007 Preprint hep-ex/0703024 
[134] Piotrzkowski K 2001 Phys. Rev. D 63071502

[135] Good M and Walker W 1960 Phys. Rev. D 1201857

[136] Frankfurt L, Miller G A and Strikman M 1993 Phys. Rev. Lett. 712859

[137] Kaidalov A B, Khoze V A, Martin A D and Ryskin M G 2003 Acta Phys. Polon. B 343163

[138] Abramovski V A, Gribov V N and Kancheli O V 1973 Yad. Fiz. 18595

[139] Frankfurt L and Strikman M 1996 Phys. Lett. B 3826

[140] Frankfurt L, Guzey V and Strikman M 2004 Phys. Lett. B 58641

[141] Klein S R, Nystrand J and Vogt R 2002 Phys. Rev. C 66044906

[142] Derrick M et al. [ZEUS Collaboration] 1995 Phys. Lett. B 356129

[143] Frankfurt L and Strikman M 1996 Proc. Workshop on Future Physics at HERA (Hamburg) 949 Preprint hep-ph/9609456

[144] Brodsky S J and Gillespie J 1968 Phys. Rev. 1731011

[145] Diehl M 1995 Z. Phys. C 66181

[146] Goulianos K [CDF Collaboration] 2002 Acta Phys. Polon. B 333467

[147] Frankfurt L and Strikman M 2006 Preprint nucl-th/0603049

[148] Bjorken J D 1992 Phys. Rev. D 47101

[149] Mueller A H and Tang W K 1992 Phys. Lett. B 284123

[150] Del Duca V and Tang W K 1993 Phys. Lett. B 312225

[151] Abe F et al. [CDF Collaboration] 1998 Phys. Rev. Lett. 815278

[152] Abramowicz H, Frankfurt L and Strikman M 1994 Proc. SLAC Summer Inst. p 539; 1997 Surv. High Energy Phys. 1151

[153] Forshaw J R and Ryskin M G 1995 Z. Phys. C 68137

[154] Bartels J et al. 1996 Phys. Lett. B 375301

[155] Derrick M et al. [ZEUS Collaboration] 1996 Phys. Lett. B 36955

[156] Adloff C et al. [H1 Collaboration] 2002 Eur. Phys. J. C 24517

[157] Chekanov S et al. [ZEUS Collaboration] 2003 Eur. Phys. J. C 26389

[158] Aktas A et al. [H1 Collaboration] 2003 Phys. Lett. B 568205

[159] Kuraev E A, Lipatov L N, and Fadin V S 1976 Sov. Phys. JETP 44 443; 197745 199; Balitsky I I and Lipatov L N 1978 Sov. J. Nucl. Phys. 28822

[160] Lipatov L N 1989 private communication

[161] Colferai D 2000 Preprint hep-ph/0008309

[162] Salam G P 2005 Preprint hep-ph/0501097

[163] Pumplin J, Stump D R, Huston J, Lai H L, Nadolsky P and Tung W K 2002 JHEP 0207012

[164] Erhan S and Schlein P E 2000 Phys. Lett. B 481177

[165] Levy A 2005 Nucl. Phys. Proc. Suppl. 14692

[166] Ashman J et al. [EMC Collaboration] 1991 Z. Phys. C 521

[167] Mandelstam S 1963 Nuovo Cim. 301148

[168] Gribov V N 2003 The Theory of Complex Angular Momenta: Gribov Lectures on Theoretical Physics (Cambridge: Cambridge Univ. Press)

[169] Bartels J, Lipatov L and Vacca G 2005 Nucl. Phys. B 706391

[170] Blok B and Frankfurt L 2006 Preprint hep-ph/0611062

[171] Blaettel B, Baym G, Frankfurt L L, Heiselberg H and Strikman M 1993 Phys. Rev. D 472761

[172] Klein S R and Nystrand J 2000 Phys. Rev. Lett. 842330

[173] Witten E 1997 Nucl. Phys. B 120189

[174] Sjostrand T, Storrow J K and Vogt A 1996 J. Phys. G 22893

[175] Frixione S, Nason P and Ridolfi G 1995 Nucl. Phys. B 4543

[176] Fritzsch H and Streng K H 1978 Phys. Lett. B 72385

[177] Klein S R, Nystrand J and Vogt R 2001 Eur. Phys. J. C 21563

[178] Emel'yanov V, Khodinov A, Klein S R and Vogt R 1997 Phys. Rev. C 562726

[179] Emel'yanov V, Khodinov A, Klein S R and Vogt R 1998 Phys. Rev. Lett. 811801

[180] Emel'yanov V, A. Khodinov, Klein S R and Vogt R 1999 Phys. Rev. C 591860 
[181] Emel'yanov V, Khodinov A, Klein S R and Vogt R 2000 Phys. Rev. C 61044904

[182] Vogt R 2001 Phys. Rev. C 64044901

[183] Martin A D, Roberts R G, Stirling W J and Thorne R S 1998 Phys. Lett. B 443301

[184] Eskola K J, Kolhinen V J and Ruuskanen P V 1998 Nucl. Phys. B 535351

[185] Eskola K J, Kolhinen V J and Salgado C A 1999 Eur. Phys. J. C 961

[186] Gluck M, Reya E and Vogt A 1992 Z. Phys. C 53127

[187] Lai H L et al. 2000 Eur. Phys. J. C 12375

[188] Glück M, Reya E and Vogt A 1992 Phys. Rev. D 461973

[189] Glück M, Reya E and Vogt A 1992 Phys. Rev. D 453986

[190] Drees M and Grassie K 1985 Z. Phys. C 28451

[191] Abramowicz H, Charchula K and Levy A 1991 Phys. Lett. B 269458

[192] Hagiwara K, Tanaka M, Watanabe I and Izubuchi T 1995 Phys. Rev. D 513197

[193] Schuler G A and Sjostrand T 1995 Z. Phys. C 68607

[194] Schuler G A and Sjostrand T 1996 Phys. Lett. B 376193

[195] Plothow-Besch H 2000 Preprint W5051 PDFLIB, 2000.04.17, CERN-ETT/TT

[196] Bartel W et al. [JADE Collaboration] 1984 Z. Phys. C 24231

[197] Jones L M and Wyld H W 1978 Phys. Rev. D 17759

[198] Smith J and van Neerven W L 1992 Nucl. Phys. B 37436

[199] Brock R et al. [CTEQ Collaboration] 1995 Rev. Mod. Phys. 67157

[200] Vogt R 2003 Heavy Ion Phys. 1775

[201] Breitweg J et al. [ZEUS Collaboration] 1998 Eur. Phys. J. C 2247

[202] Adler C et al. [STAR Collaboration] 2003 Phys. Rev. Lett. 90082302

[203] Chiu M et al. [PHENIX Collaboration] 2003 Nucl. Phys. A 715761

[204] Blyth S L et al. 2007 J. Phys. G 34271

[205] Eskola K J and Wang X N 1995 Int. J. Mod. Phys. A 103071

[206] Eskola K J, Kajantie K and Lindfors J 1989 Nucl. Phys. B 32337

[207] Eskola K J and Kajantie K 1997 Z. Phys. C 75515

[208] Field R D 1989 Applications Of Perturbative QCD (Redwood City: Addison-Wesley)

[209] Kniehl B A, Kramer G and Potter B 2000 Nucl. Phys. B 582514

[210] Kniehl B A, Kramer G and Potter B 2001 Nucl. Phys. B 597337

[211] Zhang X F, Fai G I and Levai P 2002 Phys. Rev. Lett. 89272301

[212] Gyulassy M, Levai P and Vitev I 2002 Phys. Lett. B 538282

[213] Vitev I, Gyulassy M and Levai P 2003 Heavy Ion Phys. 17237

[214] Owens J F 1987 Rev. Mod. Phys. 59465

[215] Trzcinska A, Jastrzebski J, Lubinski P, Hartmann F J, Schmidt R von Egidy T and Klos B 2001 Phys. Rev. Lett. 87082501

[216] Frankfurt L, Strikman M and Zhalov M 2005 Phys. Lett. B 61659

[217] Weigert H 2007 Nucl. Phys. A 783 165; McLerran L 2006 Int. J. Mod. Phys. A 21694

[218] Frankfurt L, Strikman M and Weiss C 2004 Phys. Rev. D 69114010

[219] Ciafaloni M 2005 Nucl. Phys. Proc. Suppl. 146129

[220] Arsene I et al. [BRAHMS Collaboration] 2004 Phys. Rev. Lett. 93242303

[221] Kuraev E A, Lipatov L N and Fadin V S 1977 Sov. Phys. JETP 45199

[222] Balitsky I and Lipatov L N 1978 Sov. J. Nucl. Phys. 28822

[223] Gribov L V, Levin E M and Ryskin M G 1983 Phys. Rept. 1001

[224] Mueller A H 1999 Nucl. Phys. B 558285

[225] Mueller A H and Qiu J-W 1986 Nucl. Phys. B 268427

[226] Blaizot J-P and Mueller A H 1987 Nucl. Phys. B 289847

[227] Mueller A H 2003 Nucl. Phys. A 71520

[228] McLerran L D and Venugopalan R 1994 Phys. Rev. D 492233

[229] McLerran L D and Venugopalan R 1994 Phys. Rev. D 493352

[230] Jalilian-Marian J, Kovner A, Leonidov A and Weigert H 1997 Nucl. Phys. B 504415 
[231] Jalilian-Marian J, Kovner A, Leonidov A and Weigert H 1999 Phys. Rev. D 59014014

[232] Balitsky I 1996 Nucl. Phys. B 46399

[233] Kovchegov Yu V 1996 Phys. Rev. D 545463

[234] Kovchegov Yu V 2000 Phys. Rev. D 61074018

[235] Jalilian-Marian J, Kovner A, McLerran L D and Weigert H 1997 Phys. Rev. D 555414

[236] Iancu E, Leonidov A and McLerran L D 2001 Nucl. Phys. A 692583

[237] Iancu E, Leonidov A and McLerran L D 2001 Phys. Lett. B 510133

[238] Weigert H 2002 Nucl. Phys. A 703823

[239] Ferreiro E, Iancu E, Leonidov A and McLerran L D 2002 Nucl. Phys. A 703489

[240] Iancu E, Itakura K and McLerran L D 2003 Nucl. Phys. A 724181

[241] Gelis F and Peshier A 2002 Nucl. Phys. A 697879

[242] Goncalves V P and Machado M V T 2006 Preprint hep-ph/0601131

[243] Gelis F and Jalilian-Marian J 2003 Phys. Rev. D 67074019

[244] Golec-Biernat K and Wüsthoff M 1999 Phys. Rev. D 59014017

[245] Golec-Biernat K and Wüsthoff M 1999 Phys. Rev. D 60114023

[246] Bartels J, Golec-Biernat K and Kowalski H 2002 Phys. Rev. D 66014001

[247] Iancu E, Itakura K and Munier S 2003 Preprint hep-ph/0310338

[248] Gotsman E, Levin E, Lublinsky M and Maor U 2003 Eur. Phys. J. C 27411

[249] Mueller A H 1998 Eur. Phys. J. A 119

[250] Gelis F and Peshier A 2002 Nucl. Phys. A 707175

[251] Goncalves V P and Machado M V T 2003 Eur. Phys. J. C 30387

[252] Goncalves V P and Machado M V T 2003 Eur. Phys. J. C 31371

[253] Baur G et al. 1996 Phys. Lett. B 368251

[254] Abulencia A et al. [CDF Collaboration] 2007 Phys. Rev. Lett. 98112001

[255] Belkacem C et al. 1997 Phys. Rev. A 562806

[256] Vane C R et al. 1992 Phys. Rev. Lett. 691911

[257] Vane C R et al. 1994 Phys. Rev. A 502313

[258] Brandt D 2000 Preprint LHC-Project-Report 450

[259] Klein S R 2001 Nucl. Instrum. Meth. A 45951

[260] Jowett J M, Jeanneret J B and Schindl K 2003 PAC 03 Portland, OR, USA p 1682 Preprint LHC-Project-Report 642

[261] Jowett J M, Bruce R and Gilardoni S 2005 PAC 05 Knoxville, TN, USA p 1306

[262] Meier H et al. 2001 Phys. Rev. A 63032713

[263] Agger C and Sorensen A H 1997 Phys. Rev. A 55402

[264] Grafstrom P et al. 1999 PAC 99

[265] Krause F et al. 1998 Phys. Rev. Lett. 801190

[266] Fatyga M, Rhoades-Brown M J and Tannenbaum M J 1990 Preprint BNL-52247

[267] Landau L D and Lifschitz E M 1934 Phys. Z. Sowjetunion 6244

[268] Racah G 1937 Nuovo Cimento 1493

[269] Bottcher C and Strayer M R 1989 Phys. Rev. D 391330

[270] Hencken K, Trautmann D, and Baur G 1999 Phys. Rev. C 59841

[271] Alscher A, Hencken K, Trautmann D and Baur G 1997 Phys. Rev. A 55396

[272] Rumrich K, Momberger K, Soff G, Greiner W, Grun N and Scheid W 1991 Phys. Rev. Lett. 66 2613

[273] Momberger K, Grün N, and Scheid W 1991 Z. Phys. D 18133

[274] Rumrich K, Soff G and Greiner W 1993 Phys. Rev. A 47215

[275] Thiel J, Hoffstadt J, Grün N, and Scheid Q 1995 Z. Phys. D 3421

[276] Baltz A J, Rhoades-Brown M J, and Weneser J 1996 Phys. Rev. A 504842

[277] Baur G 1990 Phys. Rev. A 425736

[278] Best C, Greiner W and Soff G 1992 Phys. Rev. A 46261

[279] Hencken K, Trautmann D and Baur G 1995 Phys. Rev. A 51998 
[280] Baltz A J, Gelis F, McLerran L D and Peshier A 2001 Nucl. Phys. A 695395

[281] Aste A, Baur G, Hencken K, Trautmann D and Scharf G 2002 Eur. Phys. J. C 23545

[282] Baltz A J 1997 Phys. Rev. Lett. 781231

[283] Segev B and Wells J C 1998 Phys. Rev. A 571849

[284] Baltz A J and McLerran L D 1998 Phys. Rev. C 581679

[285] Segev B and Wells J C 1999 Phys. Rev. C 592753

[286] Vane C R, Datz S, Deveney D F, Dittner P F, Krause H F, Schuch R, Gao H, and Hutton R 1997 Phys. Rev A 563682

[287] Bethe H A and Maximon L C 1954 Phys. Rev. 93768

[288] Davies H, Bethe H A and Maximon L C 1954 Phys. Rev. 93788

[289] Ivanov D Y, Schiller A, and Serbo V G 1999 Phys. Lett. B 454155

[290] Lee R N and Milstein A I 2000 Phys. Rev. A 61032103

[291] Lee R N and Milstein A I 2001 Phys. Rev. A 64032106

[292] Baltz A J 2005 Phys. Rev. C 71024901 [Erratum-ibid. C 71 039901]

[293] Hencken K, Baur G and Trautmann D 2004 Phys. Rev. C 69054902

[294] Hencken K, Kuraev E A and Serbo V G 2006 Acta Phys. Polon. B 37969

[295] Hencken K, Kuraev E A and Serbo V G 2006 Phys. Rev. C 75034903

[296] Baur G, Hencken K, and D. Trautmann 1998 J. Phys. G 241657

[297] Hencken K et al. 1996 Preprint IHEP-96-38

[298] Nystrand J and Klein S [STAR Collaboration] 1998 Preprint nucl-ex/9811007

[299] Vidovic M, Greiner M and Soff G 1995 J. Phys. G 21545

[300] Baur U, Zeppenfeld D 1989 Nucl. Phys. B 325253

[301] Diener K P, Schwanenberger C and Spira M 2002 Eur. Phys. J. C 25405

[302] Baltz A et al. 2007 Preprint hep-ph/0702212

[303] Hencken K, Kharlov Yu and Sadovsky S 2002 Preprint ALICE-INT-2002-27

[304] Dellacasa G et al. 2000 Preprint CERN/LHCC 2000-001

[305] Dellacasa G et al. 1999 Preprint CERN/LHCC 99-4

[306] Grigoriev V et al 2001 Preprint ALICE-INT-2001-38

[307] Schuler G and Sjöstrand T 1977 Z. Phys. C 73677

[308] Acciarri M et al. [L3 collaboration] 2001 Phys. Lett. B 51933

[309] Morsch A 2001 Preprint ALICE-INT-2001-10

[310] Barklow T 1990 Preprint SLAC-PUB-5364

[311] Shamov A G and Telnov V I, talk by Maslennikov A 1998 Proceedings of the Lund Workshop on Photon Interactions and Photon Structure, Lund, Sweden

[312] Budnev V M et al. 1972 Phys. Lett. B 39526

[313] Abdallah J et al. [DELPHI Collaboration] 2003 Eur. Phys. J. C 31481

[314] Nakazawa H et al. [BELLE Collaboration] 2005 Phys. Lett. B 61539

[315] Dobbs S et al. [CLEO Collaboration] 2006 Phys. Rev. D 73071101

[316] Kwong W et al. 1988 Phys. Rev. D 373210

[317] Bodwin G T et al. 1995 Nucl. Phys. 42306

[318] Alessandro B et al. [ALICE Collaboration] 2006 J. Phys. G 321295

[319] Adler SS et al. [PHENIX Collaboration] 2003 Phys. Rev. Lett. 91241803

[320] Adler C, Strobele H, Denisov A, Garcia E, Murray M and White S 2001 Nucl. Inst. Meth. A 461 337

[321] ALICE Collaboration 1999 Preprint ALICE TDR3, CERN/LHCC 99-5

[322] Oppedisano C 2002 Preprint ALICE-INT-2002-08.

[323] Korotkikh V L and Chikin K A 2002 Eur. Phys. J. A 14199

[324] Kharlov Yu V and Korotkikh V L 2004 Eur. Phys. J. A 21437

[325] Anchordoqui L A, Beacom J F, Goldberg H, Palomares-Ruiz S and Weiler T J 2007 Phys. Rev. Lett. 98121101

[326] Heckman H H and Lindstrom P J 1976 Phys. Rev. Lett. 3756 
[327] Olson D L, Berman B L, Greiner D E, Heckman H H, Lindstrom P J, Westfall G D and Crawford H J 1981 Phys. Rev. C 241529

[328] Scheidenberger C et al. 2002 Phys. Rev. Lett. 88042301

[329] Hill J C, Petridis A, Fadem B and Wohn F K 1999 Nucl. Phys. A 661313

[330] Aumann T, Bertulani C A and Summerer K 1995 Phys. Rev. C 51416

[331] Benesh C J, Cook B C and Vary J P 1989 Phys. Rev. C 401198

[332] Grunschloss A et al. 1999 Phys. Rev. C 60051601

[333] Hüfner J, Schäfer K and Schürmann B 1975 Phys. Rev. C 121888

[334] Emling H 1994 Prog. Part. Nucl. Phys. 33729

[335] Aumann T, Bortignon P F, Emling H 1998 Ann. Rev. Nucl. Part. Sci. 48351

[336] de Passos E J, Hussein M S, Canto L F and Carlson B V 2002 Phys. Rev. C 65034326

[337] Dekhissi H et al. 2000 Nucl. Phys. A 662207

[338] Young P G, Arthur E D, Chadwick M B 1998 Nuclear Reaction Data and Nuclear Reactors, ed A Gandini and G Reffo, vol i (World Scientific, Singapore) p 227

[339] Assmann R W, Braun H H, Ferrari A, Jeanneret J B, Jowett J M and Pshenichnov I A 2004 Preprint LHC-Project-Report-766

[340] Jowett J M, Braun H H, Gresham M I, Mahner E, Nicholson A N, Shaposhnikova E and Pshenichnov I A 2004 Preprint LHC-Project-Report-772

[341] For an extensive theoretical review of hard photoproduction, see e.g. Klasen M 2002 Rev. Mod. Phys. 741221

[342] Kessler P 1975 Acta Phys. Austr. 41141

[343] Albino S, Klasen M and Söldner-Rembold S 2002 Phys. Rev. Lett. 89122004

[344] Cacciari M and Salam G 2006 Phys. Lett. B 64157

[345] Chekanov S et al. [ZEUS Collaboration] 2003 Phys. Lett. B 5607

[346] Adloff C et al. [H1 Collaboration] 2002 Eur. Phys. J C 2513

[347] Chekanov S et al. [ZEUS Collaboration] 2004 Proceedings of the $32^{\text {nd }}$ International Conference on High-Energy Physics, vol. 2, http://www.ihep.ac.cn/data/ichep04/c_paper/6-0249.pdf

[348] Klasen M and Kramer G 2004 Eur. Phys. J. C 3893

[349] Klasen M and Kramer G 2006 Preprint hep-ph/0608235

[350] Klasen M and Kramer G 2004 Phys. Rev. Lett. 93232002

[351] Kaidalov A, Khoze V, Martin A and Ryskin M 2003 Phys. Lett. B 56761

[352] Klasen M and Kramer G 2005 J. Phys. G 311391

[353] Chekanov S et al. [ZEUS Collaboration] 2004 Proceedings of the $32^{\text {nd }}$ International Conference on High-Energy Physics, vol. 2, http://www.ihep.ac.cn/data/ichep04/c_paper/11-0333.pdf

[354] Cacciari M, Frixione S and Nason P (2001) JHEP 01036

[355] Kniehl B, Kramer G, Schienbein I and Spiesberger H 2005 Phys. Rev. D 71014018

[356] Adloff C et al. [H1 Collaboration] 2002 Eur. Phys. J C 2525

[357] Klasen M, Kniehl B, Mihaila L and Steinhauser M 2002 Phys. Rev. Lett. 89032001

[358] Frixione S 1998 Phys. Lett. B 429369

[359] Aktas A et al. [H1 Collaboration] 2005 Eur. Phys. J C 38437

[360] Harrison M, Ludlam T and Ozaki S 2003 Nucl. Instrum. Meth. A 499235

[361] http://www.agsrhichome.bnl.gov/AP/RHIC2004

[362] Ackermann K H et al. 1999 Nucl. Phys. A 61 681c

[363] Wieman H et al. 1997 IEEE Trans. Nucl. Sci. 44671

[364] Ackermann K H et al. 2003 Nucl. Instrum. Meth. A 499624

[365] Adler C et al. 2001 Nucl. Instrum. Meth. A 470488

[366] Surrow B [STAR Collaboration], Preprint hep-ex/0205090.

[367] Bieser F S et al. 2003 Nucl. Instrum. Meth. A 499766

[368] Morozov V 2003 Ph.D. Thesis UC Berkeley

[369] Baur G and Ferreira Filho L G 1991 Phys. Lett. B 25430

[370] Budnev V M, Ginzburg I F, Meledin G V and Serbo V G 1974 Phys. Rept. 15181 
[371] Vidovic M, Greiner M, Best C and Soff G 1993 Phys. Rev. C 472308

[372] Meissner F and Morozov V B [STAR Collaboration] 2004 Nucl. Phys. B Proc. Suppl. 12659

[373] Söding P 1966 Phys. Lett. 19702

[374] Groom D E et al. 2000 Eur. Phys. J C 151

[375] Ross M and Stodolsky L 1966 Phys. Rev. 1491172

[376] Klein S 2005 AIP Conf. Proc. 792 532, Preprint nucl-ex/0506013

[377] Alvensleben H et al. 1970 Phys. Rev. Lett. 24792

[378] Timoshenko S 2005 Preprint nucl-ex/0501010

[379] Armstrong T A et al. 1998 Nucl. Instr. Meth. A 406227

[380] Klein S and Vogt R 2003 Phys. Rev. C 68017902

[381] Glauber R J 1955 Phys. Rev. 991515

[382] Adler S S et al.[PHENIX Collaboration] 2006 Phys. Rev. Lett. 96012304

[383] Adler S S et al [PHENIX Collaboration] 2003 Phys. Rev. Lett. 91072303

[384] Sikler F [CMS Collaboration] 2007 Preprint physics/0702193

[385] CMS Collaboration 2007 "CMS Physics TDR: High Density QCD with Heavy-Ions", Preprint CERN-LHCC-2007-009, CMS TDR 8.2-Add1, to appear in J. Phys. G

[386] Carminati F et al. [ALICE Collaboration] 2004 J. Phys. G 301517

[387] Alscher A, Hencken K, Trautmann D and Baur G 1997 Phys. Rev. A 55396

[388] Dellacasa G et al. 1999 Preprint CERN/LHCC 99-12

[389] Dellacasa G et al. [ALICE Collaboration] 2000 Prerpint CERN-OPEN-2000-183

[390] Hencken K, Kharlov Y, Sadovsky S. 2002 Preprint ALICE-INT-2002-11

[391] Pi H 1992 Comput. Phys. Commun. 71173

[392] Engel R, Ranft J and Roesler S 1997 Phys. Rev. D 556957

[393] ATLAS Collaboration 1999 Preprint CERN/LHCC 99-14.

[394] CMS Collaboration 1997 Preprint CERN/LHCC 97-31

[395] CMS Collaboration 1997 Preprint CERN/LHCC 97-32

[396] CMS Collaboration 1997 Preprint CERN/LHCC 97-33

[397] CMS Collaboration 1998 Preprint CERN/LHCC 98-6 